\title{
Multi-Scale Investigation of the Formation and Breakdown of Passive Films on Carbon Steel Rebar in Concrete
}

\author{
A thesis submitted to \\ the Faculty of Graduate Studies and Research \\ in Partial Fulfillment of the requirements for the degree \\ Doctor of Philosophy
}

by

\section{Pouria Ghods}

\author{
Department of Civil and Environmental Engineering \\ Carleton University
}

Ottawa-Carleton Institute of Civil and Environmental Engineering

February 2010

C2010 Pouria Ghods 
Library and Archives

Canada

Published Heritage

Branch

395 Wellington Street

Ottawa ON K1A ON4

Canada
Bibliothèque et

Archives Canada

Direction du

Patrimoine de l'édition

395, rue Wellington

Ottawa ON K1A ON4

Canada
Your file Votre référence

ISBN: 978-0-494-67885-5

Our file Notre reférence

ISBN: 978-0-494-67885-5
NOTICE:

The author has granted a nonexclusive license allowing Library and Archives Canada to reproduce, publish, archive, preserve, conserve, communicate to the public by telecommunication or on the Internet, loan, distribute and sell theses worldwide, for commercial or noncommercial purposes, in microform, paper, electronic and/or any other formats.

The author retains copyright ownership and moral rights in this thesis. Neither the thesis nor substantial extracts from it may be printed or otherwise reproduced without the author's permission.
AVIS:

L'auteur a accordé une licence non exclusive permettant à la Bibliothèque et Archives Canada de reproduire, publier, archiver, sauvegarder, conserver, transmettre au public par télécommunication ou par l'Internet, prêter, distribuer et vendre des thèses partout dans le monde, à des fins commerciales ou autres, sur support microforme, papier, électronique et/ou autres formats.

L'auteur conserve la propriété du droit d'auteur et des droits moraux qui protège cette thèse. Ni la thèse ni des extraits substantiels de celle-ci ne doivent être imprimés ou autrement reproduits sans son autorisation.
In compliance with the Canadian Privacy Act some supporting forms may have been removed from this thesis.

While these forms may be included in the document page count, their removal does not represent any loss of content from the thesis.
Conformément à la loi canadienne sur la protection de la vie privée, quelques formulaires secondaires ont été enlevés de cette thèse.

Bien que ces formulaires aient inclus dans la pagination, il n'y aura aucun contenu manquant.

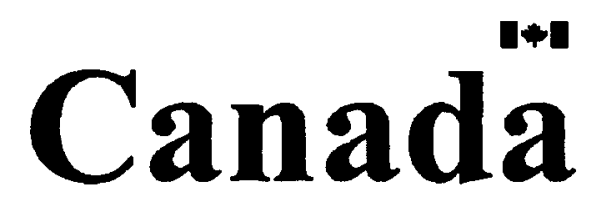




\section{Abstract}

The multi-scale investigation presented in this thesis was carried out to understand better the mechanisms of passivation and chloride-induced depassivation of carbon steel reinforcement in concrete. The study consisted of electrochemical experiments (electrochemical impedance spectroscopy, linear polarization resistance, free corrosion potential, anodic polarization), microscopic examinations (scanning electron microscopy, transmission electron microscopy, selected area diffraction, convergent beam electron diffraction), numerical modeling (finite element method), and spectroscopic studies (xray photoelectron, energy dispersed $\mathrm{x}$-ray, electron energy loss).

Electrochemical and microscopic studies showed that the composition of the pore solution and the surface conditions of the rebar affect the passivity and depassivation of carbon steel in concrete. It was demonstrated that crevices between mill scale and steel may become potential sites for depassivation and pit nucleation. The numerical investigation that was carried out to test this hypothesis confirmed that the ratio of chloride to hydroxide concentrations, $\mathrm{Cl}^{-} / \mathrm{OH}^{-}$, in crevices increased to levels higher than that of the bulk pore solution, making crevices more vulnerable to depassivation. Therefore, it was concluded that the variability associated with reported chloride thresholds might be attributed, at least in part, to the variability in mill scale properties resulting from the variability in manufacturing.

The nano-scale microscopic and spectroscopic studies indicated the formation of 4$10 \mathrm{~nm}$-thick passive oxide films on carbon steel in simulated concrete pore solutions, and these films consisted of two layers separated with an indistinct border. The inner layer was mainly composed of protective $\mathrm{Fe}^{2+}$-rich oxides that are in epitaxial relationship with the underlying steel surface; while the outer layer mostly consisted of (possibly porous) $\mathrm{Fe}^{3+}$-rich oxides, through which chlorides can penetrate. It was proposed that, in the presence of chlorides, $\mathrm{Fe}^{+2}$-rich oxides in the inner layer transform into $\mathrm{Fe}^{+3}$-rich oxides and potentially become un-protective. Although how this transformation occurs is still subject of future research, there are evidences showing that the process most likely leads to the formation of local anodic and cathodic sites on the steel surface. 


\section{Acknowledgements}

First, I would like to sincerely thank my supervisor, Professor O. Burkan Isgor, for his unrestricted support. Without him, this research would not have been possible. He is not only a very friendly advisor, but also a very intelligent and enthusiastic person in solving problems. He inspired me in every step of my work from the very early stages to the end and enhanced my knowledge as a student and also as a researcher. I owe an unforgettable debt of gratitude to him forever.

I would like to thank Professor Glenn McRae for his technical support and collaboration in this research; without his constructive comments, this research would not be in the shape it is today.

I am also thankful to Dr. Jim Beaudoin for his contributions as a member of my advisory committee and his support throughout this research.

I wish to express my deep appreciation to research scientists from CANMET/MTL Dr. Gordon Ping Gu, Dr. Jian Li, Dr. Graham Carpenter, Dr. Jim Brown, Dr. Winston Revie and Dr. Tom Malis, who provided me with their expertise in very specialized areas of work. Without their help, I would not be able to carry out the highly specialized experimental procedures presented in this thesis. I am also thankful for many hours of technical help provided by Ms. Renata Zavadil, Ms. Pei Liu, Mr. Clinton DeRushie, and Mr. Alex Doiron during my experimental studies at CANMET/MTL. Special thanks must be extended to Ms. Catherine Bibby for her skilled assistance in the TEM investigation.

I am grateful to Dr. Farid Bensebaa and Mr. David Kingston of National Research Council (NRC) of Canada for their collaboration in the XPS study and to Dr. Jieying Zhang for her help during the AFM investigation of the surface topography of the steel specimens.

I would like to express my gratitude to the administrative staff of the Department of Civil and Environmental Engineering at Carleton University and also to the manager of environmental engineering laboratory, Dr. Marie Jose Tudoret-Chow, for their assistance throughout the years. Here, I should also acknowledge the assistance of 
two co-op undergraduate students, Mr. Brian Foo and Mr. Mohamed Bouzoubaa, for their help during the summer months. I would also like to thank my fellow graduate students Mohammad Pour-Ghaz, Kostantinos Karadakis and Thomas Miller for the enjoyable moments that I had in working with them.

Words can not express how I am indebted to my family, especially to my father and mother, who have been unconditionally supporting me from the beginning of my life. They unwaveringly encouraged me to pursue my academic studies in the doctoral level. Without doubt, none of my success would be possible without their dedication.

Finally, I would like to convey my special appreciation and gratitude to my wife, Sahar Soleimani, for her unconditional love, patience, and dedication. She has been personally helping me at all times when I was faced with difficult situations. Her emotional support and encouragement during the preparation of this thesis was admirable. I cannot imagine my life without her, and I am very lucky to be her husband.

The financial supports provided in the forms of Ontario Graduate Scholarships (OGS), NACE Foundation Scholarship and Carleton University's generous financial assistance, including several scholarships, grants, fellowships, and teaching assistantships, are gratefully acknowledged. This project was financially supported by Natural Sciences and Engineering Research Council of Canada (NSERC) and CANMETMTL, through the Resource for the Innovation of Engineered Materials (RIEM) program; both contributions are greatly appreciated. 


\section{Table of Contents}

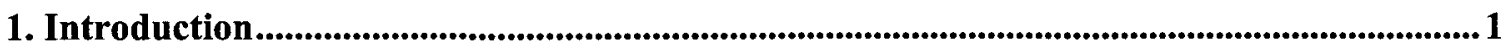

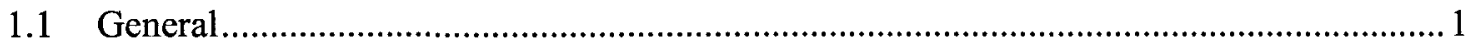

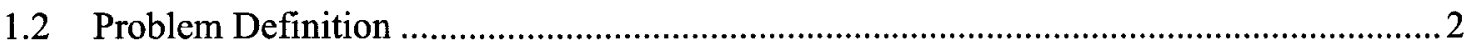

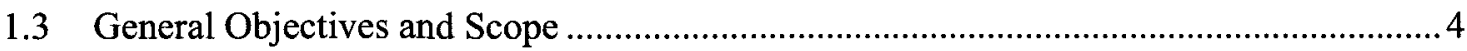

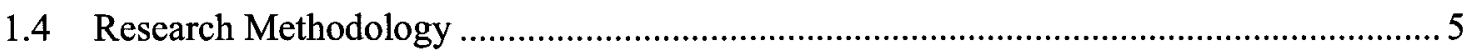

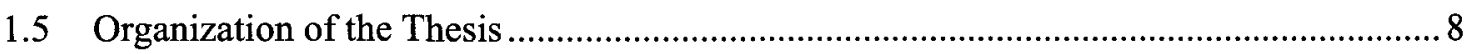

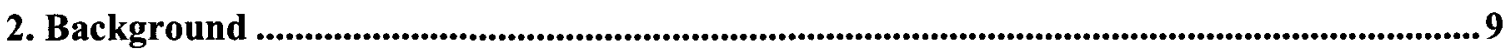

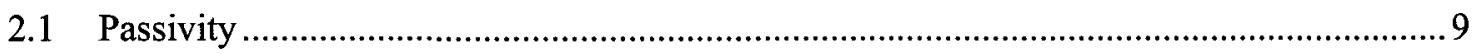

2.2 Nature and Properties of Passive films on Iron......................................................... 12

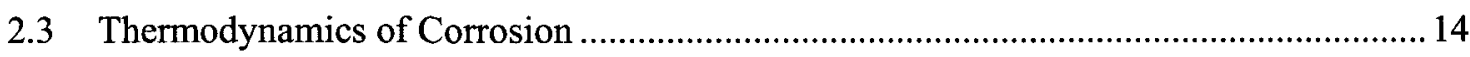

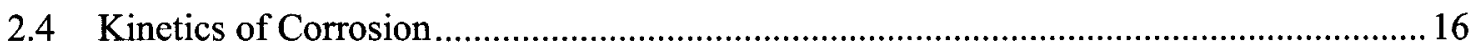

2.5 Causes of Corrosion Initiation of Steel in Concrete ................................................ 19

3. Literature Review ................................................................................................................................26

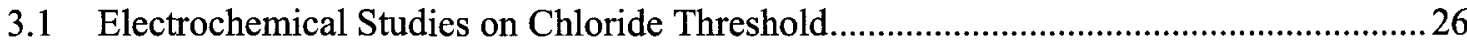

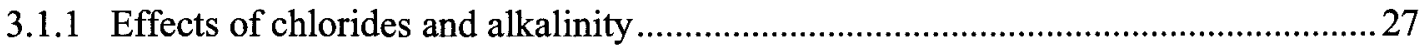

3.1.2 Effects of rebar surface condition and rebar/concrete interface ................................. 32

3.1.3 Oxygen availability, relative humidity and water content in concrete ........................35

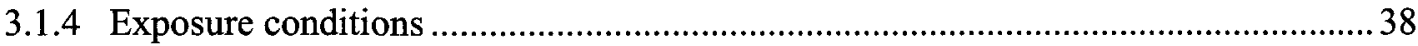

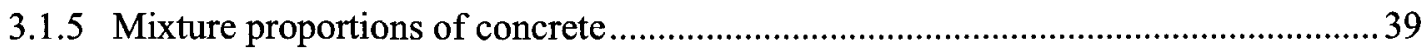

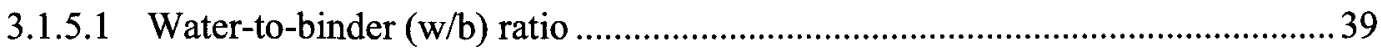

3.1.5.2 Supplementary cementitious materials (SCMs) ............................................. 40

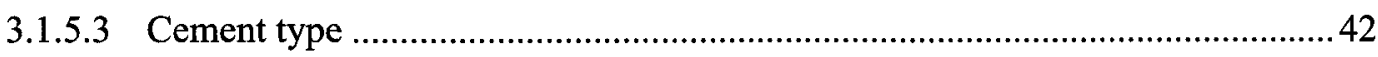

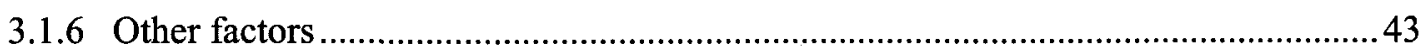

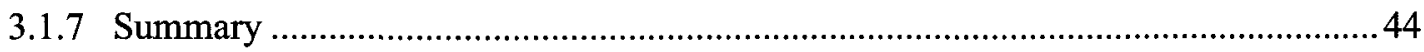

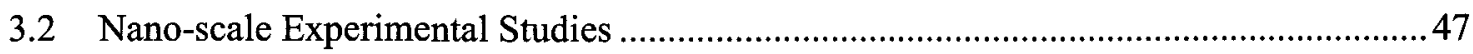

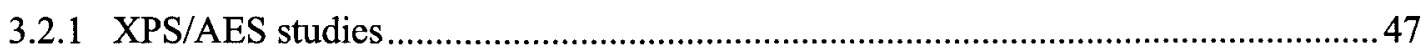

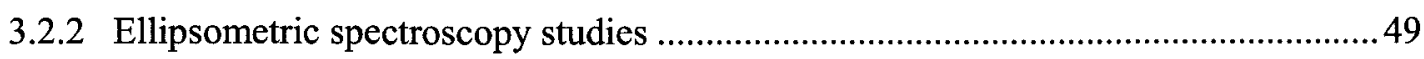

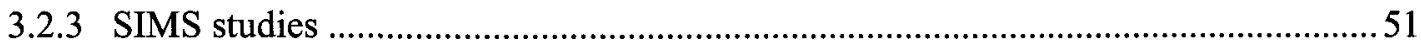

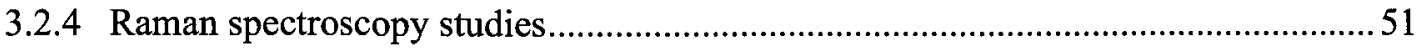

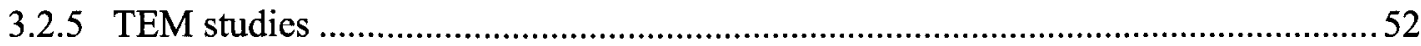




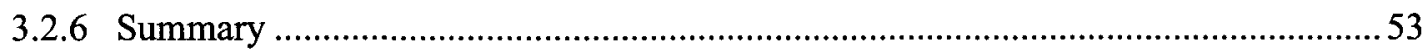

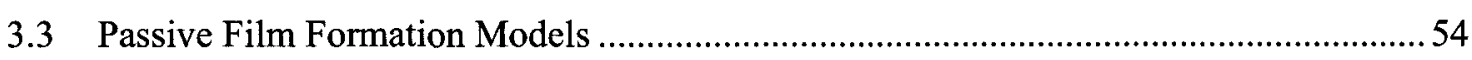

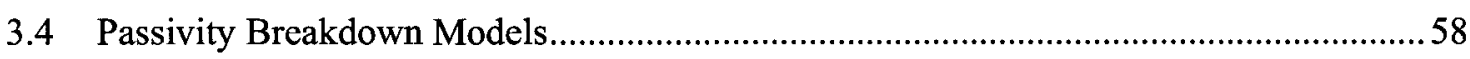

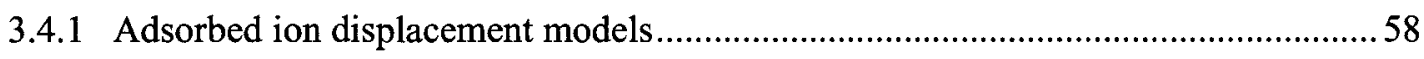

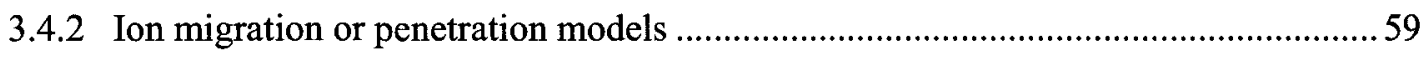

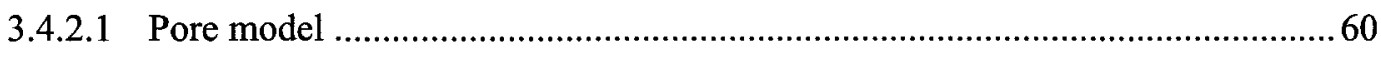

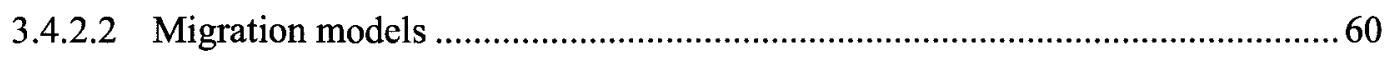

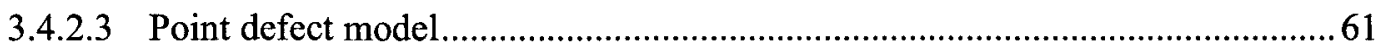

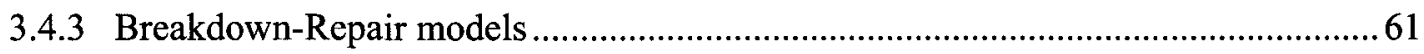

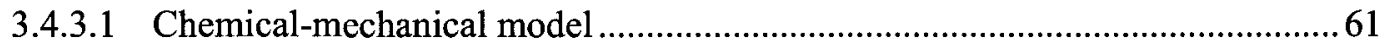

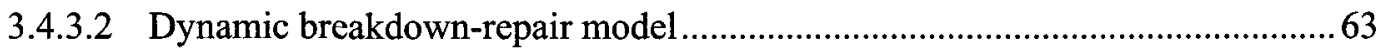

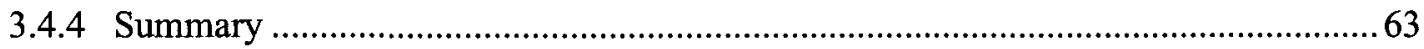

3.5 Key Questions Originating From the Literature Review ..........................................65

4. Electrochemical Study on Passivity of Rebar: Effect of Pore Solution.................................67

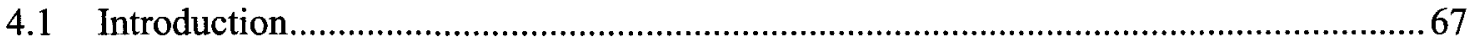

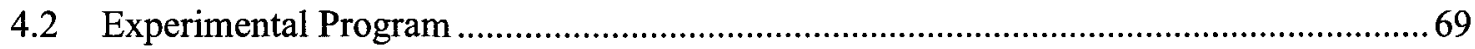

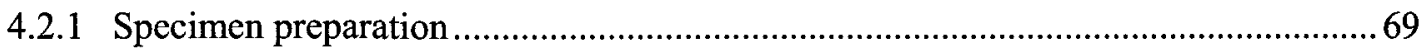

4.2.2 Preparation of the synthetic pore solutions ....................................................... 71

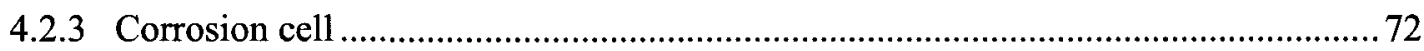

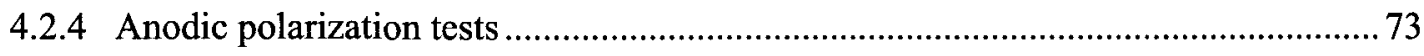

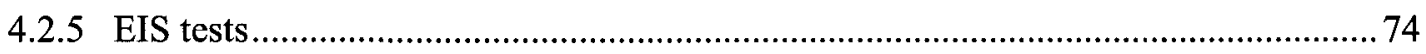

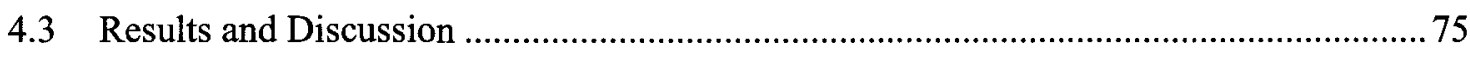

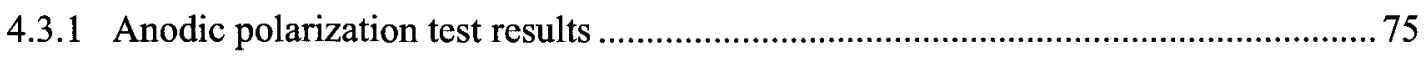

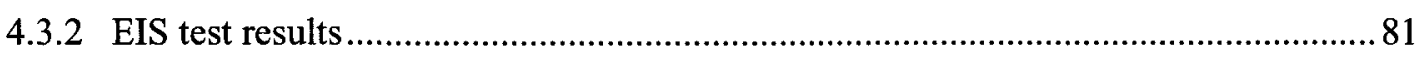

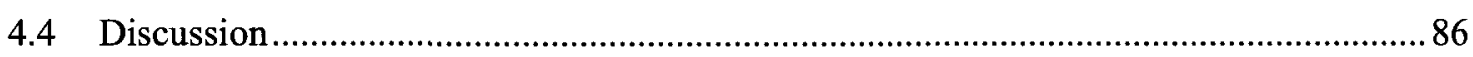

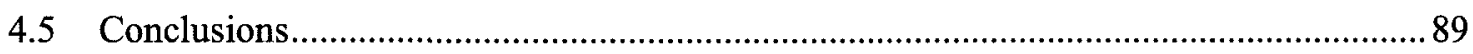

5. Electrochemical Study on Chloride-induced Depassivation of Rebar ...................................91

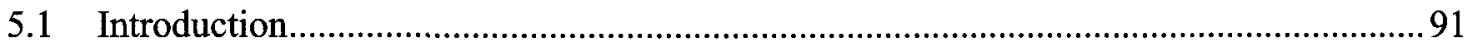

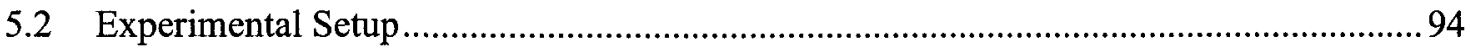

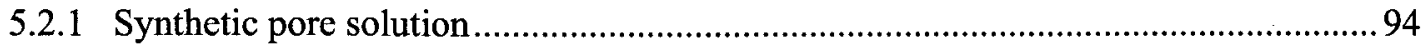

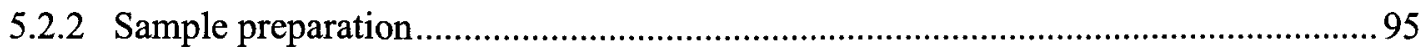

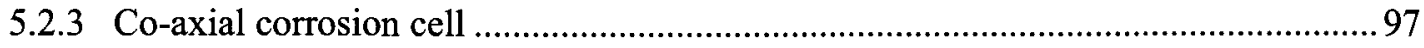

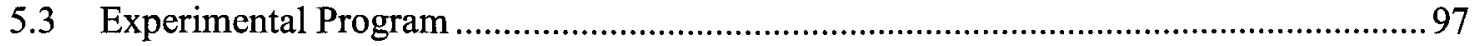

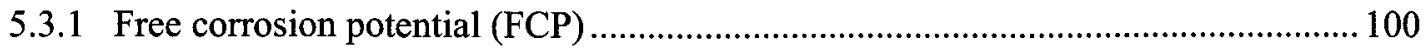




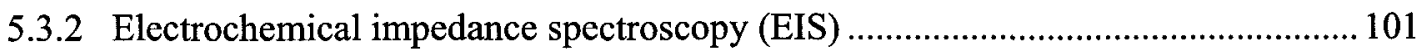

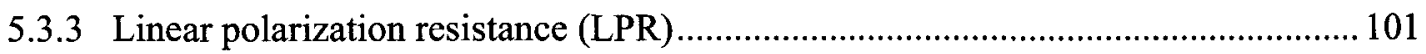

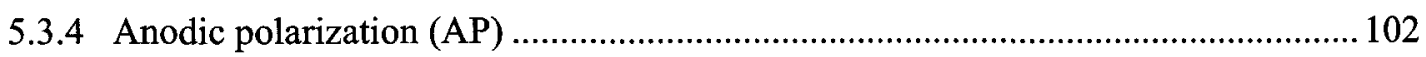

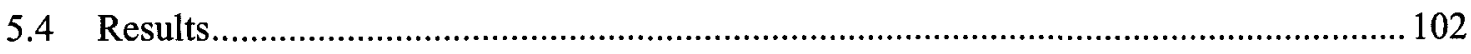

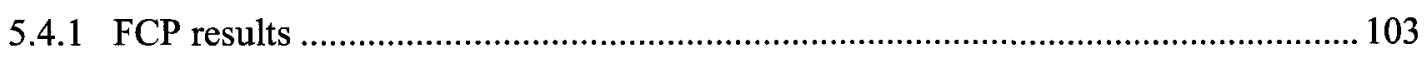

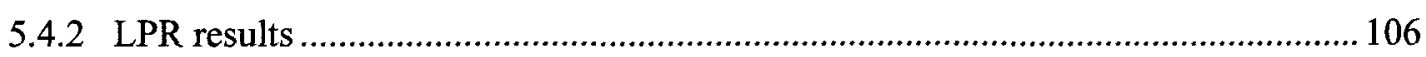

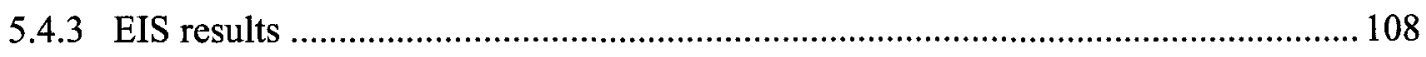

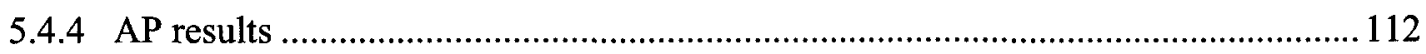

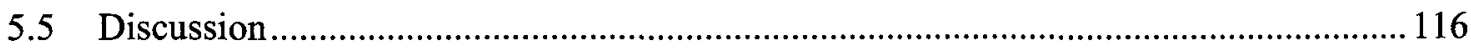

5.5.1 Chloride threshold determination technique ..................................................... 116

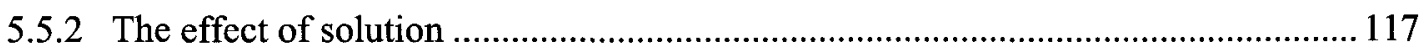

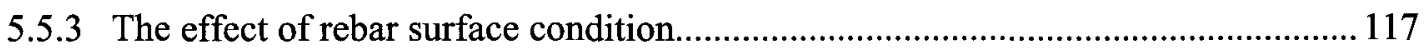

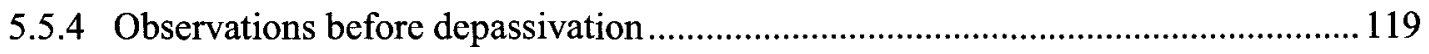

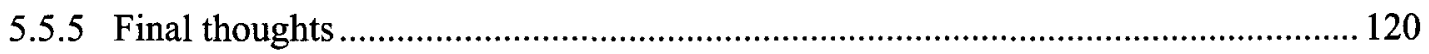

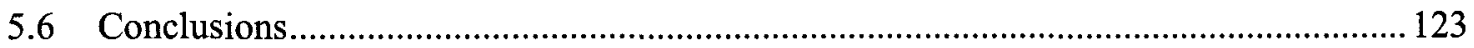

6. SEM and Numerical Studies on the Effect of Mill Scale on Chloride-induced

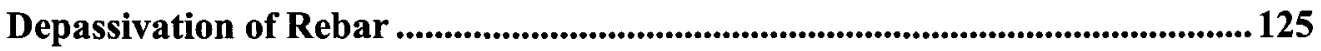

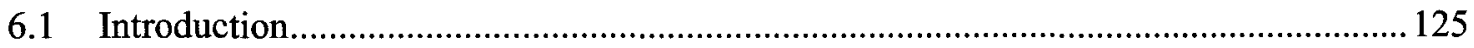

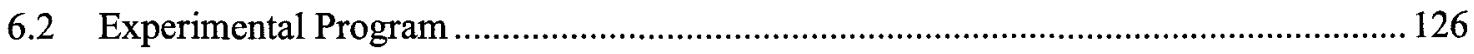

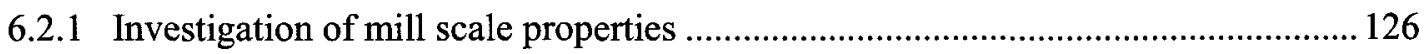

6.2.2 Investigation of the effect of mill scale on the chloride-induced depassivation....... 127

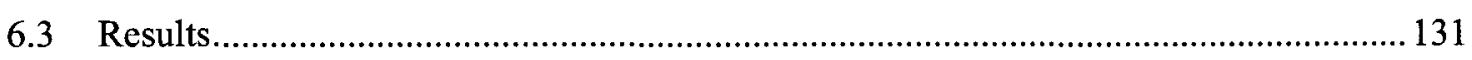

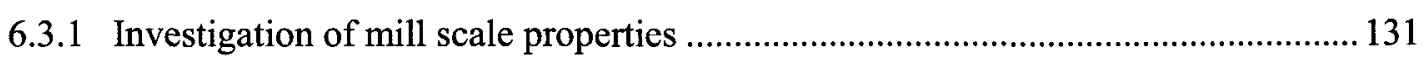

6.3.2 Investigation of the effect of mill scale on the chloride-induced depassivation....... 135

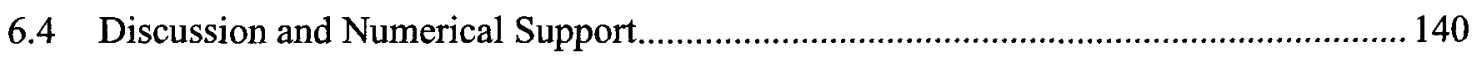

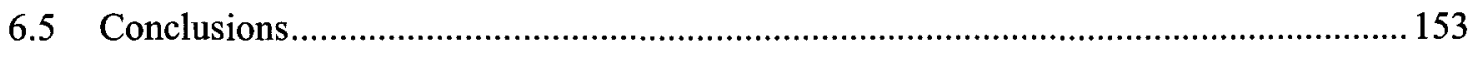

7. TEM Study on Passive Film and Chloride-induced Depassivation of Rebar .................... 155

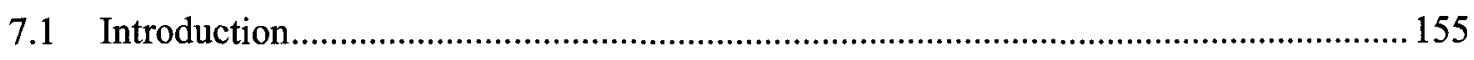

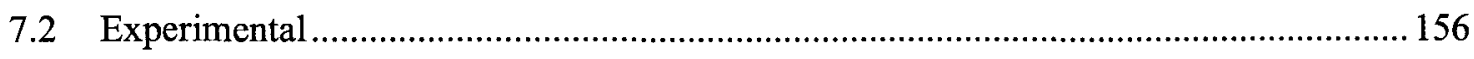

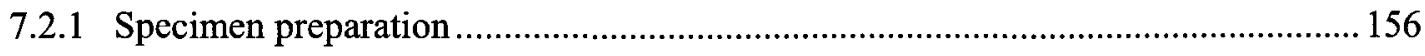

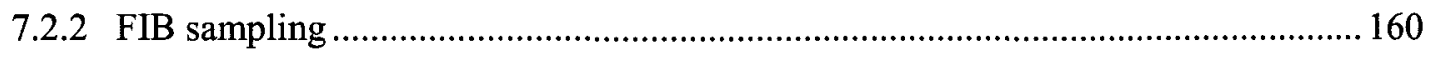

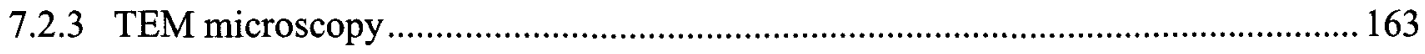

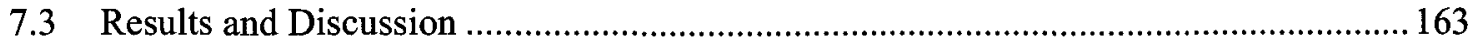

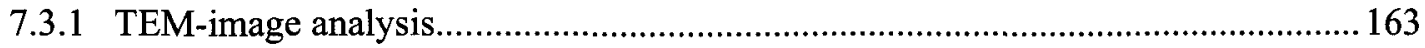


7.3.1.1 Samples passivated in chloride-free solutions: CH-0 and CP-0.

7.3.1.2 Samples exposed to chlorides in amounts below depassivation thresholds: $\mathrm{CH}-1$ and $\mathrm{CP}-1$

7.3.1.3 Samples exposed to chlorides in amounts above depassivation thresholds: $\mathrm{CH}-2$ and $\mathrm{CP}-2$

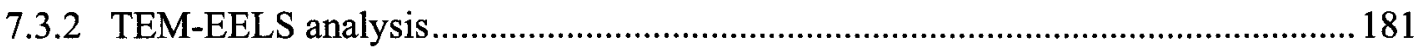

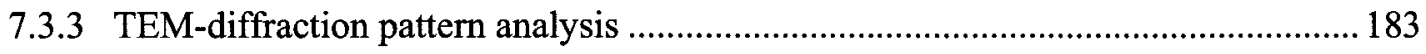

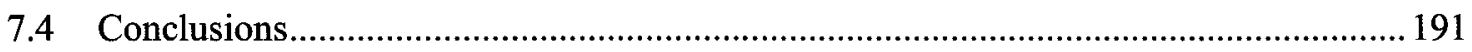

8. XPS Depth Profiling Study of Passive Film on Rebar and the Role of Chloride in

Depassivation ............................................................................................................................... 193

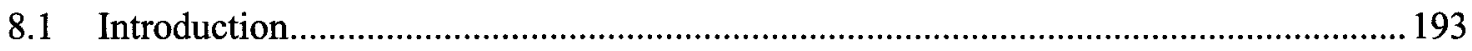

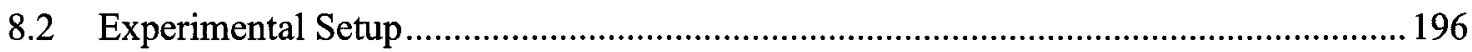

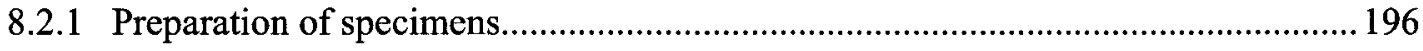

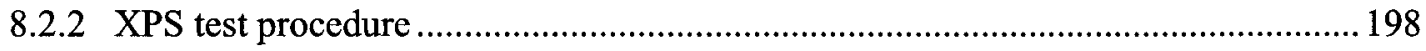

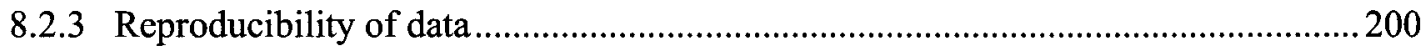

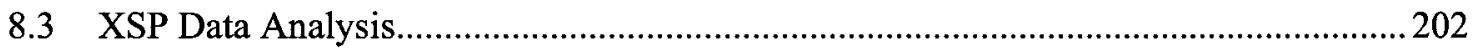

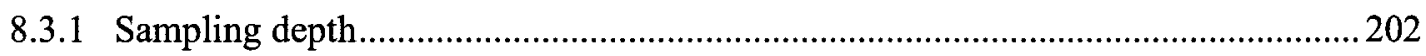

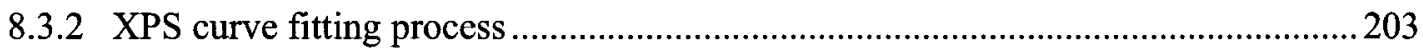

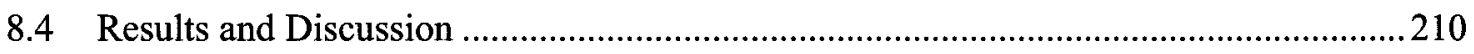

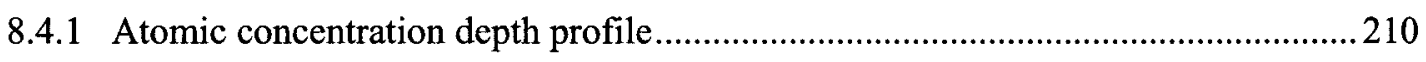

8.4.2 Fe 2p XPS spectra variation with the depth of oxide film.....................................215

8.4.3 The effect of exposure conditions on the thickness of the oxide film ......................218

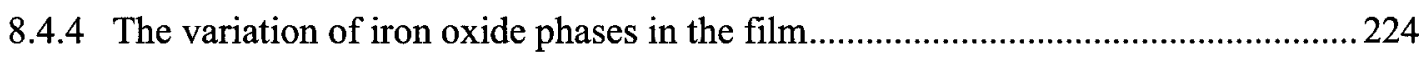

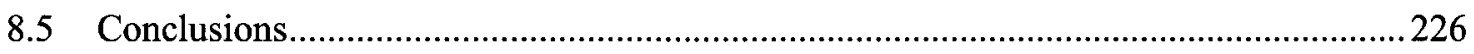

9. Angle-resolved XPS Study of Passive Film on Rebar and the Role of Chloride in

Depassivation ....................................................................................................................... 228

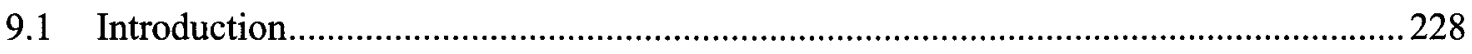

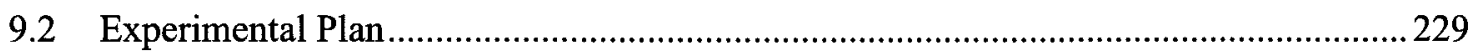

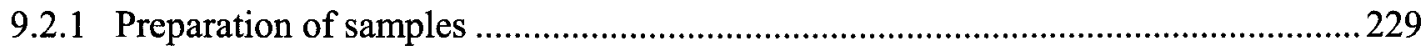

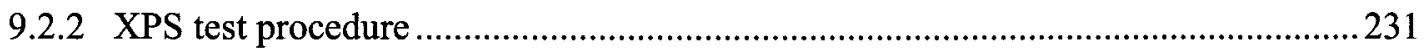

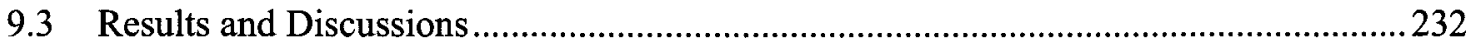

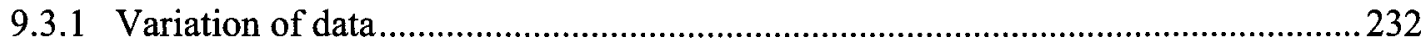

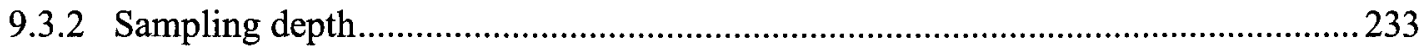

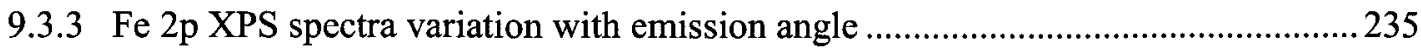




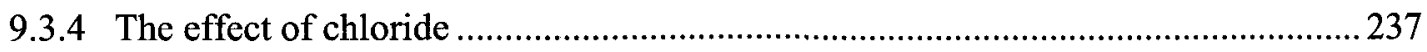

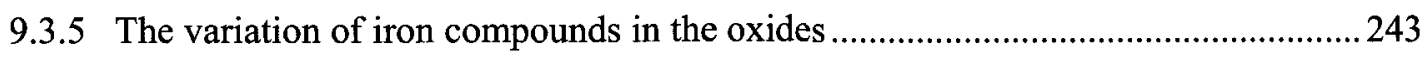

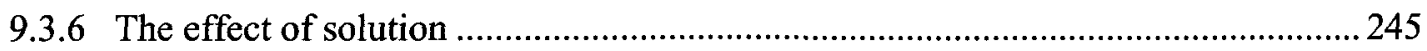

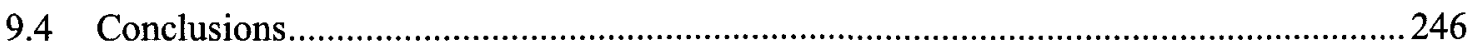

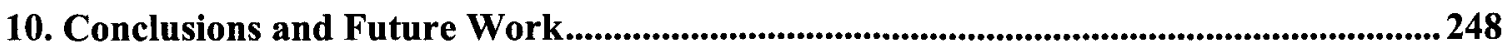

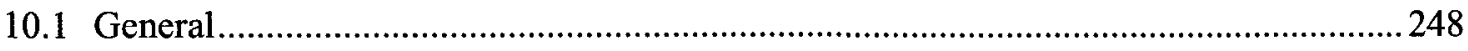

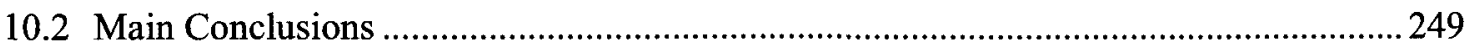

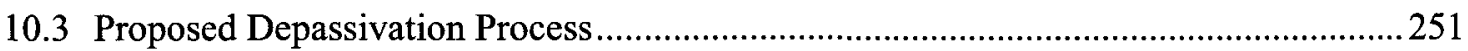

10.4 Future Work Originating from Present Research ..................................................... 252

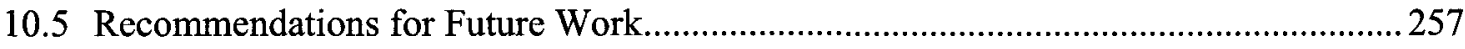

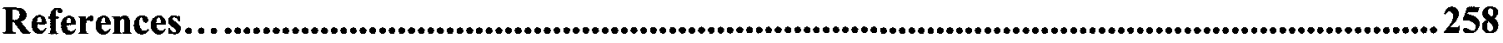

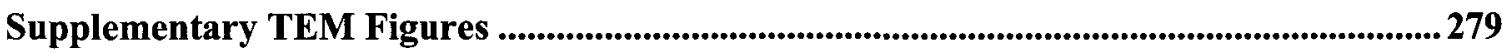

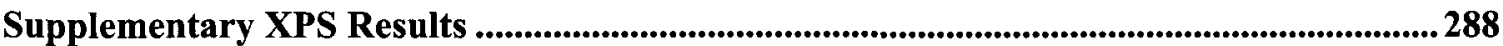




\section{List of Tables}

Table 3.1: Summary of reported chloride thresholds (reproduced from Angst et al. 2009). Abbreviations are given in Table 3.2.

Table 3.2: Abbreviations in Table 3.1 and Figure 3.2 (reproduced from Angst et al. 2009).

Table 3.3: Effect of exposure temperature on chloride thresholds by Rasheeduzzafar et al. (1992).

Table 3.4: Chloride thresholds used in various concrete building design codes (Oh et al. 2004). 45

Table 3.5: The effect of various parameters on chloride threshold (taken from Angst et al.2009)46

Table 3.6: Thickness of passive films on iron in $0.05 \mathrm{M} \mathrm{Na}(\mathrm{OH})$ at 600 s (Zakroczymski et al.

1985a).

Table 3.7: Characteristics of passive film used in the comparison of the models.

Table 3.8: The literature review summary: The model's capability of explaining the characteristics listed in Table 3.7.

Table 4.1: Steel elemental composition (Average of three spectrographic results - Instrument model: OneSpark).

Table 4.2: Concentrations of the chemicals added and the ions measured in each solution using ICP-AES: the solutions were saturated with calcium hydroxide.

Table 4.3: Measured values of dissolved oxygen, $\mathrm{pH}$ and conductivity of the synthetic pore solutions. 72

Table 4.4: Rankings of oxide film quality. 87

Table 5.1: Concentrations of the species in the two test solutions ( $\mathrm{CP}$ and $\mathrm{CH})$ 96

Table 5.2: Steel elemental composition. 96

Table 5.3: The concentration of total chloride at each 7-day interval of the test for two solutions. These intervals correspond to the incremental shading used in Figs. 5.3-5.5. 100

Table 5.4: Chloride thresholds obtained by different electrochemical techniques. 116

Table 6.1: Elemental composition of the rebar used in the SEM investigation studying the effect of mill scale on the chloride-induced depassivation. 128

Table 6.2: Concentrations of species in the simulated concrete pore solution (CP). The actual concentrations of the anions and cations in solution were measured using Inductively Coupled Plasma - Atomic Emission Spectroscopy (ICP-AES) (Model: Varian Vista RL).

Table 6.3: Transport properties of all species in the concrete pore solutions (Karadakis 2009) . 151 
Table 7.1: Elemental composition of the rebar used in the TEM investigation (Average of three spectrographic results)

Table 7.2: Concentrations of species and properties of the simulated concrete pore solutions $\mathrm{CH}$ and CP. The actual concentrations of the anions and cations in solution were measured using Inductively Coupled Plasma - Atomic Emission Spectroscopy (ICP-

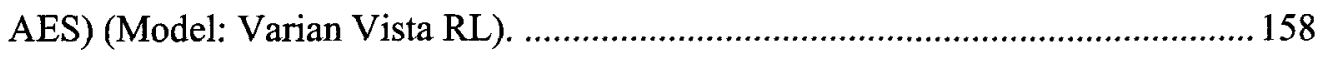

Table 7.3: Rebar specimen designations and corresponding chloride exposure......................... 160

Table 7.4: The camera constant calculation for the CP-0 diffraction patterns in Fig. 7.21........ 186

Table 7.5: The comparison of the CH-0 oxide d-spacings from Fig. 7.22 with FeOOH d-spacings. Four interplanar spacings (001), (011), (020), (210) and (220) matched those of FeOOH. For all other phases, the calculated error was larger than $5 \%$; therefore they are not reported here $(\lambda \mathrm{L}=24.6 \AA . \mathrm{mm})$.

Table 7.6: The comparison of the CP-0 oxide d-spacings from Fig. 7.23 with FeOOH d-spacings. Four interplanar spacings (110), (001), (101), (001) matched those of FeOOH. For all other phases, the calculated error was larger than 5\%; therefore they are not reported here $(\lambda \mathrm{L}=24.6 \AA ̊ . \mathrm{mm})$.

Table 8.1: Steel elemental composition (Average of three spectrographic results).................... 198

Table 8.2: The calculation of sampling depth (d) for iron oxide $\left(\theta=0^{\circ}\right.$ and $\left.h_{\mathrm{v}}=1487 \mathrm{eV}\right) \ldots \ldots \ldots .203$

Table 8.3: The peak parameters of ionic species for different elements used in this study.........206

Table 9.1: Concentrations of species in the simulated concrete pore solution (CP)...................231

Table 9.2: Steel elemental composition (Average of three spectrographic results).....................231

Table 9.3: The calculated sampling depth at various emission angles. ….................................. 235

Table 9.4: The peak parameters of Fe 2p XPS spectra used in this study................................236 


\section{List of Figures}

Figure 1.1: Techniques used in multi-scale investigation carried out in this research.................... 7

Figure 2.1: Corrosion behaviour of a metal that does not show passive behaviour...................... 11

Figure 2.2: Corrosion behaviour of a metal showing active-passive transition........................... 12

Figure 2.3: Simplified potential-pH equilibrium diagram (Pourbaix diagram) for iron-water (Revie 2000).

Figure 2.4: The intersection of three possible cathodic polarization curves (straight lines A, B, and C) with an anodic polarization curve for a system capable of exhibiting passivity (Revie 2000).

Figure 2.5: The intersection of anodic with cathodic polarization curves at $E_{\text {corrA }}$ at which breakdown occurs and at $\mathrm{E}_{\text {corr }}$ that results in no breakdown (Revie 2000).

Figure 2.6: Potentiodynamic anodic polarization data of mild steel in cement paste of water to cement ratio of 0.4 (taken from Page 2002). (Increasing percentages ( 0 to $8 \%$ of cement weight) show the increasing levels of chloride concentration).

Figure 2.7: Schematic representation of the anodic polarization curve of steel in concrete with different chloride contents (Ramachandran and Beaudoin 2001). Curves A1 to A4 are anodic polarization curves with increasing chloride concentrations. 22

Figure 2.8: The effect of carbonation on thermodynamic behaviour of passive film of steel in concrete (reproduced from Poursaee 2007). 23

Figure 2.9: The effect of carbonation on kinetic behavior of steel passive film in concrete (reproduced from Ramachandran and Beaudoin 2001). .25

Figure 2.10: The effect of $\mathrm{pH}$ drop on the cathodic polarization reaction after the breakdown of passive layer. 25

Figure 3.1: Reported chloride threshold levels plotted as a function of hydroxide concentration of the pore solution in the cover zone (taken from Hansson and Sorensen 1990). SF: silica fume, OPC: ordinary portland cement, SRPC: sulfate resistant portland cement , PFA: pulverised fly ash, RHPC: rapid hardening portland cement.

Figure 3.2: Summary of reported chloride thresholds (taken from Angst et al. 2009). Abbreviations are given in Table 3.2.

Figure 3.3: Chloride threshold for corrosion initiation as a function of volume fraction of interfacial voids (taken from Glass and Buenfeld 2000). (OPC: ordinary portland 
cement; SRPC: sulfate-resistant portland cement; PFA: pulverized fly ash; GGBS: ground granulated blast furnace slag).

Figure 3.4: Schematic cathodic polarization curves in alkaline concrete: (a) aerated and semi-dry (high oxygen availability); (b) wet (medium oxygen availability); (c) completely saturated with water (low oxygen availability) (taken from Bertolini et al. 2004).... 36

Figure 3.5: The effect of relative humidity on the chloride threshold level in laboratory exposed mortars, water to cement ratio $(\mathrm{w} / \mathrm{c})=0.50$ (taken from Pettersson 1996b). 37

Figure 3.6: Chloride thresholds measured in submerged concrete or mortar taken from Pettersson and Sandberg (1997). (OPC: ordinary portland cement; SRPC: sulfate resistant portland cement; MS: micro silica)

Figure 3.7: Chloride threshold levels and corrosion rates for OPC and fly ash concrete exposed in a marine splash zone in Canada (taken from Thomas 1996).

Figure 3.8: Chloride thresholds, represented as total chloride, versus $\mathrm{C}_{3} \mathrm{~A}$ content ( $\%$ of cement) (reproduced from Oh et al. 2004 and Hussain et al. 1995).

Figure 3.9: Schematic of physicochemical processes that occur for passive film formation according to the point defect model taken from Kamrunnahar et al. (2005)...............56

Figure 3.10: Place exchange model of passive formation (taken from Sato and Cohen 1964)....57

Figure 3.11: The bipolar model of passive film formation reproduced from Sakashita and Sato (1978). 57

Figure 3.12: Various stages of pit nucleation process according to the PDM (taken from Macdonald 1992). 62

Figure 4.1: An illustration of the rebar specimens. 70

Figure 4.2: (a) Schematic of the test setup used to carry out anodic polarization and EIS measurements; (b) the test setup. 73

Figure 4.3: Anodic polarization plots for three rebar specimens immersed in Solution 3 for different times. After 8 days the polarization plots did not change significantly with further immersion; similar results were seen for rebar immersed in the other solutions. .76

Figure 4.4: The anodic polarization curves of rebar specimens that have been immersed in different synthetic pore solutions for at least 14 days. 78

Figure 4.5: Graphical definition of passive current density and free corrosion potential used in this study.

Figure 4.6: The free-corrosion potential is correlated with the passive current density determined from anodic polarization tests. The line in the figure is a guide for the eye. .80 
Figure 4.7: Typical plots of (a) Impedance vs. Frequency; (b) Phase angle vs. Frequency; (c) Imaginary vs. Real Impedance (Nyquist).

Figure 4.8: Examples showing data at representative frequencies used in the calculations of the Pearson Correlation Coefficients shown in Figure 4.9. The r values are 0.91, 0.91, 0.91 and -0.96 for plots (a), (b), (c) and (d), respectively.

Figure 4.9: Pearson correlation coefficients, $r$, between free-corrosion potential and (a) impedance magnitude; (b) real impedance; (c) imaginary impedance; and (d) phase angle. Correlation coefficients with $|r|>0.45$ have p-values (i.e,. the probability of an observed result happening by chance under the null hypothesis) less than $0.05 \ldots \ldots . .85$

Figure 4.10: SEM images of rebar surface after immersion for 14 days in (a) Solution 10; (b)

Solution 9.

Figure 5.1: Schematic illustration of rebar specimen: (a) pull-out diagram; (b) cross section......98

Figure 5.2: Schematic illustration of the co-axial corrosion cell.

Figure 5.3: Average free corrosion potential of three as-received rebars in the CP solution. Shading in the figure corresponds to the increments of chloride addition in Table 5.3.

Figure 5.4: Average free corrosion potential of three as-received rebars in the $\mathrm{CH}$ solution. Shading in the figure corresponds to the increments of chloride addition in Table 5.3.

Figure 5.5: Average free corrosion potential of three turned-and-polished rebars in CP solution. Shading in the figure corresponds to the increments of chloride addition in Table 5.3.

Figure 5.6: Polarization resistance of rebar samples obtained by LPR for (a) as-received in CP solution; (b) as-received in $\mathrm{CH}$ solution; (c) turned-and-polished in $\mathrm{CP}$ solution. Note that the data for as-received rebar are more scattered about the trend lines when compared with the data from the turned-and-polished specimens.

Figure 5.7: Typical EIS results obtained for an as-received rebar sample in the $\mathrm{CH}$ solution with chloride concentrations (a) below and (b) above the chloride threshold

Figure 5.8: Equivalent electrical circuit (Randles circuit) used to model the EIS spectra. 110

Figure 5.9: Polarization resistance $\left(\mathrm{R}_{\mathrm{p}}\right)$ determined from EIS measurements and equivalent circuit modelling for as-received rebar samples in (a) $\mathrm{CP}$ solution; (b) $\mathrm{CH}$ solution. Note that the data for as-received rebar are more scattered about the trend lines when compared with the data from the turned-and-polished specimens as illustrated in Fig. 5.10 . 
Figure 5.10: Polarization resistance $\left(\mathrm{R}_{\mathrm{p}}\right)$ determined from EIS measurements and equivalent circuit modelling for turned-and-polished rebar samples in CP solution.

Figure 5.11: Anodic polarization curves of as-received rebar in $\mathrm{CH}$ and $\mathrm{CP}$ solutions: (a) without chloride; (b) with chloride.

Figure 5.12: Pitting potential of as-received rebar in the $\mathrm{CH}$ and $\mathrm{CP}$ solution as function of chloride.

Figure 5.13: Pitting potentials from the current study and those reproduced from $\mathrm{Li}$ and Sagues (2002) and Mammoliti et al. (1996). As-received rebar specimens pit at lower $\mathrm{Cl}^{-}$ $/ \mathrm{OH}^{-}$ratios than polished rebar specimens. 119

Figure 5.14: Cumulative probability of depassivation for as-received rebar as a function of chloride concentration. The curves in the figure were calculated from the cumulative log-normal probability distribution function, equation 5.6, and values of $\mathrm{pCl}_{\mathrm{T}}$ and $\sigma$, as described in Section 5.5.5. 122

Figure 6.1: A typical cold-mounted rebar specimen for SEM investigation. 128

Figure 6.2: Optical images of rebar samples with two different surface conditions used in this study: (a) as-received with mill scale; (b) turned-and-polished. Banding in Fig.6.2 (b) is an artifact of the light reflection form the mirror-polished surface of rebar... 130

Figure 6.3: Mill scale on rebar specimens from three different manufacturers (i, ii, iii). (a) optical images of rebar surface from different manufacturers; Figures (b) to (d) provide SEM micrographs with increasing magnification for each rebar type. Rebar (i): $\sim 30 \mu \mathrm{m}-$ thick uniform mill scale; Rebar (ii): $\sim 2 \mu \mathrm{m}$-thick non-uniform mill scale; Rebar (iii) $\sim 40 \mu \mathrm{m}$-thick non-uniform mill scale. (Note: M: Mill scale, S: Steel).

Figure 6.4: EDS analysis of mill scale of rebar (i): (a) dark phase; (b) light phase 133

Figure 6.5: Crystal grains and their boundaries for steel and mill scale under the FIB microscope (a) low magnification micrograph; (b) high magnification micrograph 134

Figure 6.6: SEM cross-section images of turned-and-polished and as-received rebar samples before exposure: (a) micrograph of turned-and-polished sample; (b) lower magnification micrograph of as-received sample; (c) higher magnification micrograph of as-received sample. 136

Figure 6.7: SEM surface images of as-received rebar samples before exposure. The smallest dark features are in the order of microns that correspond to the crack openings in mill scale.

Figure 6.8: SEM images of representative mill-scale cross sections taken from as-received rebar with mill scale after immersion in the simulated concrete pore solution plus $0.15 \mathrm{M}$ 
$\mathrm{NaCl}$ : (a) lower magnification image showing the location of corrosion initiation; (b) higher magnification image of area " $D$ " marked in Fig. 6.8(a).

Figure 6.9: EDS analysis of pits at spots marked in Figure 6.8: (a) point A; (b) point $B$; (c) point C.

Figure 6.10: SEM images of corrosion propagation stages taken from as-received rebar with mill scale after immersion in the simulated concrete pore solution plus $0.15 \mathrm{M} \mathrm{NaCl}$. (a) an image showing corrosion propagation at the interface of steel/mill-scale; (b) EDS analysis at "Area E" marked in Fig. 6.10(a); (c) an image showing the growth of corrosion site (i.e., pit) penetrating the steel; (d) EDS analysis at "Area F" marked in Fig. 6.10(c); (e) an image showing the mill-scale pop-out. Pits can be observed on steel surface well after depassivation can be observed electrochemically. 141

Figure 6.11: Schematic illustration of crevice corrosion at the mill-scale/steel interface in two stages: (a) Stage 1: before oxygen depletion; (b) Stage 2: after oxygen depletion. 144

Figure 6.12: The solution domain representing the crack in the mill scale and the crevice between the surface of steel and mill scale (Karadakis 2009). 150

Figure 6.13: $\mathrm{pH}$ and $\mathrm{Cl}^{-} / \mathrm{OH}^{-}$along boundaries 2 and 3 (from point $\mathrm{B}$ to point $\mathrm{A}$ ).. 153

Figure 7.1: A cold-mounted rebar specimen that was used in the FIB-TEM study. The TEM samples were extracted from the cross section of the rebar. 159

Figure 7.2: Schematic illustration of the stages used for TEM sample preparation using FIB H-bar lift-out technique: (a) cutting using Gallium ion beam, (b) lifting the sample using a micro-manipulator; (c) placing the sample on a TEM grid; (d) further thinning using Gallium ion beam; (e) the TEM sample. 161

Figure 7.3: FIB images of TEM sample preparation process from the cross section of the rebar specimen: (a) gold-coated surface of steel and a Tungsten strap; (b) a released TEM sample (see Fig. 7.2(b)); (c) process of thinning the TEM sample in the FIB microscope using the Gallium ion beam (see Fig. 7.2(d)). 162

Figure 7.4: TEM images of the samples passivated in chloride-free solutions: (a) $\mathrm{CH}-0$ (film thickness: 5 to $8 \mathrm{~nm}$ ); (b) CP-0 (film thickness: 8 to $13 \mathrm{~nm}$ ). 165

Figure 7.5: Typical EDS spectra of oxide film formed in the chloride-free (a) $\mathrm{CH}$ solution; b) $\mathrm{CP}$ solution. 166

Figure 7.6: EDS line scan inside the oxide film parallel to the steel surface of the $\mathrm{CH}-0$ sample: (a) TEM image showing the locations of the analysis; (b) EDS line-scan results corresponding to the points shown in the TEM image. For clarity, only elements with the atomic $\%$ higher than $1 \%$ are presented. 
Figure 7.7: EDS line scan inside the oxide film parallel to the steel surface of the CP-0 sample: (a) TEM image showing the locations of the analysis; (b) EDS line-scan results corresponding to the points shown in the TEM image. 168

Figure 7.8: EDS analyses conducted on the $\mathrm{CH}-0$ sample across the oxide film perpendicular to the steel surface: (a) TEM image showing the locations of the analyses; (b) EDS results corresponding to the points shown in the TEM image. 169

Figure 7.9: EDS analyses conducted on the CP-0 sample across the oxide film inclined to the steel surface: (a) TEM image showing the locations of the analysis; (b) EDS results corresponding to the points shown in the TEM image. 170

Figure 7.10: TEM images of (a) the $\mathrm{CH}-1$ sample exposed to $\mathrm{CH}$ solution with $0.05 \mathrm{M}$ chloride; (b) the CP-1 sample exposed to CP solution with $0.45 \mathrm{M}$ chloride. 171

Figure 7.11: EDS analysis of the oxide film of the $\mathrm{CH}-1$ sample, which was exposed to $\mathrm{CH}$ solution plus $0.05 \mathrm{M}$ chloride: (a) TEM image showing the locations of the EDS scans; (b) EDS scan along the oxide film; (c) EDS scan perpendicular to the oxide film.

Figure 7.12: EDS analysis conducted across the oxide film inclined to the steel surface in the CP solution plus $0.45 \mathrm{M}$ chloride (CP-1): (a) TEM image showing the locations of the EDS scans; (b) EDS scan along the oxide film; (c) EDS san perpendicular to the oxide film. 174

Figure 7.13: Typical TEM images of the oxide film from different parts of the $\mathrm{CH}-2$ sample showing: (a) non-uniform oxide film; (b) varying thickness of oxide film; (c) thinned oxide film. 175

Figure 7.14: Typical TEM images of the oxide film from different parts of the CP-2 sample showing: (a) non-uniform oxide; (b) no sign of oxide film; (c) thinned oxide film (2-5 $\mathrm{nm})$.

Figure 7.15: EDS analysis conducted across the oxide film of the CH-2 sample: (a) TEM image showing the scan location; (b) EDS results corresponding to the points shown in the TEM image. 178

Figure 7.16: EDS analysis conducted across a region where no oxide film was visible on the CP-2 sample: (a) TEM image showing the scan location; (b) EDS results corresponding to the points shown in the TEM image. 178

Figure 7.17: EDS analysis on the CH-2 sample: (a) TEM image showing the EDS scan locations; (b) EDS scan at the location close to a pit as shown in Fig. 7.17(a); (c) EDS scan at 
the location between two pits shown in Fig. 7.17(a); (d) EDS scan at the bottom of the pit shown in Fig. 7.17(a).

Figure 7.18: TEM images of the steel near the surface for the CP-2 sample: (a) cementite $\left(\mathrm{Fe}_{3} \mathrm{C}\right)$ lamellae in steel in a region of pearlite that intersects the surface; (b) diffraction pattern of cementite $\left(\mathrm{Fe}_{3} \mathrm{C}\right)$ from a location marked "A" in Fig. 7.18(a); (c) cavities on the surface of steel along the openings of cementite lamellae; (d) a higher magnification of a cavity along a cementite lamellae.

Figure 7.19: EELS spectra taken from the oxide film on the CP-0 sample (plots CP-0-1 and CP-02), and EELS spectra of different iron oxides for comparison purposes (Chen et al. 2009).

Figure 7.20: TEM dark-field images of the oxides films of (a) the CH-0 sample; (b) the CP-0 sample. The bright phases indicate the incorporation of nano-crystalline particles in the oxide film. 184

Figure 7.21: Gold diffraction pattern used for the calculation of the camera constant at the locations. 186

Figure 7.22: Diffraction patterns of three different areas (nano-crystalline particles) in the oxide film of the CH-0 sample (see Fig. 7.20(a): (a) SAD pattern; (b) and (c) CBED patterns.

Figure 7.23: (a)-(d) SAD patterns of four different areas (nano-crystalline particles) in the oxide film of the CP-0 sample (see Fig. 7.20(b)). $\square$ : reflections from the Fe substrate, O: reflections from oxide film. Note that the iron and oxide reflections are almost in the same direction (i.e., on a radial line, less than $5^{\circ}$ deviation). 189

Figure 8.1: The Fe $2 \mathrm{p}$ XPS spectra obtained from two $800-\mu \mathrm{m}$-diameter spots on the CH-Cl specimen: (a) pre-sputter; (b) Argon ion sputter $2.5 \mathrm{~nm}$; c) Argon ion sputter $5 \mathrm{~nm}$; (d) Argon ion sputter $12.5 \mathrm{~nm}$. 201

Figure 8.2: Typical curve fitting conducted for $\mathrm{C} 1 \mathrm{~s}$ high resolution XPS spectrum. 205

Figure 8.3: Typical curve fitting conducted for Fe $2 \mathrm{phigh}$ resolution XPS spectrum. 207

Figure 8.4: The peak $E_{b}$ position data obtained from other studies (NIST Standard Reference Database 20, V. 3.5) for iron compounds at the surface of steel. 207

Figure 8.5: Typical curve fitting conducted for $\mathrm{O} 1 \mathrm{~s} \mathrm{high}$ resolution XPS spectrum. 208

Figure 8.6: The peak $E_{b}$ position data obtained from other studies (NIST Standard Reference Database 20, V. 3.5) for oxygen compounds at the surface of steel. $\mathrm{OH}$ assignment) 209

Figure 8.7: Typical curve fitting conducted for $\mathrm{Ca} 2 \mathrm{p}$ high resolution XPS spectrum. 209 
Figure 8.8: The peak $\mathrm{E}_{\mathrm{b}}$ position data obtained from other studies (NIST Standard Reference Database 20, V. 3.5) for calcium compounds at the surface of steel.

Figure 8.9: XPS depth profiles (At\%) for (a) Iron (b) Oxygen (c) Carbon and (d) Calcium recorded for the $\mathrm{AE}, \mathrm{CH}-2, \mathrm{CH}-9$ and $\mathrm{CH}-\mathrm{Cl}$ specimens.

Figure 8.10: SEM images from the steel surface exposed to the $\mathrm{CH}$ solution for 9 days (CH-9) following the XPS analysis: (a) low magnification SEM image of precipitates from the ion sputtered area; (b) high magnification SEM image of precipitates from the sputtered area; (c) EDS spectrum recorded of the area shown in Fig. 8.10(a).......213

Figure 8.11: (a) high magnification SEM image from a typical clean steel surface following the XPS analysis; (b) EDS spectrum recorded of the area shown in Fig. 8.11(a).........214

Figure 8.12: Schematic model showing the surface conditions of the XPS specimen: (a) before sputtering; (b) after sputtering. (Note: Relative dimensions of the precipitates and the oxide film are not drawn to scale) 215

Figure 8.13: Fe 2p high resolution XPS spectra at selected sputtered depths for (a) AE specimen; (b) $\mathrm{CH}-2$ specimen; (c) $\mathrm{CH}-9$ specimen; (d) $\mathrm{CH}-\mathrm{Cl}$ specimen. 218

Figure 8.14: Comparison of $\mathrm{Fe} 2 \mathrm{p}$ spectra before sputtering for the four specimens..................220

Figure 8.15: Comparison of XPS Fe 2p spectra at a sputter depth of $5 \mathrm{~nm}$.................................220

Figure 8.16: Comparison of the (Iron oxide)/(Iron metal) at the selected sputtered depths .....223

Figure 8.17: Comparison of the $\mathrm{Fe}^{3+} / \mathrm{Fe}^{2+}$ at the selected sputtered depths. ..............................225

Figure 8.18: Comparison of the Fe-2 phase present at the selected sputtered depths................226

Figure 9.1: The Fe 2p XPS spectra obtained from two different areas at the surface of samples showing the variation of data: (a) CP-0 sample; (b) CP-1 sample; (c) CP-2 sample.

Figure 9.2: Fe $2 p$ high resolution XPS spectra for the CP-0 sample at different emission angles.

Figure 9.3: Fe 2p high resolution XPS spectra for the CP-1 sample at different emission angles.

Figure 9.4: Fe 2p high resolution XPS spectra for the CP-2 sample at different emission angles.

Figure 9.5: Comparison of the Fe 2p spectra for three samples at (a) $0^{\circ}$ emission angle; (b) $40^{\circ}$ emission angle; (c) $60^{\circ}$ emission angle. 240

Figure 9.6: Comparison of the (Iron oxide)/(Iron metal) at the different emission angles.......... 242

Figure 9.7: Comparison of the $\mathrm{Fe}^{2+} / \mathrm{Fe}^{3+}$ at the different emission angles.................................244 
Figure 9.8: The effect of solution (i.e., $\mathrm{CP}$ solution vs. $\mathrm{CH}$ solution) on the $\mathrm{Fe} 2 \mathrm{p}$ spectra at $0^{\circ}$ emission angle.

Figure A.1: Schematic illustration of TEM sample preparation procedure carried out in this study using FIB technique: a) gold coated, cross-sectional view b) Tungsten sputtered, cross-sectional view c)TEM sample taken from the bulk specimen, a 3-D view (Not to scale)

Figure A.2: TEM images of oxide film in the $\mathrm{CH}$ solution (CH-0): a) a digital TEM micrograph; b) a low magnification TEM micrograph; c) a high magnification TEM micrograph. 280

Figure A.3: TEM images of oxide film in the CP solution (CP-0): a) a digital TEM micrograph; b) a low magnification TEM micrograph c) a high magnification TEM micrograph. 281

Figure A.4: TEM images of the CH-1 sample: (a) a low magnification TEM micrograph; (b) a high magnification TEM micrograph. .282

Figure A.5: TEM images of the CP-1 sample: (a) low magnification TEM micrograph; (b) high magnification TEM micrograph. 283

Figure A.6: TEM images of the steel near the surface for the CP-2 sample: a) a cavity (void) on the surface of steel along the openings of cementite lqmellae; b) dimensions of the cavity (void); c) Fe k $\alpha$ map of Fig. A.6 (a). 284

Figure A.7: Steel near the surface for the CP-2 sample: a) a TEM micrograph showing the cementite lamellae on steel; b) Gold (Au) map of Fig. A.7(a); c) Tungsten (W)map of Fig. A.7(a); d) Iron (Fe) map of Fig. A.7(a). 285

Figure A.8: TEM dark-field images of the CH-0 sample: a) a single exposure dark-field micrograph; b) a multi-exposure dark-field micrograph. The bright phases indicate the incorporation of nano-crystalline particles in the oxide film. 286

Figure A.9: TEM dark-field images of the CP-0 sample: a) a single exposure dark-field micrograph; b) a multi-exposure dark-field micrograph. The bright phases indicate the incorporation of nano-crystalline particles in the oxide film. 287

Figure B.1: $\mathrm{O}$ 1s high resolution XPS spectra at selected sputtered depths for AE specimen and the binding energy position of various oxygen-incorporated compounds that are present in the spectra. 288

Figure B.2: $\mathrm{O}$ 1s high resolution XPS spectra at selected sputtered depths for $\mathrm{CH}-2$ specimen and the binding energy position of various oxygen-incorporated compounds that are present in the spectra. 289 
Figure B.3: O 1s high resolution XPS spectra at selected sputtered depths for CH-9 specimen and the binding energy position of various oxygen-incorporated compounds that are present in the spectra.

Figure B.4: $\mathrm{O}$ 1s high resolution XPS spectra at selected sputtered depths for $\mathrm{CH}-\mathrm{Cl}$ specimen and the binding energy position of various oxygen-incorporated compounds that are present in the spectra. 291

Figure B.5: C 1s high resolution XPS spectra at selected sputtered depths for AE specimen and the binding energy position of various carbon-incorporated compounds that are present in the spectra. 292

Figure B.6: C 1s high resolution XPS spectra at selected sputtered depths for CH-2 specimen and the binding energy position of various carbon-incorporated compounds that are present in the spectra. .293

Figure B.7: C 1s high resolution XPS spectra at selected sputtered depths for $\mathrm{CH}-9$ specimen and the binding energy position of various carbon-incorporated compounds that are present in the spectra. .294

Figure B.8: $\mathrm{C}$ 1s high resolution XPS spectra at selected sputtered depths for $\mathrm{CH}-\mathrm{Cl}$ specimen and the binding energy position of various carbon-incorporated compounds that are present in the spectra. .295

Figure B.9: Ca 2p high resolution XPS spectra at selected sputtered depths for AE specimen and the binding energy position of various calcium-incorporated compounds that are present in the spectra.

Figure B.10: Ca 2p high resolution XPS spectra at selected sputtered depths for $\mathrm{CH}-2$ specimen and the binding energy position of various calcium-incorporated compounds that are present in the spectra.

Figure B.11: $\mathrm{Ca} 2 \mathrm{p}$ high resolution XPS spectra at selected sputtered depths for $\mathrm{CH}-9$ specimen and the binding energy position of various calcium-incorporated compounds that are present in the spectra. 298

Figure B.12: $\mathrm{Ca} 2 \mathrm{p}$ high resolution XPS spectra at selected sputtered depths for $\mathrm{CH}-\mathrm{Cl}$ specimen and the binding energy position of various calcium-incorporated compounds that are present in the spectra. 299

Figure B.13: AFM topography images of polished rebar cross section: (a) surface area of $1 \mu \mathrm{mx}$ $1 \mu \mathrm{m}$; (b) surface area of $10 \mu \mathrm{m} \times 10 \mu \mathrm{m}$, recorded in Contact Mode. 300 


\section{Chapter 1}

\section{Introduction}

\subsection{General}

Reinforced concrete is the most prevalent construction material used in the world; it is used to construct highways, bridges, buildings, canals, dams, airports, poles, pipelines, sewer lines, storage tanks, and many other types of structures, which constitute the infrastructure of our modern society. The ready availability of the raw materials for manufacturing portland cement, the principal ingredient of concrete, and of sand and coarse aggregate makes concrete a universal construction material. Although concrete with average quality can be produced without extensive experience or knowledge, production of high quality concrete, or high-performance concrete, requires technological and scientific expertise. Inadequate care in concrete production will lead to poorlydesigned structures, which may experience severe serviceability and safety problems.

Today, many of the concrete structures that have been exposed to aggressive environments suffer from durability issues. The problem is particularly serious in reinforced concrete structures for which the corrosion of carbon steel reinforcement (rebar) created a multi-billion-dollar infrastructure deficit. National Association of Corrosion Engineers International (NACE 2002) valued the direct corrosion cost in 
infrastructure at $\$ 22.6$ billion/year. The U.S. Strategic Highway Research Program (FHWA 2001) estimated that the unfounded liability to correct corrosion-induced distress in bridges in the United States alone is about $\$ 20$ billion dollars. In Canada, a country where corrosion is a serious problem due to the excessive use of de-icing salts, a large portion of the overall bridge maintenance cost is due to the corrosion of carbon steel rebar in concrete structures.

The corrosion of rebar in concrete takes place due to a series of electrochemical reactions. Within the alkaline environment provided by concrete $(\mathrm{pH}>13)$, steel is covered with a passive film which reduces the rates of these electrochemical reactions; hence, rebar in concrete is generally well protected against excessive metal loss. The partial or complete loss of the passive layer, known as depassivation, may lead to higher corrosion rates, which may cause serviceability and safety problems for the structure. Since the depassivation of steel plays an important role as to when and how the corrosion will start and progress, it is important for engineers to understand its mechanism accurately. Unfortunately, although significant progress has been made on the evaluation and the mitigation of corrosion problems in concrete structures, the research to date has not been fully successful in accurately predicting how rebar in concrete loses its passivity when it is exposed to chloride-bearing (e.g. de-icing or seawater) salts.

\subsection{Problem Definition}

The current approach to quantify the chloride-induced rebar depassivation in concrete is in the form of defining chloride thresholds, beyond which the steel is assumed to lose its passivity. Although there is a general acceptance of this theory qualitatively, 
there is little agreement on how to determine chloride thresholds quantitatively. A recent review by Angst et al. (2009) clearly showed that most setups used to determine chloride thresholds are not appropriate to provide realistic results, and this may be considered as one of the reasons for the widely scattered experimental data reported in the literature. Furthermore, the depassivation of steel in concrete is affected by a large number of factors including the chloride content and the alkalinity of the pore solution around the rebar, the physical and chemical properties of concrete, the surface characteristics and the chemical composition of steel, and the presence of mechanical stresses. These complexities can also be used to explain the uncertainty associated with the reported values of chloride thresholds for carbon steel rebar in concrete.

Interestingly, an article by Hausmann in the August 2007 issue of the NACE Materials Performance Magazine (Hausmann 2007), which was appropriately titled "Three myths about corrosion of steel in concrete," identifies the following problems (i.e., "myths") with our current understanding of steel corrosion in concrete: "(1) Passivating film on steel in concrete is destroyed when high concentrations of chlorides reach the steel surface; (2) to prevent chloride-induced steel corrosion in concrete, the soluble chloride content should not exceed $0.06 \%$ of cement weight in prestressed concrete and $0.15 \%$ of the cement weight in reinforced concrete exposed to chlorides in service; (3) steel potentials in concrete (measured using the half-cell technique) more positive than -200 $\mathrm{mV} / \mathrm{CSE}$ indicate $90 \%$ probability that corrosion is not occurring; potentials more negative than $-350 \mathrm{mV} / \mathrm{CSE}$ indicate $90 \%$ probability that corrosion is occurring; and between these two potential limits, the corrosion is uncertain." 
The last myth, which is not directly related to the present work, demonstrates the current challenges associated with the corrosion measurement techniques for reinforced concrete structures. These techniques (e.g. half-cell mapping, polarization resistance) do not generally provide the necessary accuracy expected from them; however, ongoing corrosion modeling studies at Carleton University have made significant progress in improving the results of these measurements (Ghods et al. 2007, Ghods et al. 2008, PourGhaz et al. 2009).

The first two myths identified by Hausmann (2007), and the recent review on the uncertainty in existing chloride threshold data by Angst et al. (2009), are clear indicators that scientists and engineers still do not have a clear understanding of why and how rebar loses its passivity in concrete. It is evident that fundamental understanding of the steel depassivation process in concrete requires fundamental studies; only when this understanding is adequately developed, reliable and robust service life modeling tools that can help engineers predict the state and performance of rebar in concrete structures can be implemented. This understanding will also allow structure owners and operators to better manage and to schedule repair, rehabilitation and replacement operations.

\subsection{General Objectives and Scope}

The main objectives of this research were to understand better the mechanisms of passivation and chloride-induced depassivation of carbon steel reinforcement in concrete and to provide robust explanations to the outstanding questions about corrosion initiation of rebar in the alkaline environment of concrete. The outcome of this study is intended to help engineers make better predictions on the conditions under which the 
carbon steel rebar will lose its passivity, and, estimate the so called "time-to-initiation-ofcorrosion" more accurately. Specific objectives of the research have been identified at the end of the literature review (Chapter 4).

In order to achieve these general goals, a multi-scale experimental/numerical investigation was carried out. The investigation consists of four components: electrochemical experiments, microscopic examinations, numerical modeling, and spectroscopic studies. These four components cover length scales from macro to micro/nano to provide fundamental explanations for engineering observations about the depassivation of carbon steel rebar in concrete.

All the experiments in this study were carried out in synthetic concrete pore solutions representing the aqueous electrolyte (i.e., pore solution) in concrete. For most of the electrochemical experiments, as-received carbon steel rebar specimens were used in order to relate better the outcome of this study to reinforced concrete structures that are in service; however, in some cases, polished carbon steel rebar specimens were also tested to observe the effects of rebar surface conditions on the depassivation process. For nanoscale studies, only samples from highly polished carbon steel rebar cross sections were analyzed to make sure that the investigation could be carried out on high-quality passive films. The passive films that form on other types of steel (e.g. stainless, galvanized, micro-composite) were not in the scope of this study.

\subsection{Research Methodology}

Figure 1.1 illustrates the techniques used in each of the four components of the research, which were designed to complement each other. For example, the results from 
the electrochemical studies on the effect of surface conditions of rebar on the depassivation process are complemented by microscopic studies and verified by numerical modeling. Both transmission electron microscopy (TEM) and X-ray photoelectron Spectroscopy (XPS) were used to investigate the structure of the passive film and the role of chlorides in the depassivation process, but from different view points.

To investigate the kinetics of the passive film formation and chloride-induced depassivation of rebar at the macro level, electrochemical methods such as free corrosion potential (FCP), anodic polarization (AP), electrochemical impedance spectroscopy (EIS) and linear polarization (LP) techniques were used on both as-received and polished rebars in simulated concrete pore solutions.

Scanning electron microscopy (SEM) was used to investigate the effect of surface conditions of rebar, particularly of mill scale, during the depassivation process. The role of rebar surface conditions on the local pore solution chemistry was also studied using the finite element method (FEM) to provide numerical support for the macro-scale electrochemical and micro-scale SEM observations.

Microscopic (TEM) and spectroscopic (Energy Dispersive X-ray Spectroscopy EDS) techniques on nano-scale samples obtained from carbon steel rebar specimens using the focused ion beam (FIB) method were used for the visual investigation of the oxide films and for the determination of their chemical composition at micro/nano levels, respectively. Selected area diffraction (SAD) and convergent beam electron diffraction (CBED) analyses were also conducted on the oxide film in the TEM microscope to determine the nano-structural composition and the stoichiometry of the films. Electron energy loss spectroscopy (EELS) was employed to verify the results of the SAD and 
CBED techniques. Finally, both sputtered and angle-resolved X-ray photoelectron spectroscopy (XPS) were used to complement the nano-scale observations obtained from TEM, EELS, CBED and SAD analyses.

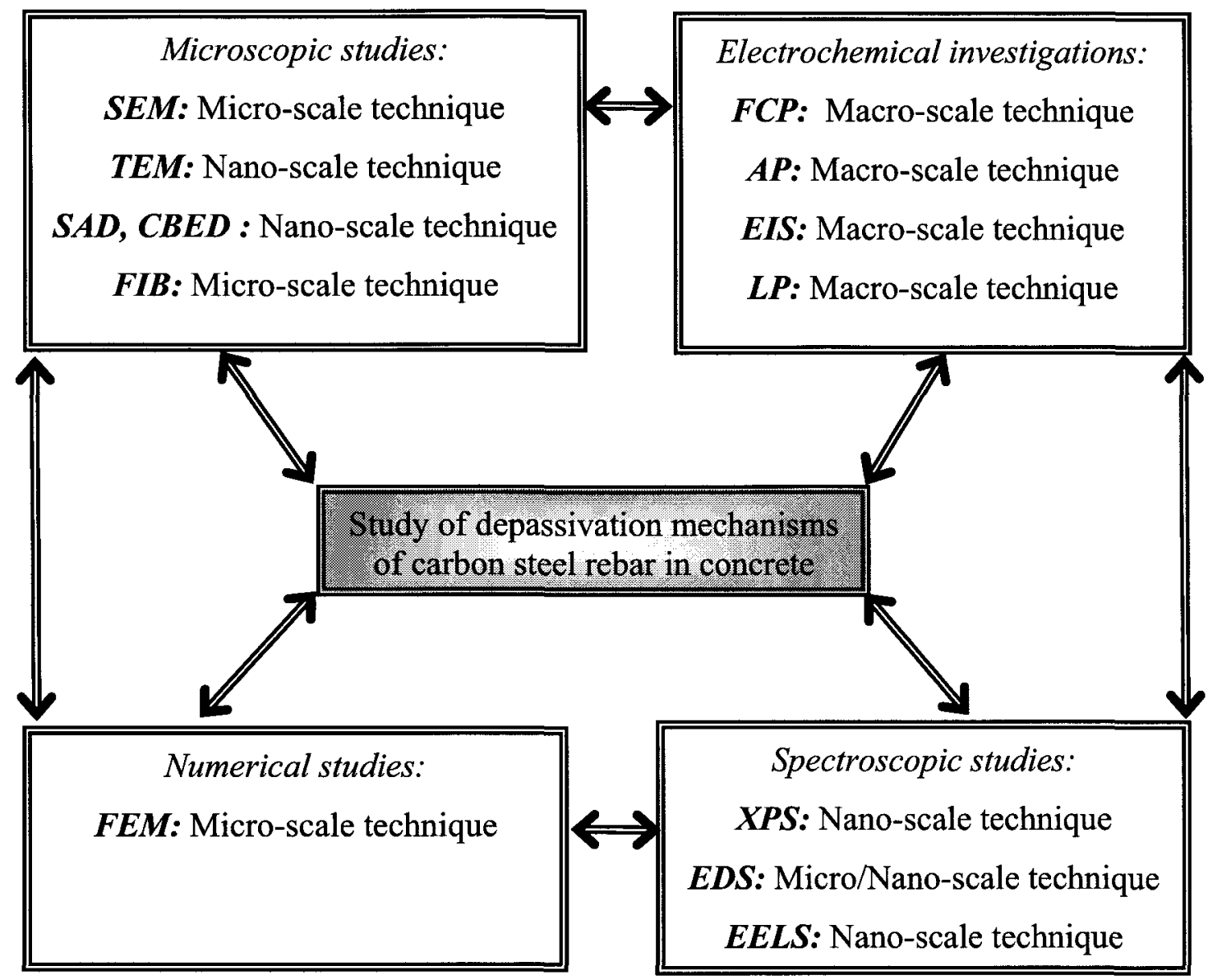

SEM: Scanning Electron Microscopy

EIS: Electrochemical Impedance Spectroscopy

TEM: Transmission Electron Microscopy

LP: Linear Polarization

SAD: Selected Area Diffraction

FEM: Finite Element Modeling

$C B E D$ : Convergent Beam Electron Diffraction EDS: Energy Dispersive Spectroscopy

FIB: Focused Ion Beam Microscopy

XPS: X-ray Photoelectron Spectroscopy

FCP: Free Corrosion Potential

EELS: Electron Energy Loss Spectroscopy

AP: Anodic Polarization

Figure 1.1: Techniques used in multi-scale investigation carried out in this research. 


\subsection{Organization of the Thesis}

The thesis is organized in ten chapters. Chapter 1 presents the problems definition, general objectives, scope and the methodology of the research.

Chapter 2 is intended for general readers and provides a background on the basic concepts of corrosion and passivity.

Chapter 3 presents the literature review related to this research to identify the specific objectives of the study.

Chapter 4 presents the electrochemical study on the effect of concrete pore solution chemistry on the passive film properties of carbon steel rebar.

Chapter 5 presents the electrochemical study on the chloride-induced depassivation of carbon-steel rebar in simulated concrete pore solutions.

Chapter 6 presents the SEM investigation on the properties of mill scale on carbon steel rebars and its effects on corrosion initiation. The numerical finite element investigation to support the SEM observations is also provided in this chapter.

Chapter 7 presents the findings of the TEM study, as well as the SAD and the EELS analyses, on the oxide films of the nano-scale FIB samples obtained from carbonsteel rebar specimens.

Chapters 8 and 9 respectively present the XPS depth profiling and angleresolved investigations of oxide film formed on the steel samples in synthetic concrete pore solutions.

The main conclusions of this study along with recommendations for future work are presented in Chapter 10. Supplementary TEM figures and XPS results are presented in Appendix A and Appendix B, respectively. 


\section{Chapter 2}

\section{Background}

In this chapter, a brief background on the basic concepts and definitions used in this research are presented. The literature on the corrosion of pure metals and alloys is vast; therefore, it is not the goal of this chapter to provide a detailed review of all concepts. Rather, it is intended that this chapter will introduce the important terminology used in this research to establish a common understanding for the readers. For more detailed information on the topics presented in this section, the readers should refer to more general books on corrosion and electrochemistry such as the ones by Fontana (1986), Jones (1995), Ramachandran and Beaudoin (2001), and Revie and Uhlig (2008).

\subsection{Passivity}

The phenomenon of metallic passivity has been studied by many researchers and scientists for over 100 years since the days of Faraday. According to Fontana's (1986) simple definition, passivity can refer to the loss of chemical reactivity experienced by certain metals and alloys under particular conditions. Many engineering and structural metals (e.g. iron, nickel, silicon, chromium, titanium) and their alloys (e.g. steel), and a 
large number of other industrial metals (e.g. zinc, cadmium, tin, uranium, thorium) may show passive behaviour under particular conditions and act like noble metals such as platinum and gold (Revie 2000).

Revie and Uhlig (2008) classified passivity in two groups: In Type A, a metal is passive if it substantially resists corrosion in a given environment owing to noticeable anodic polarization (i.e., low corrosion rate, noble potential); and in Type B, a metal is passive if it substantially resists corrosion in a given environment despite a marked thermodynamic tendency to react (i.e., low corrosion rate, active potential). Iron, nickel, chromium, titanium, and in oxidizing environments, stainless steels exhibit Type A passivity. Lead in sulfuric acid and iron in an inhibited pickling acid can be given as examples of Type B passivity. Only Type A passivity is considered within the scope of this thesis, because iron in highly alkaline solutions substantially resists corrosion at high noble potential values (i.e., under anodic polarization).

Although a common mechanistic definition of passivity is difficult to provide, it can be quantitatively described by characterizing the behaviour of metals that show this behaviour. First, consider a metal which does not show passivity: The behaviour of such a metal is shown in Fig. 2.1. If the metal is immersed in a deaerated solution and kept there for a while, a stable oxidizing potential and an associated corrosion rate will be reached; this point is marked as " $\mathrm{A}$ " in Fig. 2.1. If the potential of the metal is increased by applying a potential, the corrosion rate of the metal will increase exponentially; the increase can be plotted on a semi-logarithmic scale, yielding the, so-called, anodic polarization curve, as shown in Fig. 2.1 (Fontana 1986). 


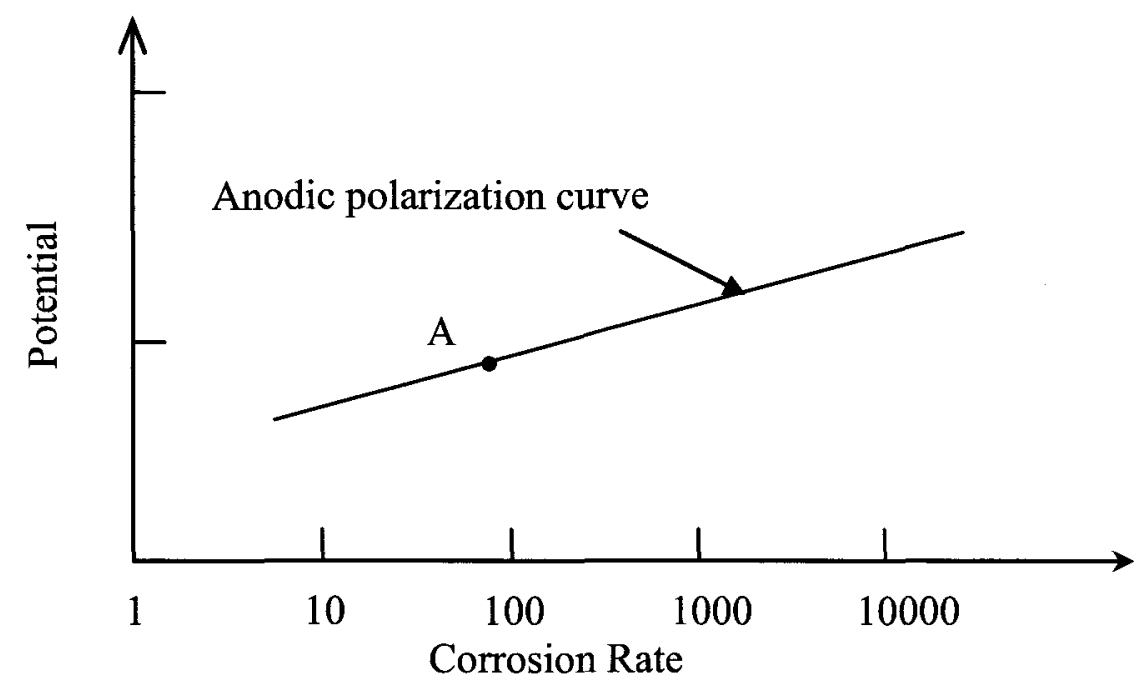

Figure 2.1: Corrosion behaviour of a metal that does not show passive behaviour.

In Fig. 2.2, the typical behaviour of a metal that shows passivity is demonstrated. The behaviour of this metal can be divided into three phases: active, passive and transpassive. In the active region, the behaviour of this metal is similar to that of a metal that does not exhibit passive behaviour: a slight increase in the potential causes a rapid increase in the corrosion rate. However, for a metal that shows passive behaviour, further increase of the potential will cause a sudden decrease in the corrosion rate (the onset of the passive region). Further increase in the potential produces little, if any, change in the corrosion rate. Finally, at very high potentials the corrosion rate starts increasing again; this region is called the transpassive region.

Although Figure 2.2 is not plotted for a specific metal, it is important to note that it is typical to observe 3 to 6 orders of magnitude reduction in corrosion rate during the transition from the active to the passive state. The actual cause of this behaviour (i.e., active-passive transition) is different for different metals, and it is not understood well for some. It is generally believed that the formation of a thin protective film on the surface of the metal results in a kind of electrical insulator which is stable against a significant range 
of potential; however, this film would eventually break down if the potential is further increased. The exact nature of this film is not completely understood for a large number of metals and alloys. However, for most engineering purposes, polarization curves such as the one in Fig. 2.2 have been useful in explaining corrosion phenomena even though the exact nature of the passive film may not be known.

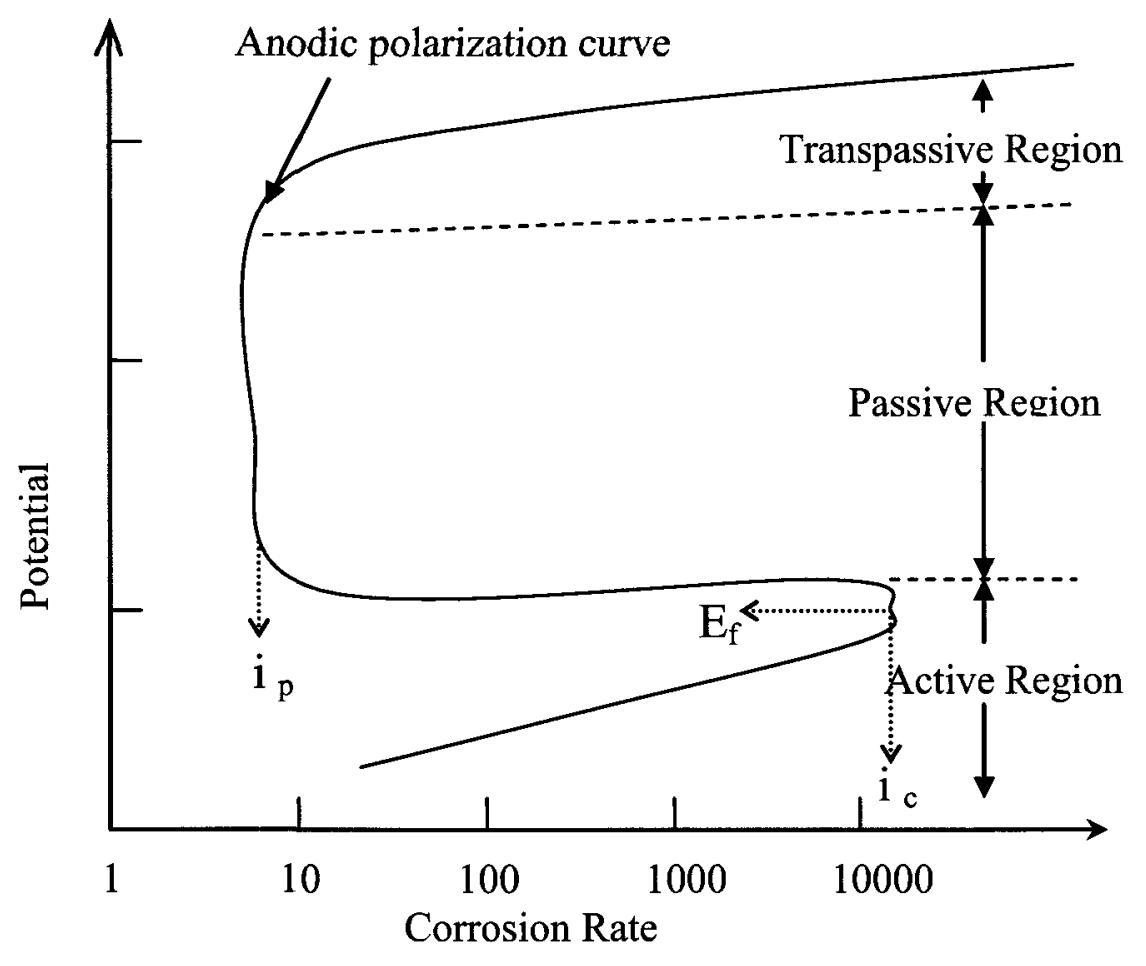

Figure 2.2: Corrosion behaviour of a metal showing active-passive transition.

\subsection{Nature and Properties of Passive films on Iron}

Since the subject of this research is carbon steel, the discussion presented here will be related to the passive films that form on iron or carbon steel. The structure favoured by most investigators for the thin passive film on iron is an inner layer of $\mathrm{Fe}_{3} \mathrm{O}_{4}$ under an outer layer of $\gamma-\mathrm{Fe}_{2} \mathrm{O}_{3}$ (Jones 1995). In earlier studies, basing his arguments on 
thermodynamics, Vetter (1958) argued for the two-layer passive film. Cohen (1978) also found evidence for two layers, but also suggested that the boundary between the layers is not sharp and is in the form of a cubic lattice with decreasing concentration of iron away from the metallic surface. Supporting Cohen's results are the studies of Ord and DeSmet (1976) and Kruger (1988).

Using ex-situ electron diffraction techniques, Foley et al. (1967) and Kuroda et al. (1982) determined that the anodically grown passive film on iron in acidic solution has a cubic spinel structure, which is structurally very similar to $\gamma-\mathrm{Fe}_{2} \mathrm{O}_{3}$ and $\mathrm{Fe}_{3} \mathrm{O}_{4}$, but the film is less ordered than the crystalline anhydrous oxides, exhibiting a tendency toward more glassy structures. Since film dissolution, film formation, and breakdown all involve the movement of electrons and ions from the metal surface through the passive film, or from the electrolyte into the film, electronic properties of the film are important. Moreover, electron transfer reactions that occur on surfaces with passive films relate to the electronic properties of such films (Kruger 1989). Whether the thin passive film that forms on iron acts as a semiconductor or an insulator is a controversial subject. For example, a number of studies, such as the one conducted by Schultze and Stimming (1975), indicate that the passive film usually demonstrates the behaviour of an n-type semiconductor with excess negative charge, while others suggest that the passive film has low electronic conductivity, and part of it may consist of an insulating material (Kruger 1989).

Despite the large number of studies that have been carried out on passive films, still, the nature and properties of different passive films, particularly on iron or carbon steel in highly alkaline environments, are controversial subjects of research. Discussion 
on this complex topic can be found in Frankenthal and Kruger (1978), Sato and Okamoto (1981), Kruger (1988), and Heusler (1995).

\subsection{Thermodynamics of Corrosion}

A useful and practical guide that describes the thermodynamic aspects of corrosion is the Pourbaix diagram, which is simply an overlay of the redox and acid-base chemistry of elements, alloys and oxides onto the water stability diagram, establishing the potential-pH relationship of the system in equilibrium (Revie 2000). In Fig. 2.3, a simplified Pourbaix diagram of iron in water is illustrated. In this figure, lines A and B are the water lines between which the water is considered to be thermodynamically stable at $1 \mathrm{~atm}$. If a metal with a potential below the B line is immersed in water, the water will be reduced $\left(\mathrm{H}_{2}\right.$ will evolve); therefore, water is not stable in this region. When a metal with a higher potential than line $\mathrm{A}$ is immersed in water, $\mathrm{O}_{2}$ evolves, and once again, water is not stable in this region. The reactions that take place below the B line tend to be cathodic (i.e., water is reduced) and the reactions that take place above the A line are anodic (i.e., water is oxidized).

In Pourbaix diagrams, three different regions that define the theoretical conditions of corrosion, immunity and passivity are established. The thermodynamic data (i.e., potential and $\mathrm{pH}$ ) determines the conditions of immunity under which the corrosion is thermodynamically unlikely to occur (see the immunity region in Fig. 2.3). In the corrosion 


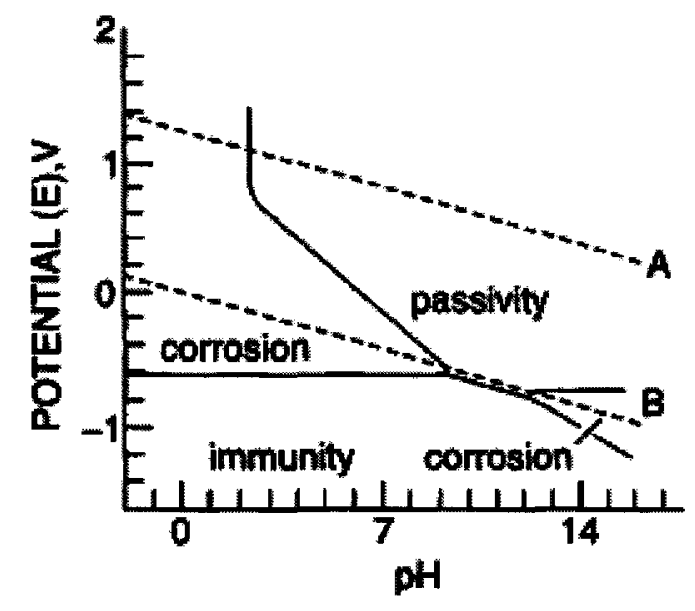

Figure 2.3: Simplified potential-pH equilibrium diagram (Pourbaix diagram) for ironwater (Revie 2000).

region, the corrosion products are soluble; both the metal and its oxides/hydroxides are unstable; therefore, corrosion is predicted to occur in these regions. In the passivity region, the oxides are stable and in equilibrium with the solution species. Generally, if the metal is covered by an oxide, and the oxide is protective (and has low solubility) then active corrosion will be prevented. In this case, the system is said to be passive. In the passivity region, the formation of corrosion products (e.g. oxides) is possible whether they can protect the metal from active corrosion or not. Pourbaix's atlas (1974) introduces a set of simplified diagrams like the one shown in Fig. 2.3 for most metals so that one can identify the conditions of corrosion, passivation, and immunity. These equilibrium diagrams could be called "road maps of the thermodynamically possible conditions for passivity" (Revie 2000).

Pourbaix diagrams can also be used to identify the active, passive and transpassive regions of active-passive polarization curves (see Fig. 2.2). For instance, the transpassive region is located at potentials above the oxygen evolution line (i.e., line $\mathrm{A}$ in Fig. 2.3). Usually, the passive regions of the polarization curves lie at potentials in the 
Pourbaix diagrams for which passive films are stable. Pourbaix diagrams cannot always be used to predict the passive regions on polarization curves. Hoar (1967) proposed the existence of metastable passive regions, in which anodic polarization curves may show passivity, while the Pourbaix diagram predicts active behaviour. Iron in sulfuric acid solutions is an example, in which metastable passive regions may be present. Furthermore, the use of Pourbaix diagrams in the study of passivity and corrosion may not be practical when complex electrolytes are considered: concrete pore solution is such a complex electrolyte; hence the use of Pourbaix diagrams of metals and alloys (e.g. carbon steel) in concrete pore solution is rather challenging.

The ability of the oxide film to protect the metal from corrosion cannot be determined by thermodynamics alone (i.e., by only using Pourbaix diagrams). In order to know whether an oxide film is protective, knowledge of the kinetics of the corrosion reactions is also required.

\subsection{Kinetics of Corrosion}

A protective oxide film on the metal surface can significantly decrease the rate of corrosion. The hypothetical anodic polarization curve is shown in Fig. 2.2. Anodic polarization curves of metals that exhibit active-passive behaviour usually consist of three general features (Fig. 2.2): (1) the current initially increases with a rise in potential; (2) there exists a passivating potential (i.e., flade potential) $\left(E_{f}\right)$ at which the current density reaches the maximum value of $i_{c}$; (3) the current density significantly decreases to the passive current density, $i_{p}$. As shown in Fig. 2.2, at high potentials in the passive region, the current density again starts increasing due to either entry to the transpassive 
region or the initiation of pitting corrosion. In the transpassive region, oxygen evolution and the increase in current can be observed.

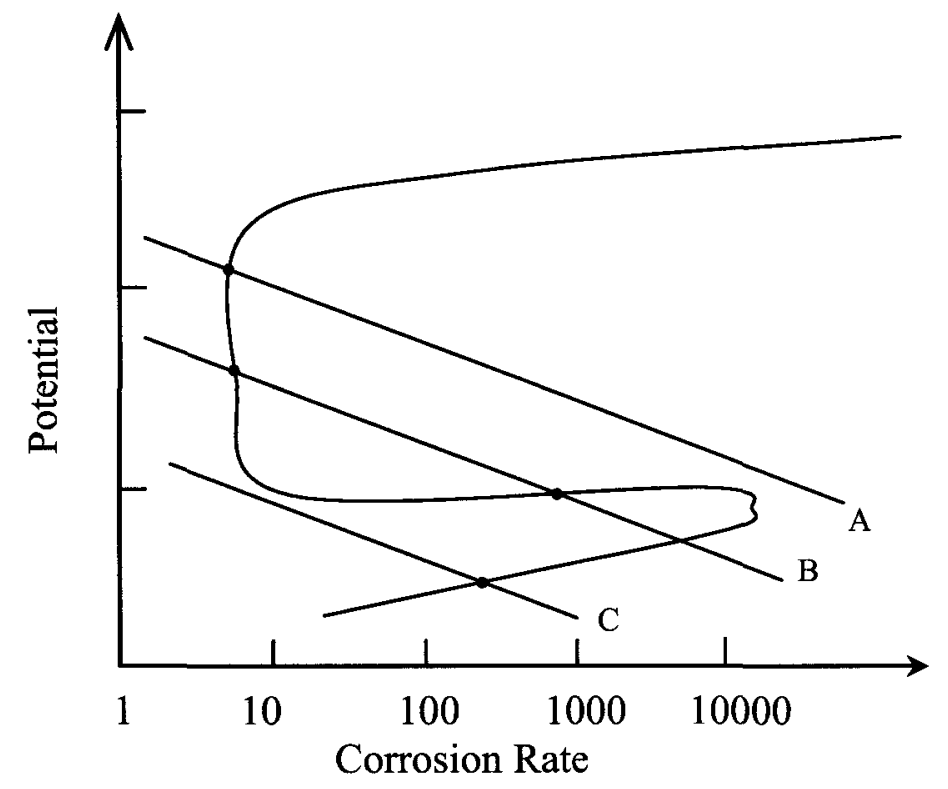

Figure 2.4: The intersection of three possible cathodic polarization curves (straight lines $A, B$, and $C$ ) with an anodic polarization curve for a system capable of exhibiting passivity (Revie 2000).

The corrosion potential of a corroding system can be determined at the intersection of the anodic and cathodic polarization curves, at which the two reactions are in dynamic equilibrium, and the rate of each reaction is equal to the corrosion rate of the metal. Hence, the corrosion rate of a metal that shows active-passive behaviour can be determined at the intersection point of cathodic and anodic polarization curves, as shown in Fig. 2.4. Three possible cases can be considered depending on the location of the intersection point: (1) cathodic polarization curve intersects the anodic polarization curve in the passive region resulting in low corrosion rates (curve A); (2) cathodic polarization curve intersects the anodic curve at multiple points (in passive and active regions) such that corrosion potential can oscillate between active and passive regions resulting in a 
metastable condition (Curve B); (3) cathodic polarization curve intersects the anodic polarization curve in the active region that results in high corrosion rates (Curve C).

Another possible intersection between anodic and cathodic polarization curves can result in the breakdown of passivity, localized attack, and pitting. As shown in Fig. 2.5 , at potentials above the pitting (or breakdown) potential, $E_{p i t}$, the breakdown of passive films start leading to a rapid increase in corrosion rate. This breakdown potential, above which the current density increases above $i_{p}$, is located below the transpassive region. The intersection of cathodic curve A with the anodic curve of a system exhibiting active-passive behaviour (i.e., $E_{\text {corrA }}$ ) leads to pitting; while the intersection of cathodic curve $\mathrm{B}$ at a potential below $\mathrm{E}_{\mathrm{pit}}\left(\right.$ i.e., $\left.E_{\text {corr }}\right)$ may not lead to the breakdown of the passive film.

Hoar (1967) identified four possible conditions that are required for passive film breakdown to initiate localized attack:

(1) The electrode potential should exceed the critical potential, $\mathrm{E}_{\mathrm{pit}}$;

(2) Damaging species (e.g. halides such as chloride) are present;

(3) There is an induction time that starts with the initiation of the breakdown process by the introduction of breakdown conditions and ends when the localized corrosion current density begins to rise;

(4) Local sites, where breakdown can take place, exist. 


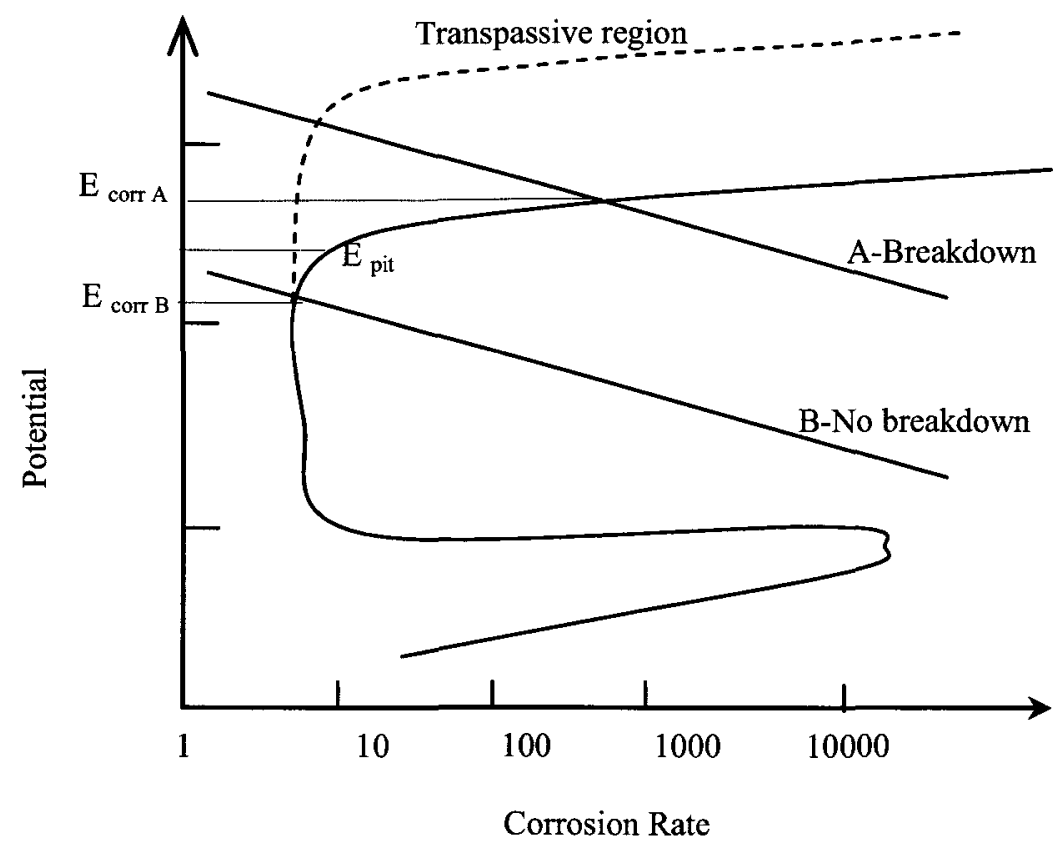

Figure 2.5: The intersection of anodic with cathodic polarization curves at $E_{c o r r A}$ at which breakdown occurs and at $E_{\text {corrB }}$ that results in no breakdown (Revie 2000).

Various explanations for the breakdown of passive film that include these four effects have been suggested in the literature (Kruger 1976, Galvele 1978, Janik-Czachor 1981). In a review, Kruger and Rhyne (1981) concluded that the breakdown of passive films can generally occur because of the chemical alteration of the passive film, or the environment, so that the films are no longer able to effectively prevent destructive local attack.

\subsection{Causes of Corrosion Initiation of Steel in Concrete}

The corrosion of steel in concrete is a result of the dissolution of iron in concrete pore solution, represented by the following half-cell reaction:

$F e \rightarrow F e^{2+}+2 e^{-}$ 
The electrons produced in this anodic reaction are consumed at the cathodic sites on the steel surface to preserve electrical neutrality. The cathodic reaction is the oxygen reduction represented by:

$$
\frac{1}{4} \mathrm{O}_{2}+\frac{1}{2} \mathrm{H}_{2} \mathrm{O}+e^{-} \rightarrow \mathrm{OH}^{-}
$$

If the anodic reaction given in Eq. 2.1 were the only reaction that took place during the corrosion process, there would be no cracking and spalling of the concrete surrounding the steel (Isgor and Razaqpur 2006). However, $\mathrm{Fe}^{2+}$ ions react with species within the pore solution to form other corrosion products. For example, the following reactions represent the formation of the so-called "red rust" after iron dissolution occurs at the anodic sites on the reinforcement (Broomfield 1997):

$$
\begin{array}{lr}
\mathrm{Fe}^{2+}+2 \mathrm{OH}^{-} \rightarrow \mathrm{Fe}(\mathrm{OH})_{2} & \text { Eq. } 2.3 \\
4 \mathrm{Fe}(\mathrm{OH})_{2}+2 \mathrm{H}_{2} \mathrm{O}+\mathrm{O}_{2} \rightarrow 4 \mathrm{Fe}(\mathrm{OH})_{3} & \text { Eq. } 2.4 \\
2 \mathrm{Fe}(\mathrm{OH})_{3} \rightarrow 2 \mathrm{H}_{2} \mathrm{O}+\mathrm{Fe}_{2} \mathrm{O}_{3} \cdot \mathrm{H}_{2} \mathrm{O} & \text { Eq. } 2.5
\end{array}
$$

The volume of corrosion products is three to six times larger than the volume of the steel with the same mass. Therefore, the formation of corrosion products cause internal stresses and generally leads to the cracking and spalling of the concrete surrounding the reinforcement.

The most common causes of the initiation of steel corrosion in concrete are: (1) localized depassivation of the reinforcing steel due to the ingress of chlorides through the concrete cover; and (2) general depassivation of the reinforcement due to the acidification of the concrete pore solution as a result of reactions of the concrete matrix with carbon dioxide that is present in the atmosphere (i.e., carbonation). It has been reported that the presence of chlorides can alter the anodic polarization curve of steel in concrete (Page 
2002): increasing chloride concentrations can decrease the breakdown (or pitting) potential, as shown in Fig. 2.6 and also schematically in Fig. 2.7. Therefore, at a certain chloride concentration, the cathodic polarization curve (of oxygen reduction) intersects the anodic polarization curve (of iron oxidation) at the potential above the pitting potential, as it was shown earlier in Fig. 2.5. When this happens, the passive film is no longer stable and may break down, resulting in a significant increase in the corrosion rate. On the other hand, when the intersection of oxygen reduction and anodic polarization curves is below the critical potential, chlorides are not very effective in breaking down the passive film.

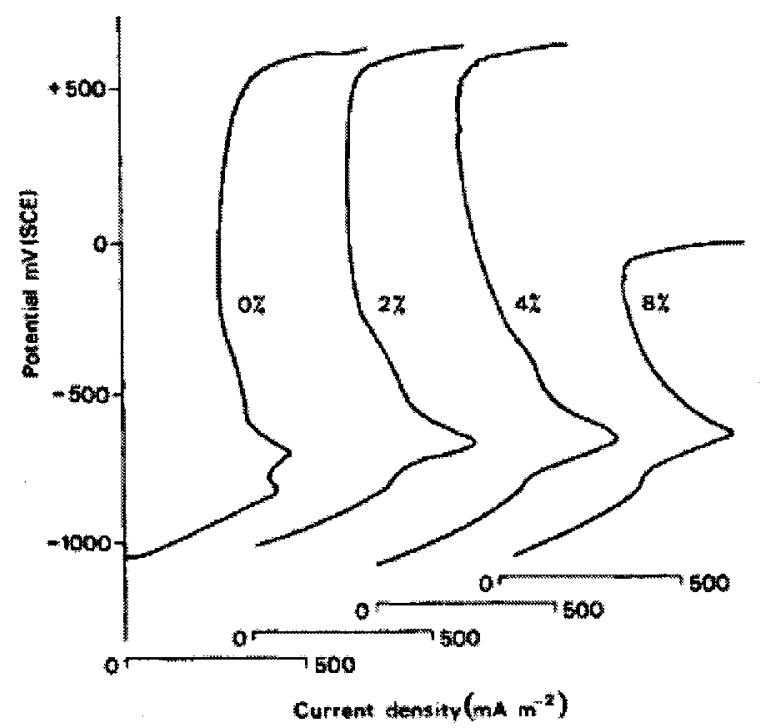

Figure 2.6: Potentiodynamic anodic polarization data of mild steel in cement paste of water to cement ratio of 0.4 (taken from Page 2002). (Increasing percentages (0 to 8\% of cement weight) show the increasing levels of chloride concentration). 


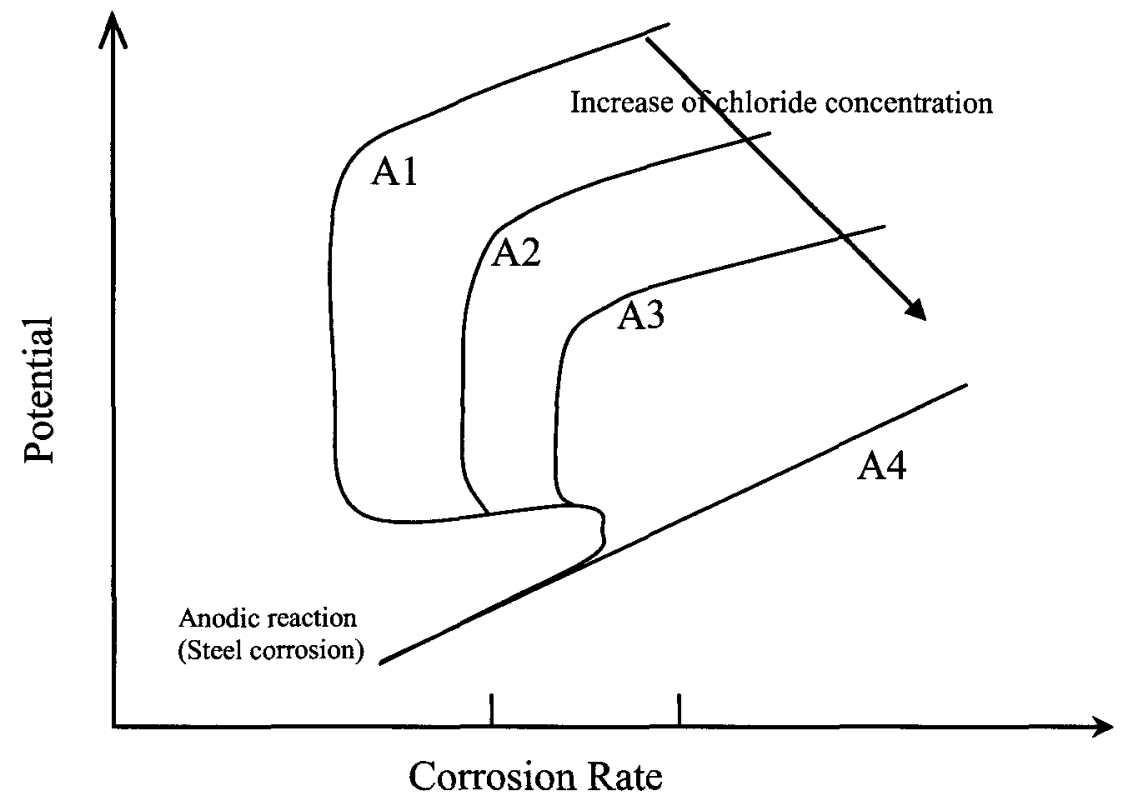

Figure 2.7: Schematic representation of the anodic polarization curve of steel in concrete with different chloride contents (Ramachandran and Beaudoin 2001). Curves A1 to $A 4$ are anodic polarization curves with increasing chloride concentrations.

For concrete, the pitting potential for steel rebar, $E_{p i t}$, is difficult to measure because of significant variations of $\mathrm{pH}$ and chloride concentrations that exist in the pore solution surrounding the surface of steel reinforcement. For a given oxygen reduction potential on steel, the highest content of chlorides compatible with conditions of passivity can be defined as the chloride threshold at that potential (Bertolini et al. 2004). More information on the chloride thresholds will be provided in the literature review.

The other mechanism associated with the breakdown of passive films on steel surface in concrete is the carbonation of concrete. Carbon dioxide present in the air can dissolve in concrete pore solution and form an acidic aqueous solution that decreases the $\mathrm{pH}$ (alkalinity) of concrete towards more neutral conditions (Bertolini et al. 2004). The alkali constituents of the cement paste that are in typical pore solutions are mostly calcium, sodium and potassium hydroxides. Concrete pore solution is always saturated 
with calcium hydroxide. When carbon dioxide comes in contact with calcium hydroxide, the following (simplified versions are presented here) reaction takes place:

$$
\mathrm{CO}_{2}+\mathrm{Ca}(\mathrm{OH})_{2} \rightarrow \mathrm{CaCO}_{3}+\mathrm{H}_{2} \mathrm{O}
$$

Similar reactions can be written for other alkali hydroxides; e.g.

$$
\mathrm{CO}_{2}+2 \mathrm{Na}(\mathrm{OH}) \rightarrow \mathrm{Na}_{2} \mathrm{CO}_{3}+\mathrm{H}_{2} \mathrm{O}
$$

Carbonation results in the $\mathrm{pH}$ drop of concrete pore solution from 13 and above towards more neutral conditions (e.g. below 10) (Ramachandran and Beaudoin 2001). As can be observed in the Pourbaix's diagram of iron (Fig. 2.8), while steel is stable in highly alkaline conditions, at lower alkalinity, the oxide film may no longer protect the reinforcement against active corrosion.

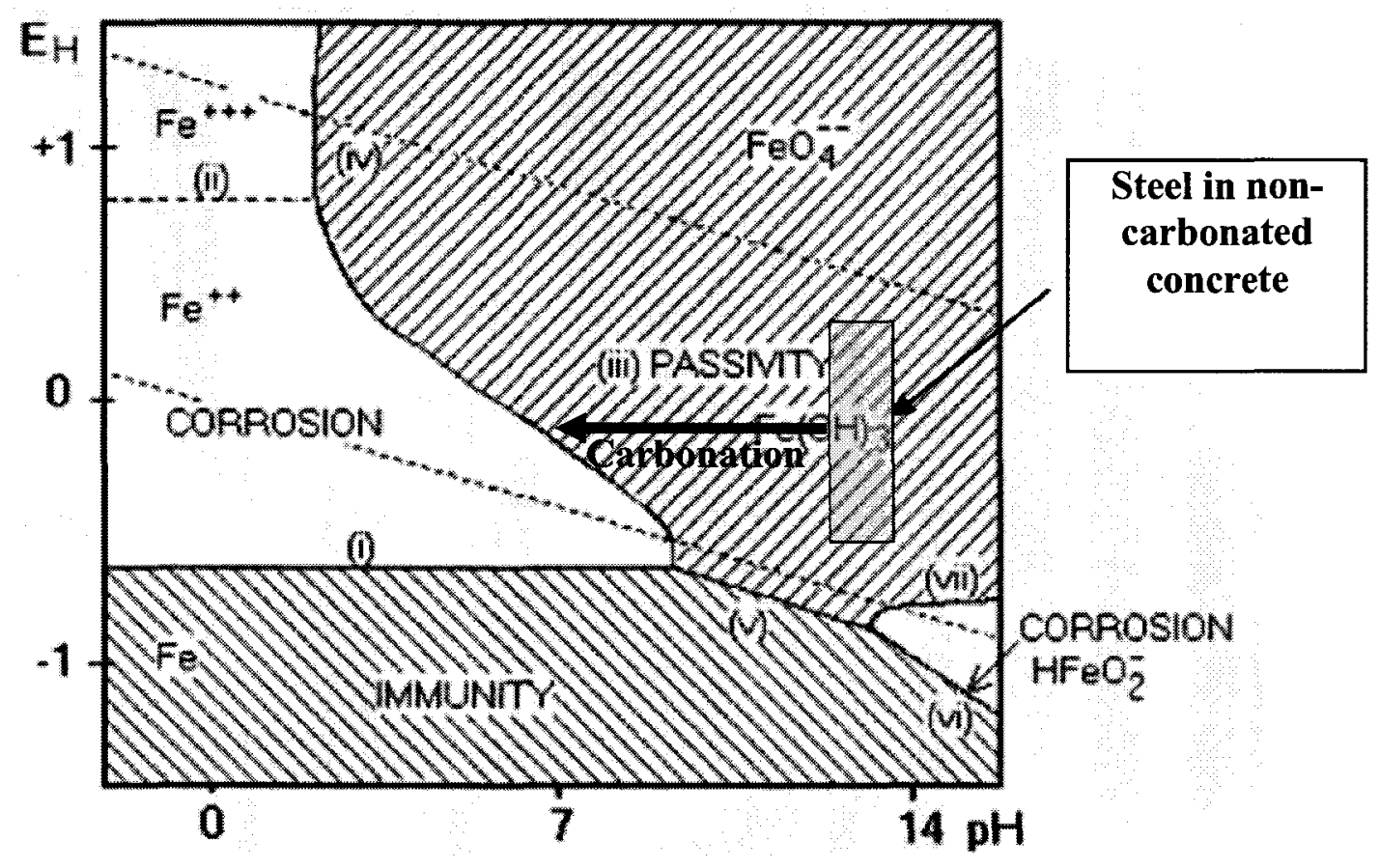

Figure 2.8: The effect of carbonation on thermodynamic behaviour of passive film of steel in concrete (reproduced from Poursaee 2007). The zone of steel in noncarbonated concrete is approximate. 
Figure 2.9 is an Evans diagram showing the kinetic behaviour of the passive film of steel in carbonated concrete. Curve " $\mathrm{B}$ " is the cathodic reaction for oxygen reduction and the "A" curves are anodic reaction curves that show the passive polarization of steel in non-carbonated concrete. As $\mathrm{pH}$ of concrete pore solution surrounding the steel decreases, the anodic reaction curve shifts to the right from $\mathrm{A} 1$ to $\mathrm{A} 2$ (Gu et al. 1997). Subsequently, the corrosion potential drops off from $E_{\text {corr }}$ to $E_{\text {corr }}$ while the corrosion rate increases from $I_{\text {corr }}$ to $I^{\prime}$ corr. However, after the complete breakdown of the steel passive film, the anodic polarization curve of steel in concrete transforms to the straight line " $\mathrm{A}$ ", as illustrated in Fig. 2.10. In this condition, the cathodic polarization curve shows a trend similar to that observed in non-carbonated concrete $(\mathrm{pH}>10)$, but its slope decreases (typically from $250 \mathrm{mV} / \mathrm{dec}$ to $160 \mathrm{mV} / \mathrm{dec}$ as per Garces et al. 2005) which would result in an increase in the corrosion rate (see Fig. 2.10). Moreover, the cathodic curve shifts towards more positive potentials because the equilibrium potential of oxygen reduction at the $\mathrm{pH}$ of carbonated concrete (i.e., $\mathrm{pH}<10$ ) is approximately $200 \mathrm{mV}$ higher than that of non-carbonate concrete (i.e., $\mathrm{pH}>10$ ) (Bertolini et al. 2004) (Fig. 2.10), and this also increases the rate of corrosion. Considering these variations of the anodic and cathodic curves, the corrosion initiation or depassivation of steel in carbonated concrete could be investigated from both kinetic and thermodynamic points of view.

In summary, corrosion of steel rebar in concrete can initiate either by the penetration of chloride into concrete, and subsequent localized depassivation of steel, and/or by the carbonation of concrete, and subsequent $\mathrm{pH}$ reduction and uniform corrosion of steel. Although both causes are important in corrosion studies of rebar in 
concrete structures, only the mechanism of depassivation of carbon steel rebar due to chloride attack is investigated in the present work.

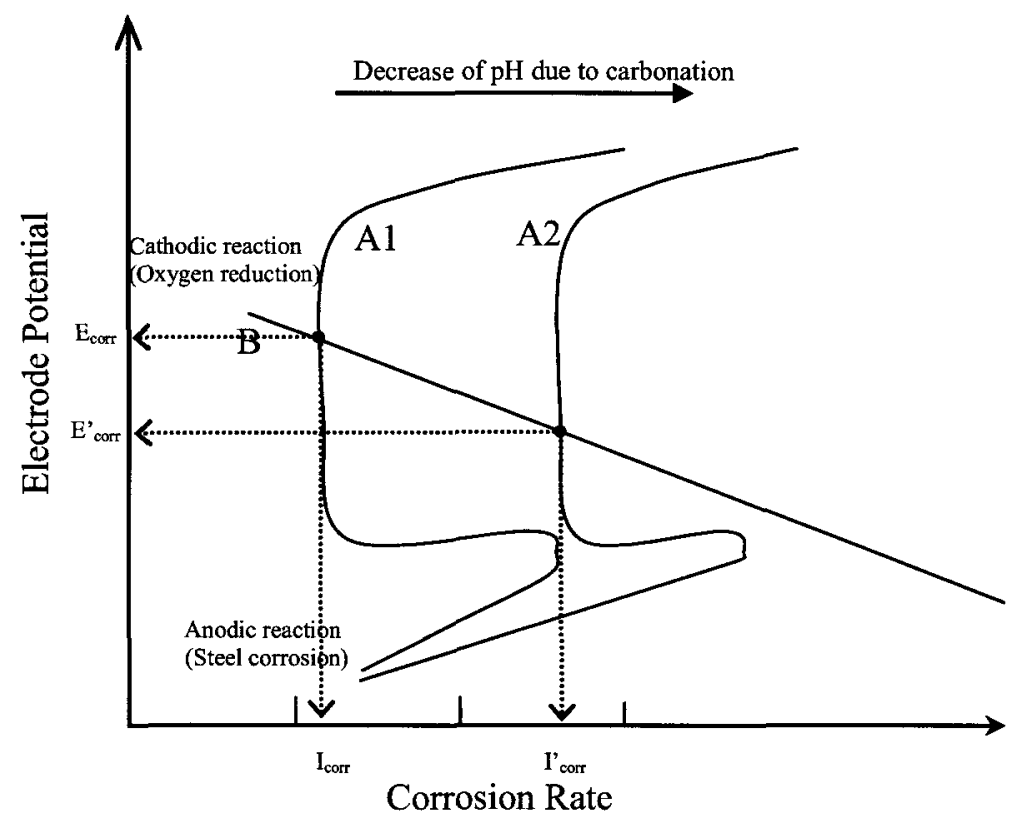

Figure 2.9: The effect of carbonation on kinetic behavior of steel passive film in concrete (reproduced from Ramachandran and Beaudoin 2001).

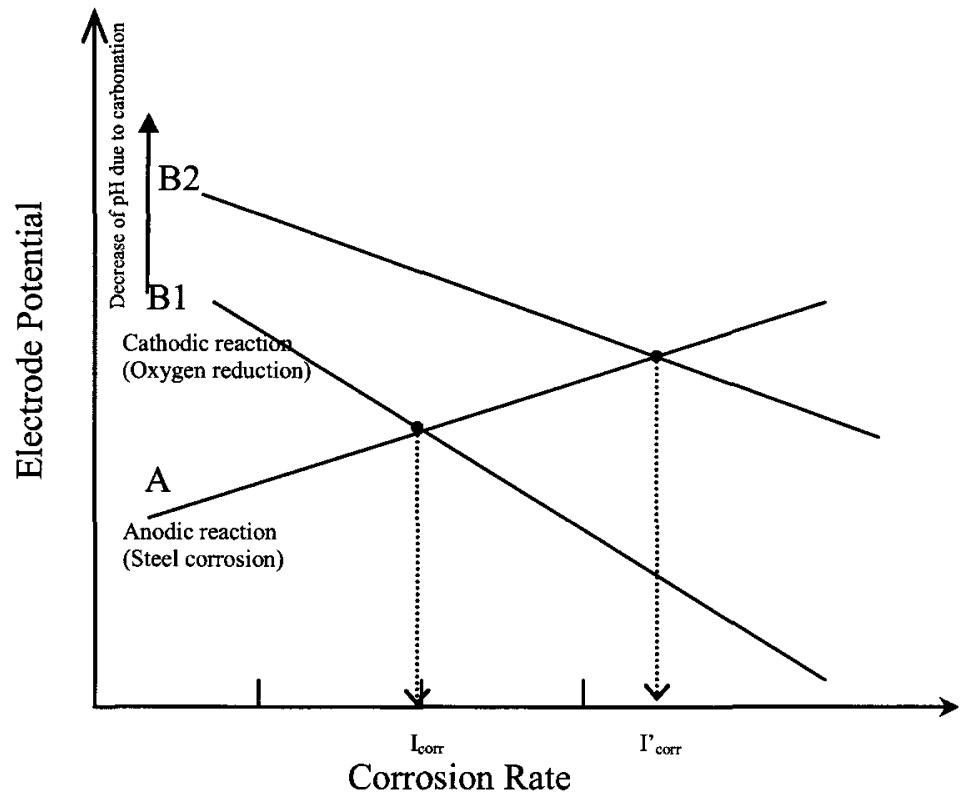

Figure 2.10: The effect of $\mathrm{pH}$ drop on the cathodic polarization reaction after the breakdown of passive layer. Corrosion rate is in logarithmic scale. 


\section{Chapter 3}

\section{Literature Review}

This literature review covers specific topics pertinent to the current studies. Unless otherwise specified, the literature review presented here focuses on carbon steel rebars. The conducted research requires background on the general subjects of corrosion science/engineering, electrochemistry, material science and cement/concrete chemistry. Due to the large scope of these general subjects, the readers are referred to more general references on the topics such as the ones provided by Bard and Faulkner (2001), Ramachandran et al. (1981), Fontana (1986), Neville (1996), Mehta and Monterio (2005), and Revie and Uhlig (2008).

\subsection{Electrochemical Studies on Chloride Threshold}

Chlorides in concrete pore solution are well known to cause depassivation of steel if they are present in concentrations beyond a threshold value. Although there is no common definition for chloride threshold, it may be defined as the chloride concentration in concrete at the depth of rebar that results in the loss of passivity and subsequent increase in corrosion rate. The influential parameters affecting chloride threshold are relatively well known due to the existence of large amount of electrochemical data 
obtained from rebar in large-scale concrete or mortar specimens or in simulated concrete pore solutions. These electrochemical studies mostly provide macro-scale bulk observations about the passivation and depassivation of rebar and have provided invaluable data that are commonly used in current engineering practice. It has been reported in these studies that passivity is affected by the alkalinity of the pore solution, the type and the surface conditions of rebar, concrete mix proportions (i.e., pore solution composition), temperature, relative humidity and oxygen availability in concrete. In this section, based on the available experimental evidence, these factors are discussed.

\subsubsection{Effects of chlorides and alkalinity}

Chlorides in concrete can be found in three forms: (1) free in the pore solution, (2) physically adsorbed to the concrete pore walls, and (3) chemically bound to hydration products. The main form of chemical binding is through the reaction of chlorides with the cement compound tricalcium aluminate $\left(\mathrm{C}_{3} \mathrm{~A}\right)$ to form Friedel salt $\left(3 \mathrm{CaO} \cdot \mathrm{Al}_{2} \mathrm{O}_{3} \cdot \mathrm{CaCl}_{2} \cdot 10 \mathrm{H}_{2} \mathrm{O}\right)$ (Neville 1996). A similar reaction of chlorides with the cement compound tetracalciulm aluminoferrite $\left(\mathrm{C}_{4} \mathrm{~A}\right)$ results in calcium chloroferrite $\left(3 \mathrm{CaO} \cdot \mathrm{Fe}_{2} \mathrm{O}_{3} \cdot \mathrm{CaCl}_{2} \cdot 10 \mathrm{H}_{2} \mathrm{O}\right)($ Neville 1996). From the stoichiometry of these reactions, it can be calculated that one gram of $\mathrm{C}_{3} \mathrm{~A}$ will consume 0.263 grams of chloride, while one gram of $\mathrm{C}_{4} \mathrm{AF}$ will consume 0.146 grams of chloride (Neville 1996).

The chloride threshold is commonly presented as the total chloride content expressed relative to the mass of cement. This approach is favoured due to the availability of relatively simple techniques to measure the total chloride concentrations in concrete, as documented in the standards ASTM C114 (2009) and BS1881:Part 124 (1988). Glass and Buenfeld (1997) supported this presentation of chloride thresholds 
because they believed that bound chloride may participate in the depassivation process when the $\mathrm{pH}$ of concrete pore solution surrounding the rebar drops locally, causing the release of bound chlorides. In fact, it has been documented that $\mathrm{pH}$-reducing processes, such as carbonation of the concrete cover, may result in the release of the chemicallybound chlorides to the pore solution (Izquierdo et al. 2004). This means that bound chlorides can increase the free chloride content and hence the probability of corrosion. However, in an early work, Hausmann (1967) argued that chloride threshold is best expressed as chloride-to-hydroxide concentration ratio $\left(\mathrm{Cl}^{-} / \mathrm{OH}^{-}\right)$or as free chloride concentration in the concrete pore solution. It has also been shown by other researchers (Goni and Andrade 1990, Venu et al.1965, Li and Sagues 2001) that the chloride threshold and the quality of the passive film on the steel surface vary with the alkalinity of the pore solution, which changes with the maturation of cement paste, and depends on the type and content of cement and on the presence of supplementary cementitious materials (SCMs), such as silica fume and fly ash.

Concrete's ability to resist local acidification (i.e., drops in $\mathrm{pH}$ below 10) has been shown to be important in the passivity of rebar (Alonso et al. 2000). However, the hydroxide concentration in the concrete pore solution, which is largely determined by the alkali metal hydroxides (e.g. calcium hydroxide, sodium hydroxide and potassium hydroxide), is not an adequate measure of the inhibitive properties of concrete against steel corrosion. The experimental data produced by Hansson and Sorensen (1990) suggest that a loose correlation exists between the chloride threshold, expressed as total chloride content (rather than free chloride concentration or $\mathrm{Cl}^{-} / \mathrm{OH}^{-}$), and the $\mathrm{pH}$ of the pore solution (see Fig. 3.1). They noted that the positive effect of the hydroxides should be 
considered along with the negative effects of the chlorides when studying the depassivation of steel in concrete. Therefore, it was suggested that $\mathrm{Cl}^{-} / \mathrm{OH}^{-}$is a better criterion for chloride threshold than total chloride concentration.

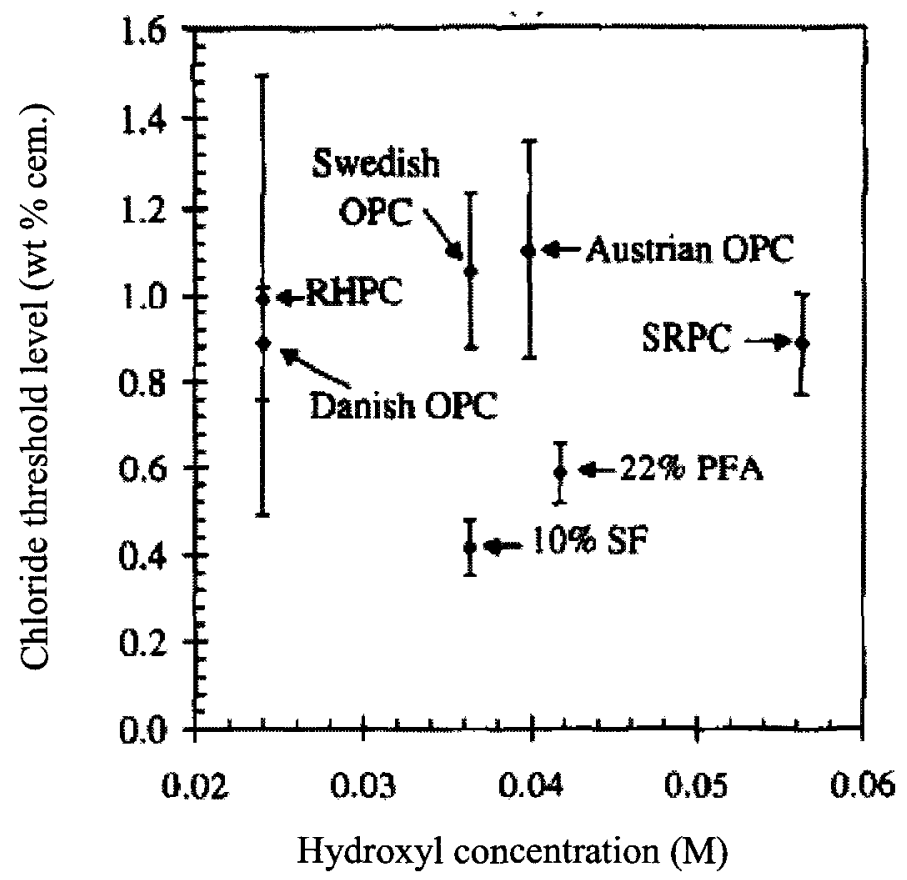

Figure 3.1: Reported chloride threshold levels plotted as a function of hydroxide concentration of the pore solution in the cover zone (taken from Hansson and Sorensen 1990). SF: silica fume, OPC: ordinary portland cement, SRPC: sulfate resistant portland cement, PFA: pulverised fly ash, RHPC: rapid hardening portland cement.

In spite of the efforts of numerous studies to establish a common chloride threshold for rebar depassivation, as documented by a recent review by Angst et al. (2009) that is presented in Table 3.1 and Fig. 3.2 (abbreviations in the table and figure are given in Table 3.2), the literature shows significant variability in chloride thresholds (represented as $\mathrm{Cl}^{-} / \mathrm{OH}^{-}$). As it can be seen in Table 3.1 and Fig. 3.2, the reported threshold $\mathrm{Cl}^{-} / \mathrm{OH}^{-}$values are scattered in a wide range from 0.01 to 45 . 
Table 3.1: Summary of reported chloride thresholds (reproduced from Angst et al. 2009). Abbreviations are given in Table 3.2.

\begin{tabular}{|c|c|c|c|c|}
\hline Reference & $\begin{array}{c}\text { Steel } \\
\text { condition }\end{array}$ & $\begin{array}{l}\text { Corrosion } \\
\text { detection }\end{array}$ & $\begin{array}{c}\text { Exposure } \\
\text { medium }\end{array}$ & $\mathrm{Cl}^{-} / \mathrm{OH}^{-}$ \\
\hline Rajagopalan et al. (1962) & $\mathrm{ABR}, \mathrm{CL}$ & GP & $\mathrm{pH} 12.6$ & $0.02-0.13$ \\
\hline Venu et al. (1965) & $\mathrm{ABR}, \mathrm{CL}$ & GP & $\mathrm{pH} 13$ & 1.0 \\
\hline Hausmann (1967) & $S M, P, C L$ & $E, V I$ & $\mathrm{pH} 11.6-13.2$ & 0.6 \\
\hline \multirow[t]{2}{*}{ Yonezawa et al. (1988) } & \multirow[t]{2}{*}{$P$} & \multirow[t]{2}{*}{ LPR, E } & $\mathrm{pH} 13.8$ & 4.9 \\
\hline & & & OPC & $7-45$ \\
\hline Goñi and Andrade (1990) & $P, C L$ & LPR, E & $\mathrm{pH} 11.64-13.22$ & $0.25-0.8$ \\
\hline Lambert et al. (1991) & $\mathrm{SM}, \mathrm{SB}, \mathrm{CL}$ & LPR, E, WL & OPC, SRPC & $3-20$ \\
\hline Pettersson (1995) & RIB & LPR & OPC, SF & $2.5-6$ \\
\hline Breit (1998) & $\mathrm{CL}$ & PC & $\mathrm{pH} 13.5$ & 0.26 \\
\hline Alonso et al. (2000) & RIB, SM & LPR, E & OPC & $1.17-3.98$ \\
\hline Zimmermann (2000) & $\mathrm{SB} ; \mathrm{CL}$ & E, MC & $\mathrm{pH} 13.2-13.5$ & $0.7-1.7$ \\
\hline \multirow[t]{3}{*}{ Li and Sagüés (2001) } & \multirow[t]{3}{*}{$A R, S B, P R$} & \multirow[t]{3}{*}{ E, EIS } & $\mathrm{pH} 12.6$ & $0.01-0.04$ \\
\hline & & & $\mathrm{pH} 13.3$ & $0.2-0.8$ \\
\hline & & & $\mathrm{pH} 13.6$ & $1.0-2.5$ \\
\hline Alonso et al. (2002) & RIB, MIL & PC & OPC, SRPC, FA & $1.67 \pm 0.3$ \\
\hline Castellote et al. (2002) & RIB & LPR, E & SRPC & $1.5-2.0$ \\
\hline Oh et al. (2003) & SM & $E, V I$ & $\begin{array}{l}\text { OPC, SRPC, } \\
\text { GGBS, FA }\end{array}$ & $0.16-0.27$ \\
\hline Trejo and Pillai (2003) & $\mathrm{CL}$ & LPR & OPC & $0.09-0.62$ \\
\hline \multirow[t]{2}{*}{ Moreno et al. (2004) } & \multirow[t]{2}{*}{$\mathrm{CL}$} & \multirow[t]{2}{*}{ PDP } & $\mathrm{pH} 12.5$ & 0.178 \\
\hline & & & $\mathrm{pH} 13.9$ & 0.313 \\
\hline
\end{tabular}



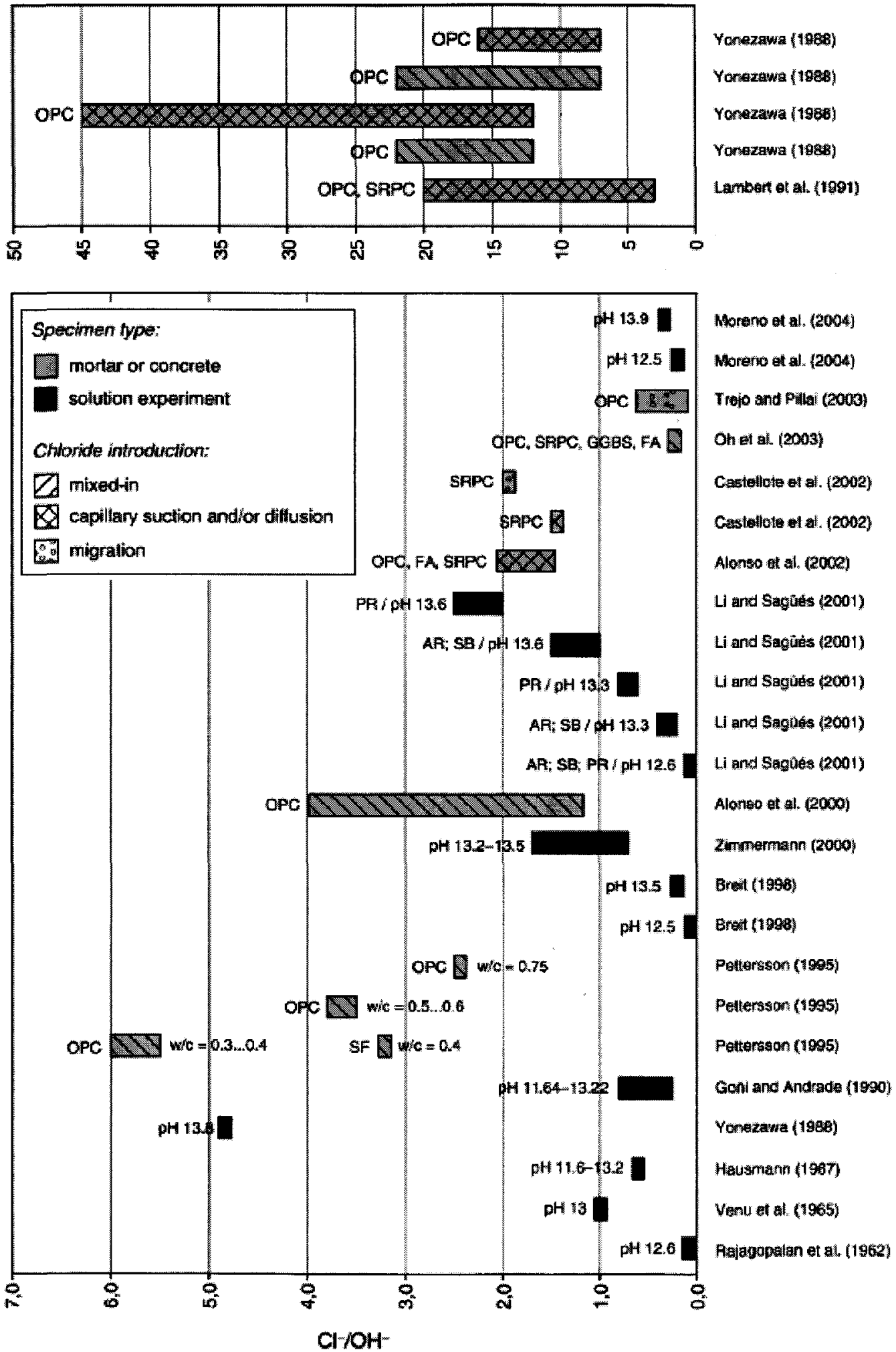

Figure 3.2: Summary of reported chloride thresholds (taken from Angst et al. 2009).

Abbreviations are given in Table 3.2. 
Table 3.2: Abbreviations in Table 3.1 and Figure 3.2 (reproduced from Angst et al. 2009).

\begin{tabular}{|l|l|l|}
\hline Exposure medium & \multicolumn{1}{|c|}{ Steel condition } & \multicolumn{1}{c|}{ Corrosion detection } \\
\hline$F A$ - Fly ash & $A B R$ - Abraded & E - Potential \\
GGBS - Ground & $A R$ - As-received & EIS - Electrochemical impedance \\
granulated blast & $C L$ - Cleaned or & spectroscopy \\
furnace slag & degreased & GP - Galvanostatic polarisation \\
OPC - Ordinary & MIL - Mill-scaled & LRP - Linear polarisation resistance \\
portland cement & $P$ - Polished & $M C$ - Macro-cell current \\
SF - Silica fume & $P R$ - Pre-rusted & $P C$ - Potentiostatic control \\
SRPC - Sulfate & $R I B$ - Ribbed steel bars & $P D P$ - Potentiodynamic polarisation \\
resistant Portland & $S B$ - Sandblasted & $V I$ - Visual inspection \\
cement & $S M$ - Smooth steel bars & WL - Weight loss \\
\hline
\end{tabular}

A widely accepted observation among these studies is that chloride threshold is highly correlated with the alkalinity of the pore solution: the higher the alkalinity, the higher the chloride threshold. Also in Table 3.1 and Fig. 3.2 is that the variability in the chloride thresholds is larger for steel in concrete or mortar than for steel in simulated concrete pore solutions. Similar earlier reviews by Alonso et al. (2000) and Glass and Buenfeld (1997) also reported the same observations.

\subsubsection{Effects of rebar surface condition and rebar/concrete interface}

Although surface conditions of rebar are generally known to influence pitting and subsequent rates of corrosion (Fontana 1986, Hansson and Sorensen 1990, Jones 1995), only a few studies exist that quantify the effect of the surface finish of rebar on corrosion initiation. A study by Mammoliti (1995) on the effectiveness of chemical inhibitor admixtures on chloride concentration to initiate corrosion came to the rare conclusion that corrosion could not be initiated in highly polished samples of steel rebar, which were tested in synthetic pore solutions, even though the specimens were exposed to unrealistically high chloride concentrations. This suggests that the lack of control on 
the surface condition of rebar may be one of the factors responsible for the fact that rebars in some concretes containing high chloride concentrations do not exhibit corrosion, whereas rebars in other structures, with much less chloride concentrations, may corrode rapidly.

Mammoliti et al. (1996) conducted an investigation on the effect of steel surface conditions on chloride threshold in synthetic pore solutions. Three different surface conditions were studied: (i) as-received, degreased, deformed rebar; (ii) polished and smooth rebar; (iii) polished cross section of deformed rebar. Results from this study indicated that the surface condition of the steel has a major influence on chloride threshold; however, they did not provide detailed conclusions regarding this observation.

Mohammed and Hamada (2006) also studied rebars with different surface conditions (e.g. as as-received rebar with mill scale, polished rebar, brown-rusted rebar, black-rusted rebar and pre-passivated rebar covered with cement paste). The highest chloride threshold was obtained for pre-passivated rebar while the lowest was reported for as-received rebar with mill scale.

Pillai and Trejo (2005) determined the chloride thresholds of five types of uncoated steel reinforcement (low-alloy steel - ASTM A706, carbon steel - ASTM A615, microcomposite - type not specified, stainless steel - SS304, and stainless steel SS316LN) with both as-received and polished surface conditions. The results of this research indicated that milling and polishing the surface of low-alloy (ASTM A706), microcomposite, and SS304 rebar specimens increased, albeit in different amounts, the chloride thresholds of these reinforcement types. However, the chloride thresholds of the ASTM A615 and SS316LN rebar decreased, again in different amounts, with the removal 
of the as-received surface. The researchers suggested that this variable behaviour of different steel types with surface modification could be due to the removal of mill scales, which are different for different steel types. For example, the reduction in the chloride threshold of the SS316LN reinforcement was thought to be a possible result of the removal of a strong and corrosion resistant film during the mill scale removal process. Further investigation of the role of mill scale in the chloride-induced depassivation of carbon steel rebar is carried out in the current research and presented in Chapters 5 and 6.

The chloride threshold has been also found to be dependent on the presence of macroscopic voids in the concrete near the steel surface (Glass and Buenfeld 2000). Voids and cracks in concrete that are normally found in real structures due to incomplete compaction, low concrete workability, the ribbing on the surface of steel rebars, and the orientation of rebar with respect to the casting direction may weaken the layer of cement hydration products deposited at the steel/concrete interface (Horne et al. 2007, Mohammed and Hamada 2001), and thus may favour local acidification that is required for sustained propagation of pits. For instance, it was shown that by decreasing the volume of entrapped air in the steel-concrete interfacial zone from $1.5 \%$ to $0.2 \%$, the chloride threshold increased from $0.2 \%$ to $2 \%$ by mass of cement, as illustrated in Fig. 3.3 (Glass and Buenfeld 1997). Page (2002) also reported that air voids and microcracks along the rebar-concrete interface may cause lower chloride thresholds in real structures when compared with those found in well-compacted similar laboratory specimens. 


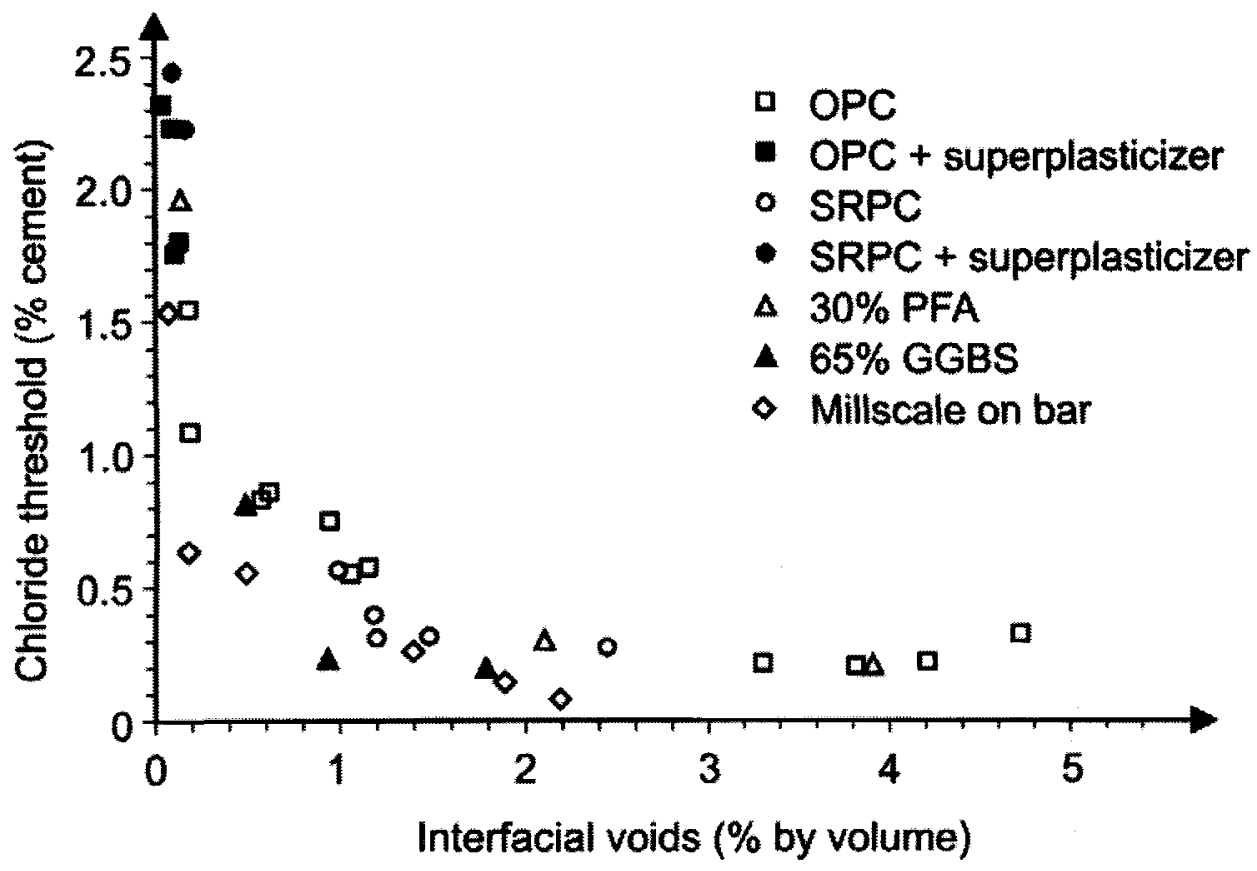

Figure 3.3: Chloride threshold for corrosion initiation as a function of volume fraction of interfacial voids (taken from Glass and Buenfeld 2000). (OPC: ordinary portland cement; SRPC: sulfate-resistant portland cement; PFA: pulverized fly ash; GGBS: ground granulated blast furnace slag).

\subsubsection{Oxygen availability, relative humidity and water content in concrete}

Although the oxygen availability in concrete at the rebar surface has been suggested to be one of the factors affecting the chloride thresholds of rebar in concrete (Hausmann 1967, Gouda 1970, Pourbaix 1973, Alonso et al. 2000), there remains a lack of experimental data for quantitative evaluation of such a correlation. Moisture and oxygen are necessary for corrosion of steel in concrete, thus there are some exposure conditions in which corrosion rates will be inconsequentially small even though the chloride levels may exceed the threshold values. For example, reinforced concrete that is continuously submerged in seawater rarely exhibits corrosion because of insufficient oxygen at the rebar level. In this condition, the only cathodic process possible is hydrogen evolution, as 
shown by the lower portions of the cathodic polarization curves in Fig.3.4 (Bertolini et al. 2004). In this figure, the cathodic polarization curve slopes downward as the oxygen concentration of concrete decreases. As a result, the open-circuit potential of rebar in concrete, which is an influential factor on chloride threshold, reduces; the effect of opencircuit potential on chloride threshold will be explained further in Section 3.1.6.

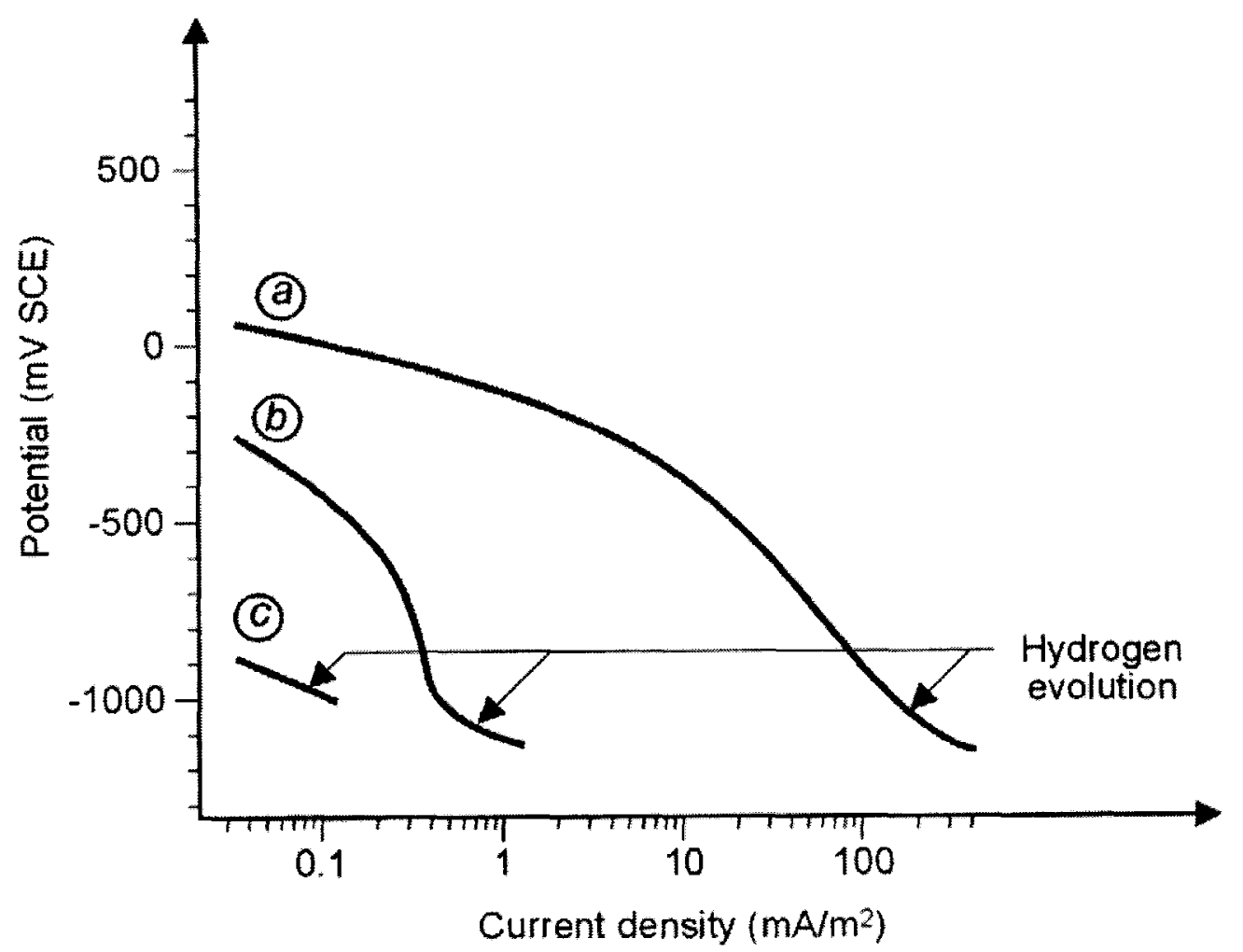

Figure 3.4: Schematic cathodic polarization curves in alkaline concrete: (a) aerated and semi-dry (high oxygen availability); (b) wet (medium oxygen availability); (c) completely saturated with water (low oxygen availability) (taken from Bertolini et al. 2004).

Changes in relative humidity concurrently influence the resistivity of concrete, the oxygen concentration at the rebar surface, and the water content of concrete required for corrosion initiation. All these factors affect chloride thresholds. The effect of relative humidity on the chloride threshold for mortars tested by Pettersson (1996b) is shown in 
Fig. 3.5. In this figure, the chloride thresholds decrease due to decreasing electrical resistivity as the relative humidity increases up to $80-90 \%$; however, beyond this level, the chloride threshold increases with increasing relative humidity as the oxygen diffusion in concrete (i.e., concentration polarization) becomes the controlling mechanism of corrosion, even though the electrical resistivity of concrete is low.

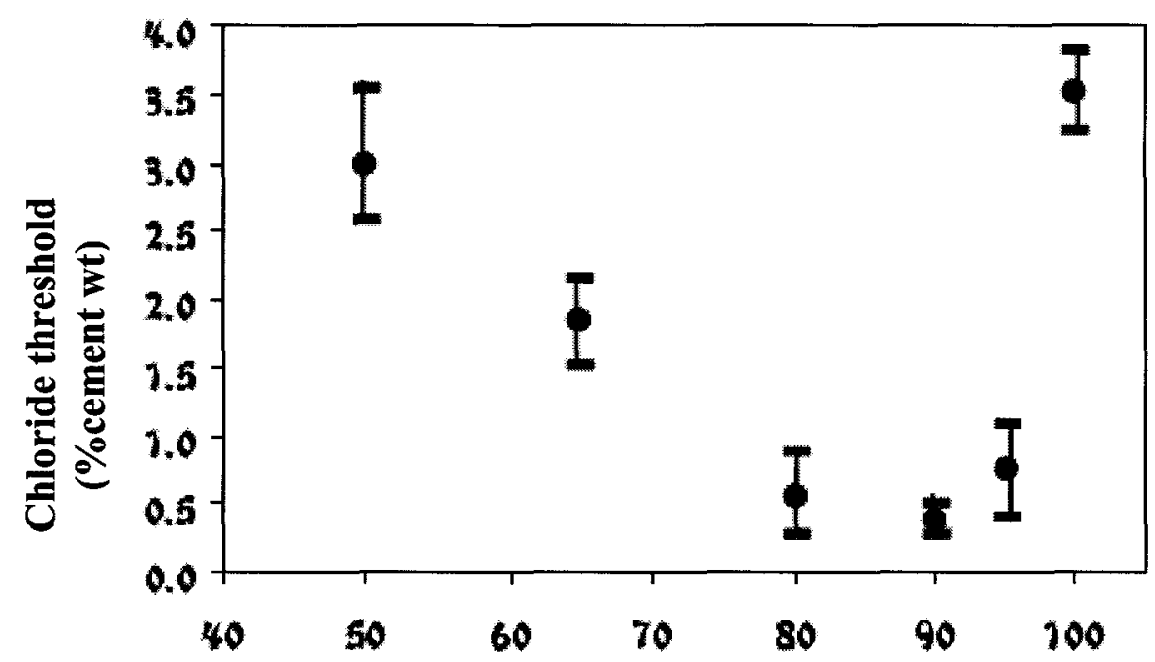

Relative humidity \%

Figure 3.5: The effect of relative humidity on the chloride threshold level in laboratory exposed mortars, water to cement ratio $(w / c)=0.50$ (taken from Pettersson $1996 \mathrm{~b}$ ).

Cyclic wetting and drying of concrete increases the risk of corrosion (Page et al. 1991) since this type of exposure increases the amount of chlorides penetrating into concrete. Wetting and drying cycles also result in the decrease in concrete resistivity and the increase in oxygen concentration around steel available for the cathodic reaction. Rehm et al. (1981) reported that corrosion did not initiate when the resistivity of the concrete was greater than $5 \times 10^{4} \mathrm{ohm}-\mathrm{cm}$, a condition that can occur when the relative humidity in the pores is less than $40-50 \%$. The effect of exposure conditions will be discussed further in the next section. 


\subsubsection{Exposure conditions}

Considering the location of a structure with respect to seawater, different exposure conditions can be defined (e.g. splash, tidal, submerged and aerated zones). There are also other types of exposure conditions that can be specified for structures that are frequently exposed to deicing salts (e.g. bridges in Canada). In each case, the influencing environmental factors are different in terms of humidity, temperature, wind and solar radiation, and the circumstances under which the corrosion initiates are directly dependent on these conditions. For example, Frederiksen (2000) has studied the effects of exposure conditions on concrete structure with different water-to-binder ratios and SCMs and suggested chloride thresholds in various Nordic exposure zones.

The initiation of reinforcement corrosion and its corrosion rate are temperature dependent (Elsener 2005). The rise in temperature usually contributes to the increase of chloride solubility in the pore solution of concrete and consequently to the breakdown of the passive layer. For example, the solubility of $\mathrm{CaCl}_{2}$ in water increases by $55 \%$ when the ambient temperature rises from $0^{\circ} \mathrm{C}$ to $30^{\circ} \mathrm{C}$ (Zivica et al. 1997). It is also known that, if there is an increase in the ambient temperature, the mobility of chlorides in concrete would also increase, and this may cause excessive accumulation of chlorides around rebars. The oxygen concentration and moisture content in concrete, which are required for corrosion (specifically cathodic reactions) to take place, are also sensitive to the ambient temperature; i.e., the oxygen concentration in the pore solution and the water content of concrete decrease by increasing ambient temperature. For instance, when the ambient temperature increases from room temperature to $80^{\circ} \mathrm{C}$, the oxygen concentration in concrete decreases by about $75 \%$ (Akimov 1953). Therefore, the temperature has an 
inverse relationship with the cathodic reaction rate (Zivica et al. 1997). This is contrary to the effect of the ambient temperature on the rate of the anodic reaction.

Tuutti (1982) considered the effect of ambient temperature on oxygen diffusion in concrete as a controlling factor affecting the corrosion process. It was shown that, between -20 and $+20^{\circ} \mathrm{C}$, the corrosion rate increased linearly by around two orders of magnitude; in the interval between +10 and $+20^{\circ} \mathrm{C}$, the corrosion rate increased only seven times. Schiessl and Raupach $(1988,1990)$ showed that by an increase in ambient temperature from +15 to $+20^{\circ} \mathrm{C}$, corrosion rate increased by around $50 \%$.

Rasheeduzzafar et al. (1992) studied the effect of temperature on the chloride threshold level. The results of these experiments on various cement pastes are shown in Table 3.3. This investigation reported that a notable increase in temperature would decrease the chloride thresholds and increase chloride ingress and corrosion rates. It was concluded that an increase in temperature from $20^{\circ} \mathrm{C}$ to $70^{\circ} \mathrm{C}$ caused a fivefold reduction in chloride thresholds.

\subsubsection{Mixture proportions of concrete}

\subsubsection{Water-to-binder $(\mathrm{w} / \mathrm{b})$ ratio}

It has been shown that chloride threshold increases by decreasing the water-tobinder (w/b) ratio of concrete (Hansson and Sorensen 1990, Poupard et al. 2004, Pettersson and Sandberg 1997). Pettersson and Sandberg (1997) investigated the impact of the $\mathrm{w} / \mathrm{b}$ on the chloride threshold of steel rebar in concrete with $15-20 \mathrm{~mm}$ of cover thickness. 
Table 3.3: Effect of exposure temperature on chloride thresholds by Rasheeduzzafar et al. (1992).

\begin{tabular}{|c|c|c|c|}
\hline No. & $\begin{array}{c}\mathrm{C}_{3} \text { A content of cement } \\
\text { (\% by weight) }\end{array}$ & Temperature & $\begin{array}{c}\text { Threshold chloride } \\
\text { (\% by weight of cement) }\end{array}$ \\
\hline 1 & 2.43 & 20 & 0.35 \\
2 & 2.43 & 70 & 0.04 \\
3 & 7.59 & 20 & 0.62 \\
4 & 7.59 & 70 & 0.09 \\
5 & 14.00 & 20 & 1.00 \\
6 & 14.00 & 70 & 0.19 \\
\hline
\end{tabular}

The corresponding results for different types of concretes are shown in Fig. 3.6. Increasing the $w / b$ from 0.3 to 0.75 , the chloride threshold first slightly increases, whereas after a value of 0.4 , it gradually decreases. It should be noted in this figure that the effect of different concrete types produced with different cementitious materials is not clearly evident; but their trends are similar with respect to the w/b .

\subsubsection{Supplementary cementitious materials (SCMs)}

Concrete in its basic form is a mixture of ordinary portland cement (OPC), fine and coarse aggregate, and water. The principle cementitious material in ordinary concrete is portland cement. However, today, most concrete produced for structural applications contains SCMs that form part of the cementitious components in addition to ordinary portland cement. These materials provide concrete with various desirable properties, sometimes in fresh state (e.g. workability, fresh density, etc.), but more often in hardened concrete (e.g. increased durability, strength, density, etc.) (Neville 1996). Fly ash, silica fume, metakaolin, (ground granulated) blast furnace slag and natural pozzolans are commonly used SCMs in concrete. 


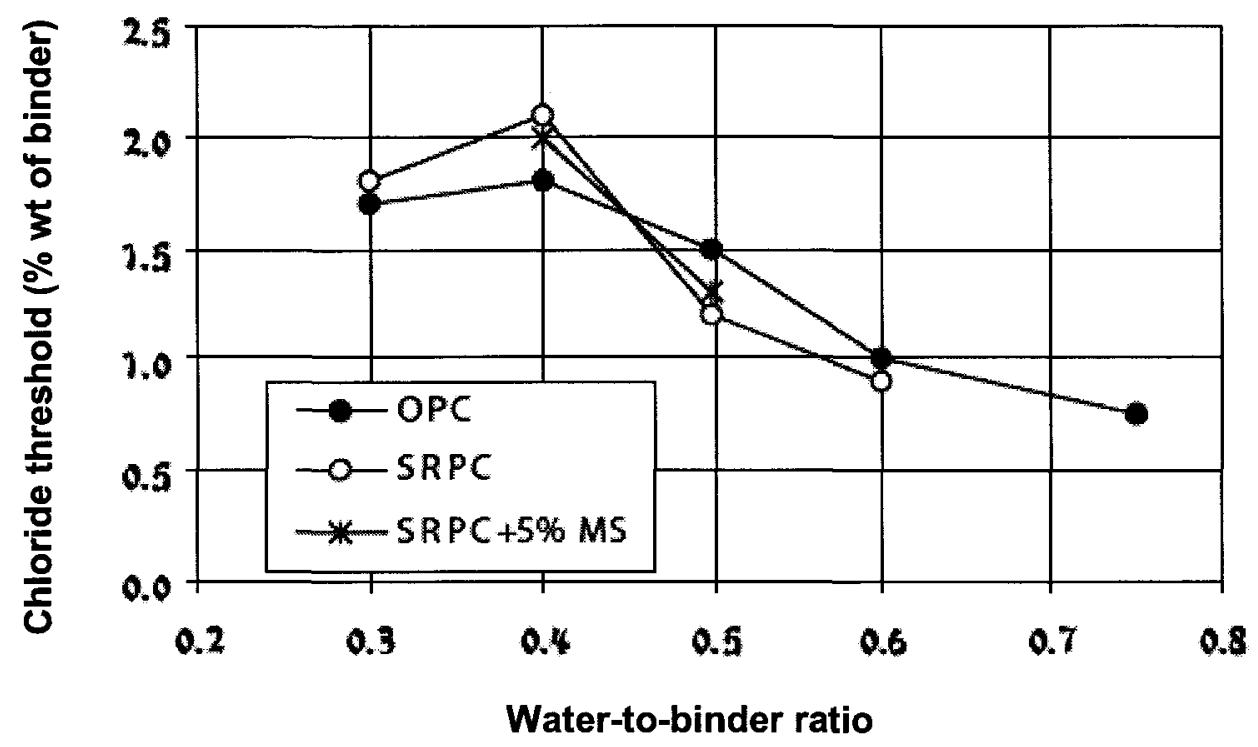

Figure 3.6: Chloride thresholds measured in submerged concrete or mortar taken from Pettersson and Sandberg (1997). (OPC: ordinary portland cement; SRPC: sulfate resistant portland cement; $M S$ : micro silica).

SCMs have been known to decrease the steel corrosion rates in concrete. The main reason of this positive effect can be attributed to the fact that the resistivity of concretes produced with SCMs are generally higher than OPC concretes at the same temperature and relative humidity. However, these findings are, in most cases, not directly related to depassivation studies that focus on chloride thresholds; rather, they are obtained from studies investigating active corrosion.

Thomas (1996), on the other hand, demonstrated that SCMs, such as fly ash, decrease the chloride thresholds in concrete used in marine environments; as illustrated in Fig. 3.7: Higher amounts of fly ash replacement resulted in lower chloride thresholds. Pettersson $(1993,1996$ a) reported similar results for fly ash and silica fume on the chloride thresholds of steel in concrete. However, Sandberg et al. (1995) stated that in some cases the presence of SCMs led to an increase in chloride thresholds. This negative 
effect of SCMs on the chloride threshold was attributed to the decrease in alkalinity of the concrete pore solution (as a result of SCM addition), and to the decrease in the amount of calcium hydroxide at the steel/concrete interface.
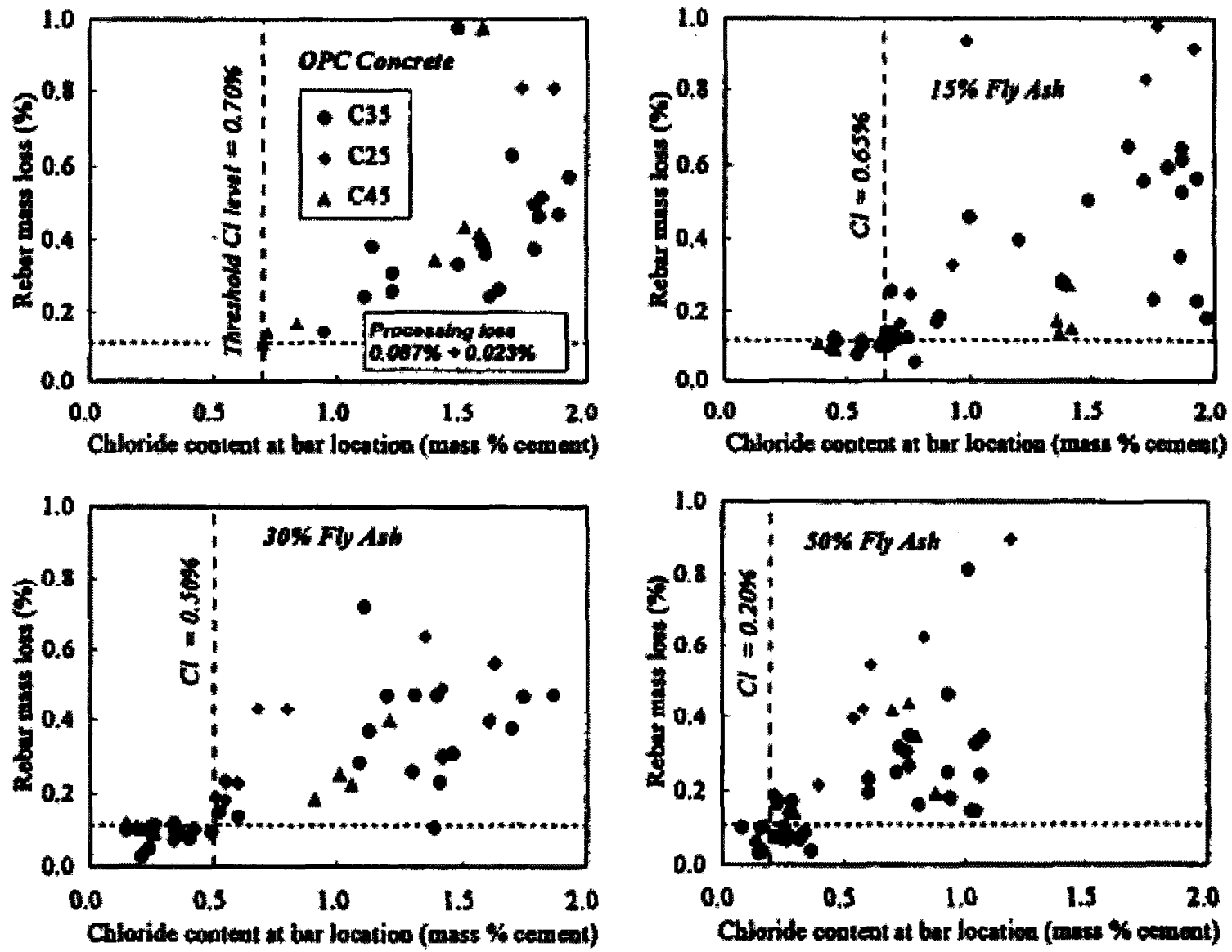

Figure 3.7: Chloride threshold levels and corrosion rates for $O P C$ and fly ash concrete exposed in a marine splash zone in Canada (taken from Thomas 1996).

\subsubsection{Cement type}

Hussain et al. (1995) studied the effect of variable $C_{3} A$ contents of cement with different levels of alkali and sulfate contents on chloride thresholds. Using pore solution composition data, they found that increased $\mathrm{C}_{3} \mathrm{~A}$ content of cement has a beneficial effect 
on chloride thresholds and the corrosion resistance of rebars: an increase in $\mathrm{C}_{3} \mathrm{~A}$ content of cement from $2.43 \%$ to $14 \%$ raises chloride thresholds by $2-3$ times. Oh et al. (2004) also showed the chloride thresholds (in terms of total chlorides) were in the range of $0.45-0.97 \%$ by mass of cement with various $\mathrm{C}_{3} \mathrm{~A}$ contents. Fig. 3.8 shows the comparison between the results of Oh et al. (2004) and Hussain et al. (1995). Although the results are not identical, the trend in both studies is similar; i.e., the higher $\mathrm{C}_{3} \mathrm{~A}$ contents results in higher chloride thresholds.

\subsubsection{Other factors}

As discussed in Chapter 2, the open-circuit potential (OCP) of steel rebar in concrete plays an important role in corrosion initiation. The experimental results of Alonso et al. (2002) have shown that the chloride threshold of rebar in concrete increases by decreasing open-circuit potential for potential ranges lower than $-200 \mathrm{mV} / \mathrm{SCE}$. The potential of steel rebar in concrete structures exposed to atmosphere generally is in the range of +100 to $-200 \mathrm{mV} / \mathrm{SCE}$, while for submerged structures it varies between -400 and $-200 \mathrm{mV} / \mathrm{SCE}$ (Bertolini et al. 2004). Thus, the chloride threshold is usually higher for submerged reinforced concrete structures than aerated ones. 


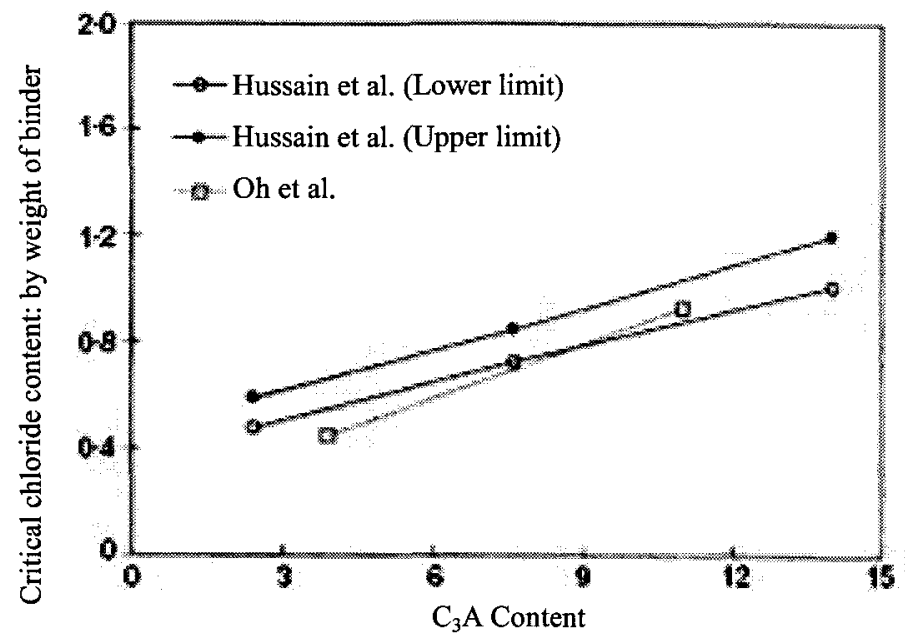

Figure 3.8: Chloride thresholds, represented as total chloride, versus $C_{3} A$ content (\% of cement) (reproduced from Oh et al. 2004 and Hussain et al. 1995).

The corrosive effect of different types of chloride salts such as $\mathrm{NaCl}, \mathrm{CaCl}_{2}, \mathrm{KCl}$ are not the same. It was reported that the chloride thresholds determined using $\mathrm{CaCl}_{2}$ is lower than that determined using $\mathrm{NaCl}$ or $\mathrm{KCl}$ (Andrade and Page 1986, Hansson et al. 1985). Furthermore, several studies revealed that the amount of bound chloride increases when $\mathrm{CaCl}_{2}$ is used as the source of chloride (Andrade and Page 1986, Arya et al. 1990, Tritthart 1989).

\subsubsection{Summary}

The factors affecting chloride thresholds of rebar have been reviewed. It has been shown that the reported chloride thresholds cover a wide range and subject to a high degree of uncertainty. This uncertainty is also obvious in the range of chloride thresholds specified in major reinforced concrete building design codes, as shown in Table 3.4. The most influential parameters that affect the chloride thresholds are summarized in Table 3.5 (Angst et al. 2009). This table also shows the qualitative effects of these parameters on chloride thresholds. 
Table 3.4: Chloride thresholds used in various concrete building design codes (Oh et al. 2004).

\begin{tabular}{|l|l|l|}
\hline \multirow{2}{*}{ Code } & \multicolumn{2}{c|}{$\begin{array}{c}\text { Threshold chloride, } \\
\% \text { by cement weight }\end{array}$} \\
\cline { 2 - 3 } & $\begin{array}{c}\text { Free } \\
\text { (water-soluble) }\end{array}$ & $\begin{array}{c}\text { Total } \\
\text { (acid-soluble) }\end{array}$ \\
\hline ACI 201 & $0.10-0.15$ & \\
ACI 222 & & 0.20 \\
ACI 318 & $0.15-0.30$ & 0.20 \\
BS 8110 & & 0.40 \\
Australia & & 0.6 \\
RILEM & & 0.4 \\
Norway & & 0.6 \\
\hline
\end{tabular}


Table 3.5: The effect of various parameters on chloride threshold (taken from Angst et al.2009)

\begin{tabular}{|c|c|c|c|}
\hline \multirow[t]{2}{*}{ Factor } & \multicolumn{3}{|c|}{ Effect on critical chloride content } \\
\hline & $\begin{array}{l}\text { Total } \mathrm{Cl}^{-} \\
\text {\% cem wt }\end{array}$ & $\mathrm{C}^{-} \mathrm{OH}^{-}$ratio & Free $\mathrm{Cl}^{-}$ \\
\hline $\begin{array}{l}\text { Steel condition } \\
\text { Defiects at steel-concrete } \\
\text { interface }\end{array}$ & 1 & 1 & 1 \\
\hline Polishing, sandblasting & $\uparrow$ & $\uparrow$ & $\uparrow$ \\
\hline $\begin{array}{l}\text { Steel potential } \\
\qquad(>-200 \mathrm{mV} \text { SCE })\end{array}$ & 0 & 0 & 0 \\
\hline $\begin{array}{l}\text { Steel potential } \\
\text { (<-200 mV SCE) }\end{array}$ & 1 & 1 & 1 \\
\hline Concrete and binder prope & ties & & \\
\hline$w / b$ ratio & 1 & 1 & 1 \\
\hline Chloride binding & $t$ & o & 0 \\
\hline pH & $\uparrow$ & 1 & $\uparrow$ \\
\hline Electrical resistivity & $\uparrow$ & $\uparrow$ & $\uparrow$ \\
\hline $\begin{array}{l}\text { SF } \\
\text { FA }\end{array}$ & $\frac{1}{110^{\circ}}$ & $10^{\circ}$ & $10^{b}$ \\
\hline GGBS & $110^{\mathrm{b}}$ & 0 & 0 \\
\hline $\begin{array}{l}\text { SRPC (low } C_{3} A+ \\
C_{A} A F \text { content) }\end{array}$ & 1 & c & $c$ \\
\hline Extemal factors: & & & \\
\hline $\begin{array}{l}\text { Moisture in rather } \\
\text { dry concrete }\end{array}$ & $\downarrow$ & (l) & (1) \\
\hline $\begin{array}{l}\text { Moisture in nearly } \\
\text { saturated concrete }\end{array}$ & $\uparrow$ & 1 & 1 \\
\hline Moisture variations & 1 & (1) & (1) \\
\hline Oxygen availability & 1 & $\downarrow$ & 1 \\
\hline Temperature & 1 & 1 & $\downarrow$ \\
\hline
\end{tabular}

Note: $\uparrow(\downarrow)$ indicates an increase (decrease) in chloride threshold with an increase of the corresponding factor; $\mathrm{O}$ means no influence on chloride threshold.

a Based on theoretical considerations.

$\mathrm{b}$ Contradictory results reported in the literature.

c No results reported. 


\subsection{Nano-scale Experimental Studies}

Experimental techniques such as X-ray photoelectron spectroscopy (XPS), Auger electron spectroscopy (AES), secondary ion mass spectrometry (SIMS), ellipsometric spectroscopy, Raman spectroscopy, and transmission electron microscopy (TEM) have been used for decades to study the surface characteristics of materials at the nanometer scale. In this section, nano-scale studies on the characteristics of iron oxide surface films that have been carried out using these experimental techniques are reviewed. Since theoretical and operational principles of these nano-scale techniques (and devices) are well known and explained in detail in several reference publications (e.g. Cherepin 1987, Suetakaand and Yates 1995, Briggs and Seah 1990, Williams and Carter 2009), they are not included in this review; readers are referred to the aforementioned publications for detailed theoretical and operational information.

\subsubsection{XPS/AES studies}

XPS/AES technique has been used to study iron oxide films grown in a variety of environments (McIntyre and Zetaruk 1977, Allen et al. 1982, Haupt et al. 1986, Fujii et al. 1999, Grosvenor et al. 2004, Poter et al. 2005).

Fujii et al. (1999) carried out an in-situ XPS analysis of various iron oxide films grown by $\mathrm{NO}^{2-}$ assisted molecular-beam epitaxy. They reported that at room temperature $\mathrm{Fe}_{3} \mathrm{O}_{4}$ was highly conductive, because of rapid electron hopping between $\mathrm{Fe}^{2+}$ and $\mathrm{Fe}^{3+}$, while $\gamma-\mathrm{Fe}_{2} \mathrm{O}_{3}$, which had a higher oxidized structure, was an insulator. Their results also confirmed that the satellite structure of Fe $2 \mathrm{p}$ core-level was very sensitive to the type of iron oxide compound, and the XPS Fe $2 p$ core-level spectra of $\alpha-\mathrm{Fe}_{2} \mathrm{O}_{3}$ and $\gamma-\mathrm{Fe}_{2} \mathrm{O}_{3}$ were almost identical although their crystal structures were significantly different. It was 
reported that the observed XPS spectra of various iron oxide films were in good agreement with XPS spectra of bulk iron oxide substances. They found that the binding energies of the $\mathrm{Fe} 2 \mathrm{p}_{3 / 2}$ main peak for $\alpha-\mathrm{Fe}_{2} \mathrm{O}_{3}, \gamma-\mathrm{Fe}_{2} \mathrm{O}_{3}$ and $\mathrm{Fe}_{3} \mathrm{O}_{4}$ films were located at $710.9 \mathrm{eV}, 710.7 \mathrm{eV}$ and $710 \mathrm{eV}$, respectively.

Allen et al. (1982) studied the reaction of highly pure iron foil with oxygen at a temperature of $600 \mathrm{~K}$; they showed that it was possible to distinguish $\mathrm{Fe}^{2+}$ and $\mathrm{Fe}^{3+}$ components of iron oxide using a monochromatic X-ray source. The same finding was also reported earlier by Brundle et al. (1977) based on the experiments conducted on iron oxide powder. In a study carried out on the effect of chloride on the iron oxide film properties, Khalil et al. (1985) found that, in the pH 5 buffer solution, the local or general thinning of passive film due to chloride led to the breakdown of passive film on iron.

Although numerous studies have been carried out using XPS on iron oxide films, only a few XPS studies have been reported on oxide film formed in highly alkaline solutions (Montemor et al. 1998, Miserque at al. 2006, Galicia et al. 2006, Haupt et al. 1986), particularly for steel in simulated or real concrete pore solutions. Using XPS, Haupt and Strehblow (1987) showed that the passive film on iron grown in $1 \mathrm{M} \mathrm{NaOH}$ consists of two parts: an inner $\mathrm{Fe}^{2+}$-rich layer and an outer $\mathrm{Fe}^{3+}$-rich layer. Miserque et al. (2006) studied the passivation of mild steel by XPS in two different solutions: $0.1 \mathrm{M}$ $\mathrm{NaOH}$ solution $(\mathrm{pH} 12.8)$ and saturated $\mathrm{Ca}(\mathrm{OH})_{2}$ solution $(\mathrm{pH} 12.5)$. They reported that the structures of iron oxides grown in the two solutions were similar, and after around 30 days of immersion, both oxide films attained a thickness of about $6 \mathrm{~nm}$. They also stated that it was difficult to differentiate between $\mathrm{Fe}_{2} \mathrm{O}_{3}$ and $\mathrm{FeOOH}$ using the XPS $\mathrm{Fe} 2 p$ spectra. 
The AES/XPS studies conducted by Montemor et al. (1998) on steel in simulated concrete pore solution showed that chloride could change the stoichiometry of the oxide film, and the composition of the concrete pore solution affected the oxide properties. They also found that the thickness of passive films formed in cement paste solution was relatively large $(\sim 100 \mathrm{~nm})$, which was a controversial observation, and its outermost layer was mainly composed of $\mathrm{FeOOH}$.

The effect of applied potential and passivation time on the structure of passive iron oxide film in $1 \mathrm{M} \mathrm{NaOH}$ was investigated by Haupt et al (1986); it was shown that the thickness of passive film linearly increased with increasing potential, and also by the logarithm of passivation time. Furthermore, $\mathrm{Fe}^{2+}$, which formed the inner layer of passive films, initially increased by increasing potential, but after a certain level of potential, it started decreasing. They also observed that the $\mathrm{Fe}^{2+} / \mathrm{Fe}^{3+}$ continuously increased with the passivation time although the $\mathrm{Fe}^{2+}$ attained a maximum and decreased for a longer passivation time.

\subsubsection{Ellipsometric spectroscopy studies}

Zakroczymski et al. (1985a) conducted kinetic studies on the formation of passive films on iron in $0.05 \mathrm{M} \mathrm{Na}(\mathrm{OH})$ solution (pH 12). Based on the ellipsometry test results, they determined the thickness of passive films on iron at $-0.290 \mathrm{mV} / \mathrm{SCE}$ potential after $600 \mathrm{~s}$ immersion in the solution. They also calculated the thickness of different possible iron oxide compounds, as presented in Table 3.6. They defined the long-term growth rate of passive films as $\sim 0.3 \mathrm{~nm}$ per time decade. 
Table 3.6: Thickness of passive films on iron in 0.05M Na(OH) at 600s (Zakroczymski et al. 1985a).

\begin{tabular}{|c|c|c|c|c|c|c|}
\hline Possible compound & $\mathrm{FeOOH}$ & $\mathrm{Fe}_{3} \mathrm{O}_{4}$ & $\mathrm{Fe}(\mathrm{OH})_{2}$ & $\mathrm{Fe}(\mathrm{OH})_{3}$ & $\mathrm{Fe}_{2} \mathrm{O}_{3}$ & Average \\
\hline Passive film thickness (nm) & 3.69 & 8.92 & 7.04 & 5.27 & 5.41 & 6.07 \\
\hline
\end{tabular}

Sarver and Szklarska-Smialowska (1984) reported that the formation of passive films on iron in two alkaline solutions of $\mathrm{pH} 12$ (i.e., $0.05 \mathrm{M} \mathrm{Na}_{2} \mathrm{SO}_{4}$ plus $\mathrm{NaOH}$ and 0.1 $\mathrm{M} \mathrm{NaOH}$ ) consisted of four stages. During the first two stages, which lasted approximatelly one second, a non-protective oxide layer formed rapidly on the surface of iron, while in the final two stages the growth of the oxide film reached a steady state, and passivation was observed afterwards. The thickness of oxide films in the first two stages was $60 \%$ of the steady-state thickness of the film. The film growth in the last two stages of oxide formation (i.e., passivation) followed a function of the square root of time. For the $\mathrm{NaOH}$ solution $(\mathrm{pH} \mathrm{12)}$ the total thickness of film was found to be around $6 \mathrm{~nm}$; however, in $0.05 \mathrm{M} \mathrm{Na}_{2} \mathrm{SO}_{4}(\mathrm{pH} \mathrm{12})$ the thickness increased up to $16 \mathrm{~nm}$.

The ellipsometric studies of passive films on iron in borate buffer ( $\mathrm{pH} 8.4$ ) conducted by Chen and Cahan (1982) showed that potentiostatically-formed oxide films had optical properties consistent with the presence of only a layer of $\mathrm{Fe}^{3+}$. Kruger and Calvert (1967) also used the ellipsometry technique to study the kinetics of passive film growth in the solutions with $\mathrm{pH}$ ranging from 7.4 to 9.1 . They detected three different stages of film growth: in stage 1, the growth was limited by diffusion process in the solution; in stage 2 , the combination of several processes were concurrently involved in the growth of film, in stage 3 , the film growth was determined by either logarithmic or inverse logarithmic functions of time. The third stage implies that the outer layer of 
passive film was poorly conductive for electrons so that a high electric field, which helps the movement of cations through the film to the bulk solution, could build up.

\subsubsection{SIMS studies}

The passivation of iron in $\mathrm{pH} 8.4$ borate buffer solution at $0.0 \mathrm{~V} / \mathrm{SHE}$ was studied by Goetz et al. (1986) both in the presence and in the absence of $0.5 \mathrm{M}$ chloride. The results showed that chloride did not affect the passive current decay with time, and neither AES nor SIMS technique was able to detect any chloride in the film. They concluded that the incorporation of chloride in the passive film was not a precursor for pit initiation. In a SIMS study carried out by Graham et al. (1990) on the nature of passive films on iron in borate buffer solution of $\mathrm{pH} 8.4$ plus chloride, it was also confirmed that chloride neither changes the thickness of passive films nor was incorporated in the structure of passive films. The SIMS results also showed that hydroxide was not incorporated in the iron passive film, and the film was mainly composed of smallparticle-size crystalline $\gamma-\mathrm{Fe}_{2} \mathrm{O}_{3}$.

Landolt et al. (1990) studied the effect of chloride on passive films on ironchromium alloies in acidic solution by the SIMS and XPS techniques. They found that the thickness of the film was not affected by chloride, but chloride penetrated into the film so that its concentration at the outer layer of oxide film was higher than the inner one.

\subsubsection{Raman spectroscopy studies}

In-situ Raman spectroscopy and rotating split-ring disk electrode were used by Hugot-Le Goff et al. (1990) to identify iron oxides that form on the surface of iron in $1 \mathrm{M}$ $\mathrm{NaOH}$. $\mathrm{Fe}_{3} \mathrm{O}_{4}$ started to form at the first peak of anodic polarization sweep and grew 
continuously at more noble potentials. It was found that $\delta-\mathrm{FeOOH}$ formed at the third peak of the anodic polarization sweep, and $\gamma-\mathrm{Fe}_{2} \mathrm{O}_{3}$ was produced after prolonged polarization at a potential of $0.27 \mathrm{~V} / \mathrm{SCE}$. Therefore, they suggested that the passive film on iron in $1 \mathrm{M} \mathrm{NaOH}$ was composed of an inner layer of $\mathrm{Fe}_{3} \mathrm{O}_{4}$ covered with an outer layer of other iron oxides. A similar conclusion was obtained by Joiret et al. (2002) in the experiments carried out on iron passivated in $1 \mathrm{M} \mathrm{NaOH}$, also using Raman spectroscopy.

Gui and Devine (1994) investigated the influence of sulphates on the passive film formed on iron in mildly alkaline $(\mathrm{pH} \mathrm{10)}$ and acidic $(\mathrm{pH}$ 5) aqueous solutions of sodium sulfate using surface-enhanced Raman spectroscopy. They demonstrated that, in the $\mathrm{pH} 10$ solution, sulfate ions were adsorbed onto the surface of oxides, and this affected the structure and the nature of the passive films. They also found that sulphates covalently bonded to the passive film that was formed in the $\mathrm{pH} 5$ solution; therefore they were incorporated in the film. Observations of Gui and Devine (1994) suggest that the presence of specific anions in the solution can change the characteristics of passive films on iron.

\subsubsection{TEM studies}

TEM electron diffraction pattern analysis is an ex-situ technique that can be used to determine the structure of oxide films. Using this technique, Foley et al. (1967) and Kuroda et al. (1982) found that the passive films anodically grown on iron in $0.1 \mathrm{M}$ $\mathrm{NaOH}$ solution had a cubic spinel structure, which is structurally very similar to the structures of $\gamma-\mathrm{Fe}_{2} \mathrm{O}_{3}$ and $\mathrm{Fe}_{3} \mathrm{O}_{4}$; but the films were less ordered than the crystalline anhydrous oxides, exhibiting a tendency toward more vitreous structures. 
Tjong (1985) carried out TEM electron diffraction pattern analysis on the passive film that formed on the iron-chromium alloys in a borate buffer solution ( $\mathrm{pH} 8.4$ ). Using selected area diffraction (SAD) patterns, the structure of passive films at various chromium contents were investigated. The results showed that the film on $\mathrm{Fe}-3 \% \mathrm{Cr}$ alloy consisted of a large number of randomly oriented fine grains, whereas the structure of passive film on the $\mathrm{Fe}-18 \% \mathrm{Cr}$ alloy was amorphous. McBee and Kruger (1972) also reported that the electron diffraction patterns of passive films on the low-chromium iron alloy had a spinel structure; however, as the chromium content of the alloy increased, the film structure became more amorphous.

\subsubsection{Summary}

Despite the fact that different techniques have been used for nano-scale studies of iron passive films, the results are not consistent and strongly depend on the type of exposure conditions. However, it is generally accepted that the protective oxide films on iron consists of two layers: an inner $\mathrm{Fe}^{2+}$-rich layer and an outer $\mathrm{Fe}^{3+}$-rich layer.The mechanism of the formation of oxide film, and its breakdown in the presence of halides (e.g. chloride), have not been clearly understood; it is commonly believed that halides cause a general or local thinning of oxide film, even though limited information is available to support this concept. It should be noted that there are a number of challenges associated with these techniques that can account for the lack of consistent and more informative data; these can be listed as: (1) sample preparation that minimizes damage to the specimens; (2) conducting in-situ test; (3) interpretation of results; (4) inherited limitations of the devices used in these techniques. Even though the minimization of the errors associated with these challenges may provide an insight into the properties of 
passive film and its behaviour under circumstances comparable to the actual exposure conditions, it is not practically possible to eliminate all these experimental limitations; therefore, multiple techniques that complement each other must be used to study the phenomena.

\subsection{Passive Film Formation Models}

According to Pou et al. (1984), models explaining the nature of the passive films that form on iron can be summarized under two categories: the crystalline oxide models and the hydrated polymeric oxide models. In the crystalline oxide models, the protective nature of the film, which forms on the surface of a metal following a particular form of oxidation, is related to the crystal structure of the film. Inherent in the hydrated polymeric model is the essential role played by water in transforming the properties of iron oxide to those of the protective passive film; particular function of water varies for each model.

Among the passive film formation models, some explain film formation under an over-potential (electric field-assisted passive models). For example, Hopping model (Cabrera and Mott 1949, Kruger 1988) is a basic treatment of anodic film formation that has served as the starting point for other electric field-assisted passive film formation models. It was adopted specially for passive film formation in neutral solutions by modifying the Mott-Caberra theory (Cabrera and Mott 1949) for gaseous oxidation to enable its use for anodic oxidation. The Hopping model describes ionic motion in the forming of the oxide film by the hopping of cations to find empty sites in oxygen lattice. The cations move under the electric field created by the potential difference that exists across the growing anodic or passive film. Induced space charge model, which is a 
modification of the basic Hopping model described above, was developed by Fromhold and Kruger (1973). It proposes that the ionic space charge induced in the passive film can reduce, by orders of magnitude, the ionic current that is responsible for film growth (e.g. the reduction was calculated to be one order of magnitude for a film with the thickness of $3 \mathrm{~nm})$.

Chao et al. (1981) have developed the point defect model (PDM) for film growth by ionic movement controlled by an electric filed. They undertook this task to obtain a more consistent agreement between the film growth model and the kinetics of growth of passive films. The PDM provides an analytical description of the growth and breakdown of passive films on reactive metal surfaces on the atomic (sub-nanometer) scale. In this model, there are seven reactions that are related to passive film formation, as shown in Fig. 3.9. Cation vacancies are produced at the barrier layer/solution interface but are annihilated at the metal/film interface. Likewise, oxygen vacancies and interstitial cations are formed at the metal/barrier layer interface but are annihilated at the barrier layer/solution interface. Consequently, the reactions 3 and 7 (see Fig. 3.9) are responsible for the growth of the barrier layer (passive film), and any analysis of the stability of the film must focus on these two reactions.

Unlike the models described above, the place exchange model (Sato and Cohen 1964) does not require an electric field to move ions through the growing film. This model attributes film thickening to a process whereby oxygen atoms adsorbed on the surface of metal exchange places with underlying metal atoms. The oxide film continues to grow with the adsorption of a second layer of oxygen, which exchanges place with the underlying metal atoms; this exchange occurs simultaneously with a place exchange of 
Metal | Barrier Oxide Layer | Solution

(1) $m+V_{M}^{X^{\prime}} \stackrel{k_{i}}{\longrightarrow} M_{M}+v_{m}+\not e^{\prime}$

(4) $M_{M} \stackrel{k_{1}}{\longrightarrow} M^{T_{+}}+V_{M}+(T-\chi) e^{-}$

(2) $m \stackrel{k_{z}}{\longrightarrow} M_{i}^{X^{+}}+y_{m}+\not e^{-}$

(5) $M_{i}^{\chi^{+}} \stackrel{k_{s}}{\longrightarrow} M^{\Gamma+}+(\Gamma-\chi) e^{*}$

(3) $m \stackrel{\boldsymbol{k}_{y}}{\longrightarrow} \boldsymbol{M}_{M}+\frac{\chi}{2} \boldsymbol{V}_{\ddot{o}}+\not e^{-}$

(6) $V_{o}^{*}+H_{2} O \stackrel{\boldsymbol{k}_{i}}{\longrightarrow} O_{O}+2 H^{+}$

(7) $M O_{x / 2}+\not H^{*} \stackrel{k_{s}}{\longrightarrow} M^{\Gamma+}+\frac{\chi}{2} H_{2} O+(\Gamma-\chi) e^{-}$

$\left(x=L_{s s}\right)$

$(x=0)$

* $\mathrm{m}=$ metal atom, $V_{M}{ }^{x^{\prime}}=$ cation vacancy on the metal sublattice of the barrier layer, $M_{i}^{x+}=$ interstitial cation, $M_{M}=$ metal cation on the metal sublattice of the barrier layer, $V_{O}^{* *}=$ oxygen vacancy on the oxygen sublattice of the barrier layer, $O_{O}=$ oxygen anion on the oxygen sublattice of the barrier layer, $M^{T+}=$ metal cation in solution

Figure 3.9: Schematic of physicochemical processes that occur for passive film formation according to the point defect model taken from Kamrunnahar et al. (2005).

the first formed underlying M-O layer. Film thickening can then continue with further place exchange of three M-O layers (see Fig. 3.10). Sato and Cohen (1964) used the place exchange mechanism to determine the parameters in the empirical equation:

$$
i=K^{\prime} \exp \left(m E-Q_{t} / n\right)
$$

where $i$ is the measured current, $E$ is the applied potential, $Q_{t}$ is the accumulated charge in the film, and $K^{\prime}, m, n$ are empirical parameters. By integrating the above equation, Sato and Notoya (1967) obtained the direct logarithmic rate law:

$x=A+B \ln \left(t-t_{0}\right)$

Eq. 3.2

where $x$ is the thickness of passive film, $t$ is the passivation time during steady growth of passive film and $t_{0}$ is the time required for the transient growth of passive film. 


\begin{tabular}{|c|c|c|c|c|}
\hline 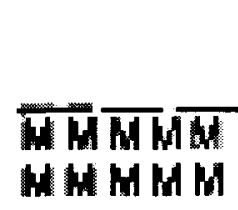 & $\begin{array}{l}\text { OOOOO } \\
\text { MMMMH } \\
\text { MMHMH }\end{array}$ & $\begin{array}{l}\text { MAMTM } \\
\text { ODOO } \\
\text { MHMHH }\end{array}$ & $\begin{array}{l}00000 \\
\text { MNMWM } \\
\text { OOOOO } \\
\text { MNMHA }\end{array}$ & 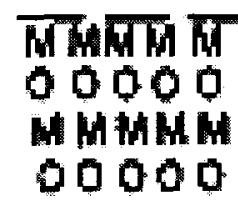 \\
\hline$\{1\}$ & (z) & (3) & $14\}$ & $(5)$ \\
\hline
\end{tabular}

Figure 3.10: Place exchange model of passive formation (taken from Sato and Cohen 1964).

The Bipolar fixed charge induced model, proposed by Sakashita and Sato (1978), suggests that there exists a bipolar film on a passivated surface. This film consists of an anion selective layer on the metal side and a cation selective layer on the solution side (hence the term "bipolar") (see Fig. 3.11). This bipolar film can reduce ionic migration by retarding the anodic current in the anodic direction. Such a layer serves as a fixed charge hydrated oxide barrier and can be either anion selective (if it is positive) or cation selective (if it is negative) (see Fig. 3.11 for an application of this model).

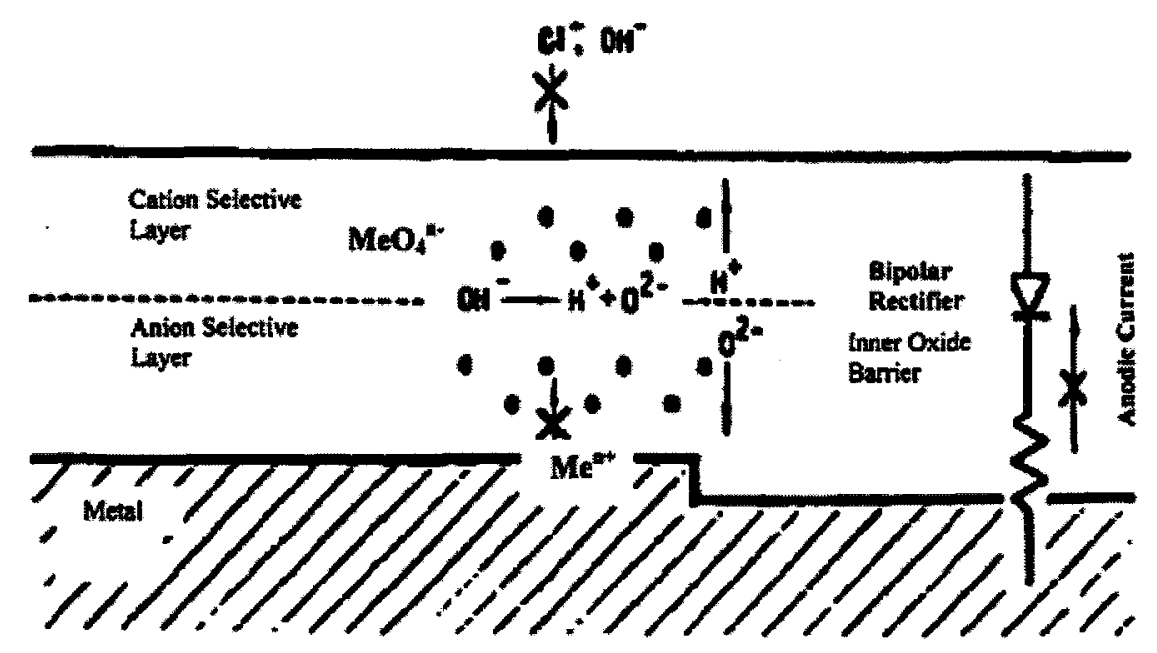

Figure 3.11: The bipolar model of passive film formation reproduced from Sakashita and Sato (1978). 


\subsection{Passivity Breakdown Models}

Many theories or models have been introduced to describe the events leading to pit or crevice corrosion initiation. The following criteria are usually considered to be associated with the breakdown of the passive film; hence they need to be explained by the models (Hoar 1967, Kruger 1988):

a) Damaging species (e.g. chlorides) are needed to initiate and to propagate breakdown;

b) Highly localized sites, where breakdown occurs, are needed;

c) A certain pitting potential, $E_{\text {pit }}$, must be exceeded;

d) An induction time, which starts with the provision of required conditions for breakdown and ends with the completion of the process, must exist.

The models in the literature for passive film breakdown can be classified in three major groups: Adsorbed ion displacement models, ion migration or penetration models, and breakdown-repair models. The summary of these are described in the following sections.

\subsubsection{Adsorbed ion displacement models (Kolotyrkin 1961, Leckie and Uhlig}

Two types of adsorbed ion displacement models have been proposed: In the first model (Kolotyrkin 1961), the passive film is considered to be an adsorbed film (probably a monolayer) of oxygen. Breakdown occurs when a damaging anion (in this research, chloride) displaces the oxygen forming the passive film. After the anion is adsorbed on the surface, the breakdown process is initiated because the bonding of the metal ions to the metal lattice is weakened. The model interprets the pitting potential as the potential 
above which the anion is adsorbed on the surface causing breakdown. Ions such as chloride are effective in promoting breakdown because they are more readily adsorbed than oxygen in the competition for surface sites. Although the effect of damaging ion concentrations on the breakdown of passive film can be explained by this model, the important influence of the passive film thickness on the induction time can not be readily described. Finally, this model accommodates the requirement of localized attack since adsorbed ion displacement will probably occur at local sites, defects and inclusions, where ion displacement is more probable.

The second ion displacement model (Leckie and Uhlig 1966) involves the formation of a transitional complex compound. It bears some similarity to some of the ion migration models, which will be described later. It postulates the adsorption of halide ions like chlorides on the surface of the passive film around a lattice cation. Considering that the probability of the formation of such a high energy complex compound is small, the process requires a high activation energy of formation. Once the complex compound is formed, the halide ions will remove the cations from the passive film lattice as soluble compounds. Therefore, the passive film becomes thinner at some sites and a larger ionic field now exists at the thinned sites compared to other locations. This field will pull another cation through the thinned site of the film, where it will meet more halide ions, reacts with them to form a complex compound, and thereby enter the solution. Once started, the passive film is locally thinned, and breakdown proceeds.

\subsubsection{Ion migration or penetration models (Kruger 1988)}

In these models damaging anions penetrate through the passive film; the breakdown process is complete once an anion reaches the metal/film interface. All of 
these models consider the passive film to be three dimensional with certain thickness. The main difference is in their proposed mode of penetration.

\subsubsection{Pore model (Evans et al. 1931, Richardson and Wood 1970)}

This model assumes the existence of pores in the passive film. In this model (Evans et al. 1931), the breakdown of the passive film occurs because there always exists defects in the film that allow instant penetration and hence the immediate initiation of localized corrosion. This leads to the conclusion that there is no induction time for the breakdown process to begin because there are no breakdown processes. However, additional research (Richardson and Wood 1970) explained the existence of an induction period by suggesting that the currents are so low at the small bare metal sites, at the base of pores or film defects, that it appears breakdown not initiated, even though it goes on from the instant that the surface is exposed to damaging anions.

\subsubsection{Migration models (McBee and Kruger 1972, Hoar et al. 1965)}

The other type of penetration models covers mechanisms involving migration of the damaging anion through a lattice, via defects, or via some type of an ion exchange process. Ion migration in a lattice can occur in a variety of ways. For example, ion migration process can involve the exchange of oxygen atom or $\mathrm{OH}^{-}$with the damaging anion. The following equation shows that such a process can also create anion vacancies that will further enhance the migration of damaging anions to the film/metal interface:

$\mathrm{Cl}_{\text {Solution }}^{-}+\square_{o^{-2}}+2 \mathrm{OH}_{\text {lattice }}^{-} \rightarrow \mathrm{Cl}_{\text {lattice }}^{-}+\mathrm{OH}_{\mathrm{OH}^{-}}+2 \mathrm{OH}_{\text {Solution }}^{-} \quad$ Eq. 3.3

In the presence of halides, the rapid increase of anion lattice at the film/metal interface can not be compensated by the slow process of film formation leading to the breakdown 
of film. Therefore, the anion lattice migration models can explain the phenomenology of breakdown. In migration models, the pitting potential is the potential required for adsorption, although in this case, it is on the outer interface of the passive film.

3.4.2.3 Point defect model (Lin et al. 1981, MacDonald and Urquidi-MacDonald 1990) Another ion migration model requiring vacancies is the PDM model, which attributes breakdown or pit initiation to the pile-up of metal holes (cation vacancies) at the metal/film interface during the passive film growth process. This collection of metal holes results in a growing void at the metal/film interface, which causes the passive film to suffer a local collapse when the void reaches a critical size at the end of induction period (see Fig. 3.12). Using this explanation, equations for induction time, film thickness and pitting potential have been developed, and these have been shown to be in quantitative agreement with the experimental data for the pitting of iron and nickel in halide-containing solutions.

\subsubsection{Breakdown-Repair models}

These theoretical models involve chemically-induced mechanical disruption of the passive film. Two of these models are briefly described here.

\subsubsection{Chemical-mechanical model (Kruger 1976, Sato 1971)}

Chemical-mechanical model suggests that the adsorption of a damaging anion on the surface of the passive film lowers the interfacial surface tension (i.e., surface free energy) at the solution interface because of the mutual repulsion of the adsorbed anions. When the repulsive forces are sufficiently large, the passive film cracks and damaging 
anions attack the exposed metal unless conditions exist whereby rapid repair of the film can occur (repassivation).

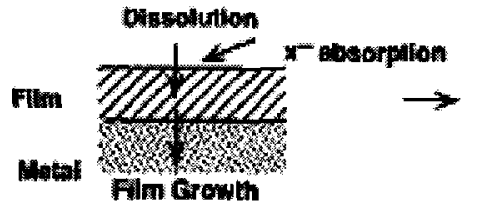

(a) Steady Stale

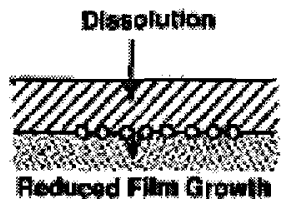

(b) Vacancy Condensatlon

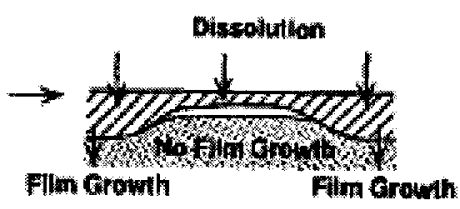

(c) Local Film Detachment
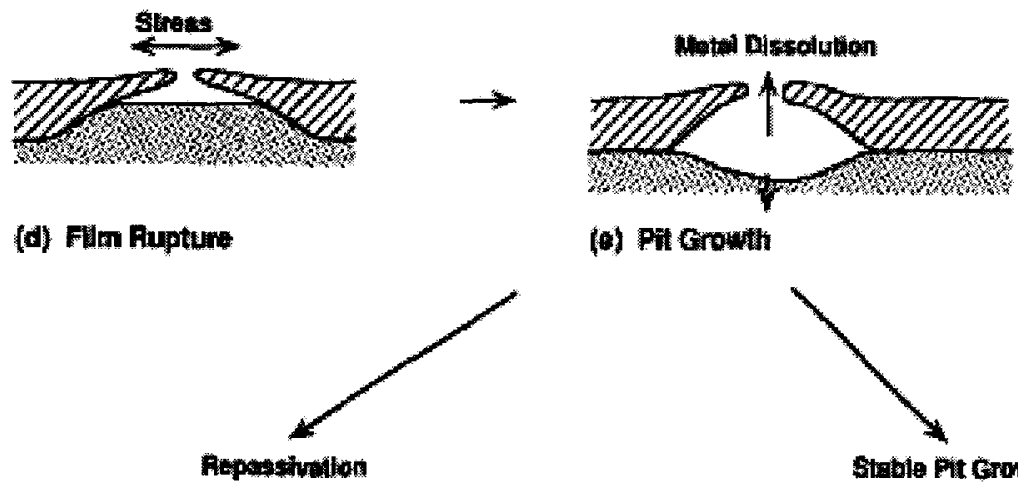

(0) Pin Growth

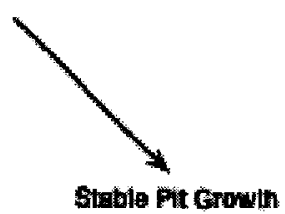

Figure 3.12: Various stages of pit nucleation process according to the PDM (taken from Macdonald 1992).

This concept of chemical-mechanical breakdown was then refined by Sato (1971), who provided a more detailed examination of the model. Sato proposed that large electric fields could lead to the mechanical rupture of thin films by electrostriction pressures exceeding the compressive fracture strength of the film. This generates the socalled film pressure, which is given by:

$$
p-p_{0}=\frac{\varepsilon(\varepsilon-1) E^{2}}{8 \pi}-\frac{\gamma}{L}
$$

where $p$ is the film pressure, $p_{0}$ is atmospheric pressure, $\varepsilon$ is the film dielectric constant, $E$ is the electric field, $\gamma$ is the surface tension, and $L$ is the film thickness. 
It is probable that ions that are considered aggressive in promoting localized corrosion attack increase film pressure by decreasing the surface tension term in the Eq. 3.4. The experimental results support this possibility as they have shown that halides decrease the solid/liquid surface energy. Based on this theory, whether a pit nucleates or dies depends not only on breakdown, but equally on film repair. This concept forms the basis upon which all models in the group are developed.

\subsubsection{Dynamic breakdown-repair model (Galvele 1978)}

This model is based on the localized acidification model that assumes a transient breaking of passive layer occurs through mechanical or electrochemical means; hydrolysis then occurs during the reaction between the exposed metal and environment. The following reactions produce localized acidification at the site of the cracked film:

$$
\begin{aligned}
& \mathrm{Me} \rightarrow \mathrm{Me}^{z+}+z e^{-} \\
& \mathrm{Me}^{z+}+z \mathrm{H}_{2} \mathrm{O} \rightarrow \mathrm{Me}(\mathrm{OH})_{z}+z H^{+}
\end{aligned}
$$

The amount of acidification necessary to prevent repassivation varies for each metal and alloy. If the acidification is sufficient to prevent repassivation, pitting initiates. In this model the induction time is the time needed to achieve a critical $\mathrm{pH}$ in a broken passive film. The pitting potential is that potential needed to cause the current flowing to the initiated pit to reach a value that causes a critical $\mathrm{pH}$ that renders repassivation impossible.

\subsubsection{Summary}

To compare the presented passive film breakdown models, indicative characteristics of passivity are defined in Table 3.7 , and are labelled from "a" to "o". 
However, only certain characteristics are crucial in the explanation of depassivation of rebar in concrete; therefore, these properties are defined in Table 3.7. Considering these factors, the models have been evaluated in Table 3.8. It is clear that the PDM is applicable to nearly all characteristics that are deemed important in modelling the breakdown of passive film on the surface of rebar in concrete. However, a negative aspect of this model is that a large number of parameters should be determined experimentally prior to using it for an engineering application. In addition to the PDM model, the absorbed ion displacement and dynamic breakdown-repair models have also the potential to explain the passivity of rebar in concrete.

Table 3.7: Characteristics of passive film used in the comparison of the models.

\begin{tabular}{|r|l|r|}
\hline a & Consider the effect of temperature. & $\sqrt{ }$ \\
$\mathrm{b}$ & Consider the effect of pH. & $\sqrt{ }$ \\
$\mathrm{c}$ & Consider the effect of potential. & $\sqrt{ }$ \\
$\mathrm{d}$ & Explain the passive layer breakdown due to chloride attack. & $\sqrt{ }$ \\
$\mathrm{e}$ & Describe the breakdown potential. & $\sqrt{ }$ \\
$\mathrm{f}$ & Explain the inverse logarithmic growth of passive film in low pH. \\
$\mathrm{g}$ & Consider if anion transportation is responsible for growth of film. & $\mathbf{x}$ \\
$\mathrm{h}$ & Explain the steady state in barrier layer thickness and passive current density. & $\sqrt{ }$ \\
$\mathrm{i}$ & Consider breakdown induction time. & $\sqrt{ }$ \\
$\mathrm{j}$ & Thickness of passive film. & $\sqrt{ }$ \\
$\mathrm{k}$ & Describe highly localized sites of breakdown. & $\sqrt{ }$ \\
$\mathrm{I}$ & Applicable to all kind of metals' passive film. & $\mathrm{x}$ \\
$\mathrm{m}$ & Capable to develop equations. & $\sqrt{ }$ \\
$\mathrm{n}$ & Explain the logarithmic growth of passive film. & $\mathbf{x}$ \\
$\mathrm{o}$ & It is applicable for modelling the rebar depassivation in concrete & $\sqrt{ }$ \\
\hline
\end{tabular}

$\sqrt{ }$ : Important for modeling of depassivation of steel in concrete

$x$ : Insignificant for modeling of depassivation of steel in concrete 
Table 3.8: The literature review summary: The model's capability of explaining the characteristics listed in Table 3.7.

\begin{tabular}{|c|c|c|c|c|c|c|c|c|c|c|c|c|c|c|c|}
\hline Model & $\underline{a^{*}}$ & $\underline{\mathbf{b}}$ & $\underline{\mathbf{c}}$ & $\underline{\text { d }}$ & $\underline{\mathbf{e}}$ & f & $\mathbf{g}$ & $\underline{\mathbf{h}}$ & $\underline{\mathbf{I}}$ & I & $\underline{\mathbf{k}}$ & I & $\underline{m}$ & $\mathbf{n}$ & 으 \\
\hline Hopping model & $Y$ & $\mathrm{~N}$ & Y & NA & NA & $\mathbf{N}$ & $\mathrm{N}$ & $N$ & NA & $\mathrm{Y}$ & NA & $Y$ & Y & $\mathrm{Y}$ & $N$ \\
\hline Induced space charge model & Y & $N$ & $Y$ & NA & NA & $N$ & N & $N$ & NA & $\mathrm{Y}$ & NA & Y & Y & $\mathrm{Y}$ & $\mathrm{N}$ \\
\hline Point defect model & Y & Y & Y & Y & Y & Y & $Y$ & $Y$ & Y & Y & $Y$ & Y & Y & $Y$ & $Y$ \\
\hline Place exchange model & $N$ & $N$ & Y & NA & $N$ & N & N & $N$ & $N$ & $\mathrm{Y}$ & $N$ & Y & Y & $\mathrm{Y}$ & $N$ \\
\hline $\begin{array}{l}\text { Bipolar fixed charge induced } \\
\text { model }\end{array}$ & $\mathbf{N}$ & $N$ & $?$ & NA & $?$ & $\mathrm{~N}$ & $Y$ & $N$ & $\mathrm{~N}$ & $\mathrm{Y}$ & $N$ & Y & $\mathrm{N}$ & $N$ & $N$ \\
\hline Polymeric hydrated oxide model & $\mathbf{N}$ & Y & $Y$ & NA & $N$ & $\mathrm{~N}$ & ? & $\mathrm{N}$ & NA & $Y$ & $N$ & Y & $N$ & $\mathrm{~N}$ & $N$ \\
\hline $\begin{array}{l}\text { Adsorbed ion displacement } \\
\text { models }\end{array}$ & $\mathbf{N}$ & $N$ & $Y$ & Y & Y & N & $\mathrm{N}$ & $N$ & Y & Y & $\mathrm{Y}$ & Y & $\mathrm{N}$ & $N$ & $Y$ \\
\hline Pore model & $N$ & $N$ & $N$ & N & $\mathrm{N}$ & $\mathrm{N}$ & $\mathrm{N}$ & $\mathrm{N}$ & Y & Y & $N$ & Y & N & $\mathrm{N}$ & $N$ \\
\hline Migration model & $\mathrm{Y}$ & $N$ & $Y$ & Y & Y & $\mathrm{N}$ & $Y$ & $\mathrm{~N}$ & Y & Y & $N$ & Y & Y & $\mathrm{N}$ & $\mathrm{N}$ \\
\hline Chemical-mechanical model & Y & $\mathrm{Y}$ & $Y$ & $Y$ & Y & $\mathrm{N}$ & $\mathrm{N}$ & $\mathbf{N}$ & $N$ & $N$ & Y & Y & Y & $N$ & $\mathrm{~N}$ \\
\hline Dynamic breakdown-repair model & $Y$ & $\mathrm{Y}$ & Y & $N$ & Y & $\mathrm{N}$ & $\mathrm{N}$ & $\mathrm{N}$ & Y & Y & Y & Y & $N$ & $\mathbf{N}$ & \\
\hline
\end{tabular}

* Underlying indicates the corresponding characteristics are important for modeling of steel depassivation in concrete.

\subsection{Key Questions Originating From the Literature Review}

The general objectives of this research are to understand better the mechanisms of passivation and chloride-induced depassivation of carbon steel reinforcement in concrete and to provide robust explanations to the outstanding questions about corrosion initiation of rebar in the alkaline environment of concrete. The literature review presented in this chapter has given rise to a number of key questions. The current research seeks answers to these specific questions, which are listed below: 
1) Which experiment(s) can be used to obtain reliable results for chloride thresholds in reinforced concrete?

2) Why is there a wide range of variation within the reported chloride thresholds?

3) Does the breakdown of rebar passivity in concrete occur abruptly after a set chloride threshold, or is depassivation a gradual/transient process?

4) What is the effect of rebar surface characteristics, specifically mill scale, on chloride thresholds?

5) What is the influence of chloride on the oxide film properties of rebar?

6) Do chlorides penetrate into the oxide film on the passivated rebar surface?

7) How does the $\mathrm{pH}$ of solution influence the oxide film formed on the surface of rebar steel?

8) What is the chemical composition of oxide film formed on rebar steel in the highly alkaline environment of concrete pore solution?

9) What is the effect of concrete pore solution composition on the passive film properties?

10) How does the passivation time affect the formation of passive film?

11) How does the $\mathrm{pH}$ of the concrete pore solution affect the chloride-induced depassivation of steel in concrete?

12) Do any of the existing models for passive film formation and destruction explain the properties of the passive film and its breakdown on carbon steel in concrete pore solutions? 


\section{Chapter 4}

\section{Electrochemical Study on Passivity of Rebar: Effect of Pore Solution}

\subsection{Introduction}

The current study focuses on how differences in the chemical compositions of pore solutions affect the quality of the protective passive oxide formed on reinforcing steel. Traditionally, electrochemical studies on reinforcing steel in simulated electrolytes have been performed in saturated calcium hydroxide solutions (Blanco et al. 2006, Saremi and Mahallati 2002, Monticelli et al. 2000). Although ordinary portland cement concrete pore solutions are saturated with calcium hydroxide, $\mathrm{Ca}(\mathrm{OH})_{2}$, they also contain different auxiliary ions depending on the type of cement and supplementary cementing materials (e.g. fly ash, slag, and silica fume) (Moragues et al. 1987, Andersson et al. 1989, Page and Vennesland 1983). The major ionic species include cations such as $\mathrm{Ca}^{2+}$, $\mathrm{Na}^{+}$and $\mathrm{K}^{+}$and anions such as $\mathrm{OH}^{-}$and $\mathrm{SO}_{4}{ }^{2-}$ : Sodium and potassium ions originate from alkali oxides, such as $\mathrm{Na}_{2} \mathrm{O}$ and $\mathrm{K}_{2} \mathrm{O}$, that exist in cement; and sulfate ions can come from gypsum added during cement production, or from contaminated aggregates, or mixing water (Neville 1996). It is generally understood that the complex and variable nature of concrete pore solutions requires the use of representative electrolytes for studying 
reinforcement corrosion (Goni and Andrade 1990, Macias and Andrade 1983, Poursaee and Hansson 2007, Mammoliti and Hansson 2005, Rondelli et al. 2006); however, how the composition of the pore solution affects the quality of the protective oxide is not well understood. Montemor et al. (1998) used Auger Electron Spectroscopy (AES) and X-ray Photoelectron Spectroscopy (XPS) to show that the compositions of various extracted concrete pore solutions can affect the nature of the passive film. Similarly, Gui and Devine (1994) investigated the passive film formed on iron in mildly alkaline aqueous solutions of sodium sulfate using Raman Spectroscopy, and demonstrated that the structure and the nature of the passive films are affected by the presence of sulfate ions. These studies show that the composition of the concrete pore solution affects how the passive film on rebar is formed and degraded.

In the present study, the quality of the passive oxide films, which is a measure of the ability to protect the steel from corrosion, was related to the composition of the pore solution in which the films were grown. Anodic polarization and electrochemical impedance spectroscopy (EIS) were used to characterize the oxide films on the surface of deformed carbon steel rebar specimens. The tests were conducted in 10 solutions designed to encompass concentrations of $\mathrm{Ca}^{2+}, \mathrm{Na}^{+}, \mathrm{K}^{+}$and $\mathrm{SO}_{4}{ }^{2-}$ determined from previous concrete solution-extraction studies (Morague et al. 1987, Andersson et al. 1989, Page and Vennesland 1983, Marcotte 2001). To mimic in-service conditions, the tests described in this study were conducted on as-received carbon steel rebar specimens with mill scale, without special surface preparation. Recent studies by Mahallati and Saremi (2006) on carbon steel showed that pickling, sand-blasting, etc., change the protective properties of the passive layer, and $\mathrm{Li}$ and Sagues (2001) demonstrated that 
chloride thresholds for carbon steel depassivation are affected by the surface finish. Hence, the current study uses as-received carbon steel rebar specimens with mill scale to relate better to the majority of reinforced concrete structures in service.

\subsection{Experimental Program}

\subsubsection{Specimen preparation}

Rebar specimens were prepared from a 1-m long as-received deformed carbon steel rebar, measuring $10 \mathrm{~mm}$ in nominal diameter. The composition of the carbon steel used in this study is provided in Table 4.1. The rebar was cut into $30 \mathrm{~mm}$ segments and a steel wire (Alloy: ER316L, Diameter: 0.035") was spot welded to one end of each specimen, and a plastic tube was then placed around the steel wire (see Fig. 4.1). The middle of each specimen was then wrapped tightly with electrical tape so that $2-3 \mathrm{~mm}$ was left exposed at the top and bottom. The specimens were then submerged in epoxy three times. After curing the epoxy, the electrical tape was removed. As a result, the top, bottom, and edges of the specimens were covered with epoxy, and the middle was uncovered (see Fig. 4.1). The average area of uncovered rebar was $7.2 \pm 0.2 \mathrm{~cm}^{2}$, which is a comparatively large area for anodic polarization measurements, but in accord with the standard ASTM G5-94 (2004). The large area was chosen with regard to the results of Li and Sagues (2004) that suggest larger areas provide more representative average behaviour and thus better simulate the in-service conditions of steel in concrete. Finally, the exposed surfaces of the specimens were rinsed with acetone and then with distilled water to remove any residue from the electrical tape. The specimens were stored in a desiccator until testing. In addition to the as-received rebar specimens described above, 
additional specimens were prepared in the same fashion, except that the deformities and the mill scale were removed, to a depth of $1 \mathrm{~mm}$, and the surface polished to $600 \mathrm{Grit}$.

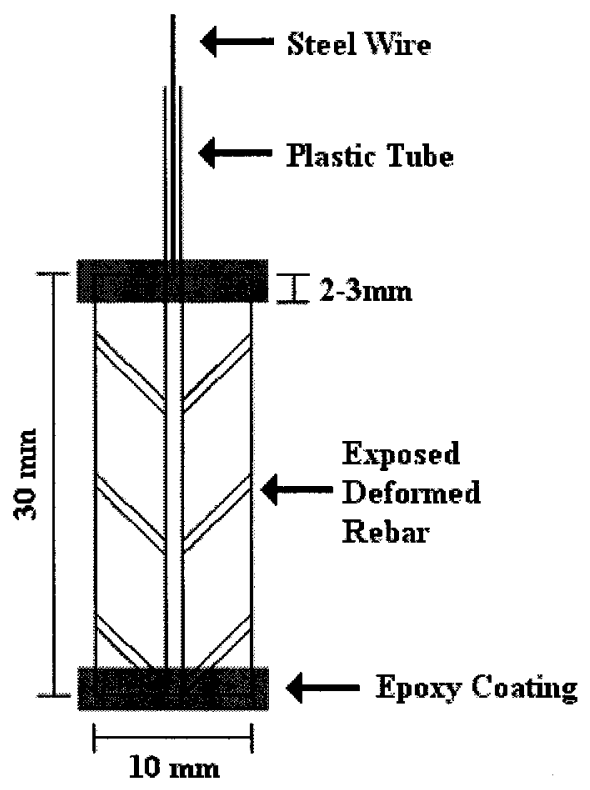

Figure 4.1: An illustration of the rebar specimens.

Table 4.1: Steel elemental composition (Average of three spectrographic results Instrument model: OneSpark).

\begin{tabular}{|l|c|}
\hline Element & $\%$ \\
\hline $\mathrm{C}$ & 0.26 \\
$\mathrm{Si}$ & 0.27 \\
$\mathrm{Mn}$ & 1.10 \\
$\mathrm{Cr}$ & 0.05 \\
$\mathrm{Ni}$ & 0.07 \\
$\mathrm{Mo}$ & $<0.01$ \\
$\mathrm{Cu}$ & 0.21 \\
$\mathrm{Al}$ & $<0.005$ \\
$\mathrm{Nb}$ & $<0.01$ \\
$\mathrm{~V}$ & $<0.005$ \\
$\mathrm{Ti}$ & $<0.005$ \\
$\mathrm{~B}$ & $<0.0005$ \\
$\mathrm{P}$ & 0.01 \\
$\mathrm{~S}$ & 0.03 \\
$\mathrm{~W}$ & $<0.01$ \\
$\mathrm{Sn}$ & 0.02 \\
$\mathrm{Co}$ & 0.01 \\
$\mathrm{Zr}$ & $<0.01$ \\
$\mathrm{Fe}$ & balance \\
\hline
\end{tabular}




\subsubsection{Preparation of the synthetic pore solutions}

Synthetic pore solutions were prepared from $\mathrm{Ca}(\mathrm{OH})_{2}, \mathrm{NaOH}, \mathrm{KOH}$, and $\mathrm{Ca}(\mathrm{SO})_{4} \cdot 2 \mathrm{H}_{2} \mathrm{O}$. All solutions were saturated with $\mathrm{Ca}(\mathrm{OH})_{2}$ to simulate conditions in ordinary portland cement concrete. Using factorial experimental design, 10 solutions were prepared with low, medium and high concentrations of the remaining three components (Raktoe et al 1981). Table 4.2 shows the concentrations of the chemicals added to make the solutions, as well as the ion concentration of each solution measured by Inductively Coupled Plasma - Atomic Emission Spectroscopy (ICP-AES) (Model: Varian Vista RL).

Table 4.2: Concentrations of the chemicals added and the ions measured in each solution using ICP-AES: the solutions were saturated with calcium hydroxide.

\begin{tabular}{|l|l|l|l|l|l|l|l|l|}
\cline { 2 - 9 } \multicolumn{4}{c|}{} & \multicolumn{4}{c|}{ Added Compound (g/l) } & \multicolumn{4}{c|}{ Measured lon (M) } \\
\hline Sol. & $\mathbf{C a}(\mathbf{O H})_{2}$ & $\mathbf{N a}(\mathbf{O H})$ & $\mathbf{K}(\mathbf{O H})$ & $\mathbf{C a}(\mathbf{S O})_{4}+\mathbf{2 H}_{\mathbf{2}} \mathbf{O}$ & $\mathbf{C a}^{{ }^{+}}$ & $\mathbf{N a}^{+}$ & $\mathbf{K}^{+}$ & $\left(\mathbf{S O}_{4}\right)^{2-}$ \\
\hline 1 & Sat. & 4.00 & 11.22 & 13.77 & 0.006 & 0.089 & 0.159 & 0.073 \\
2 & Sat. & 8.00 & 22.44 & 27.55 & 0.003 & 0.177 & 0.315 & 0.146 \\
3 & Sat. & 0.40 & 0.56 & 0.00 & 0.012 & 0.009 & 0.007 & 0.000 \\
4 & Sat. & 8.00 & 22.44 & 0.00 & 0.000 & 0.170 & 0.306 & 0.000 \\
5 & Sat. & 0.40 & 22.44 & 0.00 & 0.001 & 0.009 & 0.310 & 0.000 \\
6 & Sat. & 0.40 & 22.44 & 27.55 & 0.013 & 0.009 & 0.322 & 0.132 \\
7 & Sat. & 8.00 & 0.56 & 0.00 & 0.002 & 0.173 & 0.007 & 0.000 \\
8 & Sat. & 8.00 & 0.56 & 27.55 & 0.011 & 0.180 & 0.007 & 0.065 \\
9 & Sat. & 0.40 & 0.56 & 27.55 & 0.023 & 0.010 & 0.007 & 0.014 \\
10 & Sat. & 0.00 & 0.00 & 0.00 & 0.016 & 0.000 & 0.000 & 0.000 \\
\hline
\end{tabular}

The $\mathrm{pH}$ of the solutions varied between 12.3 (Solution 9) and 13.3 (Solution 4) as shown in Table 4.3. The conductivities of all of the solutions were high: the highest conductivity was $44.7 \mathrm{mS} / \mathrm{cm}$ for Solution 4 , which had high concentrations of $\mathrm{Na}^{+}$and $\mathrm{K}^{+}$ions in addition to being saturated with calcium hydroxide; and the lowest was 3.74 $\mathrm{mS} / \mathrm{cm}$ for Solution 10 , which was saturated calcium hydroxide with no other ions added (see Table 4.3). The oxygen concentration of the stagnant solutions was monitored and 
recorded during the experiments. The results, which are presented in Table 4.3 , indicate that the concentration of oxygen was sufficiently high in the solutions ( $>1 \mathrm{mg} / \mathrm{L}$ ) for the oxygen reduction reaction to take place; therefore, oxygen bubbling in the solutions was not required.

Table 4.3: Measured values of dissolved oxygen, $p H$ and conductivity of the synthetic pore solutions.

\begin{tabular}{|l|l|l|l|}
\hline Sol. \# & $\begin{array}{c}\text { Dissolved } \mathbf{O}_{\mathbf{2}} \\
\mathbf{m g} / \mathbf{l}\end{array}$ & $\mathbf{p H}$ & $\begin{array}{c}\text { Conductivity } \\
\mathbf{m S} / \mathbf{c m}\end{array}$ \\
\hline 1 & 3.07 & 12.9 & 17.5 \\
2 & 5.28 & 13.2 & 29.8 \\
3 & 6.30 & 12.5 & 4.41 \\
4 & 5.38 & 13.3 & 44.7 \\
5 & 3.74 & 13.2 & 32.9 \\
6 & 3.73 & 12.8 & 19.3 \\
7 & 5.56 & 12.9 & 17.8 \\
8 & 4.47 & 12.6 & 12.0 \\
9 & 6.43 & 12.3 & 4.72 \\
10 & 6.35 & 12.4 & 3.74 \\
\hline
\end{tabular}

\subsubsection{Corrosion cell}

Figs. 4.2(a) and 4.2(b) show the cylindrical corrosion cell used to carry out both the anodic polarization and EIS tests. The $30 \mathrm{~mm}$ as-received carbon steel rebar specimen is the working electrode in the figures. The counter electrode was a co-axial platinum mesh cylindrical cage, which provided uniformly distributed electric fields around the rebar specimens. This configuration of the co-axial counter electrode allows the as-received cylindrical rebar to be examined over a reasonably large surface area that includes the deformity ribbing and local imperfections, as it would appear in service. A saturated calomel reference electrode (Model: Accumet) was placed in the port connected to the main body of the cell. As shown in Fig. 4.2(a), the port includes a glass tube with a 
tip that was situated between the working and counter electrodes, and $5 \mathrm{~mm}$ away from the surface of the specimens.

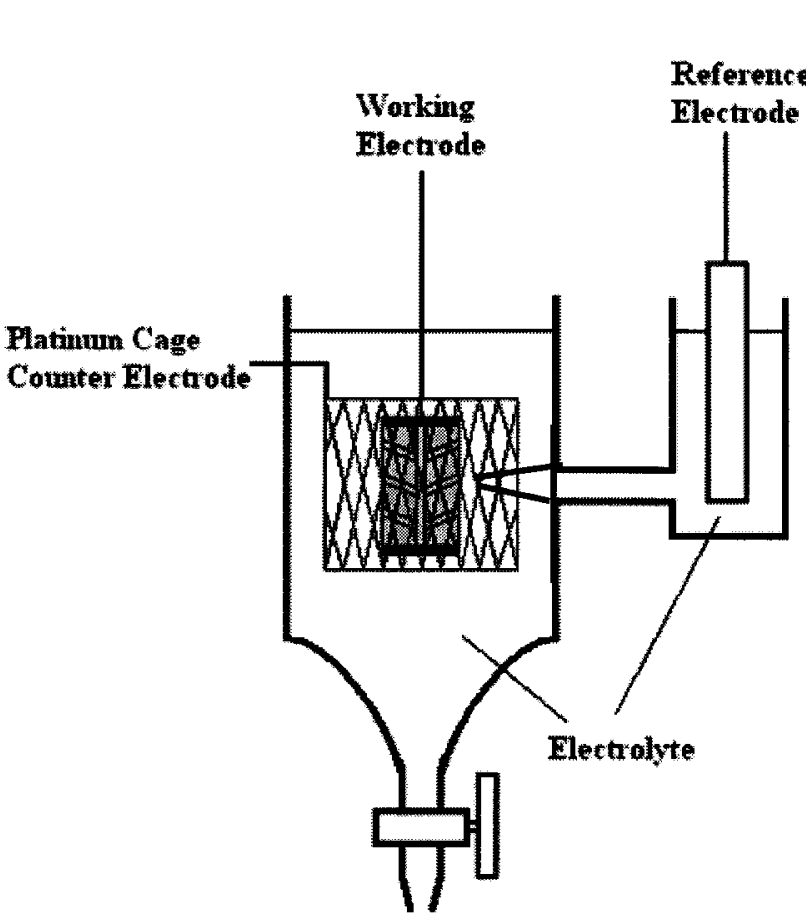

(a)

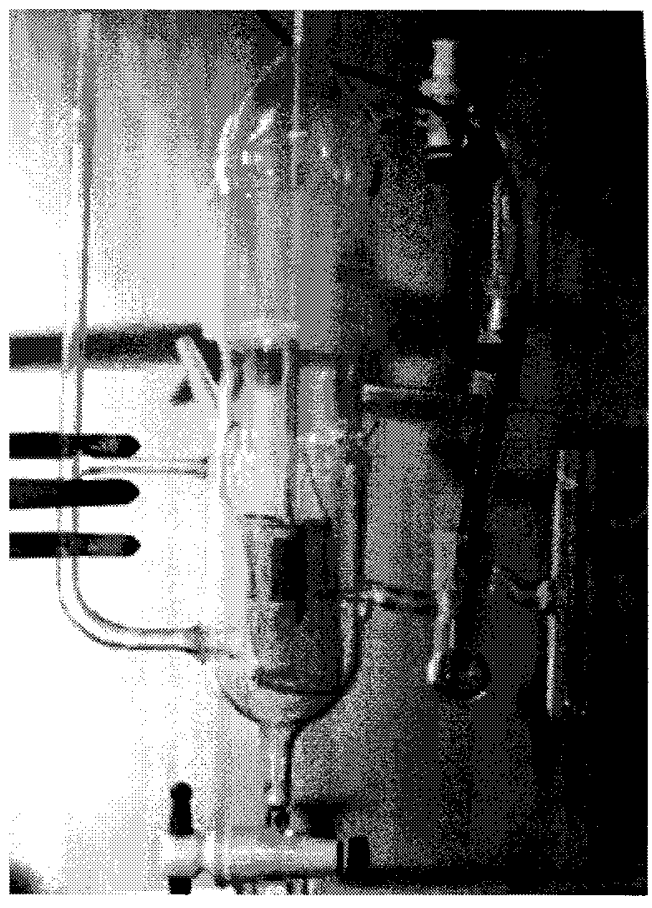

(b)

Figure 4.2: (a) Schematic of the test setup used to carry out anodic polarization and EIS measurements; (b) the test setup.

\subsubsection{Anodic polarization tests}

Three replicate rebar specimens were immersed in approximately $100 \mathrm{ml}$ of each synthetic pore solution; the 10 solution containers were then sealed with parafilm in order to prevent water evaporation. For each solution, the anodic polarization experiment was carried out for each of the three rebar specimens. Anodic polarization tests were run for the first replicate specimen from each set of three in a solution (rebar 1) after five days of immersion; the second specimen (rebar 2) was tested after eight days; and the third specimen (rebar 3) was tested after at least fourteen days in each solution. 
Prior to the anodic polarization experiment, the rebar specimens were removed from their solutions and then placed inside the co-axial platinum cage counter electrode (see Section 4.2.3). In each instance, the measurement cell was filled with the same (but filtered to remove solids) solution in which the rebar was initially immersed. The freecorrosion potential was measured with a Gamry PC4/300 Potentiostat/Galvanosatat/ZRA, relative to the saturated calomel reference electrode. It took approximately one hour before the free-corrosion potential would no longer fluctuate significantly (fluctuations were less than $1 \mathrm{mV} / \mathrm{min}$ ). Subsequently, anodic polarization curves were obtained starting $10 \mathrm{mV}$ below the free potential and finishing at $800 \mathrm{mV} / \mathrm{SCE}$, using a scan rate of $0.166 \mathrm{mV} / \mathrm{s}$, as prescribed in ASTM G5 (2004). The choice of scan rate is a compromise between accuracy and the time to complete the measurement. It was found in this study that slower scan rates did not significantly change the results, which is consistent with other studies (Bird et al.1988, Li and Sagues 2002, Leckie 1970).

The $\mathrm{pH}$, dissolved oxygen, and conductivity of the solutions were measured with a VWR SympHony SP90M5. No differences in the readings were observed during immersion, or before and after each anodic polarization measurement; the results of the measurements are given in Table 4.3.

\subsubsection{EIS tests}

Two replicate rebar specimens were placed in covered beakers containing $\sim 100$ $\mathrm{ml}$ of each of the 10 synthetic pore solutions for at least 10 days, to allow the growth of stable oxide films. The rebar specimens were then placed inside the corrosion cell and EIS measurements were done in $\sim 200 \mathrm{ml}$ of the same solution, which was filtered to remove solids. 
The free-corrosion potential and impedance spectrum for each rebar specimen were measured with a Gamry PC4/300 Potentiostat/Galvanosatat/ZRA, relative to the saturated calomel reference electrode. The free potentials were monitored for two hours before EIS measurements to ensure that the oxide films had re-stabilized after transfer to the corrosion cell. The EIS measurements were done at the free potential, with an AC voltage of $10 \mathrm{mV}$ RMS, between $10^{5}$ to $10^{-3} \mathrm{~Hz}$ at 10 equally spaced points per decade.

No differences were observed in $\mathrm{pH}$, dissolved oxygen, and conductivity of the solutions, measured with a VWR SympHony SP90M5, before and after each EIS measurement; the measurements were essentially the same as the ones reported in Table 4.3 .

\subsection{Results and Discussion}

\subsubsection{Anodic polarization test results}

Anodic polarization experiments that were carried out after 5, 8 and 14 days of immersion suggest that 8 days are required for the oxides to stabilize. In Fig. 4.3, as an example, typical anodic polarization curves are plotted for the three sets of as-received rebar specimens immersed in solution 8 for 5,8 and 14 days. All of the anodic polarization curves have the same general appearance; however, the curve for rebar 1, which only experienced 5 days of immersion, is shifted to higher currents compared to the curves for rebars 2 and 3, which essentially overlap. (For the second and third rebar specimens, the estimated reproducibility is $\pm 60 \mathrm{mV}$ for the free potential and $\pm 30 \%$ for the passive current density). The inference is that the oxide films on the rebar specimens became relatively stable after 8 days of immersion in the synthetic pore solutions. Similar 
conclusions were drawn from observations of the free potential: large drifts of $>180$ $\mathrm{mV} /$ hour during the first few days became much smaller $(<0.4 \mathrm{mV} /$ hour $)$ after about 8 days. These results are consistent with previous work by Poursaee and Hansson (2007). The stability of the tests after 8 days of immersion indicates that reliable data can be obtained with as-received rebar.

Anodic polarization tests were also done on two specimens that had their deformities and mill scale removed and their surfaces subsequently polished. Similar polarization curves were obtained for these polished specimens; however, they were shifted to lower currents (by approximately a decade) relative to the curves for the asreceived rebar specimens. These shifts are large enough to suggest that modifying the surfaces by polishing will result in conditions not representative of service. Hence, the remainder of the discussion will focus on results obtained for as-received specimens.

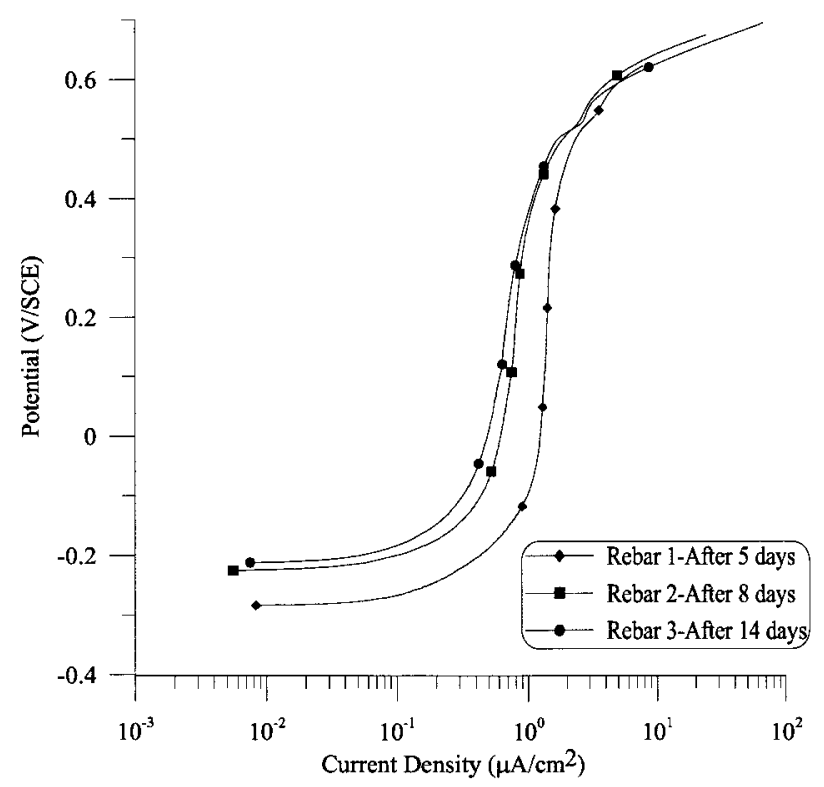

Figure 4.3: Anodic polarization plots for three rebar specimens immersed in Solution 3 for different times. After 8 days the polarization plots did not change significantly with further immersion; similar results were seen for rebar immersed in the other solutions. 
Anodic polarization curves obtained for as-received rebar specimens immersed for at least 14 days (i.e., rebar 3 for each solution) in the 10 simulated pore solutions are shown in Fig. 4.4. The large differences indicate that the characteristics of the oxide film formed in the various solutions may not be the same. For instance, consider the current density corresponding to the inflection point in the polarization plot in Fig. 4.5; in this study, this current density is equated to the passive current density, $\mathrm{i}_{\mathrm{p}}$. Figure 4.4 shows over two orders of magnitude difference in the passive current density for the different simulated pore solutions. These passive current densities are not significantly correlated with $\mathrm{pH}$, conductivity or oxygen content of the solutions shown in Table 4.2, within the reported ranges of the values. This suggests more complicated functional dependencies, and/or that differences observed in the anodic polarization curves might originate with the oxide films that form on the rebar surface, and that the quality of the oxide film is influenced by the pore solution in which it was grown: higher quality is characterized by lower passive current density, and vice versa. Higher passive current densities would extrapolate to higher metal-loss rates and, thus these oxides would be less protective and thus of lesser quality. Figure 4.4 also shows that the passive current densities for oxides grown in solutions with $\mathrm{SO}_{4}{ }^{2-}$ were always higher than for those grown in solutions without $\mathrm{SO}_{4}{ }^{2-}$.

The iron Poubaix diagram shows that for the $\mathrm{pH}$ range found in concrete, and in this study, iron forms a passive protective oxide as it corrodes; hence, it is reasonable to assume that all rebar specimens grown in the different synthetic pore solutions are in a passive state. A simple Evan's diagram description (plotting potential against log of the current density) of the present experiment would show all of the corrosion potentials in 
Fig. 4.4 occurring at the intersections of the cathodic line for the reduction of oxygen and the various metal oxidation lines, which would all be 'vertical' in accord with the passive state conditions. Oxides that are not as protective would passivate (become vertical on the Evan's diagram) at higher current densities and consequently the intersection of the oxygen reduction line with the passive region occurs at lower potentials, in accord with the negative Tafel slope relating the reduction overpotential to the logarithm of the current density (Revie an Uhlig 2008). Thus the anodic polarization curves in Fig. 4.4 move down (to lower corrosion potentials) and to the right (to higher current densities) as the passive films become less protective.

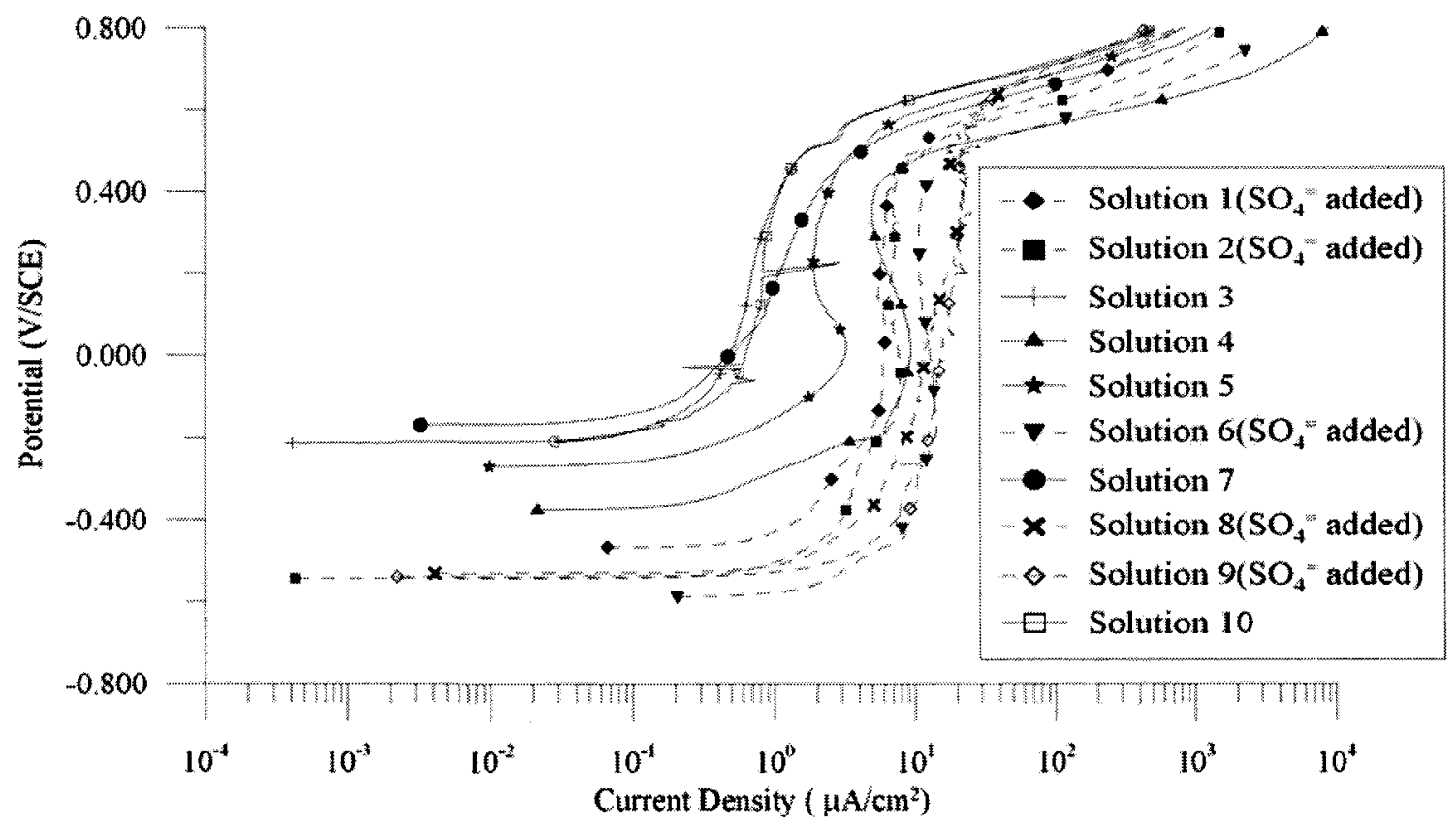

Figure 4.4: The anodic polarization curves of rebar specimens that have been immersed in different synthetic pore solutions for at least 14 days. 


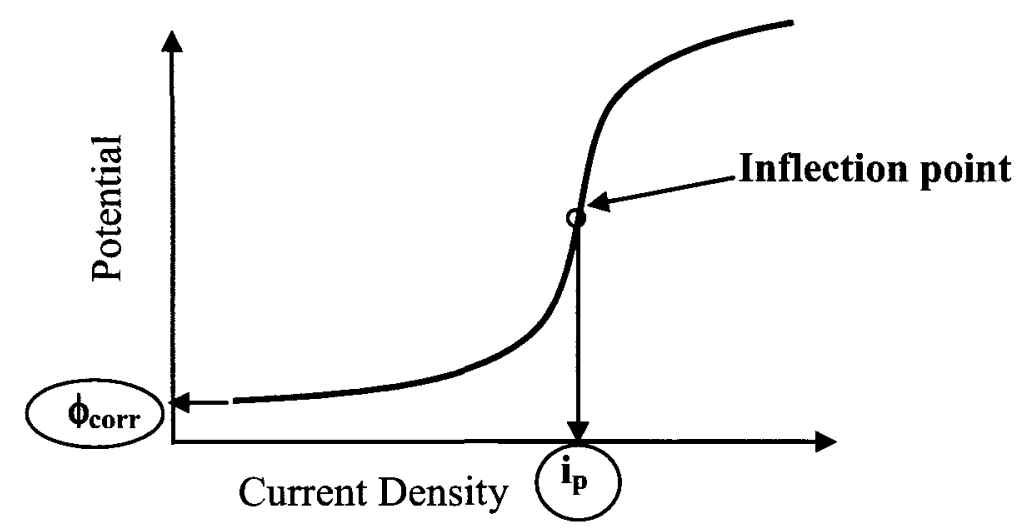

Figure 4.5: Graphical definition of passive current density and free corrosion potential used in this study.

The present experiment provides a set of oxide films with different values for the passive current density, $i_{p}$, that can be paired with the free-corrosion potential, $\phi_{c o r r}$, to determine the Tafel relation for the oxygen reduction reaction on as-received carbon steel at high $\mathrm{pH}$, because associated values of $i_{p}$ and $\phi_{c o r r}$ are points on the reduction line. Fig. 4.6 shows the correlation between free-corrosion potential and passive current density; implicit in this plot is the $\mathrm{pH}$ dependence, which is small. These data can be fitted to the Tafel equation:

$\phi_{\text {corr }}=\beta_{c} \log \left(i_{p} / i_{o}\right)+\phi_{c}^{o}$

where $\beta_{c}$ is the cathodic Tafel slope, $i_{o}$ is the cathodic exchange current density, and $\phi_{c}^{o}$ is the equilibrium oxygen reduction potential. Assuming the oxygen reduction reaction is reversible, then (Revie an Uhlig 2008)

$\phi_{c}^{o}[\mathrm{mV} / \mathrm{SCE}]=988-59.2 \mathrm{pH}$

Substituting Eq. 4.2 into Eq. 4.1 yields the regression equation used to determine the Tafel parameters from the free-corrosion potentials, passive current densities and solution pH measurements: 
$\beta_{c}=-245 \pm 30 \mathrm{mV} /$ decade; $i_{o}=(1.4 \pm 0.9) \times 10^{-2} \mu \mathrm{A} / \mathrm{cm}^{2}$.

Eq.4.3

The exchange current density determined in this study is comparable to earlier experimental values (Conway 1952). The value for the determined Tafel slope is consistent with previously reported cathodic Tafel slopes for oxygen reduction in alkaline solutions and in mortars determined in the standard way from the linear region of the cathodic polarization versus log current density plot (Garcés et al. 2005, . Jäggi et al. 2000, Brem 2004). The method introduced in this study for determining $\beta_{c}$ is complementary to the standard method, which is based on cathodic polarization; however, it does not have the same difficulties with defining the extent of the linear region.

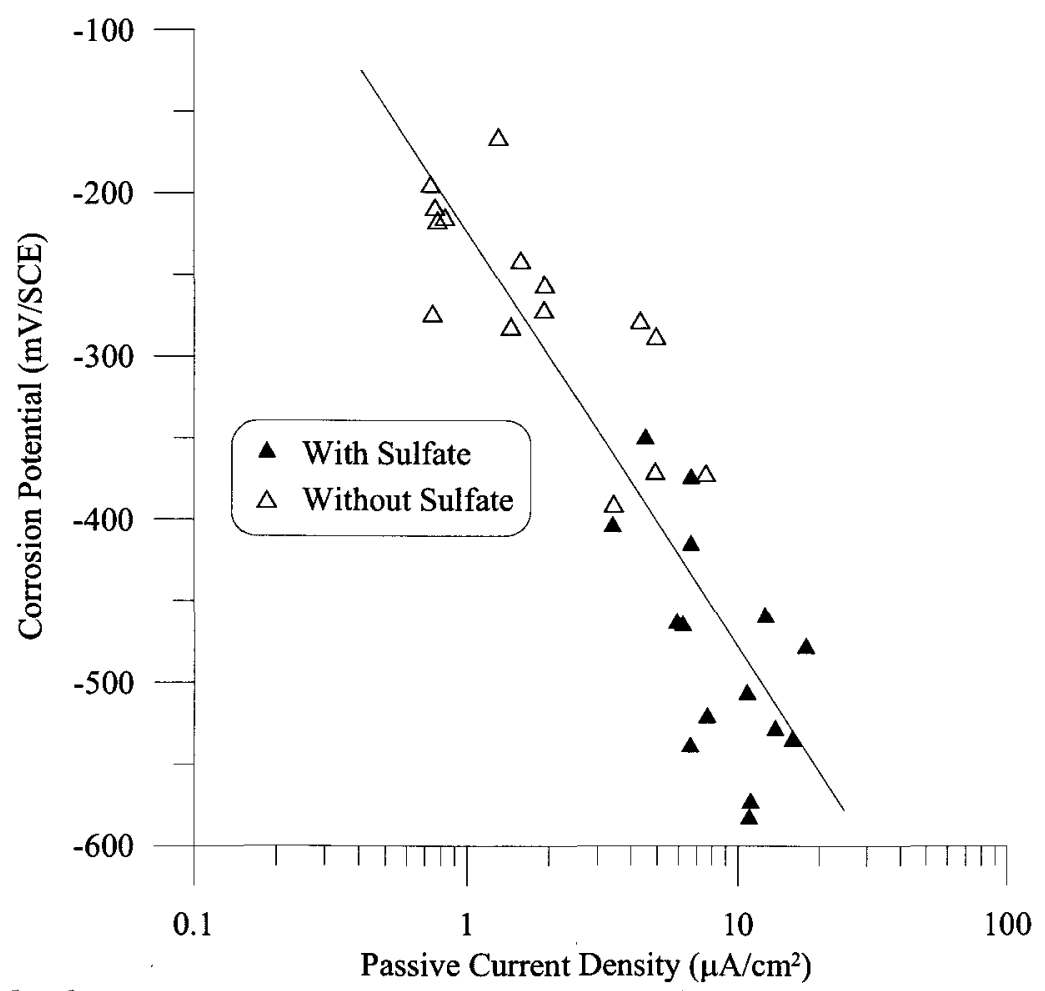

Figure 4.6: The free-corrosion potential is correlated with the passive current density determined from anodic polarization tests. The line in the figure is a guide for the eye. 


\subsubsection{EIS test results}

The anodic polarization results described in Section 4.3.1 suggested that the quality of the protective oxide film may be significantly influenced by the pore solution in which it was grown. In this section, EIS is used to explore the electrochemical properties of the oxides grown in the various simulated pore solutions.

Figure 4.7 shows impedance spectra plotted different ways for rebar specimens in the ten synthetic pore solutions; replicate specimens show similar results and are not plotted. Fig. 4.7(a) shows relatively large differences in the magnitude of the impedance at low frequencies $(<1 \mathrm{~Hz})$; these can be used to distinguish differences in the oxide film properties that form in different solutions. As an example, Fig. 4.7(a) shows the effect of adding sulfate ions. At low frequencies $(<1 \mathrm{~Hz})$, rebar oxides grown in $\mathrm{SO}_{4}{ }^{2-}$ containing solutions have lower impedance magnitudes compared with those grown in solutions without $\mathrm{SO}_{4}{ }^{2-}$ ions. (In the plots, the sulfate-containing solutions are shown as dashed lines, and the spectral points for the solutions without sulfate are connected with solid lines.) This is consistent with Direct Current measurements that show films with higher electrical resistances generally are more resistant to corrosion (Mansfeld 1976), and this, in turn, supports the observations made during the anodic polarization tests that the passive oxide films were less protective if they were grown in solutions containing $\mathrm{SO}_{4}{ }^{2-}$ ions. It can also be observed in Fig. 4.7(b) that the phase angles in different solutions show large variations. For example, in the mid-frequency region (i.e., $1 \mathrm{~Hz}$ to $10^{2} \mathrm{~Hz}$ ) the phase angles of the specimens grown in solutions containing $\mathrm{SO}_{4}{ }^{2-}$ are generally larger than those for specimens grown in solutions without $\mathrm{SO}_{4}{ }^{2-}$ ions. 


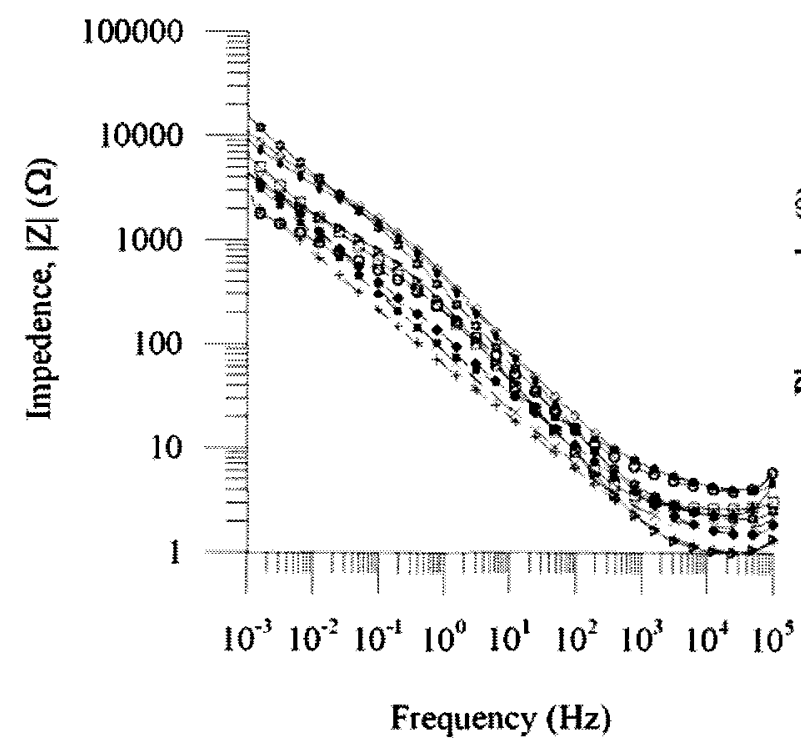

(a)

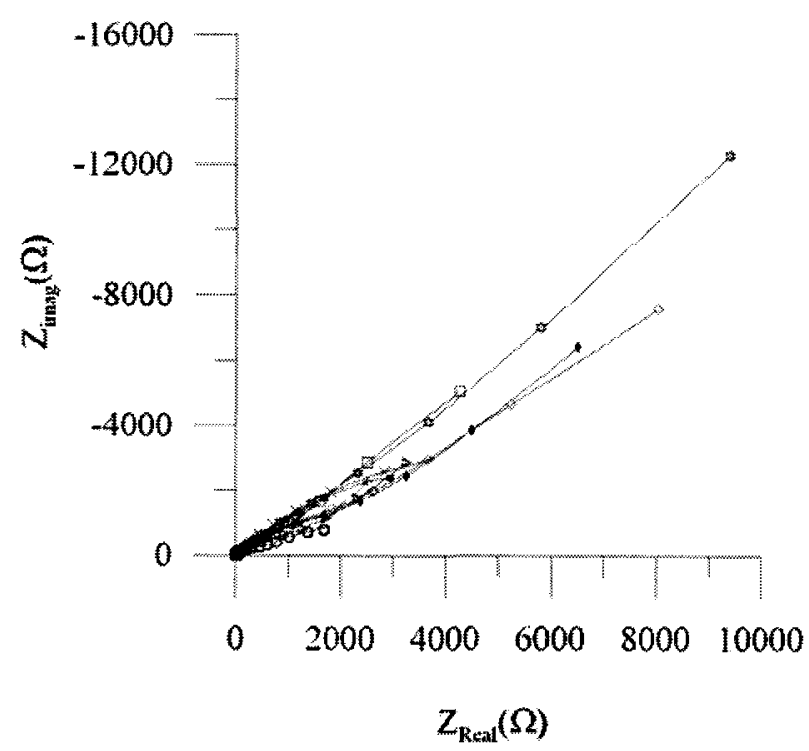

(c)

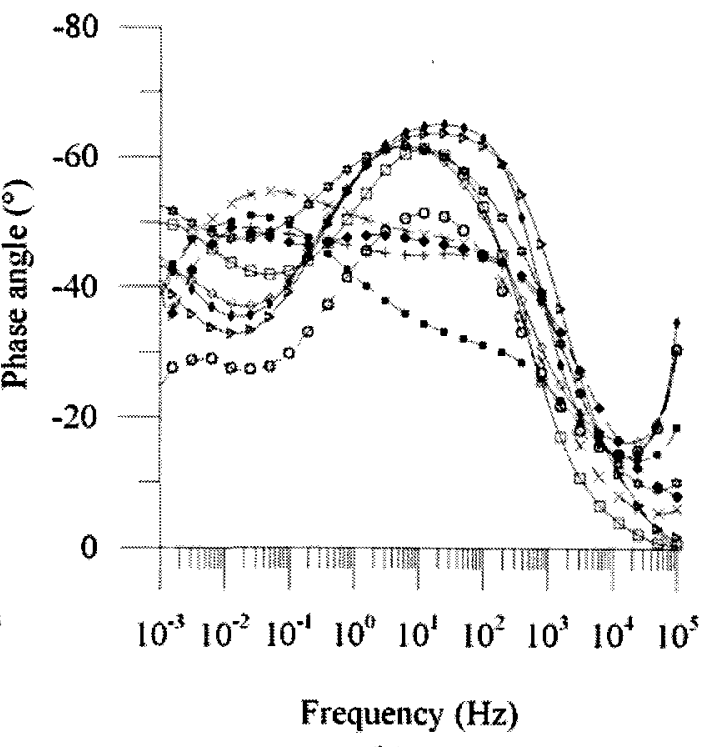

(b)

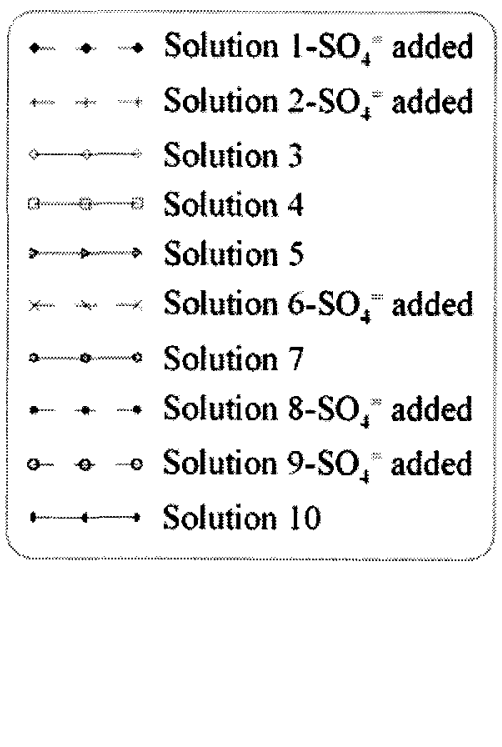

Figure 4.7: Typical plots of (a) Impedance vs. Frequency; (b) Phase angle vs.

Frequency; (c) Imaginary vs. Real Impedance (Nyquist). 
The observations described above can be made quantitative by calculating Pearson Correlation Coefficients, $r$, between the measured rebar free-corrosion potentials and the various EIS measures (magnitude of the impedance, real and imaginary components, and phase angle) at each measured frequency. Figure 4.8 shows typical examples of plots of free-corrosion potential versus EIS measures at specific frequencies, along with the calculated correlation coefficients.

Similar correlation calculations were done at all frequencies using all the EIS measures from replicate sets of specimens in the ten synthetic pore solutions. The results are shown in Fig. 4.9. The free-corrosion potential tends to correlate with impedance at low frequencies, and phase angle at intermediate frequencies. The correlations with freecorrosion potential are positive and largest at approximately $0.1 \mathrm{~Hz}$ with the impedance magnitude, and at $\approx 0.05 \mathrm{~Hz}$ with the real component; the correlations are negative and smallest at $\approx 0.01 \mathrm{~Hz}$ with the imaginary impedance, and at $\approx 10 \mathrm{~Hz}$ with the phase angle. Because the free-corrosion potential can be related to the passive current density, as shown in the previous section, these correlations with the free-corrosion potential suggest that low-frequency impedances, and mid-frequency phase angles, can also be used in to rank the quality of the oxide films. 


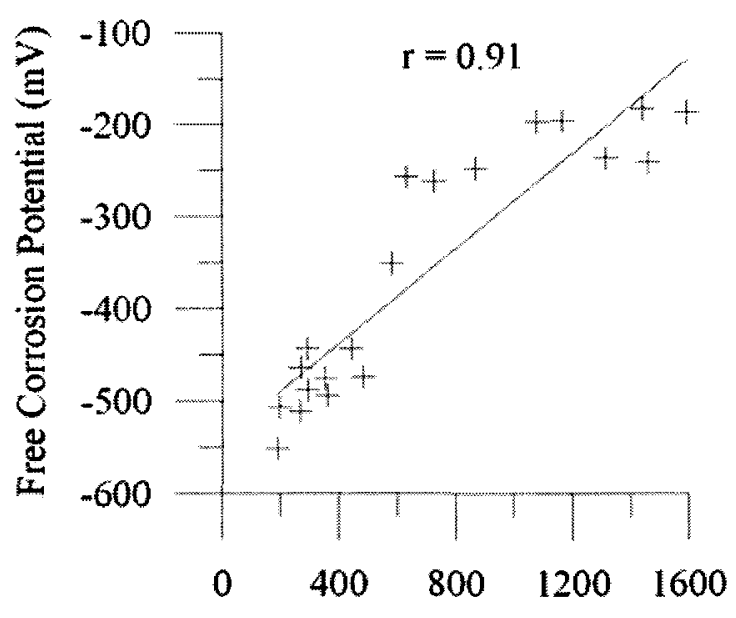

Impedance, $|\mathrm{Z}|(\Omega)$ at $0.128 \mathrm{~Hz}$

(a)

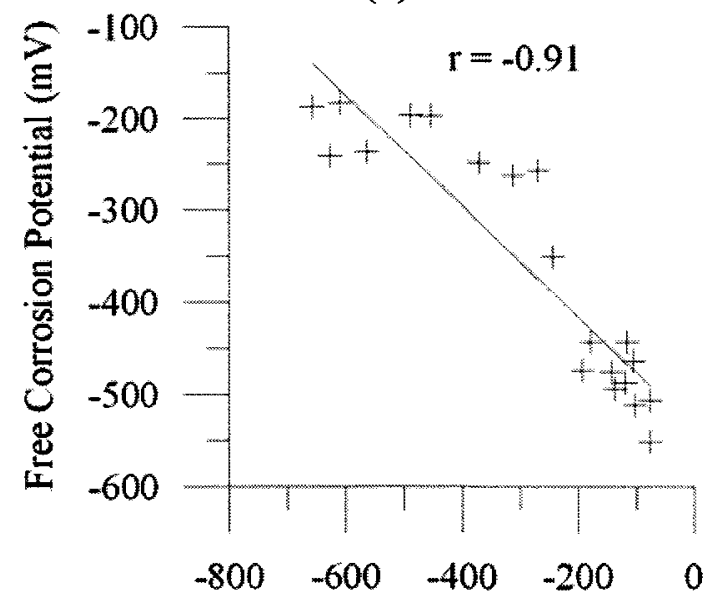

Imaginary Impedance, $Z_{\text {imag. }}$ at $0.3982 \mathrm{~Hz}$

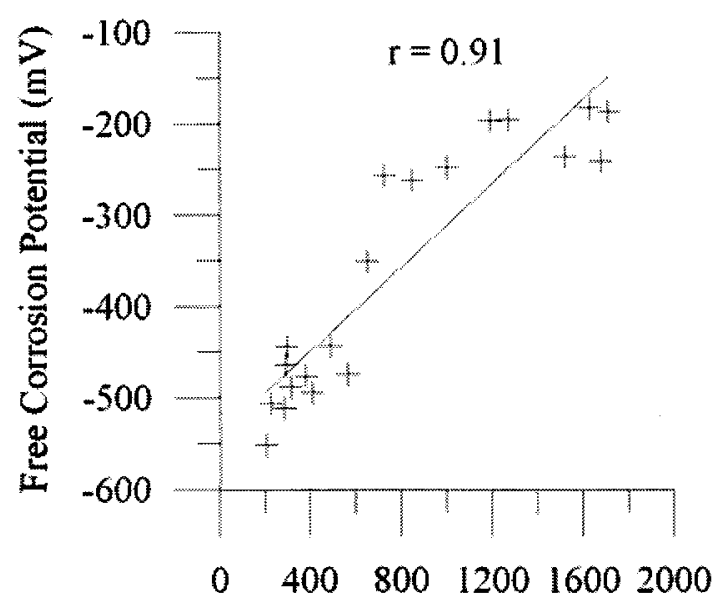

Real Impedance, $Z_{\text {Real }}(\Omega)$ at $0.05 \mathrm{~Hz}$

(b)

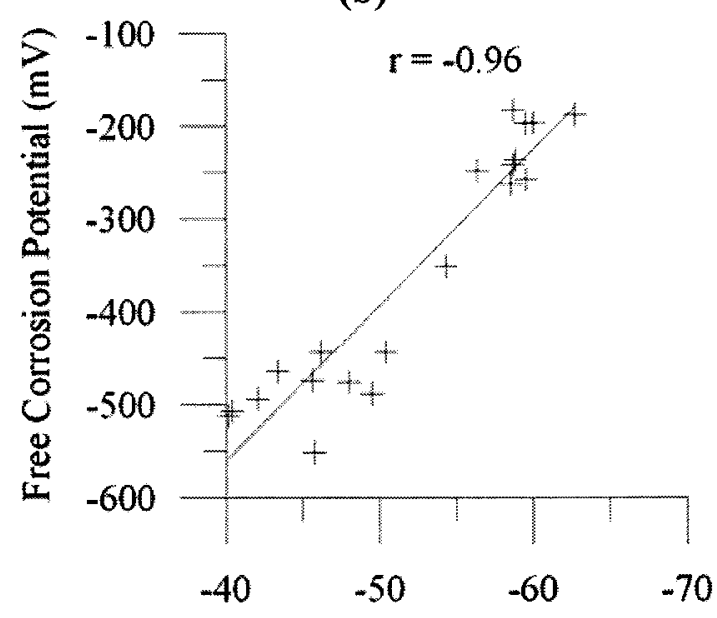

Phase angle $\left(^{\circ}\right)$ at $1.58 \mathrm{~Hz}$

(d)

Figure 4.8: Examples showing data at representative frequencies used in the calculations of the Pearson Correlation Coefficients shown in Figure 4.9. The r values are 0.91, 0.91, -0.91 and -0.96 for plots (a), (b), (c) and (d), respectively. 


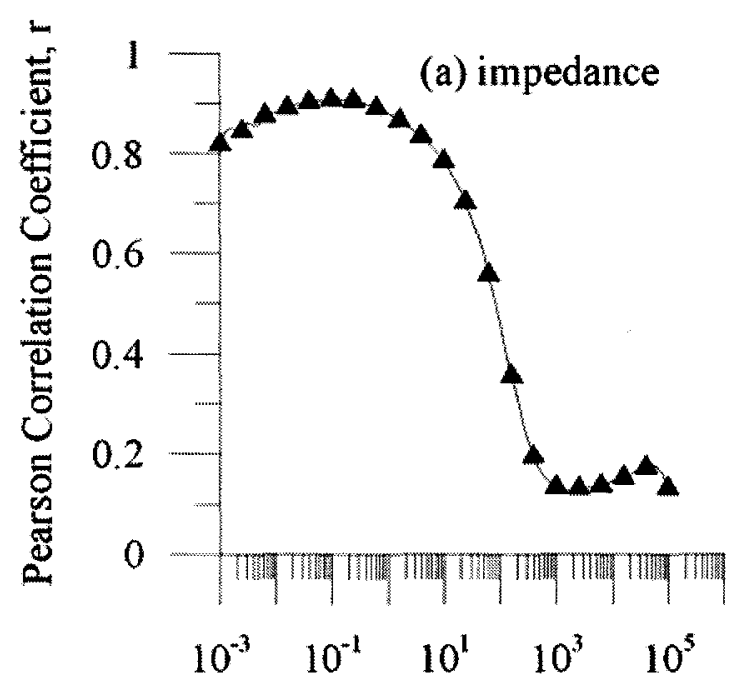

Frequency $(\mathrm{Hz})$

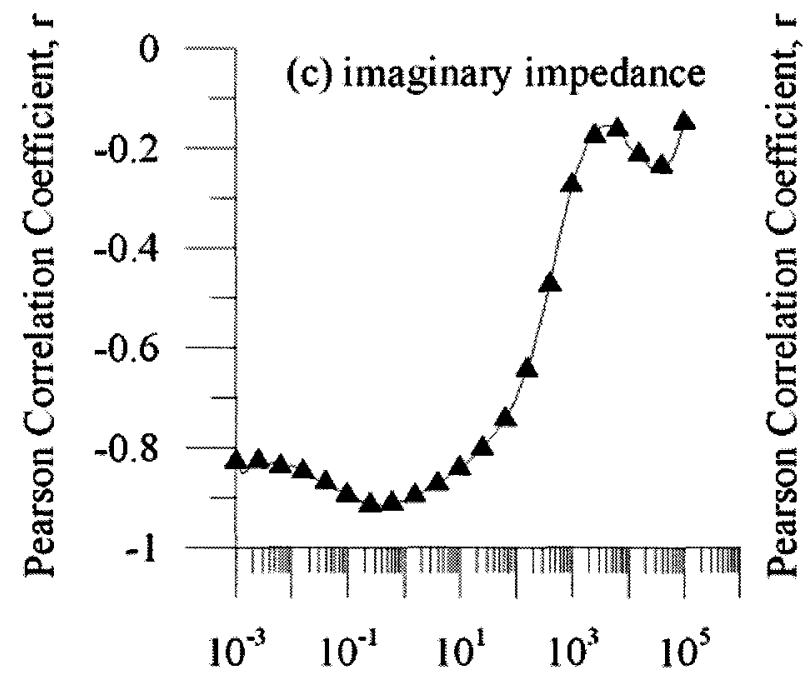

Frequency $(\mathrm{Hz})$

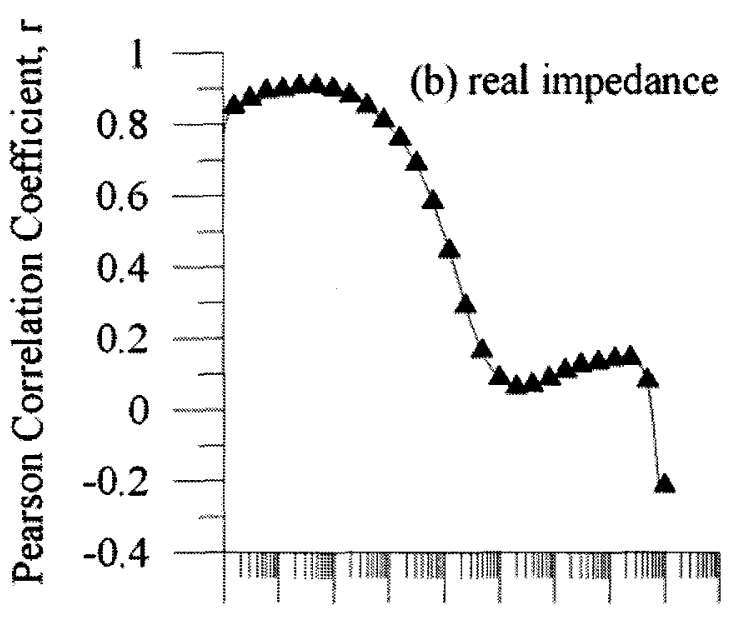

$\begin{array}{lllll}10^{-3} & 10^{-1} & 10^{1} & 10^{3} & 10^{5}\end{array}$

Frequency $(\mathrm{Hz})$

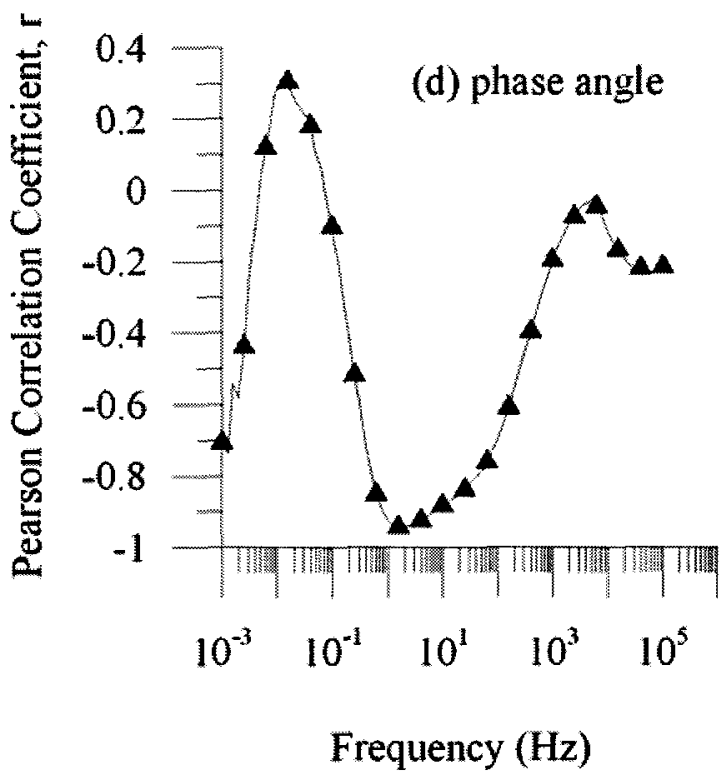

Figure 4.9: Pearson correlation coefficients, $r$, between free-corrosion potential and (a) impedance magnitude; (b) real impedance; (c) imaginary impedance; and (d) phase angle. Correlation coefficients with $|r|>0.45$ have p-values (i.e,. the probability of an observed result happening by chance under the null hypothesis) less than 0.05 . 


\subsection{Discussion}

Rankings of the quality of the protective oxides grown on rebar immersed in the various synthetic pore solutions are shown in Table 4.4. The anodic polarization experiments suggested that high quality oxides have low passive current densities and high corrosion potentials; hence the quality of the oxides can be ranked from high to low by ordering them according to low to high values of $i_{p}$, or high to low values of $\phi_{\text {corr }}$. In addition, the EIS correlations suggest that higher quality films have higher values of the real impedance at $\approx 0.05 \mathrm{~Hz}$ and, hence, the quality of the films can be similarly ranked. Finally, phase angles at $\approx 10 \mathrm{~Hz}$ can be used to indicate the quality of the oxides: phase angles closer to $-90^{\circ}$ tend to be of higher quality. It can be seen in Table 4.4 that these measures derived from both anodic polarization and EIS techniques provided very similar rankings for the oxide films These measures are not all independent, so it is not surprising that they lead to similar rankings; however, this shows that any of the measures can be used to provide an estimate of the quality of the protective oxide film. This could prove useful in practical situations where some measurements might be easier to make than others.

There were insufficient data to determine the functional dependencies of the passive film quality (as measured by the free-corrosion potential or the passive current density) on the concentrations of the ions, $\mathrm{Ca}^{2+}, \mathrm{Na}^{+}, \mathrm{K}^{+}$and $\mathrm{SO}_{4}{ }^{2-}$, examined in this study. In addition, the passive current density was not correlated with $\mathrm{pH}$ (conductivity or oxygen content of the solutions), which suggested that $\mathrm{pH}$ might not be an important factor in determining film quality. However, $\mathrm{pH}$ does play a role by relating passive- 
current density to free-corrosion potential through Eqs. 4.1 and 4.2, hence, passivecurrent density and free-corrosion potential are strictly not independent indicators of passive film quality. This is a reminder that simple correlations can be misleading when the underlying mechanism is complex. More detailed studies are required to understand the effects on the quality of the passive film of $\mathrm{pH}$, conductivity and oxygen content, and also the contributions of different amounts of various ions in the pore solution.

Table 4.4: Rankings of oxide film quality.

\begin{tabular}{|c|c|c|c|c|}
\hline \multirow{3}{*}{ Rank } & \multicolumn{2}{|c|}{ Anodic polarization } & \multicolumn{2}{|c|}{ EIS } \\
\hline & $\begin{array}{c}\text { Based on } \\
i_{p}\end{array}$ & $\begin{array}{c}\text { Based on } \\
\phi_{c}\end{array}$ & $\begin{array}{l}\text { Based on } \\
Z_{\text {real }} \\
\text { at } 0.05 \mathrm{~Hz}\end{array}$ & $\begin{array}{c}\text { Based on } \\
\text { phase } \\
\text { angle at } 10 \mathrm{~Hz}\end{array}$ \\
\hline & $\begin{array}{l}\text { (low } i_{p} \\
\left.\text { to high } i_{p}\right)\end{array}$ & $\begin{array}{l}\text { (high } \phi_{c} \\
\text { to low } \phi_{c} \text { ) }\end{array}$ & $\begin{array}{l}\text { (high } Z_{\text {real }} \\
\text { to low } Z_{\text {real }}\end{array}$ & $\left(-90^{\circ}\right.$ to $\left.0^{\circ}\right)$ \\
\hline $\begin{array}{l}\text { (Highest quality) } \\
\text { (Lowest quality) }\end{array}$ & $\begin{array}{l}3 \\
10 \\
7 \\
5 \\
4 \\
1^{*} \\
2^{*} \\
6^{*} \\
8^{*} \\
9^{*}\end{array}$ & $\begin{array}{l}7 \\
10 \\
3 \\
5 \\
4 \\
1^{*} \\
6^{*} \\
9^{*} \\
8^{*} \\
2^{*}\end{array}$ & $\begin{array}{l}7 \\
3 \\
10 \\
5^{\star *} \\
4^{* *} \\
6^{*} \\
1^{*} \\
8^{*} \\
9^{*} \\
2^{*}\end{array}$ & $\begin{array}{l}10 \\
5 \\
3,7,4^{\star \star} \\
3,7,4^{\star \star} \\
3,7,4^{\star \star} \\
9^{\star} \\
6^{*} \\
1^{*} \\
2^{\star} \\
8^{*}\end{array}$ \\
\hline
\end{tabular}

(*) Solutions with $\left(\mathrm{SO}_{4}\right)^{2-}$

$(* *)$ Designates a tie 
Finally, SEM images of as-received carbon steel rebar specimens exposed to Solution 10 and Solution 9 are presented in Fig. 4.10; the oxides formed in these solutions have low and high passive current densities, respectively. Figure 4.10(a) shows that the rebar surface in Solution 10 is uniform without cracks and pores. Figure $4.10(\mathrm{~b})$ shows Solution 9 has cracks distributed over the surface of the rebar. In general, the SEM investigation revealed that relatively crack-free surfaces have higher corrosion potentials and lower passive current densities compared to those with more cracked surfaces. However, these results are preliminary and further microscopic investigations of the oxide film morphology and composition using other methods are required.

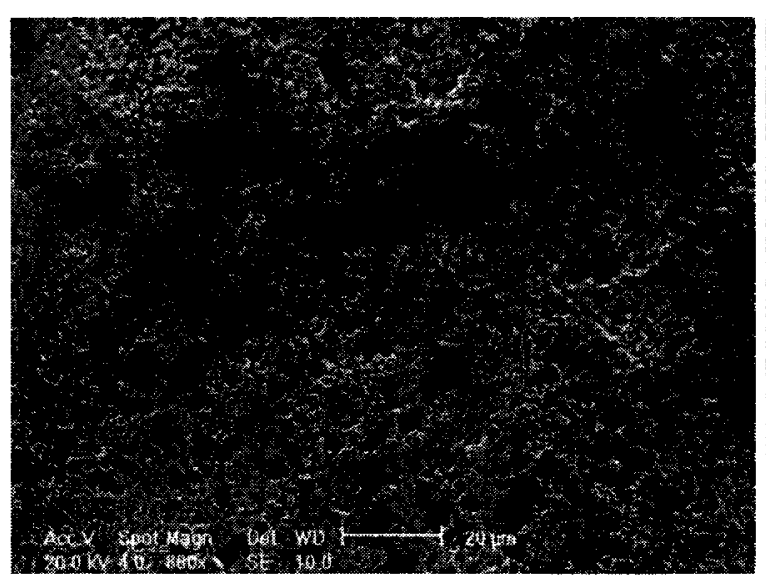

(a) $i_{p}=0.83 \mathrm{~mA} / \mathrm{cm}^{2}, \phi_{c}=-214 \mathrm{mV}$

Figure 4.10: SEM images of rebar surfac

Figure 4.10: SEM
10; (b) Solution 9.

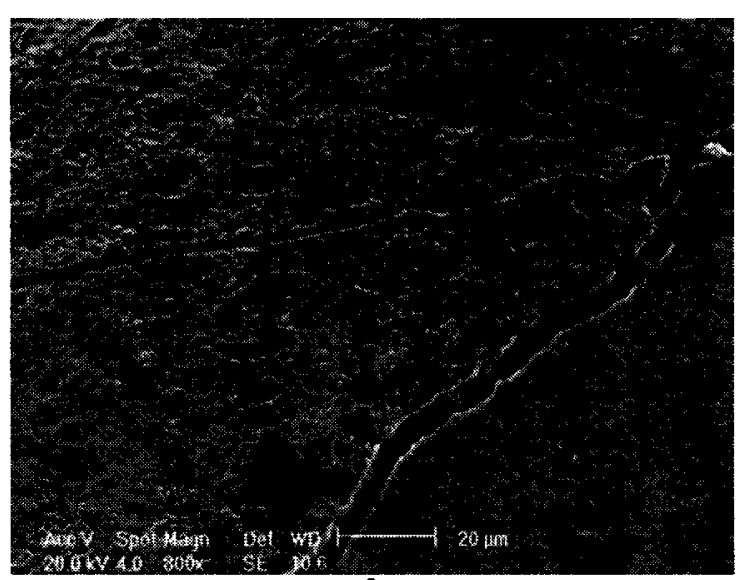

(b) $i_{p}=15.95 \mathrm{~mA} / \mathrm{cm}^{2}, \phi_{c}=-563 \mathrm{mV}$ 


\subsection{Conclusions}

The following conclusions can be drawn from the study presented in this chapter:

- Ions commonly found in concrete pore solutions, such as $\mathrm{Ca}^{2+}, \mathrm{Na}^{+}, \mathrm{K}^{+}$and $\mathrm{SO}_{4}{ }^{2-}$, can affect the passive oxide film formed on common carbon reinforcing steel. Hence, even though concrete pore solutions are overwhelmingly composed of calcium hydroxide, the current results suggest the importance of these auxiliary ions during the formation of the oxide films. Further investigations are required to determine the functional details regarding how the quality of the passive film depends on different amounts of various ions in the pore solution, and on $\mathrm{pH}$, conductivity and oxygen content.

- In particular, it has been observed by both anodic polarization and EIS that the presence of $\mathrm{SO}_{4}{ }^{2-}$ ions in the pore solution has a significant negative effect on the protective properties of passive oxide films.

- The experiments in this chapter were designed to simulate in-service conditions of steel rebar in concrete; hence, no special surface preparation technique was used. Anodic polarization experiments that were carried out after 5,8 and 14 days of immersion suggest that 8 days are required for the oxides to stabilize. The stability of the tests after 8 days of immersion indicates that reliable data can be obtained with as-received rebar, without special surface preparation, such as polishing.

- The analysis of anodic polarization curves obtained from different oxide films has led to the determination of the Tafel parameters for the oxygen reduction 
reaction on carbon steel rebar in synthetic concrete pore solutions $\left(\beta_{c}=-245 \pm\right.$ $30 \mathrm{mV} /$ decade, $\left.i_{o}=(1.4 \pm 0.9) \times 10^{-2} \mu \mathrm{A} / \mathrm{cm}^{2}\right)$. These values are consistent with previously reported cathodic Tafel slopes for oxygen reduction in alkaline solutions and in mortars. The method introduced in this study determines $\beta_{\mathrm{c}}$ from the passive current densities of a number of passive oxides and their corresponding free-corrosion potentials, which were used to map the cathodic activation polarization. This method is complementary to the standard approach, which relies on finding the linear region in a plot of the cathodic polarization versus log current density; however, it does not have the same difficulties with defining the extent of the linear region.

- The protective properties of passive oxide films can be estimated with EIS at frequencies where there is a strong correlation between the free-corrosion potential and the measured impedances and phase angles: higher impedances at low frequencies $\left(<1 \mathrm{~Hz}\right.$ ), and lower phase angles at mid frequencies (1 to $10^{2}$ $\mathrm{Hz}$ ) are good indicators of high-quality protective oxides. 


\section{Chapter 5}

\section{Electrochemical Study on Chloride-induced Depassivation of Rebar}

\subsection{Introduction}

Chlorides are well known to cause depassivation, if they are present in sufficient concentration; however, reported values of threshold chloride concentrations for rebar depassivation in concrete cover a wide range and have a large degree of uncertainty (Glass and Buenfeld 1997, Alonso et al. 2000, Angst et al. 2009). Understanding the underlying variability in threshold values is important for service-life-based design and for the prediction of the remaining service life of concrete structures.

Currently, there is no comprehensive explanation for the variability of measured chloride thresholds, but, as presented in the literature review, it is believed to depend on a large number of factors: the alkalinity of the pore solution (Gouda 1970, Goni and Andrade 1990, Pettersson 1996b, Mammoliti et al. 1996, Breit 1998, Li and Sagues 2001, Oh et al. 2003); the presence of auxiliary ions such as sulfates, sodium and potassium (Bird et al. 1988, Hansson and Sorensen 1990, Hussain et al. 1995, Thomas 1996, Andrade and Page 1986, Kayyali and Haque 1995, Ghods et al. 2009); rebar surface conditions (Mammoliti et al. 1996, Li and Sagues 2001, Pillai and Trejo 2005); the 
properties of the rebar-concrete interface (Gonzalez et al. 1993, Glass and Buenfeld 2000a, Sagues et al. 2000); chloride binding in concrete (Rasheeduzzafar et al. 1991, Hussain and Al-Gahtani 1996, Glass and Buenfeld 2000b); and oxygen availability around the reinforcement (Mangat and Molloy 1992, Gonzalez et al. 1993, Pettersson 1996b, Glass and Buenfeld 2000b).

Experimental studies to isolate the mechanism of chloride depassivation of carbon steel rebar in concrete are challenging because it is difficult to control confounding variables, and because of the long waiting times (months) required due to the slow ingress of chloride into concrete, and because of the difficulty in measuring chloride concentrations in the vicinity of the rebar surface. Therefore, most experimental studies of chloride thresholds have been done using relatively small-scale bench-top devices in which the sections of rebar are submerged in solutions that simulate the chemical environment most often found in extracted pore solutions. These bench-top experiments usually include standard electrochemical procedures, which can be done relatively quickly under conditions for which the control of variables is relatively easy; thus, bench-top experiments are widely used to study chloride threshold of steel in concrete. However, it is often difficult to relate the results of these experiments to rebar in service.

For example, a large portion of chloride threshold data have been obtained from laboratory experiments in simulated solutions on highly-processed (e.g. polished and/or pickled) steel specimens or on relatively small exposure surfaces (typically smaller than 2 $\mathrm{cm}^{2}$ ), that were machined from rebar samples (Abd El Aal et al. 2009, Saremi and Mahallati 2002, Goni and Andrade 1990, Choi et al. 2006, Bird et al. 1988). These 
experiments provide information on the effects of solution chemistry on the pristine steel surface, but, because the surfaces are processed to eliminate the deformities (ribbing), defects and mill-scale found in service, these experiments cannot be used to draw practical conclusions about rebar depassivation in concrete (Li and Sagues (2001, 2004), Mahallati and Saremi (2006), Mammoliti et al. 1996). Fortunately, it is not necessary to test rebar of lengths typically used in service, which can be several meters, because the resistivity of concrete is relatively high (typically larger than $100 \Omega-\mathrm{m}$ ), so that the electrochemical processes on the rebar surface take place on a scale of several centimetres (Pour-Ghaz et al. 2009, Li and Sagues 2004). Hence, rebar specimens of a few centimetres provide representative lengths, provided they are long enough to contain sufficient sampling of all the surface characteristics (e.g. ribbing, defects and mill scale).

Cutting specimens to lengths of a few centimetres introduces freshly cut surfaces that are relatively large fractions of the total specimen surface area. These freshly cut surfaces will most likely have a different and confounding chloride threshold, so some care must be taken to isolate the ends of the specimens. Effective coating of the ends would eliminate this problem; however, initial attempts to coat the ends with a nonconductive epoxy were foiled by corrosion in the crevices between the rebar and the epoxy in the presence of chloride. Similar difficulties have been reported by others (Gonzalez et al. 1993, Sagues et al. 2000, Li and Sagues 2001). In this study, a method to coat the cut ends of rebar specimens that effectively eliminates crevice corrosion is reported.

The cylindrical nature of rebar suggests that the standard flat counter electrodes used in most other studies, in which the specimens are generally small, polished and flat, 
be replaced with a co-axial cylindrical electrode. This co-axial geometry provides a relatively uniform radial electric field at the rebar surface. Thus, in this study an experimental electrochemical setup is introduced such that a short section of as-received rebar, coated at both ends, is immersed in simulated concrete pore solution, and surrounded by a co-axial counter electrode. In addition, in this study the reference electrode was connected by a very small glass tip that was designed to minimize the distortion of the electric field.

The main objective of this work was to measure chloride thresholds of carbon steel rebar under conditions reasonably comparable with those found in service. Various electrochemical techniques was used to follow passivation and depassivation of asreceived rebar specimens, with and without surface modification, in simulated pore solutions as a function of chloride concentration. The result of the study presented in Chapter 4 showed the importance of pore solution composition for the passivation of rebar; in this chapter, this result is extended to depassivation. The electrochemical techniques included free corrosion potential (FCP) measurements, electrochemical impedance spectroscopy (EIS), linear polarization resistance (LPR) and anodic polarization (AP). In addition, some suggestions are provided on how to report chloride thresholds in terms of probabilities.

\subsection{Experimental Setup}

\subsubsection{Synthetic pore solution}

Two synthetic solutions were used in this study to simulate the pore solutions found in concrete. The label "CH" refers to a saturated calcium hydroxide solution, 
where " $\mathrm{C}$ " stands for calcium and " $\mathrm{H}$ " stands for hydroxide; this is typically used as a pore solution surrogate. The solution referred as "CP", where " $\mathrm{C}$ " stands for concrete and "P" stands for pore, was prepared with typical concentrations of different anions and cations that can be found in ordinary portland cement concrete (OPC) pore solution (Page and Vennesland 1983, Moragues et al. 1987, Andersson et al. 1989, Marcotte 2001). The CP solution contains small amounts of sulfate ions at the level that is typically present in concrete pore solutions. In Table 5.1, the concentrations of the different compounds that have been added to distilled water to produce the CP solution are presented. The actual concentrations of the anions and cations in solution were measured using Inductively Coupled Plasma - Atomic Emission Spectroscopy (ICP-AES) (Model: Varian Vista RL).

\subsubsection{Sample preparation}

Two types of specimens were prepared for this study: 'as-received', and 'turned and polished'. Both specimen types were cut to $30 \mathrm{~mm}$ lengths from deformed carbon steel rebar, of $10 \mathrm{~mm}$ nominal diameter. The as-received surfaces were not prepared further. The turned-and-polished specimens were turned on a lathe to remove the deformities and the mill-scale, and then polished to 600 Grit. The average area of the exposed rebar surfaces was $10.6 \pm 0.2 \mathrm{~cm}^{2}$, which was chosen in accordance with the recommendations of $\mathrm{Li}$ and Sagues (2004). The elemental composition of the carbon steel used in this study is shown in Table 5.2. 
Table 5.1: Concentrations of the species in the two test solutions (CP and CH).

\begin{tabular}{|c|c|c|c|c|c|c|c|c|c|c|c|}
\hline \multirow[b]{2}{*}{ Solution } & \multicolumn{4}{|c|}{$\begin{array}{l}\text { Added compounds } \\
\text { (M) }\end{array}$} & \multicolumn{4}{|c|}{$\begin{array}{c}\text { Measured ions } \\
(\mathrm{mg} / \mathrm{l})\end{array}$} & \multirow{2}{*}{$\mathbf{p H}$} & \multirow{2}{*}{$\begin{array}{c}\sigma^{*} \\
(\mathrm{~ms} / \mathrm{cm}) \\
\end{array}$} & \multirow{2}{*}{$\begin{array}{l}\mathrm{Do}_{2}{ }^{\star \star *} \\
(\mathrm{mg} / \mathrm{l})\end{array}$} \\
\hline & $\mathrm{Ca}(\mathrm{OH})_{2}$ & $\mathrm{Na}(\mathrm{OH})$ & $\mathrm{K}(\mathrm{OH})$ & $\mathrm{CaSO}_{4}$ & $\mathrm{Ca}^{2+}$ & $\mathrm{Na}^{+}$ & $\mathbf{K}^{+}$ & $\mathrm{SO}_{4}{ }^{2}$ & & & \\
\hline $\mathrm{CP}$ & 0.1 & 0.1 & 0.2 & 0.003 & 3 & 2232 & 8059 & \begin{tabular}{|l|}
9277 \\
\end{tabular} & 13.3 & 64.9 & 2.27 \\
\hline $\mathrm{CH}$ & 0.1 & 0 & 0 & 0 & 814 & 3 & 0.4 & 2 & 12.5 & 8.82 & 2.28 \\
\hline
\end{tabular}

* Conductivity of the solution

** Dissolved oxygen

Table 5.2: Steel elemental composition.

\begin{tabular}{|c|c|}
\hline Element & Weight \% \\
\hline $\mathrm{C}$ & 0.26 \\
$\mathrm{Si}$ & 0.27 \\
$\mathrm{Mn}$ & 1.10 \\
$\mathrm{Cr}$ & 0.05 \\
$\mathrm{Ni}$ & 0.07 \\
$\mathrm{Mo}$ & $<0.01$ \\
$\mathrm{Cu}$ & 0.21 \\
$\mathrm{Al}$ & $<0.005$ \\
$\mathrm{Nb}$ & $<0.01$ \\
$\mathrm{~V}$ & $<0.005$ \\
$\mathrm{Ti}$ & $<0.005$ \\
$\mathrm{~B}$ & $<0.0005$ \\
$\mathrm{P}$ & 0.01 \\
$\mathrm{~S}$ & 0.03 \\
$\mathrm{~W}$ & $<0.01$ \\
$\mathrm{Sn}$ & 0.021 \\
$\mathrm{Co}$ & 0.01 \\
$\mathrm{Zr}$ & $<0.01$ \\
$\mathrm{Fe}$ & Balance \\
\hline
\end{tabular}


The ends of the rebar specimens were isolated to avoid the confounding effects of freshly cut surfaces, and in a way that was not susceptible to crevice corrosion. Initial attempts to eliminate crevice corrosion by coating the ends with epoxy or silicon glue proved unsuccessful. It was found that a Teflon washer held tight to the surface by plastic screws fitted into threaded holes machined into the specimen ends, as shown schematically in Fig. 5.1, eliminated all visible signs of crevice corrosion in this study. The top screw had a hold drilled axially in the middle to accommodate a steel wire (Alloy: ER316L, Diameter: 0.035") that was spot welded to the bottom of the threaded hole and encased in a plastic tube (see Fig. 5.1b).

\subsubsection{Co-axial corrosion cell}

The cylindrical geometry of the rebar was complemented with a co-axial platinum mesh counter electrode that was used to produce a uniformly-distributed electric field around the exposed surface of the rebar specimens. The mesh counter electrode was longer than the rebar specimens as indicated in Fig. 5.2. The reference potential was provided by a saturated calomel electrode (SCE) placed in the port connected to the main body of the cell. The port was linked to the test region by a glass tube that terminated with a thin tip situated between the working and counter electrodes, and $5 \mathrm{~mm}$ away from the surface of the specimens.

\subsection{Experimental Program}

A number of standard electrochemical techniques were used to determine chloride thresholds for the depassivation of the surface oxide films on rebar: free 
corrosion potential (FCP), electrochemical impedance spectroscopy (EIS), linear polarization resistance (LPR) and anodic polarization (AP).
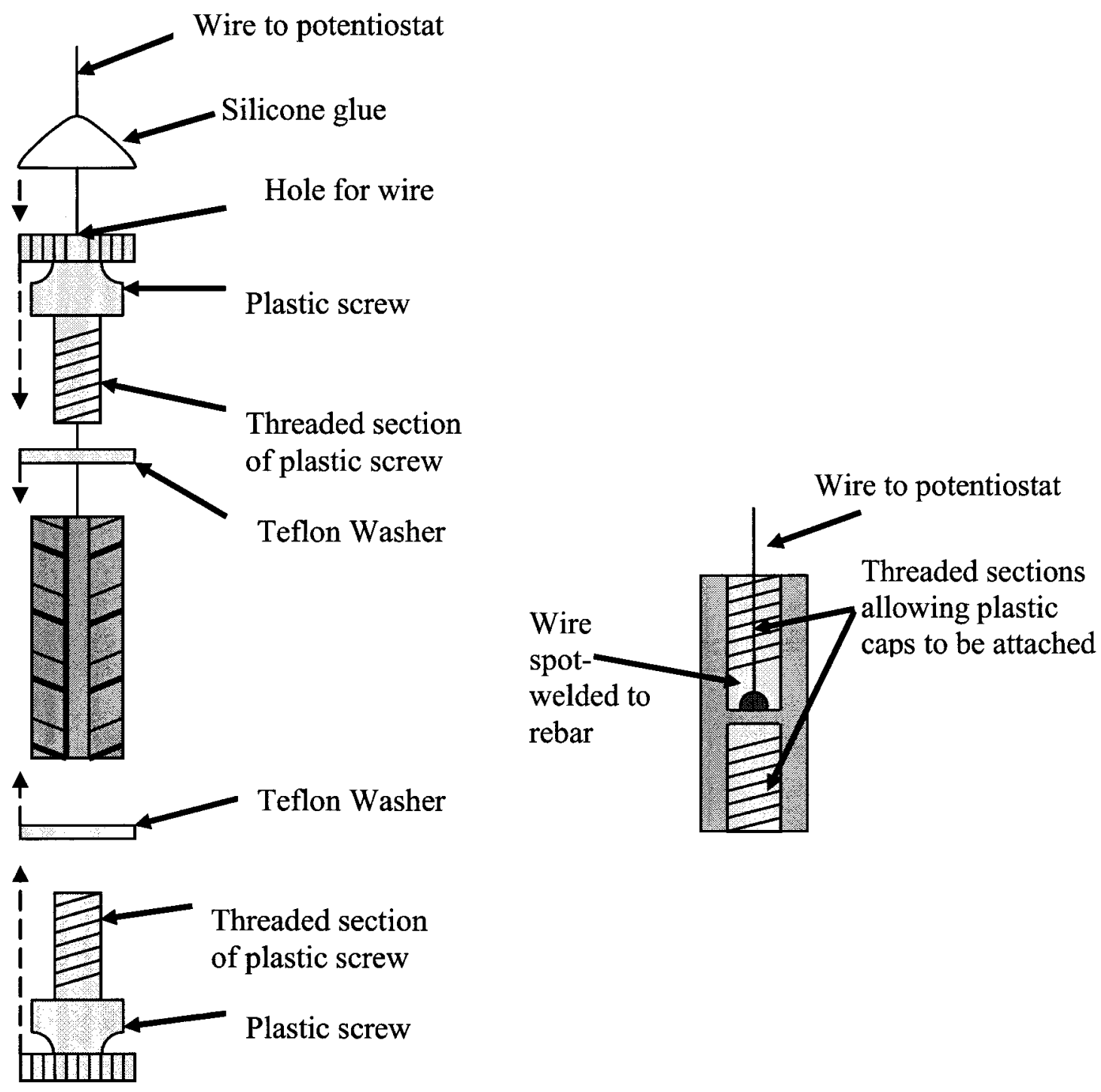

(a)

(b)

Figure 5.1: Schematic illustration of rebar specimen: (a) pull-out diagram; (b) cross section. 


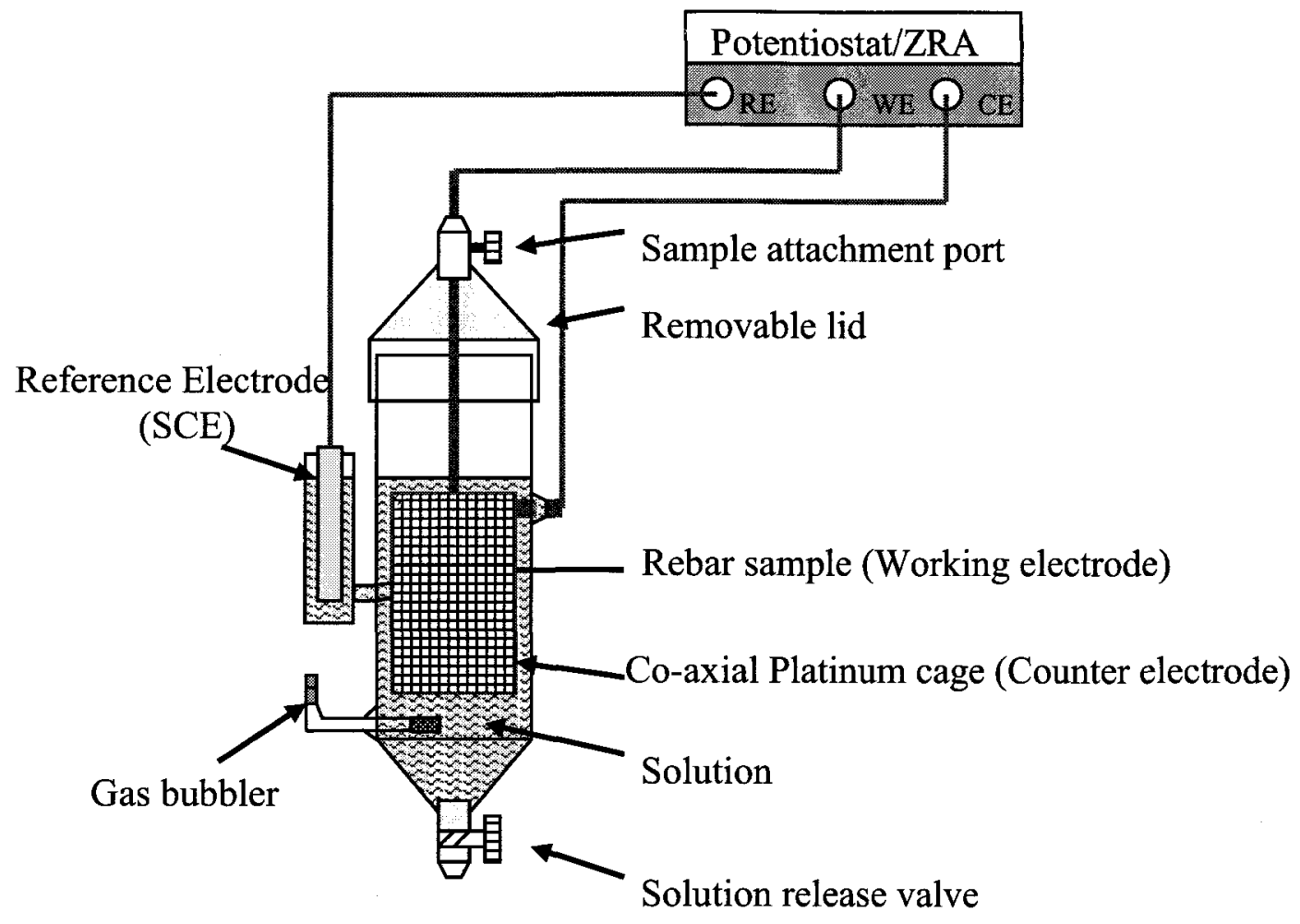

Figure 5.2: Schematic illustration of the co-axial corrosion cell.

The rebar specimens were passivated in two beakers containing the CP solution and another two containing the $\mathrm{CH}$ solution. In each beaker, 24 as-received and 24 turned-and-polished rebar specimens were immersed for two weeks. The immersion time was selected based on the results of previous tests that have shown at least 8 days is required to achieve stable passive films on rebar surfaces (Ghods et al. 2009, Poursaee and Hansson 2007).

After the initial two-week passivation, the chloride concentrations in the solutions were increased at 7-day intervals for two months (1400 hours), as shown in Table 5.3, by adding sodium chloride $(\mathrm{NaCl}$, reagent grade) and stirring. Before each chloride addition, three replicate specimens of each type (as-received and turned-andpolished) were removed and then immersed in the same solution in the co-axial corrosion cell for sequential examination by EIS, LPR, and AP. In parallel, the free corrosion 
potentials of other rebar specimens in the $\mathrm{CP}$ and $\mathrm{CH}$ solutions were monitored continuously. The $\mathrm{pH}$, conductivity and dissolved oxygen of the solutions were measured with a VWR SympHony SP90M5 before and after each chloride addition; there were no significant changes in these parameters during the tests; the averages of these measurements are presented in Table 5.3. Further details of all the tests carried out in the study are presented in the following sections.

Table 5.3: The concentration of total chloride at each 7-day interval of the test for two solutions. These intervals correspond to the incremental shading used in Figs. 5.3-5.5.

\begin{tabular}{|l|l|l|l|l|l|l|l|l|l|}
\cline { 3 - 10 } \multicolumn{1}{c|}{} & \multicolumn{6}{|c|}{ Chloride addition increments (M) } \\
\hline Surface condition & Solution & Start & 1st & 2nd & 3rd & 4th & 5th & 6th & 7th \\
\hline \multirow{2}{*}{ As-received } & $\mathrm{CP}$ & 0 & 0.03 & 0.05 & 0.1 & 0.15 & 0.3 & 0.5 & 1 \\
& $\mathrm{CH}$ & 0 & 0.003 & 0.005 & 0.01 & 0.02 & 0.05 & 0.1 & 0.3 \\
Turned-and-Polished & $\mathrm{CP}$ & 0 & 0.01 & 0.05 & 0.45 & 1.25 & 2 & 3 & -- \\
\hline
\end{tabular}

\subsubsection{Free corrosion potential (FCP)}

A sudden drop of FCP of the rebar in the simulated pore solutions is a simple indication of depassivation. The FCP in the passive state is generally between $-100 \mathrm{mV}$ and $-200 \mathrm{mV} / \mathrm{SCE}$, whereas after complete depassivation, it drops below $-350 \mathrm{mV} / \mathrm{SCE}$. In this study, free corrosion potentials of three replicate as-received rebar specimens in the $\mathrm{CP}$ and $\mathrm{CH}$ solutions, and three replicate turned-and-polished samples in the $\mathrm{CP}$ solution, were recorded at 60-second intervals using a Gamry PC4/300 Potentiostat/Galvanosatat/ZRA and a Gamry Multiplexer. A saturated calomel electrode (Model: Accumet) was used as a reference electrode. 


\subsubsection{Electrochemical impedance spectroscopy (EIS)}

EIS tests were carried out on replicate rebar specimens (i.e., 3 replicate asreceived specimens that were immersed in the $\mathrm{CH}$ solution, 3 as-received from the $\mathrm{CP}$ solution, and 3 turned-and-polished specimens from the CP solution) 7 days after each chloride addition, using a Gamry PC4/300 Potentiostat/Galvanosatat/ZRA. To avoid extensive exposure to air, rebar specimens were transferred within seconds from the breakers to the co-axial corrosion cell, which contained the same solution. The FCP was measured for 60 minutes before the EIS measurement to ensure the stability of the electrochemical system. If the FCP varied more than $10 \mathrm{mV}$ over an hour, the measurement was postponed until the potential stabilized. The EIS measurements were done at the free potential, with an AC voltage of $10 \mathrm{mV} \mathrm{RMS}$, between $10^{5}$ and $10^{-3} \mathrm{~Hz}$ at 10 equally spaced frequencies per decade.

\subsubsection{Linear polarization resistance (LPR)}

After completion of each EIS test, LPR measurements were conducted on each specimen without modification of the experimental arrangement with a Gamry PC4/300 Potentiostat/Galvanosatat/ZRA. The FCP was monitored for 60 minutes before the LPR measurement to ensure the stability of the electrochemical system, and if it varied by more than $10 \mathrm{mV}$, the measurement was postponed until the potential stabilized. The LPR measurements started $15 \mathrm{mV}$ below, and ended $15 \mathrm{mV}$ above, the FCP of each specimen. The scan rate was $0.166 \mathrm{mV} / \mathrm{s}$, as prescribed in ASTM G5 (2004). Slower scan rates did not affect the results, which is in accord with earlier work (Bird et al. 1988, Leckie 1970). 


\subsubsection{Anodic polarization (AP)}

After the LPR measurements, anodic polarization curves of each specimen were obtained without modification of the experimental arrangement using a Gamry PC4/300 Potentiostat/Galvanostat/ZRA. As for the EIS and LPR measurements, the FCP was monitored for 60 minutes before the EIS test to ensure stability. The AP measurements started at the FCP for each specimen. The potentials were raised to $600 \mathrm{mV}$ (SCE) in the forward (anodic) direction, and then lowered to $-100 \mathrm{mV}$ below the FCP in the backward (cathodic) direction. The scan rate in both directions was $0.166 \mathrm{mV} / \mathrm{s}$, which is as prescribed by ASTM G5 (2004). The choice of scan rate is a compromise between accuracy and the time to complete the measurement. It was found in this study that slower scan rates did not change the results, which is consistent with other studies (Leckie 1970, Bird et al.1988, Li and Sagues 2002).

\subsection{Results}

In this section, the results of four electrochemical techniques used to determine chloride thresholds are described. In each case, negligible changes were observed in the electrochemical responses up to a certain chloride concentration. Upon further addition of chloride (as per Table 5.3) large changes were observed followed by visible pits on the rebar surfaces. These changes define chloride thresholds for rebar: reported chloride thresholds in this section are thus upper limits for the chloride concentrations associated with the large change. 


\subsubsection{FCP results}

Figures 5.3 and 5.4 present the FCP measurements, as averages of three replicate as-received specimens, in the $\mathrm{CP}$ and $\mathrm{CH}$ solutions, respectively. Figure 5.5 presents the FCP measurements, as averages of three replicate turned-and-polished specimens, in the CP solution. In all instances, the FCP increases gradually during the early stages of immersion and becomes relatively constant after approximately 10 days (i.e., 240 hours). This is consistent with earlier studies that showed the passive film on rebar stabilizes after approximately 10 days (Poursaee and Hansson 2007, Ghods et al. 2009).

Once the passive films stabilized, the average FCP of the as-received rebar was $169 \mathrm{mV} / \mathrm{SCE}$ (see Fig. 5.3) in the CP solution, and $-156 \mathrm{mV} / \mathrm{SCE}$ (see Fig. 5.4) in the $\mathrm{CH}$ solution. The average FCP of the turned-and-polished rebar specimens in the CP solution after stabilization was $-220 \mathrm{mV} / \mathrm{SCE}$ (Fig. 5.5), which is significantly lower than the average FCP for the as-received specimens in the same solution (i.e.,- $169 \mathrm{mV} / \mathrm{SCE}$ ).

Figures 5.3 and 5.4 show that the FCP remains relatively constant with increasing chloride concentration until a threshold, $[\mathrm{Cl}]_{\mathrm{T}}$, is reached, and then the FCP decreases sharply: the threshold chloride concentrations are $0.1 \mathrm{M}$ and $0.05 \mathrm{M}$ for the $\mathrm{CP}$ and the $\mathrm{CH}$ solutions, respectively. 


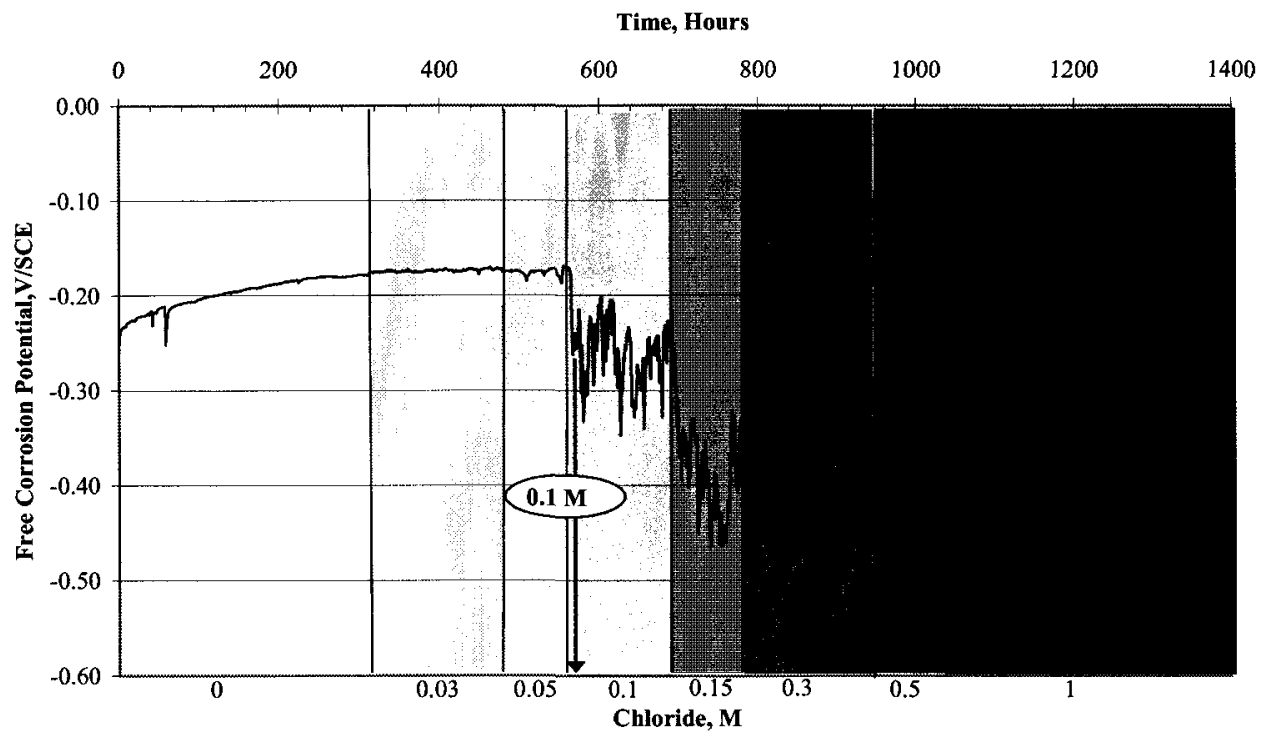

Figure 5.3: Average free corrosion potential of three as-received rebars in the $C P$ solution. Shading in the figure corresponds to the increments of chloride addition in Table 5.3.

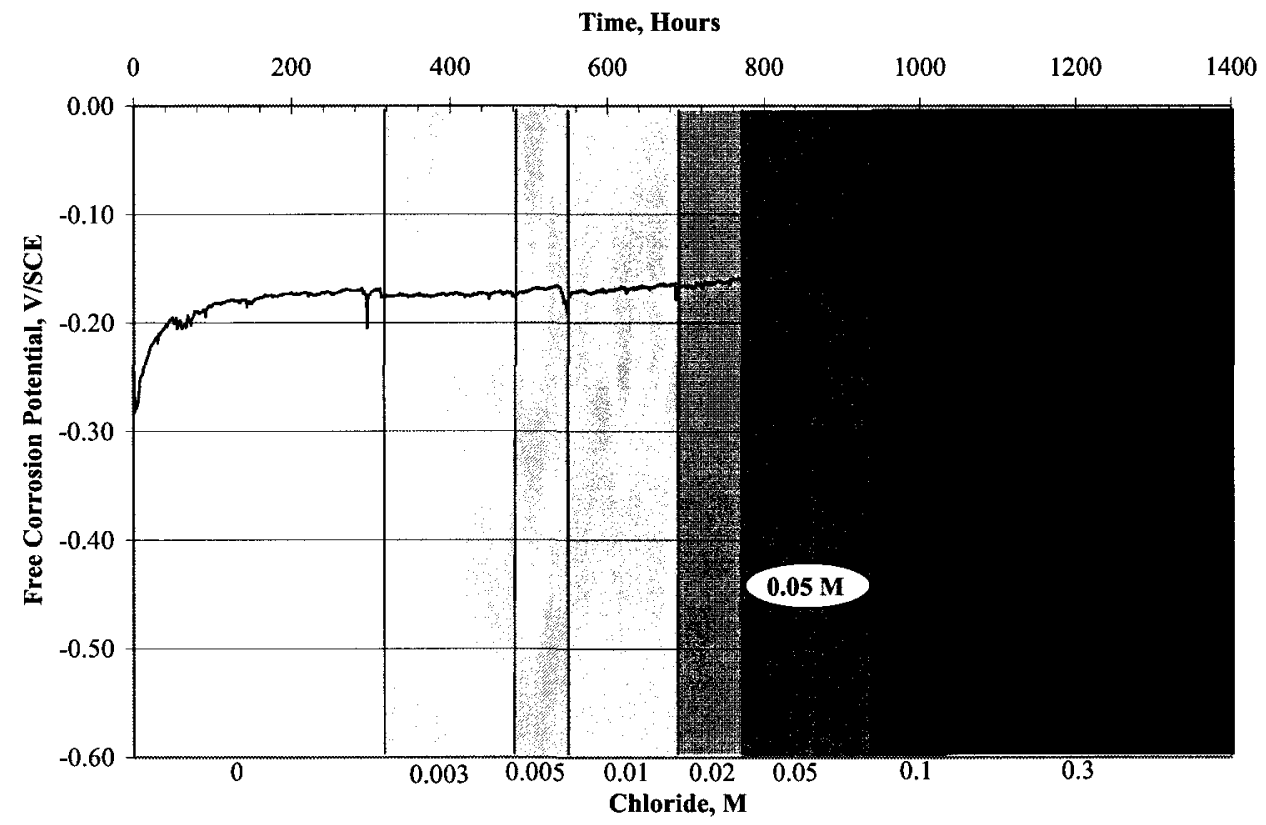

Figure 5.4: Average free corrosion potential of three as-received rebars in the $\mathrm{CH}$ solution. Shading in the figure corresponds to the increments of chloride addition in Table 5.3. 


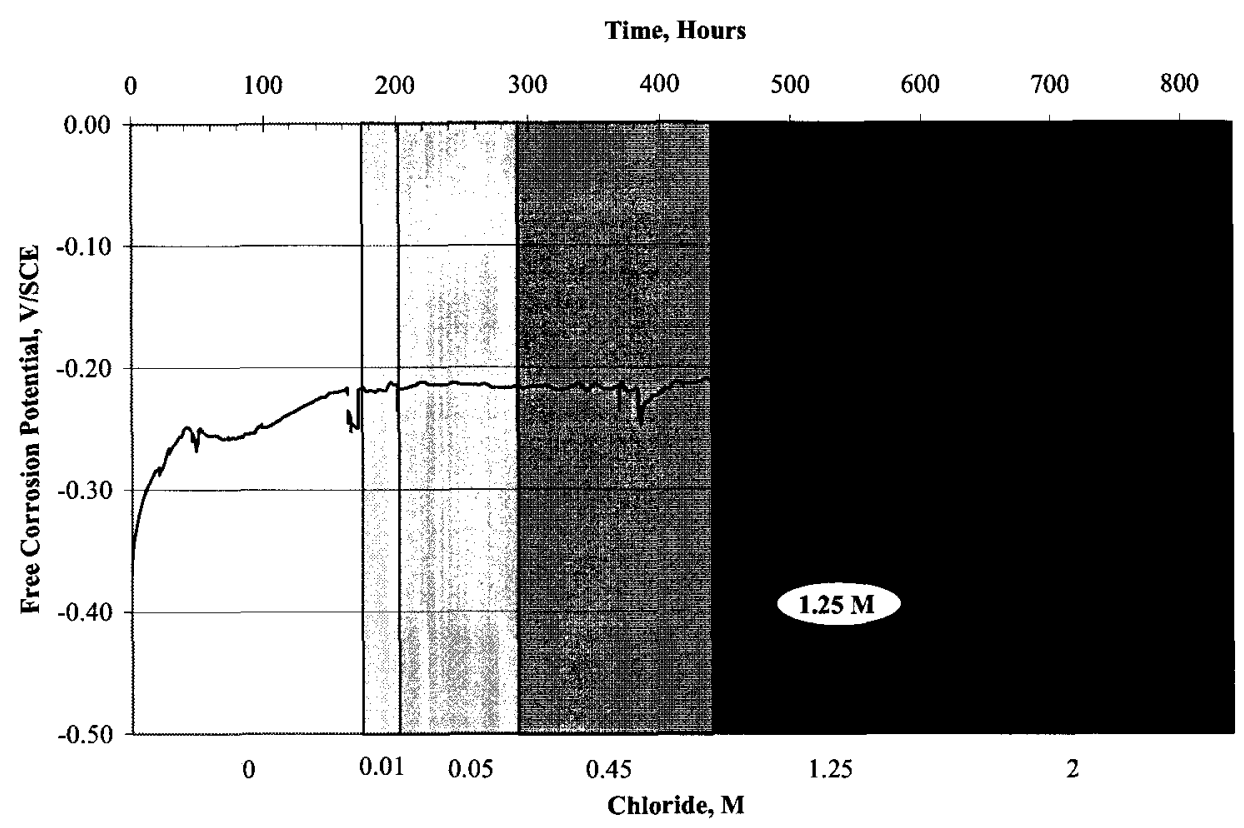

Figure 5.5: Average free corrosion potential of three turned-and-polished rebars in CP solution. Shading in the figure corresponds to the increments of chloride addition in Table 5.3.

The FCP of the turned-and-polished specimens in the CP solution as a function of chloride concentration is shown in Fig. 5.5. In this case, the threshold occurs at higher chloride concentrations, and the FCP does not fluctuate as much for chloride concentrations above the threshold when compared with the corresponding results for the as-received specimens shown in Fig. 5.3. The chloride threshold of the polished rebar specimens is approximately $1.25 \mathrm{M}$, which is significantly higher than the $0.1 \mathrm{M}$ threshold observed for the as-received specimens in the same solution (Fig. 5.3). This result is consistent with earlier work that has shown polishing increases the chloride threshold of rebar in concrete (Mammoliti et al. 1996, Li and Sagues 2001, Mahallati and Saremi 2006). 


\subsubsection{LPR results}

Polarization resistance was determined from the slope of the polarization curve (voltage versus current) for applied voltages of $\pm 15 \mathrm{mV}$ relative to the FCP. The polarization resistances of the rebar specimens measured with LPR are shown in Fig. 5.6. The chloride thresholds of as-received rebar in the $\mathrm{CP}$ and $\mathrm{CH}$ solutions are $0.15 \mathrm{M}$ and $0.05 \mathrm{M}$, respectively. The threshold for the turned-and-polished specimens in the CP solution is $1.25 \mathrm{M}$.

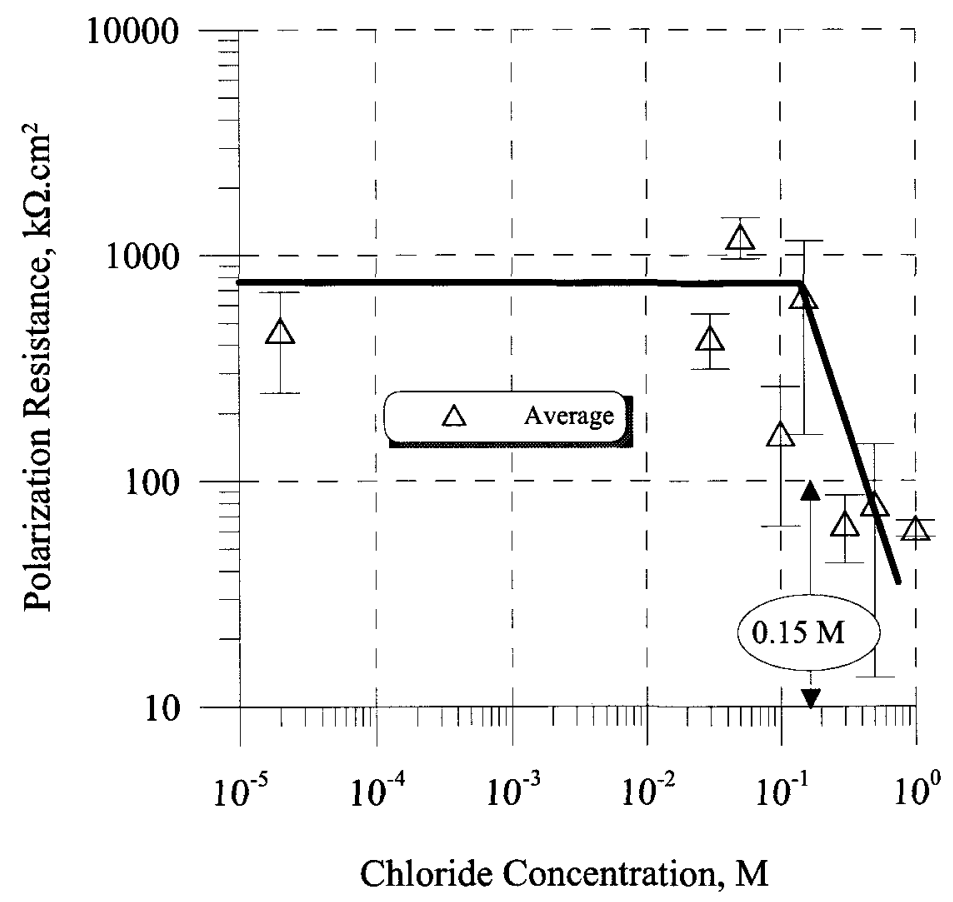

(a) As-received in CP solution 


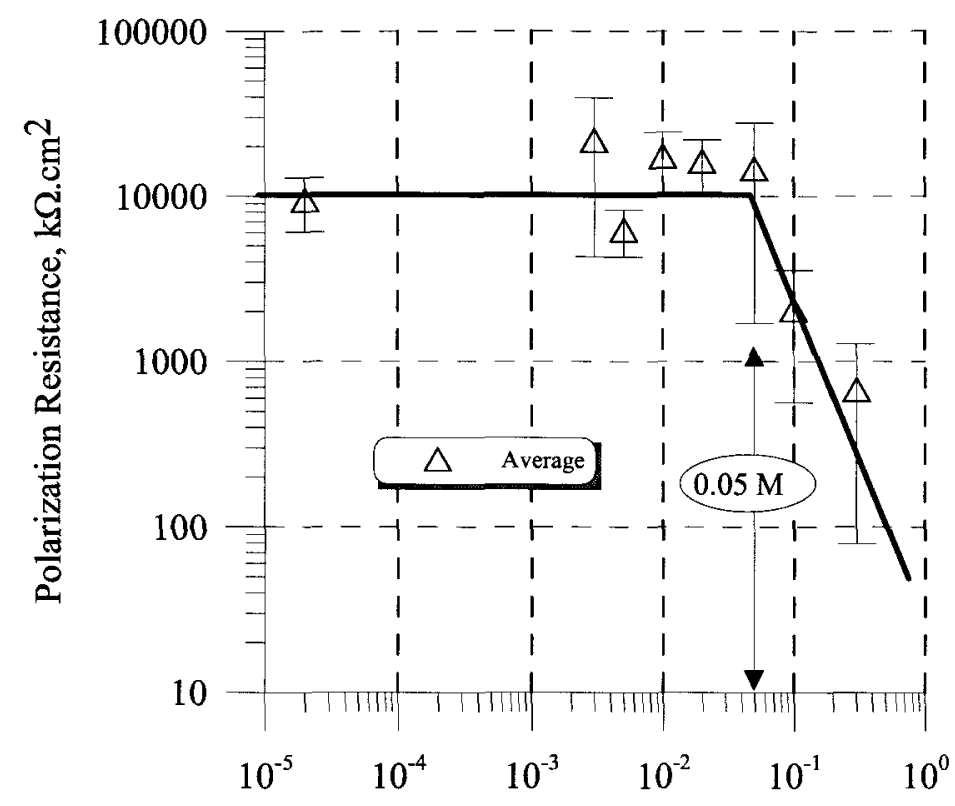

Chloride Concentration, $\mathrm{M}$

(b) As-received in $\mathrm{CH}$ solution

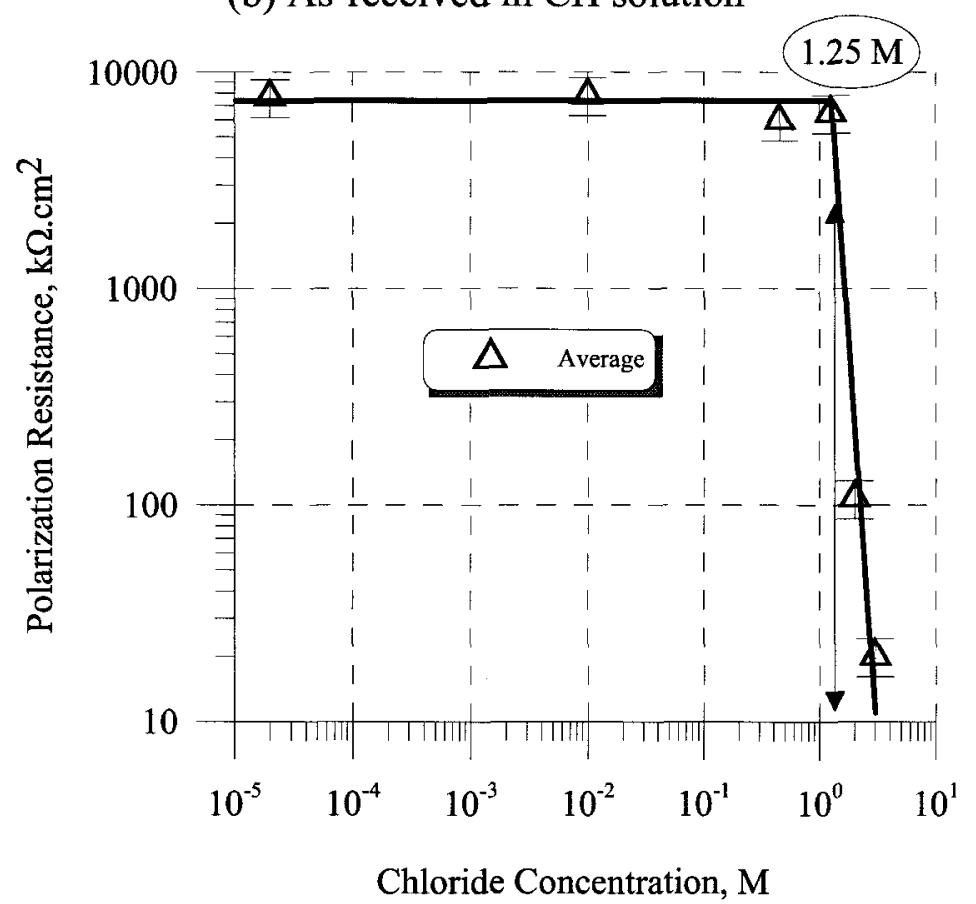

(c) Turned-and-polished rebar in CP solution

Figure 5.6: Polarization resistance of rebar samples obtained by LPR for (a) as-received in CP solution; (b) as-received in CH solution; (c) turned-and-polished in CP solution. Note that the data for as-received rebar are more scattered about the trend lines when compared with the data from the turned-and-polished specimens. 


\subsubsection{EIS results}

Figure 5.7 shows distinctly different Bode and Nyquist plots for a typical asreceived specimen in the $\mathrm{CH}$ solution containing chloride at concentrations below (Fig. 5.7(a)), and above (Fig. 5.7(b)), the threshold value. Below the chloride threshold, the impedance $(Z)$ increases with decreasing frequency, as shown in the Bode plot on the left of Fig. 5.7(a). Above the chloride threshold the impedance is relatively constant at low frequencies in the Bode plot of Fig. 5.7(b), and the Nyquist plot changes from 'linear' in Fig. 5.7(a) to 'semicircular' in Fig. 5.7(b). These EIS spectra are typical of passivated and depassivated rebar ( $\mathrm{Li}$ and Sagues 2001).

Modelling of the EIS results to equivalent electrical circuits was used to reduce the data shown in Fig. 5.7. Figure 5.8 shows the equivalent circuit model that was used (Berke and Hicks 1990, Feliu et al. 1998, Li and Sagues 2001, Nmai 2004, Trabanelli et al. 2005, Choi et al. 2006). In this model, $R_{s}$ is the solution resistance $(\sim 100 \Omega), R_{p}$ is the polarization resistance and $C_{C P E}$ is a constant phase element. Using Gamry Echem Analyst V5.3 software, the model parameters were determined by nonlinear least-squares fitting of the real and imaginary components of the impedance to the functional forms derived from the equivalent circuit model.

The ability of the model to reproduce the measured spectra was evaluated with the goodness-of-fit, $E^{2}$, defined in the Gamry Echem Analyst software (V5.3) (Tait et al. 1993, Siegel and Castellani 1988).

$E^{2}=\frac{\sum_{i=1}^{n}\left[\frac{\left(y_{i}-f\left(v_{i}\right)\right)^{2}}{y_{i}^{2}}\right]}{n-m}$ Eq.5.1 
where $y_{i}$ is the measured impedance at the $i^{\text {th }}$ frequency, $v_{i} ; f\left(v_{i}\right)$ is the impedance calculated from the model; $n$ is the number of data points; and $m$ is the number of adjustable parameters in the model. The standard-deviation errors in the measured impedances are assumed to be constant fractions of the measurements, i.e., $\sigma_{i}=\varepsilon \cdot y_{i}$ Hence, the goodness-of-fit, $E^{2}$, can be related to the reduced chi-square error, $\chi^{2}$ :

$$
E^{2}=\varepsilon^{2} \cdot \chi^{2}
$$

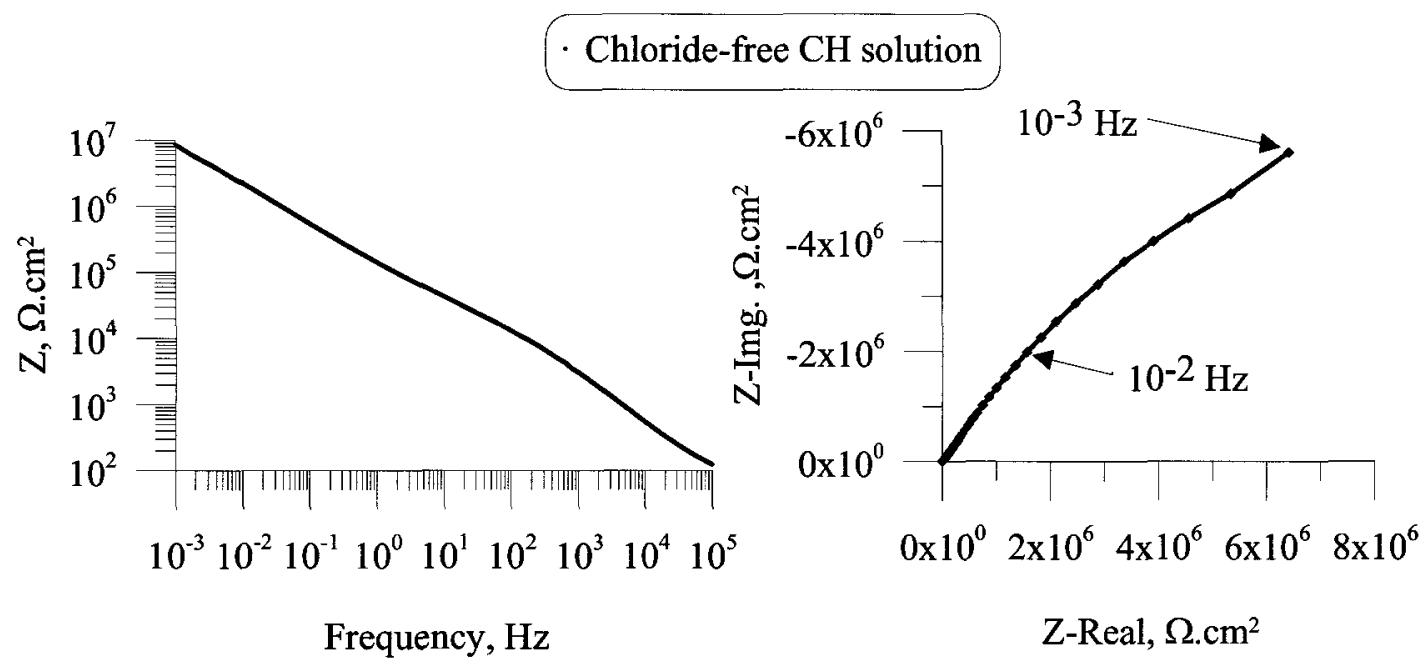

(a)

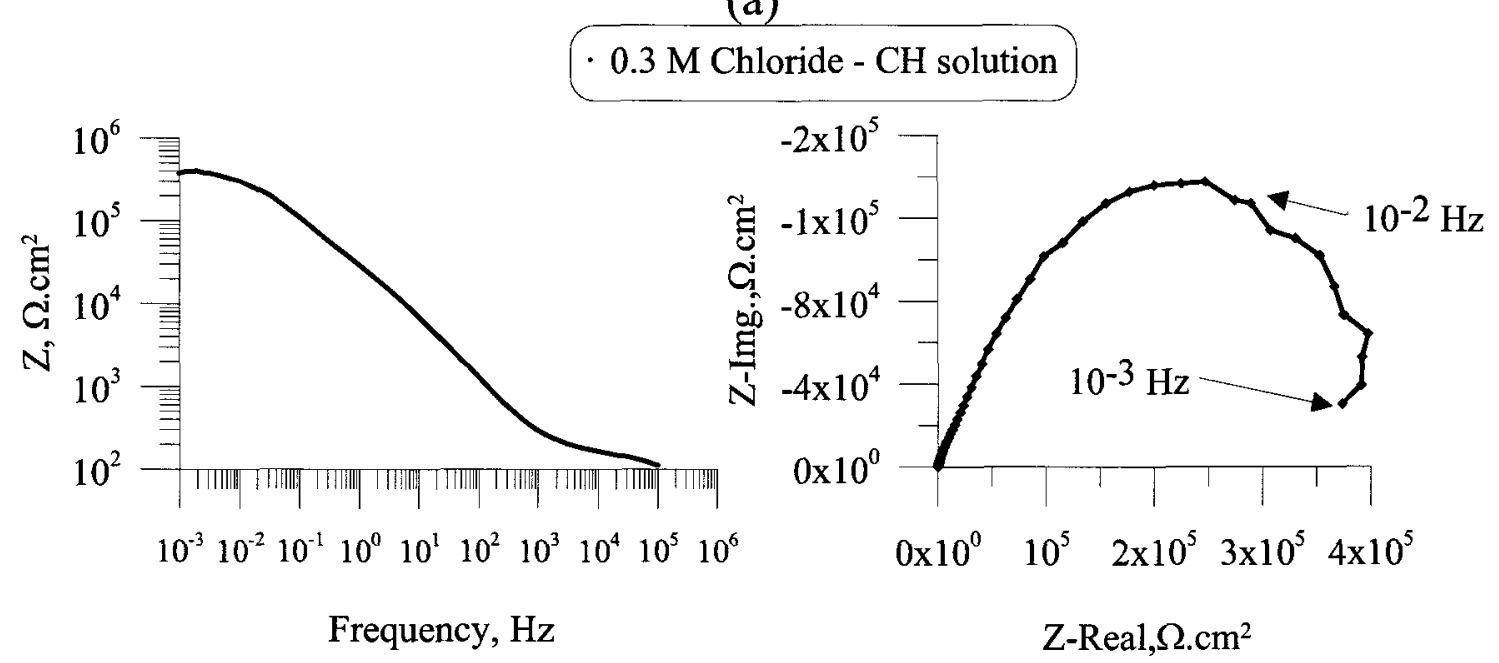

(b)

Figure 5.7: Typical EIS results obtained for an as-received rebar sample in the CH solution with chloride concentrations (a) below and (b) above the chloride threshold. 


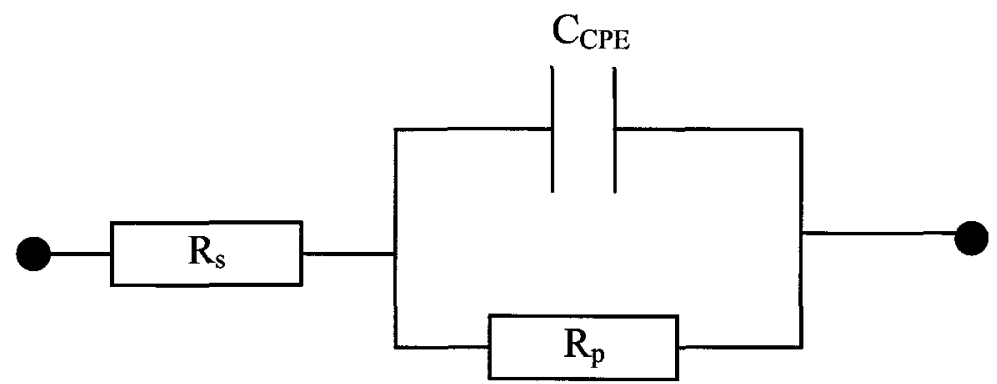

Figure 5.8: Equivalent electrical circuit (Randles circuit) used to model the EIS spectra.

Repeated EIS measurements on the same specimen showed reproducibility approaching $6 \%$, however, measurements on triplicate specimens varied up to $15 \%$; hence in this study, $\varepsilon$ is assumed to be 0.15 . For each spectrum there are 80 data points and 4 model parameters, therefore 76 degrees of freedom. Typical $\chi^{2}$ values were 1.3 and thus $P$ values were less than 0.05 .

Determined polarization resistances of as-received rebar as a function of chloride concentration are shown in Fig. 5.9. The polarization resistances in this figure are relatively constant as chloride concentration is increased until a threshold value is reached, after which the resistances drop significantly. The thresholds for as-received rebar are $0.15 \mathrm{M}$ and $0.05 \mathrm{M}$ in the $\mathrm{CP}$ and the $\mathrm{CH}$ solutions, respectively. The polarization resistance of turned-and-polished rebar in the CP solution are shown in Fig. 5.10. The chloride threshold is $1.25 \mathrm{M}$. 


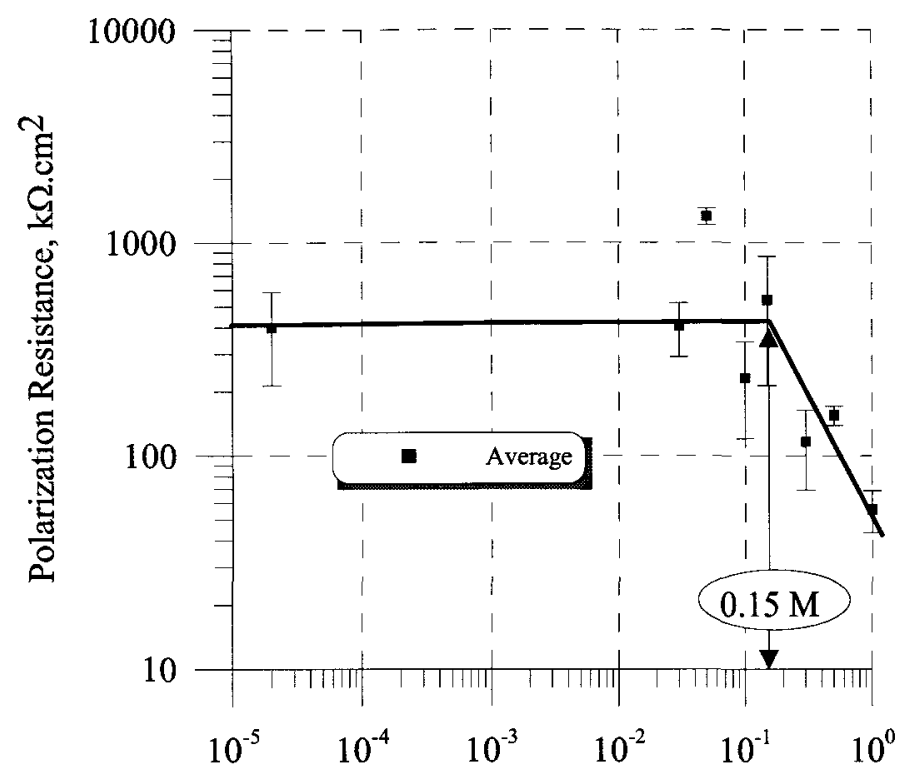

Chloride Concentration, $\mathrm{M}$

(a) As-received in CP solution

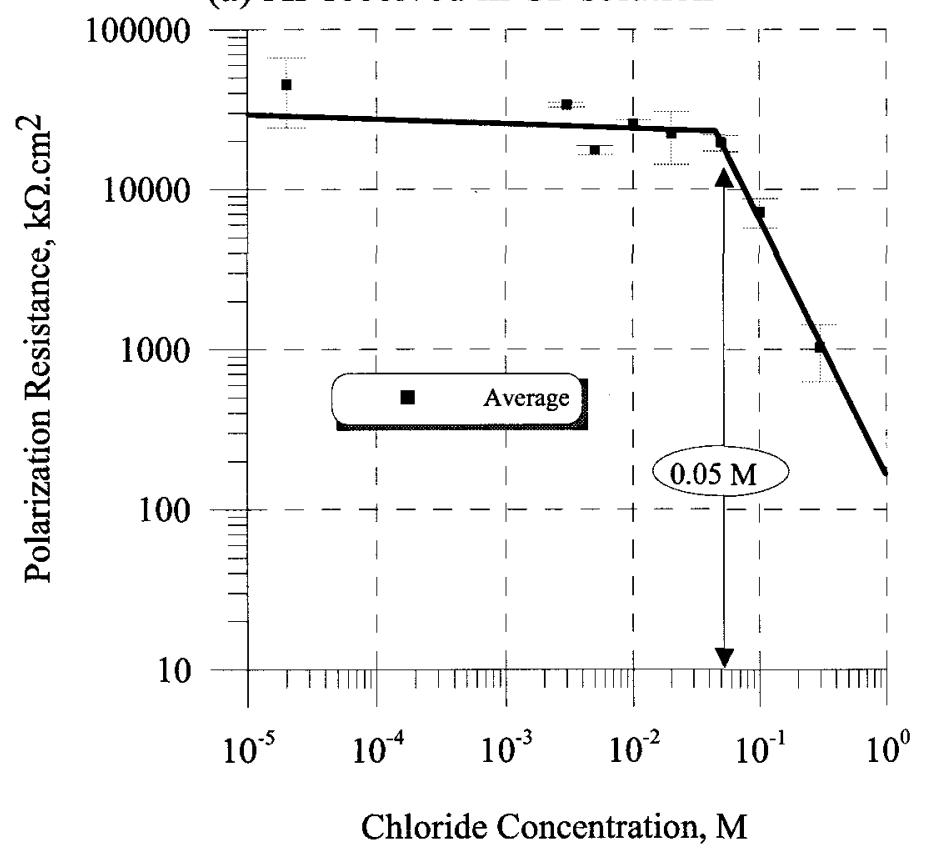

(b) As-received in $\mathrm{CH}$ solution

Figure 5.9: Polarization resistance $\left(R_{p}\right)$ determined from EIS measurements and equivalent circuit modelling for as-received rebar samples in (a) CP solution; (b) $\mathrm{CH}$ solution. Note that the data for as-received rebar are more scattered about the trend lines when compared with the data from the turned-and-polished specimens as illustrated in Fig. 5.10 . 


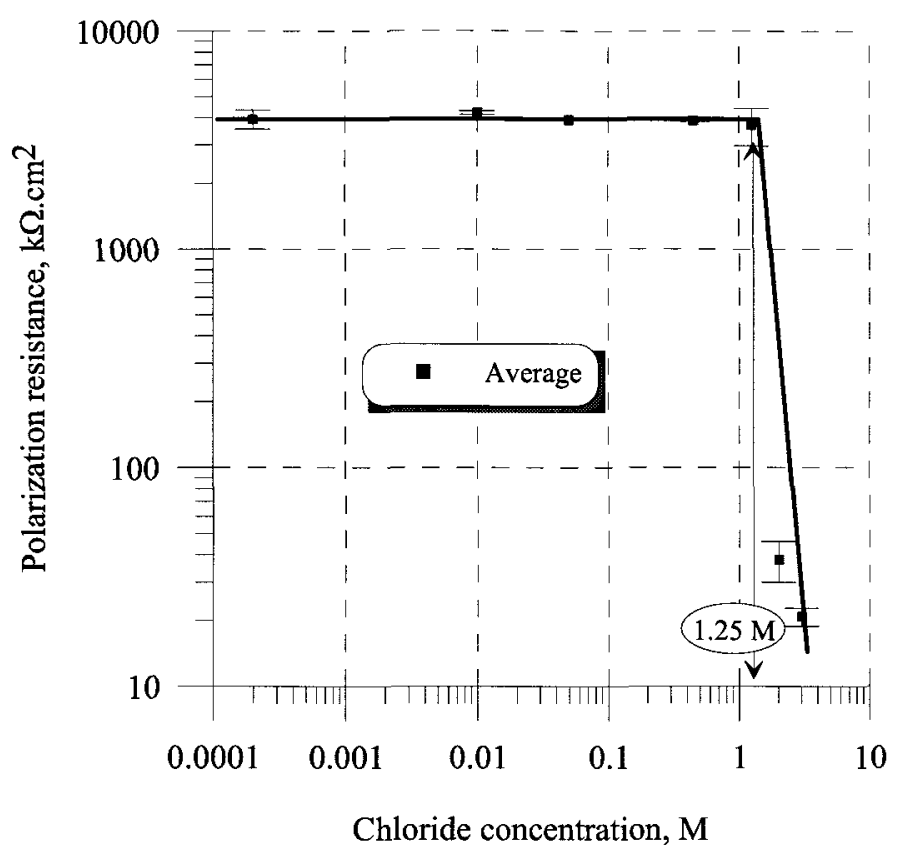

Figure 5.10: Polarization resistance $\left(R_{p}\right)$ determined from EIS measurements and equivalent circuit modelling for turned-and-polished rebar samples in CP solution.

The magnitudes of the polarization resistances obtained from EIS are generally higher than those from LPR measurements; however, a strong correlation exists between the two. Thus, both measurements are able to capture the chloride threshold. The relationship between the polarization resistances measured by EIS $\left(R_{p}{ }^{E I S}\right)$ and by LPR $\left(R_{p}{ }^{L P R}\right)$ can be expressed as:

$\ln \left(R_{p}^{L P R}\right)=0.90 \times \ln \left(R_{p}^{E I S}\right)+0.31$

with an $R^{2}$ value of 0.97 .

\subsubsection{AP results}

Anodic polarization curves taken before chloride addition, reported as the average of three replicates, are shown in Fig. 5.11(a) for the $\mathrm{CP}$ and $\mathrm{CH}$ solutions. In Fig. 5.11, FCP is the starting potential for the scan, and $i_{p}$ is the passive current density 
corresponding to the inflection point of the anodic polarization curve for the forward scan. The repassivation potential $\left(E_{r p}\right)$ is the potential in the backward scan when the applied potential is removed. The forward and backward scan directions are identified by arrows. As the chloride concentration in the solution is increased, a pitting potential $\left(E_{p}\right)$ can be defined in the forward scan as the potential corresponding to the sharp increase in current density that is accompanied by the formation of pits on the rebar surface (see Fig. 5.11(b)).

If the potential is not high enough during the forward scan to cause pitting, then the repassivation potential $\left(E_{r p}\right)$ is higher than the initial FCP (Fig. 5.11(a)), which is generally explained to be because of the growth of a thicker, more protective, oxide during the anodic scan. On the other hand, if pitting does occur, then $E_{r p}$ is lower than the initial FCP as shown in Fig. 5.11(b). In this case, pitting results in local destruction of the oxide so that it is less protective when the applied anodic potential is removed. Thus, the occurrence of pitting can be determined by comparing the magnitude of $E_{r p}$ with initial FCP.

For the turned-and-polished specimens in the CP solution, pitting could not be observed until large amounts of chloride were added; for these specimens, the average pitting potential was $-512 \mathrm{mV}$, which occurred after $1.25 \mathrm{M}$ chloride addition. The average FCP and the passive current density $\left(i_{p}\right)$ for as-received rebar specimens after the formation of stable passive films were $-169 \mathrm{mV}$ and $1 \mu \mathrm{A} / \mathrm{cm}^{2}$ for the CP solution, and $156 \mathrm{mV}$ and $0.02 \mu \mathrm{A} / \mathrm{cm}^{2}$ for the $\mathrm{CH}$ solution (Figs 5.3 and 5.4). The pitting potentials identified in Fig. 5.11(b) decrease as the chloride concentration increases. In addition, the pitting potential of rebar in $\mathrm{CP}$ is higher than in $\mathrm{CH}$. 


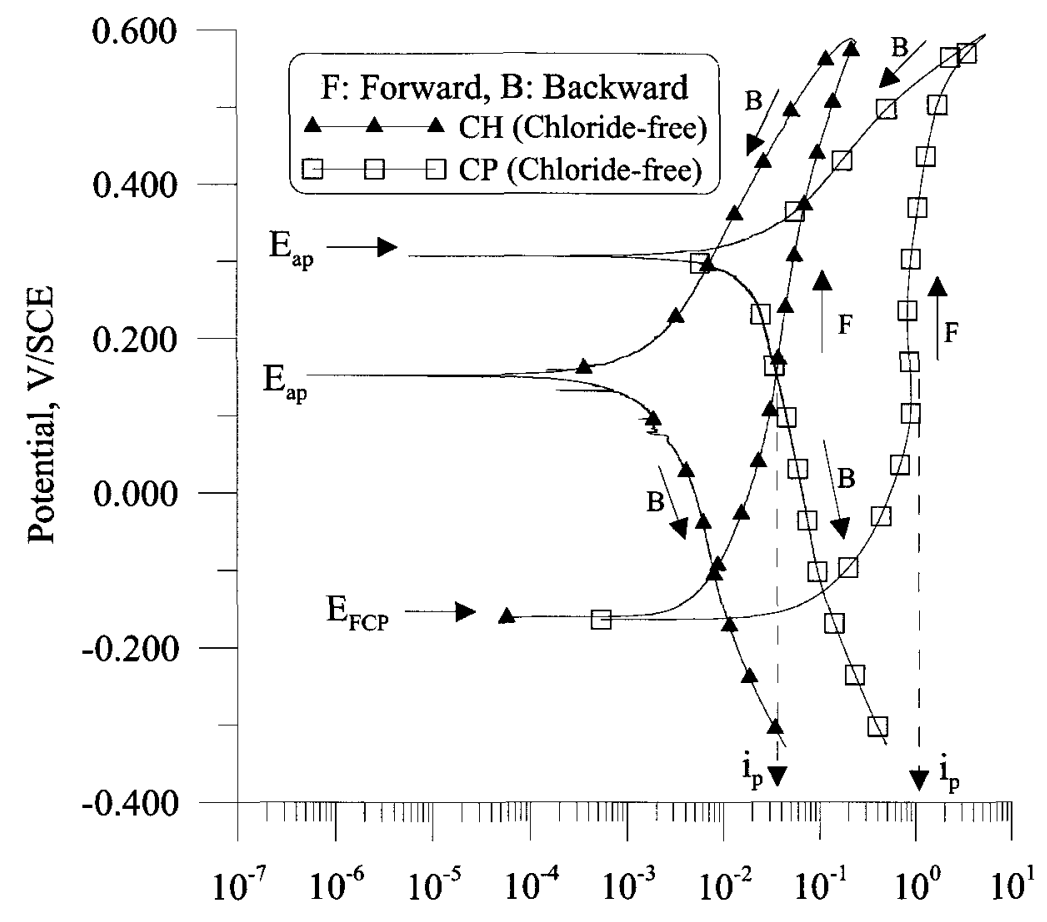

Current Density, $\mu \mathrm{A} / \mathrm{cm}^{2}$

(a)

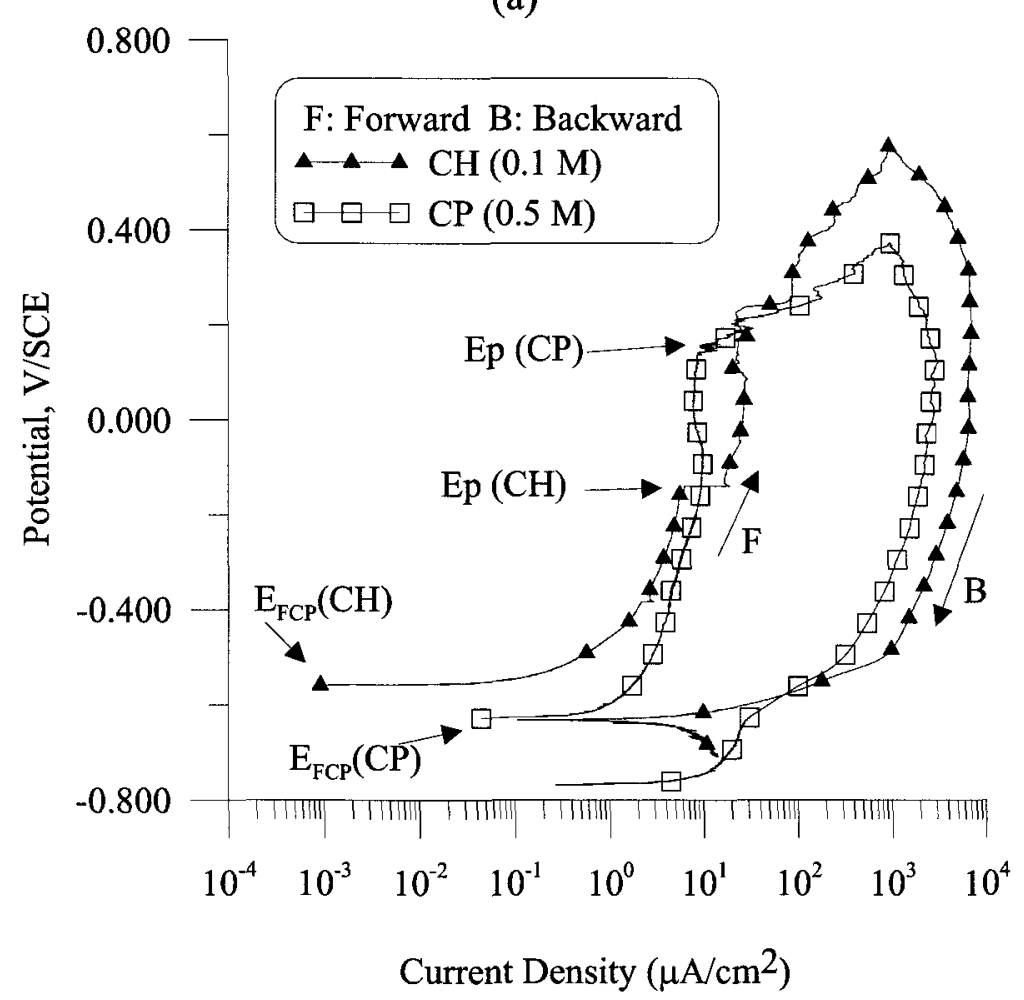

(b)

Figure 5.11: Anodic polarization curves of as-received rebar in $\mathrm{CH}$ and $\mathrm{CP}$ solutions:

(a) without chloride; (b) with chloride. 
A relationship between pitting potential and chloride concentration was obtained by fitting to a logarithmic function of chloride concentration (Freiman and Kolotyrkin 1969, Janik-Czachor 1981, Heusler and Fischer 1976, Strehblow and Titze 1977, Alvarez and Galvele 1984, Bird et al. 1988, Ergun and Turan 1991, Li \& Sagues 2002). The results shown in Fig. 5.12 give a reasonable correlation with $R^{2}$ values of 0.92 and 0.83 for the $\mathrm{CH}$ and $\mathrm{CP}$ solutions, respectively.

Assuming that pitting occurs once the FCP exceeds the pitting potential, the pitting threshold, $[\mathrm{Cl}]_{\mathrm{AP}}$, can be calculated using the equations shown in Fig. 5.12 as follows:

$$
\begin{array}{lll}
-0.156=-0.51 \times \log \left([C l]_{A P}\right)-0.44 \Rightarrow[C l]_{A P}=0.28 \mathrm{M} & \text { (CH solution) } & E q .5 .4 \\
-0.169=-0.58 \times \log \left([C l]_{A P}\right)-0.18 \Rightarrow[C l]_{A P}=0.96 \mathrm{M} & \text { (CP solution) } & \text { Eq. 5.5 }
\end{array}
$$

Note that the pitting thresholds are significantly higher than the threshold concentrations for depassivation determined by the other methods described previously.

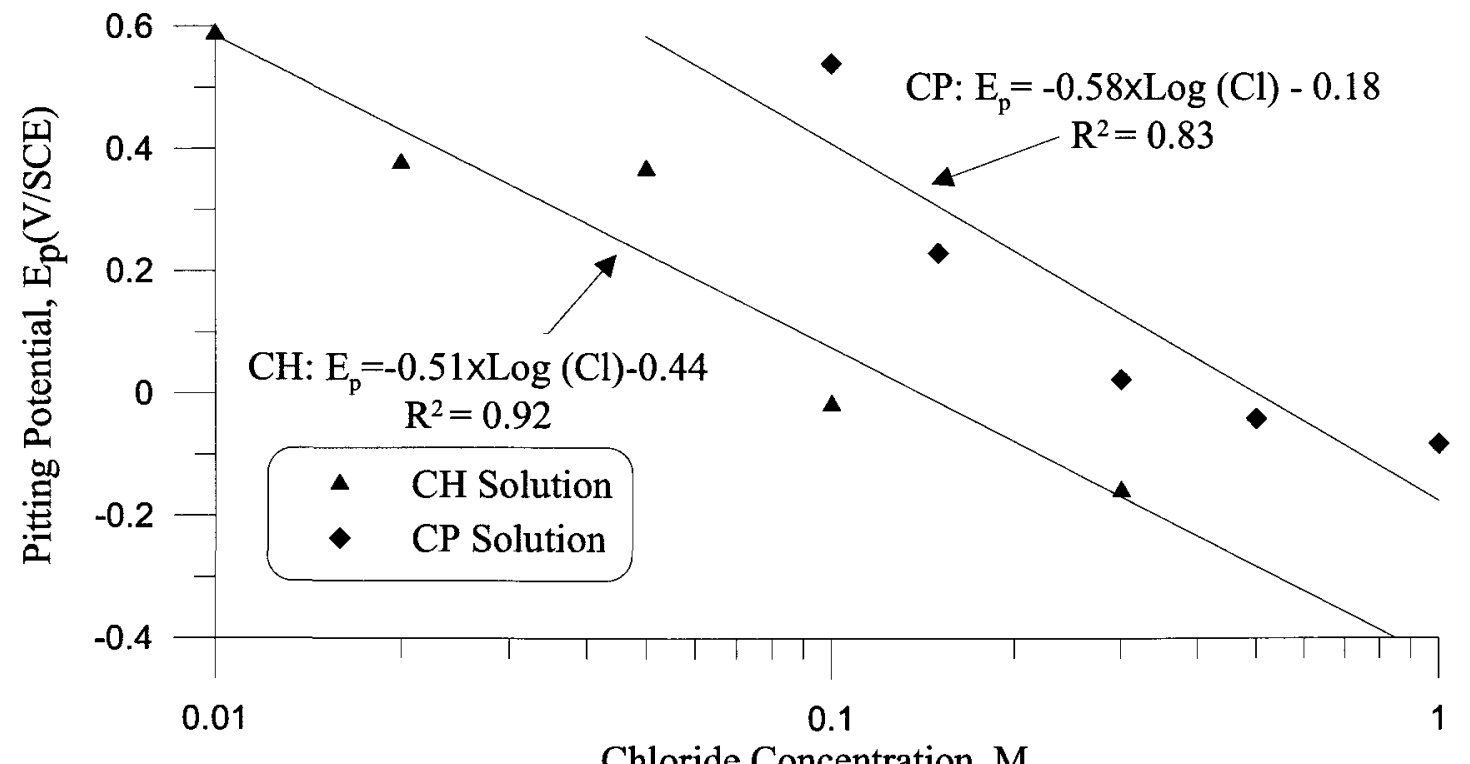

Chloride Concentration, $\mathrm{M}$

Figure 5.12: Pitting potential of as-received rebar in the $C H$ and $C P$ solution as function of chloride. 


\subsection{Discussion}

5.5.1 Chloride threshold determination technique

The chloride thresholds for depassivation obtained by the different electrochemical techniques used in this study are presented in Table 5.4. The thresholds determined by FCP, EIS and LPR are similar and much smaller than the chloride thresholds for pitting determined with AP. Similar observations have been reported by $\mathrm{Li}$ and Sagues (2002). The AP thresholds are higher because the damaging effect of chloride is offset by the formation and repair of the oxide by the applied anodic potential. Because applied potentials are not routinely used in service, the threshold values obtained from the FCP, EIS and LPR techniques should provide more representative chloride thresholds for rebar in service conditions.

Table 5.4: Chloride thresholds obtained by different electrochemical techniques.

\begin{tabular}{|l|l|l|c|c|l|}
\cline { 3 - 6 } \multicolumn{2}{c|}{} & \multicolumn{4}{c|}{ Chloride threshold, [Cl] (M) } \\
\hline $\begin{array}{l}\text { Rebar Surface } \\
\text { Preparation }\end{array}$ & $\begin{array}{c}\text { Solution } \\
\text { (Table 5.2) }\end{array}$ & FCP & EIS & LPR & \multicolumn{1}{|c|}{ AP* $^{*}$} \\
\hline As-received & $\mathrm{CP}$ & $0.05-0.1$ & $0.1-0.15$ & $0.1-0.15$ & 0.96 \\
As-received & $\mathrm{CH}$ & $0.02-0.05$ & $0.02-0.05$ & $0.02-0.05$ & 0.28 \\
$\begin{array}{l}\text { Turned-and- } \\
\text { Polished }\end{array}$ & $\mathrm{CP}$ & $0.45-1.25$ & $0.45-1.25$ & $0.45-1.25$ & $\mathrm{~N} / \mathrm{A}$ \\
\hline
\end{tabular}

$\mathrm{FCP}=$ free corrosion potential; EIS = electrochemical impedance spectroscopy; LPR $=$ linear polarization resistance; $\mathrm{AP}=$ anodic polarization

* Calculated from the AP results as shown in Section 5.4.4 $\left([\mathrm{Cl}]_{\mathrm{AP}}\right)$ 


\subsubsection{The effect of solution}

The chloride threshold values for as-received rebar specimens in the CP solution were consistently 2-3 times higher than those in the $\mathrm{CH}$ solution. This is consistent with earlier work showing chloride thresholds increase as the $\mathrm{pH}$ of the solution increases (Gouda 1970, Mammoliti et al. 1996, Li and Sagues 2001). In this study, 2-3 times higher thresholds were observed in $\mathrm{CP}(\mathrm{pH} 13.3)$ compared with $\mathrm{CH}(\mathrm{pH} 12.5)$, but, this increase is lower than expected: larger changes in thresholds (up to 10 times) have been reported in previous studies for similar pH variations (Hausmann 1967, Breit 1998, Li and Sagues 2001). However, in these studies, the simulated pore solutions did not contain sulfate ions. Chloride thresholds tend to be lower in solutions with sulfate ions (Bird et al. 1988, Abd El Aal et al. 2009), and the passive films grown in the presence of sulfate ions have different characteristics (Gui \& Devine 1994, Ghods et al. 2009). The current study re-emphasizes the importance of the composition of the simulated pore solutions in electrochemical studies of rebar corrosion.

\subsubsection{The effect of rebar surface condition}

The chloride thresholds measured by FCP, EIS, and LPR are all lower for asreceived rebar when compared with turned-and-polished rebar. Li and Sagues (2001) reported similar results for rebar specimens that had been sandblasted to remove the millscale. Figure 5.13 shows pitting potentials determined in the current study, and those from previous work, in terms of the $\mathrm{Cl}^{-} / \mathrm{OH}^{-}$concentration ratio. Generally, modifying the rebar surfaces leads to higher chloride thresholds. The implication is that if the goal is to determine thresholds that will be representative for service-life-based design and end-of-life calculations, then any surface modification - turning, polishing, sandblasting 
- should be avoided. The significance of surface condition can be further demonstrated with reference to Figs. 5.6, 5.9 and 5.10. Compared with the turned-and-polished rebar, the as-received rebar showed greater variability in polarization resistance. For example, in the figures the data for as-received rebar are more scattered about the trend lines when compared with the data from the turned-and-polished specimens. Similar behaviour can be observed in the FCP above the threshold for the specimens in the CP solution; in Figs. 5.3 and 5.5 the FCP for the as-received specimens fluctuates much more than it does for the turned-and-polished specimens. The inference is that the turned-and-polished surfaces are uniformly the same everywhere, whereas the as-received surfaces are locally much more diverse and complex.

The importance of the as-received rebar surface suggests an explanation for the wide range and uncertainty of reported in-service chloride thresholds measured in similar concrete types under the same environmental conditions (Glass and Buenfeld 1997, Alonso et al. 2000). The variability associated with the chloride thresholds may be attributed to the variability in the surface characteristics of the steel resulting from the variability in fabrication and production; there is usually no strict quality control for the mill-scale on rebar. The results of studies detailed in Chapter 6 implicate the mill-scale as part of the explanation for the lower thresholds for as-received rebar. 


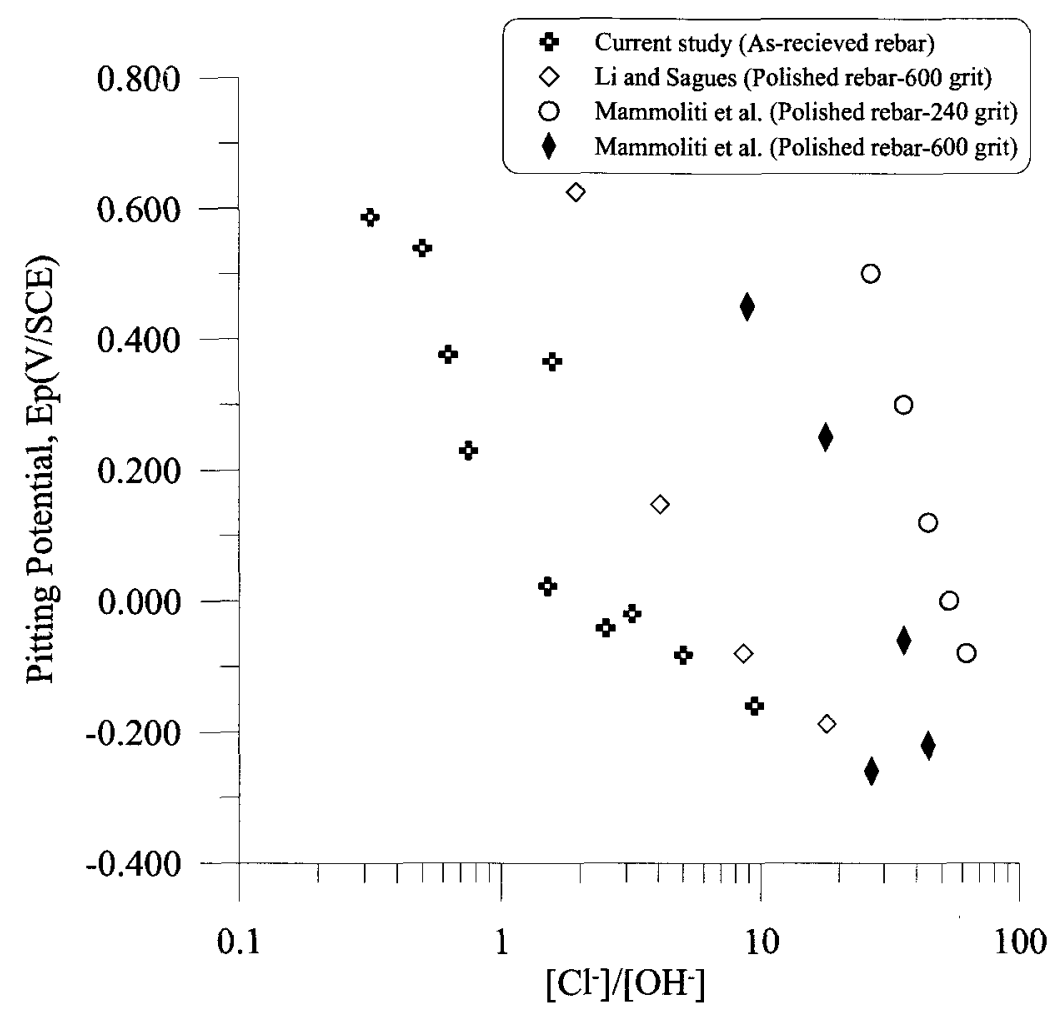

Figure 5.13: Pitting potentials from the current study and those reproduced from Li and Sagues (2002) and Mammoliti et al. (1996). As-received rebar specimens pit at lower Cl $1 \mathrm{OH}$ ratios than polished rebar specimens.

\subsubsection{Observations before depassivation}

The average passive current densities $\left(i_{p}\right)$ of rebar measured in the AP tests before chloride addition were significantly different in the $\mathrm{CP}$ and $\mathrm{CH}$ solutions: 1 $\mu \mathrm{A} / \mathrm{cm}^{2}$ and $0.02 \mu \mathrm{A} / \mathrm{cm}^{2}$, respectively, as shown in Fig. 5.11(a). In addition, the polarization resistances measured by LPR and EIS were higher in CH solutions (Fig 5.6, 5.9 and 5.10). Lower passive current densities and higher polarization resistances for the passive films in the $\mathrm{CH}$ solution may suggest more resistance to corrosion. However, the passive film in $\mathrm{CH}$ solution had a lower chloride threshold, which shows that the resistance of the passive film to corrosion does not necessarily mean that the film will be more resistant to chloride. 
The FCP does not change significantly with chloride addition up to threshold concentrations, as can be seen in Figs. 5.3-5.5. Similarly, polarization resistances shown in Figs. 5.9 and 5.10 remain relatively constant until the chloride thresholds and then drop. Thus, chloride is not systematically changing the electrical properties of the film. For instance, if chloride were thinning the passive oxide film or making it more porous, a decrease in the oxide film resistance should have been observed; however it remains relatively constant below the threshold. Similar conclusions were drawn for iron depassivation due to chloride in mildly alkaline solution (Goetz et al.1986). Hence, chloride-induced depassivation of rebar in highly alkaline solutions is more likely caused by critical chemical conditions that lead to depassivation. These critical conditions (thresholds) occur at lower chloride concentrations, as measured in the bulk solution, for as-received specimens than turned-and-polished specimens, which suggests that the local chemistry at the surface of the as-received rebar may be different from that in the bulk solution.

\subsubsection{Final thoughts}

Reconciling measurements of chloride thresholds for carbon steel rebar are complicated by a number of factors that include differences in specimen surface preparations, differences in synthetic pore solutions, and different experimental methods. However, even when the same experimental methods and specimen preparation techniques are used, there is a high degree of variability in the chloride threshold values. This suggests that in addition to reporting chloride thresholds, some indication of the variability is also required. 
Figure 5.14 shows the cumulative probability of depassivation, $P_{D}$, which is defined as the ratio of the number of depassivated specimens to the total number of specimens, $N=24$, for a given chloride concentration. (A specimen was defined to be depassivated when its FCP dropped below $-250 \mathrm{mV}$. This value was chosen because pits initiate on the surface for specimens when FCP is lower than $-250 \mathrm{mV}$. Varying this value by $50 \mathrm{mV}$ did not affect the probability distribution.) Figure 5.14 shows that the probability of depassivation increases with chloride addition, and that the probabilities are higher in the $\mathrm{CH}$ solution than in the $\mathrm{CP}$ solution.

The results shown in Fig. 5.14, were fitted to the cumulative log-normal probability distribution function:

$$
P_{D}=0.5\left(1+e r f\left(\frac{\log _{10}[C l]+\mathrm{pCl}_{\mathrm{T}}}{\sigma \sqrt{2}}\right)\right)
$$

where $[C l]$ is the chloride concentration, $\sigma$ is the logarithmic standard deviation and $p C l_{T}$ is defined as

$$
p C l_{T}=-\log _{10}[C l]_{T}
$$

In the regressions each datum was weighted by the squared inverse of its estimated error, which was calculated in accord with Poisson counting statistics. The $p C l_{T}$ and $\sigma$ obtained for the $\mathrm{CH}$ solution were $1.16 \pm 0.07$ and $0.45 \pm 0.11$, respectively, whereas for the CP solution, $p C l_{T}$ and $\sigma$ were $0.74 \pm 0.1$ and $0.4 \pm 0.14$, respectively. The $P$ values for the regressions were less than 0.05 , and the reported errors are standard deviations. 


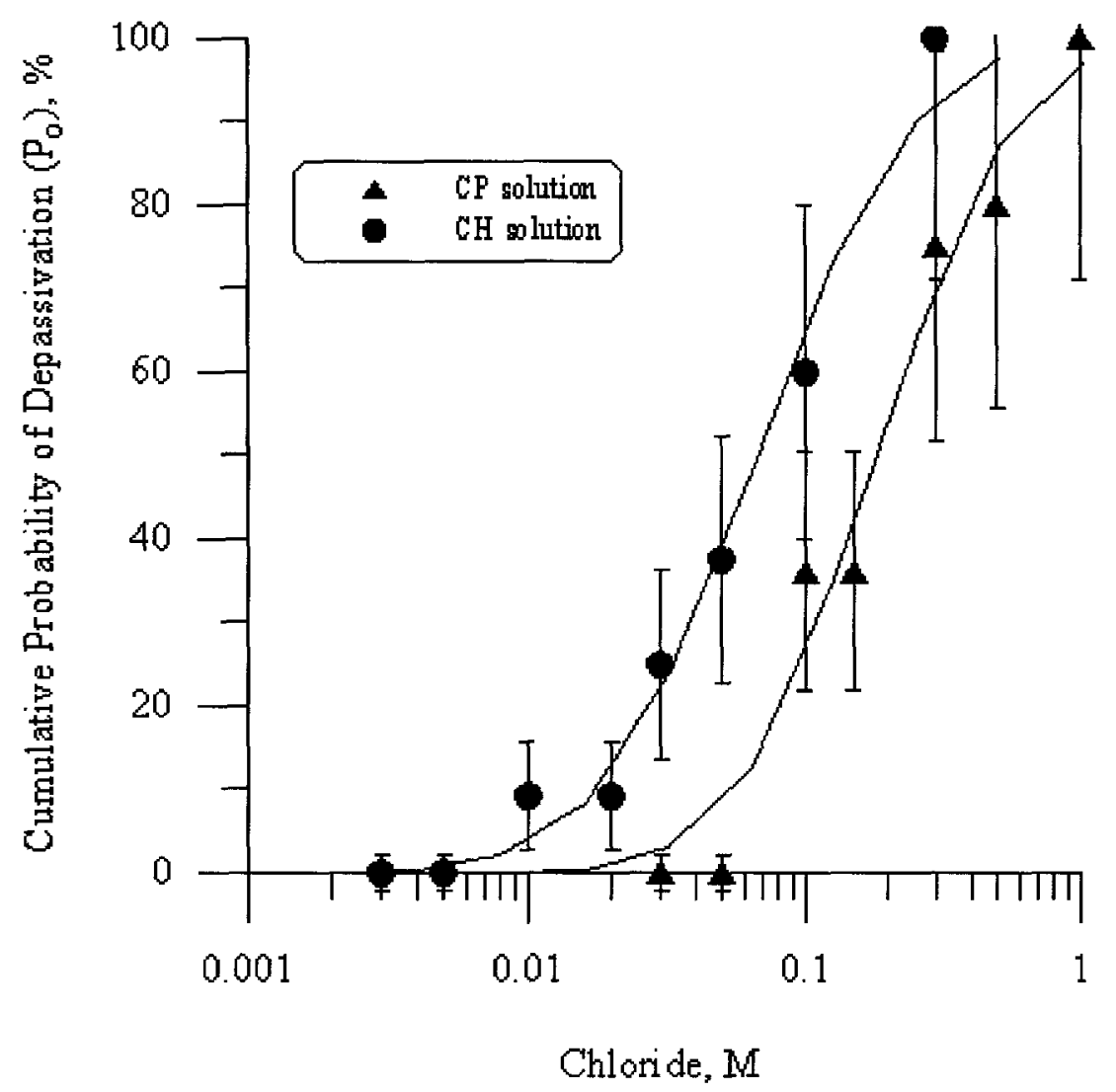

Figure 5.14: Cumulative probability of depassivation for as-received rebar as a function of chloride concentration. The curves in the figure were calculated from the cumulative log-normal probability distribution function, equation 5.6, and values of $\mathrm{pCl}_{T}$ and $\sigma$, as described in Section 5.5.5.

These values of $p \mathrm{Cl}_{T}$ are the most probable logarithmic chloride thresholds (i.e., the modes of the distributions), as well as the medians and means because the probability of depassivation is distributed symmetrically (i.e., normally) in terms of the logarithm of chloride concentration. However, if instead the independent variable was simply chloride concentration, the distributions would be skewed, and the mode, median and mean would no longer be the same: the modes for the $\mathrm{CH}$ and $\mathrm{CP}$ solution are $0.023 \mathrm{M}$ and $0.08 \mathrm{M}$, respectively; the medians for the $\mathrm{CH}$ and $\mathrm{CP}$ solution are $0.07 \mathrm{M}$ and $0.18 \mathrm{M}$, respectively; and the respective means are $0.12 \mathrm{M}$ and $0.29 \mathrm{M}$. This begs the question: 
which of these means, modes and medians should be used to characterize the threshold? The answer is that any of these will do, as long as one other parameter is included to complete the description of the distribution, (i.e., some parameterization of the width/variability). This suggests that simple averages of chloride threshold measurements might not provide reliable indicators of depassivation. For the purposes of this research, $\mathrm{pCl}_{T}$ and $\sigma$, and Eq. 5.6, were used to predict the probability of exceeding the chloride threshold.

\subsection{Conclusions}

In this study, FCP, LPR, EIS and AP were used to determine chloride thresholds in an electrochemical cell in which short specimens of as-received rebar were immersed in simulated concrete pore solution, and surrounded by a co-axial counter electrode. A method to coat the cut ends of the rebar specimens that effectively eliminates confounding crevice corrosion is proposed.

Two types of rebar surface condition were studied: as-received and turned-andpolished. Modification of the rebar surfaces, by turning and polishing, led to higher depassivation thresholds: the as-received specimens had lower chloride thresholds. The implication is that if the goal is to determine thresholds that will be representative for service-life-based design and end-of-life calculations, then any experimental surface modification should be avoided. Modifying the rebar surface also led to reduced variability in polarization resistance, and reduced fluctuations in FCP above the depassivaton threshold. These results of surface modification suggest that the variability associated with reported chloride thresholds may be attributed to the variability in the 
surface characteristics of the rebar resulting from the variability in fabrication and production.

The variability in chloride thresholds for as-received rebar was found to be well represented by a log-normal distribution. This suggests that simple averages of chloride threshold measurements, without reference to the underlying distribution, might not provide reliable indicators of depassivation. In this study, probabilities of depassivation are reported in addition to chloride thresholds.

The relative constancy of electrochemical measurements below the thresholds, and the dependence of the thresholds on the state of the surface, suggests that chlorideinduced depassivation of rebar in highly alkaline solutions is likely caused by the occurrence of local critical chemical conditions at the surface of the rebar. 


\section{Chapter 6}

\section{SEM and Numerical Studies on the Effect of Mill Scale on Chloride-induced Depassivation of Rebar}

\subsection{Introduction}

This chapter focuses on the contribution of the rebar surface, in particular the mill scale, to the chloride-induced depassivation. Previous studies showed that the removal of mill scale from the surface of the rebar by means of sandblasting, polishing or pickling increases chloride thresholds, in some cases, as much as one order of magnitude (Mangat and Molloy 1992, Pettersson 1996b, Mammoliti et al. 1996, Li and Sagues 2001, Pillai and Trejo 2005). In a rare case, Mammoliti (1995) reported that corrosion did not even initiate in highly-polished rebar even after the specimens were exposed to chloride concentrations in excess of that is typically found in sea water.

In Chapter 5, the chloride thresholds of as-received and turned-and-polished rebar specimens (which were turned on a lathe to remove the deformities and the mill scale and then polished to $600 \mathrm{Grit}$ ) in a simulated pore solution were compared using an experimental apparatus designed to simulate service conditions. A number of electrochemical techniques that included FCP, EIS, LPR and AP were used. It was reported that surface modification led to higher chloride thresholds. More important, 
modifying the rebar surface also led to reduced variability in polarization resistance and reduced fluctuations in free corrosion potential above the depassivation threshold. The inference was that the turned-and-polished surfaces were relatively uniform compared to that of the as-received surfaces.

In summary, previous electrochemical studies on the effect of surface modification of rebar on chloride thresholds suggest that the variability in chloride thresholds may be, at least in part, attributed to the variability in the surface characteristics of the rebar that originate from the manufacturing process. Although the actual mechanism of this effect is not well understood, the variability associated with mill scale that exists on rebar surface requires attention. The main objective of the present study is to carry out an SEM study that will help us better understand why mill scale on the surface of rebar makes the steel more susceptible to corrosion initiation. The study was carried out in two parts: In the first part, the properties of mill scale on rebars from different manufacturers are investigated; the second part focuses on the effect of mill scale on chloride-induced depassivation.

\subsection{Experimental Program}

\subsubsection{Investigation of mill scale properties}

In the first part of the investigation, mill scales on as-received deformed rebars from three different producers were studied using an SEM and an FIB microscope. Rebars were cut to $10-\mathrm{mm}$ lengths from as-received deformed carbon steel rebars of 10 $\mathrm{mm}$ nominal diameter. One specimen from each producer was mounted in low shrinkage Epoxy under vacuum (see Fig. 6.1) so that their cross sections could be polished to 1200 
grit. After polishing, all specimens were rinsed with distilled water and kept in a desiccator until microscopy studies. Mill scale around the cross section of each specimen was studied using a Philips XL30 SEM and a Micrion 2500 FIB microscope.

In addition to the cold-mounted specimens, three $10-\mathrm{mm}$ as-received rebar samples were cleaned with acetone and then with alcohol, and they were kept in a desiccator for the SEM investigation of mill scale from the surface of the rebar.

\subsubsection{Investigation of the effect of mill scale on the chloride-induced} depassivation

The second part of the investigation was carried out in parallel with an electrochemical study carried out in Chapter 5 so that the electrochemical observations (using FCP, EIS, LPR and AP) can be linked to the SEM data. Both electrochemical and SEM investigations were done on rebar specimens cut to $30-\mathrm{mm}$ lengths from as-received deformed carbon steel rebar with $10 \mathrm{~mm}$ nominal diameter. The chemical composition of the steel is given in Table 6.1. Further details about the specimen preparation for electrochemical experiments can be obtained from Chapter 5.

As previously explained in Chapter 5, twenty four rebar specimens were kept in a beaker containing a simulated concrete pore (CP) solution ( $\mathrm{pH} \sim 13.3$ ) for 8 days to allow the formation of stable passive film on the steel surface. The CP solution was prepared by dissolving calcium hydroxide $\left(\mathrm{Ca}(\mathrm{OH})_{2}\right)$, sodium hydroxide $(\mathrm{NaOH})$, potassium hydroxide $(\mathrm{KOH})$ and calcium sulfate $\left(\mathrm{Ca}\left(\mathrm{SO}_{4}\right)\right)$ in distilled water. The concentrations of the ions in the solution are presented in Table 6.2. The actual concentrations of the anions and cations in solution were measured using Inductively Coupled Plasma - Atomic Emission Spectroscopy (ICP-AES) (Model: Varian Vista RL). 


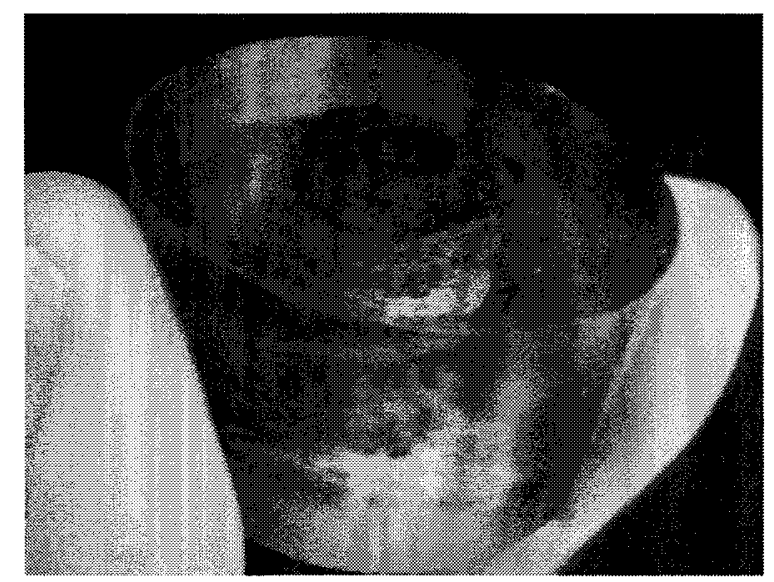

Figure 6.1: A typical cold-mounted rebar specimen for SEM investigation.

Table 6.1: Elemental composition of the rebar used in the SEM investigation studying the effect of mill scale on the chloride-induced depassivation.

\begin{tabular}{|c|c|}
\hline Element & Weight \% \\
\hline $\mathrm{C}$ & 0.26 \\
$\mathrm{Si}$ & 0.27 \\
$\mathrm{Mn}$ & 1.10 \\
$\mathrm{Cr}$ & 0.05 \\
$\mathrm{Ni}$ & 0.07 \\
$\mathrm{Mo}$ & $<0.01$ \\
$\mathrm{Cu}$ & 0.21 \\
$\mathrm{Al}$ & $<0.005$ \\
$\mathrm{Nb}$ & $<0.01$ \\
$\mathrm{~V}$ & $<0.005$ \\
$\mathrm{Ti}$ & $<0.005$ \\
$\mathrm{~B}$ & $<0.0005$ \\
$\mathrm{P}$ & 0.01 \\
$\mathrm{~S}$ & 0.03 \\
$\mathrm{~W}$ & $<0.01$ \\
$\mathrm{Sn}$ & 0.021 \\
$\mathrm{Co}$ & 0.01 \\
$\mathrm{Zr}$ & $<0.01$ \\
$\mathrm{Fe}$ & Balance \\
\hline
\end{tabular}


Following the initial passivation period, the chloride concentration in each beaker was increased incrementally at 7-day intervals for two months by adding reagentgrade sodium chloride. Before each chloride addition, three replicate specimens were removed from the beaker for electrochemical investigation in a corrosion cell to determine if depassivation had taken place. Once electrochemical data indicated depassivation, which typically occurred after $0.15 \mathrm{M} \mathrm{NaCl}$ addition, the specimen was removed from the corrosion cell and cold-mounted to be used later in the SEM investigation. A typical cold-mounted rebar specimen is shown in Fig. 6.1. Three asreceived and three turned-and-polished rebar samples that were not exposed to the pore solution were also cold-mounted to compare with the depassivated specimens. The optical images of these two surface conditions, as-received and turned-and-polished, are shown in Fig. 6.2(a) and (b), respectively.

Table 6.2: Concentrations of species in the simulated concrete pore solution (CP).

The actual concentrations of the anions and cations in solution were measured using Inductively Coupled Plasma-Atomic Emission Spectroscopy (ICP-AES) (Model: Varian Vista $R L)$.

\begin{tabular}{|c|c|c|c|c|c|c|c|c|}
\hline \multicolumn{4}{|c|}{$\begin{array}{l}\text { Added compounds } \\
(\mathrm{M})\end{array}$} & \multicolumn{4}{|c|}{$\begin{array}{c}\begin{array}{c}\text { Measured ions } \\
(\mathrm{mg} / \mathrm{l})\end{array} \\
\end{array}$} & \multirow[t]{2}{*}{ pH } \\
\hline $\mathrm{Ca}(\mathrm{OH})_{2}$ & $\mathrm{Na}(\mathrm{OH})$ & $\mathrm{K}(\mathrm{OH})$ & $\mathrm{CaSO}_{4}$ & $\mathrm{Ca}^{2+}$ & $\mathrm{Na}^{+}$ & $\mathrm{K}^{+}$ & $\mathrm{SO}_{4}{ }^{2-}$ & \\
\hline 0.1 & 0.1 & 0.2 & 0.003 & 3 & 2232 & 8059 & 277 & 13.3 \\
\hline
\end{tabular}


The cold-mounted rebar specimens were cut in half to $15-\mathrm{mm}$ long specimens; they were then placed in a vacuum desiccator for 24 hours for drying; the cut surfaces were polished to 1200 grit for microstructure examinations. It should be noted that the main reason for cold-mounting the rebar specimens was mainly to prevent the possible damage on the rebar surface near the cross section and minimize the effect of cutting process on the interface between steel and mill scale.

All SEM specimens were examined under an SEM equipped with an EDAX light element energy dispersive X-ray spectrometer for chemical composition analyses.

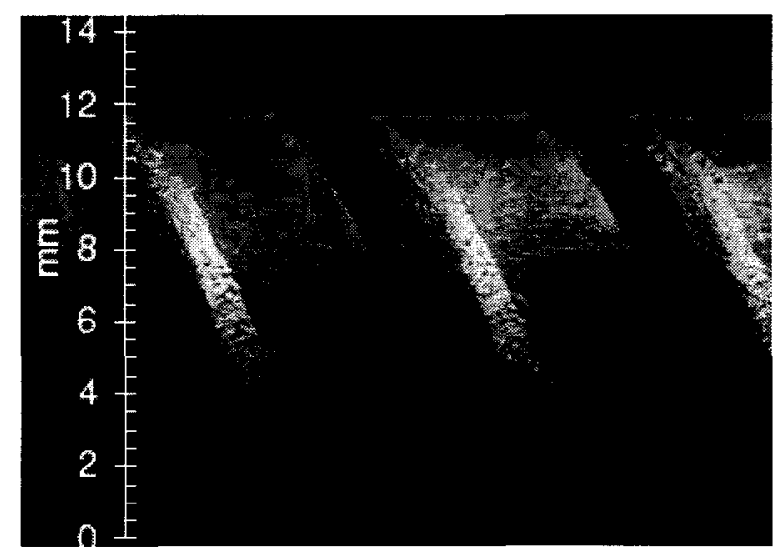

(a)

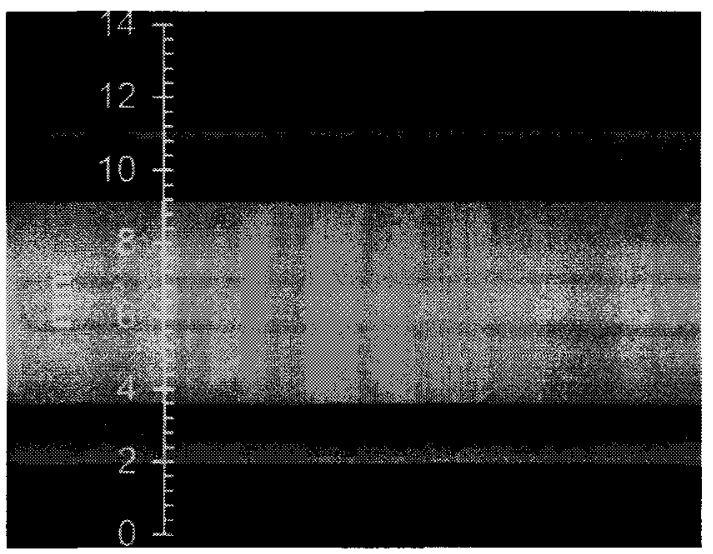

(b)

Figure 6.2: Optical images of rebar samples with two different surface conditions used in this study: (a) as-received with mill scale; (b) turned-and-polished. Banding in Fig. 6.2 (b) is an artifact of the light reflection form the mirror-polished surface of rebar. 


\subsection{Results}

\subsubsection{Investigation of mill scale properties}

In Fig. 6.3, cross-sectional SEM micrographs of rebar specimens from three manufacturers are presented in increasing magnification. As shown in Fig. 6.3(i-b to i-d), the surface of rebar (i) is covered with a thick $(\sim 30 \mu \mathrm{m})$ and relatively uniform layer of mill scale; however, localized cracks that connect the surface of steel to the free surface of rebar, crevices between the steel surface and mill scale and voids within the mill scale are visible. Figures 6.3(ii-b to ii-d) illustrate the mill scale on the surface of rebar (ii), which is mostly in the form of non-uniform patches with a maximum thickness of $\sim 2 \mu \mathrm{m}$. There are some areas on the steel surface where there is no mill scale formation. For the third rebar type, as illustrated in Fig. 6.3(iii-b to iii-d), the mill scale is quite thick $(\sim 40 \mu \mathrm{m})$, but unlike rebar (i), it does not cover the surface of the steel completely.

Similar to rebar (i), the mill scale on the surface of rebar (iii) also contains cracks, crevices and voids.

Higher magnification SEM micrographs shown in Fig. 6.3(i-d) and Fig.6.3 (iiid) indicate that mill scale consists of two phases, which can be clearly seen in the backscattered electron images with a darker outer layer and a lighter inner layer. The EDS analyses shown in Figs. 6.4(a) and (b) indicate that these two layers mainly consist of iron and oxygen. The intensity ratios of iron to oxygen ( $\mathrm{Fe}$ and $\mathrm{O}$ ), which is related to the chemical composition of the phases, are quite different. 
(i)

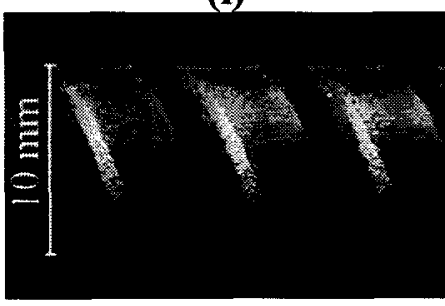

(a)

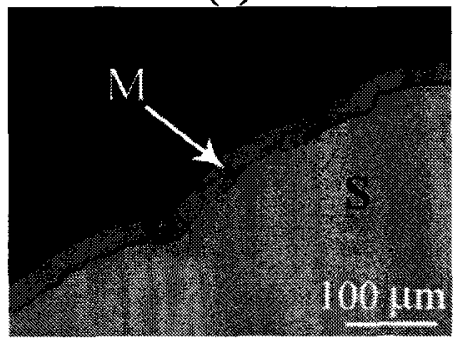

(b)

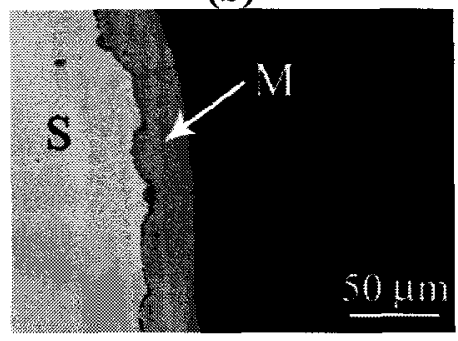

(c)

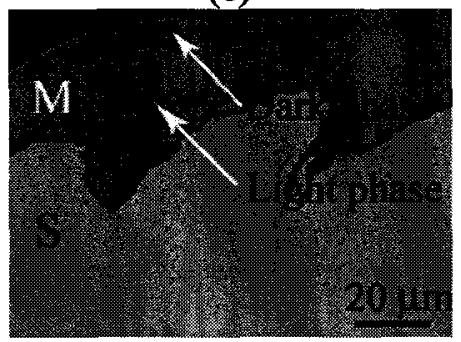

(d) (ii)

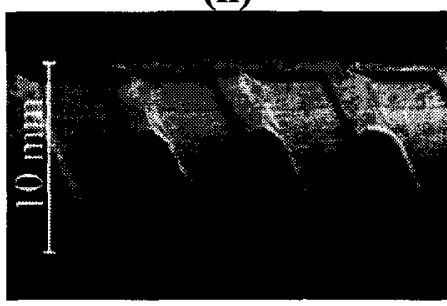

(a)

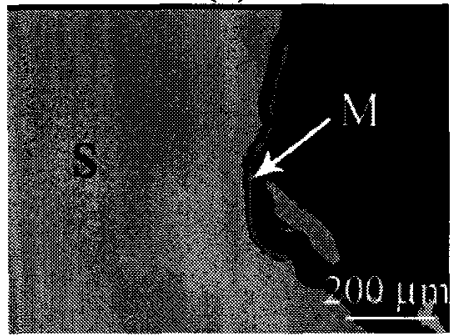

(b)

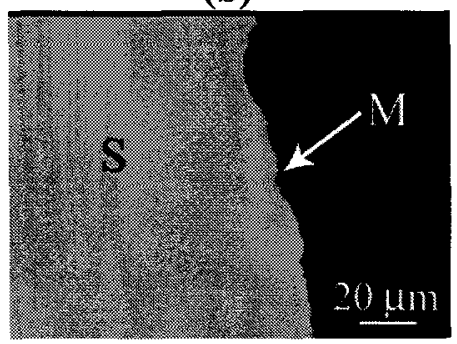

(c)

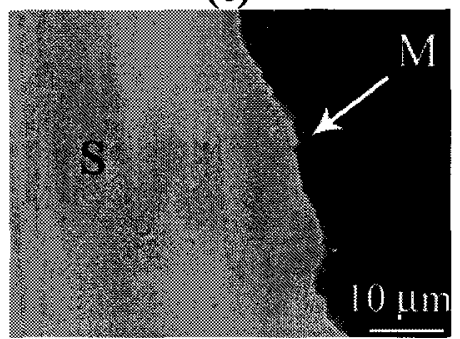

(d) (iii)

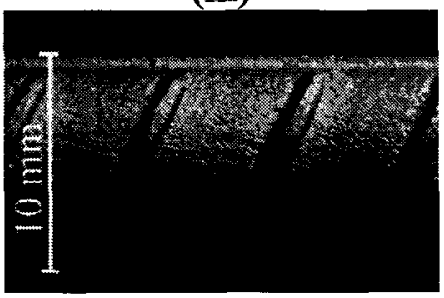

(a)

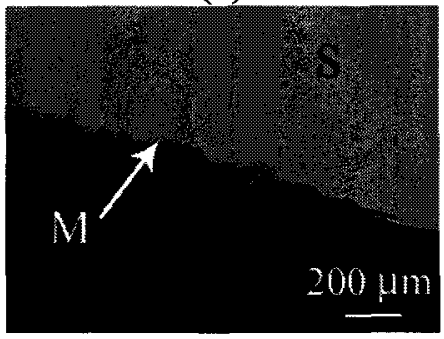

(b)

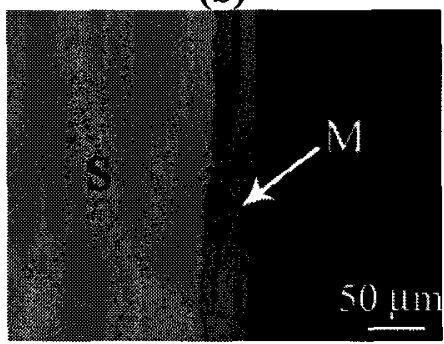

(c)

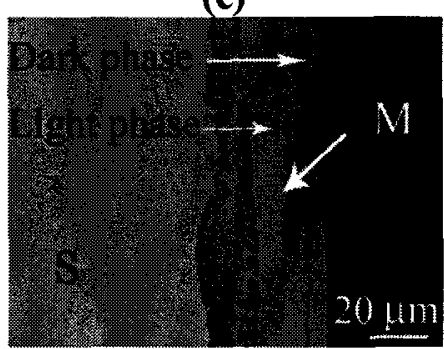

(d)

Figure 6.3: Mill scale on rebar specimens from three different manufacturers $(i, i i, i i i)$.

(a) optical images of rebar surface from different manufacturers; Figures (b) to (d) provide SEM micrographs with increasing magnification for each rebar type. Rebar (i):

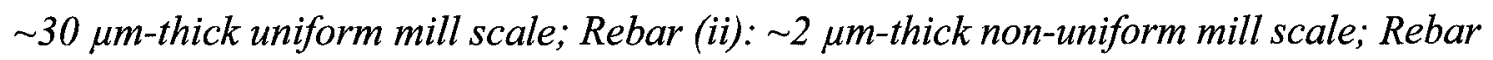
(iii) 40 4 m-thick non-uniform mill scale. (Note: M: Mill scale, S: Steel).

Figure 6.5 shows two FIB micrographs obtained from the cross section of an asreceived rebar before exposure to concrete pore solution. In Figure 6.5(a) the crystal grains of steel and mill scale and their boundaries are shown. It can be seen from this 
figure that the crystal structure of mill scale on the steel surface is very similar to that of the steel. Mill scale has a crystalline structure and in a higher magnification micrograph (Fig. 6.5(b)) the average size of the crystal grains of mill scale with the thickness of $\sim 20$ $\mu \mathrm{m}$ appears slightly larger than that of steel.

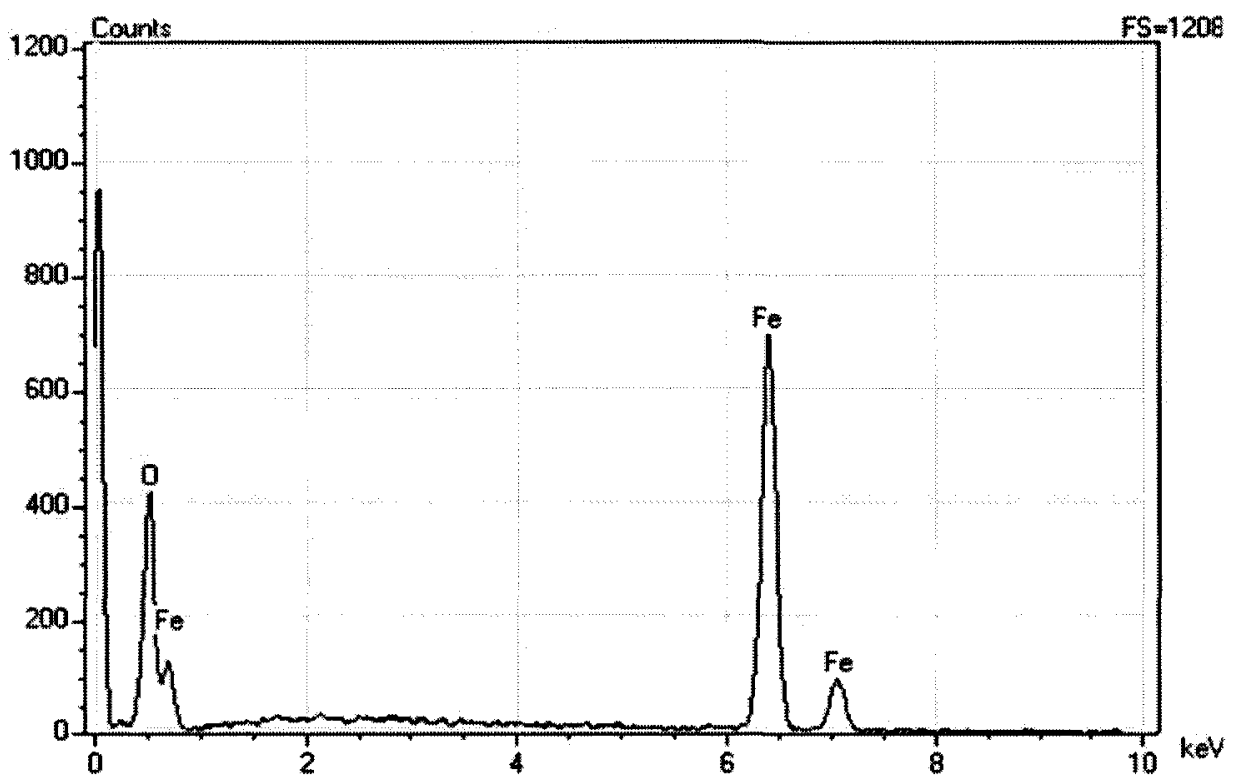

(a)

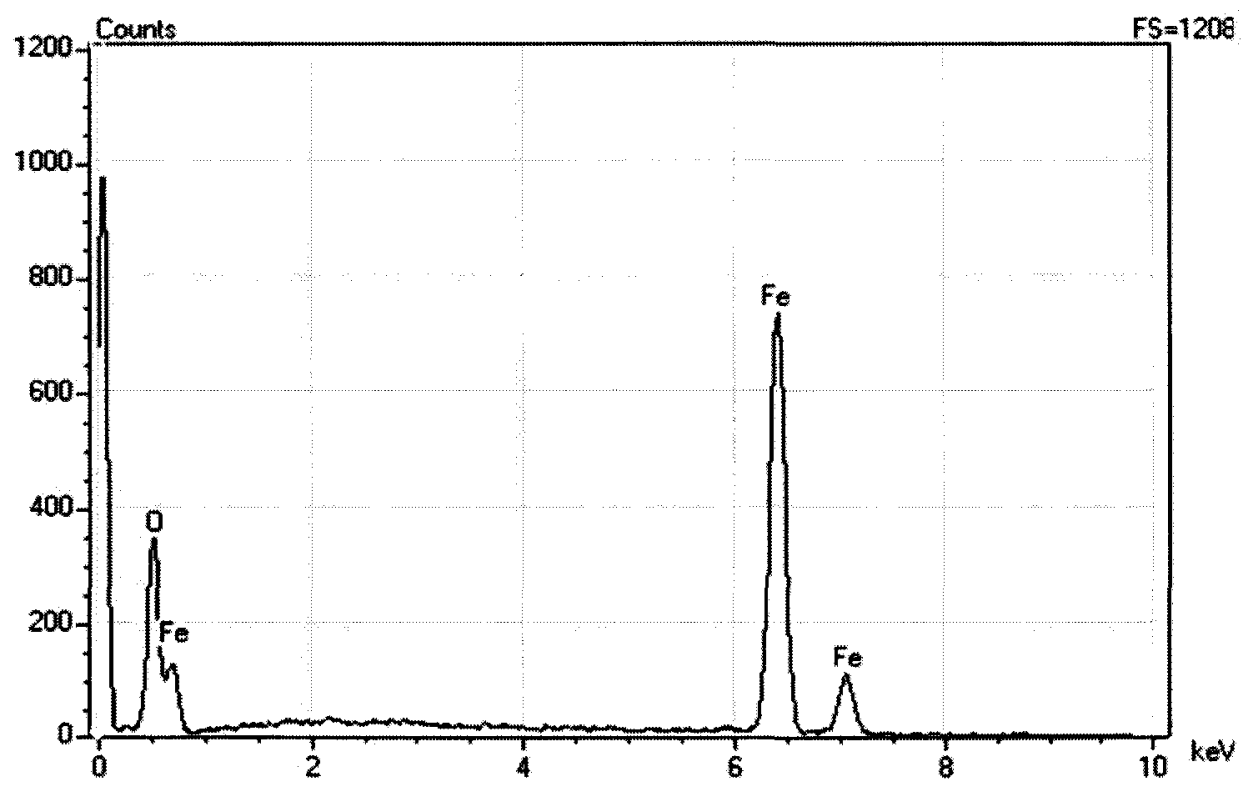

(b)

Figure 6.4: EDS analysis of mill scale of rebar (i): (a) dark phase; (b) light phase. 


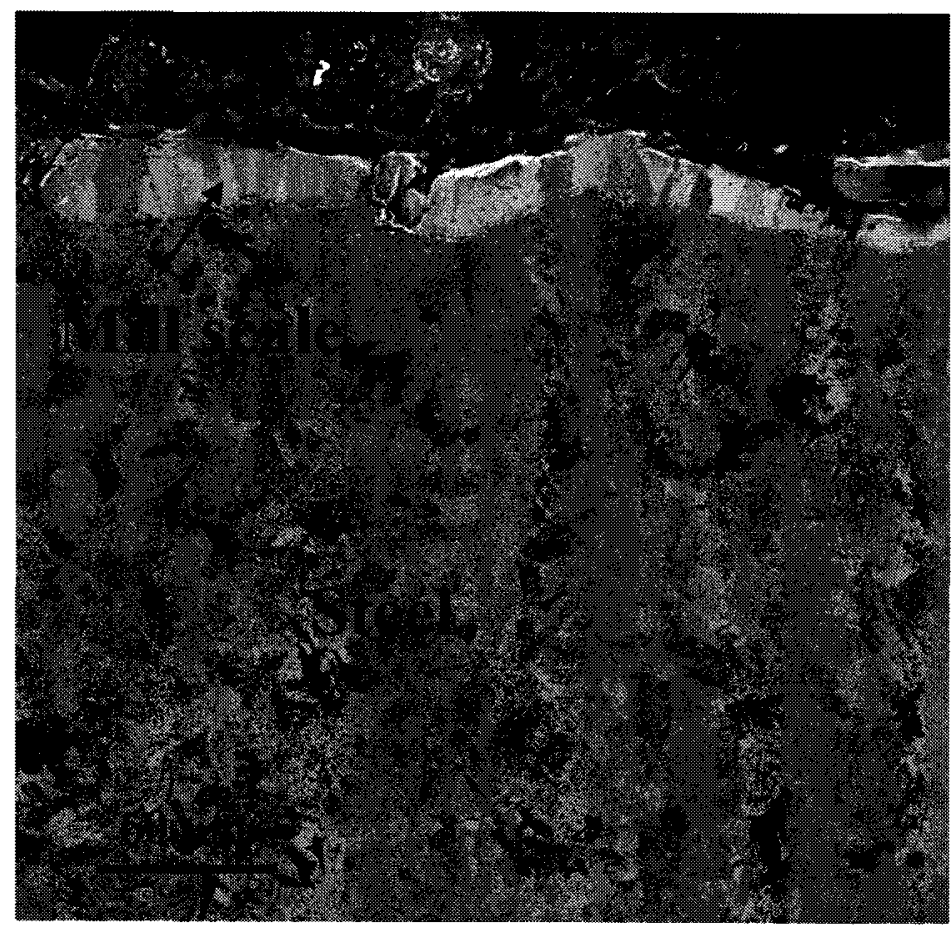

(a)

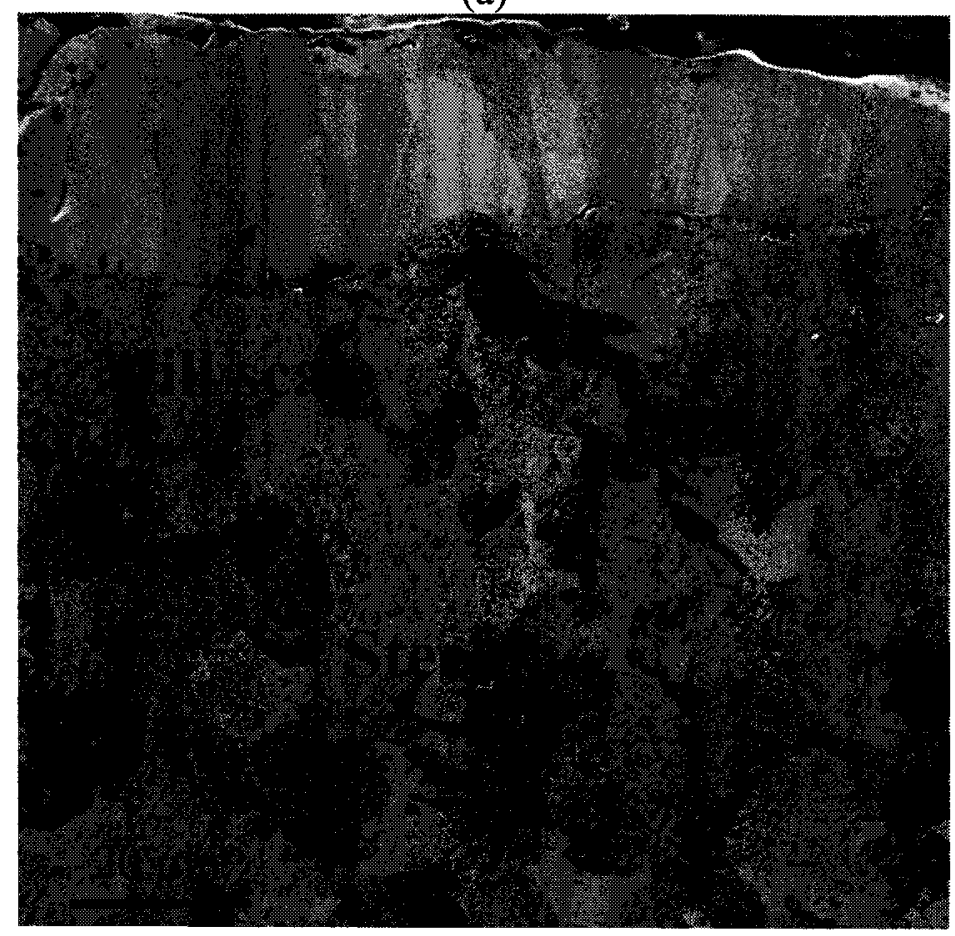

(b)

Figure 6.5: Crystal grains and their boundaries for steel and mill scale under the FIB microscope (a) low magnification micrograph; (b) high magnification micrograph. 


\subsubsection{Investigation of the effect of mill scale on the chloride-induced depassivation}

The SEM micrographs of the cross sections of the turned-and-polished and asreceived rebar specimens before immersion in the simulated concrete pore solution are shown in Fig. 6.6. As expected, the surface of steel for turned-and-polished specimen is smooth and free of any visible oxide (Figs. 6.6(a)). In contrast, the surface of the asreceived specimen is covered with a thick layer of mill scale (Figs. 6.6(b) and (c)). The thickness of mill scale on the surface of the as-received rebar specimen was found to vary between $20 \mu \mathrm{m}$ and $40 \mu \mathrm{m}$ (Fig. 6.6(b)). SEM micrograph of the mill scale with higher magnification (Fig. 6.6(c)) revealed that the interface of steel and mill scale is filled with crevices with the lengths as large as $500 \mu \mathrm{m}$, and the mill scale contains cracks with widths as large as $1 \mu \mathrm{m}$. In some locations around the steel surface, these cracks transversely link the crevices to the free surface (Fig. 6.6(c)), hence, providing pathways for the concrete pore solution to penetrate into the voids and the crevices.

SEM images of as-received rebar surfaces before exposure to the CP solution or chloride are shown in Fig. 6.7. The smallest surface features in Fig. 6.7 are in the order of micrometers that correspond to the size of the crack openings observed in Fig. 6.6(c). In this figure two phases are clearly observed: dark phase and light phase. SEM analyses conducted on these two phases indicate that iron to oxygen ratio in the light phase is quite larger than that in the dark phase area. Similar observations on two different phases of mill scale are reported in the cross sectional studies of the mill scale before exposure (Section 6.3.1). 


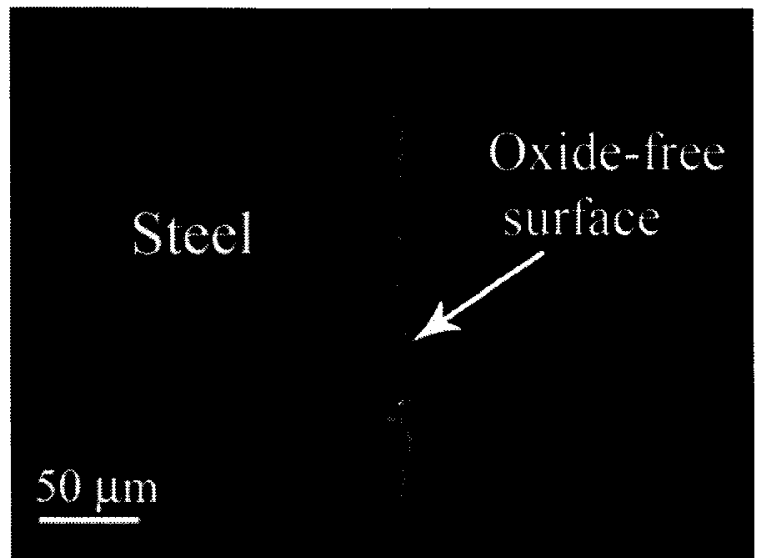

(a)

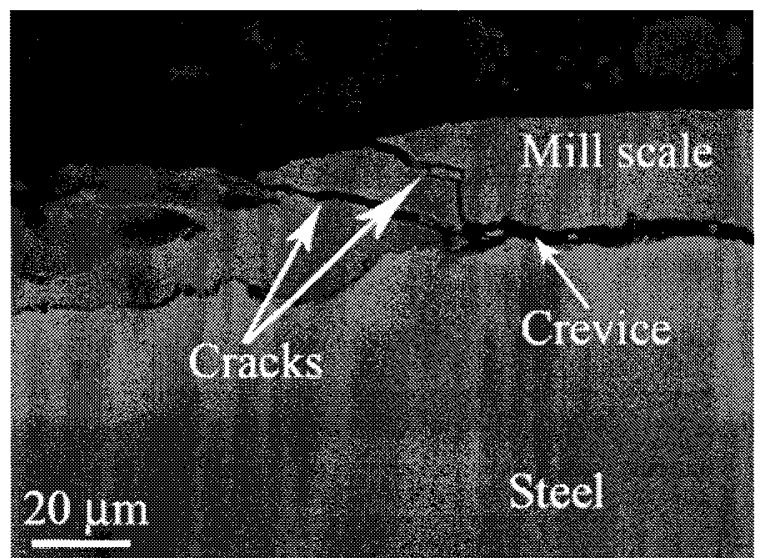

(c)

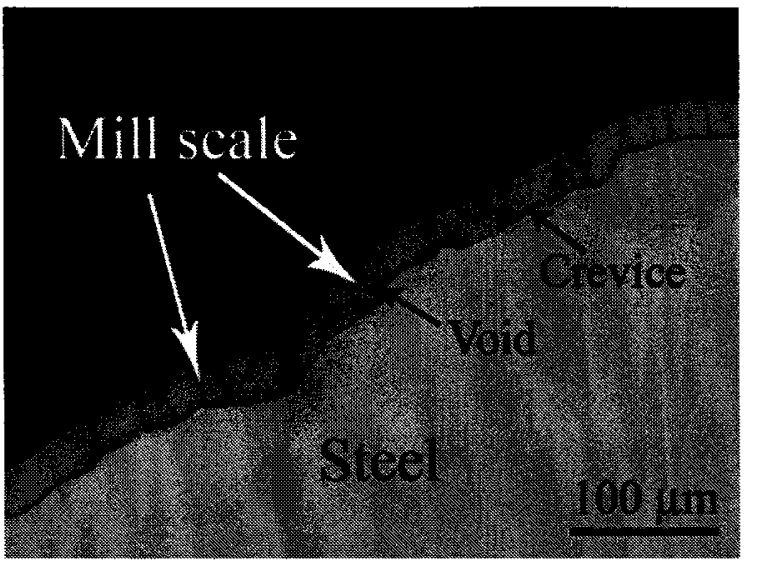

(b)

Figure 6.6: SEM cross-section images of turned-and-polished and as-received rebar samples before exposure: (a) micrograph of turned-and-polished sample; (b) lower magnification micrograph of as-received sample; (c) higher magnification micrograph of as-received sample.

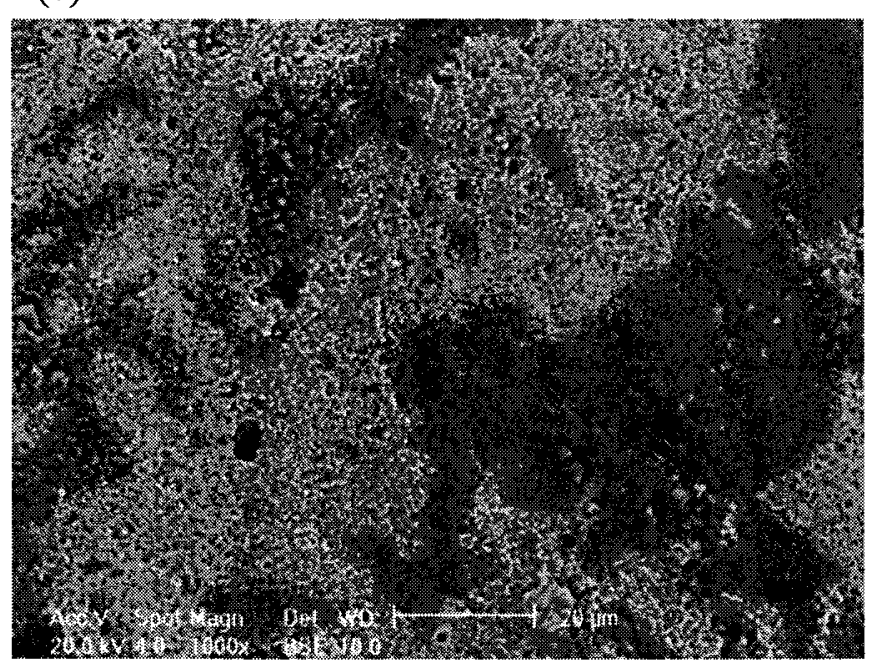

Figure 6.7: SEM surface images of as-received rebar samples before exposure. The smallest dark features are in the order of microns that correspond to the crack openings in mill scale. 
In Fig. 6.8(a), the SEM image of part of the cross section of an as-received rebar specimen that was exposed to $0.15 \mathrm{M} \mathrm{NaCl}$ is presented. In this figure, there are two cracks connecting the confined crevice between the steel and the mill scale to the free surface, providing passage for chlorides. A section of Fig. 6.8(a) is enlarged in Fig. 6.8(b). EDS analysis has been carried out at sites A, B and C; the results are presented in Figs. 6.9(a), (b) and (c), respectively. The existence of iron and oxygen in the spectra confirms the presence of iron oxide (with $\mathrm{Fe} / \mathrm{O}$ varying between 1 and 2 ) at these sites. In addition, the presence of calcium, sodium, potassium and chloride indicates that concrete pore solution contributed to the formation of these oxides; therefore, it can be stated that these are pit initiation sites. Although these pits are under the mill scale and are not visible when the surface of the rebar is observed, their formation is identified electrochemically. Within the crevice other corrosion pits at different stages are also visible (Fig. 6.8(a)). The crevice has a width that varies between 5 to $10 \mu \mathrm{m}$, and some parts of the crevice have narrowed down due to the formation of these corrosion products. It was also observed that at locations without mill scale, there are no corrosion pits. This suggests that for as-received rebar, crevices between the underlying steel and the mill scale provide an environment for pit nucleation.

Figure 6.10 illustrates an advanced-stage of pitting. These pits can be observed on the rebar surface, well after the electrochemical observation of pit initiation (depassivation). In Fig. 6.10(a), it can be observed that products have expanded and destroyed the mill scale. The EDS analysis of the corroded site (i.e., "Area E") shown in Fig. 6.10(b) confirms the presence of corrosion products. Figures $6.10(\mathrm{c})$ and (d) show the propagation of a pit penetrating the steel and the EDS analysis of the corresponding 
location (site F), respectively. EDS results confirm the corrosion product and the presence of chloride in this area. Finally in Fig. 6.10(e) an advanced propagation stage of rebar corrosion, in which the mill scale popped out, is well in progress.

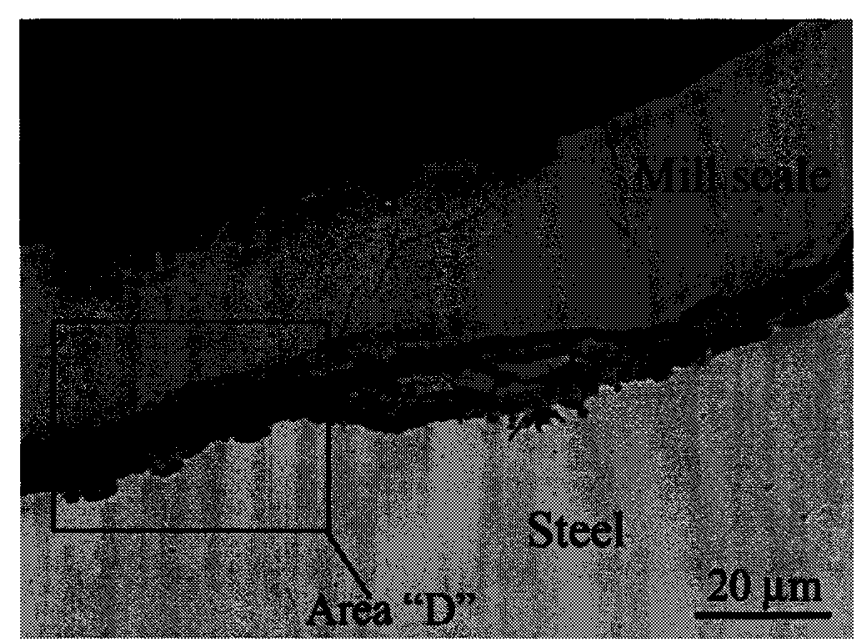

(a)

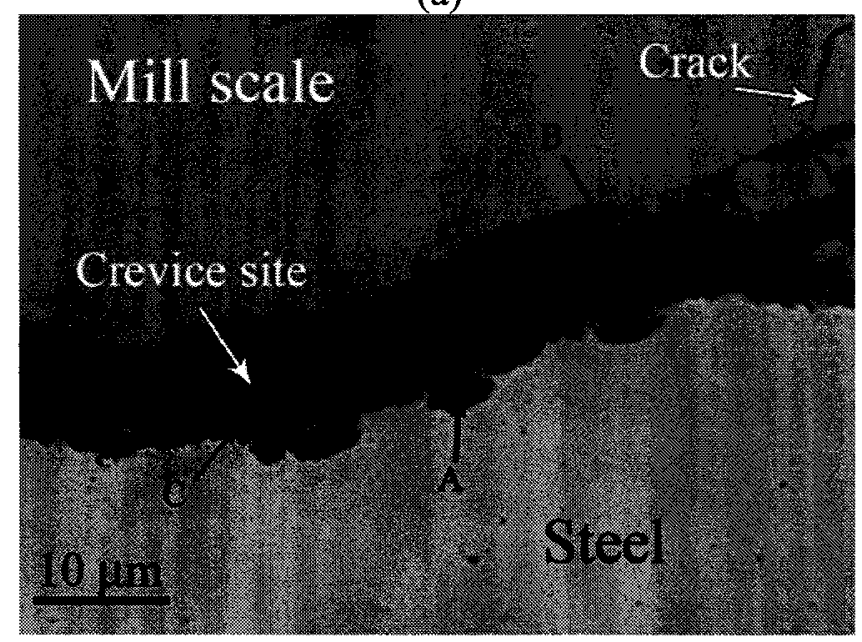

(b)

Figure 6.8: SEM images of representative mill-scale cross sections taken from asreceived rebar with mill scale after immersion in the simulated concrete pore solution plus $0.15 \mathrm{M} \mathrm{NaCl}$ : (a) lower magnification image showing the location of corrosion initiation; (b) higher magnification image of area " $D$ " marked in Fig. $6.8(a)$ 


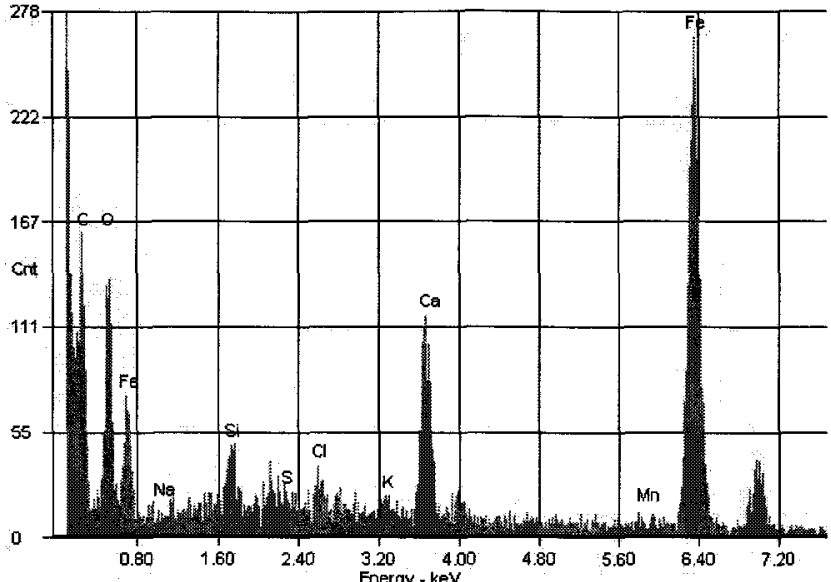

(a)

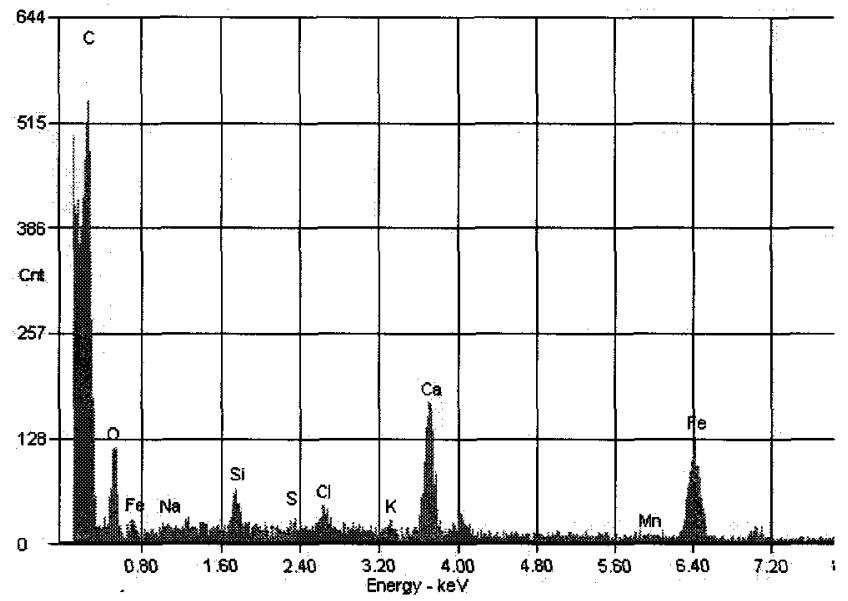

(b)

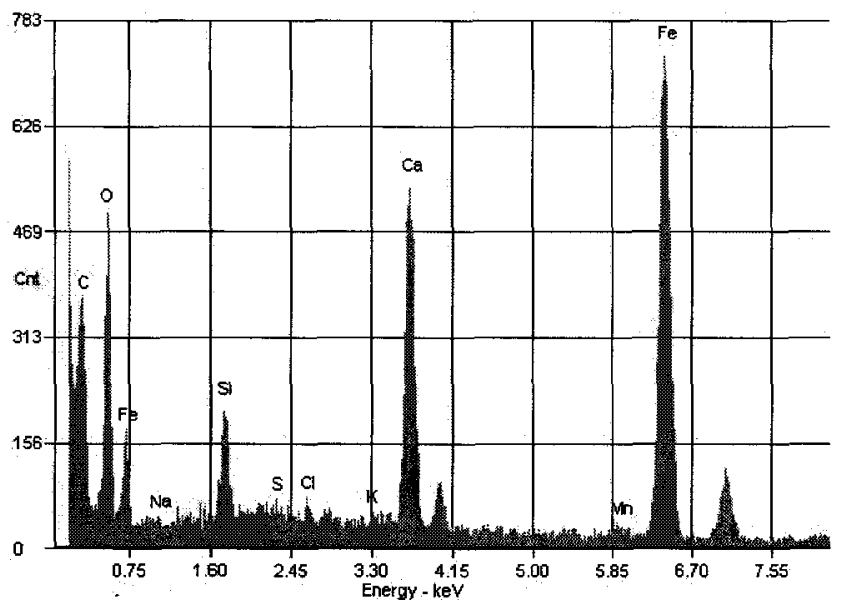

(c)

Figure 6.9: EDS analysis of pits at spots marked in Figure 6.8: (a) point A; (b) point $B$; (c) point $C$. 


\subsection{Discussion and Numerical Support}

Mill scale is an iron oxide layer that forms on the steel surface during the hot rolling process of steel production; therefore, it is expected that the properties of mill scale vary with the type of production and the rolling temperature. The present investigation supports this expectation because three rebars produced in three different mills had considerably different physical properties in terms of thickness, uniformity and microstructure. Even for the same rebar specimen, mill scale was not uniform and was full of randomly-distributed cracks, voids and crevices.

Furthermore, the chemical composition of mill scale was found to be typically composed of two layers. Schweitzer (1989) reported that mill scale is generally composed of magnetite $\left(\mathrm{Fe}_{3} \mathrm{O}_{4}\right)$, and smaller quantities of other oxides. Assuming the large portion of mill scale (referring to the light phase in this study) is composed of $\mathrm{Fe}_{3} \mathrm{O}_{4}$ (Fe/O 0.75), as reported by Schweitzer (1989), the outer layer of mill scale (i.e., the dark phase) should have composition with a lower $\mathrm{Fe} / \mathrm{O}$ than that of $\mathrm{Fe}_{3} \mathrm{O}_{4}$ since the darker composition corresponds to lower Fe content in backscattered SEM images. Therefore, $\mathrm{Fe}_{2} \mathrm{O}_{3}$ with $\mathrm{Fe} / \mathrm{O}$ of 0.66 is a potential candidate as the main composition of the outer layer of the mill scale. Raman spectroscopy studies of mill scale by Marcotte (2001) also supports that the mill scale is largely composed of magnetite $\left(\mathrm{Fe}_{3} \mathrm{O}_{4}\right)$ with a surface layer of hematite $\left(\alpha-\mathrm{Fe}_{2} \mathrm{O}_{3}\right)$ and goethite $(\alpha-\mathrm{FeOOH})$. However, these suggestions should be confirmed by other characterization techniques such as XPS, SAD, and CBED analysis. Regardless of the exact composition of the two phases, it is observed that relative volume fractions of these two phases differ in rebars from different suppliers; even for the same rebar, different cross sections show different volume fractions for the two phases. 


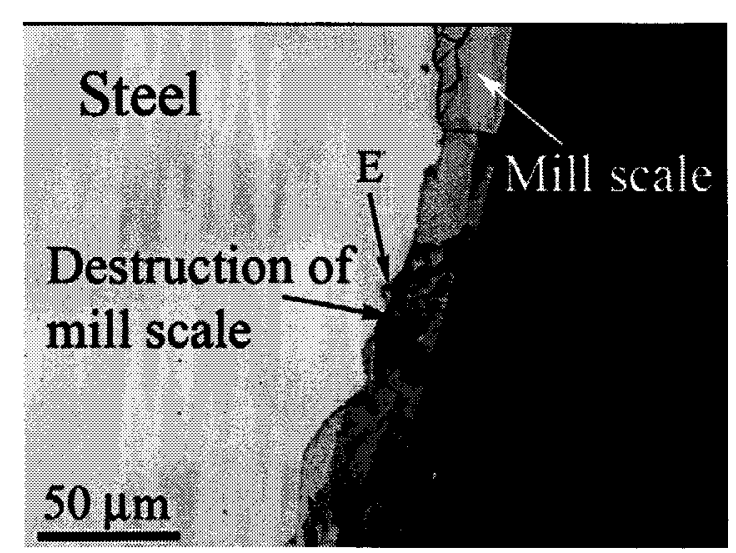

(a)

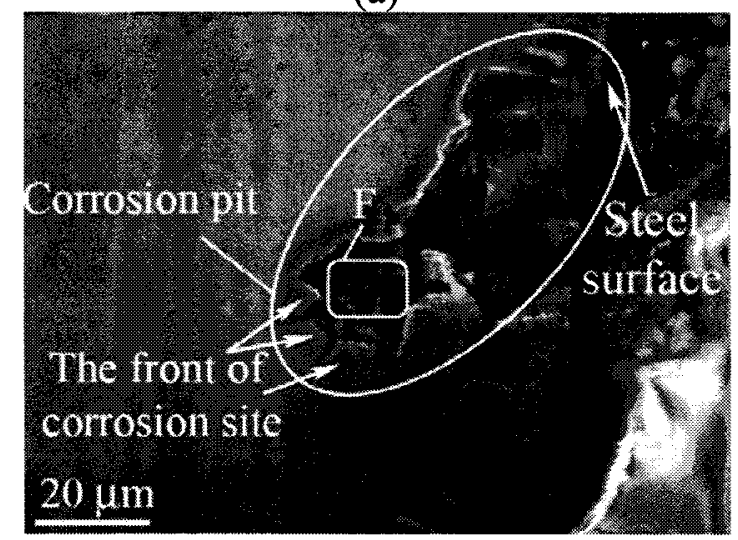

(c)

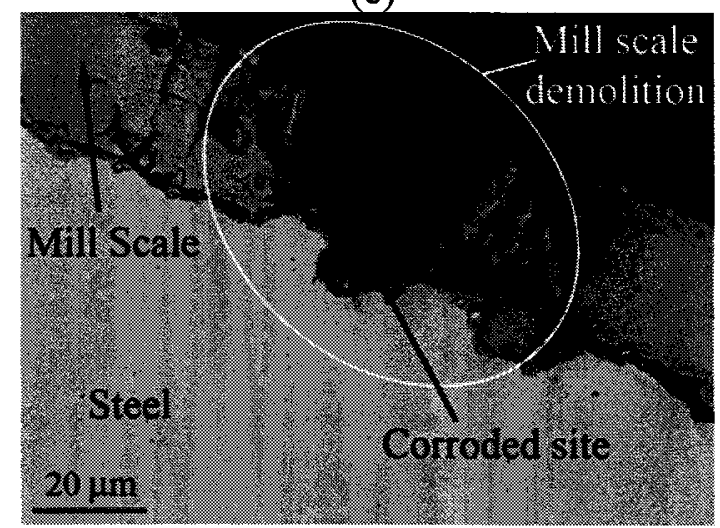

(e)

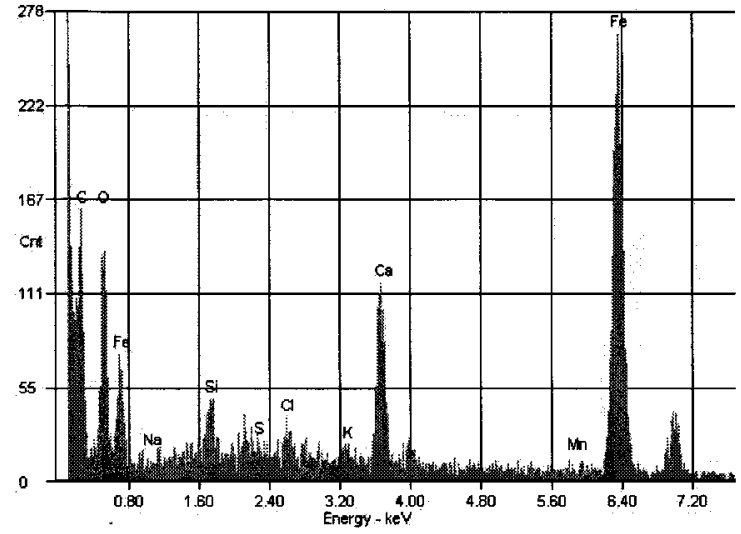

(b)

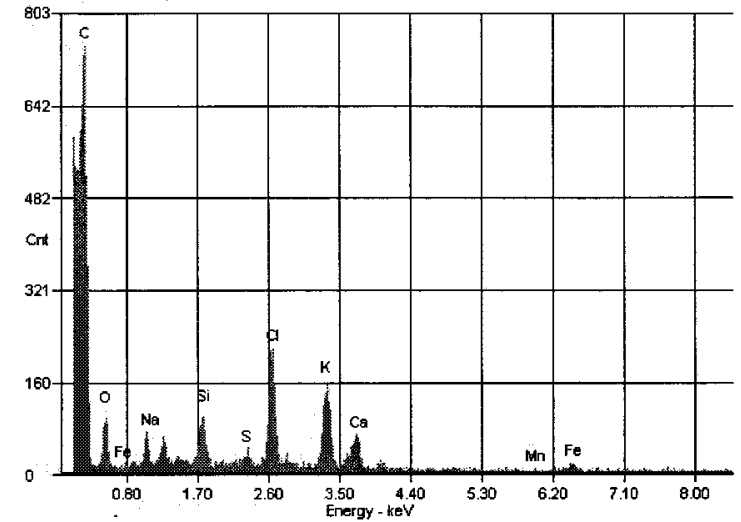

(d)

Figure 6.10: SEM images of corrosion propagation stages taken from as-received rebar with mill scale after immersion in the simulated concrete pore solution plus 0.15 $\mathrm{MNaCl}$. (a) an image showing corrosion propagation at the interface of steel/millscale; (b) EDS analysis at "Area E" marked in Fig. 6.10(a); (c) an image showing the growth of corrosion site (i.e., pit) penetrating the steel; (d) EDS analysis at "Area $F$ " marked in Fig. 6.10(c); (e) an image showing the mill-scale pop-out. Pits can be observed on steel surface well after depassivation can be observed electrochemically. 
The second part of the investigation that focused on the effect of mill scale on chloride-induced depassivation of as-received rebar specimens has demonstrated that mill scale on the surface of rebar makes the steel more susceptible to corrosion initiation. This observation is in agreement with previous electrochemical studies, which reported that when mill scale is removed, chloride thresholds increase (Mammoliti, et al. 1996, Li and Sagues 2001, Pillai and Trejo 2005, Chapter 5). In addition, as reported in Chapter 5, modifying the rebar surface also leads to reduced variability and reduced fluctuations in electrochemical measurements (e.g. polarization resistance and free corrosion potentials), which can be explained by the hypothesis that modified surfaces are uniformly the same everywhere, whereas the as-received surfaces are locally much more diverse and complex due to the presence of mill scale. These results suggest that the variability associated with reported chloride thresholds may be attributed to the variability in mills scale properties resulting from the variability in manufacturing.

The present study provides clues that can be used to explain why as-received rebar is more susceptible to corrosion initiation than rebar without mill scale. Crosssectional SEM images of rebar specimens showed that hollow areas, such as voids and crevices, exist at the interface between steel and mill scale. Some of these voids and crevices are connected to the pore solution by transverse cracks, which serve as pathways for concrete pore solution to reach the surface of steel; hence allow ion exchange between the pore solution and the crevices through electrical migration and diffusion. Over time, the chemical composition of the pore solution within the crevices begins to differ from that of the bulk solution through a process similar to the suggested mechanisms of typical 
crevice corrosion (Jones 1995, Fontana 1986). Here a brief description of one of the proposed mechanisms is discussed.

In Fig. 6.11, the schematic illustrations of a crevice connected to a crack at the interface of steel/mill scale are provided. In this figure, the effect of mill scale on the corrosion initiation mechanism of as-received rebar is divided into two stages. The first stage refers to the condition in which oxygen is extensively available on the surface of steel beneath the mill scale (Fig. 6.11(a)); in the second stage oxygen is completely depleted due to the cathodic reaction (Fig. 6.11(b)).

In the first stage (Fig. 6.11(a)), due to the anodic reaction at the surface of steel, iron dissolves into the concrete pore solution via (these equations have been presented before, but repeated here for clarity):

$$
\mathrm{Fe} \rightarrow \mathrm{Fe}^{2+}+2 e^{-}
$$

The rate of iron dissolution before corrosion initiation is controlled by passive current density since steel is in the passive state. At the same time, in the presence of oxygen, the cathodic reaction also takes place on the steel surface via:

$$
\frac{1}{2} \mathrm{O}_{2}+\mathrm{H}_{2} \mathrm{O}+2 e^{-} \rightarrow 2 \mathrm{OH}^{-}
$$

Since the solubility of ions in alkaline solutions is very low $\left(\mathrm{K}_{\mathrm{sp}} \sim 4.87 \times 10^{-17}\right)$ (Dean 1999), iron ions react with hydroxides and precipitate as iron hydroxide, $\left(\mathrm{Fe}(\mathrm{OH})_{2}\right)$. During this stage, the concentrations of anions and cations in the crevice are the same as the concentrations in the bulk concrete pore solution. In particular, $\mathrm{Cl}^{-} / \mathrm{OH}^{-}$, which is known to be a main factor in the depassivation of steel in concrete, of the bulk solution and of the solution in the crevice is identical. As the anodic and cathodic reactions proceed, oxygen consumed in the cathodic reaction can not be compensated by 
the transport of oxygen from the bulk solution; therefore, after a while, oxygen is completely depleted in the crevice.

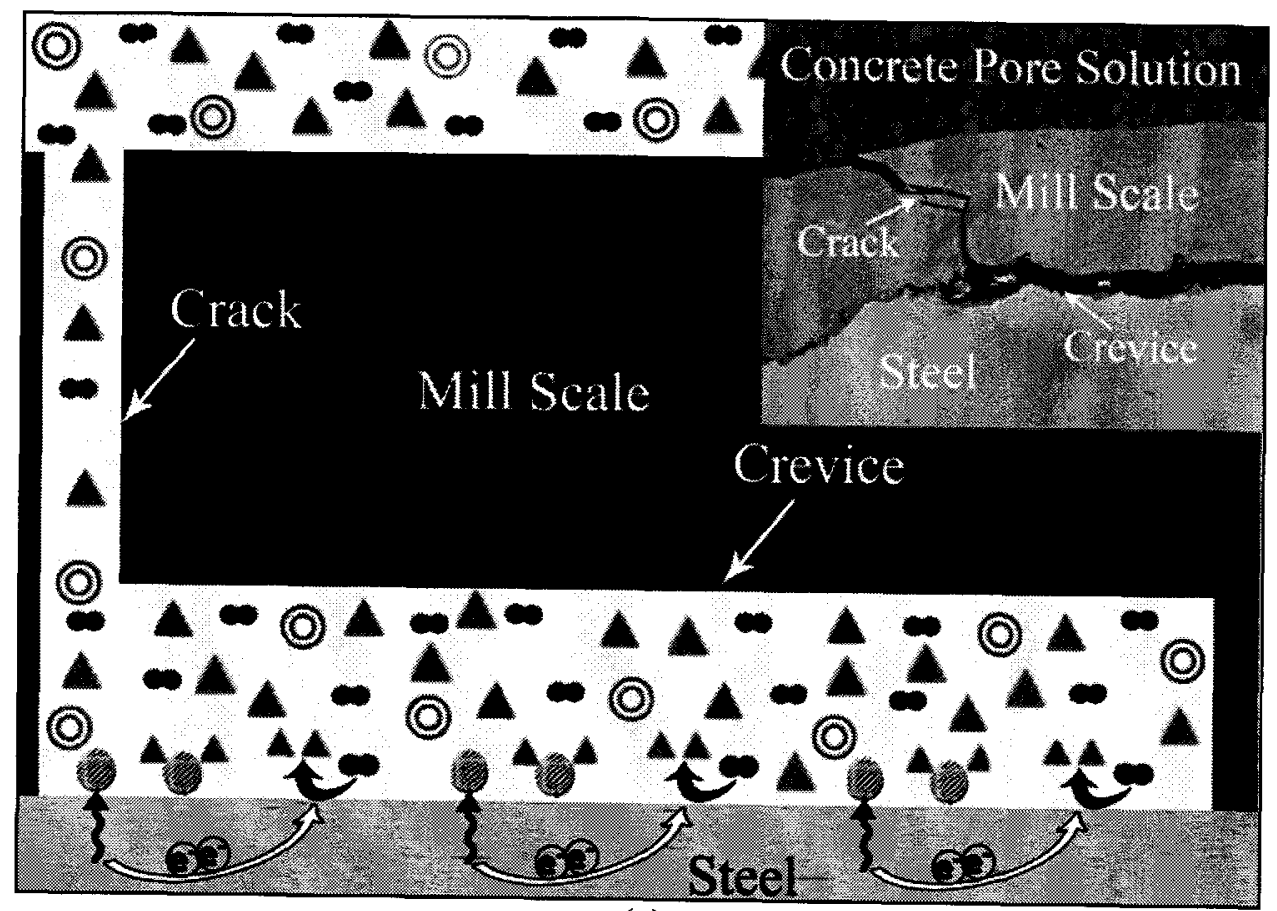

(a)

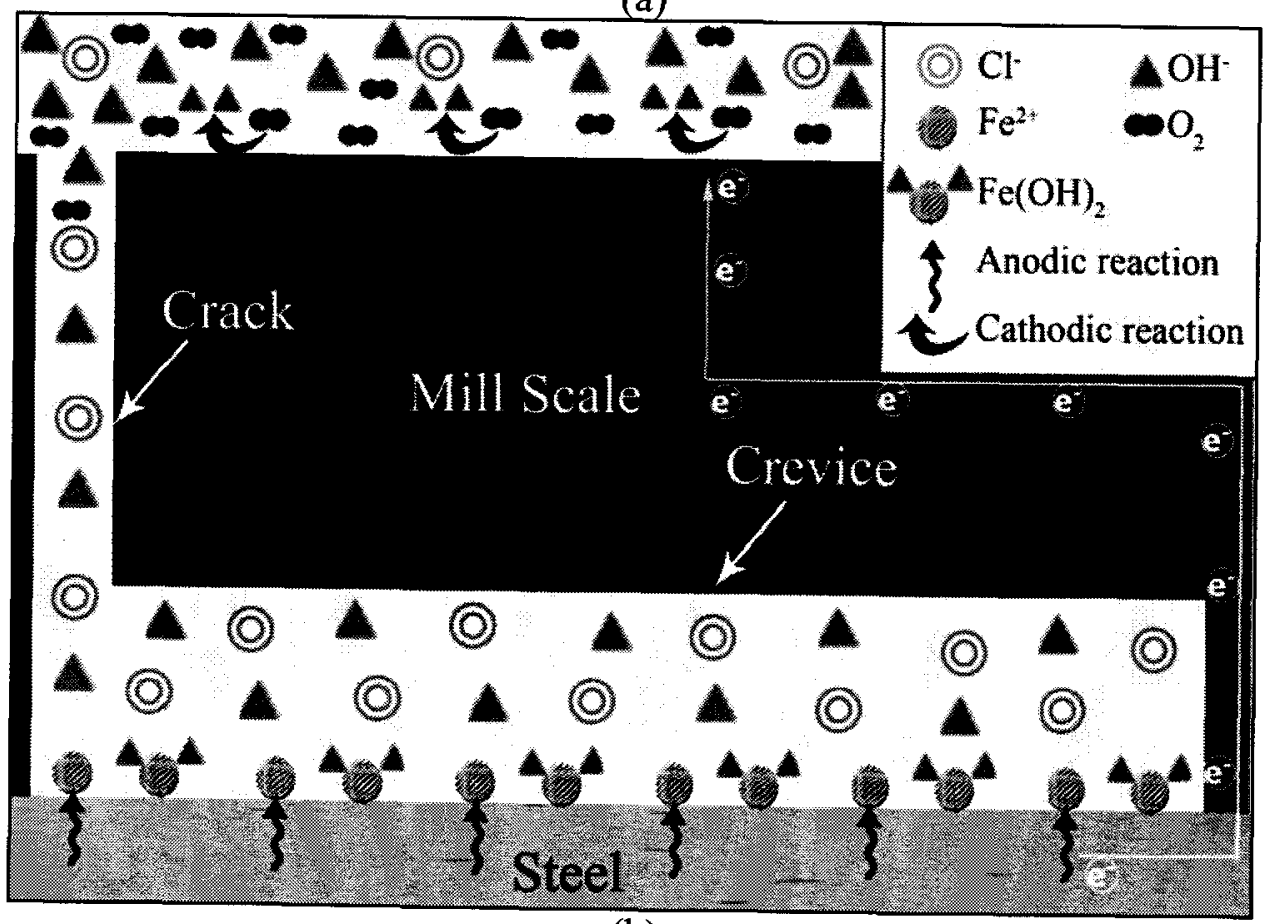

(b)

Figure 6.11: Schematic illustration of crevice corrosion at the mill-scale/steel interface in two stages: (a) Stage 1: before oxygen depletion; (b) Stage 2: after oxygen depletion. 
Following the depletion of oxygen the second stage starts. In this stage, the cathodic reaction ceases in the crevice but continues to take place outside the crevice (see Fig. 6.11(b)); hence hydroxides are no longer produced inside the crevice. Due to the continuous formation of iron hydroxide, $\mathrm{Fe}(\mathrm{OH})_{2}$, the concentration of hydroxide decreases inside the crevice, while an electrochemical gradient forms between the bulk and crevice pore solutions. The requirement of electro-neutrality dictates that negatively changed ions (e.g. chloride) migrate from the bulk solution into the crevice via cracks; therefore, the chloride concentration inside the crevice starts increasing as the hydroxide concentration decreases (Fig. 6.11(b)), causing $\mathrm{Cl}^{-} / \mathrm{OH}^{-}$to increase within the crevice.

This proposed mechanism supports the electrochemical findings in Chapter 5. It has been suggested that chloride-induced depassivation of rebar in highly alkaline solutions is more likely caused by critical chemical conditions that occur at lower chloride concentrations, as measured in the bulk solution, for as-received specimens than turned-and-polished specimens. This conclusion suggests that the local chemistry at the surface of the as-received rebar (i.e., within crevices) may be different from that in the bulk solution. In order to test this suggestion, a numerical investigation of the abovementioned mechanism has been carried out in collaboration with Karadakis (2009), who reports detailed findings of this study in his Master's thesis. Here, a part of this numerical investigation is presented to demonstrate that local chemistry, specifically $\mathrm{Cl}^{-}$ $/ \mathrm{OH}^{-}$, within the mill scale crevices of rebar at the surface of the as-received rebar may be different from that in the bulk solution.

The transport of species in concrete pore solution can be modelled by the extended Nernst-Planck equation (Samson et al. 1999): 
$\frac{\partial c_{i s}}{\partial t}+\frac{\partial c_{i}}{\partial t}+\nabla \cdot(-\underbrace{D_{i} \nabla c_{i}}_{\text {Diffusion }}-\underbrace{\frac{D_{i} z_{i} F}{R T} c_{i} \nabla \varphi}_{\text {Electrical Migration }}-\underbrace{D_{i} c_{i} \nabla\left(\ln \gamma_{i}\right)}_{\text {Chemical Activity }})=0 \quad$ Eq. 6.3

where subscript $i$ is the index representing each ionic (in this study: $\mathrm{OH}^{-}, \mathrm{Fe}^{2+}, \mathrm{Ca}^{2+}, \mathrm{Cl}^{-}$, $\mathrm{Na}^{+}, \mathrm{K}^{+}$), and gaseous (in this study: $\mathrm{O}_{2(\mathrm{~g})}$ ) species modelled, $\mathrm{c}_{i s}\left(\mathrm{~mol} / \mathrm{m}^{3}\right)$ is the concentration of the stationary species in solid state (i.e., the components $\mathrm{Fe}_{(\mathrm{s})}$ and $\mathrm{OH}_{(\mathrm{s})}$ as they exist in $\left.\mathrm{Fe}(\mathrm{OH})_{2}\right), D_{i}\left(\mathrm{~m}^{2} / \mathrm{s}\right)$ is the diffusion coefficient for the species in water, $R$ is the ideal gas constant $(8.3143 \mathrm{~J} / \mathrm{mol} / \mathrm{K}), T(\mathrm{~K})$ is the temperature, $z_{i}$ is the valence of the ionic species, $F$ is Faraday's constant $(96,488 \mathrm{C} / \mathrm{mol}), \phi(\mathrm{V})$ is the electric potential, and $\gamma_{i}$ is the chemical activity coefficient for the various ionic species in water, and $t(\mathrm{~s})$ is time. $\partial c_{i s} / \partial t$ term in the equation is the sink/source that accounts for the exchange between the solid and ionic species in the concrete pore solution; i.e., the exchange between $\mathrm{Fe}_{(\mathrm{s})}$ and $\mathrm{Fe}^{+2}$, and the exchange between $\mathrm{OH}_{(\mathrm{s})}$ and $\mathrm{OH}^{-}$. For all other species, $\partial c_{i s} / \partial t$ can be set to zero if a simplification is made such that the dominant solid species in the pore solution are limited to $\mathrm{Fe}(\mathrm{OH})_{2}$ and $\mathrm{Ca}(\mathrm{OH})_{2}$.

In Eq. 6.3, there are three mechanisms that are used to describe the flux of species in concrete pore solution: diffusion, electrical migration and chemical activity. Advection flux by capillary suction was not considered as part of the total flux of species because the analysis domain was assumed to be fully saturated with the concrete pore solution. The diffusion term in the total flux expression represents the movement of species based on a concentration gradient. Although there was no external current applied to the system, the diffusion of ions within the pore solution creates electric potential gradients since different ions move at different velocities, and this, in turn, leads to an imbalance in 
the electro-neutrality of the system. These potential gradients, $\nabla \phi$, through the electrical migration term in Eq. 6.3, slows down the faster ions while accelerating the slower ions so that electro-neutrality of the system is maintained. The potential gradients change over time, and they need to be calculated for each time marching step during the numerical solution of Eq. 6.3; therefore, in this study, Eq. 6.3 was coupled with Poisson's equation that directly relates the electric potential with the electric charge distribution in the concrete pore solution (Samson and Marchand 1999):

$$
\nabla^{2} \phi+\frac{F}{\varepsilon} \sum_{i=1}^{n_{i}} z_{i} c_{i}=0
$$

where $n_{i}$ refers to the total number of ionic species and $\varepsilon\left(\mathrm{C}^{2} / \mathrm{N} / \mathrm{m}^{2}\right)$ is the permittivity of the medium, which was taken in this study as that of water $\left(7.092 \times 10^{-10} \mathrm{C}^{2} / \mathrm{N} / \mathrm{m}^{2}\right)$. Finally, the chemical activity term $\left(\gamma_{i}\right)$ in Eq. 6.3 represents the ion-ion and ion-solvent interactions that take place in an ionic solution. The effect of chemical activity on the overall transport of species is investigated further by Karadakis (2009).

For simplicity, the numerical investigation presented here was carried out in a saturated $\mathrm{Ca}(\mathrm{OH})_{2}$ solution (labelled as $\left.\mathrm{CH}\right)$ with a $\mathrm{pH}$ of 12.5 . Calcium $\left(\mathrm{Ca}^{2+}\right)$ and iron $\left(\mathrm{Fe}^{+2}\right)$ ions in the pore solution were assumed to react only with hydroxides to form solid calcium hydroxide and solid iron hydroxide as per:

$$
\begin{array}{lr}
\mathrm{Ca}_{(a q)}^{2+}+2 \mathrm{OH}_{(a q)}^{-} \stackrel{K_{s p 1}}{\longleftrightarrow} \mathrm{Ca}(\mathrm{OH})_{2(s)} & \text { Eq. 6.5 } \\
\mathrm{Fe}_{(a q)}^{2+}+2 \mathrm{OH}_{(a q)}^{-} \stackrel{K_{s p 2}}{\longrightarrow} \mathrm{Fe}(\mathrm{OH})_{2(s)} & \text { Eq. 6.6 }
\end{array}
$$

where $K_{s p 1}$ and $K_{s p 2}$ are the solubility product constants, which are taken in this study as $5500 \mathrm{~mol}^{3} / \mathrm{m}^{9}$ and $4.87 \times 10^{-8} \mathrm{~mol}^{3} / \mathrm{m}^{9}$, respectively (Dean 1999). The stoichiometric 
balance among the solid species of these two equations require that in the bulk concrete pore solution the following constraint exists:

$$
c_{F e_{(s)}}+c_{C a_{(s)}}=0.5 \times c_{O H_{(s)}}
$$

Since the solubility product constant for $\mathrm{Ca}(\mathrm{OH})_{2(\mathrm{~s})}$ is almost 11 orders of magnitude larger than that of $\mathrm{Fe}(\mathrm{OH})_{2(\mathrm{~s})}$, the concentrations of $\mathrm{Ca}_{(\mathrm{s})}$ and $\mathrm{OH}_{(\mathrm{s})}$ from $\mathrm{Ca}(\mathrm{OH})_{2(\mathrm{~s})}$ are significantly smaller in a concrete pore solution than the concentrations of $\mathrm{Fe}_{(\mathrm{s})}$ and $\mathrm{OH}_{(\mathrm{s})}$ from $\mathrm{Fe}(\mathrm{OH})_{2(\mathrm{~s})}$. Therefore, it was assumed in this numerical simulation that all $\mathrm{Ca}(\mathrm{OH})_{2(\mathrm{~s})}$ dissolved within the mill scale crack and crevice such that the following constraint can be used instead of Eq. 6.7:

$$
c_{F e_{(s)}}=0.5 \times c_{O H_{(s)}}
$$

The reaction rates of the reversible reactions in Eq. 6.5 and Eq. 6.6 were assumed to be large in comparison to the rate of transport of species involved (Rubin 1983). Accordingly, reactions 6.5 and 6.6 require the following two solubility relations to be satisfied simultaneously at every node in the domain:

$$
\begin{aligned}
& \left(\gamma_{\mathrm{Ca}^{2+}} c_{\mathrm{Ca}^{2+}}\right)\left(\gamma_{\mathrm{OH}^{-}} c_{\mathrm{OH}^{-}}\right)^{2}=K_{s p_{1}} \\
& \left(\gamma_{\mathrm{Fe}^{2+}} c_{\mathrm{Fe}^{2+}}\right)\left(\gamma_{\mathrm{OH}^{-}} c_{\mathrm{OH}^{-}}\right)^{2}=K_{s p_{2}}
\end{aligned}
$$

These two solubility relations must be satisfied at every node in the pore solution when their respective solid compound is present in the concrete pore solution.

As iron hydroxide precipitates, $\mathrm{Fe}(\mathrm{OH})_{2}$, in the concrete pore solution, it progressively occupies more space within mill scale crack and the crevice; this build-up within the mill scale crack and the crevice would cause a decrease in the rate of transport of species. To account for this effect, the diffusion coefficients were assumed to decrease 
relative to $\mathrm{Fe}(\mathrm{OH})_{2}$ concentration at any given point in the analysis domain at a given time. In this model, $\mathrm{Fe}(\mathrm{OH})_{2}$ was assumed to build up in the space within the mill scale crack and crevice until there is no more space left. Assuming the density of $\mathrm{Fe}(\mathrm{OH})_{2(\mathrm{~s})}$ (molar mass $89.86 \mathrm{~g} / \mathrm{mol}$ ) as $3400 \mathrm{~g} / \mathrm{l}$ (Dean 1999), the maximum amount of $\mathrm{Fe}(\mathrm{OH})_{2(\mathrm{~s})}$ that can be found in a given volume was calculated as $37,840 \mathrm{~mol} / \mathrm{m}^{3}$. When the $\mathrm{Fe}(\mathrm{OH})_{2(\mathrm{~s})}$ reaches $37,840 \mathrm{~mol} / \mathrm{m}^{3}$ within a given finite element, the diffusion coefficients of all species in that element were assumed to be very small, typically in a range between $10^{-10}$ and $10^{-13} \mathrm{~m}^{2} / \mathrm{s}$ (Bamforth et al. 1997). The diffusion coefficients for all other concentrations of $\mathrm{Fe}(\mathrm{OH})_{2}$ were linearly interpolated between the maximum and minimum diffusion coefficients (i.e., $D_{\max }$ and $D_{\min }$ ) at $\left[\mathrm{Fe}(\mathrm{OH})_{2(\mathrm{~s})}\right]=0$ and $\left[\mathrm{Fe}(\mathrm{OH})_{2(\mathrm{~s})}\right]=$ $37,840 \mathrm{~mol} / \mathrm{m}^{3}$, respectively.

Figure 6.12 illustrates the axi-symmetrical domain and boundary conditions of the simulation, representing the crack in the mill scale and crevice between the mill scale and the steel surface. In the figure, boundary 1 is the opening of the mill scale crack to the concrete pore solution; boundary 2 is the axis of symmetry; boundary 3 is the steel surface; and boundaries 4,5 and 6 represent the mill scale surfaces. The analysis presented here was carried out on a domain with the following dimensions, representing a realistic mill scale crack and crevice on rebar: crack length $(l)=5 \times 10^{-5} \mathrm{~m}$; crack thickness $\left(t_{l} / 2\right)=5 \times 10^{-8} \mathrm{~m}$; crevice length $(c / 2)=5 \times 10^{-4} \mathrm{~m}$; and crevice thickness $\left(t_{c}\right)=$ $1 \times 10^{-6} \mathrm{~m}$. 


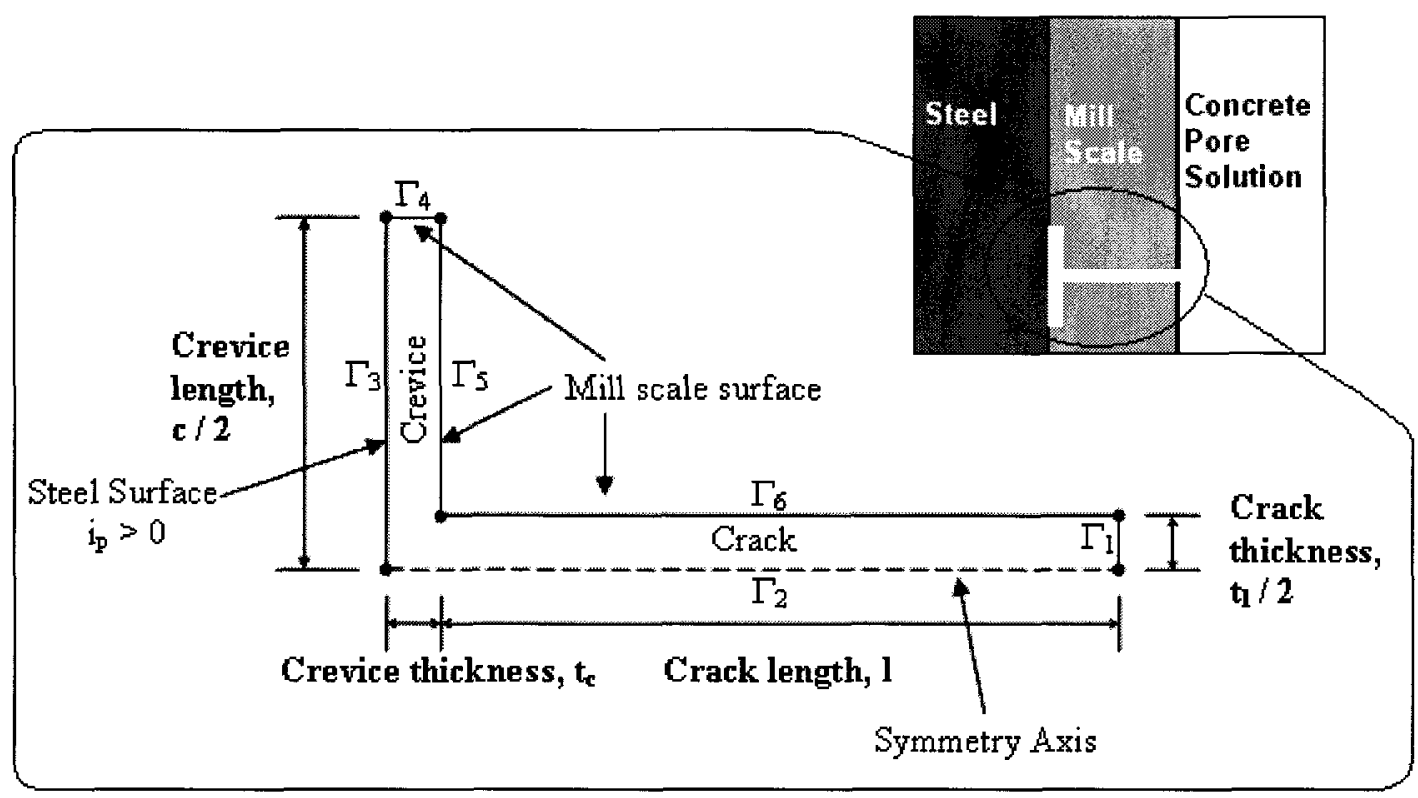

Figure 6.12: The solution domain representing the crack in the mill scale and the crevice between the surface of steel and mill scale (Karadakis 2009).

The boundary conditions for steel and mill scale surfaces (i.e., boundaries 2-6) during the solution of the extended Nernst-Planck Eq. 6.3 were defined as Neumann conditions (Ghods et al. 2007, Ghods et al. 2008):

$$
n \cdot N_{i}=\frac{i}{n_{e} F}
$$

where $n$ is the direction normal to the boundary, $n_{e}$ refers to the number of electrons exchanged in the reaction and $i$ refers to the current density, representing the rate of electrochemical reactions taking place on the steel surface.

The solution of Poisson's Eq. 6.4 provides a potential gradient $(\nabla \phi)$ in the electrical migration term of the extended Nernst-Plank Eq. 6.3. Since it was assumed that no external current was applied, for the solution of the Poisson's equation, all boundaries, except for boundary 1 , were represented by the Neumann condition: 


$$
n \cdot \nabla \phi=0 \text { on } \Gamma_{2}, \Gamma_{3}, \Gamma_{4}, \Gamma_{5} \text { and } \Gamma_{6}
$$

Eq. 6.12

Boundary 1 was defined as a Dirichlet condition. Since the ultimate goal of the solution of the Poisson's equation is to solve for the potential gradient $(\nabla \phi)$, the value of the Dirichlet condition defined on boundary 1 is not important as long as all other boundaries are defined as "no flux" Neumann conditions; the gradients of the potentials will be the same regardless of the value of the potential used as the Dirichlet condition on boundary 1. For convenience, the Dirichlet condition on boundary 1 was defined as:

$$
\phi=0 \mathrm{~V} \text { on } \Gamma_{1}
$$

The simulations were carried out in the $\mathrm{CH}$ solution; the chloride concentration in the bulk $\mathrm{CH}$ solution was assumed to be $500 \mathrm{~mol} / \mathrm{m}^{3}$, a chloride concentration that would likely to cause depassivation of rebar. The passive current density on the steel surface was assumed to be equal to $0.01 \mathrm{~A} / \mathrm{m}^{2}$. Diffusion coefficients of species were assumed to linearly decrease from $D_{\max }$ to $D_{\min }$, as per Table 6.3.

Table 6.3: Transport properties of all species in the concrete pore solutions (Karadakis 2009)

\begin{tabular}{|l|l|l|}
\hline Species & $D_{\max _{i}} @ c_{F e_{(s)}}=0$ & $D_{\min _{i}} @ c_{F e_{(s)}} 37,840 \mathrm{~mol} / \mathrm{m}^{3}$ \\
\hline $\mathrm{OH}^{-}$ & $5.28 \times 10^{-9}$ & $5.28 \times 10^{-13}$ \\
$\mathrm{Fe}^{2+}$ & $0.72 \times 10^{-9}$ & $0.72 \times 10^{-13}$ \\
$\mathrm{Ca}^{2+}$ & $0.79 \times 10^{-9}$ & $0.79 \times 10^{-13}$ \\
$\mathrm{Cl}^{-}$ & $2.03 \times 10^{-9}$ & $2.03 \times 10^{-13}$ \\
$\mathrm{Na}^{+}$ & $1.33 \times 10^{-9}$ & $1.33 \times 10^{-13}$ \\
$\mathrm{O}_{2}$ & $2.20 \times 10^{-9}$ & $2.20 \times 10^{-13}$ \\
\hline
\end{tabular}

The numerical solution of Eqs. 6.3 and 6.4 within the domain, with the imposed constraints, initial and boundary conditions, provide the solutions for 8 concentrations 
$\left(c_{\mathrm{OH}^{-}}, c_{\mathrm{OH}_{(s)}}, c_{\mathrm{Fe}^{2+}}, c_{\mathrm{Fe}_{(s)}}, c_{\mathrm{Ca}^{2+}}, c_{\mathrm{Cl}^{-}}, c_{\mathrm{Na}^{+}}, c_{\mathrm{O}_{2}}\right)$ and electric potential $(\phi)$ over time.

This boundary value problem solved using a commercial finite element analysis software called COMSOL Multiphysics v3.5. COMSOL software is a generic finite element package, which allows the implementation of an unrestricted number of coupled partial differential equations. The software has its own mesh generation routines and offers several alternatives for nonlinear and transient solution algorithms, which are explained in more detail by Karadakis (2009).

The $\mathrm{pH}$ and $\mathrm{Cl}^{-} / \mathrm{OH}^{-}$along the mill scale crack (boundary 2) and crevice (boundary 3) are plotted in Fig. 6.13. The results of analysis were taken when the steadystate condition was achieved. The simulation shows $\mathrm{pH}$ decreasing and $\mathrm{Cl}^{-} / \mathrm{OH}^{-}$ increasing from the bulk pore solution (point B) to the tip of the crevice (point A). The $\mathrm{pH}$ in the bulk solution is around 12.5 that drops to $\sim 12.1$ inside the crevice. The $\mathrm{Cl}^{-} / \mathrm{OH}^{-}$ within the crevice area $(\sim 34)$ is remarkably higher (around three times) than $\mathrm{Cl}^{-} / \mathrm{OH}^{-}$of bulk solution $(\sim 12)$. As mentioned in Chapter $5, \mathrm{Cl}^{-} / \mathrm{OH}^{-}$is a critical factor that determines the breakdown of passive film and subsequent corrosion initiation of rebar in concrete. Therefore, the numerical results in Fig. 6.13 strongly support the proposed mechanism (see Fig. 6.11), in which the increase of $\mathrm{Cl}^{-} / \mathrm{OH}^{-}$inside the crevice at the mill scale/steel interface might be responsible for the lower chloride thresholds of as-received rebars in comparison with the turned-and-polished rebars (see Table 5.4 in Chapter 5). 


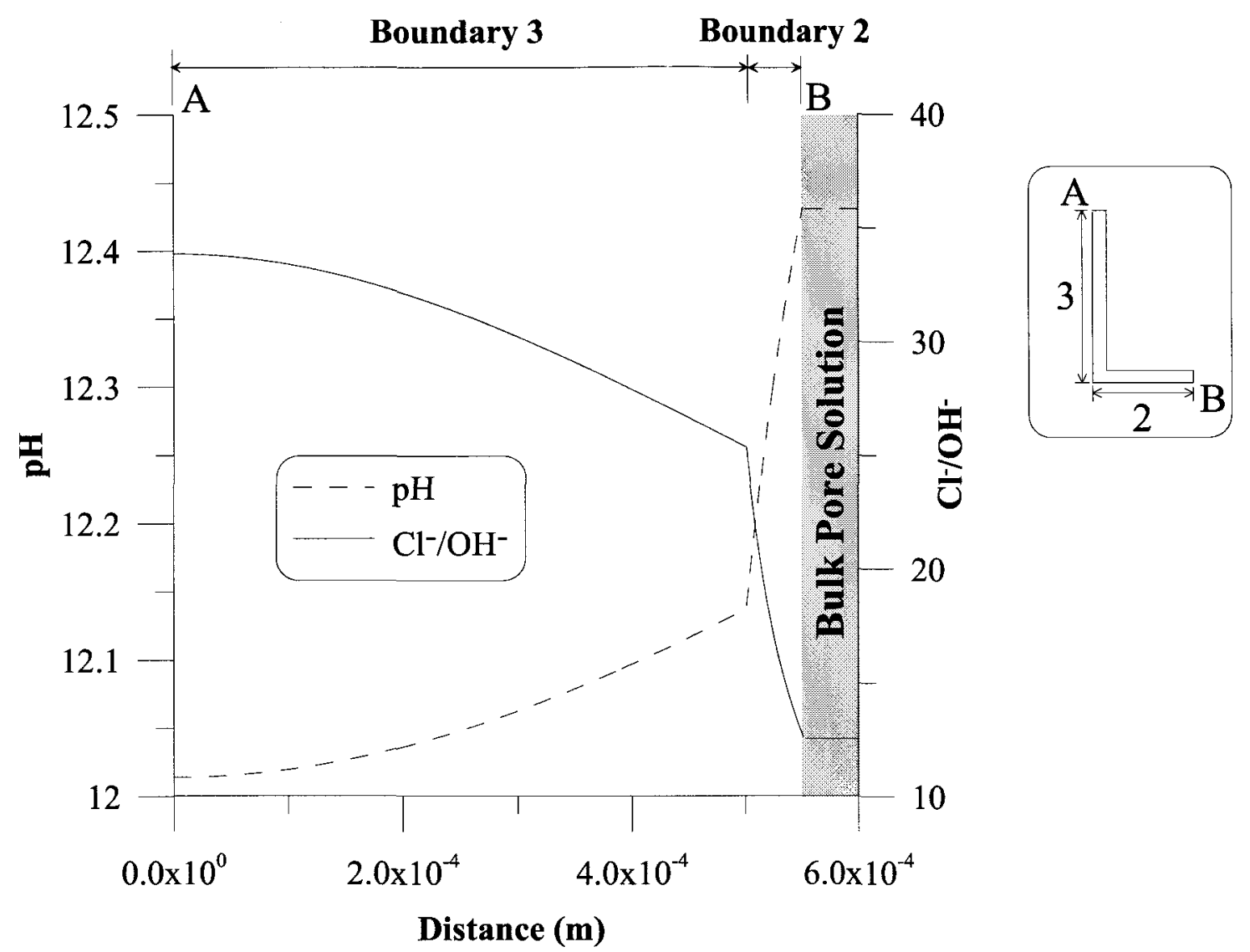

Figure 6.13: $\mathrm{pH}$ and $\mathrm{Cl} / \mathrm{OH}$ along boundaries 2 and 3 (from point $B$ to point $A$ ).

\subsection{Conclusions}

In this chapter, a microscopic investigation was carried out to study the properties of mill scale that form on rebar surface and the confounding role of chlorides in corrosion initiation. Numerical studies were also conducted to support the speculations about the effect of mill scale on corrosion initiation of rebar. The main conclusions of the study are as follows:

- The interface of steel and mill scale is filled with voids and crevices, and the mill scale contains cracks. In some locations on the steel surface, these cracks transversely link the voids and crevices to the free surface, hence, providing 
pathways for the concrete pore solution to penetrate into the voids and the crevices.

- Crevices between mill scale and steel provide sites for pit nucleation (depassivation). Although these initial pits are under the mill scale and are not visible when the surface of the rebar is observed, their formation can be identified electrochemically. Visible pits on the rebar surface form well after the depassivation can be observed electrochemically.

- Mill scale shows variable characteristics in terms of its physical and chemical properties. The results of this study suggest that the variability associated with reported chloride thresholds may be partly attributed to the variability in mill scale properties resulting from the variability originating from the manufacturing process of rebars.

- Using both SEM and finite element analysis techniques, the present study provides an explanation for as-received rebars' high susceptibility to corrosion initiation as compared to rebars without mill scale.

- The proposed mechanism, which is similar to that of typical crevice corrosion, explains that $\mathrm{Cl}^{-} / \mathrm{OH}^{-}$within crevices of as-received rebar may become larger than that of the bulk solution. 


\section{Chapter 7}

\section{TEM Study on Passive Film and Chloride-induced Depassivation of Rebar}

\subsection{Introduction}

Understanding the depassivation mechanism of carbon steel rebar in concrete due to chloride attack is key to the development of methods to mitigate problems associated with chloride-induced corrosion initiation in reinforced concrete structures. Although a number of researchers have proposed general mechanistic models (Kolotyrkin 1961, Leckie and Uhlig 1966, McBee and Kruger 1972, Hoar et al. 1965, Lin et al. 1981, Kruger 1976, Sato 1971, Alvarez and Galvele 1984), they do not explain all experimental observations regarding the depassivation of carbon steel, particularly in highly alkaline environments such as concrete pore solution; therefore, additional studies are needed.

The investigation of passive films and depassivation processes is challenging and requires the use of nano-scale techniques such as XPS, SIMS, Raman spectroscopy and TEM (Goetz et al. 1986, Landolt et al. 1990, Khalil et al. 1985). So far, only a few studies using some of these techniques have been carried out to study iron or steel passivity in highly alkaline environments (Zakroczymski et al. 1985a, Zakroczymski et al. 1985b, Sarver and Szklarska-Smialowska 1984, Joiret et al. 2002, Galicia et al. 2006, 
Schmuki et al. 1999); and, to the author's knowledge, TEM has not been used to investigate the passivity and depassivation of carbon steel in concrete pore solutions.

In this study, various TEM imaging (e.g. bright-field and dark-field) and analysis (e.g. selected area electron diffraction - SAD, convergent beam electron diffraction - CBED, energy-dispersive X-ray spectrometry - EDS, and electron energy loss spectroscopy - EELS) methods were used as a nano-scale techniques to study the characteristics of passive films on carbon steel grown in concrete pore solutions in the presence of chlorides.

\subsection{Experimental}

\subsubsection{Specimen preparation}

TEM samples were taken from the cross sections of $10 \mathrm{~mm}$ long rebar specimens cut from as-received deformed carbon steel rebar with $10 \mathrm{~mm}$ nominal diameter. The chemical composition of the steel is given in Table 7.1. Six rebar samples were mounted in low-shrinkage epoxy under vacuum. The cross sections of each coldmounted specimen (see Fig. 7.1) were polished to $1 \mu \mathrm{m}$ using anhydrous (water-free) isopropyl alcohol. After polishing, all cold-mounted specimens were kept in anhydrous alcohol to avoid exposure to air until they were transferred to the beakers containing simulated concrete pore solutions to allow the formation of passive films on the cross sections of the rebar specimens.

Two types of passivating solutions, which were labeled as $\mathrm{CH}$ and $\mathrm{CP}$, were used. The label " $\mathrm{CH}$ " refers to a saturated calcium hydroxide solution, where " $\mathrm{C}$ " stands for calcium and " $\mathrm{H}$ " stands for hydroxide, and the label " $\mathrm{CP}$ " refers to a simulated 
concrete pore solution, where "C" stands for concrete and "P" stands for pore. The $\mathrm{CH}$ solution ( $\mathrm{pH}$ 12.5) was prepared by dissolving the analytical grade calcium hydroxide in distilled water. The CP solution ( $\mathrm{pH}$ 13.3) was prepared by dissolving calcium hydroxide $\left(\mathrm{Ca}(\mathrm{OH})_{2}\right)$, sodium hydroxide $(\mathrm{NaOH})$, potassium hydroxide $(\mathrm{KOH})$ and calcium sulfate $\left(\mathrm{Ca}\left(\mathrm{SO}_{4}\right)\right)$ in distilled water. The chemical composition and properties of these two solutions are presented in Table 7.2. The actual concentrations of the anions and cations in solution were measured using Inductively Coupled Plasma - Atomic Emission Spectroscopy (ICP-AES) (Model: Varian Vista RL).

Table 7.1: Elemental composition of the rebar used in the TEM investigation (Average of three spectrographic results).

\begin{tabular}{|l|l|}
\hline Element & Weight \% \\
\hline $\mathrm{C}$ & 0.26 \\
$\mathrm{Si}$ & 0.27 \\
$\mathrm{Mn}$ & 1.10 \\
$\mathrm{Cr}$ & 0.05 \\
$\mathrm{Ni}$ & 0.07 \\
$\mathrm{Mo}$ & $<0.01$ \\
$\mathrm{Cu}$ & 0.21 \\
$\mathrm{Al}$ & $<0.005$ \\
$\mathrm{Nb}$ & $<0.01$ \\
$\mathrm{~V}$ & $<0.005$ \\
$\mathrm{Ti}$ & $<0.005$ \\
$\mathrm{~B}$ & $<0.0005$ \\
$\mathrm{P}$ & 0.01 \\
$\mathrm{~S}$ & 0.03 \\
$\mathrm{~W}$ & $<0.01$ \\
$\mathrm{Sn}$ & 0.021 \\
$\mathrm{Co}$ & 0.01 \\
$\mathrm{Zr}$ & $<0.01$ \\
$\mathrm{Fe}$ & Balance \\
\hline
\end{tabular}


Table 7.2: Concentrations of species and properties of the simulated concrete pore solutions $C H$ and CP. The actual concentrations of the anions and cations in solution were measured using Inductively Coupled Plasma - Atomic Emission Spectroscopy (ICPAES) (Model: Varian Vista RL).

\begin{tabular}{|c|c|c|c|c|c|c|c|c|c|c|c|}
\hline \multirow[b]{2}{*}{ Solution } & \multicolumn{4}{|c|}{$\begin{array}{l}\text { Added compounds } \\
(\mathrm{M})\end{array}$} & \multicolumn{4}{|c|}{$\begin{array}{c}\text { Measured ion } \\
\text { concentrations } \\
(\mathrm{mg} / \mathrm{l})\end{array}$} & \multirow{2}{*}{ pH } & \multirow{2}{*}{$\begin{array}{c}\sigma^{*} \\
(\mathrm{mS} / \mathrm{cm})\end{array}$} & \multirow{2}{*}{$\begin{array}{l}\mathrm{C}_{\mathrm{O} 2}^{* *} \\
(\mathrm{mg} / \mathrm{l})\end{array}$} \\
\hline & $\mathrm{Ca}(\mathrm{OH})_{2}$ & $\mathrm{Na}(\mathrm{OH})$ & $\mathrm{K}(\mathrm{OH})$ & $\mathrm{CaSO}_{4}$ & $\mathrm{Ca}^{2+}$ & $\mathrm{Na}^{+}$ & $\mathrm{K}^{+}$ & $\mathrm{SO}_{4}{ }^{2-}$ & & & \\
\hline $\mathrm{CH}$ & 0.1 & 0 & 0 & 0 & 814 & 3 & 0.4 & 2 & 12.5 & 8.82 & 2.28 \\
\hline CP & 0.1 & 0.1 & 0.2 & 0.003 & 3 & 2232 & 8059 & 277 & 13.36 & 64.9 & 2.27 \\
\hline
\end{tabular}

Conductivity of the solution

*** Dissolved oxygen concentration

Six cold-mounted rebar specimens were split into two groups of three, and each group was transferred to a beaker containing one of the simulated concrete pore solutions. The specimens were kept in the solutions for 15 days to allow the formation of stable passive films on the cross sections. Previous electrochemical experiments have shown that a minimum of 8 days of immersion in concrete pore solution is required for the formation of a stable passive film on the surface of rebar (Ghods et al. 2009, Poursaee and Hansson 2007). After passivation, one specimen was taken out from each solution; these specimens, labeled as "CH-0" and "CP-0", were used to investigate the passive films before exposure to chlorides. 


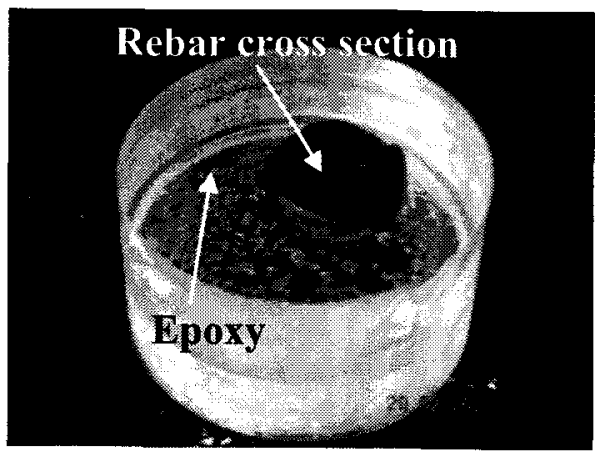

Figure 7.1: A cold-mounted rebar specimen that was used in the FIB-TEM study. The TEM samples were extracted from the cross section of the rebar.

The effect of chlorides on the passive films was investigated with two levels of chloride concentration chosen for $\mathrm{CP}$ and $\mathrm{CH}$ solutions: a 'low level' that is below the chloride threshold and a 'high level' that is above the chloride threshold. Electrochemical studies in Chapter 5 have shown that similarly polished rebar specimens in the $\mathrm{CH}$ and $\mathrm{CP}$ solutions depassivated at chloride thresholds between 0.05 and $0.15 \mathrm{M}$ and between 0.45 and 1.25 $\mathrm{M}$, respectively. Therefore, to simulate chloride exposure below the threshold level, $0.05 \mathrm{M}$ and $0.45 \mathrm{M}$ analytical-grade $(99.9 \%)$ sodium chloride $(\mathrm{NaCl})$ were added to the $\mathrm{CH}$ and $\mathrm{CP}$ solutions, respectively. The specimens were kept in these solutions for two weeks to give chlorides time to react with the passive films. After the two-week exposure period, one specimen was taken out of each solution; these specimens, labeled as "CH-1" and "CP-1", were used to investigate the passive films exposed to chloride concentrations below the threshold levels. Following this, the chloride concentrations in the $\mathrm{CH}$ and $\mathrm{CP}$ solutions containing the remaining rebar specimens were increased to $0.45 \mathrm{M}$ and $3 \mathrm{M}$, respectively, which are beyond the corresponding typical chloride thresholds. After two weeks of exposure, these final specimens, labeled as "CH-2" and "CP-2", were taken out of the solutions. The chloride 
concentrations at which the specimens were taken out of the solutions are summarized in Table 7.3.

Immediately after each rebar specimen was taken out of the solution, and dried with nitrogen gas, the cross sections of the specimens were coated with a $\sim 300 \mathrm{~nm}$ layer of sputtered gold to prevent oxidation. They were kept in a dessicator until FIB sampling.

Table 7.3: Rebar specimen designations and corresponding chloride exposure.

\begin{tabular}{|l|c|}
\hline Sample & $\begin{array}{c}\text { Chloride } \\
\text { concentration } \\
(\mathbf{M})\end{array}$ \\
\hline $\mathrm{CH}-0$ & 0 \\
$\mathrm{CP}-0$ & 0 \\
$\mathrm{CH}-1$ & 0.05 \\
$\mathrm{CP}-1$ & 0.45 \\
$\mathrm{CH}-2$ & 0.15 \\
$\mathrm{CP}-2$ & 3 \\
\hline
\end{tabular}

\subsubsection{FIB sampling}

Each specimen was transferred to the focused ion beam (FIB) microscope. A layer of tungsten (W) was sputtered on a $\sim 200 \mu \mathrm{m}$ by $\sim 50 \mu \mathrm{m}$ area of interest on the gold-covered cross section of the rebar specimen. The FIB H-bar lift-out method was used to extract the TEM sample from the gold and tungsten covered cross section of the rebar specimen. The four stages of FIB H-bar lift-out technique used in this study are schematically illustrated in Fig. 7.2. A gallium ion beam was used to make two trenches on the surface of the specimen (Fig. 7.2(a)). The edges of the area between the two trenches were then cut in order to release the sample from the bulk material (Fig, 7.2(a)). The released sample was placed on a copper TEM grid using a micro-manipulator, as shown in Figs. 7.2(b) and 7.2(c). The middle part of the sample was milled to $\sim 100 \mathrm{~nm}$ 
thickness using gallium ion beam (Fig. 7.2(d)) so that it is electron transparent. The planar dimensions of each TEM sample were $\sim 20 \mu \mathrm{m} \mathrm{x} \sim 10 \mu \mathrm{m}$, as shown in Fig. 7.2(e).

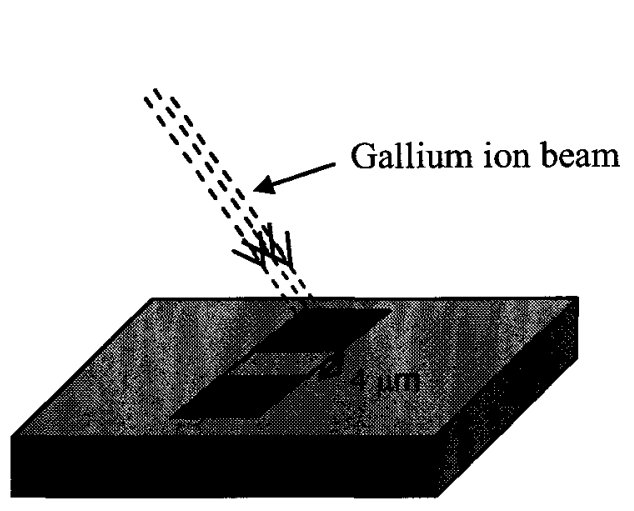

(a)

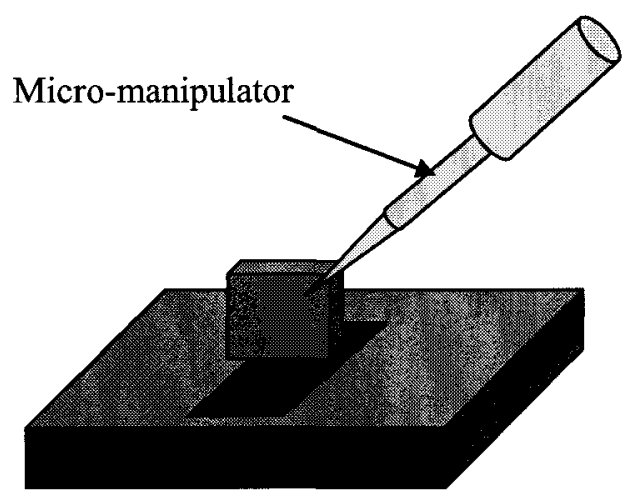

(b)

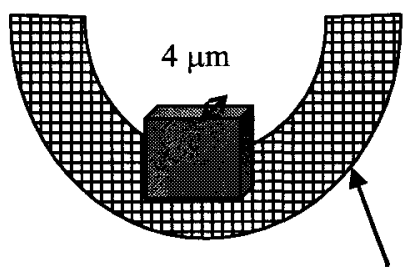

(c)

$$
\text { TEM grid }
$$

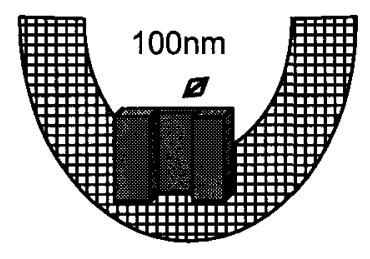

(d)

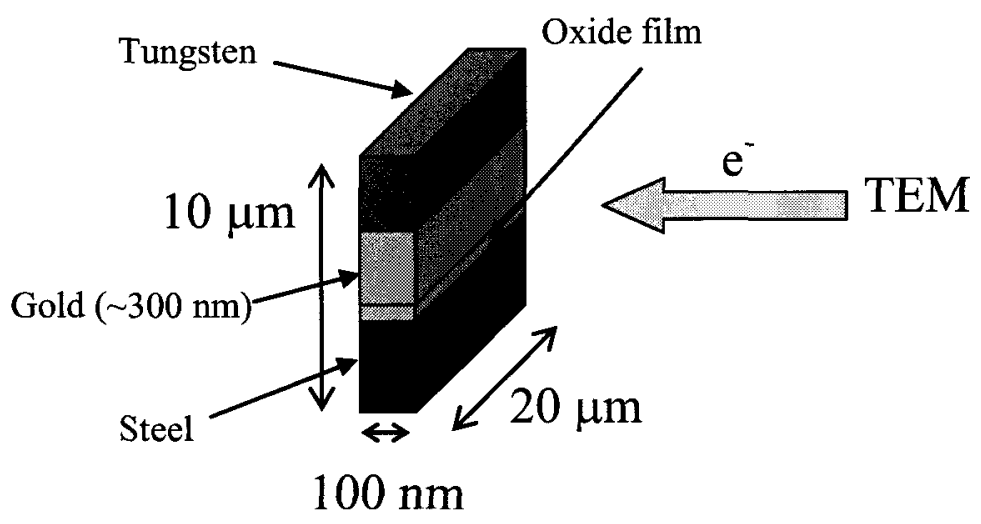

(e)

Figure 7.2: Schematic illustration of the stages used for TEM sample preparation using FIB H-bar lift-out technique: (a) cutting using Gallium ion beam, (b) lifting the sample using a micro-manipulator; (c) placing the sample on a TEM grid; (d) further thinning using Gallium ion beam; (e) the TEM sample. 


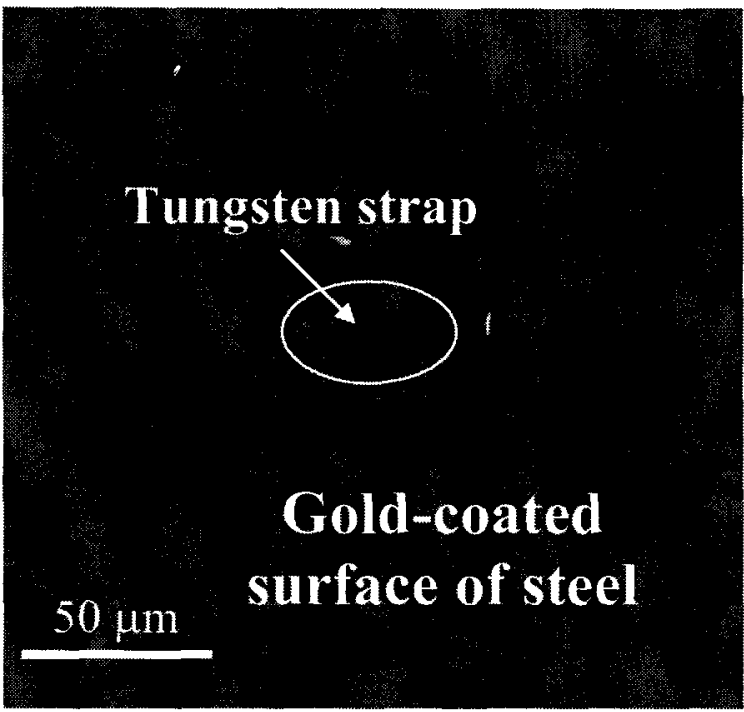

(a)

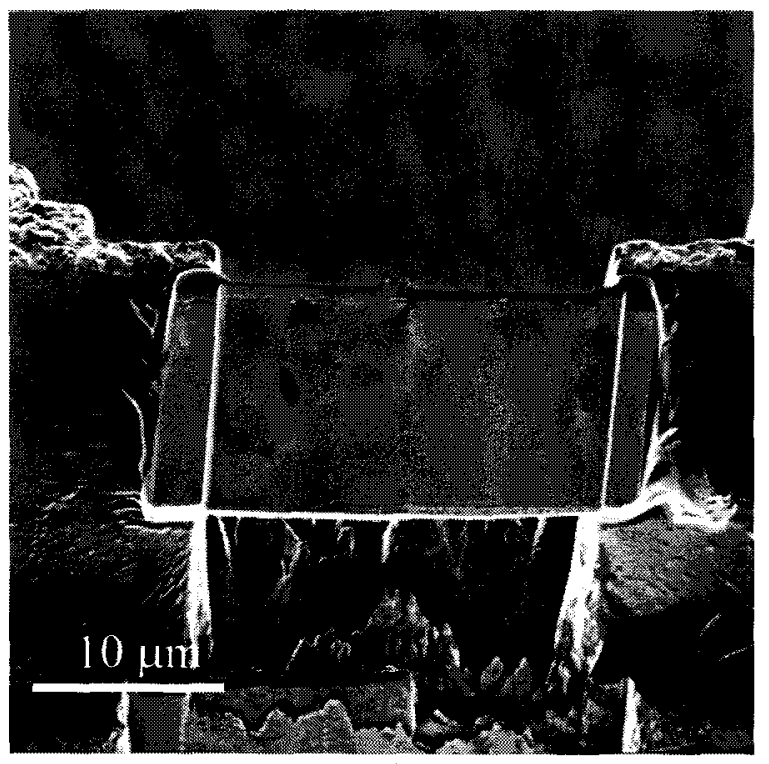

(c)

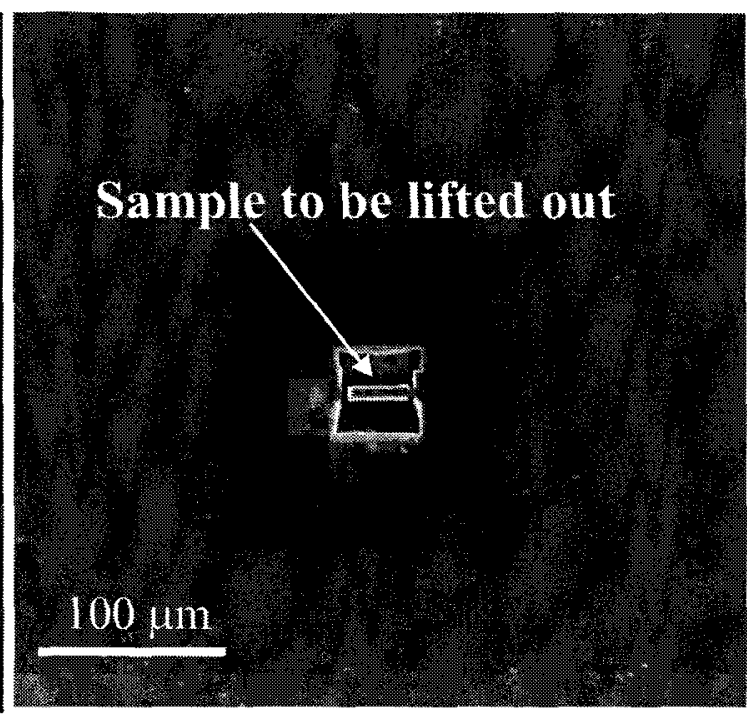

(b)

Figure 7.3: FIB images of TEM sample preparation process from the cross section of the rebar specimen: (a) goldcoated surface of steel and a Tungsten strap; (b) a released TEM sample (see Fig. 7.2(b)); (c) process of thinning the TEM sample in the FIB microscope using the Gallium ion beam (see Fig. 7.2(d)).

The FIB microscope images taken during FIB sampling are shown in Fig. 7.3. In Fig. 7.3(a), a Tungsten strap can be observed at the site of interest on the surface of steel that is already covered with a thick layer of gold. After the milling process, the released sample is ready to be lifted out for further thinning (Fig.7.3 (b)). The sample during the thinning process is presented in Fig. 7.3(c). 


\subsubsection{TEM microscopy}

The prepared TEM samples were examined in the transmission electron microscope (TEM) (Model: Philips CM20FEG) equipped with a Schottky field emission gun operated at the voltage of $200 \mathrm{kV}$. Conventional bright-field TEM imaging was used to visualize the passive film. Dark-field TEM imaging was also used to determine the amorphous and crystalline phases. The SAD and CBED techniques were employed as an aid to phase identification. Chemical analysis was performed using a thin-window EDS detector from Oxford Instruments equipped with an INCA system analyzer. A gatan imaging filter (GIF) (model 678) was used for energy-filtered imaging as well as parallel electron energy loss spectroscopy (PEELS). EDS analyses were obtained in scanning transmission electron microscopy (STEM) mode using a probe diameter of $1 \sim 2 \mathrm{~nm}$. EELS spectra were collected in diffraction mode (image coupling) with a probe diameter of $10 \mathrm{~nm}$.

\subsection{Results and Discussion}

\subsubsection{TEM-image analysis}

7.3.1.1 Samples passivated in chloride-free solutions: $\mathrm{CH}-0$ and $\mathrm{CP}-0$ TEM micrographs of the $\mathrm{CH}-0$ and $\mathrm{CP}-0$ samples exposed to chloride-free $\mathrm{CH}$ and CP passivating solutions are shown in Figs. 7.4(a) and 7.4(b), respectively. A thin oxide film between the steel substrate and the gold layer is visible in both TEM samples. Both oxide films have relatively uniform thickness; however, the oxide film formed in the $\mathrm{CP}$ solution was slightly thicker than the one in the $\mathrm{CH}$ solution. From these images, 
it is difficult to extract an exact thickness for either of the films, mainly due to the fact that the TEM samples were approximately $100 \mathrm{~nm}$ thick, and the layers that are visible in Figs. 7.4(a) and 7.4(b) represent the two dimensional projection of the oxide films across the depth of the TEM samples. Atomic force microscopy (AFM) studies (see Appendix B) conducted on the surface of specimens before exposure to the passivating solutions revealed that the topographic variation (RMS) of the polished steel surface was limited to $5 \mathrm{~nm}$; therefore, it is expected that the oxide films in both samples are thinner than the thickest part of the films in the TEM micrographs given in Fig. 7.4. The thickness of the oxide films on the $\mathrm{CH}-0$ and $\mathrm{CP}-0$ samples can be estimated to range from 5 to $8 \mathrm{~nm}$ and 8 to $13 \mathrm{~nm}$, respectively.

EDS analysis was carried out to investigate the elemental composition of the oxide films of both TEM samples. Typical EDS spectra obtained at the middle of oxide films grown in the $\mathrm{CH}$ and $\mathrm{CP}$ solutions are presented in Figs. 7.5(a) and 7.5(b), respectively. EDS analyses of different locations along the oxide film provided similar spectra. Oxygen and iron peaks in the spectra are the indication of the presence of iron oxides. Gold and copper peaks are associated with the gold coating layer on the oxide film and the TEM copper grid that was used to support the TEM samples in the FIB microscope, respectively. The gallium peak is a result of the use of gallium ion beam during the FIB milling process. In addition, trace amounts of manganese, silicon and carbon in the EDS spectra originate from the steel substrate. However, potassium and sulfur peaks observed in the spectra of the CP-0 sample suggest that potassium and sulfate ions in the $\mathrm{CP}$ solution might be incorporated into the structure of oxide film. 
Calcium was also detected in the spectra of the $\mathrm{CH}-0$ sample; this can be attributed to the presence of calcium in the structure of oxide film.
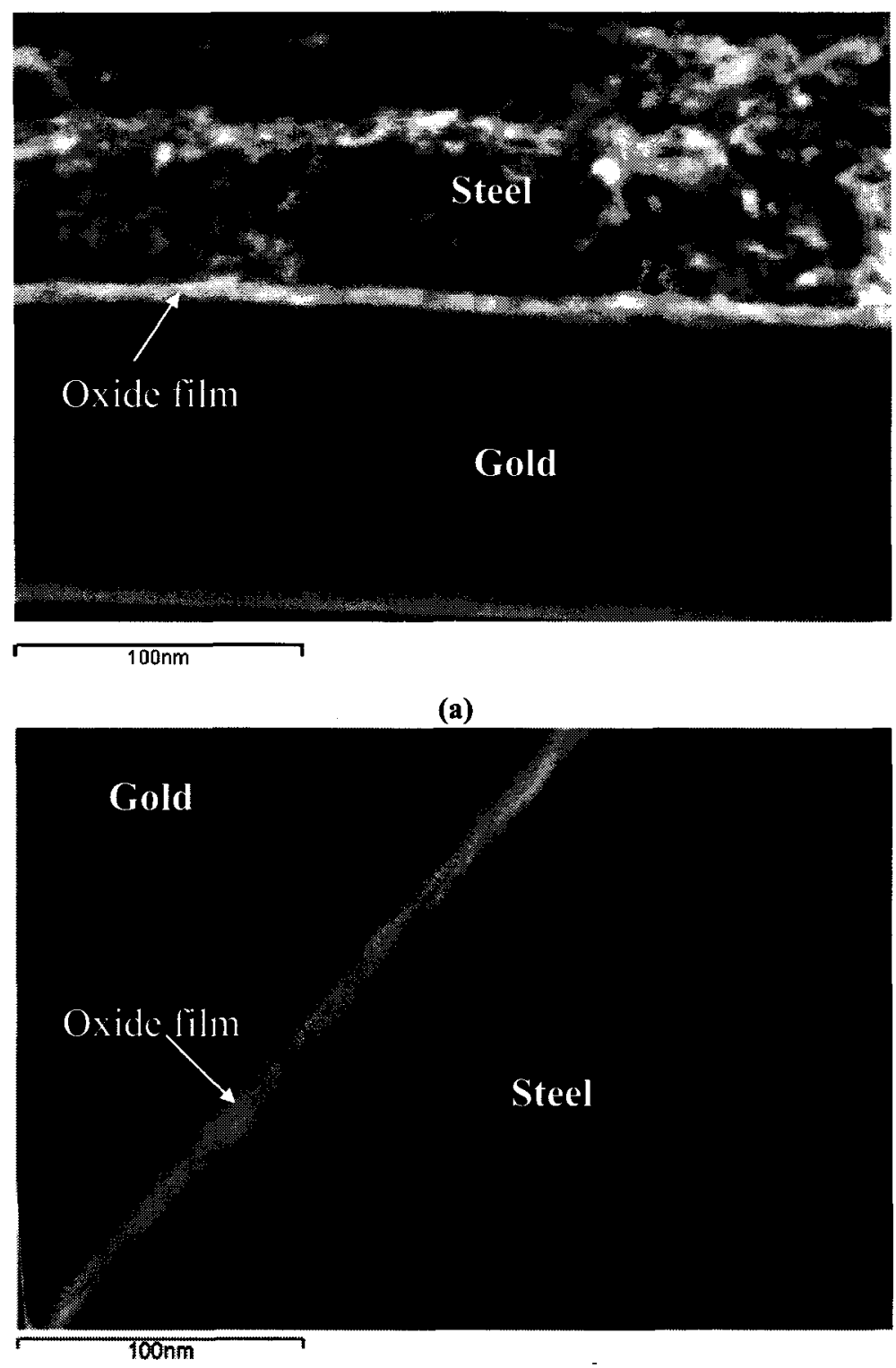

(b)

Figure 7.4: TEM images of the samples passivated in chloride-free solutions: (a) $\mathrm{CH}-0$ (film thickness: 5 to $8 \mathrm{~nm}$ ); (b) CP-O (film thickness: 8 to $13 \mathrm{~nm}$ ). 


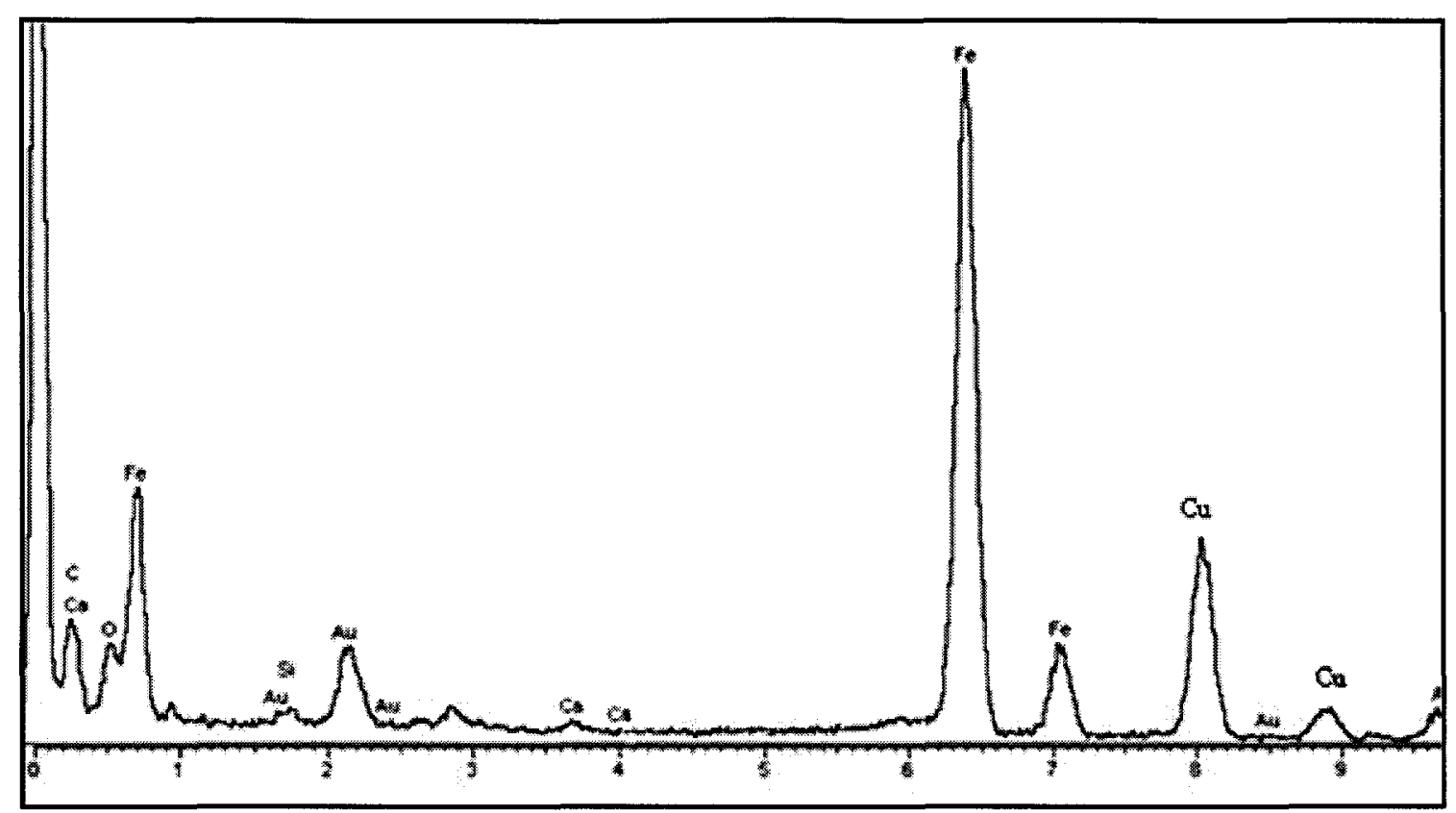

(a)

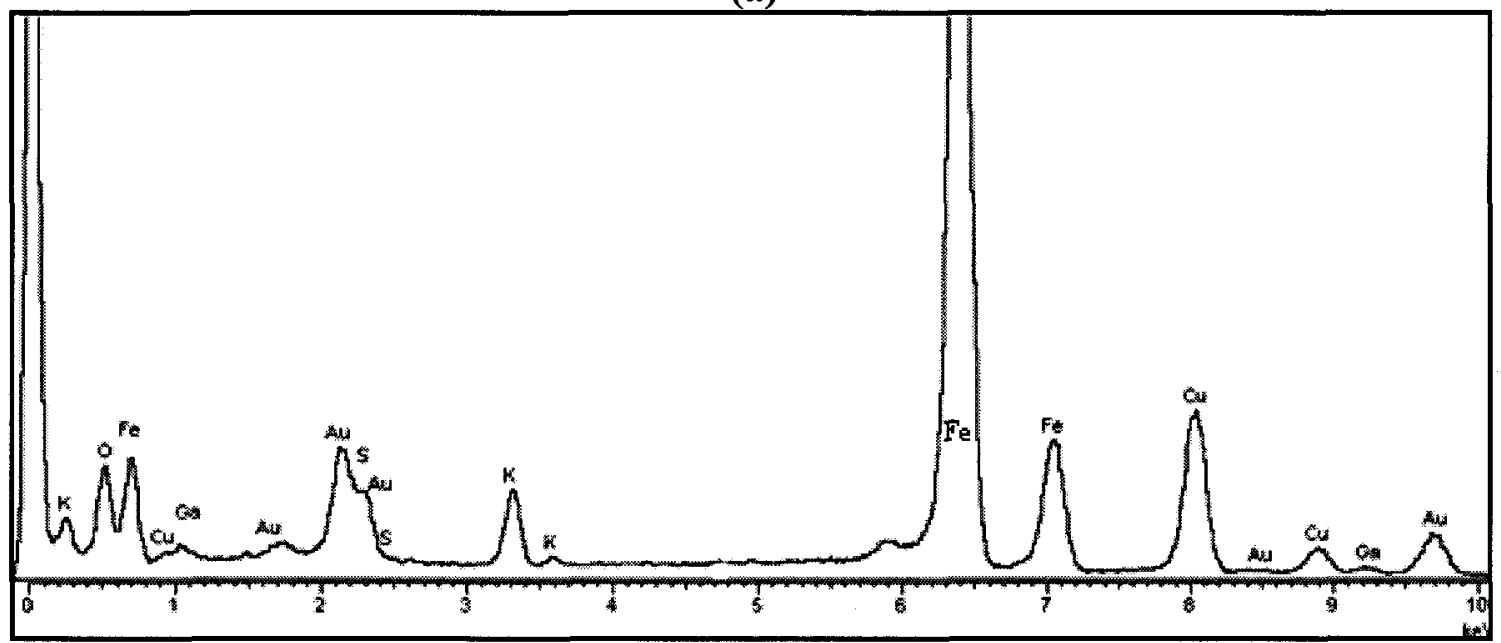

(b)

Figure 7.5: Typical EDS spectra of oxide film formed in the chloride-free (a) $\mathrm{CH}$ solution; b) CP solution.

To investigate the oxide film composition in more detail, further EDS analyses were carried out on the $\mathrm{CH}-0$ and $\mathrm{CP}-0$ samples. It must be emphasized that the relative concentrations of elements of low atomic number, such as oxygen, obtained from EDS analysis in a TEM are subject to significant errors, because of the strong absorption of low energy X-rays by heavier elements such as iron. Figure 7.6 illustrates the EDS 
analysis from a line scan that was carried out along the oxide film of the $\mathrm{CH}-0$ sample. Figure 7.6(a) shows the locations where the EDS analysis was conducted, and Fig. 7.6(b) presents the atomic percentage of the major elements along the line. The profile shows that although the $\mathrm{Fe} / \mathrm{O}$ may not be accurate, oxygen and iron concentrations in the oxide film along the line scan are relatively constant nominally at $50-60 \%$ and $20-30 \%$, respectively. Gold and silicon signals are respectively from the coated surface of the specimen and the steel substrate.
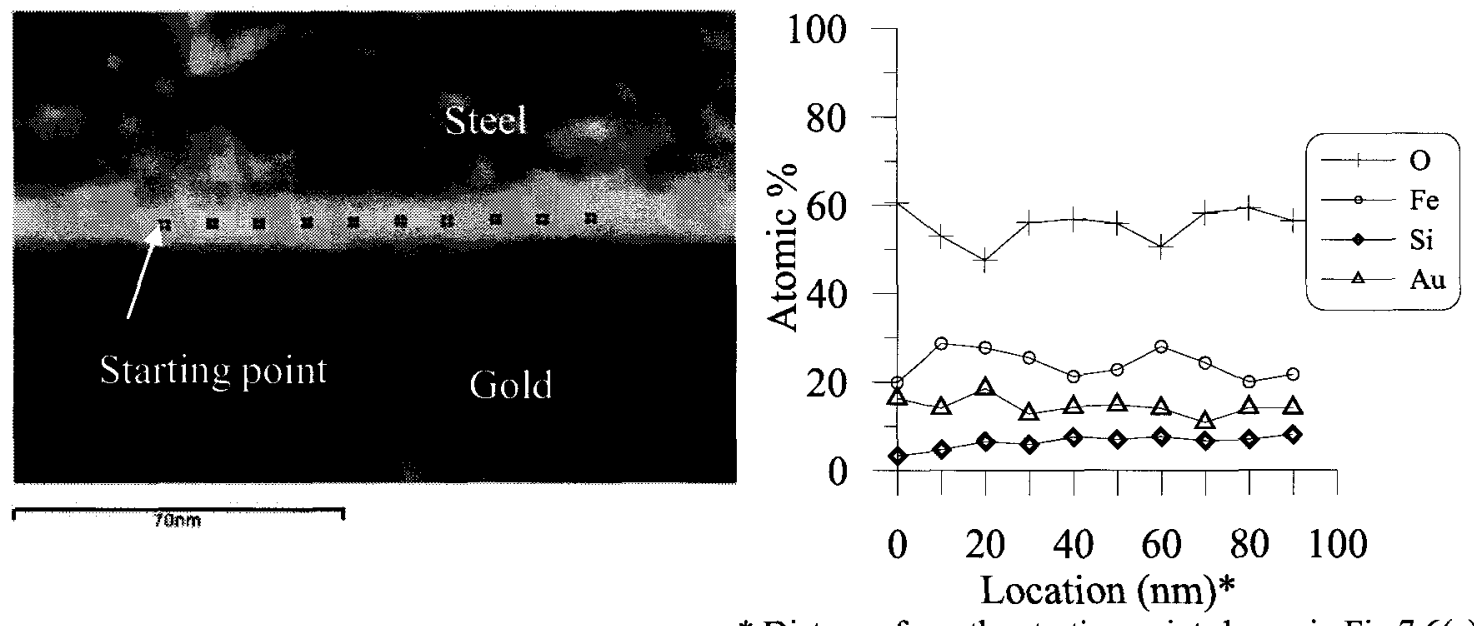

(a) (b)

Figure 7.6: EDS line scan inside the oxide film parallel to the steel surface of the CH-O sample: (a) TEM image showing the locations of the analysis; (b) EDS line-scan results corresponding to the points shown in the TEM image. For clarity, only elements with the atomic \% higher than $1 \%$ are presented. 

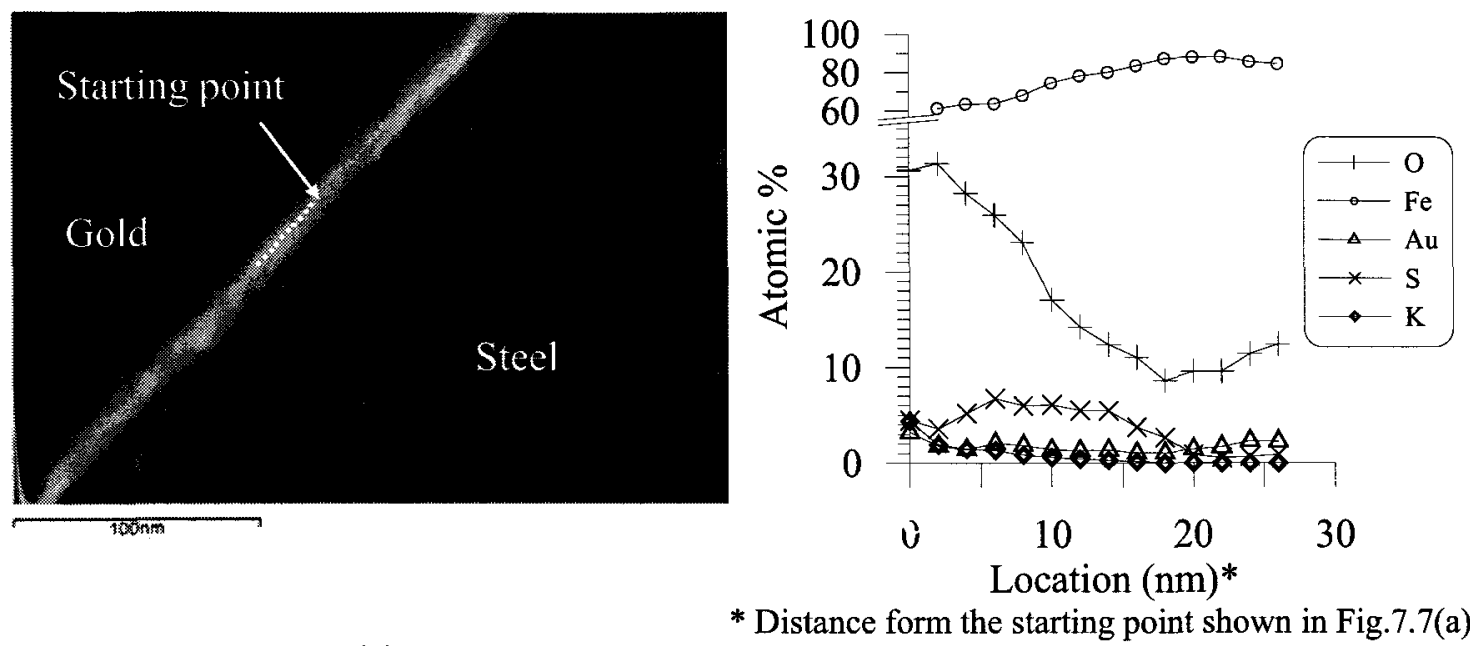

(a)

(b)

Figure 7.7: EDS line scan inside the oxide film parallel to the steel surface of the CP-O sample: (a) TEM image showing the locations of the analysis; (b) EDS line-scan results corresponding to the points shown in the TEM image.

Figure 7.7 illustrates the EDS analysis of a line scan that was carried out along the oxide film of the CP-0 sample. Figure 7.7(a) shows the locations where the EDS analysis was conducted, and Fig. 7.7(b) presents the relative atomic percentage of the major elements along the line. Unlike the CH-0 sample, the oxide film of the CP-0 sample shows variations in oxygen and iron peaks. This suggests that the oxide structure in the CP-0 might be more complex than that of the $\mathrm{CH}-0$ sample. However, this variation may also be an artifact of the variation of the surface roughness along the TEM sample; as discussed previously, the samples are around 100-nm thick, and the EDS readings at each point is the average for the entire thickness of the sample. It should also be noted that the presence of sulfur and potassium peaks also suggests that the ions in the CP solution may be involved in the structure of the film.

To study the variation of the oxide film composition with thickness, further EDS analyses across the oxide films of the $\mathrm{CH}-0$ and $\mathrm{CP}-0$ samples were carried out. The EDS results across the oxide film of the $\mathrm{CH}-0$ sample are presented in Fig. 7.8. Figure 7.8(a) 
shows the locations where the EDS analyses were conducted, and Fig. 7.8(b) presents the relative atomic percentage of the major elements along the line. As shown in Fig. 7.8(b), the intensity of the iron signal decreases, while the oxygen concentration increases, as the analysis point is further away from the steel surface. As a result, the $\mathrm{Fe} / \mathrm{O}$ decreases towards the outer layer of the oxide film. A similar trend is also seen in Fig. 7.9 for the EDS analysis of the CP-0 sample. In addition, it can be observed in Fig. 7.9(b) that sulfur and potassium signals, which are respectively the indicators of sulfate and potassium ions of the CP solution, at the outer layer of the oxide film are stronger than those at the inner layer. This may indicate that CP solution may have penetrated into the outer layer of the oxide film.
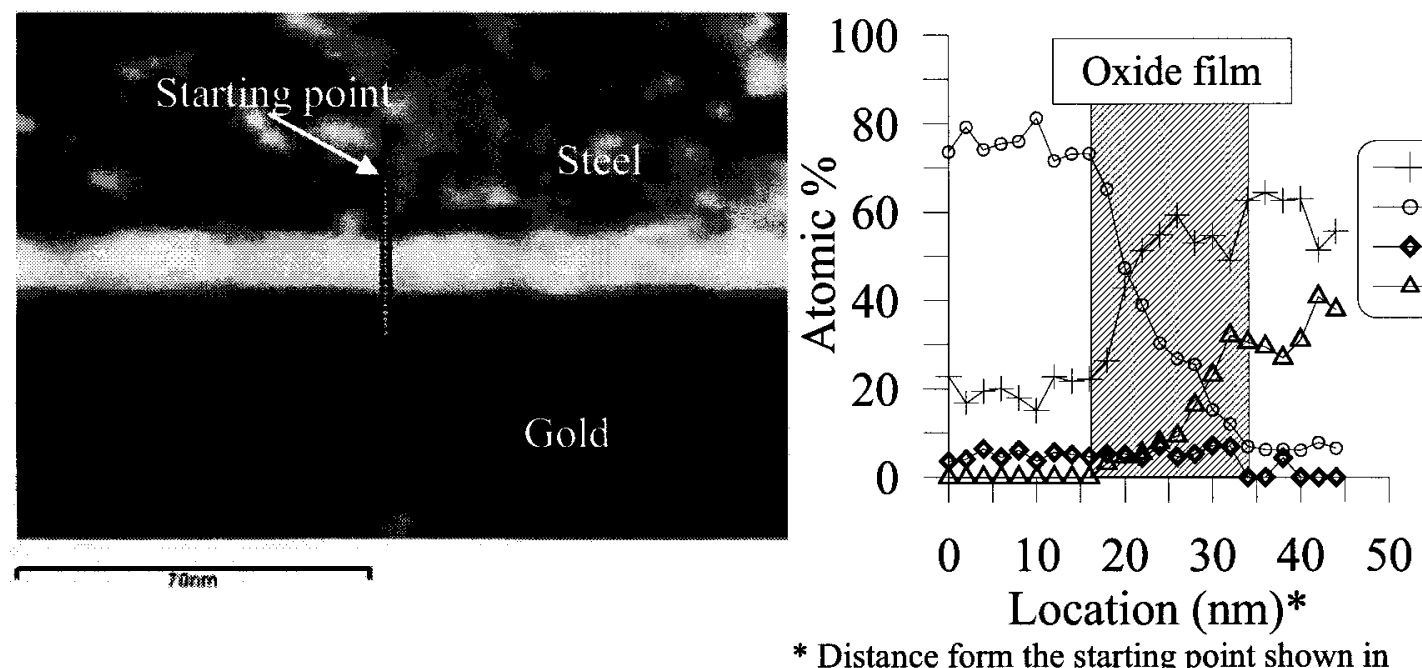

(a) Fig.7.8(a)

(b)

Figure 7.8: EDS analyses conducted on the CH-O sample across the oxide film perpendicular to the steel surface: (a) TEM image showing the locations of the analyses; (b) EDS results corresponding to the points shown in the TEM image. 


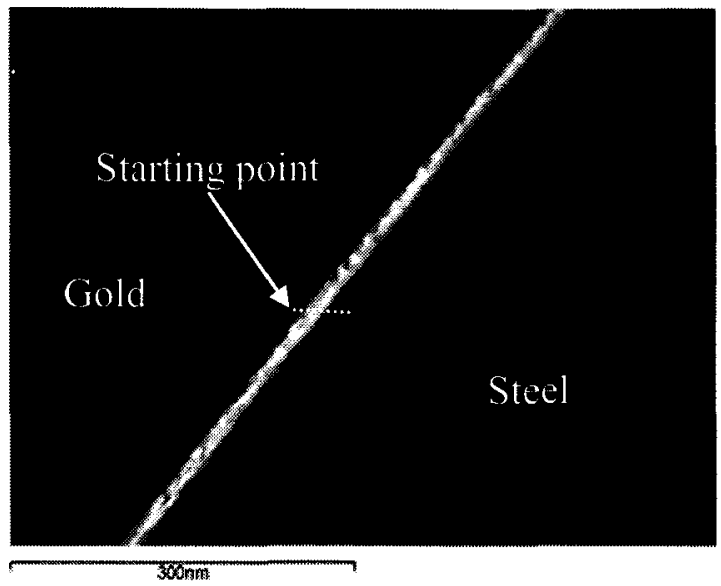

(a)

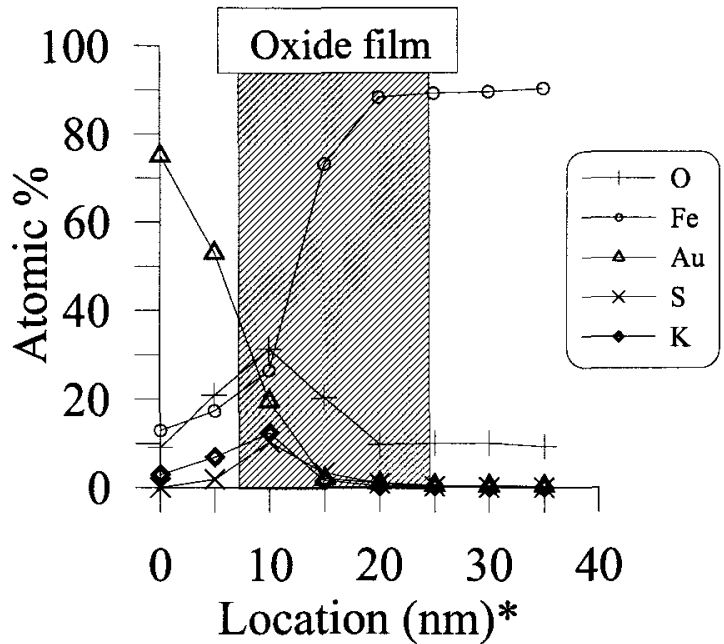

* Distance form the starting point shown in Fig.7.9(a)

(b)

Figure 7.9: EDS analyses conducted on the CP-O sample across the oxide film inclined to the steel surface: (a) TEM image showing the locations of the analysis; (b) EDS results corresponding to the points shown in the TEM image.

7.3.1.2 Samples exposed to chlorides in amounts below depassivation thresholds: $\mathrm{CH}-1$ and $\mathrm{CP}-1$

TEM micrograp'ss of the $\mathrm{CH}-1$ and $\mathrm{CP}-1$ samples exposed to $\mathrm{CH}$ solution plus $0.05 \mathrm{M}$ chloride and $\mathrm{CP}$ solution plus $0.45 \mathrm{M}$ chloride are shown in Figs. 7.10(a) and 7.10(b), respectively. It can be observed in Fig. 7.10(a) that the oxide film of the CP-0 sample is 5 to $8 \mathrm{~nm}$ thick and quite uniform. A comparison of Figs. 7.4(a) and 7.10(a) shows that the oxide film of the $\mathrm{CH}-1$ sample is very similar in thickness and uniformity to the oxide film of the $\mathrm{CH}-0$ sample. Similar observations can also be made for the oxide film of the CP-1 sample: Figures 7.10(b) shows that the oxide film of the CP-1 sample is 8 to $13 \mathrm{~nm}$ thick and quite uniform. These observations suggest that chlorides in quantities lower than the depassivation thresholds do not change the appearance of the oxide films that were grown in either of the solutions. 


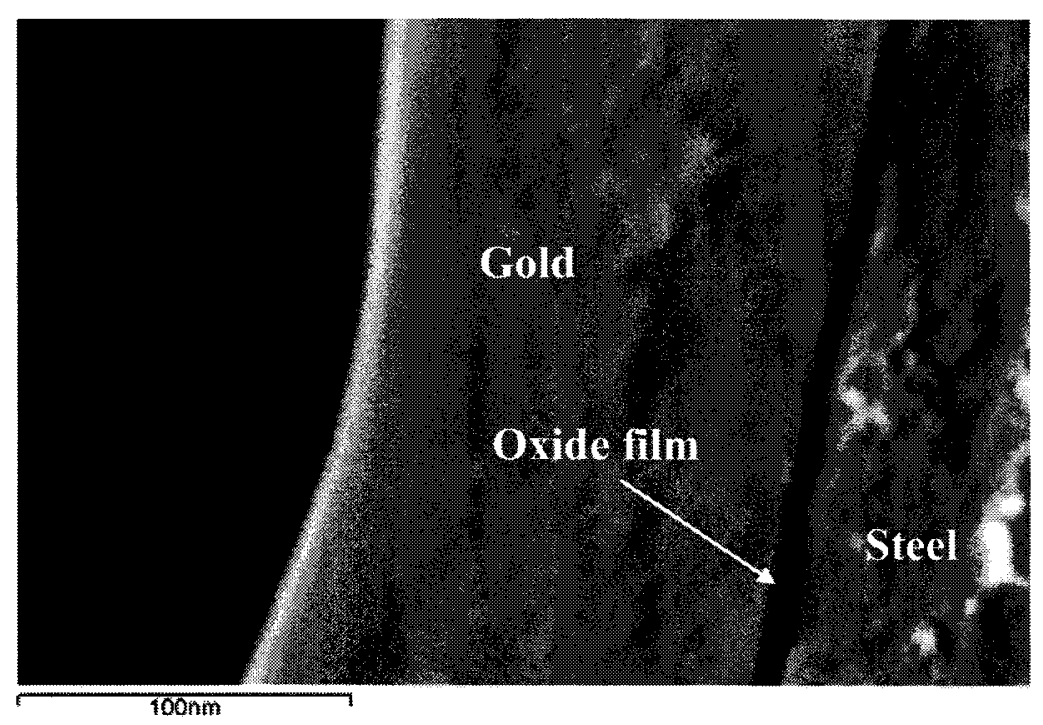

(a)

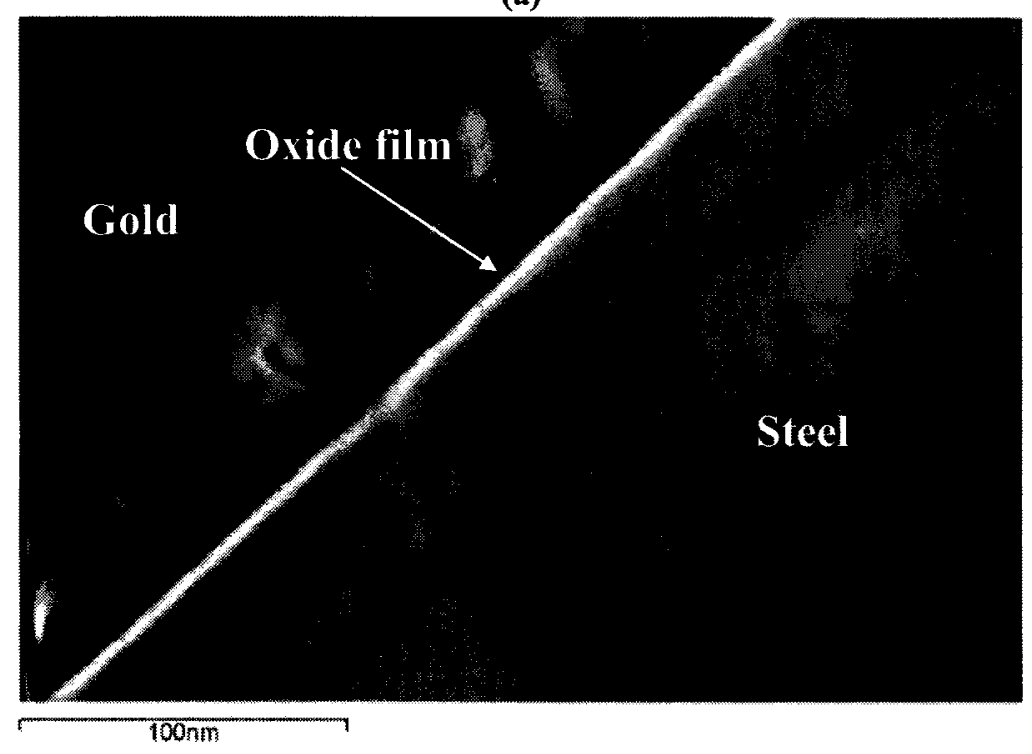

(b)

Figure 7.10: TEM images of (a) the CH-1 sample exposed to $C H$ solution with $0.05 \mathrm{M}$ chloride; (b) the CP-1 sample exposed to CP solution with $0.45 \mathrm{M}$ chloride. 
Further EDS analyses were carried out to determine if chlorides affect the elemental composition of the oxide films. The locations of the line scans that were carried out on the oxide film of the CH-1 sample are shown in Fig. 7.11(a). Figures 7.11(b) and 11(c) respectively illustrate the EDS analyses along and across the oxide film of the CH-1 sample. EDS analysis along the oxide film, as shown in Fig. 7.11(b) shows the presence of chlorine $(\sim 1-2 \%)$ and calcium $(<0.5 \%)$ inside the oxide film. Figure 7.11(c), which shows the EDS analysis across the oxide film, also show the presence of chlorine and calcium; the intensities of the chlorine and calcium signals are higher towards the outer layer of the oxide film. These observations suggest that chlorides and calcium in the $\mathrm{CH}$ solution might have penetrated into the oxide film. Although it is also possible that chlorine and calcium signals in the oxide film may be respectively originating from the $\mathrm{NaCl}$ and $\mathrm{Ca}(\mathrm{OH})_{2}$ precipitates on the surface of the sample, relatively uniform intensities of these two species observed in Fig. 7.11(b), and the fact that calcium signal was not present in the $\mathrm{CH}-0$ sample (see Figs. 7.6 and 7.8), strongly support the contention that chlorine and calcium are present in the outer porous layers of the oxide film.

The locations of the EDS line scans on the oxide film of the CP-1 sample are shown in Fig. 7.12(a). No chlorine is observed in the EDS analysis obtained along the line inside the oxide film (Fig. 7.12(b)). Figures 7.12(c) illustrates the EDS analysis across the oxide film of the CP-1 sample. Small amounts of chlorine were detected at some points; however, the intensity of the chlorine signal was not as strong as the one for the $\mathrm{CH}-1$ sample. 

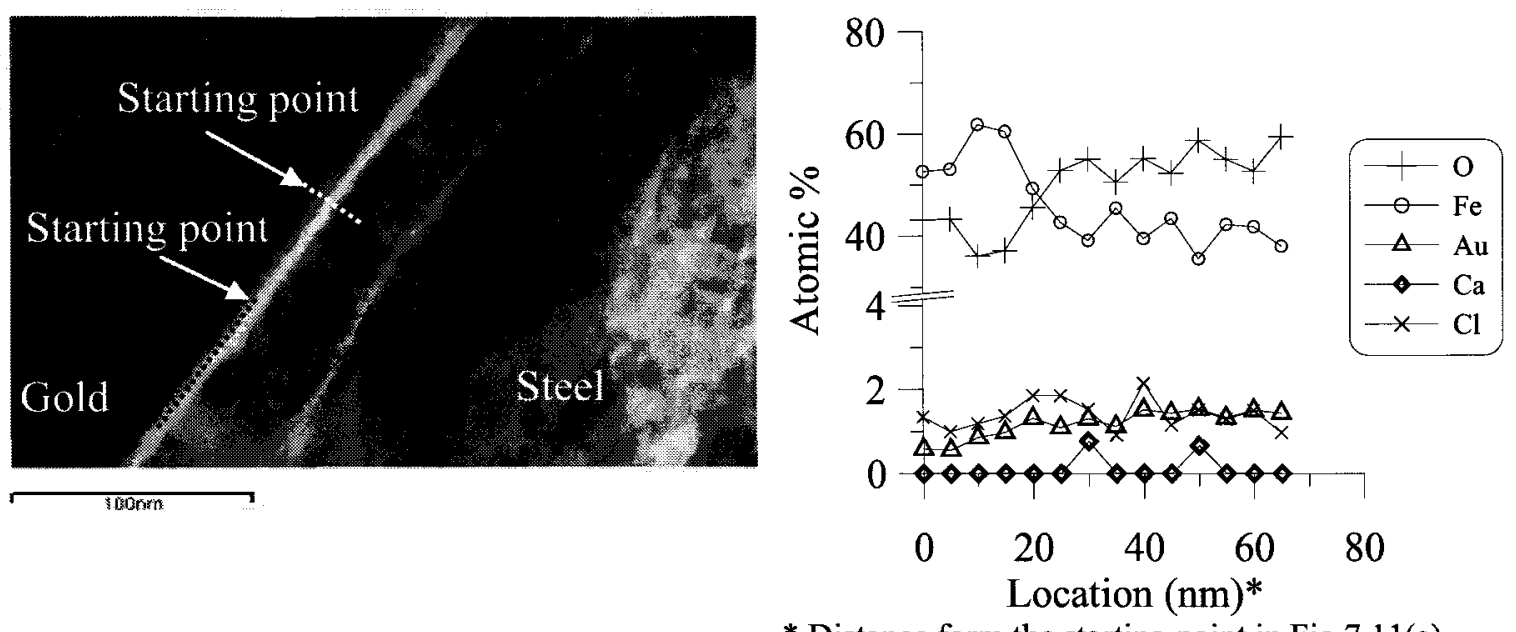

* Distance form the starting point in Fig.7.11(a)

(a)

(b)

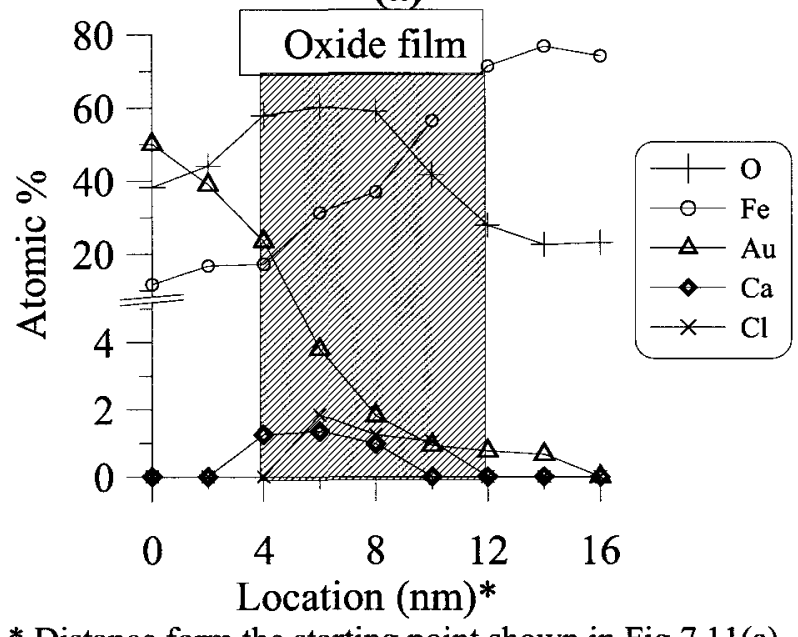

Figure 7.11: EDS analysis of the oxide film of the $\mathrm{CH}-1$ sample, which was exposed to $\mathrm{CH}$ solution plus $0.05 \mathrm{M}$ chloride: (a) TEM image showing the locations of the EDS scans; (b) EDS scan along the oxide film; (c) EDS scan perpendicular to the oxide film. (c)

7.3.1.3 Samples exposed to chlorides in amounts above depassivation thresholds: $\mathrm{CH}-2$ and CP-2

TEM micrographs of the $\mathrm{CH}-2$ and $\mathrm{CP}-2$ samples exposed to $\mathrm{CH}$ solution plus $0.45 \mathrm{M}$ chloride and $\mathrm{CP}$ solution plus $3 \mathrm{M}$ chloride are shown in Figs. 7.13 and 7.14, respectively. Fig. 7.13(a) shows that the oxide film of the $\mathrm{CH}-2$ sample has experienced changes in thickness and uniformity when compared with the oxide films of the $\mathrm{CH}-0$ and $\mathrm{CH}-1$ samples (see Fig. 7.10(a)). The average thickness of the oxide film in the $\mathrm{CH}-2$ sample is smaller than the thickness of the oxide film in the $\mathrm{CH}-0$ and $\mathrm{CH}-1$ samples. As it is seen in Fig. 7.13(b), the oxide film still covers the steel surface, albeit with 
varying thickness that ranges from $2 \mathrm{~nm}$ to $10 \mathrm{~nm}$. Fig. 7.13(c) shows part of the oxide film that appears thinner than other parts of the film $(<\sim 3 \mathrm{~nm})$.
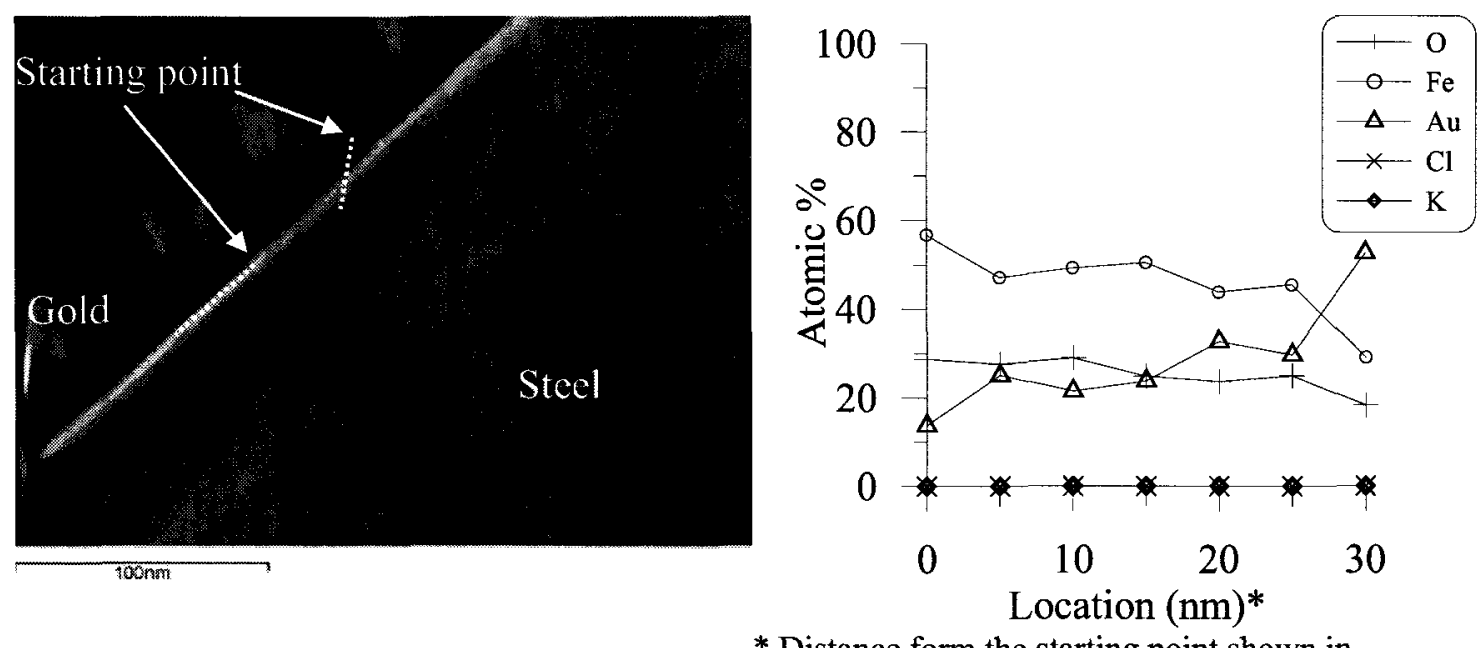

* Distance form the starting point shown in Fig.7.12(a)

(a)

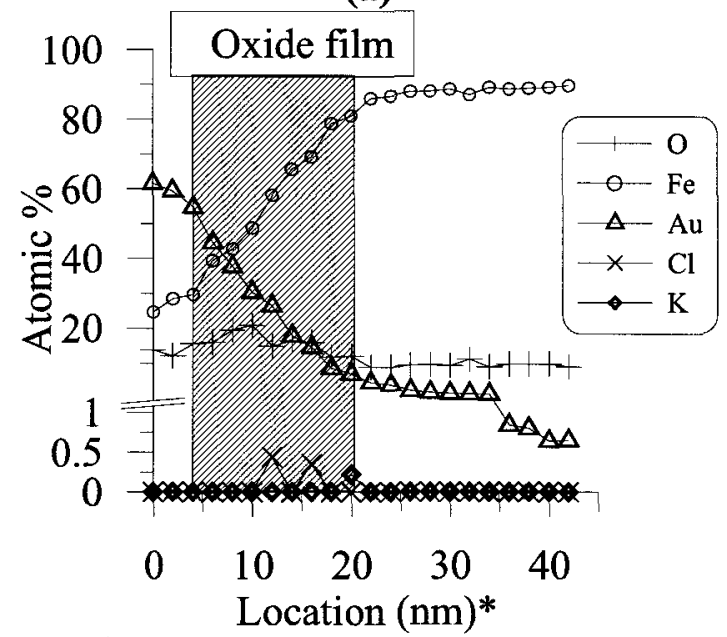

(b)

Figure 7.12: EDS analysis conducted across the oxide film inclined to the steel surface in the CP solution plus $0.45 \mathrm{M}$ chloride (CP-1): (a) TEM image showing the locations of the EDS scans; (b) EDS scan along the oxide film; (c) EDS san perpendicular to the oxide film.

* Distance form the starting point shown in Fig.7.12(a)

(c)

TEM images of the CP-2 sample are illustrated in Fig. 7.14. These images also reveal a similar story for the oxide film after chloride exposure in amounts larger than depassivation thresholds of the $\mathrm{CP}$ solution (i.e., $3 \mathrm{M}$ ). Similar to the $\mathrm{CH}-2$ sample, the oxide film of the CP-2 sample has also experienced changes in thickness and uniformity when compared with the oxide film of the CP-0 and CP-1 samples (see Fig. 7.10(b)). Some parts of the oxide film still cover the steel surface (see Fig. 7.14(a)), albeit with 
varying thickness; however, unlike the $\mathrm{CH}-2$ sample, there are also parts of the steel surface without any oxide film at all (Fig, 7.14(b)), as confirmed by EDS analysis, which is shown in Fig. 7.16. The average thickness of the remaining oxide film in the CP-2 sample is smaller than the average thickness of the oxide film in the CP-1 sample (see Figs. 7.14(c) and 7.10(b)) Since steel is not fully covered with the oxide film, the inference is that iron oxides are no longer protective after exposure to chlorides in the CP solution in amounts larger than typical depassivation thresholds.

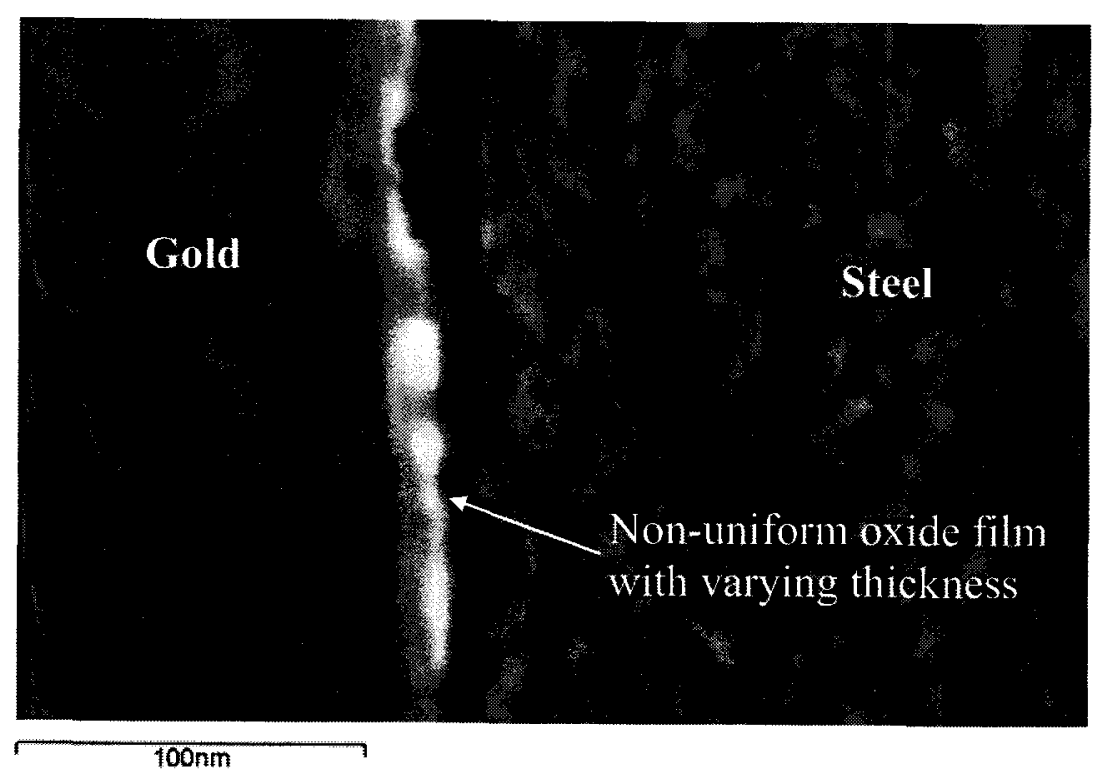

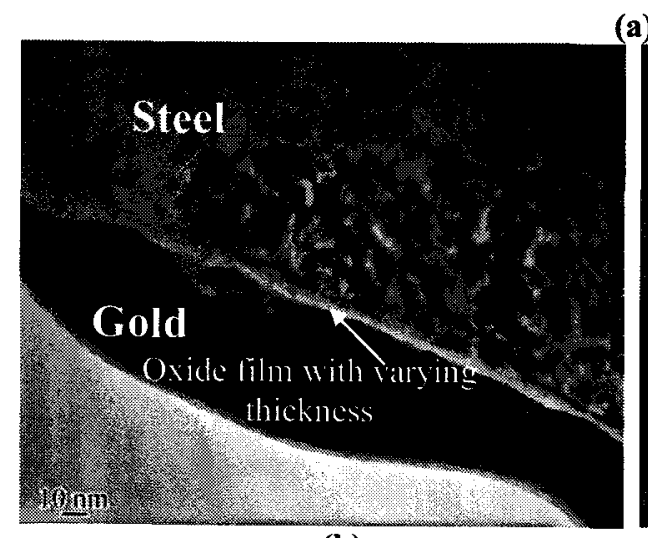

(b)

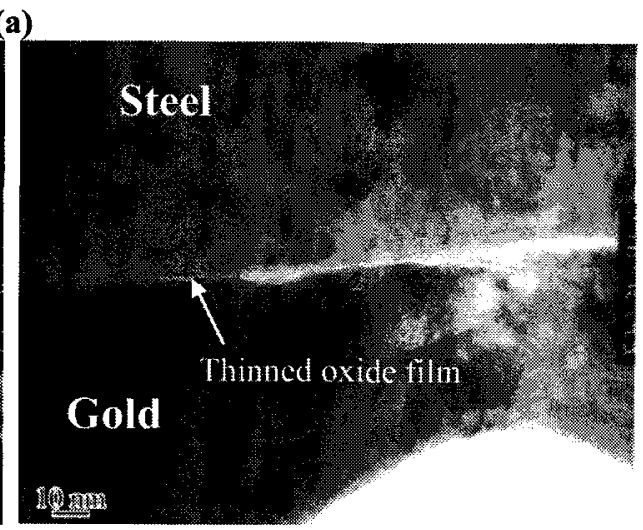

(c)

Figure 7.13: Typical TEM images of the oxide film from different parts of the $\mathrm{CH}-2$ sample showing: (a) non-uniform oxide film; (b) varying thickness of oxide film; (c) thinned oxide film. 


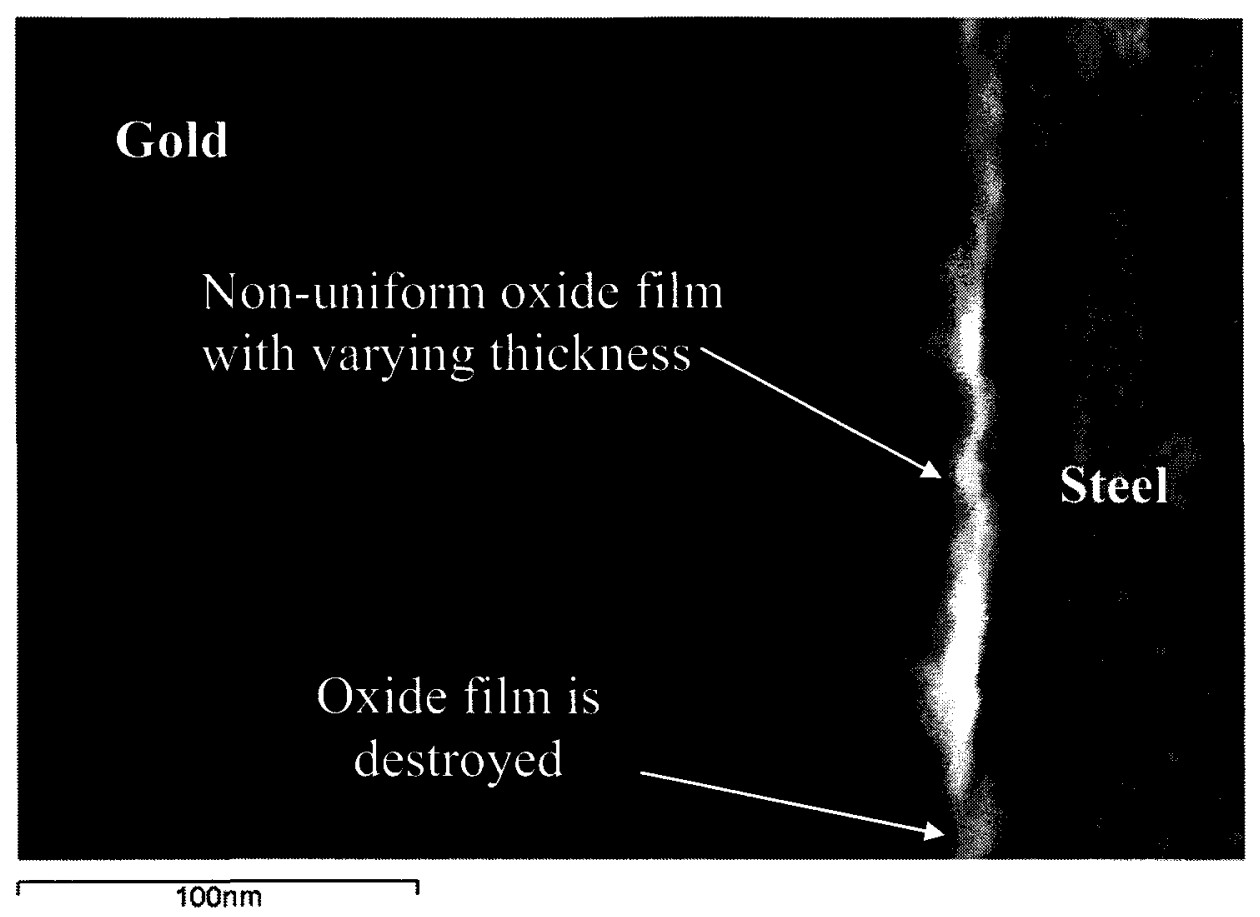

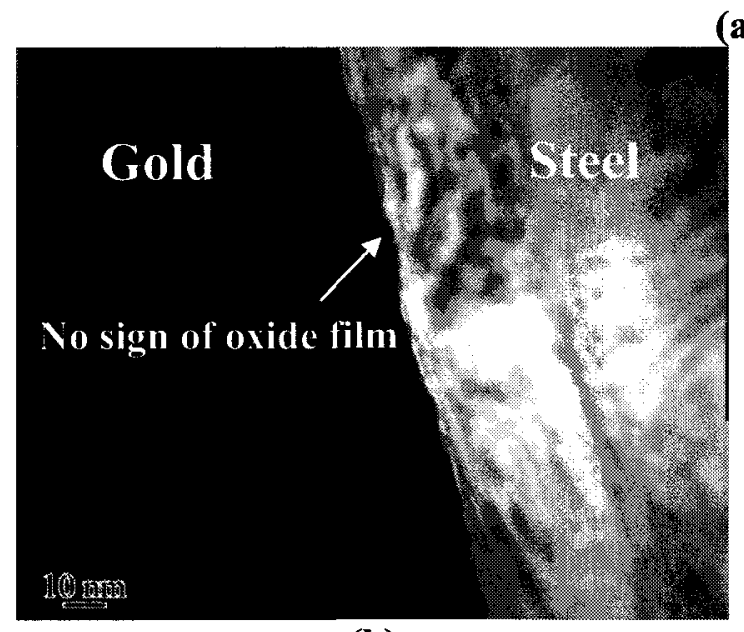

(b)

(a)

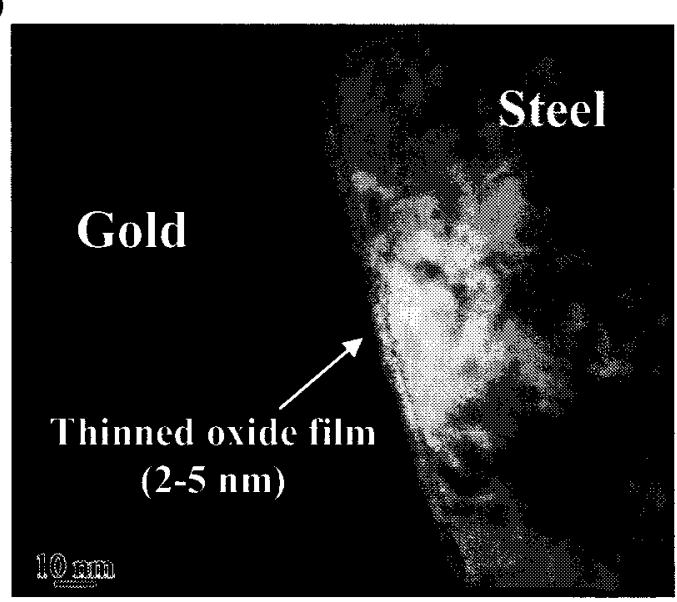

(c)

Figure 7.14: Typical TEM images of the oxide film from different parts of the CP-2 sample showing: (a) non-uniform oxide; (b) no sign of oxide film; (c) thinned oxide film (2-5 nm).

EDS analysis of the oxide film on the $\mathrm{CH}-2$ sample (Fig. 7.15) shows no indication of chlorine. This observation, suggests that the chlorine-containing parts of the oxide film, which were clearly present in the $\mathrm{CH}-1$ sample (see Fig. 7.11(b)), were destroyed and washed away in the $\mathrm{CH}$ solution plus $0.45 \mathrm{M}$ chloride during exposure. 
(EDS analysis across the steel-gold interface of the CP-2 sample, as shown in Fig. 7.16, confirms that there is no oxide film in the interface). Further examination of the $\mathrm{CH}-2$ sample also showed the presence of pits, with depths ranging from $30 \mathrm{~nm}$ to $100 \mathrm{~nm}$, on the steel surface. In Fig. 7.17 (a), two pits with different sizes are shown on the surface of the $\mathrm{CH}-2$ sample on which the oxide film is partially destroyed. Two EDS line scans at locations near these pits (see Figs. 7.17(b) and 7.17(c)) and another one inside the pit (see Fig. 7.17(d)) were carried out. The EDS scan in Fig. 7.17(b) shows that oxygen is present at the steel/gold interface, albeit localized only around $8 \mathrm{~nm}$ from the starting point of the line scan. In addition to oxygen, small amounts of calcium were found in the same region; therefore it is difficult to judge if the oxygen signal is originating from a thin layer of oxide film or from $\mathrm{Ca}(\mathrm{OH})_{2}$ precipitates. On the other hand, the line scan between the two pits, as shown in Fig. 7.17(c), shows a strong oxygen signal all along the scan length in addition to small amounts of calcium; therefore, it is more likely that the oxide film is still present at this location. The EDS analysis inside the pit, which is presented in Fig. 7.17(d), shows that the substances in this region contained calcium, iron, oxygen and chlorine. This suggests that the corrosion products containing ionic species present in the solution have been formed at the bottom of the pit, and it is likely that, due to the dissolution of iron in this region, the pit was actively propagating into the steel substrate. It can also be suggested that the regions near the pits (see Figs. 7.17(b) and 7.17(b)) may be serving as cathodes for these active pits. 

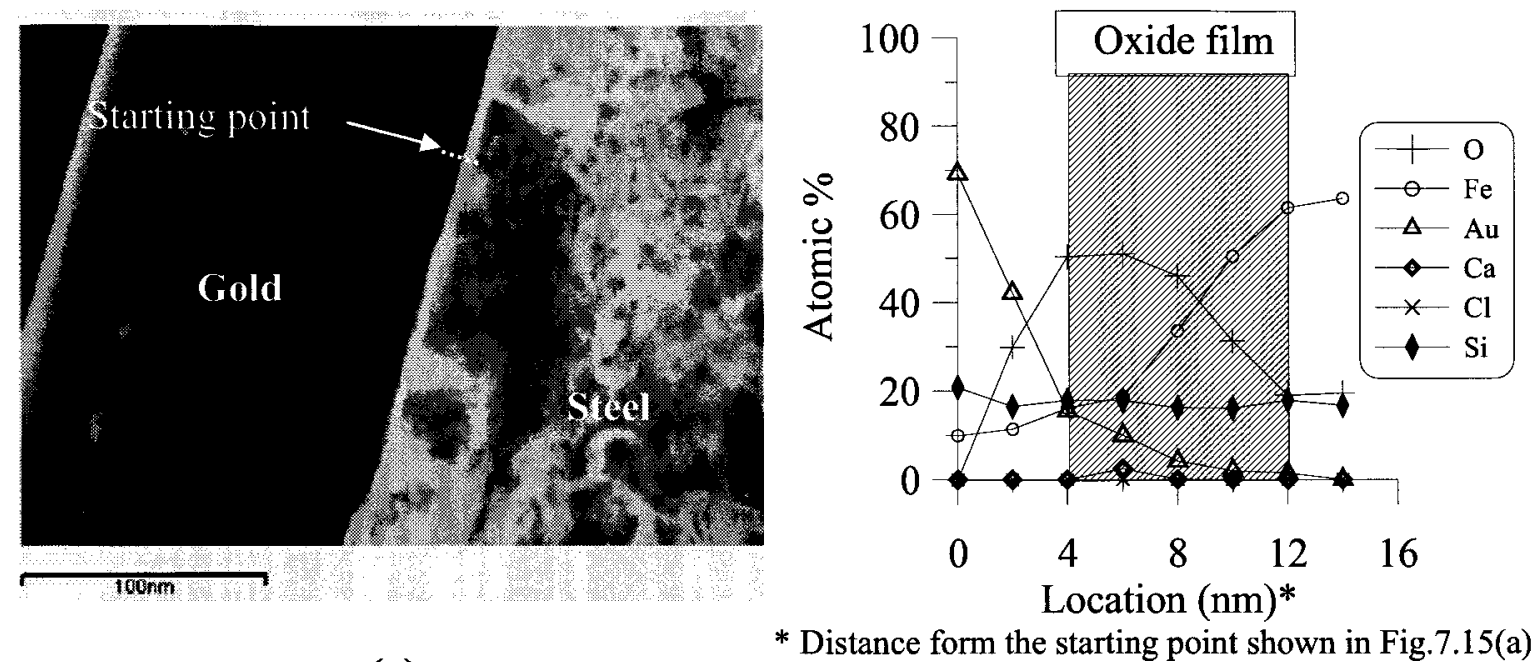

(a) (b)

Figure 7.15: EDS analysis conducted across the oxide film of the CH-2 sample: (a) TEM image showing the scan location; (b) EDS results corresponding to the points shown in the TEM image.
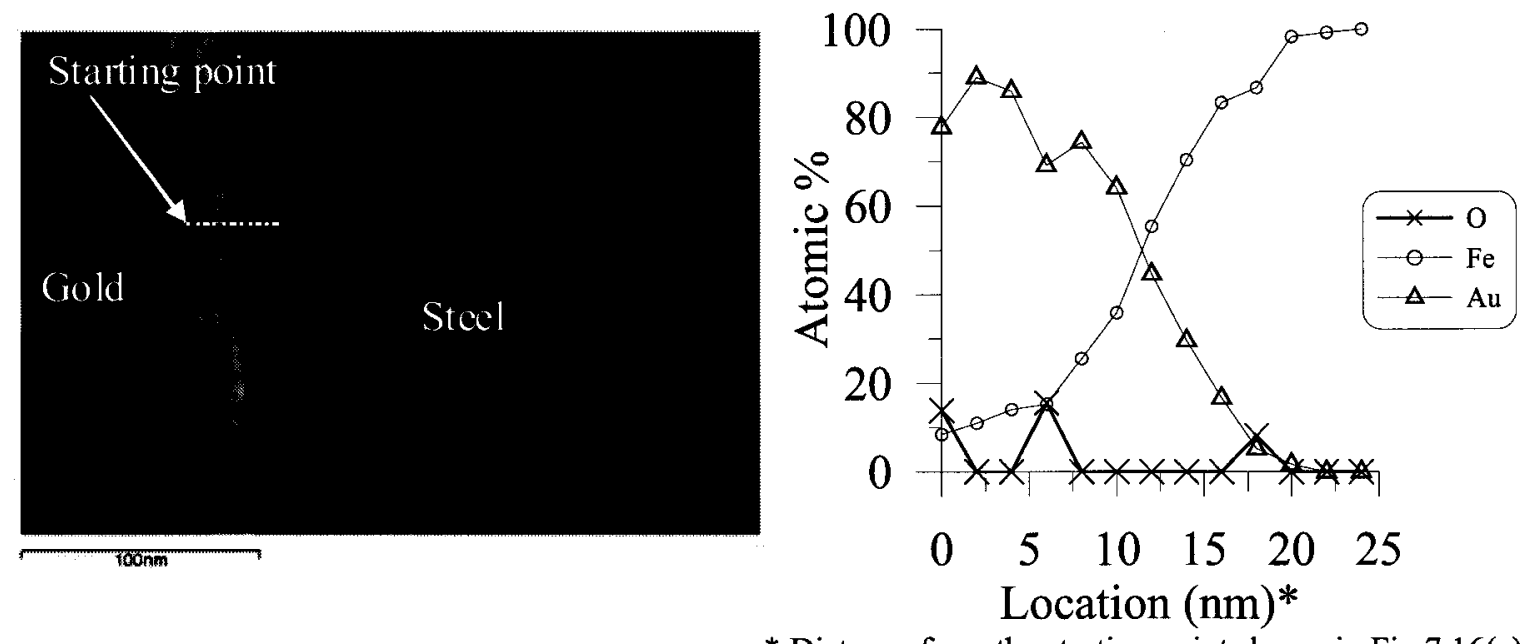

(a)

* Distance form the starting point shown in Fig.7.16(a)

(b)

Figure 7.16: EDS analysis conducted across a region where no oxide film was visible on the CP-2 sample: (a) TEM image showing the scan location; (b) EDS results corresponding to the points shown in the TEM image. 


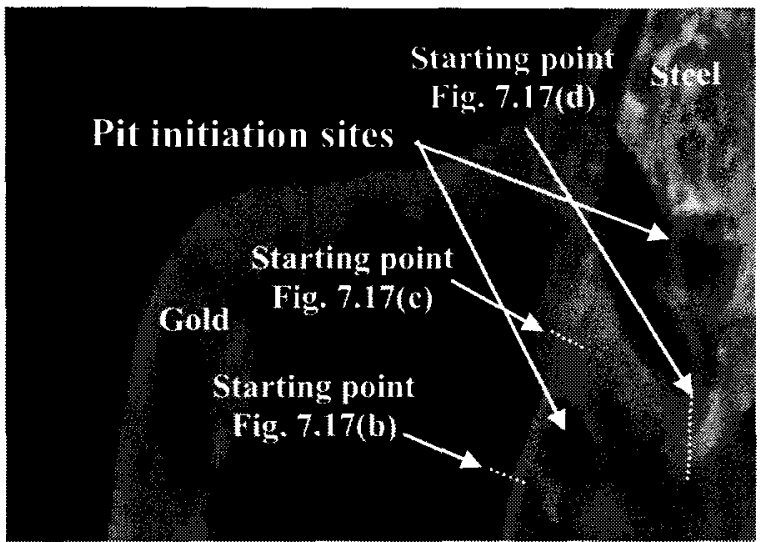

(a)

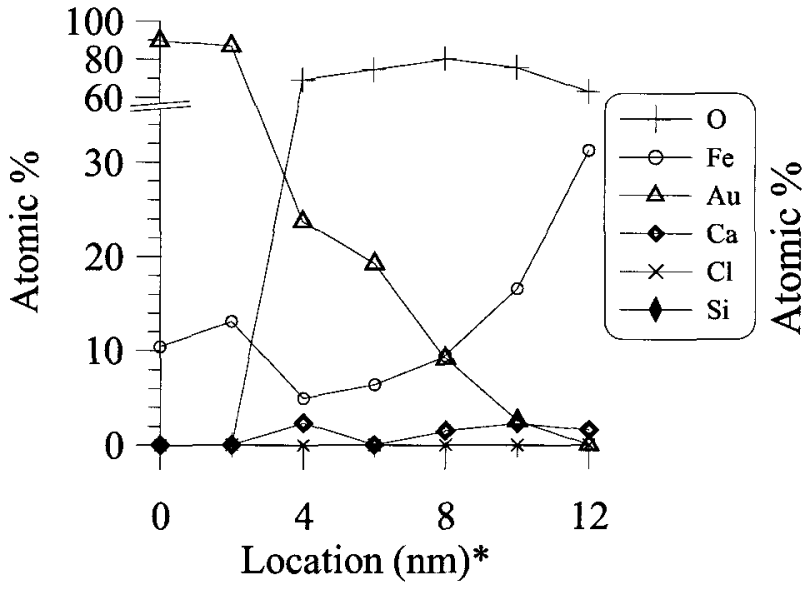

* Distance form the starting point shown in Fig.7.17(a)

(c)

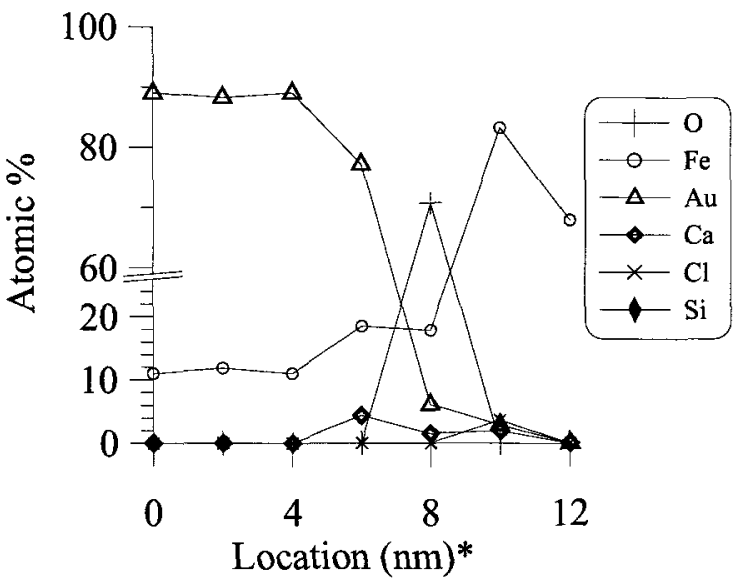

* Distance form the starting point shown in Fig.7.17(a)

(b)

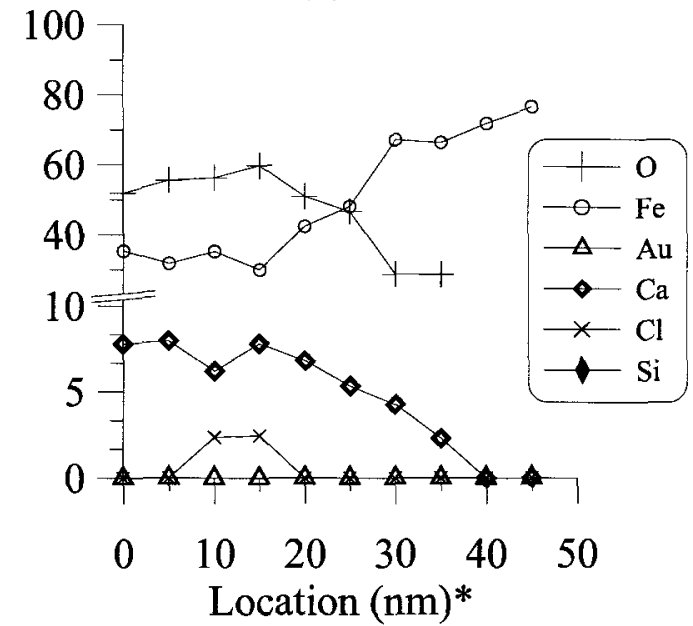

* Distance form the starting point shown in

Fig.7.17(a) (d)

Figure 7.17: EDS analysis on the CH-2 sample: (a) TEM image showing the EDS scan locations; (b) EDS scan at the location close to a pit as shown in Fig. 7.17(a); (c) EDS scan at the location between two pits shown in Fig. 7.17(a); (d) EDS scan at the bottom of the pit shown in Fig. 7.17(a).

No pit initiation sites were observed in the CP-2 sample; however this does not eliminate the existence of these sites at other locations on the steel surface that have not been investigated using the FIB-TEM technique. However, detailed investigation of the steel surface of the CP-2 sample revealed the presence of potential pit initiation sites. Figure 7.18(a) clearly shows the presence of lamellae of cementite $\left(\mathrm{Fe}_{3} \mathrm{C}\right)$ near the steel 
surface. TEM diffraction analysis carried out at location "A" in Fig. 7.18(a) confirmed that these lamellae were indeed composed of cementite (see Fig. 7.18(b)). Steel used for rebar contains carbon in concentrations large enough to exhibit a significant volume fraction of pearlite, and a mixture of ferrite $(\alpha-\mathrm{Fe})$ and cementite $\left(\mathrm{Fe}_{3} \mathrm{C}\right)$ with a lamella structure. It can be seen in Fig. 7.18(c) that some of these cementite lamellae reach the surface of steel and are exposed.

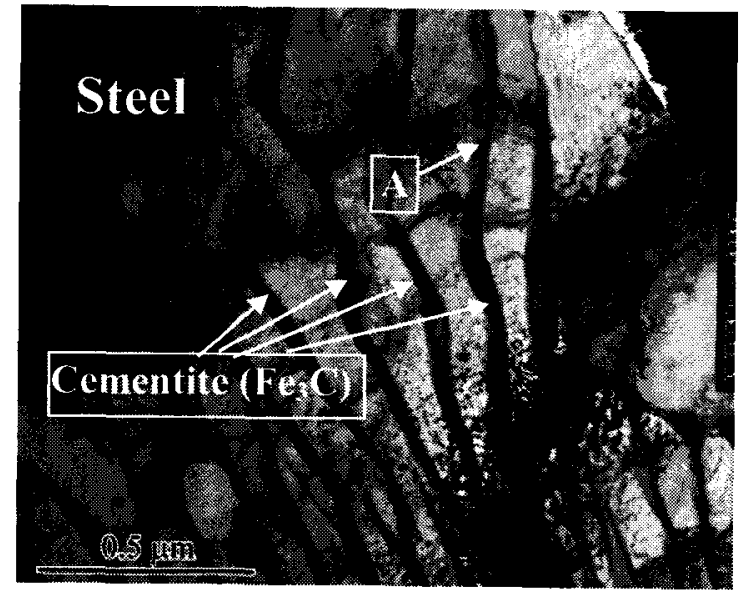

(a)

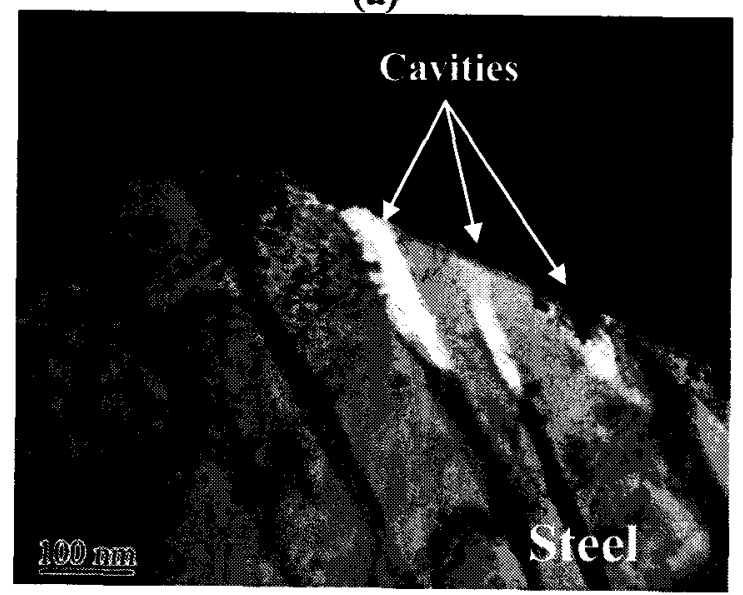

(c)

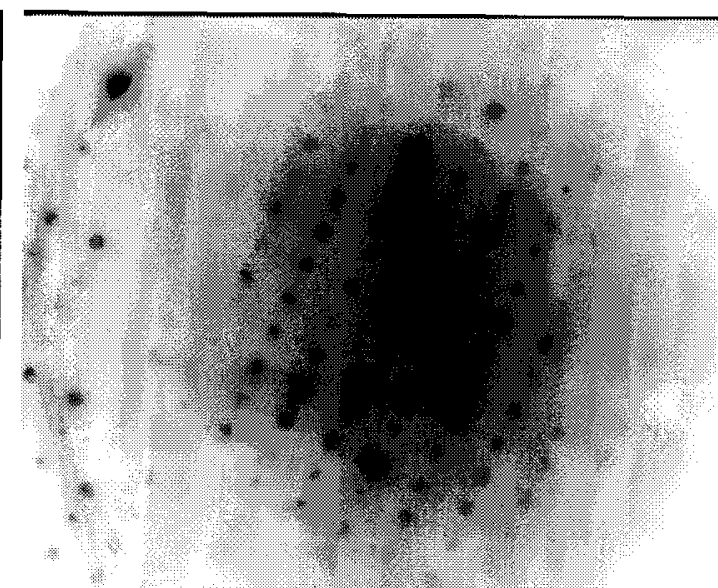

(b)

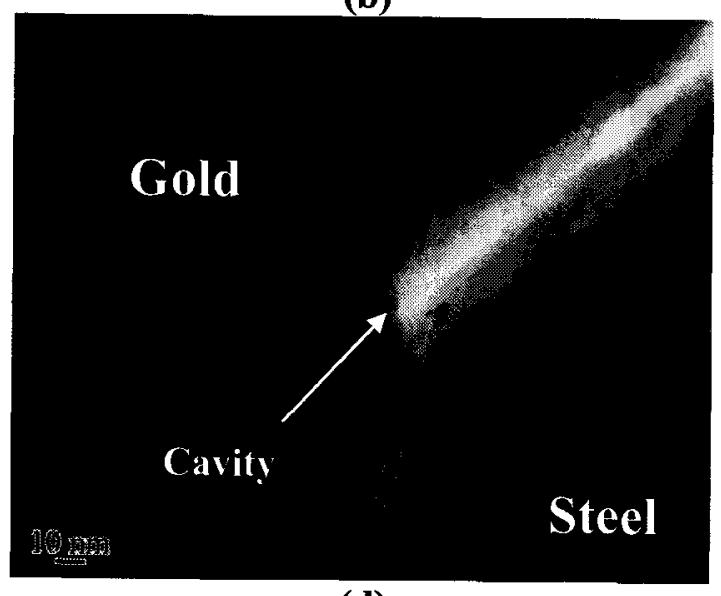

(d)

Figure 7.18: TEM images of the steel near the surface for the CP-2 sample: (a) cementite $\left(\mathrm{Fe}_{3} \mathrm{C}\right)$ lamellae in steel in a region of pearlite that intersects the surface; $(b)$ diffraction pattern of cementite ( $\mathrm{Fe}_{3} \mathrm{C}$ ) from a location marked " $A$ " in $\mathrm{Fig}$. 7.18(a); (c) cavities on the surface of steel along the openings of cementite lamellae; (d) a higher magnification of a cavity along a cementite lamellae. 
It can be observed in the same figure that a portion of cementite dissolved into the solution and cavities were formed on the surface (EDS mapping showed that these locations are indeed cavities). A higher magnification micrograph in Fig. 7.18(d) shows a detailed view of a cavity along a cementite lamella on the surface of steel. These cavities, as suggested by Hoar (1967), Kruger (1988), can serve as local sites for pit growth; but further investigation is necessary to confirm that pitting would preferentially initiate in these cavities. In a study carried out on steel rebar in concrete, Jain et al. (1977) observed that cementite accelerated corrosion of rebar in the presence of chloride: the larger amount of cementite in steel rebar resulted in a higher corrosion rates. The same observation was also made by Evans (1981) for corrosion of steel in a buffer solution. More studies are still required to understand the mechanism of cementite effect on chloride-induced corrosion of steel in highly alkaline solutions.

\subsubsection{TEM-EELS analysis}

Oxygen K-edge EELS core loss spectra taken from the oxide film of the CP-0 sample at two different locations, labeled as CP-0-1 and CP-0-2, are plotted in Fig. 7.19. In addition, EELS spectra of different iron oxides (i.e., Ferrihydrite $(\mathrm{FeOOH}), \alpha-$ Ferrihydrite $(\alpha-\mathrm{FeOOH}), \beta$-Ferrihydrite $(\beta$-FeOOH$)$, Magnetite $\left(\mathrm{Fe}_{3} \mathrm{O}_{4}\right), \alpha$-Hematite $(\alpha$ $\mathrm{Fe}_{2} \mathrm{O}_{3}$ ) and Siderite $\left(\mathrm{FeCO}_{3}\right)$ ) (Chen et al. 2009) are also plotted in Fig. 7.19 for comparison purposes. It should be noted that since the electron beam diameter used to obtain the EELS spectra was about $10 \mathrm{~nm}$, the identification of different layers in the oxide film was not possible. Therefore, it is possible that other minor constituent oxides or thin layers are present in the oxide film. 


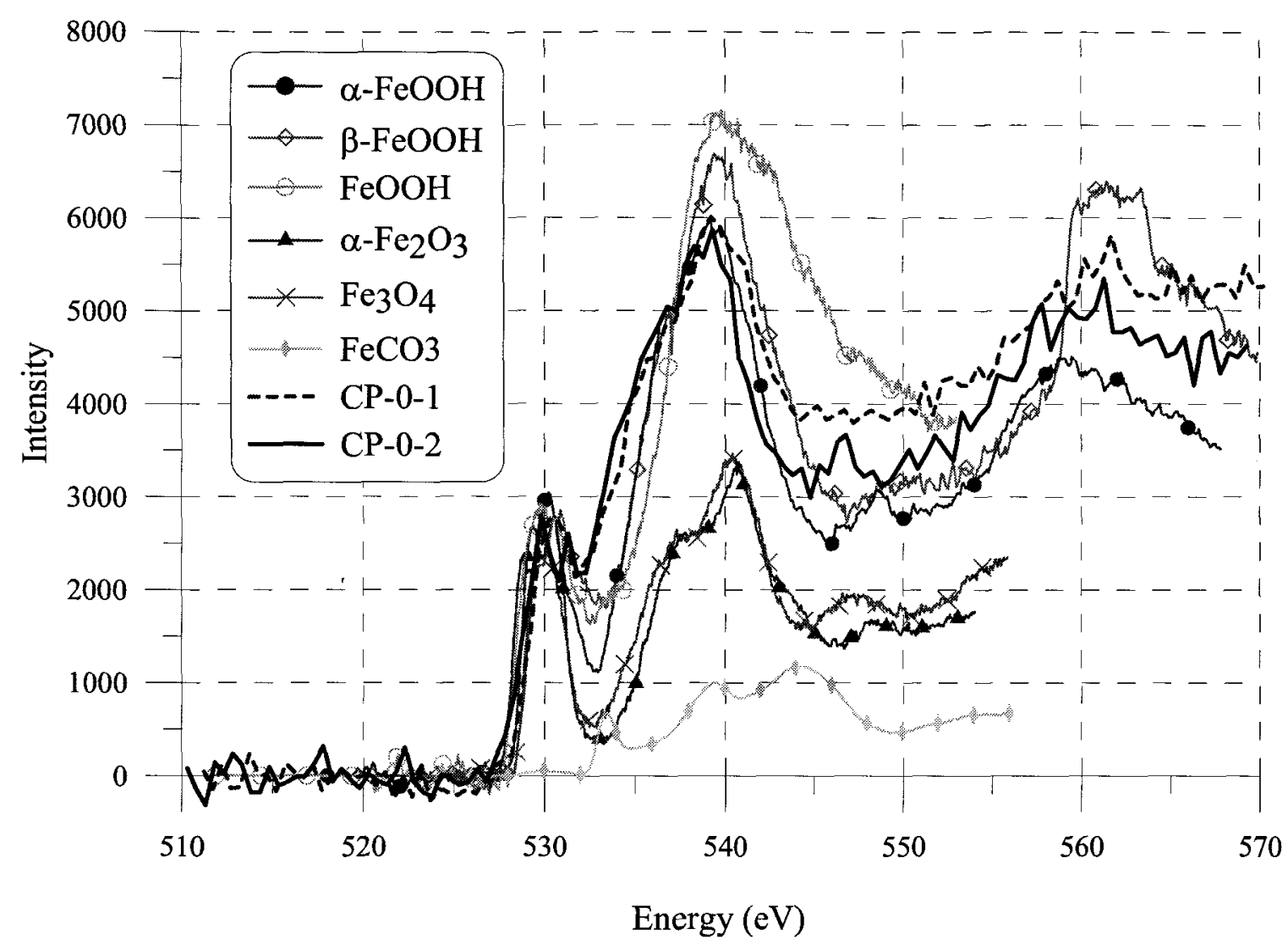

Figure 7.19: EELS spectra taken from the oxide film on the CP-0 sample (plots CP-0-1 and $C P-0-2$ ), and EELS spectra of different iron oxides for comparison purposes (Chen et al. 2009).

In general, three main peaks were observed in each spectrum. The first main peak of all spectra, except for the one for Siderite $\left(\mathrm{FeCO}_{3}\right)$, was located at $530 \mathrm{eV}$. The first peak of Siderite is located at $533 \mathrm{eV}$; therefore, it can be stated that the oxide film of the CP-0 sample does not contain a Siderite phase. In order to compare the spectra of the oxide of the CP-0 sample with the spectra of the other reference oxides, the first peak intensity of all spectra was set to an arbitrary value. It can be seen in Fig. 7.19 that the spectrum of the CP-0 sample is noticeably different from the spectra of Magnetite and $\alpha$ Hematite; the second and third peaks of the CP-0 sample spectrum are higher than those of Magnetite and $\alpha$-Hematite. In contrast, the CP-0 peaks generally match with the three 
types of Ferrihydrite compounds (i.e., $\mathrm{FeOOH}, \alpha-\mathrm{FeOOH}$, and $\beta-\mathrm{FeOOH}$ ); however, the shape of the $\alpha-\mathrm{FeOOH}$ spectrum is more similar to the shapes of the CP-0-1 and CP-0-2 spectra. This suggests that the oxide film on the CP-0 sample is mainly composed of $\alpha-$ FeOOH. Support for this observation comes from ellipsometric studies conducted by Zakroczymski et al. (1985a, 1985b) on iron passive film, which showed that the anodically grown passive film in $0.05 \mathrm{M} \mathrm{NaOH}$ solution is mainly composed of $\mathrm{FeOOH}$, and its composition does not change in the presence of chloride during the film growth. Further support can be found in Sarver and Szklarska-Smialowska's study (1984) on passive films of iron in highly alkaline $\mathrm{NaOH}$ solution $(\mathrm{pH}=12)$ using elipsometry and electrochemical techniques.

\subsubsection{TEM-diffraction pattern analysis}

Additional evidence on the nature of the oxide film was obtained using electron diffraction coupled with dark field imaging. SAD patterns were obtained using a diffraction aperture that gave a selected area of $\sim 1 \mu \mathrm{m}$ diameter, which is the minimum possible size owing to aberration effects from the microscope lenses. This area is therefore large enough to permit both the Fe substrate and the Au layer to contribute to the diffraction patterns. In order to distinguish oxide reflections from the other phases present, two procedures need to be followed: (a) the interplanar spacings corresponding to the diffraction spots must be measured and compared to standard unit cell data for $\mathrm{Fe}$, $\mathrm{Au}$ and possible oxides; and (b) dark-field images must be obtained in order to confirm the origin of the diffraction spots. In addition to the SAD patterns, some CBED patterns were obtained from some oxide films, with the electron beam focused to $\sim 5 \mathrm{~nm}$ diameter. The CBED patterns contain diffraction discs rather than the smaller spots of SAD, and 
again, it is necessary to take separate possible contributions from the adjoining phases into account.

Dark-field TEM images taken respectively from the oxide phase in the $\mathrm{CH}-0$ and the CP-0 samples are presented in Figs. 7.20. In these dark-field TEM micrographs, the bright phases, which are as large as $8 \mathrm{~nm}$, are indicators of crystalline substances that compose the nanostructure of the oxide film. From these images, it can be observed that the structure of oxide film in both samples are quite similar and consists of nanocrystalline particles in different sizes up to $8 \mathrm{~nm}$.

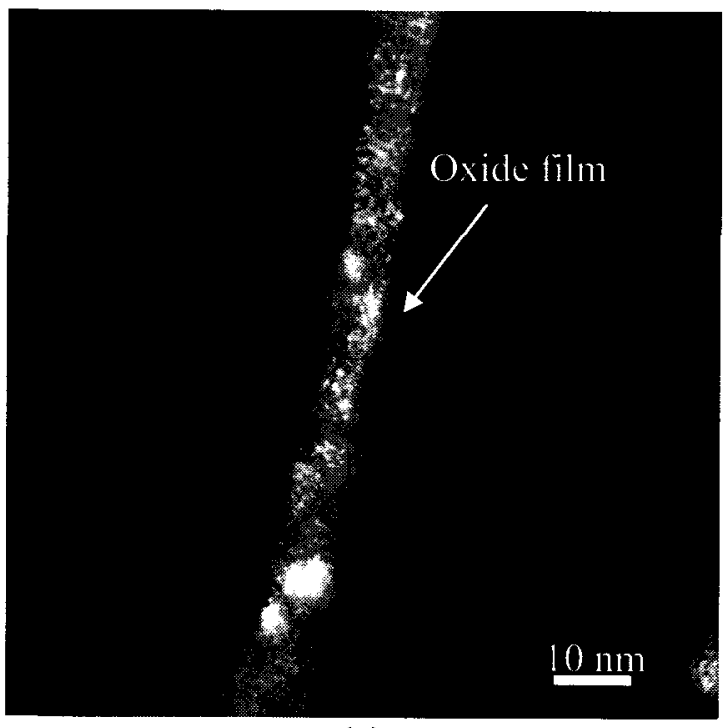

(a)

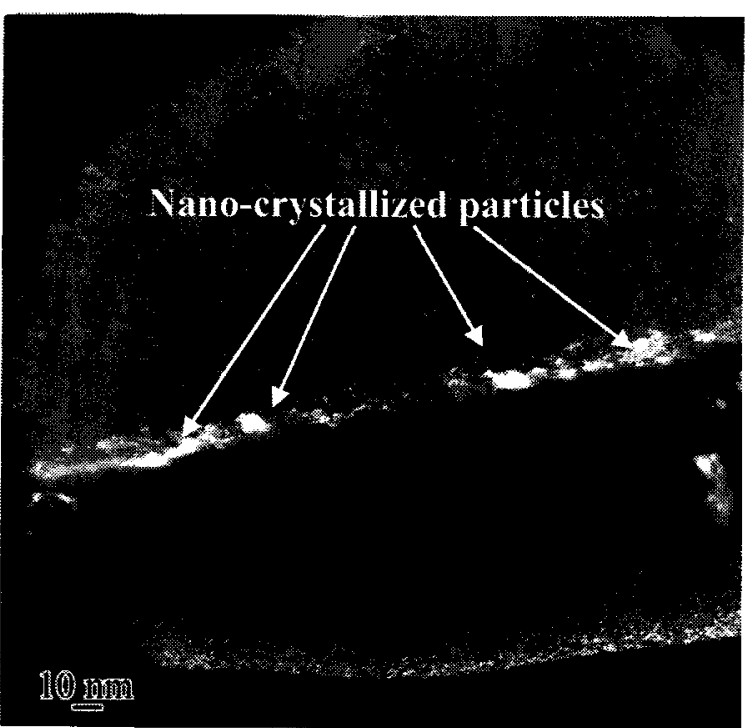

(b)

Figure 7.20: TEM dark-field images of the oxides films of (a) the CH-0 sample; (b) the CP-O sample. The bright phases indicate the incorporation of nano-crystalline particles in the oxide film.

In order to interpret the diffraction patterns, diffraction rings from the polycrystalline gold layer were used along with single crystal diffraction spots from the Fe substrate to calibrate the camera constant, $\lambda L$, of the microscope under the conditions used, where $\lambda$ is the electron wavelength and $L$ is the camera length for the microscope. 
This is necessary if the errors in the measurements of interplanar spacings $(d)$ of any unknown phases are to be minimized (typically to $1 \sim 2 \%$ ). An example of a diffraction pattern from the Au phase is shown in Fig. 7.21, from which the camera constant was calculated using:

$$
\lambda L=d_{h k l} r_{h k l}
$$

where $\lambda$ is the wavelength of radiation, $L$ is a nominal length, controlled by the lenses, $r_{h k l}$ is the measured distance of the $(h k l)$ ring diffraction from the center of the SAD pattern, and $d_{h k l}$ (i.e., d-spacing) ( $\AA$ ) is the spacing of $(h k l)$ diffracting plane. Three $r$ distances (i.e., $r_{1}, r_{2}$ and $r_{3}$ ) that were measured on the SAD pattern in Fig. 7.21 are presented in Table 7.4 along with the corresponding d-spacing of the gold pattern. The camera constant of each $r$ distance was calculated using Eq. 7.1, and are also reported in Table 7.4. The camera constant of $24.6(\AA . \mathrm{mm})$ averaged form these three measurements was used for the interpretation of the SAD patterns. A similar calculation using the iron spectra also gave the same camera constant.

The SAD patterns of the iron oxide film on the $\mathrm{CH}-0$ sample taken from three different locations are shown in Figs. 7.22(a)-(c). As it can be seen from these figures, nine diffraction spots from the oxide films were identified and the corresponding $r$ values (i.e., $r_{4}, r_{5}, r_{6}, r_{7}, r_{8}$, and $r_{9}$ ), which are presented in Table 7.5 , were determined. Table 7.5 also presents the calculated d-spacing of each measurement and the comparisons with the d-spacing of the FeOOH . The data were only compared with FeOOH because dspacing that are greater than $5 \AA$, which are characteristic of other iron oxide compounds (e.g. $\mathrm{Fe}_{2} \mathrm{O}_{3}, \mathrm{Fe}_{3} \mathrm{O}_{4}, \mathrm{FeO}, \mathrm{Fe}(\mathrm{OH})_{2}$, and $\mathrm{Fe}(\mathrm{OH})_{3}$ ), were never observed. Therefore, all other iron oxides are discarded in determining the chemical structure of the oxide film. 
As presented in Table 7.5, matches are found for lattice spacings for $(001),(011),(020)$, (210) and (220) planes, which suggests that nano-crytalline particles of oxide film are mainly composed of $\mathrm{FeOOH}$, with an orthorhombic unit cell of $\mathrm{a}=4.937 \AA, b=4.432 \AA$ and $\mathrm{c}=2.994 \AA$. The errors determined based on the percentage difference between the calculated d-spacing and FeOOH d-spacing are generally less than $2 \%$ (see Table 7.5) which is within the acceptable limits for the SAD analysis based on calibrated diffraction patterns.

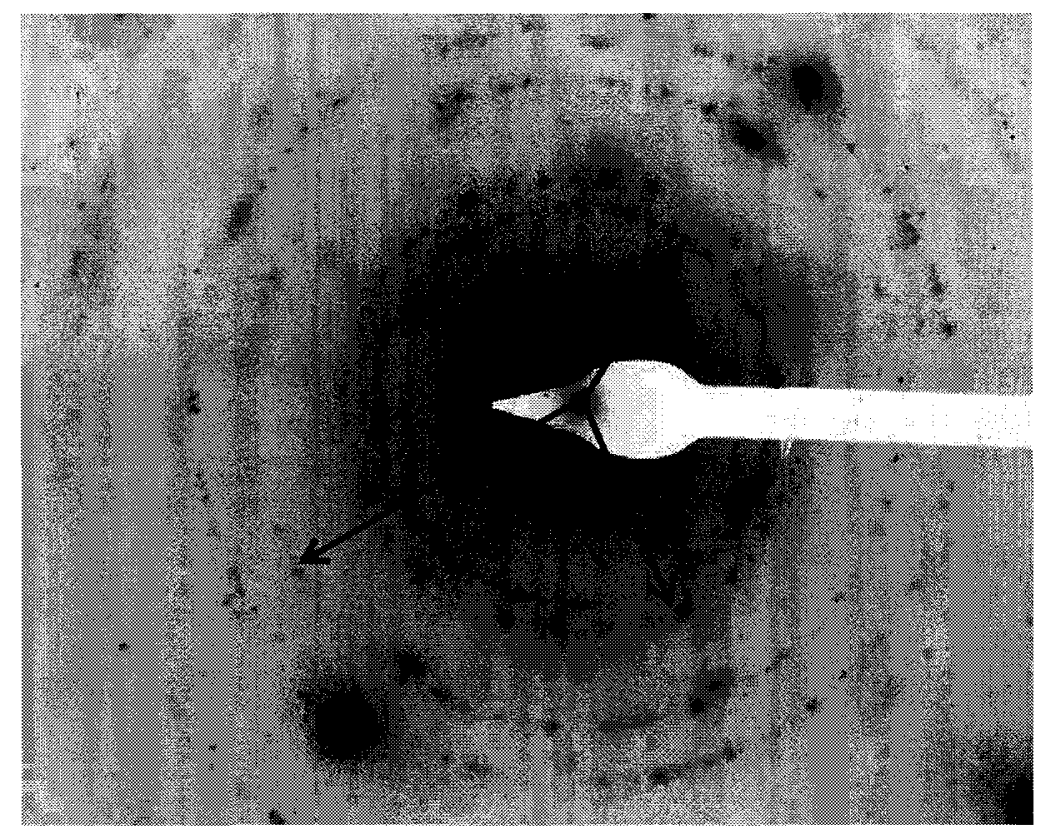

Figure 7.21: Gold diffraction pattern used for the calculation of the camera constant at the locations.

Table 7.4: The camera constant calculation for the CP-0 diffraction patterns in Fig. 7.21 .

\begin{tabular}{|c|c|c|c|}
\cline { 2 - 4 } \multicolumn{1}{c|}{} & $\boldsymbol{r}_{\mathbf{1}}{ }^{*}$ & $\boldsymbol{r}_{\mathbf{2}}{ }^{*}$ & $\boldsymbol{r}_{3}{ }^{*}$ \\
\hline$(h k l)$ & $(111)$ & $(002)$ & $(022)$ \\
$r_{h k l}(\mathrm{~mm})$ & 10.5 & 12.1 & 17.0 \\
$d_{h k l}(\AA)$ & 2.28 & 1.98 & 1.41 \\
$\lambda L(\AA . \mathrm{mm})$ & 24.73 & 24.67 & 24.51 \\
\hline Average $\lambda L(\AA . \mathrm{mm})$ & \multicolumn{3}{|c}{24.6} \\
\hline
\end{tabular}

$* r_{1}, r_{2}$ and $r_{3}$ were obtained from Fig. 7.21 . 


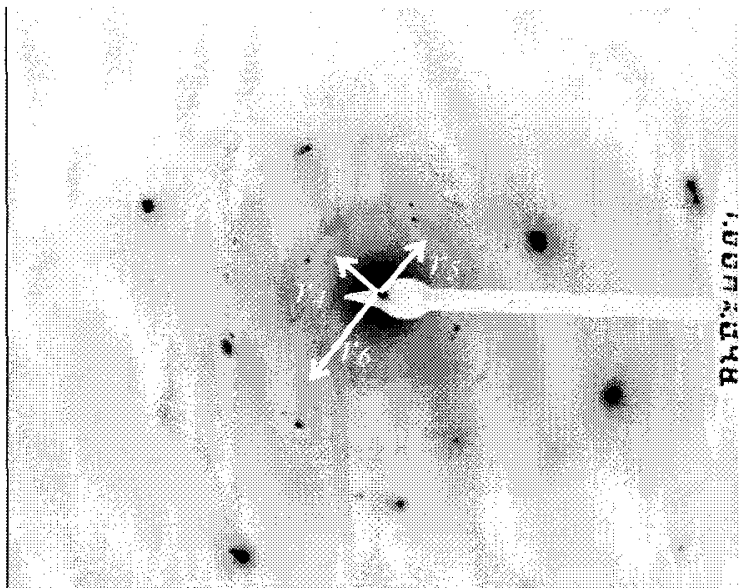

(a)

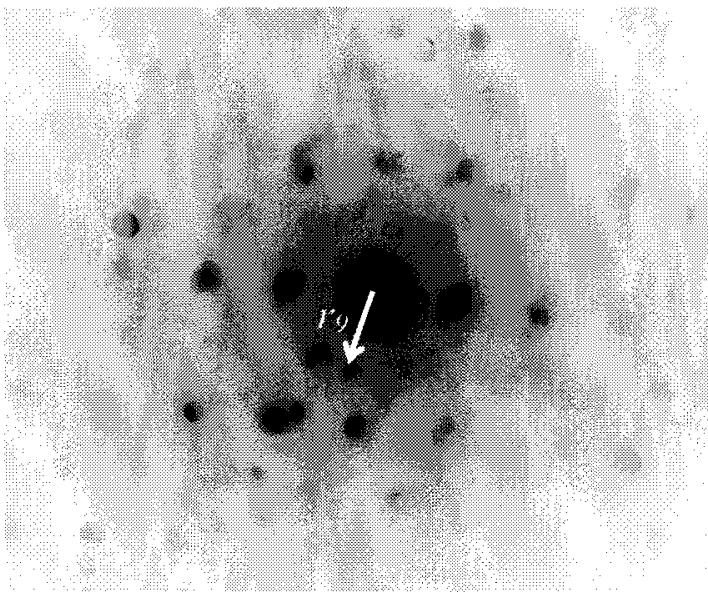

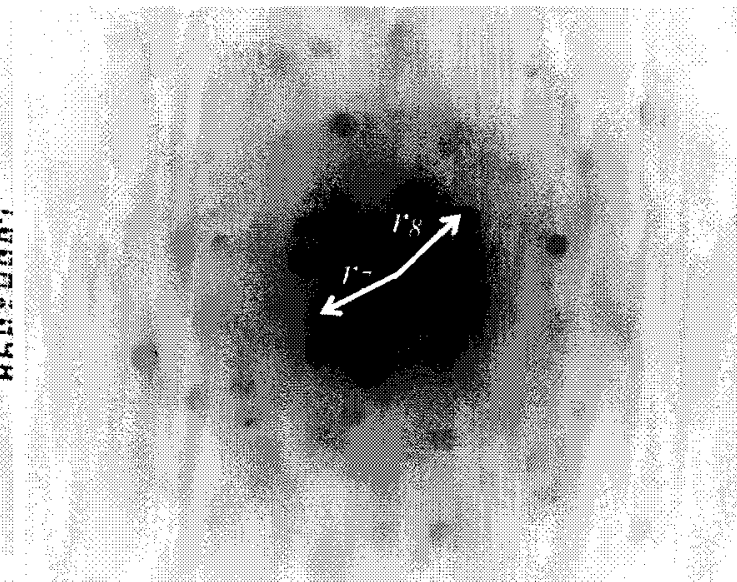

(b)

Figure 7.22: Diffraction patterns of three different areas (nano-crystalline particles) in the oxide film of the $\mathrm{CH}-\mathrm{O}$ sample (see Fig. 7.20(a): (a) SAD pattern; (b) and (c) CBED patterns.

Table 7.5: The comparison of the $\mathrm{CH}-\mathrm{O}$ oxide d-spacings from Fig. 7.22 with $\mathrm{FeOOH}$ $d$-spacings. Four interplanar spacings (001), (011), (020), (210) and (220) matched those of FeOOH. For all other phases, the calculated error was larger than $5 \%$; therefore they are not reported here $(\lambda L=24.6 \AA \mathrm{mm})$.

\begin{tabular}{|c|c|c|c|c|}
\hline$h k l$ & $\begin{array}{c}\mathrm{FeOOH} \\
d(\mathrm{~A})\end{array}$ & $\begin{array}{c}\text { Measured } \\
r(\mathrm{~mm})\end{array}$ & $\begin{array}{c}\text { Calculated } \\
d(A)\end{array}$ & $\begin{array}{c}\text { Error } \\
\text { e \% }\end{array}$ \\
\hline 001 & 2.99 & $\begin{array}{l}r_{9}=8.20 \\
r_{4}=8.34\end{array}$ & $\begin{array}{l}d_{9}=3.00 \\
d_{4}=2.95\end{array}$ & $\begin{array}{l}e_{9}=0.3 \\
e_{4}=1.35\end{array}$ \\
\hline 011 & 2.48 & $r_{5}=8.20$ & $d_{5}=2.43$ & $e_{5}=2.06$ \\
\hline 020 & 2.22 & $r_{7}=10.98$ & $d_{7}=2.24$ & $e_{7}=0.89$ \\
\hline 210 & 2.16 & $r_{8}=11.55$ & $d_{8}=2.13$ & $e_{8}=1.41$ \\
\hline 220 & 1.65 & $r_{6}=15.18$ & $d_{6}=1.62$ & $e_{6}=1.85$ \\
\hline
\end{tabular}

* Error was obtained based on the percentage difference between the calculated dspacing and $\mathrm{FeOOH}$ d-spacing 
A similar SAD analysis was also carried on the iron oxide formed on the CP-0 sample, as shown in Fig. 7.23. Figure 7.23(a)-(d) demonstrates the diffraction patterns at four different locations in the oxide film of the CP-0 sample. In these figures seven diffuse diffraction spots were identified and the corresponding $r$ values (i.e., $r_{10}, r_{11}, r_{12}$, $r_{13,} r_{14,} r_{15}$ and $r_{16}$ ), which are presented in Table 7.6, were determined. Table 7.6 also presents the calculated d-spacings of each measurement and the comparisons with the dspacing of the $\mathrm{FeOOH}$. Interestingly, in Table 7.6 four interplaner spacings (i.e., (110), (001), (101), (001)) were identified, which strongly indicate that the chemical structure of the nano-crytalline particles of iron oxide of the CP-0 sample is similar to that of the $\mathrm{CH}$ 0 sample; i.e., the iron oxide of the $\mathrm{CP}-0$ sample mostly consists of $\mathrm{FeOOH}$ phase.

The oxide reflections shown in the SAD patterns of Fig. 7.23 are rather diffuse because of the small crystallite size, but inspection shows that they tend to occur circumferentially over a small range of angles $\left(\sim 5^{\circ}\right)$. Dark field imaging, as in Fig. 7.20, revealed many small crystallites associated with these diffuse oxide reflections, far more than would be expected if the crystallites were orientated randomly. This indicates that they have a similar orientation relative to the electron beam. Furthermore, there is one set of oxide reflections that lie along a common radial direction with a prominent $\mathrm{Fe}$ reflection from the substrate, demonstrating that there is a set of parallel planes common to both substrate and oxide film. 


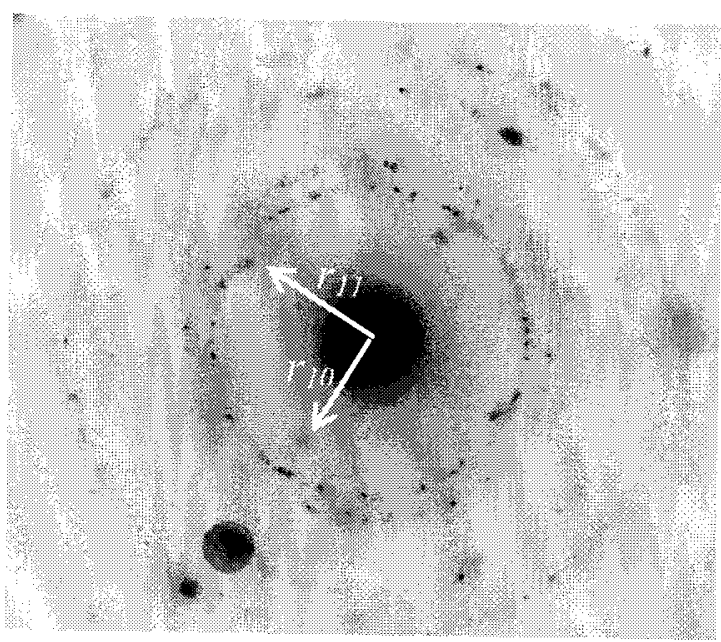

(a)

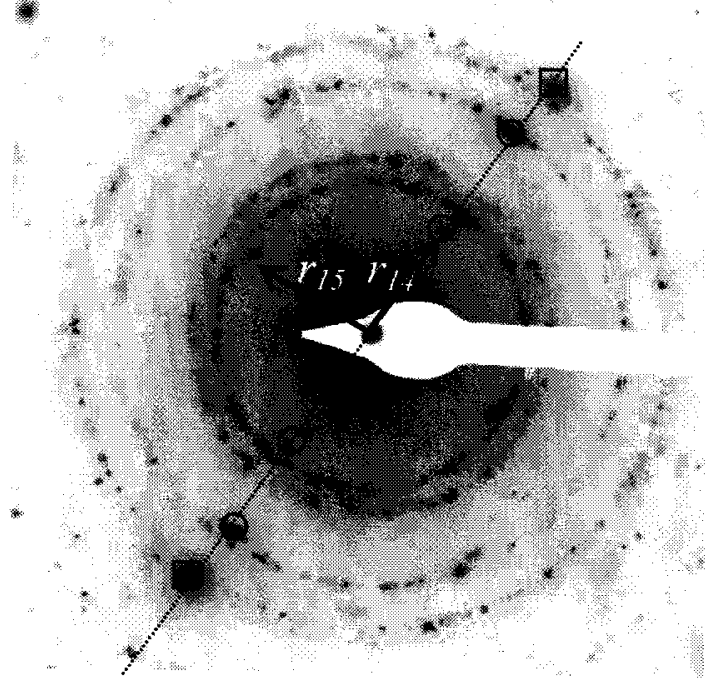

(c)

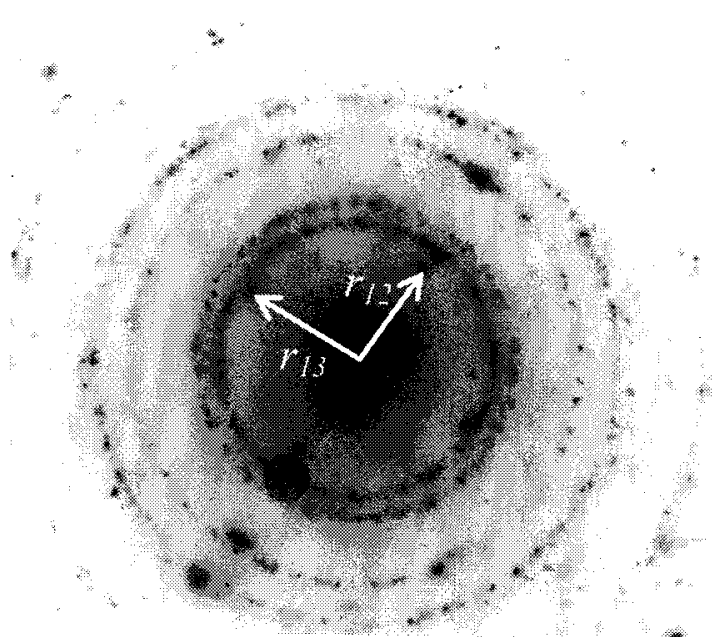

(b)

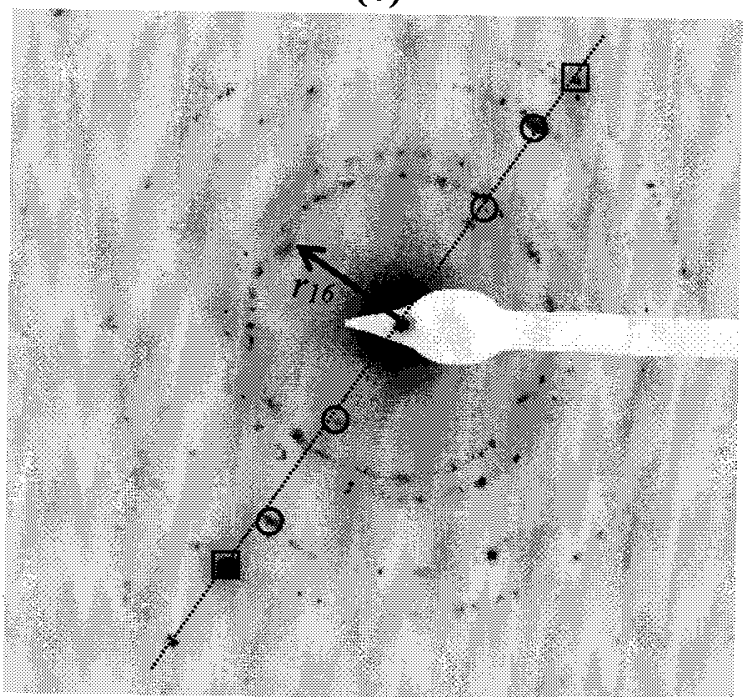

(d)

Figure 7.23: (a)-(d) SAD patterns of four different areas (nano-crystalline particles) in the oxide film of the CP-0 sample (see Fig. 7.20(b)). $\square$ : reflections from the Fe substrate, $O$ : reflections from oxide film. Note that the iron and oxide reflections are almost in the same direction (i.e., on a radial line, less than $5^{\circ}$ deviation).

These results, in general, indicate an epitaxial relationship between the oxide and substrate (i.e., steel), which would lead to a crystallographic texture in the oxide film. Examples of epitaxial growth of oxide films on a metal (including iron) surface have been previously reported in the literature (Foley et al. 1967). This implies that a rearrangement of iron and oxygen atoms to form a crystalline structure is required to 
form the oxide. Therefore, the formation of oxide film with the thickness of less than 10 $\mathrm{nm}$ seems reasonable since only at high temperatures deep oxygen diffusion would be observed. This observation is in support of the place exchange model (Sato and Cohen 1964 in section 3.3) in which the formation of film is attributed to a process whereby oxygen atoms adsorbed on the surface of metal exchange places with underlying metal atoms. The oxide film continues to grow with the adsorption of a second layer of oxygen, which exchanges places with the underlying metal atoms. Point defect model (PDM) also relates the formation of passive film to the annihilation of oxygen vacancies by oxygen atoms at the oxide/solution interface; therefore the observations above are also in agreement with the PDM model.

Table 7.6: The comparison of the CP-0 oxide d-spacings from Fig. 7.23 with $\mathrm{FeOOH}$ d-spacings. Four interplanar spacings (110), (001), (101), (001) matched those of $\mathrm{FeOOH}$. For all other phases, the calculated error was larger than 5\%; therefore they are not reported here $(\lambda L=24.6$ A.mm).

\begin{tabular}{|c|c|l|l|c|}
\hline hkl & $\begin{array}{c}\text { FeOOH } \\
\boldsymbol{d}(\boldsymbol{A})\end{array}$ & $\begin{array}{c}\text { Measured } \\
\boldsymbol{r}(\mathbf{m m})\end{array}$ & $\begin{array}{c}\text { Calculated } \\
\boldsymbol{d}(\boldsymbol{A})\end{array}$ & $\begin{array}{c}\text { Error* } \\
\boldsymbol{e} \%\end{array}$ \\
\hline 110 & 3.30 & $r_{16}=7.4$ & $d_{16}=3.32$ & $e_{16}=0.60$ \\
& & $r_{10}=8.3$ & $d_{10}=2.96$ & $e_{10}=1.01$ \\
001 & 2.99 & $r_{12}=8.3$ & $d_{12}=2.96$ & $e_{12}=1.01$ \\
& & $r_{14}=8.4$ & $d_{14}=2.93$ & $e_{14}=2.05$ \\
101 & 2.56 & $r_{13}=9.7$ & $d_{13}=2.54$ & $e_{13}=0.79$ \\
011 & 2.48 & $r_{15}=9.7$ & $d_{15}=2.54$ & $e_{15}=0.79$ \\
& & $r_{11}=9.8$ & $d_{11}=2.51$ & $e_{11}=1.19$ \\
\hline
\end{tabular}

* Error was obtained based on the percentage difference between the calculated $\mathrm{d}$-spacing and $\mathrm{FeOOH}$ d-spacing. 


\subsection{Conclusions}

The following conclusions can be drawn from the study presented in this chapter:

- The thickness of the oxide films on the $\mathrm{CH}-0$ and $\mathrm{CP}-0$ samples ranges from 5 to $8 \mathrm{~nm}$ and 8 to $13 \mathrm{~nm}$, respectively.

- For the $\mathrm{CH}$ solution, calcium was observed at some locations in the oxide film, while for the $\mathrm{CP}$ solution sulfur and potassium were detected in the structure of oxide film.

- Chlorides in quantities lower than the depassivation thresholds do not change the appearance of the oxide films grown in the $\mathrm{CH}$ and $\mathrm{CP}$ solutions.

- EDS analyses suggest that chlorides and calcium in the $\mathrm{CH}$ solution might have penetrated into the oxide film on the surface of the $\mathrm{CH}-1$ sample.

- Small amounts of chlorine were detected at some points inside the oxide film of the CP-1 sample; however, the intensity of the chlorine signal was not as strong as the one for the $\mathrm{CH}-1$ sample.

- After exposure to chloride in amounts larger than typical depassivation thresholds, iron oxide is no longer uniformly covering the surface and the average thickness of the remaining oxide film is less than the average thickness of the oxide film before exposure to chloride.

- Some pit initiation sites were formed on the surface of steel in the $\mathrm{CH}$ sample at chloride concentrations higher than depassivation thresholds. 
- Some cavities were observed on the surface of steel in the CP sample at chloride concentration higher than depassivation thresholds. These cavities might serve as local sites for pit growth.

- Both EELS and electron diffraction analysis showed that oxide films on the surface of steel are mainly composed of nano-crystalline particles of $\mathrm{FeOOH}$, with an orthorhombic unit cell of $\mathrm{a}=4.937 \AA, \mathrm{b}=4.432 \AA$ and $\mathrm{c}=2.994 \AA$. However, the presence of other oxides could not be eliminated. Additional nano-scale techniques (e.g. XPS) are necessary to identify these minor oxides.

- Diffraction analysis studies suggest that oxide films are in an epitaxial orientation relationship with the underlying $\alpha-\mathrm{Fe}$ crystals. This implies that the oxygen atoms diffuse into the iron surface, where only a small rearrangement of the iron atoms is required to form the oxide film. 


\section{Chapter 8}

\section{XPS Depth Profiling Study of Passive Film on Rebar and the Role of Chloride in Depassivation}

\subsection{Introduction}

Although it is well known that chloride is involved in the depassivation of steel in concrete, the underlying mechanism of this process is still not fully understood. One of the main reasons for this lack of understanding is the unavailability of data on the compositional characteristics of the passive oxide film before and after exposure to chlorides. Unfortunately, traditional electrochemical and most microscopic techniques do not provide such specific data; therefore, specialized nano-scale characterization techniques, such as X-ray photoelectron spectroscopy (XPS), need to be used.

The use of XPS in the characterization of oxides that form on metal and alloy surfaces is not new. A number of researchers have studied oxide formation on iron, in environments different from concrete pore solution (McIntyre and Zetaruk 1977, Khalil 1985, Allen et al. 1982, Fujii et al. 1999, Grosvenor et al. 2004a, Brundle et al. 1977). The investigation of passive film properties of steel rebar, or iron, in highly alkaline environments such as the one provided by concrete pore solution has been quite limited (Haupt et al. 1986, Galicia et al. 2006, Zakroczymski et al. 1985a and 1985b). 
Haupt et al. (1986) passivated iron specimens in $1 \mathrm{M} \mathrm{NaOH}$ solution by applying different levels of potential with time. They used XPS to study the effect of applied potential and passivation time on the structure of the passive oxide film. They showed that the thickness of passive film increased with increasing potential and passivation time at constant applied potential. The maximum thickness, which was obtained at the highest level of applied potential (i.e., $0.7 \mathrm{~V} / \mathrm{SHE}$ ) was reported to be 6 $\mathrm{nm}$. XPS investigation also showed that the passive film consisted of an inner $\mathrm{Fe}^{2+}$-rich layer and an outer $\mathrm{Fe}^{3+}$-rich layer. Although these observations provide important clues about the characteristics of the passive oxide film on iron in alkaline environments, the results cannot be easily extrapolated to carbon steel that passivates in concrete without the application of external potential. In addition, the use of $\mathrm{NaOH}$ as the passivating solution does not adequately represent the concrete pore solution, which is mainly composed of saturated calcium hydroxide.

The XPS studies conducted by Montemor et al. (1998) on carbon steel (polished to 2,400-grit) in extracted concrete pore solutions and saturated calcium hydroxide in the presence of fly ash showed that the composition of the concrete pore solution and chlorides can change the type of the oxide film on the steel surface. They reported that the outermost layers of the passive films formed on the steel in concrete pore solutions were essentially composed of three types of oxides including $\mathrm{FeOOH}$. Based on $\mathrm{Fe} / \mathrm{O}$ variation as a function of the estimated sputtering depth (Fig.5 of Montemor et al. 1998), the oxide film seems to consist of two layers: a top layer with a thickness of $5 \mathrm{~nm}$ and an intermediate layer with a thickness of about $100 \mathrm{~nm}$. The exact composition of this intermediate layer was not investigated. They also stated that the concentration of 
FeOOH increased with the presence of chlorides in the solution; chlorides also caused an increase in the thickness and water content of the passive film, which is an interesting but somewhat controversial observation. A more controversial outcome of this study, on the other hand, was regarding the thickness of the passive oxide films, which were reported to be, in some cases, in excess of $100 \mathrm{~nm}$, a value considerably larger (by an order of magnitude) than those reported in other studies using different characterization techniques (See Miserque et al. 2006, Haupt and Strehblow 1987, Zakroczymski et al. 1985a, Chapter 7 of this thesis). This unusual finding suggests that oxide film investigated by Montemor et al. (1998) may also have contained oxygen-rich precipitates on the steel surface, leading to an overestimation of the average thickness of the film, since they relied on the XPS-derived oxygen spectra data to determine the thickness.

Currently, only limited knowledge exists on the composition and structure of passive oxide films formed on carbon steel that is exposed to concrete pore solutions. The XPS study, presented in this chapter, was carried out to partly fill this gap in knowledge. The study also investigates the mechanism of chloride-induced depassivation of such films.

It is common practice to use a saturated calcium hydroxide solution to study passivity and depassivation of carbon steel in highly alkaline environments since concrete pore solution mainly contains saturated calcium hydroxide along with other auxiliary components such as sodium hydroxide, potassium hydroxide and calcium sulfate (Abd El Aal et al. 2009, Monticelli et al. 2000, Blanco et al. 2006, Saremi and Mahallati 2002). Although the auxiliary components also contribute to the passivity as well as depassivation of the steel in concrete (Goni and Andrade 1990, Ghods et al. 2009, 
Poursaee and Hansson 2007, Mammoliti and Hansson 2005), in order to eliminate the complexity associated with these auxiliary components, it was decided in this study to use saturated calcium hydroxide as the test solution. XPS studies on the effect of the simulated concrete pore solution that includes other ionic species such as calcium, sodium, potassium and sulfate have been also carried out and are presented in Chapter 9.

\subsection{Experimental Setup}

\subsubsection{Preparation of specimens}

Saturated calcium hydroxide solution (designated as " $\mathrm{CH}^{\prime)}(\mathrm{pH}=12.5$, conductivity $=8.82 \mathrm{mS} / \mathrm{cm}$ and dissolved oxygen $=2.28 \mathrm{mg} / 1$ ) was prepared by dissolving analytical grade calcium hydroxide $(99.6 \%)$ in distilled water, and was considered in this study as surrogate to concrete pore solution.

XPS analysis was performed on cross sections of carbon steel rebar specimens. The elemental composition of rebar obtained from spectrographic analysis is presented in Table 8.1. Rebar (Size designation: \#10M) was cut into four 8-mm-long specimens, which were then hot-mounted using epoxy resins (a combination of thermosetting Phemolic and Epomet Fine Grain molding compounds) to prepare for polishing. The cross sections of hot-mounted specimens were polished to $0.05 \mu \mathrm{m}$ with anhydrous (water content: $<0.05 \%$ by weight) isopropyl alcohol, which was used to avoid the oxidation of the surface. After polishing, the epoxy resin was removed from the four rebar specimens, which were then kept in anhydrous isopropyl alcohol to avoid exposure to air. 
Three of the polished rebar specimens were then immediately immersed in the $\mathrm{CH}$ solution. The first two specimens, $\mathrm{CH}-2$ and $\mathrm{CH}-9$, were removed from the $\mathrm{CH}$ solution after 2 and 9 days of immersion, respectively, and immediately placed in sealed jars containing anhydrous isopropyl alcohol until the XPS test. These two specimens were prepared to study the effect of immersion time on the structure and properties of passive film. After removing the $\mathrm{CH}-9$ specimen, sodium chloride ( $\mathrm{NaCl}$, analytical grade $99.99 \%$ ) was added to the $\mathrm{CH}$ solution to increase the chloride concentration to $0.05 \mathrm{M}$, which is an amount close to the depassivation threshold of carbon steel passivated in a saturated calcium hydroxide solution.

A third specimen ( $\mathrm{CH}-\mathrm{Cl})$ was exposed for an additional 14 days to this $\mathrm{CH}$ plus $0.05 \mathrm{M}$ chloride solution and then was removed and immersed in anhydrous isopropyl alcohol prior to XPS examination. The $\mathrm{CH}-\mathrm{Cl}$ specimen was used to study the effect of chlorides on the passive film. The two-week exposure time was chosen to give chlorides enough time to react with the passive oxide film. A fourth specimen (AE) was not immersed in the $\mathrm{CH}$ solution, but was exposed to indoor air (at room temperature) for about 10 minutes to allow oxidation of the steel surface. This specimen was prepared to compare the properties of passive film grown in the $\mathrm{CH}$ solution with the iron oxide film formed in air. 
Table 8.1: Steel elemental composition (Average of three spectrographic results).

\begin{tabular}{|c|c|}
\hline Element & Weight \% \\
\hline $\mathrm{C}$ & 0.26 \\
$\mathrm{Si}$ & 0.27 \\
$\mathrm{Mn}$ & 1.10 \\
$\mathrm{Cr}$ & 0.05 \\
$\mathrm{Ni}$ & 0.07 \\
$\mathrm{Mo}$ & $<0.01$ \\
$\mathrm{Cu}$ & 0.21 \\
$\mathrm{Al}$ & $<0.005$ \\
$\mathrm{Nb}$ & $<0.01$ \\
$\mathrm{~V}$ & $<0.005$ \\
$\mathrm{Ti}$ & $<0.005$ \\
$\mathrm{~B}$ & $<0.0005$ \\
$\mathrm{P}$ & 0.01 \\
$\mathrm{~S}$ & 0.03 \\
$\mathrm{~W}$ & $<0.01$ \\
$\mathrm{Sn}$ & 0.021 \\
$\mathrm{Co}$ & 0.01 \\
$\mathrm{Zr}$ & $<0.01$ \\
$\mathrm{Fe}$ & Balance \\
\hline
\end{tabular}

\subsubsection{XPS test procedure}

A modified Perkin-Elmer (Physical Electronics Inc.) PHI-5700-2 XPS spectrometer with an achromatic $\mathrm{Al} \mathrm{K \alpha} \mathrm{x}$-ray source and an analytical chamber with a base pressure of $10^{-6} \mathrm{~Pa}$ were used to collect XPS spectra. The x-ray gun was operated at 400 watts $(15 \mathrm{kV}, 26.7 \mathrm{~mA})$. The work function of the spectrometer was adjusted using ultra pure gold metal $(\mathrm{Au} 4 \mathrm{f7} / 2=84.0 \mathrm{eV})$ and the instrument linearity was set to yield a difference of $857.5 \mathrm{eV}$ between the $\mathrm{Cu} 2 \mathrm{p} 3 / 2$ and $\mathrm{Cu} 3 \mathrm{p}$ photoelectron lines. The data were collected using a spherical capacitor analyzer (SCA) equipped with a seven-element "Omni-Focus V" lens. The angle between the analyzer and the X-ray source was $54^{\circ}$. The X-ray emission angle was $90^{\circ}$ with respect to the specimen surface. 
Each specimen was mounted into a specimen holder and then transferred directly to the fore-chamber of the XPS spectrometer and purged with dry nitrogen gas. This chamber was evacuated to a pressure of $\sim 10^{-5} \mathrm{~Pa}$ before the specimen was transferred to the analytical chamber for examination. The analytical chamber is an ultra high vacuum (UHV) chamber with vacuum pressure of $10^{-6} \mathrm{~Pa}$. In this study, the diameter of the analyzed area was $800 \mu \mathrm{m}$. The low-energy electron flood gun for charge neutralization was not required during these analyses. XPS "survey scan" mode permits broad low-energy spectra to be collected. Survey acquisition was performed under the following conditions: energy range $=1400 \mathrm{eV}$, analyzer pass energy $=187.8 \mathrm{eV}$, step size $=$ $0.25 \mathrm{eV}$ and sweep time $=180 \mathrm{~s}$. Higher resolution XPS spectra were then collected using either 10 or $20 \mathrm{eV}$ spectral windows at an analyzer pass energy of $29.3 \mathrm{eV}$ and $0.1 \mathrm{eV}$ steps. The high-energy resolution spectra were collected for all elements assigned in the survey spectra; typically oxygen (O 1s), carbon (C 1s), iron (Fe 2p), chlorine (Cl 2p), calcium ( $\mathrm{Ca} 2 \mathrm{p})$, and sodium ( $\mathrm{Na} 1 \mathrm{~s})$.

All specimens were ion sputtered using an argon gun operating at $3 \mathrm{KeV}$ to conduct in-depth profile analysis and to determine the thickness of the oxide film. The Argon ion beam was rastered over a $2 \times 2 \mathrm{~mm}$ area $\left(\sim 150 \mu \mathrm{A} / \mathrm{cm}^{2}\right)$ yielding a sputter rate of $\sim 2.5 \mathrm{~nm} /$ minute, calibrated against a reference silicon oxide thin film on silicon substrate $\left(\mathrm{SiO}_{2} / \mathrm{Si}\right)$. These settings reduce the potential distortion of oxide film due to argon ion beam sputtering. An HP-425 Vectra personal computer with MS-WinNT was used to control all spectrometer functions and to move the five-axis sample stage. PHI Access XPS operating software (ver. 7.2C) was employed to collect and process all "survey" and "high-resolution" spectra. Optimal XPS spectra curve-fitting and resultant 
peak data set analyses were performed using Casa-XPS software (ver. 2.2.107). CasaXPS's Shirley background correction algorithm was used to determine optimal spectral baseline and peaks. Curve-resolved shapes were based on Gaussian (70\%) Lorentzian (30\%) combination function (Fairley and Carrick 2005). All peaks were calibrated to hydrocarbon (C 1s) signal set at $285.0 \mathrm{eV}$. Semi-quantitative composition information was determined using empirically derived XPS elemental sensitivity factors along with the peak areas obtained from the curve-fitted spectra.

\subsubsection{Reproducibility of data}

As shown in Fig. 8.1, Fe 2p high resolution spectra were obtained at two 800 $\mu$ m-diameter spots $(\sim 5 \mathrm{~mm}$ apart $)$ on the surface of the $\mathrm{CH}-\mathrm{Cl}$ specimen to determine any variation in oxide film stoichiometry with location. Fig. 8.1(a) shows two Fe 2 p spectra acquired before argon ion sputtering; both the Fe $2 \mathrm{p}$ spectra show nearly identical shapes and intensities. This indicates that the film stoichiometry is quite similar over the large area of the specimen.

The $\mathrm{Fe} 2 \mathrm{p}$ spectra of the $\mathrm{CH}-\mathrm{Cl}$ specimen acquired at these spots after ion sputtering to depths of $2.5 \mathrm{~nm}, 5 \mathrm{~nm}$ and $12.5 \mathrm{~nm}$, are shown in Fig. 8.1(b), 8.1(c) and 8.1(d), respectively. The average differences between the Fe $2 \mathrm{p}$ spectra for the $2.5 \mathrm{~nm}, 5$ $\mathrm{nm}$ and $12.5 \mathrm{~nm}$ sputtered depths are less than $8 \%$ indicating that the variation of oxide film stoichiometry over the surface area is not noticeable. 


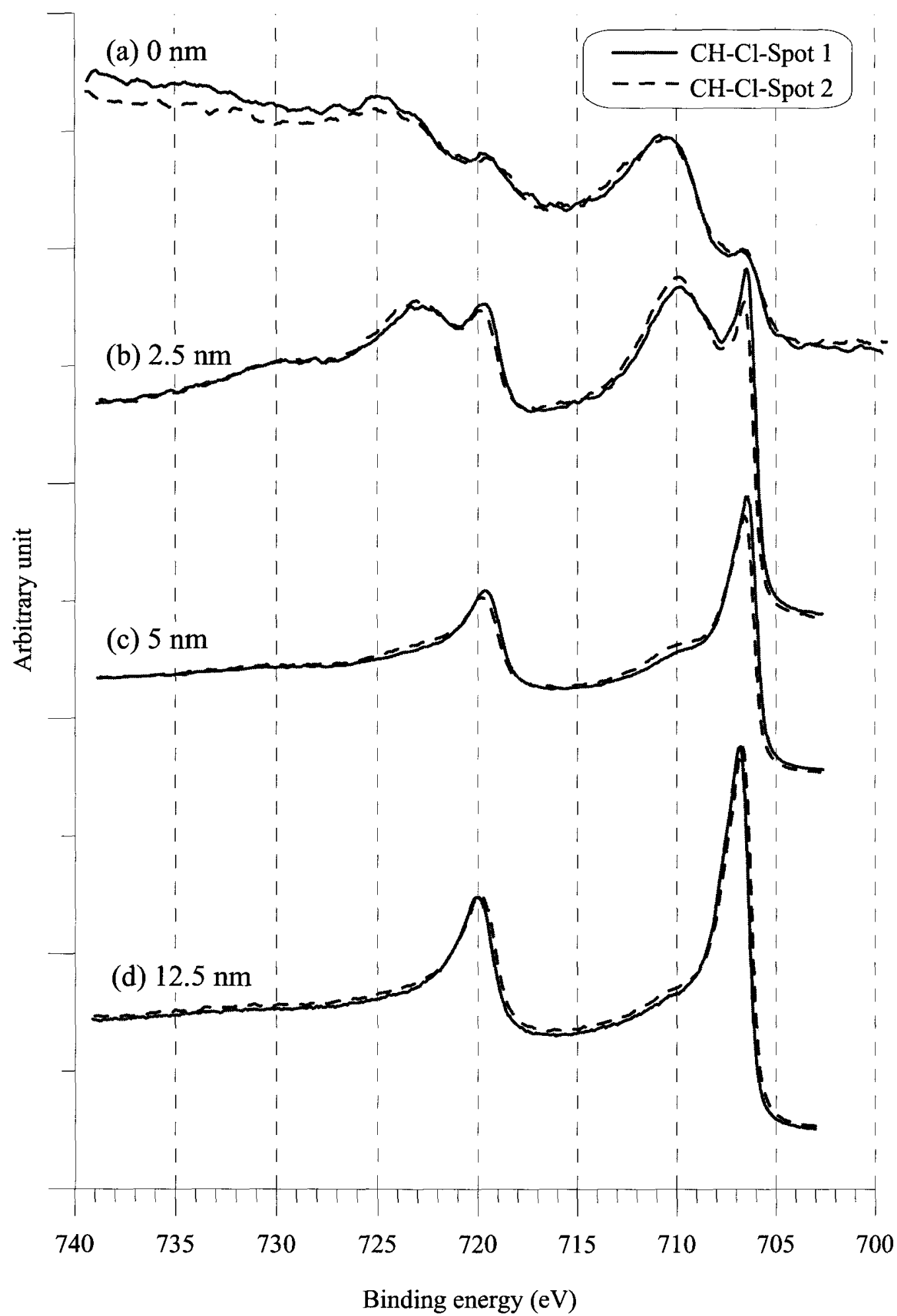

Figure 8.1: The Fe 2p XPS spectra obtained from two 800- $\mu m$-diameter spots on the $\mathrm{CH}$-Cl specimen: (a) pre-sputter; (b) Argon ion sputter $2.5 \mathrm{~nm}$; c) Argon ion sputter 5 $\mathrm{nm}$; (d) Argon ion sputter $12.5 \mathrm{~nm}$. 


\subsection{XSP Data Analysis}

\subsubsection{Sampling depth}

The sampling depth $(d)$ is defined as the thickness of a layer where $95 \%$ of the signal intensity is derived form. In XPS, the sampling depth (d) can be obtained by (Briggs and Seah 1990)

$$
d=3 \lambda \cos (\theta)
$$

where $\theta$ (degree) is the take-off angle with respect to the surface normal and $\lambda(\mathrm{nm})$ is the attenuation length. For inorganic compounds, such as iron oxides, $\lambda$ can be estimated as (Seah and Dench 1979)

$$
\lambda \approx 0.096 \sqrt{E_{k}}
$$

where $E_{k}(\mathrm{eV})$ is the kinetic energy of the ejected photoelectron. Having the core level binding energy of an individual element, the kinetic energy of that element can be calculated from

$$
E_{k}=h_{v}-E_{b}
$$

where $E_{b}(\mathrm{eV})$ is the binding core level energy of the element and $h_{v}(\mathrm{eV})$ is the characteristic energy of the X-ray photon. Using this procedure the sampling depth can be estimated for iron oxide.

In this study, since iron and oxygen elements generally form the structure of oxide film, an average attenuation length $\left(\lambda_{\text {ave. }}\right)$ of these elements was used to determine the sampling depth (Eq. 8.1). Using the core level binding energy of these elements, their kinetic energies were obtained, and the average attenuation length was therefore defined using Eqs. 8.2 and 8.3. The emission angle of $0^{\circ}$ and the X-ray photon energy source of 
$1487 \mathrm{eV}$ result in an average sampling depth of $8.5 \mathrm{~nm}$ for the iron oxide film. Summary of the results from this calculation is presented in Table 8.2.

Table 8.2: The calculation of sampling depth (d) for iron oxide $\left(\theta=0^{\circ}\right.$ and $\left.h_{v}=1487 \mathrm{eV}\right)$

\begin{tabular}{|l|l|l|l|l|}
\hline Element core level & $E_{\mathbf{b}}(\mathbf{e V})$ & $E_{\mathbf{k}}(\mathbf{e V})$ & $\lambda(\mathbf{n m})$ & $\mathbf{d}(\mathbf{n m})$ \\
\hline Fe $2 \mathrm{p}$ & 710 & 777 & 2.7 & 8.0 \\
\hline $\mathrm{O} 1 \mathrm{~s}$ & 532 & 955 & 3.0 & 8.9 \\
\hline & & Average & 2.8 & 8.5 \\
\hline
\end{tabular}

\subsubsection{XPS curve fitting process}

Curve fitting of high resolution XPS spectra is usually conducted to quantify and deconvolute the contribution of each chemical species (element associations) that comprise the spectra. Curve fitting of high resolution XPS spectra is a challenging task for which different constraints are required to be met simultaneously (Briggs and Seah 1990, Fairley and Carrick 2005). Deciding on the number of possible components (peaks) that compose an XPS spectra envelope, and accordingly assigning appropriate chemical compounds to these components (peaks), require a deep understanding of the characteristics of the material being tested. In this research, in addition to reviewing the data reported in other studies, all high resolution XPS spectra envelopes systematically curve fitted to the minimum number of peaks required for an optimum fit and to the most probable components needed for corresponding chemical assignments. A brief description of this process is provided here.

Using Casa-XPS features (Fairley and Carrick 2005) the following constraints have been introduced for optimal fitting:

- For each element the peak position of different compounds are set with respect to the average reported data in the literature. However, due to the uncertainty 
inherent in the experimental data, an adjustment of $\pm 0.5 \mathrm{eV}$ to the peak positions obtained from the literature is applied.

- All peak positions are kept constant for different specimens at all depths. For instance, the peak position for metallic iron in $\mathrm{Fe} 2 \mathrm{p}_{3 / 2}$ spectra is set to $\sim 707 \pm$ $0.5 \mathrm{eV}$ for all depths and for all specimens.

- The full width at half maximum (FHWM) value of different compounds is approximately set as the FHWM of photoelectron core level of the corresponding element. For instance, in the $\mathrm{O} 1 \mathrm{~s}$ the FHWM for different oxygen-containing compounds is considered to be $1.6 \mathrm{eV}$. However, for some cases, in which there is a possibility of the placement of two phases at a similar binding energy position, a wider FHWM value was considered.

In this work, curve fitting was carried out for iron (Fe 2p), oxygen (O 1s), carbon (C 1s) and calcium (Ca 2p) spectra set. The details are provided below for each element.

Carbon: The C 1s spectrum generally consists of seven components (i.e., C-1, C-2, C-3, C-4, C-5, C-6 and C-7) as shown in Fig. 8.2. Comparing the energy positions of these components with previous studies (Dilks 1981), a chemical state was assigned for each component, as presented in Table 8.3. Additionally, the curve parameters such as the position of peak and the FHWM of each $\mathrm{C} 1 \mathrm{~s}$ component that were used for curve fitting of all $\mathrm{C} 1 \mathrm{~s}$ spectra are also reported in Table 8.3. All high resolution spectra were calibrated to C-3 (C-C) binding energy of $285.0 \mathrm{eV}$ following the curve fitting of $\mathrm{C} 1 \mathrm{~s}$ spectra. 


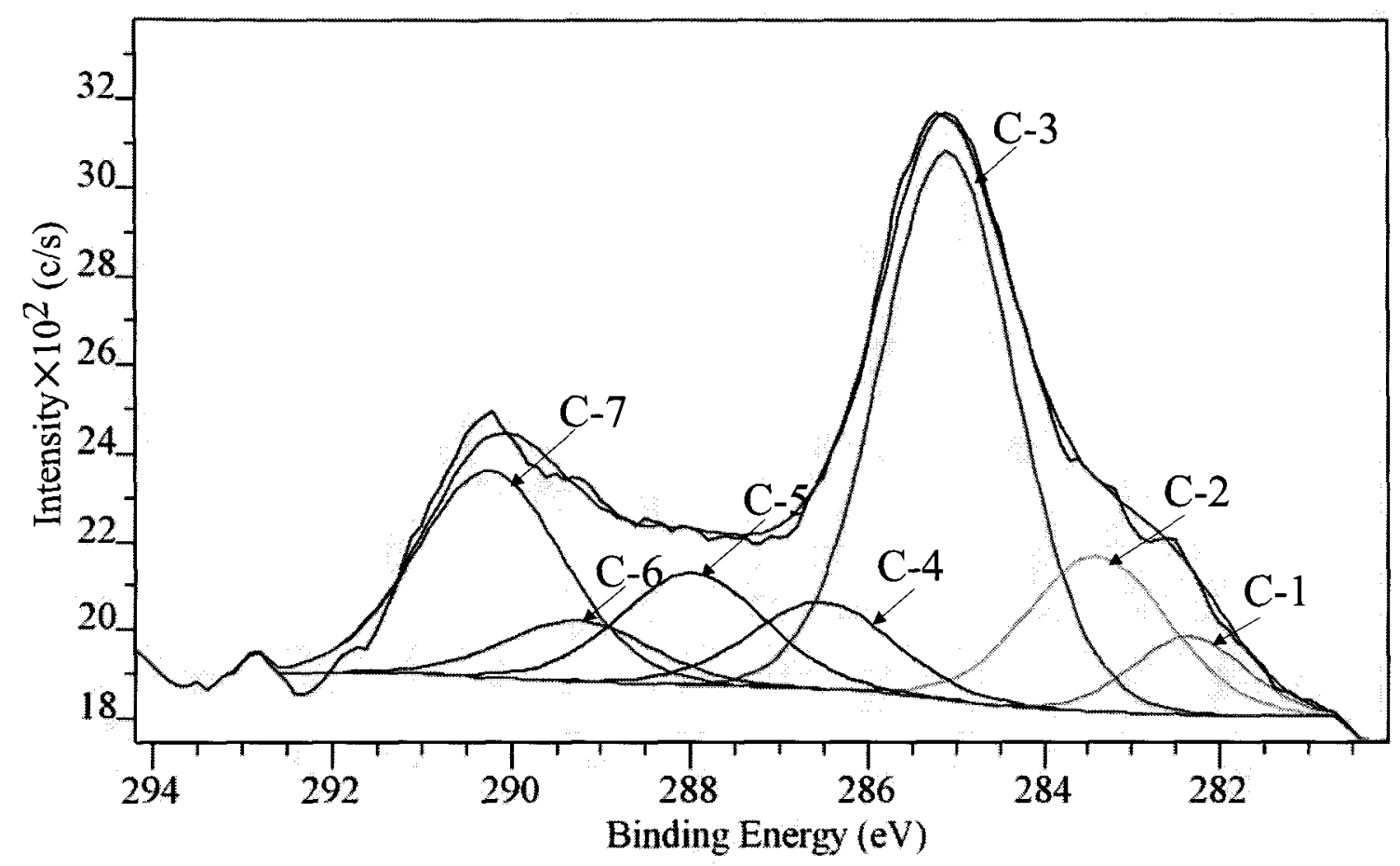

Figure 8.2: Typical curve fitting conducted for C1s high resolution XPS spectrum.

Iron: Iron $2 \mathrm{p}$ spectrum is composed of a doublet structure ( 2 peaks) due to multiplets splitting (i.e., Fe $2 \mathrm{p}_{3 / 2}$ and $\mathrm{Fe} 2 \mathrm{p}_{1 / 2}$ ) as shown in Fig. 8.3. The binding energies of different ion compounds reported in previous studies (NIST Standard Reference Database 20, V. 3.5) are shown in Fig. 8.4. In view of the average of the binding energies reported for each compound in Fig. 8.4, iron compounds can be classified into four groups as Fe-1, $\mathrm{Fe}-2, \mathrm{Fe}-3$ and $\mathrm{Fe}-4$, respectively corresponding to $\mathrm{Fe}$ metal, $\mathrm{Fe}_{3} \mathrm{C}, \mathrm{Fe}_{3} \mathrm{O}_{4} / \mathrm{FeO}\left(\mathrm{Fe}^{2+}\right)$ and $\mathrm{Fe}_{2} \mathrm{O}_{3} / \mathrm{FeOOH}\left(\mathrm{Fe}^{3+}\right)$. Peak position proximity and the difficulty in resolving these peaks is the main reason why additional peak was not considered individually. It is recognized that a much better fitting could be obtained when considering extra peaks (Grosvenor et al. 2004b). Furthermore, an additional component (Fe-5) associated with ' $\mathrm{Fe}_{2} \mathrm{O}_{3}$-Satellite was used in curve fitting analysis of Fe 2p, as suggested in other studies (Fujii et al. 1999, Grosvenor et al. 2004b, McIntyre and Zetaruk 1977). The iron $2 p$ spectra were therefore 
fitted to five iron components as illustrated in Fig. 8.3. The parameters of each component are presented in Table 8.3.

Table 8.3: The peak parameters of ionic species for different elements used in this study.

\begin{tabular}{|c|c|c|c|c|c|c|}
\hline $\begin{array}{c}\text { Element } \\
\text { (Photo } \\
\text { electron core } \\
\text { level) }\end{array}$ & \begin{tabular}{|c} 
Peak \\
ID
\end{tabular} & Assignment & $\begin{array}{c}\text { Peak } \\
\text { position } \\
2 \mathrm{P}_{312} / 1 \mathrm{~s} \\
( \pm 0.5 \mathrm{eV})\end{array}$ & $\begin{array}{c}\text { FWHM } \\
2 P_{3 / 2} \\
( \pm 0.1 \mathrm{eV})\end{array}$ & $\begin{array}{c}\text { FWHM } \\
2 P_{1 / 2} \\
( \pm 0.1 \mathrm{eV})\end{array}$ & $\begin{array}{l}\Delta \mathrm{e}(\mathrm{eV}) \\
( \pm 0.5 \mathrm{eV})\end{array}$ \\
\hline \multirow{5}{*}{$\mathrm{Fe} 2 \mathrm{p}$} & $\mathrm{Fe}-1$ & Fe metal & 707.0 & 1.8 & 1.9 & 13.5 \\
\hline & $\mathrm{Fe}-2$ & $\mathrm{Fe}_{3} \mathrm{C}$ & 708.2 & 2 & 2.3 & 13.5 \\
\hline & $\mathrm{Fe}-3$ & $\mathrm{Fe}_{3} \mathrm{O}_{4} / \mathrm{FeO}$ & 709.6 & 2 & 2.3 & 13.5 \\
\hline & $\mathrm{Fe}-4$ & $\mathrm{Fe}_{2} \mathrm{O}_{3} / \mathrm{FeOOH}$ & 711.0 & 2 & 2.3 & 13.5 \\
\hline & $\mathrm{Fe}-5$ & $\mathrm{Fe}_{2} \mathrm{O}_{3}$-Satellite & 712.7 & 2.6 & NA & 13.5 \\
\hline \multirow{4}{*}{$01 \mathrm{~s}$} & $0-1$ & Iron oxide & 529.8 & 1.6 & NA & NA \\
\hline & $0-2$ & $\mathrm{CaO}$ & 530.3 & 1.6 & NA & NA \\
\hline & $0-3$ & {$\left[\mathrm{CO}_{3}\right]^{2} / \mathrm{FeO}(\mathrm{OH})^{\star *}$} & 531.5 & 1.6 & NA & NA \\
\hline & $0-4$ & $(\mathrm{C}=0) / \mathrm{H}_{2} \mathrm{O}$ & 533.3 & 1.6 & NA & NA \\
\hline \multirow{7}{*}{ C 1s } & $\mathrm{C}-1$ & $\mathrm{Ca}_{2} \mathrm{C}$ & 282.3 & 1.5 & NA & NA \\
\hline & $\mathrm{C}-2$ & $\mathrm{Fe}_{3} \mathrm{C}$ & 283.7 & 1.5 & NA & NA \\
\hline & C-3 & $\mathrm{C}-\mathrm{C}$ & 285.0 & 1.9 & NA & NA \\
\hline & C-4 & $\mathrm{C}-\mathrm{O}$ & 286.6 & 1.9 & NA & NA \\
\hline & $C-5$ & $\mathrm{C}=\mathrm{O}$ & 287.8 & 1.9 & NA & NA \\
\hline & C-6 & $\mathrm{O}-\mathrm{C}=\mathrm{O}$ & 289.2 & 1.9 & NA & NA \\
\hline & $\mathrm{C}-7$ & $\mathrm{CaCO}_{3}$ & 290 & 1.9 & NA & NA \\
\hline \multirow{3}{*}{ Сa $2 p$} & Ca-1 & $\mathrm{Ca}_{2} \mathrm{C}$ & 345.2 & 1.8 & 1.9 & 3.5 \\
\hline & Ca-2 & $\mathrm{CaCO}_{3} / \mathrm{CaO} / \mathrm{Ca}(\mathrm{OH})_{2}$ & 346.7 & 1.8 & 1.9 & 3.5 \\
\hline & Ca-3 & $\mathrm{CaCl}_{2} / \mathrm{CaSO}_{4}$ & 348.2 & 1.8 & 1.9 & 3.5 \\
\hline $\mathrm{Na} 1 \mathrm{~s}$ & $\mathrm{Na}-1$ & $\mathrm{NaCl}$ & 1071.1 & 1.6 & NA & NA \\
\hline $\mathrm{Cl} 2 \mathrm{p}$ & $\mathrm{Cl}-1$ & $\mathrm{NaCl} / \mathrm{CaCl}_{2}$ & 196.3 & 3.2 & 3.2 & 1.6 \\
\hline
\end{tabular}

* Reported as binding energy

${ }^{* *}$ Fe-OH component in $\mathrm{FeO}(\mathrm{OH})$ 


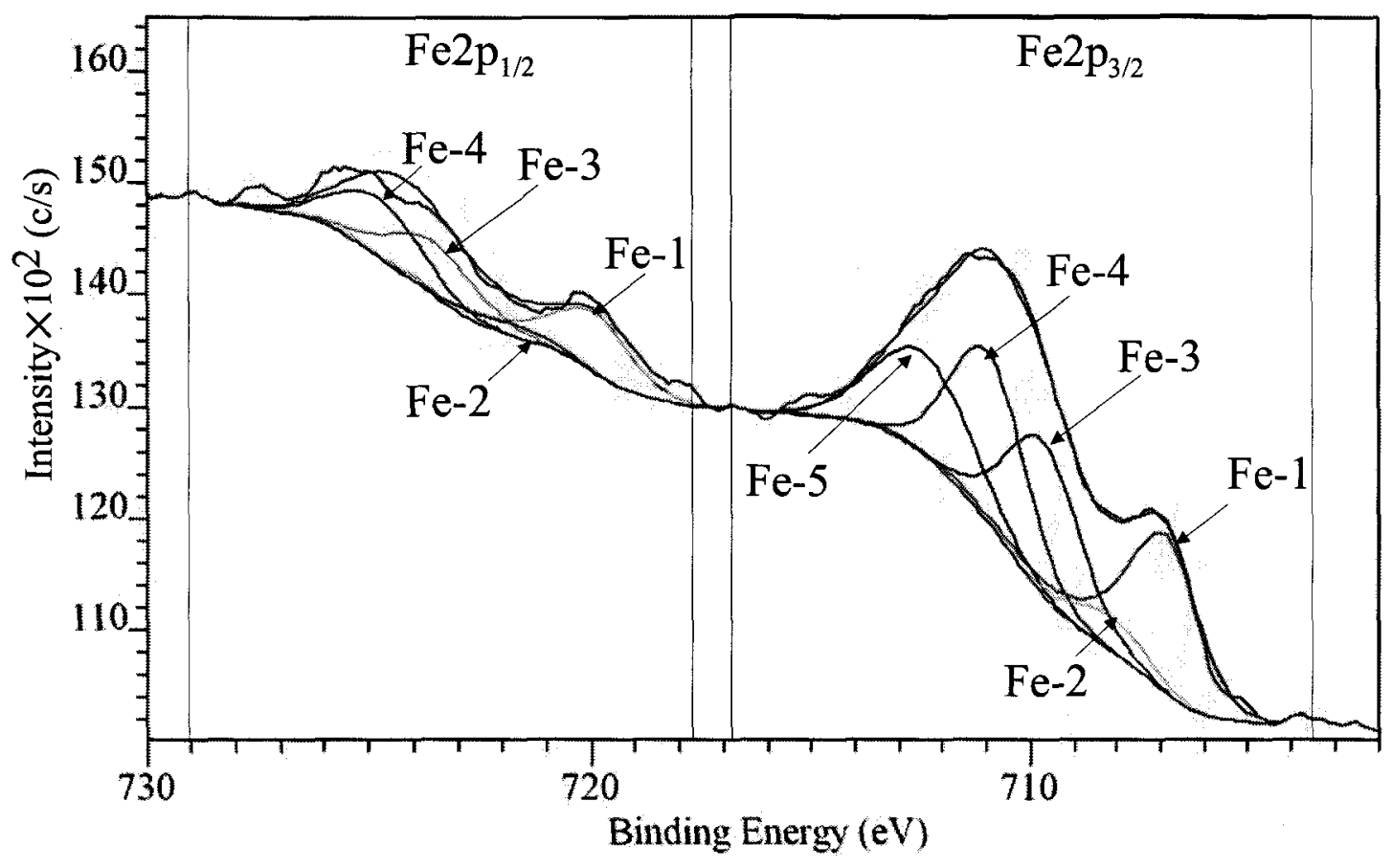

Figure 8.3: Typical curve fitting conducted for Fe 2p high resolution XPS spectrum.

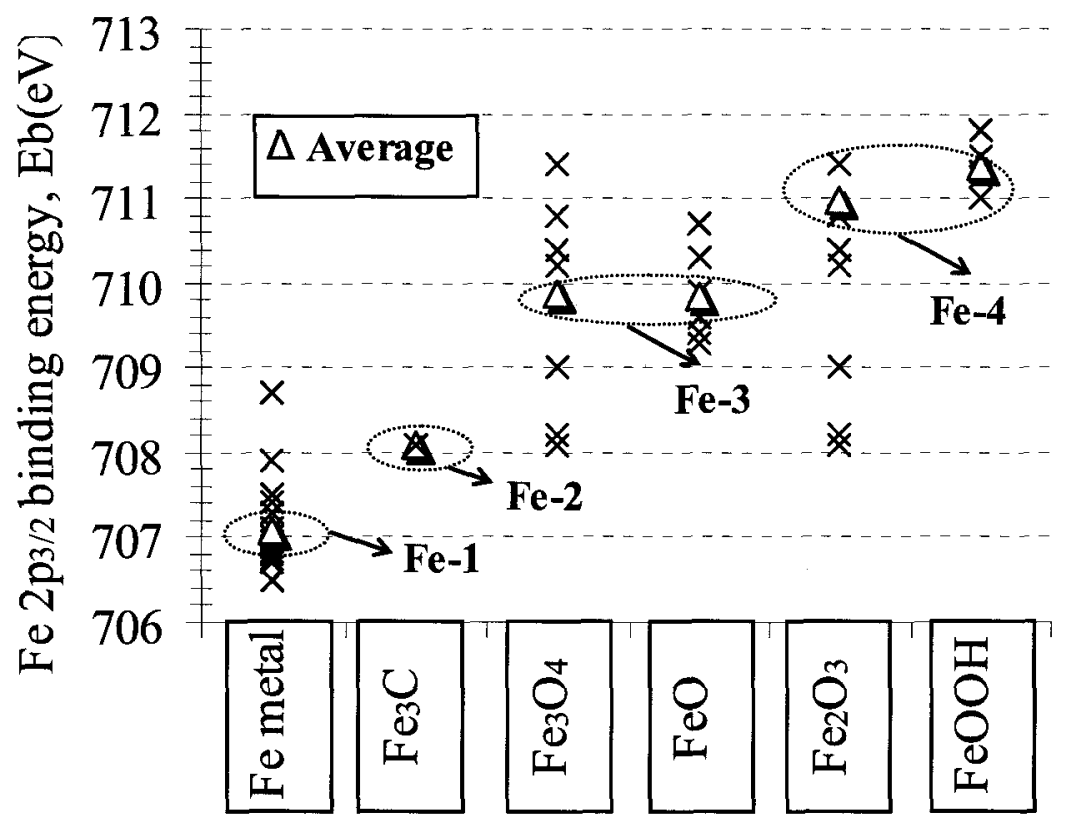

Figure 8.4: The peak $E_{b}$ position data obtained from other studies (NIST Standard Reference Database 20, V. 3.5) for iron compounds at the surface of steel. 
Oxygen: The $\mathrm{O}$ 1s spectrum can be generally fitted with four components $(\mathrm{O}-1, \mathrm{O}-2, \mathrm{O}-3$ and O-4), as shown in Fig. 8.5. Based on the results reported in other studies (NIST Standard Reference Database 20, V. 3.5) given in Fig. 8.6, these four components were assigned to different chemical compounds: O-1 is associated with iron oxide compounds, O-2 corresponds to $\mathrm{CaO}$, and $\mathrm{O}-3$ and $\mathrm{O}-4$ represent $\left[\mathrm{CO}_{3}\right]^{2-} / \mathrm{FeO}(\mathrm{OH})$ and $(\mathrm{C}=\mathrm{O}) / \mathrm{H}_{2} \mathrm{O}$, respectively. The parameters of each component are presented in Table 8.3.

Calcium: A typical high resolution XPS spectrum of $\mathrm{Ca} 2 \mathrm{p}$ is shown in Fig. 8.7. Three $\mathrm{Ca} 2 \mathrm{p}$ peak doublets (i.e., $\mathrm{Ca}-1, \mathrm{Ca}-2$ and $\mathrm{Ca}-3$ ) associated with $\mathrm{Ca}$ incorporated chemical compounds were defined and fitted to the spectra. Using the average of binding energy data obtained from other studies (NIST Standard Reference Database 20, V. 3.5) (see Fig. 8.8), it was found that $\mathrm{Ca}-1$ represents $\mathrm{Ca}_{2} \mathrm{C}$, and $\mathrm{Ca}-2$ and $\mathrm{Ca}-3$ correspond to $\mathrm{CaCO}_{3} / \mathrm{CaO} / \mathrm{Ca}(\mathrm{OH})_{2}$ and $\mathrm{CaCl}_{2} / \mathrm{CaSO}_{4}$, respectively. The $\mathrm{Ca} 2 \mathrm{p}$ spectra were therefore fitted to three calcium components as illustrated in Fig. 8.7

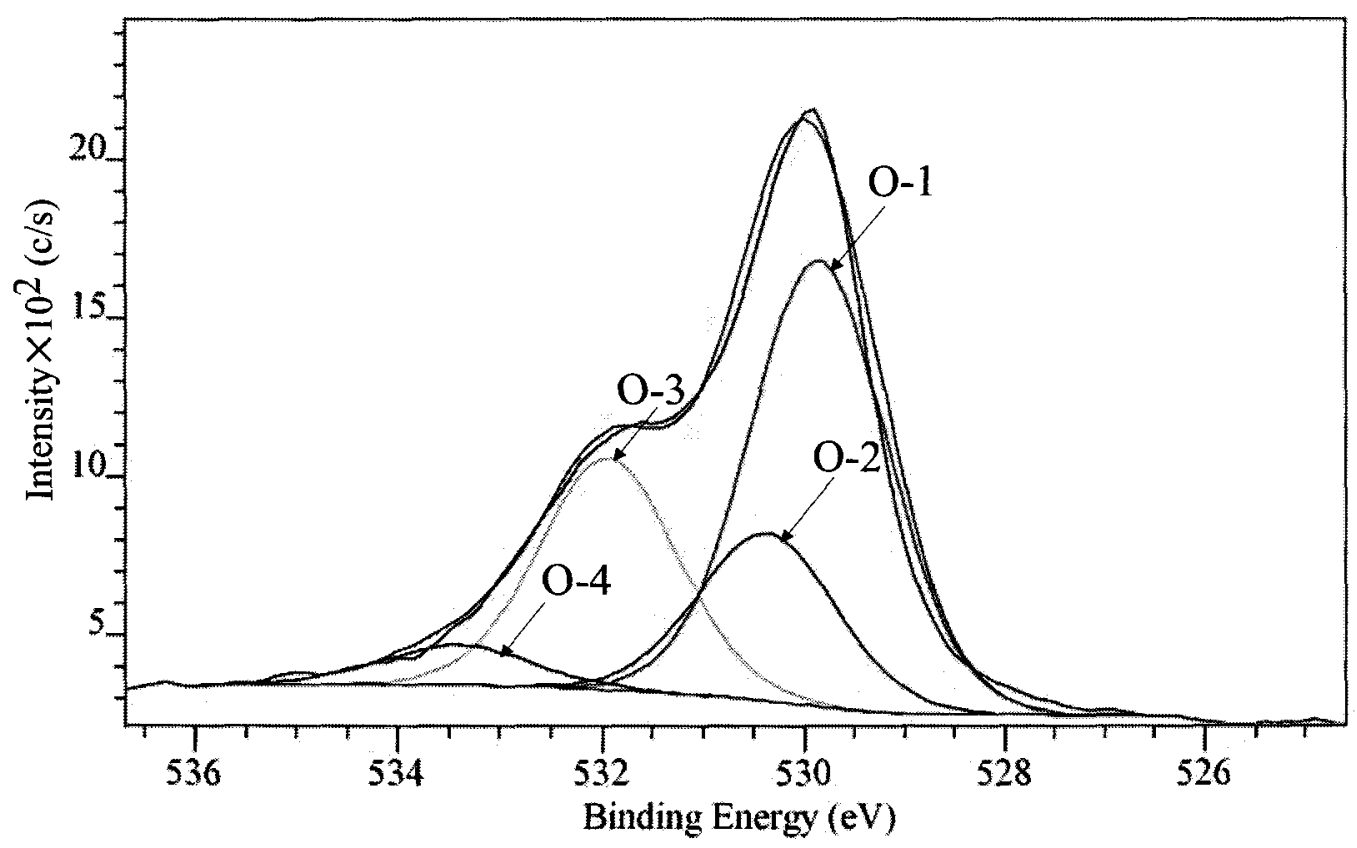

Figure 8.5: Typical curve fitting conducted for O 1s high resolution XPS spectrum. 


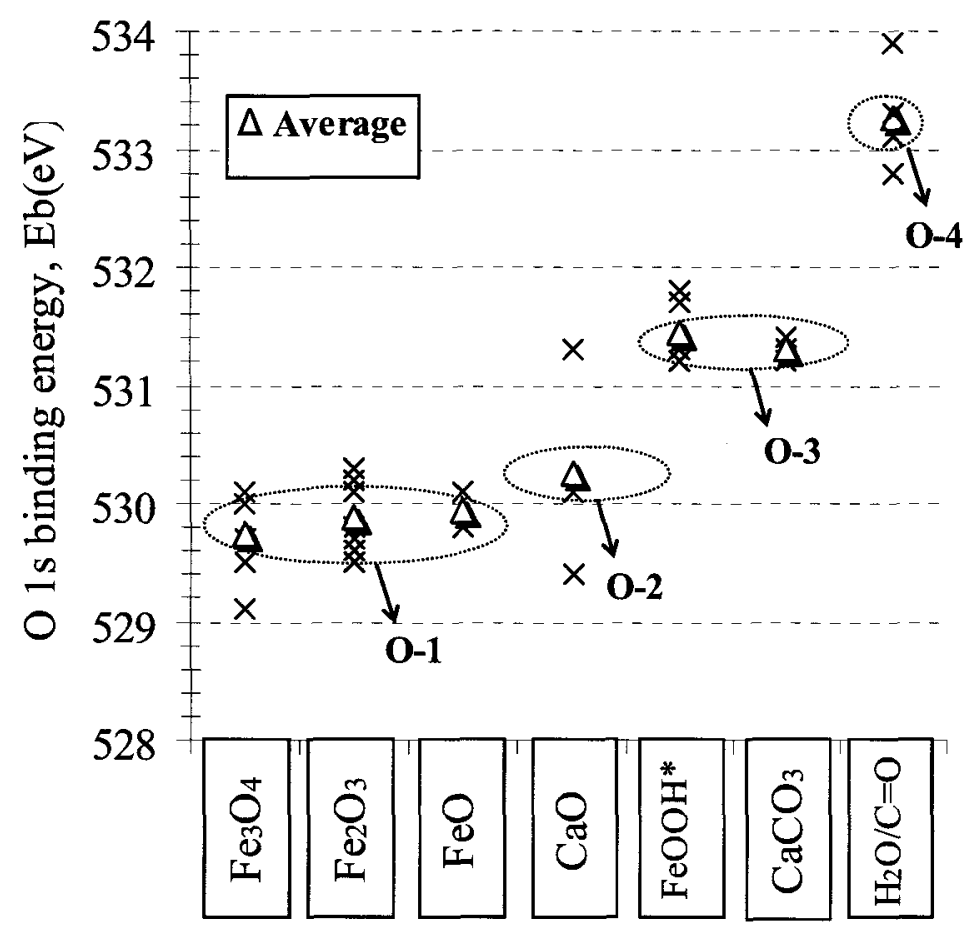

Figure 8.6: The peak $E_{b}$ position data obtained from other studies (NIST Standard Reference Database 20, V. 3.5) for oxygen compounds at the surface of steel. (* $\mathrm{Fe}-\mathrm{OH}$ assignment)

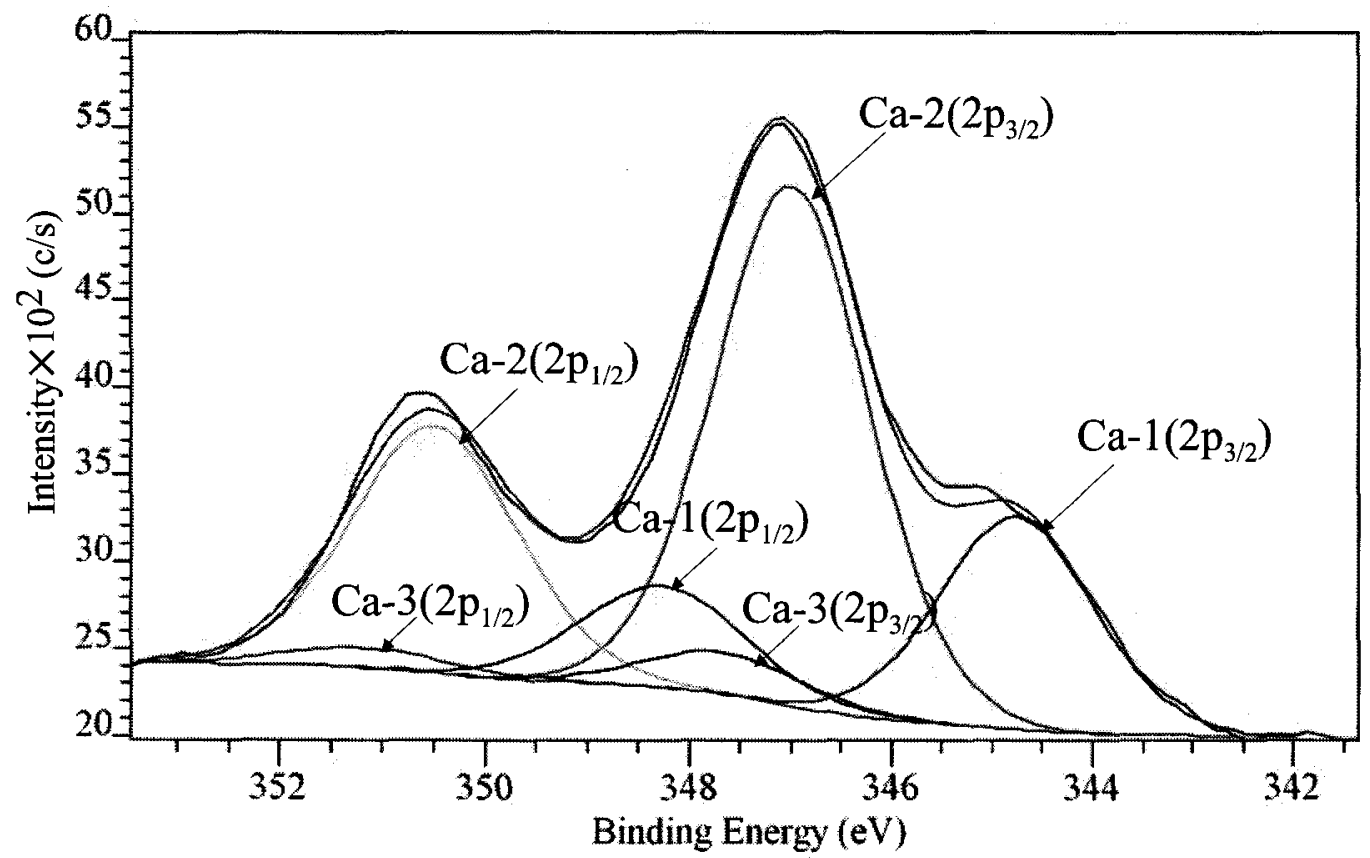

Figure 8.7: Typical curve fitting conducted for Ca 2p high resolution XPS spectrum. 


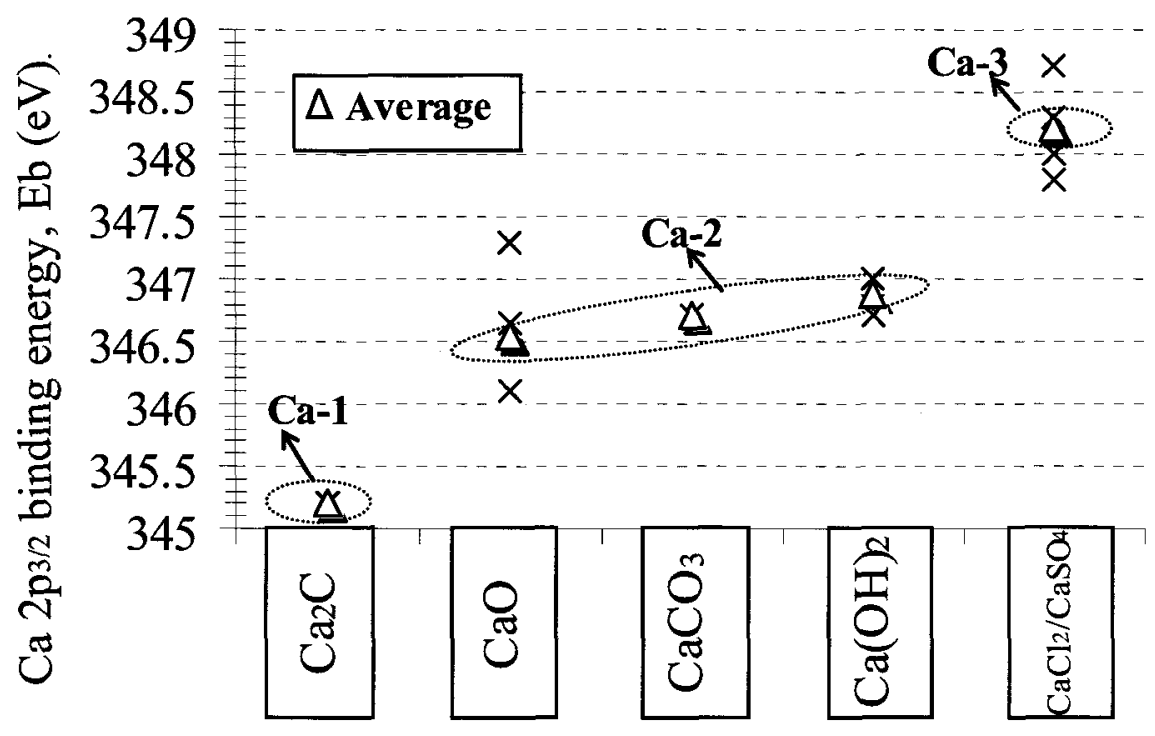

Figure 8.8: The peak $E_{b}$ position data obtained from other studies (NIST Standard Reference Database 20, V. 3.5) for calcium compounds at the surface of steel.

\subsection{Results and Discussion}

\subsubsection{Atomic concentration depth profile}

The variation in chemical composition with depth (XPS depth profiles) of iron, oxygen, carbon and calcium obtained from the XPS survey scans for the four specimens (i.e., $\mathrm{AE}, \mathrm{CH}-2$, $\mathrm{CH}-9$ and $\mathrm{CH}-\mathrm{Cl}$ ) are shown in Fig. 8.9. As expected and observed in Fig. 8.9(a), the iron concentration increases with depth. The concentration of oxygen, on the other hand, follows a sharp increase to a depth of $2.5 \mathrm{~nm}$, and then decreases approaching the steel surface, as shown in Fig. 8.9(b). 

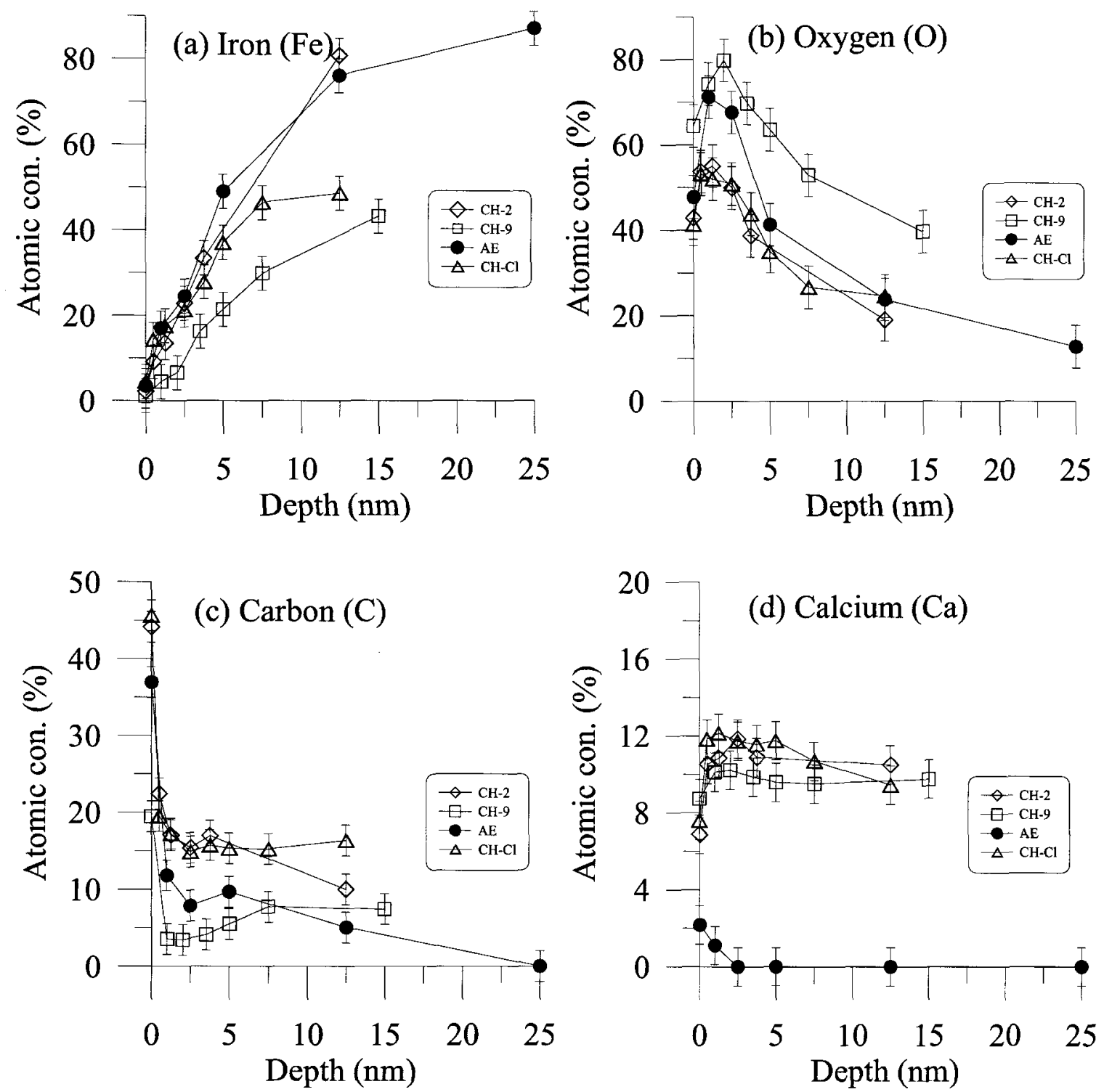

Figure 8.9: XPS depth profiles (At\%) for (a) Iron (b) Oxygen (c) Carbon and (d) Calcium recorded for the $\mathrm{AE}, \mathrm{CH}-2, \mathrm{CH}-9$ and $\mathrm{CH}-\mathrm{Cl}$ specimens.

In Fig. 8.9(c), the high carbon concentration at the surface of each specimen is mainly due to organic carbon contamination (the absorbed alcohol) during the specimen preparation and post-immersion storage; the carbon concentration decreases remarkably after briefly sputtering the surface and remains relatively constant for all specimens. 
In Fig. 8.9(d), it can be observed that the AE specimen does not contain significant amounts of calcium, which is consistent with its exposure history; small amounts of calcium at the outermost region of the depth profile of the AE specimen is most likely associated with particles embedded at the surface during the polishing process. On the other hand, calcium concentration levels for specimens exposed to the $\mathrm{CH}$ solutions (i.e., $\mathrm{CH}-2, \mathrm{CH}-9$ and $\mathrm{CH}-\mathrm{Cl}$ ) are relatively constant, typically between 8 to $12 \%$, across the thickness of the oxide films as shown in Fig. 8.9(d). This observation implies that the micro-size calcium hydroxide and/or calcium carbonate particles, which remained on the specimen surface upon drying after the specimens were removed from the $\mathrm{CH}$ solution, are not sputtered away completely.

This argument was supported by the SEM images taken from the sputtered surface $(\sim 2 \mathrm{~mm} \times \sim 2 \mathrm{~mm})$ of the specimens after the XPS examination. As shown in Figs. 8.10(a) and $8.10(\mathrm{~b})$, precipitated particles of about $1 \mu \mathrm{m}$ size are present on the surface of the CH-9 specimen, which had been sputtered $\sim 25 \mathrm{~nm}$. The EDS analysis conducted on these particles confirms that they are mainly composed of the calcium hydroxide and/or calcium carbonate (see Fig. 8.10(c)). However, in areas between the particles the surface of steel is relatively free of calcium hydroxide and/or calcium carbonate (Fig. 8.11(a)). This is also confirmed by the EDS spectrum obtained from the clean (particle-free) area shown in Fig. 11(b). Based on these observations, a model is provided in Fig. 8.12 to schematically demonstrate the surface topography of the test specimens and also to explain the effect of ion sputtering on the surface topography. Figs. 8.12(a) and 8.12(b) illustrate the surface topography of the specimens before and after sputtering, respectively. 


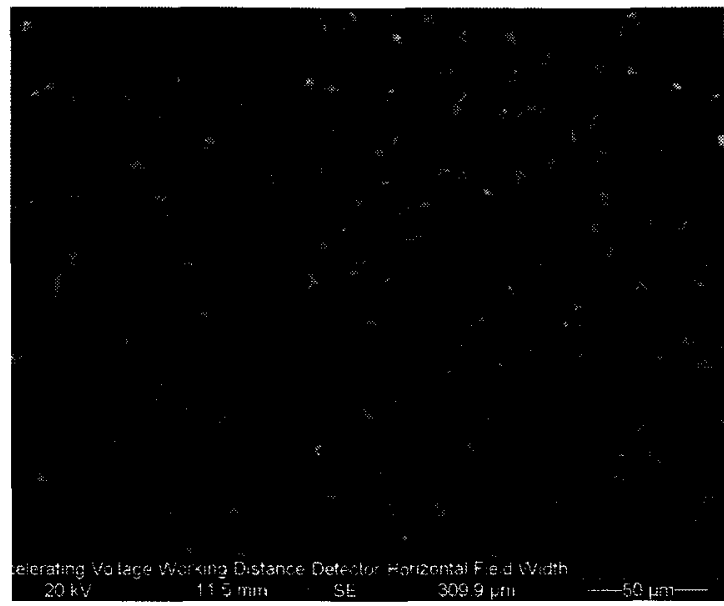

(a)

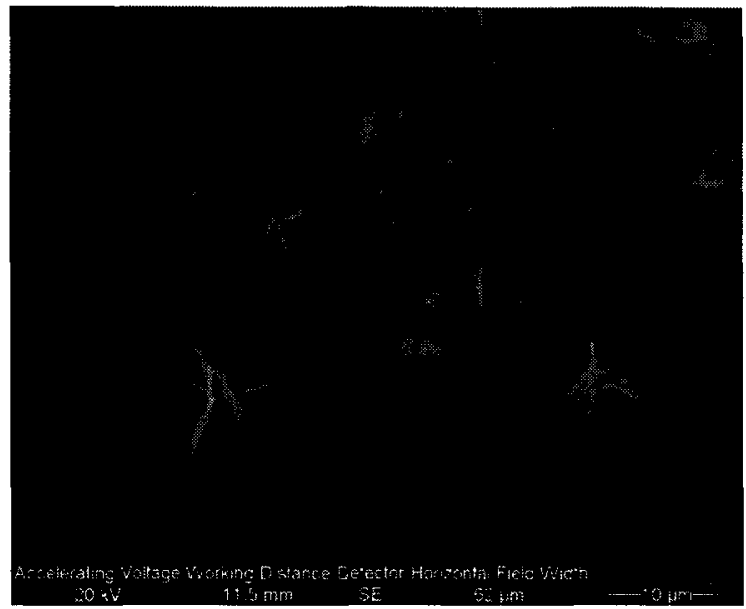

(b)

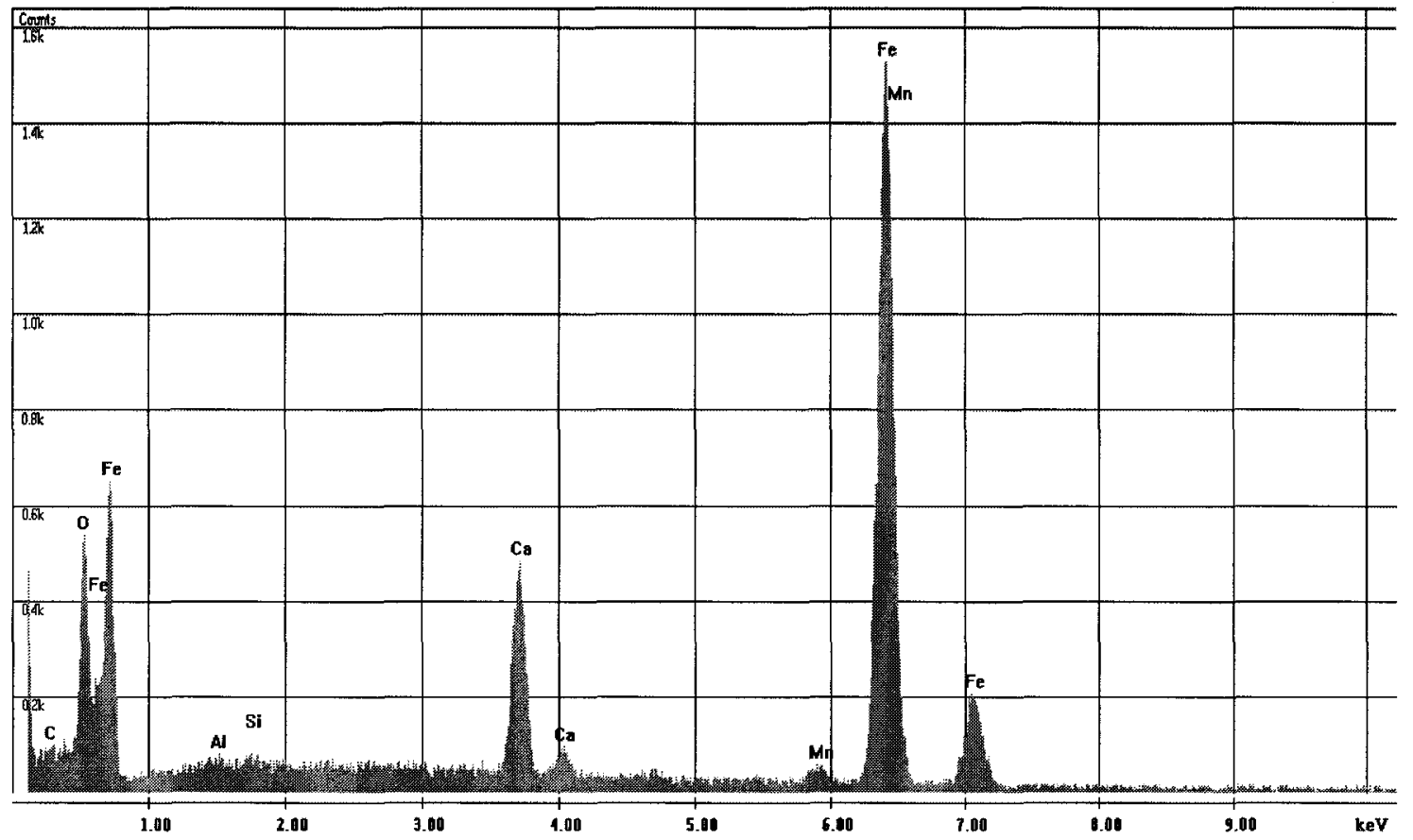

(c)

Figure 8.10: SEM images from the steel surface exposed to the CH solution for 9 days (CH-9) following the XPS analysis: (a) low magnification SEM image of precipitates from the ion sputtered area; (b) high magnification SEM image of precipitates from the sputtered area; (c) EDS spectrum recorded of the area shown in Fig. 8.10(a). 


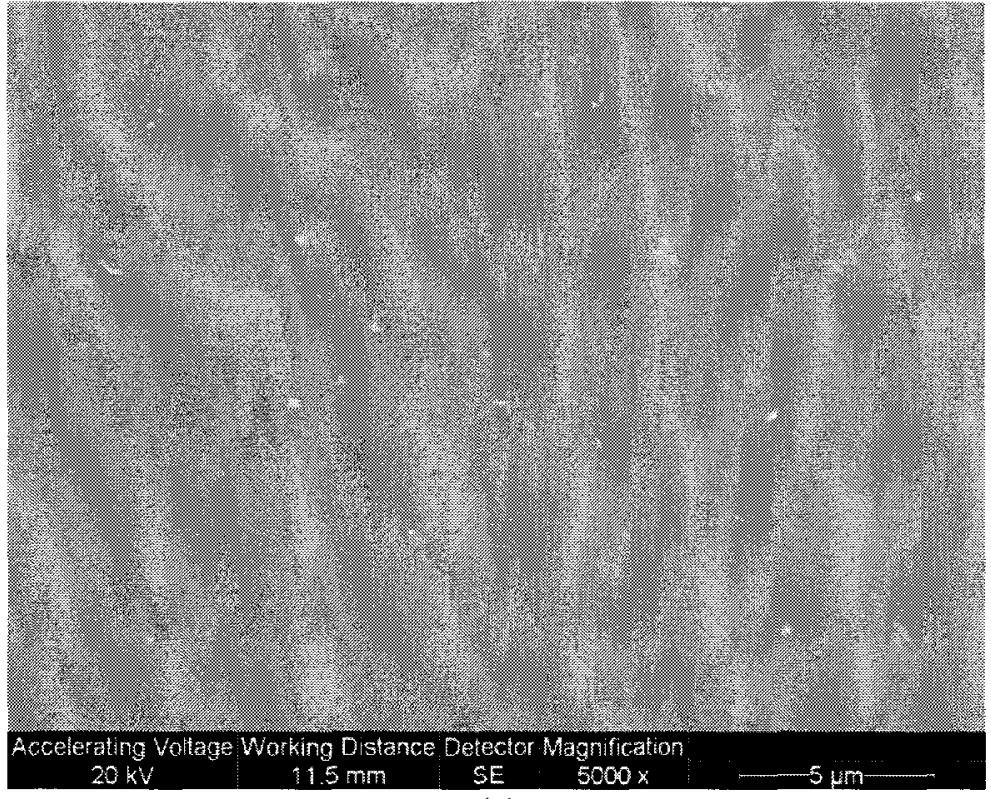

(a)

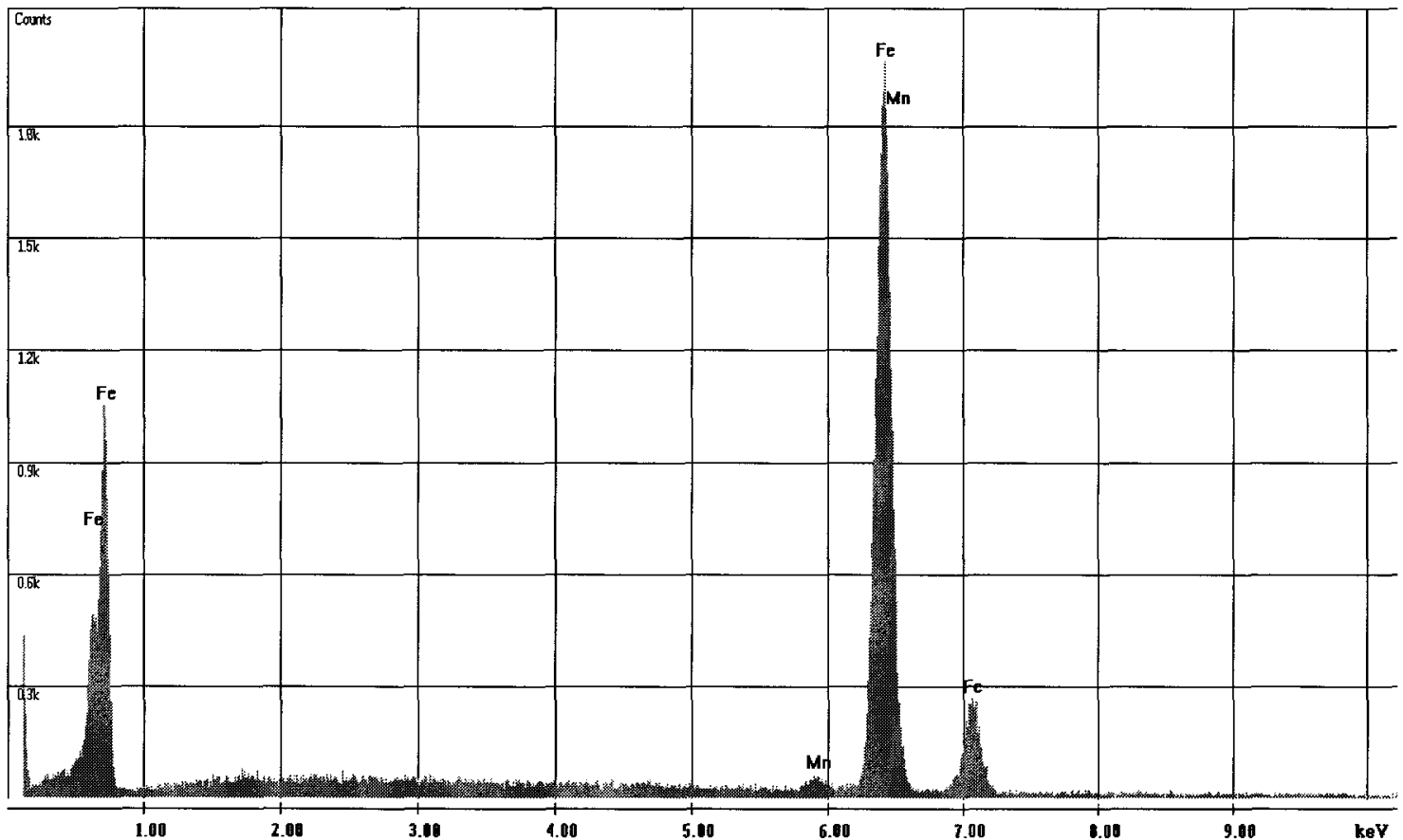

(b)

Figure 8.11: (a) high magnification SEM image from a typical clean steel surface following the XPS analysis; (b) EDS spectrum recorded of the area shown in Fig. $8.11(a)$. 


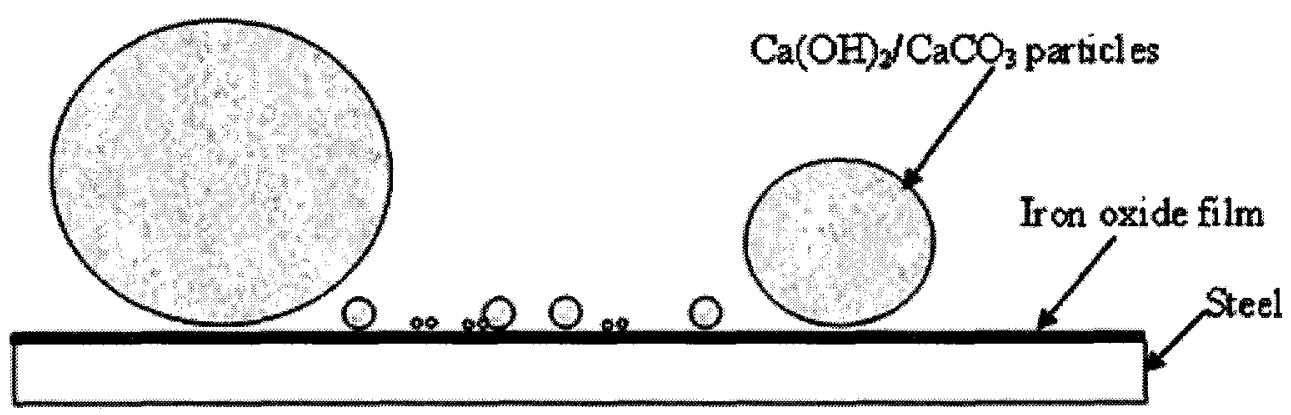

(a)

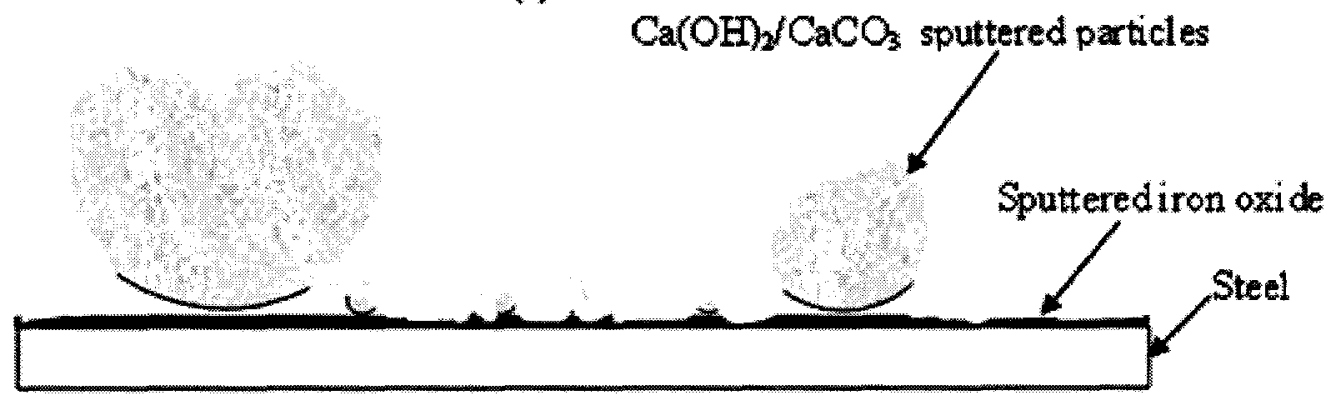

(b)

Figure 8.12: Schematic model showing the surface conditions of the XPS specimen: (a) before sputtering; (b) after sputtering. (Note: Relative dimensions of the precipitates and the oxide film are not drawn to scale).

Thus, the XPS-derived $\mathrm{C}, \mathrm{O}$ and $\mathrm{Ca}$ depth-profile results cannot be used to accurately study the atomic structure of the oxide film since these elements are also present in the precipitates on the surface of specimens. In this study, the XPS spectra of these elements are not used as a precursor to determine the oxide film structure. However, since the iron is not incorporated into the precipitates, Fe $2 p$ XPS spectra are used to investigate the characteristics of oxide film.

\subsubsection{Fe 2p XPS spectra variation with the depth of oxide film}

Fe $2 p$ high resolution XPS spectra collected at selected depths are provided in Figs. 8.13(a)-(d). The $\mathrm{Fe} 2 \mathrm{p}_{3 / 2}$ spectra of the specimens generally consist of five components. Four of these components can be assigned to iron phases such as metallic iron $(\mathrm{Fe})$, cementite $\left(\mathrm{Fe}_{3} \mathrm{C}\right)$, magnetite $\left(\mathrm{Fe}_{3} \mathrm{O}_{4}\right)$, and hematite/ferrihydrite 
$\left(\mathrm{Fe}_{2} \mathrm{O}_{3} / \mathrm{FeOOH}\right)$, for which the peak positions are shown in Figs. 8.13(a)-(d). The fifth component is assigned to $\mathrm{Fe}_{2} \mathrm{O}_{3}$ satellite structure. The peak positions of these five components are presented in Table 8.3.

For all specimens, as expected, the Fe metal peak intensity increases rapidly with ion sputtering (See Figs. 8.13(a)-(d)). This indicates that the metallic iron component assigned to these Fe $2 p$ spectra comes from the substrate. In addition, a brief ion sputter shifts the $\mathrm{Fe} 2 \mathrm{p}_{3 / 2}$ signal from $\sim 712 \mathrm{eV}$ toward $\sim 707 \mathrm{eV}$, indicating that the oxide film on the surface of steel is quite thin. This observation applies to all specimens (i.e., $\mathrm{AE}, \mathrm{CH}-2, \mathrm{CH}-9$, and $\mathrm{CH}-\mathrm{Cl}$ ) although their exposure conditions are very different (See Figs 8.13(a)-(d)).

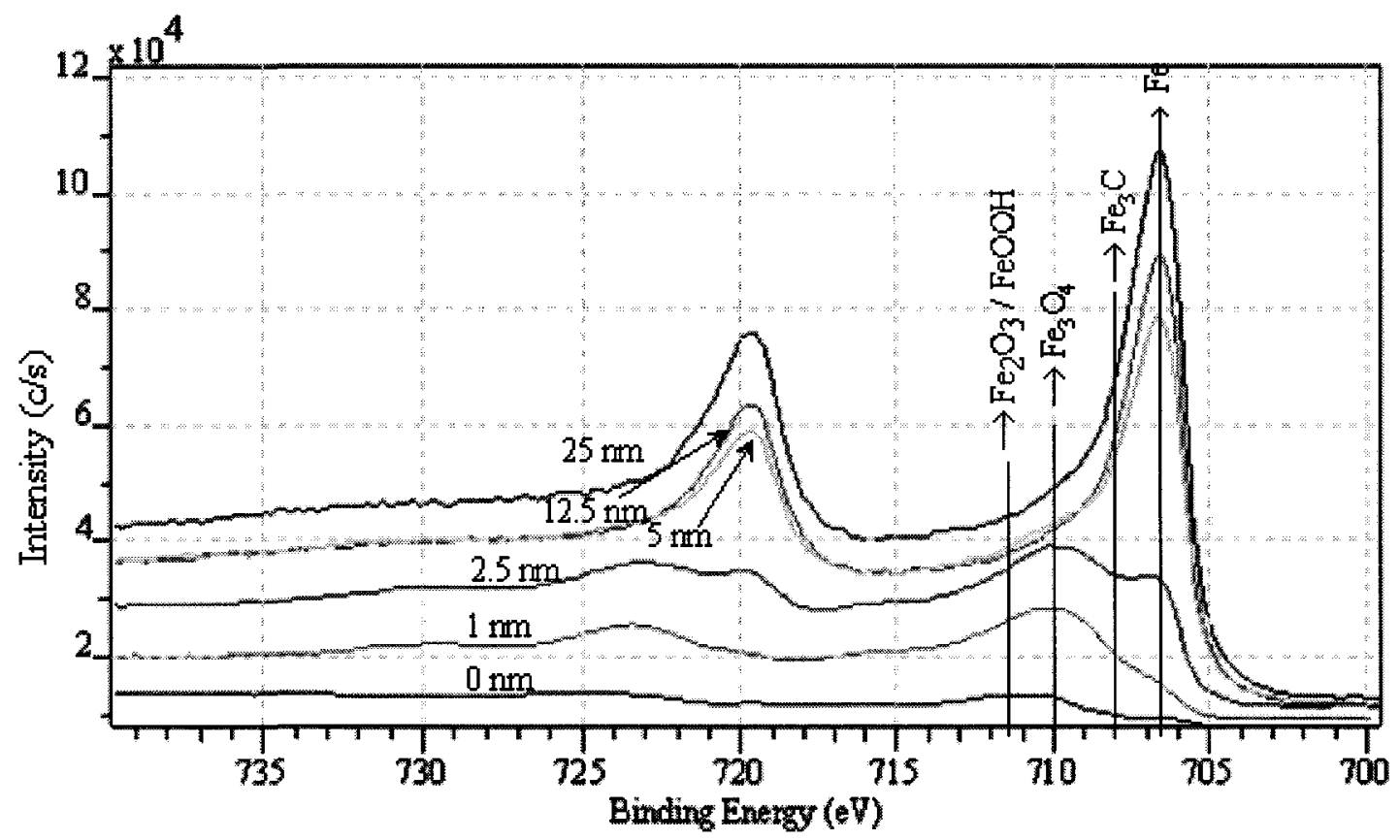

(a) 


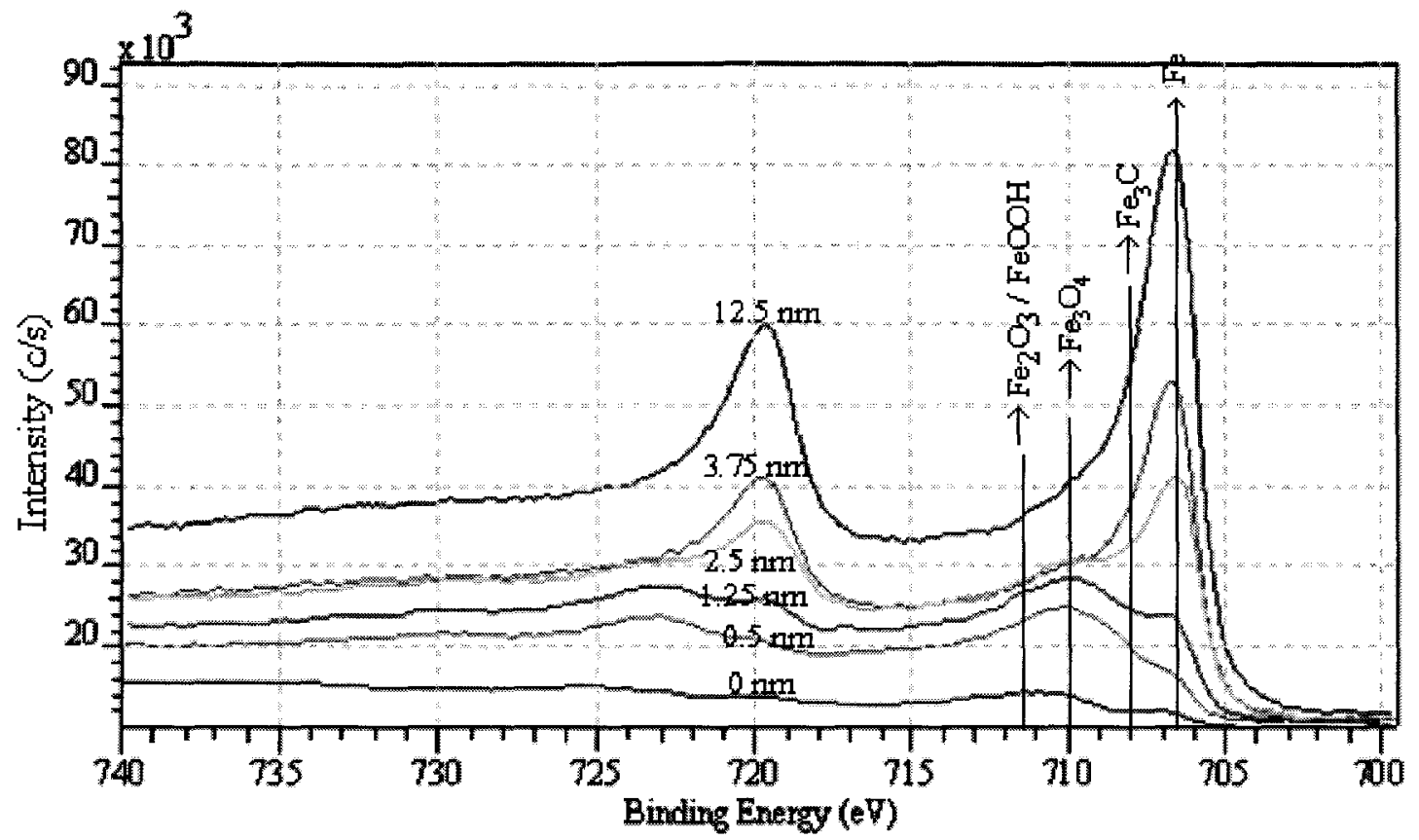

(b)

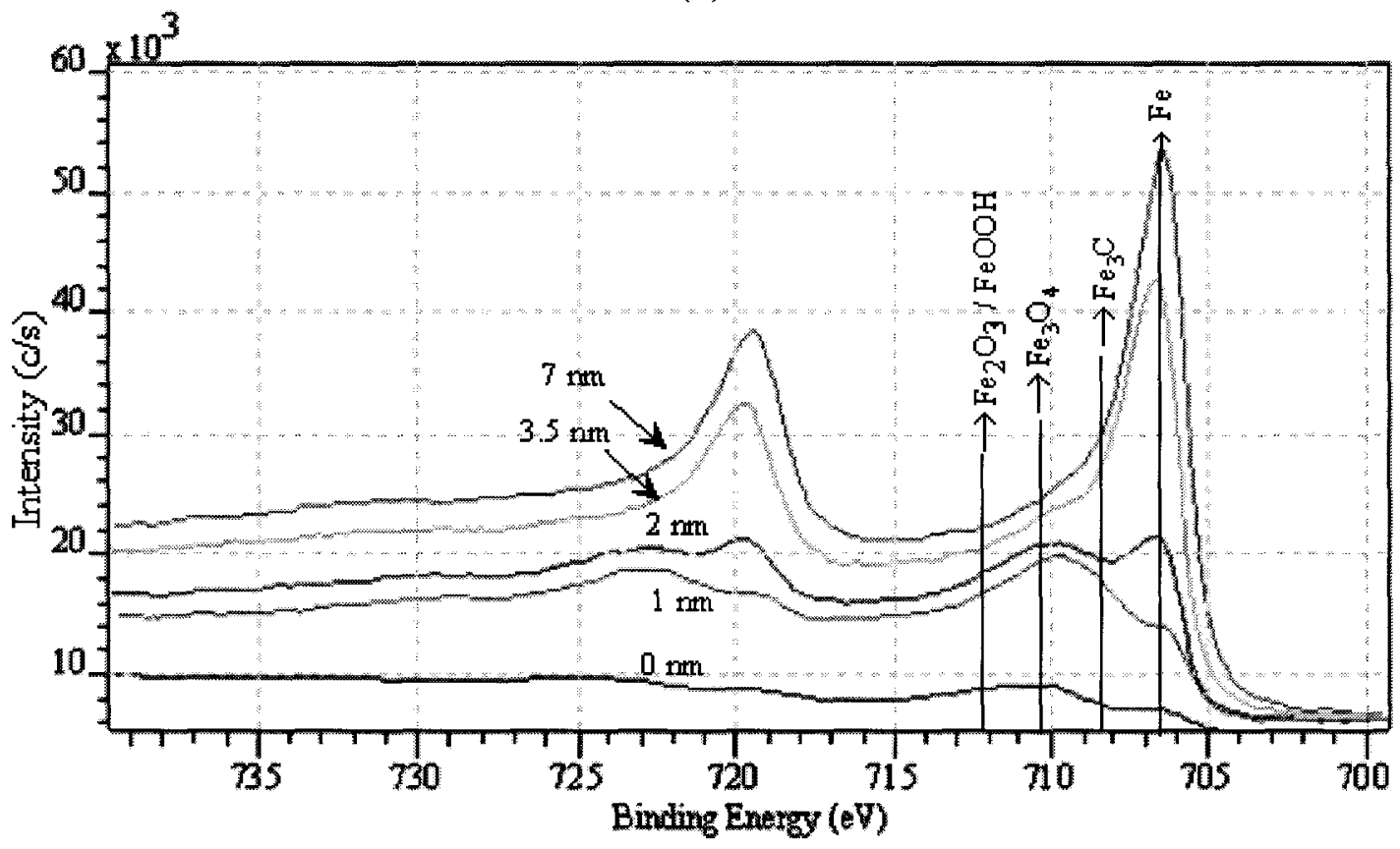

(c) 


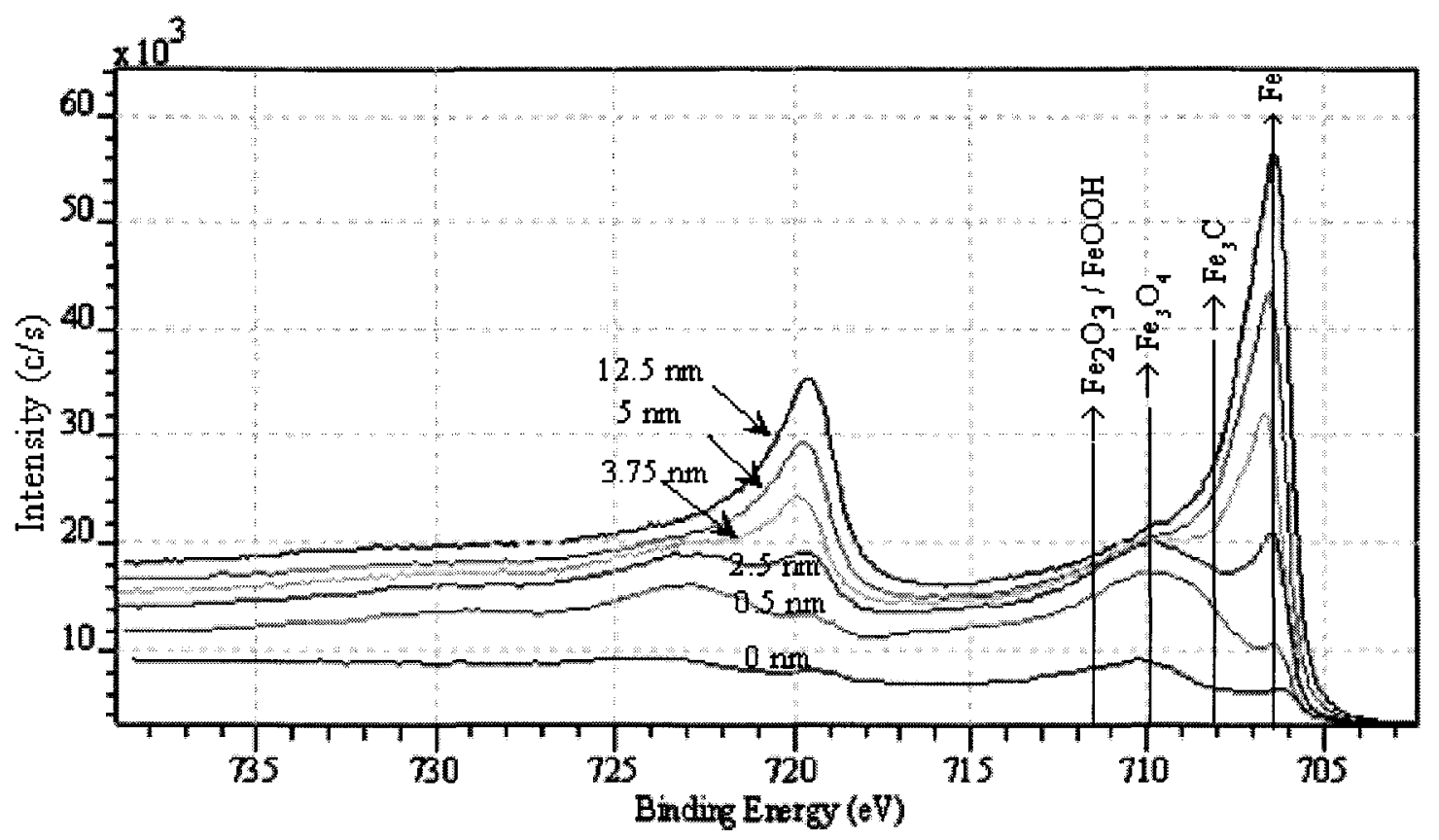

(d)

Figure 8.13: Fe 2p high resolution XPS spectra at selected sputtered depths for (a) AE specimen; (b) $\mathrm{CH}-2$ specimen; (c) $\mathrm{CH}-9$ specimen; (d) $\mathrm{CH}-\mathrm{Cl}$ specimen.

The Fe $2 p$ spectra recorded for the air exposed specimen (AE) in Fig. 8.13(a) have a similar shape at selected sputtered depths as those recorded from specimens exposed to the $\mathrm{CH}$ solution in Figs 8.13(b)-8.13(d). The depth profile of oxide formed in the highly alkaline $\mathrm{CH}$ solution is similar to the one formed in air. The inference is that the $\mathrm{CH}$ solution does not appreciably alter the film chemistry on the steel surface compared with air exposure.

8.4.3 The effect of exposure conditions on the thickness of the oxide film

The effect of exposure conditions on XPS Fe 2p spectra at surface and at a sputter depth of $5 \mathrm{~nm}$ is shown in Figs. 8.14 and 8.15, respectively. As mentioned previously, the Fe-1 component is assigned to metallic iron while $\mathrm{Fe}-3$ and $\mathrm{Fe}-4$ represent selected iron oxides (i.e., $\mathrm{Fe}_{3} \mathrm{O}_{4} / \mathrm{FeO}$ and $\mathrm{Fe}_{2} \mathrm{O}_{3} / \mathrm{FeOOH}$, respectively). Therefore, the relative height (peak intensity) of the $\mathrm{Fe}-3$ and $\mathrm{Fe}-4$ components in comparison with the 
Fe-1 peak intensity indicates the amount of iron oxides relative to the amount of metallic iron. In other words, if the metallic iron (Fe-1) peak height for $\mathrm{Fe} 2 \mathrm{p}$ spectra is set to an arbitrary level, the relative height of $\mathrm{Fe}-3$ or Fe-4 is an indicator for the relative amount of iron oxide species in the film. This qualitative concept was used to compare the thickness of iron oxide in the four specimens.

The relative amount of iron oxide on the surface of the AE specimen, Fig. 8.14, is larger than that of the other specimens (i.e., $\mathrm{CH}-2, \mathrm{CH}-9$ and $\mathrm{CH}-\mathrm{Cl}$ ). This difference may arise from two non-exclusive physical factors. The porosity, and hence atom density, of the oxide film on the AE specimen might be different from that of the other $\mathrm{CH}$ exposed oxide films; this can affect the XPS signal intensities of different films - higher porosity of oxide film results in lower XPS signal intensities. A second factor can be related to the average thickness of iron oxide on the surface of steel exposed to air which may be greater than those exposed to the $\mathrm{CH}$ solution. The larger thickness of the oxide film on the AE specimens can be attributed to its possibly expansive and/or porous structure, but it can also be argued that the oxide film of the $\mathrm{CH}$ specimens (i.e., $\mathrm{CH}-2$, $\mathrm{CH}-9$ and $\mathrm{CH}-\mathrm{Cl}$ ) might be dissolving in the solution. The $\mathrm{Fe} 2 \mathrm{p}$ spectra for the $\mathrm{CH}-2$ and CH-9 specimens are almost the same (see Fig. 8.14), which is an indication that the immersion time does not remarkably affect the thickness of iron oxide grown in the $\mathrm{CH}$ solution. The $\mathrm{CH}-\mathrm{Cl}$ spectrum has the lowest iron oxides peak compared with the other specimens; therefore, it can be stated that chloride reduces the average thickness of oxide film, possibly by increasing the rate of dissolution of oxide film in the $\mathrm{CH}$ solution. 


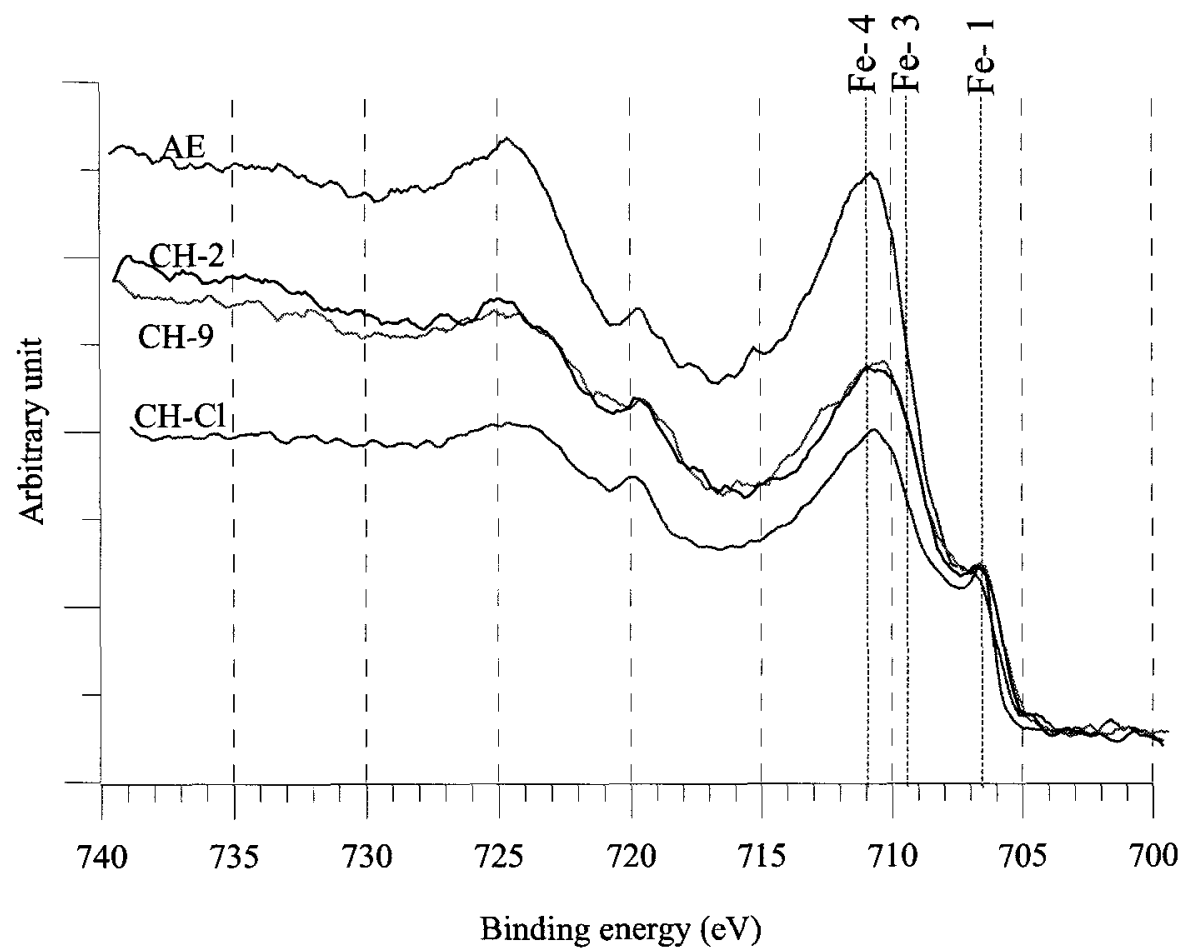

Figure 8.14: Comparison of $\mathrm{Fe} 2 p$ spectra before sputtering for the four specimens.

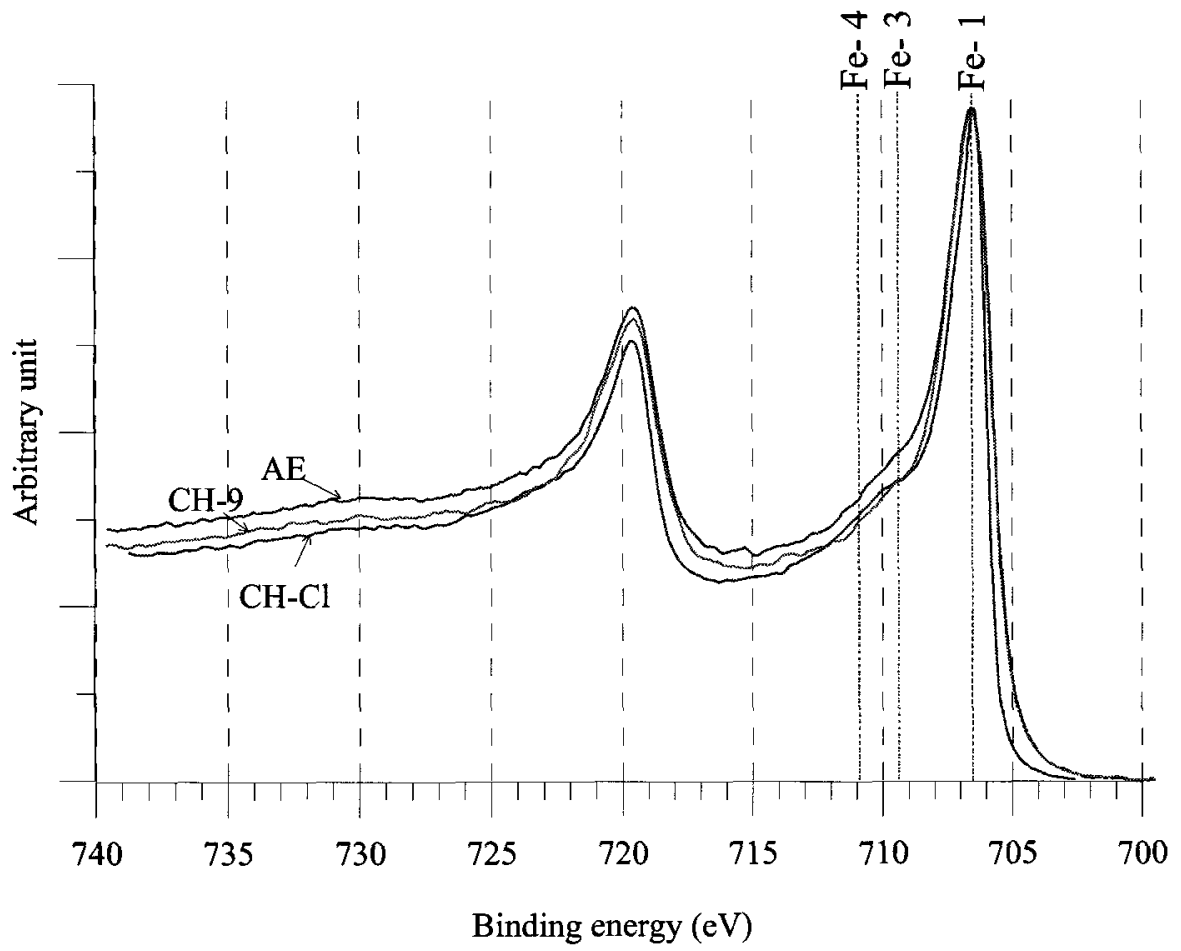

Figure 8.15: Comparison of XPS Fe 2p spectra at a sputter depth of $5 \mathrm{~nm}$.

(Note: See Table 8.3 for each assignment) 
The Fe $2 p$ spectra recorded for three specimens at a sputter depth of $5 \mathrm{~nm}$ are shown in Fig. 8.15. At this depth the difference between the three spectra are less significant than those obtained from the surface before sputtering (see Fig. 8.14) because at the depth of $5 \mathrm{~nm}$ the major portion of oxide film is sputtered away and most of the Fe $2 \mathrm{p}$ intensities are therefore associated with the steel substrate. Although the $\mathrm{CH}-9$ and $\mathrm{CH}-\mathrm{Cl}$ spectra are quite comparable, the region assigned to iron oxides (i.e., $\mathrm{Fe}-3$ and $\mathrm{Fe}-$ 4 peaks) for the $\mathrm{AE}$ specimen is slightly higher than those for the $\mathrm{CH}$ specimens. This can be attributed to the larger oxide film thickness of the AE specimens compared to the specimens that were exposed to the $\mathrm{CH}$ solution.

The ratio of the iron oxides to the metallic iron concentration $\left(\mathrm{Fe}_{\text {oxides }} / \mathrm{Fe}_{\mathrm{m}}\right)$ at each sputtered depth is determined by proportioning the total intensity of iron oxides (i.e., $\mathrm{Fe}-3, \mathrm{Fe}-4$ and $\mathrm{Fe}-5$ ) to the intensity of the metallic iron (i.e., Fe-1). In Fig. 8.16, the variation of the iron oxide to metallic iron is shown at the selected sputtered depths for each specimen. The AE specimen profile has the highest ratio compared with the other specimens, which is consistent with the thickness results reported previously. The results of the $\mathrm{CH}-2, \mathrm{CH}-9$ and $\mathrm{CH}-\mathrm{Cl}$ specimens are quite similar, but at the surface $\mathrm{Fe}_{\text {oxides }} / \mathrm{Fe}_{\mathrm{m}}$ for the $\mathrm{CH}-\mathrm{Cl}$ specimen is lower than for the other $\mathrm{CH}$ specimens, which indicates that iron oxides have dissolved into the solution after the introduction of the chlorides. Below about $5 \mathrm{~nm}$ the variation of $\mathrm{Fe}_{\text {oxides }} / \mathrm{Fe}_{\mathrm{m}}$ is relatively constant for all $\mathrm{CH}$-exposed specimens suggesting that these iron oxide films are approximately $5 \mathrm{~nm}$ thick.

To support the qualitative observations on the effect of exposure conditions on the thickness of the oxide films, a quantitative method was also used. Assuming a uniform film of iron oxide on the steel substrate, the oxide film thickness can be 
calculated using the intensity ratio of the iron oxides to the metallic iron. Accordingly, the thickness of iron oxide film can be calculated as (Briggs and Seah 1990)

$$
d_{o x}=\lambda_{o x}^{F e} \cos (\theta) \ln \left(1+\frac{I_{o x}^{F e}}{I_{m}^{F e}} \cdot \frac{N_{m}^{F e}}{N_{o x}^{F e}} \cdot \frac{\lambda_{m}^{F e}}{\lambda_{o x}^{F e}}\right)
$$

where $d_{o x}(\mathrm{~nm})$ is the thickness of iron oxide, $I_{o x}{ }^{F e}$ and $I_{m}{ }^{F e}$ are the intensities of the iron oxide and the metallic iron, respectively; $N_{o x}{ }^{F e}$ and $N_{m}{ }^{F}$ are atom densities of iron oxide and metallic iron $\left(N_{o x}^{F e}=38 \mathrm{~atm} / \mathrm{nm}^{3}, N_{m}^{F}=84 \mathrm{~atm} / \mathrm{nm}^{3}\right)$, respectively; $\lambda_{o x}{ }^{F e}$ and $\lambda_{m}{ }^{F}$ (nm) are attenuation lengths of iron oxide and metallic iron that can be obtained from (Seah and Dench 1979):

$$
\begin{aligned}
& \lambda_{o x}^{F e}=0.72\left(a_{o x}\right)^{3 / 2}\left(E_{k}\right)^{1 / 2} \\
& \lambda_{m}^{F e}=0.41\left(a_{m}\right)^{3 / 2}\left(E_{k}\right)^{1 / 2}
\end{aligned}
$$

where $E_{k}(\mathrm{eV})$ is the kinetic energy of iron $(779 \mathrm{eV}) ; a_{o x}$ and $a_{m}(\mathrm{~nm})$ are the monolayer thicknesses of iron oxide and metallic iron, respectively; these monolayer thickness can be estimated by (Seah and Dench 1979):

$$
\begin{aligned}
& a_{o x}=\left(\frac{1}{N_{o x}^{F e}}\right)^{1 / 3} \\
& a_{m}=\left(\frac{1}{N_{m}^{F e}}\right)^{1 / 3}
\end{aligned}
$$




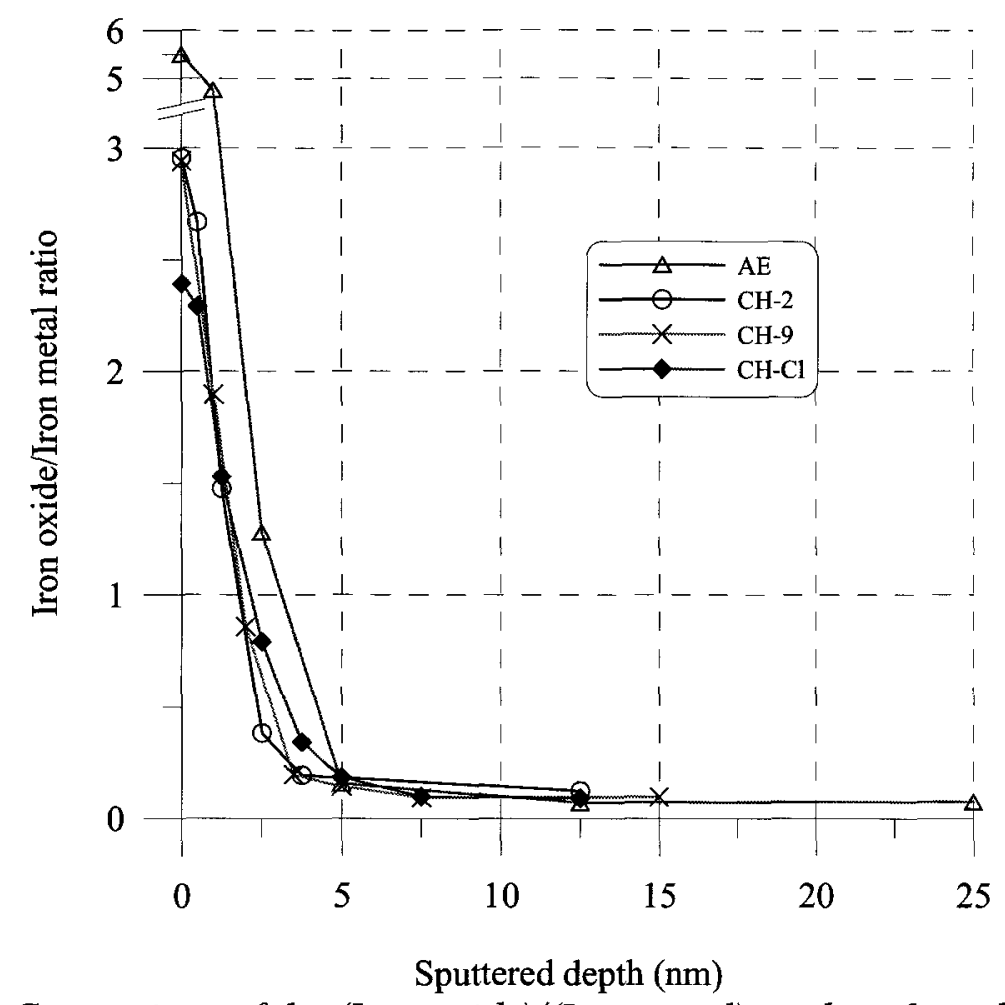

Figure 8.16: Comparison of the (Iron oxide)/(Iron metal) at the selected sputtered depths.

Using the procedure described above, the oxide film thicknesses were calculated for the $\mathrm{AE}, \mathrm{CH}-2, \mathrm{CH}-9$ and $\mathrm{CH}-\mathrm{Cl}$ specimens as $5.7,4.1,4.1$ and $3.6 \mathrm{~nm}$, respectively. These calculations are consistent with the results obtained by Miserque at al. (2006) in $0.1 \mathrm{M} \mathrm{NaOH}$; they reported that the thickness of oxide film on the surface of mild steel does not exceed $6 \mathrm{~nm}$. It is clear from these calculations that the thickness of iron oxide grown in air is larger than that of other specimens. The thicknesses of the oxide films formed in the $\mathrm{CH}$ solution are almost the same for the immersion periods of 2 and 9 days (i.e., $\mathrm{CH}-2$ and $\mathrm{CH}-9$ ), which indicates the immersion time does not change the oxide thickness. However, adding $0.05 \mathrm{M}$ chloride to the $\mathrm{CH}$ solution decreased the thickness of the film by $14 \%$, which supports the idea that part of oxide dissolved into the solution as a result of the interactions with chlorides. A similar observation was also reported by 
Khalil et al. (1985), who employed XPS to study iron oxide films grown in $\mathrm{pH} 5$ solutions containing chloride, bromide and iodide; these ions reduced the thickness of oxide film.

\subsubsection{The variation of iron oxide phases in the film}

The ratio between the concentration of $\mathrm{Fe}_{2} \mathrm{O}_{3} / \mathrm{FeOOH}\left(\mathrm{Fe}^{3+}\right)$ and $\mathrm{Fe}_{3} \mathrm{O}_{4} / \mathrm{FeO}$ $\left(\mathrm{Fe}^{2+}\right)$ can be obtained at various sputtered depths by proportioning the total XPS intensities of Fe-4 and Fe-5 to the XPS intensity of Fe-3 as shown in Fig. 8.17. For all specimens, the $\mathrm{Fe}^{3+} / \mathrm{Fe}^{2+}$ generally decreases with depth into the oxide film, which indicates that the concentration of $\mathrm{Fe}^{3+}$ decreases toward the film/substrate interface. In other words, the concentration of $\mathrm{Fe}^{3+}$ relative to $\mathrm{Fe}^{2+}$ decreases with depth in the oxide film. This observation is in agreement with the other studies that reported that the inner layers of the oxide film grown in alkaline solution are mainly composed of $\mathrm{Fe}^{2+}$ oxides while the outer layers mostly consist of $\mathrm{Fe}^{3+}$ oxides (Haupt and Strehblow 1987, HugotLe Goff et al. 1990, Joiret et al. 2002).

Comparing the XPS depth profiles given in Fig. 8.17 indicates that the $\mathrm{Fe}^{3+} / \mathrm{Fe}^{2+}$ in the films is quite similar for the $\mathrm{AE}$ and $\mathrm{CH}-2$ specimens, whereas it is smaller across the entire depth of the $\mathrm{CH}-9$ oxide film. The inference is that the amount of $\mathrm{Fe}^{2+}$ oxides produced on the steel surface during the exposure to the $\mathrm{CH}$ solution is relatively larger than the amount of $\mathrm{Fe}^{3+}$ oxides produced or present; longer immersion time results in larger concentrations of $\mathrm{Fe}^{2+}$ relative to $\mathrm{Fe}^{3+}$. Considering the fact that the calculated oxide film thickness is approximately the same for both immersion periods (i.e., $\mathrm{CH}-2$ and $\mathrm{CH}-9$ ), it can be concluded that a portion of $\mathrm{Fe}^{3+}$ has been replaced by $\mathrm{Fe}^{2+}$ during the period of immersion. 


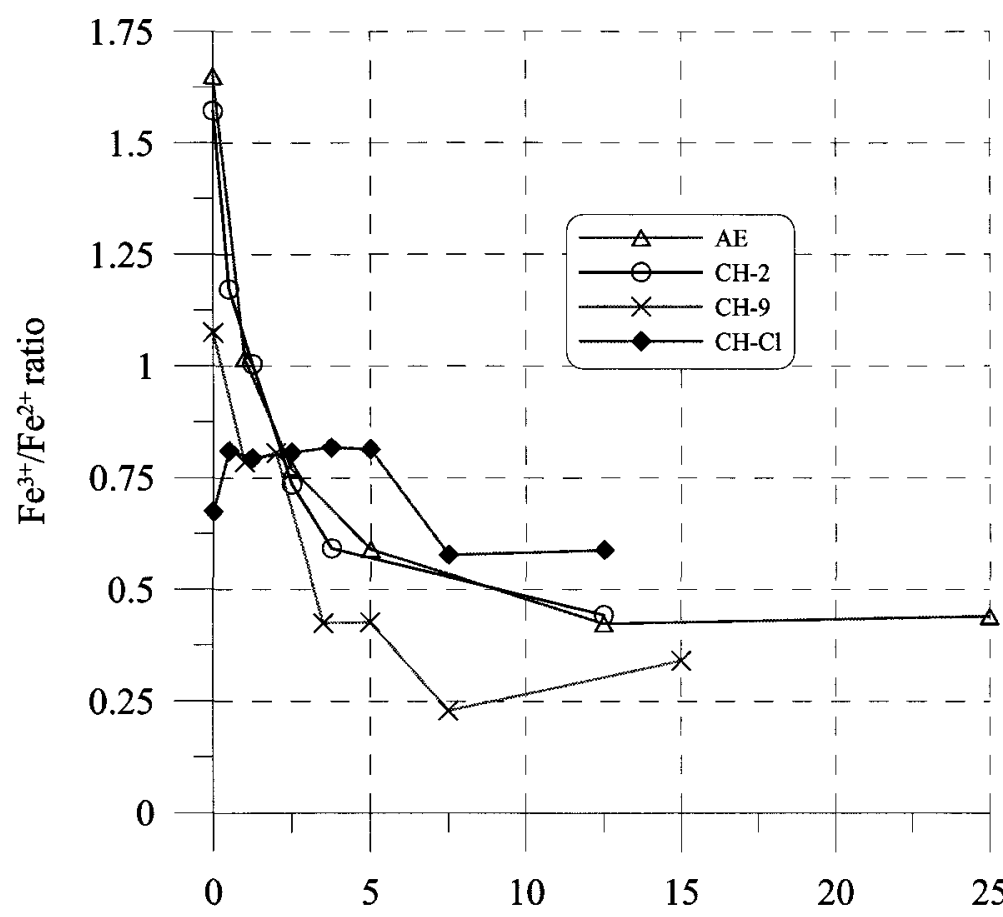

Sputtered depth (nm)
Figure 8.17: Comparison of the $\mathrm{Fe}^{3+} / \mathrm{Fe}^{2+}$ at the selected sputtered depths.

The $\mathrm{Fe}^{3+} / \mathrm{Fe}^{2+}$ for the $\mathrm{CH}-\mathrm{Cl}$ specimen exposed to $0.05 \mathrm{M}$ chloride is greater than the ratio for the other specimens that were not exposed to chloride, particularly at the sputtered depth beyond $2.5 \mathrm{~nm}$. It can be stated that the addition of chloride to the $\mathrm{CH}$ solution changes the stoichiometry of oxide film production such that within the inner layer of oxide film (i.e., sputter depth $>2.5 \mathrm{~nm}$ ) the relative concentration of $\mathrm{Fe}^{2+}$ decreases in comparison with $\mathrm{Fe}^{3+}$.

A plot of the concentration of the $\mathrm{Fe}-2$ phase assigned to $\mathrm{Fe}_{3} \mathrm{C}$ (Cementite, $\left.\mathrm{Fe}-2\right)$ with sputter depth for the four specimens is shown in Fig. 8.18; no appreciable difference is observed between the specimens. The concentration of $\mathrm{Fe}_{3} \mathrm{C}$ is the largest near to the surface of the film (i.e., sputter depth $<2.5 \mathrm{~nm}$ ), and decreases quickly below this depth. These observations suggest that the $\mathrm{Fe}_{3} \mathrm{C}$ phase tends to accumulate in the oxide film at the oxide film/air interface so that the surface of steel is therefore enriched with carbon. 
Similar observation was reported for stainless steel for which the oxide film was generally enriched by chromium during the formation of the film (Holliday and Frankenthal 1972).

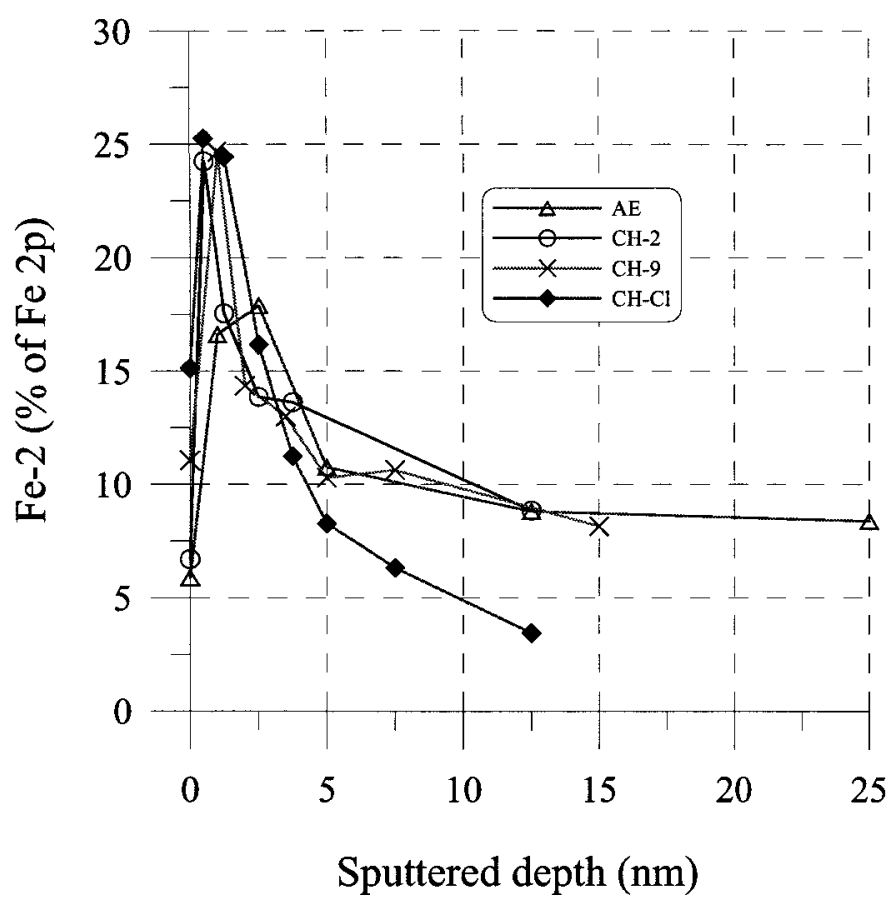

Figure 8.18: Comparison of the Fe-2 phase present at the selected sputtered depths.

\subsection{Conclusions}

In this chapter, the properties of the passive oxide film that forms on carbon steel in saturated calcium hydroxide solution and the effect of chloride on the film properties were investigated by the XPS depth profiling mode of analysis.

It was found that the surface of the passivated steel contained micro-size precipitates of calcium hydroxide and/or calcium carbonate, which could not be sputtered away completely. Therefore, the changes in atomic concentrations of calcium, carbon and oxygen across the depth of the oxide films could not be used to study accurately the atomic structure of the films since these elements were also present in the precipitates on 
the surface of specimens. Since the iron was not incorporated into the precipitates, Fe $2 p$ XPS spectra were used to investigate the characteristics of the oxide film.

The thickness of the oxide films formed in the $\mathrm{CH}$ solution was determined to be approximately $4 \mathrm{~nm}$ and was not affected by the exposure time to the $\mathrm{CH}$ solution. Further XPS analysis revealed that the oxide close to the substrate were mainly composed of $\mathrm{Fe}^{2+}$ oxides while near the free surface the oxide consisted mostly of $\mathrm{Fe}^{3+}$ oxides. The amount of $\mathrm{Fe}^{2+}$ oxides generated near the steel surface during the exposure to the $\mathrm{CH}$ solution was found to be larger than the amount of $\mathrm{Fe}^{3+}$ oxides; longer immersion time resulted in larger concentrations of $\mathrm{Fe}^{2+}$ relative to $\mathrm{Fe}^{3+}$. Since the passivation time did not affect the oxide film thickness, it can be concluded that a portion of $\mathrm{Fe}^{3+}$ was replaced by $\mathrm{Fe}^{2+}$ as the specimens were exposed to the $\mathrm{CH}$ solution.

Addition of chloride to the $\mathrm{CH}$ solution decreased the thickness of the oxide film, which supports the idea that part of oxide dissolved into the solution as a result of the interactions with chlorides. It was found that the addition of chloride to the $\mathrm{CH}$ solution changes the stoichiometry of oxide film such that at the film/substrate interface the $\mathrm{Fe}^{3+} / \mathrm{Fe}^{2+}$ increased. 


\section{Chapter 9}

\section{Angle-resolved XPS Study of Passive Film on Rebar and the Role of Chloride in Depassivation}

\subsection{Introduction}

In Chapter 8, using the XPS technique the characteristics of passive film on carbon steel rebar were investigated by sputtering the samples with Argon ion beam. It was reported in the literature that Argon ion bombardment of oxide films may have artefacts that can result in misleading data (Kim et al. 1976). For instance, Brundle et al. (1977) showed that, during the XPS test conducted on bulk $\alpha-\mathrm{Fe}_{2} \mathrm{O}_{3}$, a significant amount of $\mathrm{Fe}^{2+}$ was produced as a result of $\mathrm{Fe}^{3+}$ reduction by Argon ion beam, even though the rate of beam sputtering was only a few $\AA$ per minute. Therefore, some questions may arise as to whether the depth-profiling results obtained by ion beam sputtering indicate the "true" or "altered" nature of the oxide film.

Depth-profiling studies in Chapter 8 were carried out on oxide films grown in the saturated calcium hydroxide solution as a simplified surrogate for concrete pore solution. However, compounds such as sodium hydroxide, potassium hydroxide and calcium sulfate that are present in concrete pore solution may affect the oxide film 
properties, as demonstrated in Chapter 4, and suggested by other researchers (Montemor et al. 1998, Hansson and Sorensen 1990, Kayyali and Haque 1995). For example, it was found in Chapter 4 that the polarization resistance of the passive film formed in the saturated calcium hydroxide plus calcium sulfate solution was significantly lower than the one formed in the saturated calcium hydroxide solution in the absence of sulfate ions; this indicates that the auxiliary ions such as potassium, sodium and specially sulfate that are generally present in concrete pore solutions, may influence the properties of oxide films.

To address these two issues, angle-resolved XPS technique, a non-destructive surface analysis method, was used to study the oxide films grown in a simulated concrete pore solution that includes the ionic species that are generally present in concrete (e.g. calcium, sodium, potassium and sulfate). It is expected that this experiment provides more reliable insight into the nanostructure and properties of oxide film on carbon steel in an environment that is comparable to that of rebar in concrete. Moreover, the effect of chloride on the oxide films was also investigated in this chapter by adding sodium chloride to the simulated concrete pore solution after the formation of passive film on the samples. In general, the results of this chapter complement the results of XPS depth profiling studies reported in Chapter 8 .

\subsection{Experimental Plan}

\subsubsection{Preparation of samples}

Simulated concrete pore solution (CP) was considered in this study as the test solution, where "C" stands for concrete and "P" stands for pore. The $\mathrm{CP}$ solution was 
prepared by dissolving the analytical grade calcium hydroxide $\left(\mathrm{Ca}(\mathrm{OH})_{2}\right)$, sodium hydroxide $(\mathrm{NaOH})$, potassium hydroxide $(\mathrm{KOH})$ and calcium sulfate $\left(\mathrm{CaSO}_{4}\right)$. The chemical composition and the $\mathrm{pH}$ of the solution are presented in Table 9.1.

XPS analysis was carried out on the cross section of carbon steel rebar specimens. The elemental composition of rebar obtained from spectrographic analysis is presented in Table 9.2. Rebar (Size designation: \#10M) was cut into three 2-mm-thick disks, which were then mounted on the surface of an epoxy holder using resin glue. The cross section of mounted samples was perfectly polished to $1 \mu \mathrm{m}$ by wet polishing technique. The anhydrous isopropyl alcohol (water content $<0.05 \%$ ) was used instead of water during the polishing process to avoid the oxidation of surface. After polishing, the specimens were kept in anhydrous isopropyl alcohol to avoid exposure to air.

The steel specimens were released from the epoxy holder and were quickly immersed in the CP solution. The first sample was taken out of the solution after two weeks of immersion (CP-0) and transferred to a 20-ml-airtight container containing the same solution. Using sodium chloride analytical grade ( $\mathrm{NaCl}$, analytical grade $99.99 \%$ ), the chloride concentration was increased in the $\mathrm{CP}$ solution to $0.45 \mathrm{M}$. After two weeks, the second sample (CP-1) was transferred to the airtight container with the same solution. The same procedure was repeated for the third sample (CP-2) at $3 \mathrm{M}$ chloride concentration. The three samples were kept in the airtight containers until the XPS test. 
Table 9.1: Concentrations of species in the simulated concrete pore solution (CP).

\begin{tabular}{|c|c|c|c|c|c|c|c|c|}
\hline \multicolumn{4}{|c|}{$\begin{array}{l}\text { Added compounds } \\
(\mathrm{M})\end{array}$} & \multicolumn{4}{|c|}{$\begin{array}{c}\text { Measured ions } \\
(\mathrm{mg} / \mathrm{l})\end{array}$} & \multirow[t]{2}{*}{$\mathrm{pH}$} \\
\hline $\mathrm{Ca}(\mathrm{OH})_{2}$ & $\mathrm{Na}(\mathrm{OH})$ & $\mathrm{K}(\mathrm{OH})$ & $\mathrm{CaSO}_{4}$ & $\mathrm{Ca}^{2+}$ & $\mathrm{Na}^{+}$ & $\mathbf{K}^{+}$ & $\mathrm{SO}_{4}{ }^{2-}$ & \\
\hline 0.1 & 0.1 & 0.2 & 0.003 & 3 & 2232 & 8059 & 277 & 13.3 \\
\hline
\end{tabular}

(Note: The actual concentrations of the anions and cations in solution were determined using Inductively Coupled Plasma - Atomic Emission Spectroscopy (ICP-AES) (Model: Varian Vista $\mathrm{RL})$ )

Table 9.2: Steel elemental composition (Average of three spectrographic results).

\begin{tabular}{|c|c|}
\hline Element & $\begin{array}{c}\text { Weight } \\
\%\end{array}$ \\
\hline $\mathrm{C}$ & 0.26 \\
$\mathrm{Si}$ & 0.27 \\
$\mathrm{Mn}$ & 1.10 \\
$\mathrm{Cr}$ & 0.05 \\
$\mathrm{Ni}$ & 0.07 \\
$\mathrm{Mo}$ & $<0.01$ \\
$\mathrm{Cu}$ & 0.21 \\
$\mathrm{Al}$ & $<0.005$ \\
$\mathrm{Nb}$ & $<0.01$ \\
$\mathrm{~V}$ & $<0.005$ \\
$\mathrm{Ti}$ & $<0.005$ \\
$\mathrm{~B}$ & $<0.0005$ \\
$\mathrm{P}$ & 0.01 \\
$\mathrm{~S}$ & 0.03 \\
$\mathrm{~W}$ & $<0.01$ \\
$\mathrm{Sn}$ & 0.021 \\
$\mathrm{Co}$ & 0.01 \\
$\mathrm{Zr}$ & $<0.01$ \\
$\mathrm{Fe}$ & Balance \\
\hline
\end{tabular}

\subsubsection{XPS test procedure}

The samples were transferred from the solution to the XPS analysis chamber and were analyzed using the Kratos Axis Ultra XPS equipped with a monochromated Al Xray source $(\mathrm{hv}=1486.6 \mathrm{eV})$. Analyses were carried out using an accelerating voltage of 14 $\mathrm{kV}$ and a current of $10 \mathrm{~mA}$. Chamber pressure during analysis was typically in the order of $2.67 \mathrm{e}^{-7} \mathrm{~Pa}$. Charge build-up was compensated by using the Axis charge balancing system. 
Analyses consisted first of a survey scan performed at a pass energy of $160 \mathrm{eV}$ to identify all the species present, followed by high resolutions scans $(40 \mathrm{eV})$ of the species of interest. Survey scans were performed using an energy range of $1100 \mathrm{eV}$, analyzer pass energy of $160 \mathrm{eV}$, step size of $0.5 \mathrm{eV}$ and a total acquisition time of 660 seconds. High resolution scans were performed using analyzer pass energy of $40 \mathrm{eV}$ with a step size of $0.1 \mathrm{eV}$. Both the survey scans and high resolution scans were performed at the following series of emission angles $(\theta): 0^{\circ}, 30^{\circ}, 40^{\circ}, 50^{\circ}, 60^{\circ}, 70^{\circ}, 75^{\circ}$. Peak fitting was performed using CasaXPS (ver. 2.2.107) data processing software. Shirley background correction procedures were used as provided by CasaXPS to modify the spectra for curve fitting. Curve fitting procedures used for high resolution spectra presented in this report used a Gaussian (70\%)-Lorentzian (30\%) function. High resolution analyses were calibrated to $\mathrm{C} 1 \mathrm{~s}$ (hydrocarbon) signal of $285 \mathrm{eV}$. SemiQuantification analysis was performed on the curve fitted data using sensitivity factors provided by CasaXPS's Scofield element library.

\subsection{Results and Discussions}

\subsubsection{Variation of data}

Two repeating $\mathrm{Fe} 2 \mathrm{p}$ high resolution spectra were obtained from two different spots on the surface of the samples to determine the variation of results. Figure 9.1 shows the results for three samples (CP-0, CP-1 and CP-2) at three emission angles: $0^{\circ}, 40^{\circ}$ and $60^{\circ}$. For the CP-0 sample, two repeating spectra at different emission angles are almost identical (Fig. 9.1(a)). The variation of the results between the two spots in the CP-1 and CP-2 samples increases by increase in the emission angle; e.g. the difference of the two 
spectra for the emission angle of $60^{\circ}$ is larger than the one for the emission angle of $0^{\circ}$. No appreciable difference in the two spectra variation is seen between the CP-1 and CP-2 samples.

Comparing the CP-0 results with the results obtained from the CP-1 and CP-2 samples indicates that the variation in the CP-0 spectra (Fig. 9.1(a)) at all three emission angles are generally smaller than those for the other two samples (Figs. 9.1(b) and (c)). This suggests that the oxide film formed on the surface of steel in the CP solution is uniform, and there is no remarkable variation in the characteristics of the oxide. On the other hand, the oxide film formed on the CP-1 and CP-2 samples appears unevenly distributed on the surface in comparison with the CP-0 sample. As shown in Fig. 9.1, the discrepancy among the results is slightly larger for the CP-2 sample than that of the CP-1 sample, which shows that the dissimilarity in the characteristics of oxide film is possibly related to the concentration of chloride: samples exposed to the higher concentration of chloride (i.e., $\mathrm{CP}-2: 3 \mathrm{M} \mathrm{Cl}$ ) have greater variations in oxide film than those exposed to the lower chloride concentration (i.e., CP-1: $0.45 \mathrm{M} \mathrm{Cl}$ ).

\subsubsection{Sampling depth}

The sampling depth $(d)$ is defined as the thickness of a layer where $95 \%$ of the signal intensity is derived from (Briggs and Seah 1990). In this study, using the procedure described in Chapter 8 (Section 8.3.2), the sampling depth at different emission angles was calculated; the results are presented in Table 9.3. The largest sampling depth is 8.3 $\mathrm{nm}$ that occurs at $0^{\circ}$ emission angle, whereas for the emission angle of $75^{\circ}$, the sampling depth is limited to $2.1 \mathrm{~nm}$. 


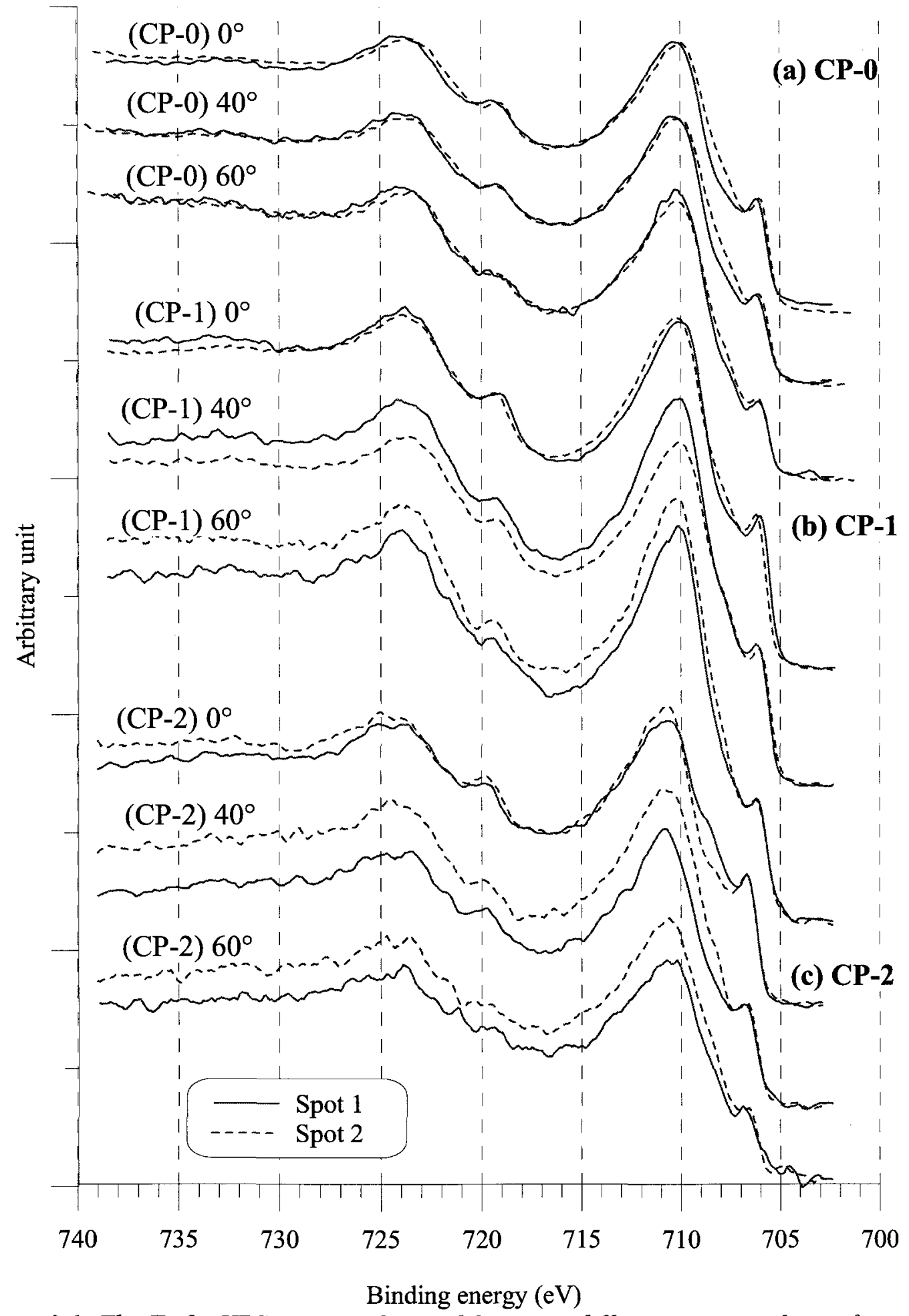

Figure 9.1: The Fe 2p XPS spectra obtained from two different areas at the surface of samples showing the variation of data: (a) CP-0 sample; (b) CP-1 sample; (c) CP-2 sample. 


\subsubsection{Fe 2p XPS spectra variation with emission angle}

The variations of the calcium, potassium, sodium, chloride, carbon and oxygen spectra versus the emission angle $(\theta)$ cannot be used to accurately determine the atomic structure of the oxide film since these elements are also present in the precipitates on the surface of the samples. The SEM examination used in this study also confirmed the presence of micro-size particles on the surface of the samples. Therefore, the XPS spectra of these elements are not considered as a precursor to determine the oxide film structure. However, since iron is not incorporated into the precipitates, only Fe 2p XPS spectra are included in the analysis of the characteristics of oxide films.

Fe $2 p$ high resolution XPS spectra of the samples conducted at the different emission angles are provided in Figs. 9.2 to 9.4 . The $\mathrm{Fe} 2 \mathrm{p}_{3 / 2}$ spectra generally consist of five components. Four of these components were assigned to iron-incorporated chemical compounds such as metallic iron $(\mathrm{Fe})$, cementite $\left(\mathrm{Fe}_{3} \mathrm{C}\right)$, magnetite/wustite $\left(\mathrm{Fe}_{3} \mathrm{O}_{4} / \mathrm{FeO}\right)$, and hematite/maghemite/ferrihydrite $\left(\alpha-\mathrm{Fe}_{2} \mathrm{O}_{3} / \gamma-\mathrm{Fe}_{2} \mathrm{O}_{3} / \mathrm{FeOOH}\right)$, and the fifth component represents the $\mathrm{Fe}_{2} \mathrm{O}_{3} / \mathrm{FeOOH}$ satellite structure, as marked in Figs. 9.2-9.4. The corresponding binding energies of these components are given in Table 9.4.

Table 9.3: The calculated sampling depth at various emission angles.

\begin{tabular}{|c|c|}
\hline Emission angle $(\boldsymbol{\theta})$ & $\mathbf{d}(\mathbf{n m})$ \\
\hline 0 & 8.3 \\
30 & 7.1 \\
40 & 6.3 \\
50 & 5.3 \\
60 & 4.1 \\
70 & 2.8 \\
75 & 2.1 \\
\hline
\end{tabular}

(Note: The calculated average attenuation length $\left(\lambda_{\text {ave. }}\right)$ is $2.75 \mathrm{~nm}$.) 
Table 9.4: The peak parameters of Fe $2 p X P S$ spectra used in this study.

\begin{tabular}{|c|c|c|c|c|c|c|}
\hline \begin{tabular}{|c|} 
Element \\
(Photo \\
electron \\
core \\
level)
\end{tabular} & Peak ID & Assignment & $\begin{array}{c}\text { Peak } \\
\text { position* } \\
2 \mathrm{P}_{3 / 2} / 1 \mathrm{~s} \\
( \pm 0.5 \mathrm{eV})\end{array}$ & $\begin{array}{c}\text { FWHM } \\
2 P_{3 / 2} \\
( \pm 0.2 \mathrm{eV})\end{array}$ & $\begin{array}{c}\text { FWHM } \\
2 \mathrm{P}_{1 / 2} \\
( \pm 0.2 \mathrm{eV})\end{array}$ & $\begin{array}{c}\Delta \mathrm{e}(\mathrm{eV}) \\
( \pm 0.5 \mathrm{eV})\end{array}$ \\
\hline Fe $2 p$ & $\begin{array}{l}\mathrm{Fe}-1 \\
\mathrm{Fe}-2 \\
\mathrm{Fe}-3 \\
\mathrm{Fe}-4 \\
\mathrm{Fe}-5\end{array}$ & $\begin{array}{l}\text { Fe metal } \\
\mathrm{Fe}_{3} \mathrm{C} \\
\mathrm{Fe}_{3} \mathrm{O}_{4} / \mathrm{FeO} \\
\mathrm{Fe}_{2} \mathrm{O}_{3} / \mathrm{FeOOH} \\
\mathrm{Fe}_{2} \mathrm{O}_{3} \text {-Satellite }\end{array}$ & $\begin{array}{l}707.0 \\
708.2 \\
709.6 \\
711.0 \\
712.7 \\
\end{array}$ & $\begin{array}{l}1.5 \\
2 \\
2 \\
2 \\
2.5 \\
\end{array}$ & $\begin{array}{l}1.9 \\
2.3 \\
2.3 \\
2.3 \\
\text { NA }\end{array}$ & $\begin{array}{l}13.5 \\
13.5 \\
13.5 \\
13.5 \\
13.5\end{array}$ \\
\hline
\end{tabular}

\footnotetext{
Reported as binding energy
}

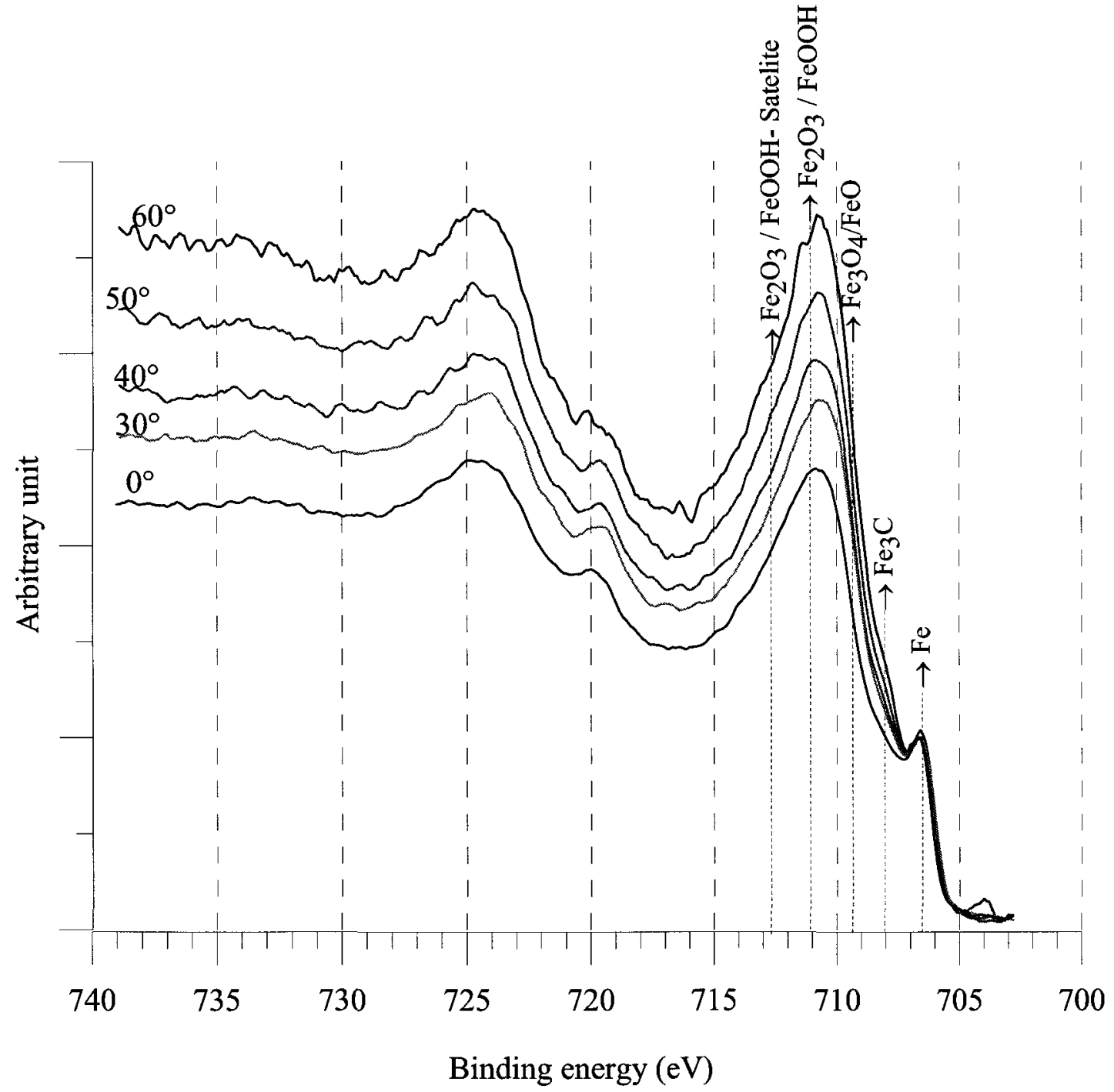

Figure 9.2: Fe 2p high resolution XPS spectra for the CP-0 sample at different emission angles. 
In this study, in order to qualitatively compare the amount of iron oxide in each Fe $2 p$ spectrum, the metallic iron (Fe-1) peaks for various $\mathrm{Fe} 2 \mathrm{p}$ spectra were set to an arbitrary level. This qualitative approach is considered to compare and to analyze the XPS results of the CP-0, CP-1 and CP-2 samples demonstrated in Fig. 9.2, Fig. 9.3 and Fig. 9.4, respectively. As seen in these figures, the intensities of iron oxides peaks (i.e., $\mathrm{Fe}-3, \mathrm{Fe}-4$ and $\mathrm{Fe}-5)$ are higher than iron metallic peak intensity for all three samples at different emission angles. Moreover, the height of the region assigned to iron oxides increases by increasing the emission angle in each sample. Due to the fact that a larger emission angle results in a smaller sampling depth, as presented in Table 9.3, the rise of iron oxide peaks with the increase of emission angle is consistent with the presence of a film of iron oxide covering the surface of steel of all three samples. The relative changes in the spectra by increasing the emission angle appear similar for all three samples.

\subsubsection{The effect of chloride}

The Fe $2 p$ spectra of three samples (i.e., CP-0, CP-1 and CP-2) are compared at three different angels (i.e., $0^{\circ}, 40^{\circ}$, and $60^{\circ}$ ) in Fig. 9.5. As mentioned previously, the Fe1 component represents the metallic iron while the Fe-3 and Fe-4 represent different types of iron oxides (i.e., $\mathrm{Fe}_{3} \mathrm{O}_{4} / \mathrm{FeO}$ and $\mathrm{Fe}_{2} \mathrm{O}_{3} / \mathrm{FeOOH}$, respectively). Therefore, the ratio of the heights of $\mathrm{Fe}-3$ and/or $\mathrm{Fe}-4$ components relative to the height of $\mathrm{Fe}-1$ indicates the ratio of the amounts of iron oxides to iron metal. Since all the iron metal peaks are placed at a constant arbitrary height, only the height of iron oxides region can be considered as an indicator for the amount of iron oxide on the surface of steel. In the following, this concept will be used for interpretation of the XPS spectra. 


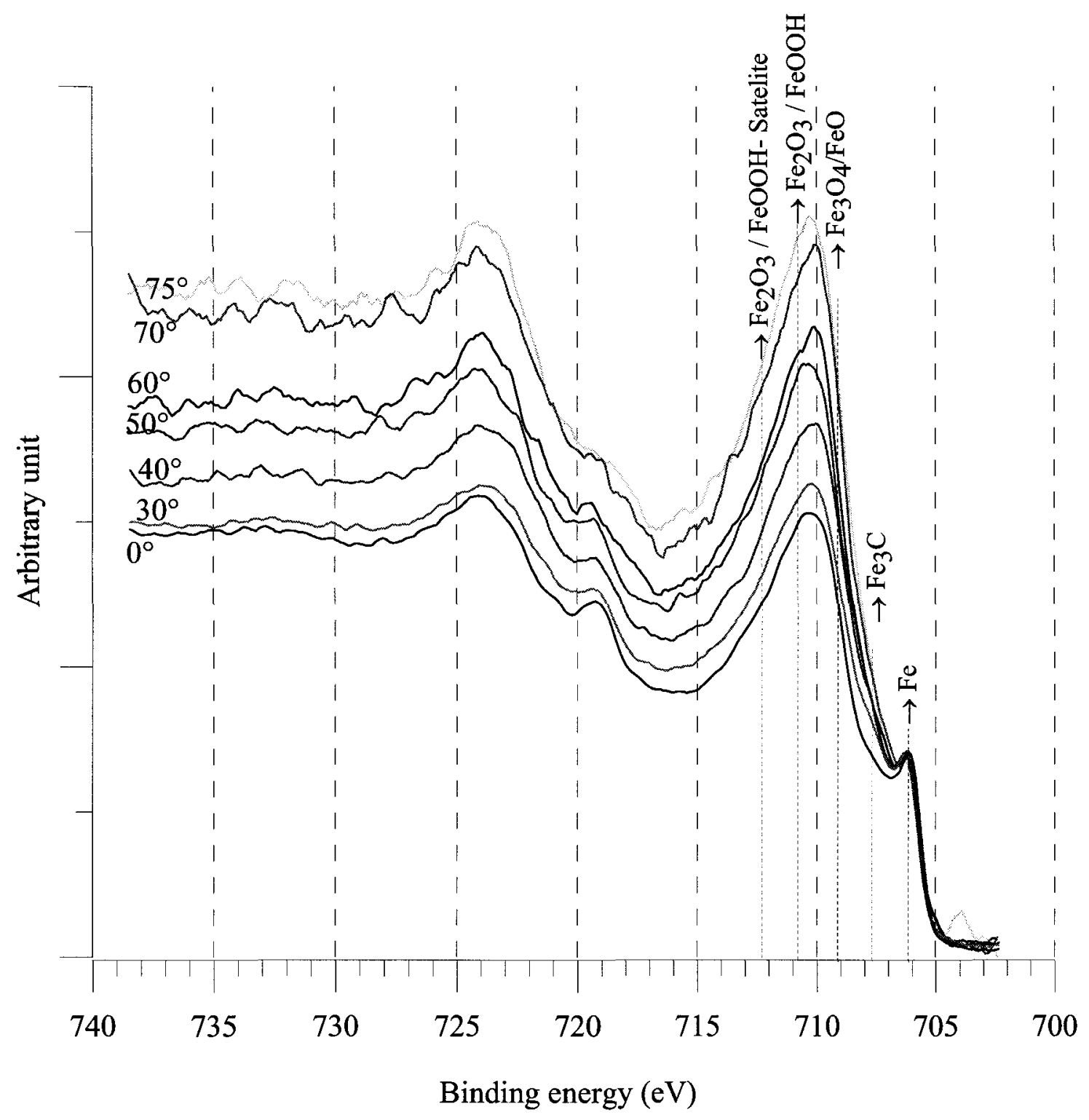

Figure 9.3: $\mathrm{Fe} 2 p$ high resolution XPS spectra for the CP-1 sample at different emission angles. 


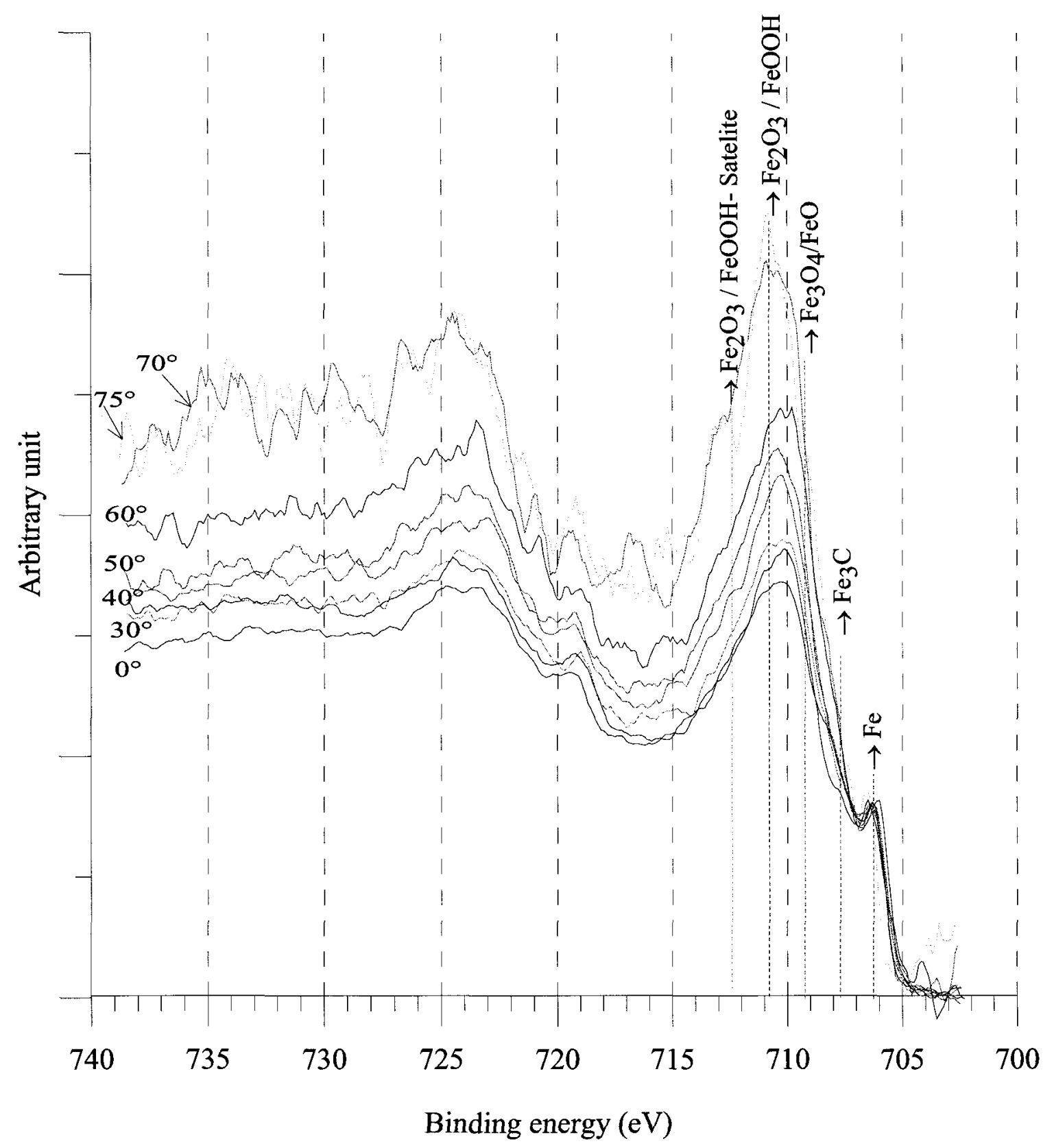

Figure 9.4: Fe 2p high resolution XPS spectra for the CP-2 sample at different emission angles. 


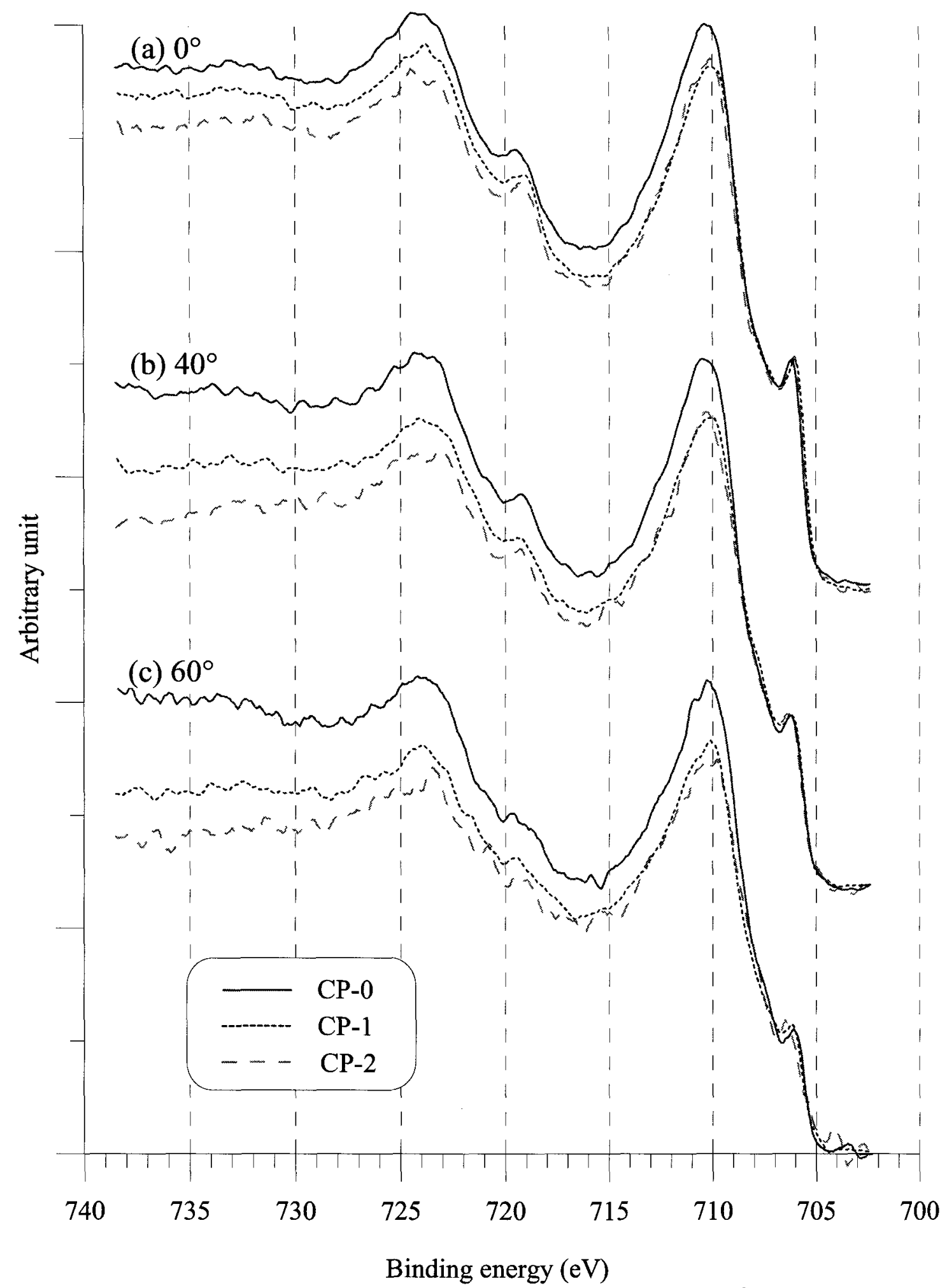

Figure 9.5: Comparison of the Fe $2 p$ spectra for three samples at (a) $0^{\circ}$ emission angle; (b) $40^{\circ}$ emission angle; (c) $60^{\circ}$ emission angle. 
It can be observed in Fig. 9.5(a) that the amount of iron oxide on the surface of the CP-0 sample is larger than that of the other two samples (i.e., CP-1 and CP-2). This suggests that the amount of iron oxide, and hence the average thickness of iron oxide, on the surface of steel for the samples that have been in contact with chloride are smaller than the ones exposed to the free-chloride solution. The smaller thickness of the oxide film on the CP-1 and CP-2 samples can be possibly attributed to the dissolution of iron oxides into the solution due to the presence of chloride. Similar observations were made in the XPS depth-profiling studies of oxide film in the saturated calcium hydroxide solution presented in Chapter 8 as well as the TEM studies of oxide film described in Chapter 7.

In Fig. 9.5(a) the Fe 2p spectra for the CP-1 and CP-2 samples are quite similar, indicating that the high concentration of chloride $(3 \mathrm{M})$ does not remarkably affect the thickness of iron oxide compared with the low chloride concentration $(0.45 \mathrm{M})$. Furthermore, the Fe $2 \mathrm{p}$ spectra at the emission angles of $40^{\circ}$ and $60^{\circ}$ have been respectively compared in Figs. 9.5 (b) and (c). The results at these angles which mostly reflect the characteristics of the outer layer of iron oxides reveal a very similar trend compared with the ones demonstrated for the emission angle of $0^{\circ}$.

The curve fitting analyses of the Fe $2 p$ XSP spectra were carried out using procedure similar to that described in Chapter 8 (Section 8.3.3). The ratio of the amount of iron oxides to the metallic iron at each emission angle can be therefore determined by proportioning the total intensity of iron oxides (i.e., Fe-3, Fe-4 and Fe-5) to the intensity of the metallic iron (i.e., Fe-1). In Fig. 9.6, this ratio, which is determined based on the average of two spots for each sample, is plotted at various emission angles for three 
samples. The CP-0 profile has the highest ratio compared with the other two samples, which is consistent with the thickness results reported previously. The results of the CP-1 and CP-2 samples are quite similar, but at $0^{\circ}$, the ratio for the CP-1 sample is slightly greater than that for the CP-2 sample.

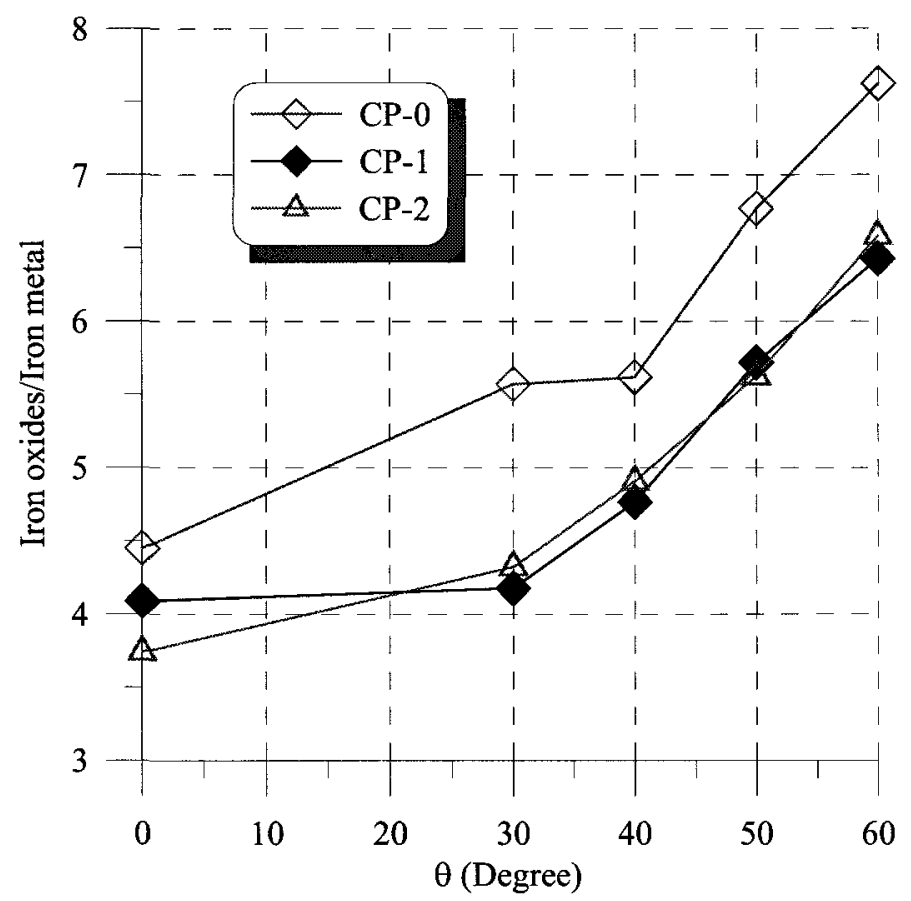

Figure 9.6: Comparison of the (Iron oxide)/(Iron metal) at the different emission angles.

To support the qualitative observations of the chloride effect on the thickness of the oxide films, a quantitative method was also used. Assuming a uniform film of iron oxide on the steel surface, the iron oxide thickness can be calculated using the intensity ratio of iron oxides to metallic iron, as described in Chapter 8 (Section 8.4.3). Therefore, the average thickness of oxide film (calculated from two different test spots for each sample) for the CP-0, CP-1 and CP-2 samples was found to be $4.8,4.6$ and $4.4 \mathrm{~nm}$, respectively. These results support the second type of ion displacement mechanism (Kolotyrkin 1961, Leckie and Uhlig 1966), explained in Chapter 3 (Section 3.4.2), in 
which the local thinning of passive film in the presence of chloride will lead to the breakdown of passive film.

\subsubsection{The variation of iron compounds in the oxides}

Using the curve fitting results, the ratio between the concentration of $\mathrm{Fe}_{3} \mathrm{O}_{4} / \mathrm{FeO}$ $\left(\mathrm{Fe}^{2+}\right)$ and $\mathrm{Fe}_{2} \mathrm{O}_{3} / \mathrm{FeOOH}\left(\mathrm{Fe}^{3+}\right)$ was obtained at various emission angles by proportioning the XPS intensity of Fe-3 to the total XPS intensities of $\mathrm{Fe}-4$ and $\mathrm{Fe}-5$, as demonstrated in Fig. 9.7. For all samples, the $\mathrm{Fe}^{2+} / \mathrm{Fe}^{3+}$ generally decreases by increasing the emission angle, which indicates that within the outer part of the film the concentration of $\mathrm{Fe}^{3+}$ is higher than that within the inner part. The XPS angle-resolved studies in high pH by Haupt and Strehblow (1987) also proposed the same structure for the passive film formed on iron in $1 \mathrm{M} \mathrm{NaOH}$ solution. This observation is also in agreement with the XPS depth profiling studies in Chapter 8 , in which it was demonstrated that the inner layers of the oxide film are mainly composed of $\mathrm{Fe}^{2+}$ oxides whereas its outer layers mostly consist of $\mathrm{Fe}^{3+}$ oxides.

Comparing the profiles given in Fig. 9.7, it is also observed that the $\mathrm{Fe}^{2+} / \mathrm{Fe}^{3+}$ ratios at emission angles larger than $50^{\circ}$ are quite comparable in all three samples, whereas for the lower angles $\left(\theta<50^{\circ}\right)$ the $\mathrm{Fe}^{2+} / \mathrm{Fe}^{3+}$ of $\mathrm{CP}-0$ sample is greater than that of the others (i.e., CP-1 and CP-2). The inference is that the chemical structure of the outermost layer of iron oxides for three samples are quite similar, but the amount of $\mathrm{Fe}^{2+}$ oxides in the inner layers of oxide films that have been in contact with chloride (i.e., CP1 and CP-2 solution) is relatively smaller than the amount of $\mathrm{Fe}^{3+}$ oxides. Furthermore, the $\mathrm{Fe}^{2+} / \mathrm{Fe}^{3+}$ for the $\mathrm{CP}-1$ sample exposed to $0.45 \mathrm{M}$ chloride is greater than that of the CP-2 sample that was exposed to $3 \mathrm{M}$ chloride, particularly at the emission angles 
between $0^{\circ}$ and $50^{\circ}$, indicating that the chloride in the CP solution changes the stoichiometry of oxide film such that the higher concentration of chloride in the solution results in a lower concentration of $\mathrm{Fe}^{2+}$ in the inner layer of iron oxide. This shows that chloride affects the $\mathrm{Fe}^{2+}$-incorporated compounds, which are believed to be the protective part of the oxide film against corrosion (Haupt and Strehblow 1987, Hugot-Le Goff et al. 1990, Joiret et al. 2002). Therefore, the mechanism of the breakdown of passive film in the presence of chloride can be attributed to the further oxidation of protective layer of the film, which is mainly composed of $\mathrm{Fe}^{2+}$ compounds, to the non-protective layer of $\mathrm{Fe}^{3+}$ compounds. This can explain the role of chloride in depassivation of steel in highly alkaline environments. These observations are also consistent with the second type of ion displacement mechanism (Kolotyrkin 1961, Leckie and Uhlig 1966) explained in Chapter 3 (Section 3.4.2). Further studies are required to support these observations.

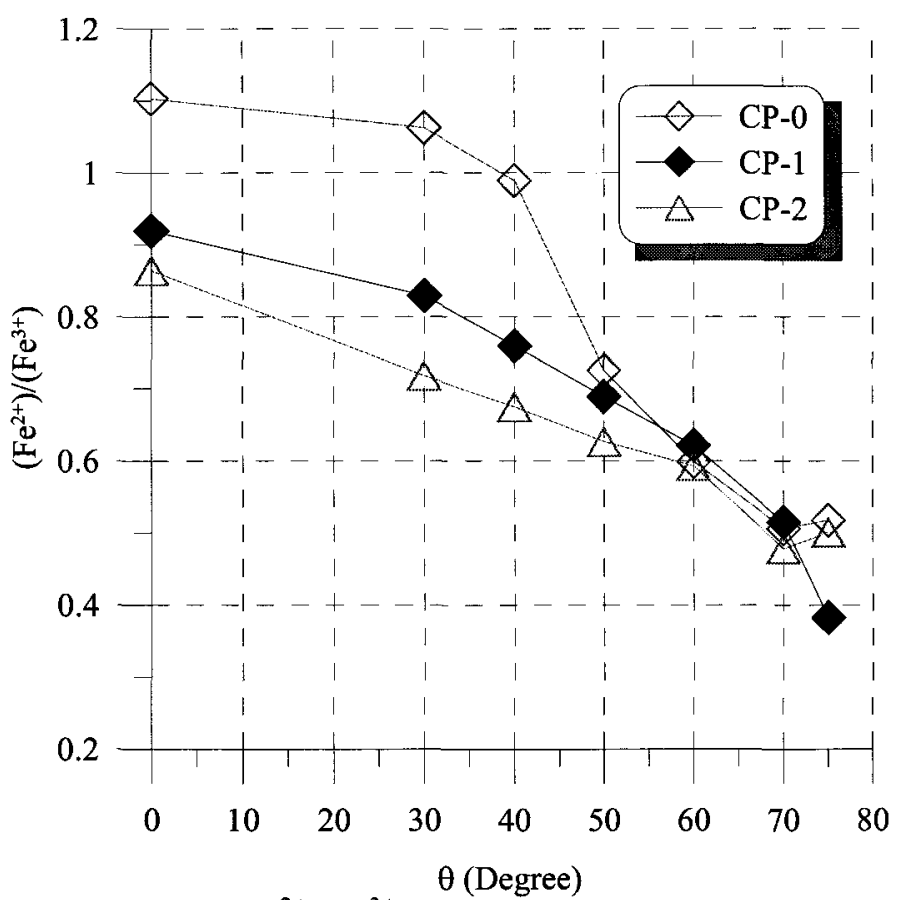

Figure 9.7: Comparison of the $\mathrm{Fe}^{2+} / \mathrm{Fe}^{3+}$ at the different emission angles. 


\subsubsection{The effect of solution}

The effect of solution on the properties of oxide film was investigated using the XPS spectrum of the CH-9 sample (presented in Chapter 8), along with that of the CP-0 sample. In Fig. 9.8, the Fe 2p XPS spectrum of the CP-0 sample exposed to the CP solution $(\mathrm{pH}=13.3)$ is compared with the one of the $\mathrm{CH}-9$ sample exposed to the $\mathrm{CH}$ solution $(\mathrm{pH}=12.5)$. As seen in this figure, the amount of iron oxides relative to iron metal for the CP-0 sample is larger than that for the CH-9 sample (4.45 vs. 2.94), which suggests that the thickness of iron oxide formed in the CP solution is greater than that of the iron oxide formed in the $\mathrm{CH}$ solution. In contrast, the $\mathrm{Fe}^{3+} / \mathrm{Fe}^{2+}$ for the $\mathrm{CP}-0$ sample is lower than the one for the CH-9 sample (0.91 vs. 1.07$)$, indicating that the relative concentration of $\mathrm{Fe}^{2+}$ is larger for the oxide formed in the $\mathrm{CH}$ solution than that of the oxide formed in the $\mathrm{CP}$ solution.

This can possibly explain the higher chloride threshold of the $\mathrm{CP}$ solution in comparison with the $\mathrm{CH}$ solution, as reported in Chapter 5 and in the works of other researchers (Gouda 1970, Goni and Andrade 1990, Pettersson 1995, Mammoliti et al. 1996, Breit 1998, Li and Sagues 2001, Oh et al. 2003, Hausmann 1967), since the $\mathrm{Fe}^{2+}$ incorporated iron oxides in passive film (e.g. $\mathrm{Fe}_{2} \mathrm{O}_{3}$ and $\mathrm{FeO}$ ) can prevent the dissolution of iron into the solution. The implication is that the higher concentration of $\mathrm{Fe}^{2+}$ in oxide films that form in the CP solution probably contributes to a better protection of steel against chloride attack; this observation is consistent with the mechanism described in Section 9.3.5. 


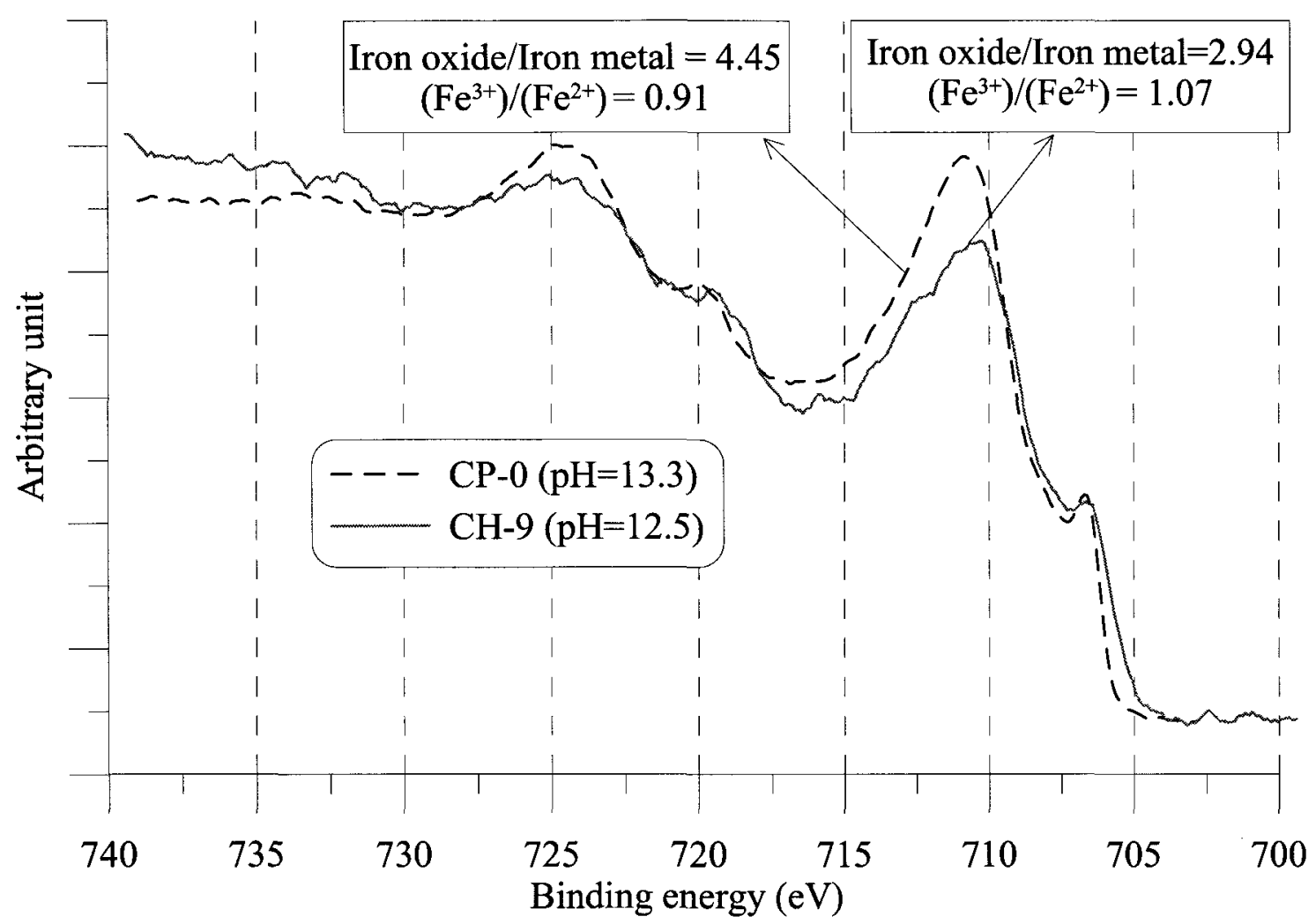

Figure 9.8: The effect of solution (i.e., CP solution vs. CH solution) on the Fe $2 p$ spectra at $0^{\circ}$ emission angle.

\subsection{Conclusions}

Based on the angle-resolved XPS investigation carried out on the compositional characteristics of iron oxide films in the simulated concrete pore solution, the following conclusions can be drawn:

- The amount of iron oxide, and hence the average thickness of iron oxide, on the surface of steel for the samples that have been in contact with chloride (i.e., CP-1 and CP-2) are smaller than the ones exposed to the free-chloride solution (i.e., CP-0).

- The average thickness of oxide film calculated for the CP-0, CP-1 and CP-2 samples is $4.8,4.6$ and $4.4 \mathrm{~nm}$, respectively. 
- In all samples, the $\mathrm{Fe}^{2+} / \mathrm{Fe}^{3+}$ generally decreases by increasing the emission angle, indicating that the concentration of $\mathrm{Fe}^{3+}$ is higher in the outer layers than inner layers.

- The $\mathrm{Fe}^{2+} / \mathrm{Fe}^{3+}$ of the outermost layer of iron oxides for three samples are quite similar, but the relative amount of $\mathrm{Fe}^{2+}$ oxides in the inner layers of oxide films that have been in contact with chloride (i.e., CP-1 and CP-2) are smaller than the inner layers of oxides that have never been exposed to chloride (i.e., CP-0).

- The relative concentration of $\mathrm{Fe}^{2+}$ as well as the thickness of iron oxide formed in the CP solution ( $\mathrm{pH}=13.3$ ), i.e., $\mathrm{CP}-0$, is greater than the one formed in the CH solution $(\mathrm{pH}=12.5)$, i.e., $\mathrm{CH}-9$.

- The higher concentration of $\mathrm{Fe}^{2+}$ in oxide films that form in the $\mathrm{CP}$ solution can be possibly related to a better protection of steel against the chloride attack.

- In the view of the results obtained in this study, the mechanism of the breakdown of passive film in the presence of chloride can be attributed to the further oxidation of protective inner layer of the oxide film, which is mainly composed of $\mathrm{Fe}^{2+}$ compounds, to the non-protective $\mathrm{Fe}^{3+}$ compounds. This can explain the role of chloride in depassivation of steel in high alkaline environment.

- The results obtained by angle-resolved XPS technique from oxide films in the CP solution generally confirm those obtained by depth-profiling technique in Chapter 8. No remarkable discrepancy was observed between the overall outcomes of these two techniques. 


\section{Chapter 10}

\section{Conclusions and Future Work}

\subsection{General}

The study presented in this thesis was carried out to better understand the mechanisms of passivation and chloride-induced depassivation of carbon steel reinforcement in concrete and to provide robust explanations to the outstanding questions about corrosion initiation of steel rebar in the alkaline environment of concrete. In order to achieve these goals, a multi-scale investigation was carried out. The investigation consisted of four components: electrochemical experiments, microscopic examinations, numerical modeling, and spectroscopic studies. These four components cover length scales from macro to micro/nano to provide fundamental explanations for engineering observations about the depassivation of carbon steel reinforcement in concrete. This chapter presents the main conclusions of this research, a proposed process for chloride-induced depassivation of carbon steel in highly alkaline environments similar to those provided by concrete, and proposals for future work originating from and related to the current study. 


\subsection{Main Conclusions}

The electrochemical studies revealed that concrete pore solution composition has an effect on the protective properties of the passive oxide films on carbon steel rebars and, in particular, sulfate ions $\left(\mathrm{SO}_{4}\right)^{2-}$ have been shown to have a negative influence on the film quality. The electrochemical experiments on the depassivation of carbon steel rebars also supported the role of pore solution chemistry: the chloride thresholds in the simulated concrete pore solution with a pH of 13.3 were higher than those in the saturated calcium hydroxide solution with a $\mathrm{pH}$ of 12.5. Higher chloride thresholds were observed for rebars without mill scale than those measured for asreceived rebars. It was also found that modifying the rebar surface led to reduced variability and reduced fluctuations in electrochemical measurements, which can be explained by the fact that modified surfaces are uniformly the same everywhere, whereas the as-received surfaces are locally much more diverse and complex due to the presence of mill scale.

SEM studies have shown that mill scale on carbon steel rebar is poorly and non-uniformly attached to the steel surface, and it contains randomly distributed cracks that link the free surface of the mill scale to the underlying crevices on the steel surface. It was inferred that when as-received rebar surfaces are exposed to concrete pore solution contaminated with chlorides, transverse mill scale cracks serve as pathways for concrete pore solution to reach the surface of steel and allow ion exchange between the pore solution and the crevices. It was suggested that, over time, the chemical composition of the pore solution within the crevices differs from that of the bulk solution through a process similar to the suggested mechanisms of typical 
crevice corrosion. Therefore, the crevices between mill scale and steel are proposed potential sites for depassivation and pit nucleation. The numerical investigation that was carried out to test this hypothesis confirmed that $\mathrm{Cl}^{-} / \mathrm{OH}^{-}$inside crevices increased to levels higher than that of the bulk pore solution, making crevices more vulnerable to depassivation. Therefore, it was concluded that the variability associated with reported chloride thresholds could be attributed, at least in part, to the variability in mill scale properties resulting from the variability in manufacturing.

Nano-scale experimental techniques (i.e., TEM and XPS) were used to investigate the properties of oxide films formed on highly polished steel surfaces in simulated concrete pore solutions and to study the role of chlorides during the depassivation process. TEM observations on samples obtained using the FIB technique provided the following main conclusions: (1) chlorides in quantities lower than the depassivation thresholds did not change the appearance of the oxide film that was grown in simulated concrete pore solutions; (2) small amounts of chlorine, potassium, sulfur and calcium were detected inside the outer layers of the oxide film; (3) after exposure to chlorides in amounts larger than typical depassivation thresholds, oxide films were no longer uniform: the average thickness of the remaining film was smaller than the thickness of the oxide film before exposure to chlorides, and some pit initiation sites formed on the surface of steel.

Both EELS and TEM diffraction analysis showed that the oxide film on the surface of steel was mainly composed of nano-crystalline particles of $\mathrm{FeOOH}$, with an orthorhombic unit cell of $\mathrm{a}=4.937 \AA, \mathrm{b}=4.432 \AA$ and $\mathrm{c}=2.994 \AA$. Nevertheless, possible presence of other minor constituent oxides could not be eliminated. The 
diffraction analysis of the oxide film indicated that the oxide is in an epitaxial orientation relationship with the underlying $\alpha$-Fe crystals. This epitaxial relationship would lead to a crystallographic texture in the oxide suggesting that the oxide film on the surface of steel is a fairly coherent layer.

XPS analyses also provided insights into the properties of oxide film and the role of chlorides during the depassivation process. It was found that $\mathrm{Fe}^{3+} / \mathrm{Fe}^{2+}$ increased from the inner parts of the oxide film near the surface of the steel towards the outer parts. The relative concentration of $\mathrm{Fe}^{2+}$ of oxide film in the simulated concrete pore solution $(\mathrm{pH}=13.3)$ was greater than that of the oxide film in the saturated calcium hydroxide solution $(\mathrm{pH}=12.5)$. After exposure to chlorides, the $\mathrm{Fe}^{3+} / \mathrm{Fe}^{2+}$ of the $\mathrm{Fe}^{+3}$-rich outer layer did not change; however, the $\mathrm{Fe}^{3+} / \mathrm{Fe}^{2+}$ of the $\mathrm{Fe}^{+2}$-rich inner layer increased (i.e., relative concentration of $\mathrm{Fe}^{2+}$ in the inner layer decreased). In addition, the amount of iron oxide, and hence its average thickness of the film, decreased after exposure to chlorides.

\subsection{Proposed Depassivation Process}

The nano-scale studies indicated that the 4-10 nm-thick passive oxide film formed on carbon steel in highly alkaline environments such as the one provided by concrete consists of two layers separated with an indistinct border. The thinner inner layer is mainly composed of protective $\mathrm{Fe}^{2+}$-rich oxides that are in epitaxial relationship with the underlying steel surface; while the outer layer mostly consists of (possibly porous) $\mathrm{Fe}^{3+}$-rich oxides. Chlorides in the concrete pore solution can penetrate though the outer layer to reach the inner layer. In the presence of chlorides, 
$\mathrm{Fe}^{+2}$-rich oxides in the inner layer transform into $\mathrm{Fe}^{+3}$-rich oxides and become unprotective. Although how this transformation occurs is still subject to future research (see Section 10.4), there are some evidences showing that the process most likely leads to the formation of local anodic and cathodic sites on the steel surface.

The characteristics of the passive oxide film in this research can be explained by the crystalline oxide film models reviewed in Chapter 3 since it was observed that the oxide film on the surface of steel is mainly composed of nano-crystalline particles with the size up to $8 \mathrm{~nm}$. In particular, the Place Exchange Model (Sato and Cohen 1964) and the PDM model (Chao 1981) can explain the epitaxial relationship of the nano-crystalline structure of oxide film with underlying steel. Furthermore, the depassivation process has similarities to the depassivation processes explained by the Pore Model (Evans et al. 1931, Richardson and Wood 1970), Dynamic Breakdownrepair Model (Galvele 1978), and particularly, by the second type of Absorbed Ion Displacement Model (Kolotyrkin 1961, Leckie and Uhlig 1966). Although these three depassivation models can be used to partly explain certain experimental observations presented in this research, it was also found that the chloride-induced depassivation of carbon steel rebar is unique, and no existing model provides a comprehensive explanation for all multi-scale experimental observations.

\subsection{Future Work Originating from Present Research}

It has been shown in this study that chlorides, the $\mathrm{pH}$ of solution, the presence of localized sites, and applied potential play significant roles in the breakdown of passive film on carbon steel in simulated concrete pore solutions. 
Furthermore, it has been reported in literature that temperature and oxygen availability also affect chloride thresholds. Therefore, a successful mechanistic model for passive film breakdown should be able to explain the effects of all these six factors. The development of such a mechanistic model and its verification constitute a major future research originating from the present study. In this section, working hypotheses that would help the development of such a mechanistic model are presented to give direction to future research.

The depassivation process proposed in Section 10.3 is based on the observation that chlorides in the concrete pore solution penetrate though the outer oxide layer and transform the $\mathrm{Fe}^{+2}$-rich oxides in the inner layer into $\mathrm{Fe}^{+3}$-rich oxides; this transformation leads to the loss of protective nature of the inner layer. Although this assumption can be justified in part by the experimental evidences gathered in this study, the governing mechanism of chloride ingress through the outer layer (i.e., whether the ingress is in the through diffusion, migration or vacancy/iron exchange process) and the chemical and physical interactions of chlorides with the outer layer are not well understood. Atomistic modeling techniques (e.g. density functional theory) and additional nano-scale experiments are needed to provide explanations for these two outstanding issues.

Even though it was experimentally established that chlorides can alter the $\mathrm{Fe}^{2+}$-rich inner layer of the passive film, the underlying mechanism of this transformation is not yet known. Here a working hypothesis for the breakdown of the inner layer by chlorides is provided for future researchers to explore. 
The transformation of $\mathrm{Fe}^{2+}$-rich oxides of the inner layer to $\mathrm{Fe}^{3+}$-rich oxides may take place through an electrochemical process similar to the three (among other possible reactions) provided below:

$$
\begin{array}{lr}
\mathrm{Fe}_{x} \mathrm{O}+(2 x) \mathrm{Cl}^{-}+\mathrm{H}_{2} \mathrm{O} \rightarrow x \mathrm{Fe}(\mathrm{OH}) \mathrm{Cl}_{2}+(2-x) \mathrm{OH}^{-}+(3 x-2) e^{-} & E q .10 .1 \\
\mathrm{Fe}_{x} \mathrm{O}+(x) \mathrm{Cl}^{-}+\mathrm{H}_{2} \mathrm{O} \rightarrow x \mathrm{Fe}(\mathrm{OH})_{2} \mathrm{Cl}+(2-2 x) \mathrm{OH}^{-}+(3 x-2) e^{-} & E q .10 .2 \\
\mathrm{Fe}_{x} \mathrm{O}+(3 x) \mathrm{Cl}^{-}+\mathrm{H}_{2} \mathrm{O} \rightarrow x \mathrm{FeCl}_{3}+2 \mathrm{OH}^{-}+(3 x-2) e^{-} & \text {Eq. } 10.3
\end{array}
$$

Although all these electrochemical reactions can be used to explain the role of the influential factors (i.e., chloride, $\mathrm{pH}$, temperature, potential, and oxygen availability) in depassivation process, as presented later in this section, there are some evidences indicating the presence of $\mathrm{Fe}(\mathrm{OH}) \mathrm{Cl}_{2}$ and $\mathrm{Fe}(\mathrm{OH})_{2} \mathrm{Cl}$ in the structure of green rust, which is an early-stage corrosion product of iron in alkaline solutions (Refait and Genin 1993, Refait et al. 1998).

The electric potential of an electrochemical reaction (such as the one presented in Eqs.10.1-10.3) can be obtained by using the Nernst equation (Revie and Uhlig 2008):

$$
\phi=\phi^{\circ}+\frac{R T}{z F} \ln (K)
$$

where $\phi^{\circ}$ is the standard potential of an electrochemical reaction when all reactants and products are in their standard states (activities equal to unity), $F$ ( $\approx 96500 \mathrm{C} / \mathrm{mole}$ ) is the Faraday's constant, $R(\approx 8.314 \mathrm{~J} /($ mole. $\mathrm{K}))$ is the universal gas constant, $T$ is temperature $\left({ }^{\circ} \mathrm{K}\right), z$ is the number of electrons participating in the electrochemical reaction and $K$ is the equilibrium constant defined by: 


$$
K=\frac{\sum a_{\text {products }}}{\sum a_{\text {reactants }}}
$$

where $a_{\text {products }}$ and $\mathrm{a}_{\text {reactants }}$ are the activites of the products and the reactants, respectively. The standard potential $\left(\phi^{\circ}\right)$ of an electrochemical system can be also obtained from the standard Gibbs free energy of the electrochemical reaction $\left(\Delta G^{\circ}\right)$ via

$$
\phi^{\circ}=\frac{\Delta G^{\circ}}{z F}
$$

Using the procedure explained above, the electric potential of the electrochemical reactions presented in Eqs. 10.1-10.3 can be derived respectively as

$$
\begin{aligned}
& \phi=\frac{\Delta G^{\circ}}{(3 x-2) F}+\frac{R T}{(3 x-2) F} \log \left(\frac{\gamma_{O H}\left[O H^{-}\right]^{2-x}}{\gamma_{C l^{-}}\left[C l^{-}\right]^{2 x}}\right) \\
& \phi=\frac{\Delta G^{\circ}}{(3 x-2) F}+\frac{R T}{(3 x-2) F} \log \left(\frac{\gamma_{O H}\left[O H^{-}\right]^{2-2 x}}{\gamma_{C l}\left[C l^{-}\right]^{x}}\right) \\
& \phi=\frac{\Delta G^{\circ}}{(3 x-2) F}+\frac{R T}{(3 x-2) F} \log \left(\frac{\gamma_{O H}\left[O H^{-}\right]^{2}}{\gamma_{C l}\left[C l^{-}\right]^{3 x}}\right)
\end{aligned}
$$

In these equations it is quite obvious that either decrease in hydroxide concentration or increase in chloride concentration decreases the electric potential, which is the potential required to make the electrochemical reactions given in Eqs. 10.1-10.3 thermodynamically possible. Therefore, the roles of chloride and hydroxide (i.e., $\mathrm{pH}$ ) can be clearly identified using any of these three electrochemical reactions.

Let's assume that the electrochemical reaction in Eq. 10.1 is responsible for the breakdown of the passive film. For a steel electrode covered by a passive film in a highly alkaline solution with a certain amount of chloride, the potential required to 
destroy the passive film (i.e., pitting potential) can be calculated by using the corresponding Nernst equation defined in Eq. 10.7. When the potential of steel exceeds the calculated potential, the electrochemical reaction in Eq. 10.1 can thermodynamically take place, and hence, the destruction of passive film is highly probable. On the other hand, at potentials lower than this calculated pitting potential, the breakdown of passive film on the steel electrode is not thermodynamically possible. As a result, this model is able to consider the effect of potential on the breakdown of the passive film.

The effect of temperature can be seen directly in the Nernst equation (Eq. 10.4), and indirectly in the Gibbs free energy as well as the in the activity coefficients. The availability of oxygen also has an indirect effect on the depassivation mechanism through the potential of steel in the solution: it is well known that the depletion of oxygen around steel decreases its open circuit potential; thus, the thermodynamic conditions required for passive film destruction (determined by the electrochemical reactions) would be affected indirectly by the availability of oxygen around the surface of steel.

It is also important to keep in mind that the kinetics of the reactions given in Eqs. 10.1-10.3 needs to be also considered along with the thermodynamics. If the reaction rate is too slow, the electrochemical reaction will not have an effect on depassivation, even though the reaction is thermodynamically possible. As a result, it is suggested that more experimental studies are carried out on the kinetics of the possible electrochemical reactions (e.g. Eq.10.1-10.3) explaining the transformation of the $\mathrm{Fe}^{2+}$-rich oxides of the inner layer to $\mathrm{Fe}^{3+}$-rich oxides. 


\subsection{Recommendations for Future Work}

In addition to the future work originating from the present study, other research topics related to the present work are recommended here for future investigation. These topics are listed as follows:

- The study of the role of cementite phases of carbon steel on the breakdown of the passive film of rebar in concrete and the subsequent pit initiation process.

- The effect of sulfate on the passive film formation and depassivation of carbon steel rebar in concrete.

- The effect of concrete/rebar interface on the chloride-induced depassivation of carbon steel rebar.

- The effect of temperature on the chloride-induced depassivation of carbon steel rebar in concrete.

- The effect of carbonation on the passivity and chloride-induced depassivation of carbon steel rebar in concrete.

- Passivity studies on other types of rebars (e.g. stainless, galvanized and micro-composite) in concrete.

- The elimination of the mill scale on the surface of carbon steel during rebar production. 
Abd El Aal, E. E., Abd El Wanees, S., Diab, A., \& Abd El Haleem, S. M. (2009). Environmental factors affecting the corrosion behavior of reinforcing steel III. Measurement of pitting corrosion currents of steel in $\mathrm{Ca}(\mathrm{OH})_{2}$ solutions under natural corrosion conditions. Corrosion Science, 51(8), p. 1611.

Akimov, G. V. (1953). Theory and testing methods of corrosion of metals. SNTL, Praha (in Czech).

Allen, G. C., Tucker, P. M., \& Wild, R. K. (1982). Characterization of iron/oxygen surface reactions by $\mathrm{X}$-ray photoelectron spectroscopy. Philosophy Magazine B, 46(5), p. 411.

Alonso, C., Andrade, C., Castellote, M., \& Castro, P. (2000). Chloride threshold values to depassivate reinforcing bars embedded in a standardized OPC mortar. Cement and Concrete Research, 30(7), p.1047.

Alonso, C., Castellote, M., \& Andrade, C. (2002). Chloride threshold dependence of pitting potential of reinforcements. Electrochimica Acta, 47(21), p. 3469.

Alvarez, M. G., \& Galvele, J. R. (1984). The mechanism of pitting of high purity iron in $\mathrm{NaCl}$ solutions. Corrosion Science, 24(1), p. 27.

Andersson, K., Allard, B., Bengtsson, M., \& Magnusson, B. (1989). Chemical composition of cement pore solutions. Cement and Concrete Research, 19(3), p. 327.

Andrade, C., \& Page, C. L. (1986). Pore solution chemistry and corrosion in hydrated cement systems containing chloride salts: A study of cation specific effects. British Corrosion Journal, 21(1), p. 49. 
Angst, U., Elsener, B., Larsen, C. K., \& Vennesland, Ø. (2009). Critical chloride content in reinforced concrete-A review. Cement and Concrete Research, I39(12), p. 1122.

Arya, C., Buenfeld, N. R., \& Newman, J. B. (1990). Factors influencing chloride-binding in concrete. Cement and Concrete Research, 20(2), p. 291.

ASTM C1 14 (2009). Standard Test Methods for Chemical Analysis of Hydraulic Cement chemical analysis, compositional analysis, hydraulic cements, Alkalies content cement, Aluminum oxide (Al) content, Vinsol resin content, Water-soluble-matter content. Annual Book of ASTM Standards, American Society for Testing and Materials, Philadelphia, USA.

ASTM G 5-94 (2004). Standard Reference Test Method for Making Potentiostatic and Potentiodynamic Anodic Polarization Measurements, Annual Book of ASTM Standards, American Society for Testing and Materials, Philadelphia, USA.

Bamforth, P. B., Price, W. F., and Emerson, M. (1997). An international review of chloride ingress into structural concrete, Thomas Telford.

Bard, A. J., \& Faulkner, L. R. (2001). Electrochemical methods: Fundamentals and applications. John Wiley \& Sons, Inc., New York, NY.

Berke, N. S., \& Hicks, M. C. (1990). Electrochemical methods of determining the corrosivity of steel in concrete. Corrosion Testing and Evaluation, Eds: Baboian, R., \& Dean, S. W., p. 425.

Bertolini, L., Elsener, B., Pedeferri, P., \& Polder, R. (2004). Corrosion of steel in concrete prevention, diagnosis, repair. WILEY-VCH.

Bird, H. E. H., Pearson, B. R., \& Brook, P. A. (1988). The breakdown of passive films on iron. Corrosion Science, 28(1), p. 81.

Blanco, G., Bautista, A., \& Takenouti, H. (2006). EIS study of passivation of austenitic and duplex stainless steels reinforcements in simulated pore solutions. Cement and Concrete Composites, 28(3), p. 212. 
Breit, W. (1998). Kritischer chloridgehalt-untersuchungen an stahl in chloridhaltigen alkalischen LoÈsungen. Materials and Corrosion, 49(8), p. 539.

Brem M. (2004) Numerische Modellierung der Korrosion an Stahlbetonbauten. Ph.D. dissertation, ETH Zürich, No. 15567.

Briggs, D., \& Seah, M. P. (1990). Practical surface analysis: Volume 1-Auger and X-ray photoelectron spectroscopy. Wiley.

Broomfield, J. P. (1997). Corrosion of steel in concrete: Understanding, investigation and repair, E \& FN Spon Press, New York, NY.

Brundle, C. R., Chuang, T. J., \& Wandelt, K. (1977). Core and valence level photoemission studies of iron oxide surfaces and the oxidation of iron. Surface Science, 68, p. 459.

BS 1881: Part 124 (1988). Testing concrete: Methods for analysis of hardened concrete, BSI.

Cabrera, N., \& Mott, N. F. (1949). Theory of the oxidation of metals. Reports and Progress in Physics, 163.

Castellote, M., Andrade, C., \& Alonso, C. (2002). Accelerated simultaneous determination of the chloride depassivation threshold and of the non-stationary diffusion coefficient values. Corrosion Science, 44(11), p. 2409.

Chao, C. Y. (1981). A point defect model for anodic passive films. I. film growth kinetics. Journal of the Electrochemical Society, 128(6), p. 1187.

Chen, C. T., \& Cahan, B. D. (1982). The nature of the passive film on iron. I. automatic ellipsometric spectroscopy studies. Journal of the Electrochemical Society, 129(1), p. 17.

Chen, S. Y., Gloter, A., Zobelli, A., Wang, L., Chen, C. H., \& Colliex, C. (2009). Electron energy loss spectroscopy and ab initio investigation of iron oxide nanomaterials grown by a hydrothermal process. Physical Review B, 79(10), p. 104. 
Cherepin, V. T. (1987). Secondary ion mass spectroscopy of solid surfaces. VNU Science Press BV.

Choi, Y. S., Kim, J. G., \& Lee, K. M. (2006). Corrosion behavior of steel bar embedded in fly ash concrete. Corrosion Science, 48(7), p. 1733.

Cohen, M. (1978). The passivity and breakdown of passivity on iron. "The Passivity of Metals”, Eds.: Frankenthal R.P., Kruger J., Electrochemical Society (ECS), 521.

Conway, B. E. (1952). Electrochemical data. Elsevier, Amsterdam.

Dean, J.A. (1999). Lange's Handbook of Chemistry, 15th Edition, Table 3.2 Physical Constants of Inorganic Compounds, McGraw-Hill.

Dilks, A. (1981). Polymer surfaces. Analytical Chemistry, 53(7), p. 802.

Elsener, B. (2005). Corrosion rate of steel in concrete-Measurements beyond the tafel law. Corrosion Science, 47(12), p. 3019.

Ergun, M., \& Turan, A. Y. (1991). Pitting potential and protection potential of carbon steel for chloride ion and the effectiveness of different inhibiting anions. Corrosion Science, 32(10), p. 1137.

Evans, U. R. (1981). An Introduction to Metallic Corrosion (3 ${ }^{\text {rd }}$ ed.). Edward Arnold Publishers.

Evans, U. R., Bannister, L. C., \& Britton, S. C. (1931). The velocity of corrosion from the electrochemical standpoint. Proceedings of the Royal Society of London: Series A Containing Papers of a Mathematical and Physical Character, p. 355.

Fairley, N., \& Carrick, A. (2005). The casa cookbook: Recipes for XPS data processing. Acolyte science.

Feliu, V., González, J. A., Andrade, C., \& Feliu, S. (1998). Equivalent circuit for modelling the steel-concrete interface. I. experimental evidence and theoretical predictions. Corrosion Science, 40(6), p. 975. 
FHWA-RD-01-156 (2001). Corrosion Costs and Preventive Strategies in the United States. Federal Highway Administration (FHWA), Office of Infrastructure Research and Development.

Foley, C. L., Kruger, J., \& Bechtoldt, C. J. (1967). Electron diffraction studies of active, passive, and transpassive oxide films formed on iron. Journal of the Electrochemical Society, 114, 994.

Fontana, M. G. (1986). Corrosion engineering, McGraw Hill.

Frankenthal, R. P., \& Kruger, J., Eds. (1978). Passivity of metals. $4^{\text {th }}$ International Symposium on Passivity, Electrochemical Society, Warrenton, US.

Frederiksen, J. M. (2000). Method for determination of chloride threshold values for steel in concrete. Nordtest Report, TR500.

Freiman, L., \& Kolotyrkin, Y. (1969). Iron passivation potential and solution composition. Protection of Metals, 5(2), p.113.

Fromhold Jr, A. T., \& Kruger, J. (1973). Space-Charge and Concentration-Gradient effects on anodic oxide film formation. Journal of the Electrochemical Society, 120 , p. 722 .

Fujii, T., De Groot, F. M. F., Sawatzky, G. A., Voogt, F. C., Hibma, T., \& Okada, K. (1999). In situ XPS analysis of various iron oxide films grown by $\mathrm{NO}_{2}$-assisted molecular-beam epitaxy. Physical Review-Series B, 59, p. 3195.

Galicia, P., Batina, N., \& Gonzalez, I. (2006). The relationship between the surface composition and electrical properties of corrosion films formed on carbon steel in alkaline sour medium: An XPS and EIS study. The Journal of Physical Chemistry B, $110(29)$, p. 14398.

Galvele, J. R. (1978). In Passivity of metals. Eds: Frankenthal, R. P. \& Krager, J., Electrochemical Society, New Jersey, p. 285. 
Garcés, P., Andrade, M. C., Saez, A., \& Alonso, M. C. (2005). Corrosion of reinforcing steel in neutral and acid solutions simulating the electrolytic environments in the micropores of concrete in the propagation period. Corrosion Science, 47(2), p. 289.

Ghods, P., Isgor, O. B., \& Pour-Ghaz, M. (2007). A practical method for calculating the corrosion rate of uniformly depassivated reinforcing bars in concrete. Materials and Corrosion, 58(4), p. 265.

Ghods, P., Isgor, O. B., \& Pour-Ghaz, M. (2008). Experimental verification and application of a practical corrosion model for uniformly depassivated steel in concrete. Materials and Structures, 41(7), p. 1211.

Ghods, P., Isgor, O. B., McRae, G., \& Miller, T. (2009). The effect of concrete pore solution composition on the quality of passive oxide films on black steel reinforcement. Cement and Concrete Composites, 31(1), p. 2.

Glass, G. K., \& Buenfeld, N. R. (1997). The presentation of the chloride threshold level for corrosion of steel in concrete. Corrosion Science, 39(5), p.1001.

Glass, G. K., \& Buenfeld, N. R. (2000a). The inhibitive effects of electrochemical treatment applied to steel in concrete. Corrosion Science, 42(6), p. 923.

Glass, G. K., \& Buenfeld, N. R. (2000b). The influence of chloride binding on the chloride induced corrosion risk in reinforced concrete. Corrosion Science, 42(2), p. 329.

Goetz, R., MacDougall, B., \& Graham, M. J. (1986). An AES and SIMS study of the influence of chloride on the passive oxide film on iron. Electrochim.Acta, 31(10), p. 1299.

Goni, S., \& Andrade, C. (1990). Synthetic concrete pore solution chemistry and rebar corrosion rate in the presence of chlorides. Cement and Concrete Research, 20(4), p. 525. 
Gonzalez, J. A., Otero, E., Feliu, S., \& Lopez, W. (1993). Initial steps of corrosion in the steel/Ca(OH)ZCl system: The role of heterogeneities on the steel surface and oxygen supply. Cement and Concrete Research, 23(1), p. 33.

Gouda, V. K. (1970). Corrosion and corrosion inhibition of reinforcing steel: 1Immersion in alkaline solution. British Corrosion Journal, 5, p. 198.

Graham, M. J., Bardwell, J., Goetz, R., Mitchell, D. F., \& Macdougall, B. (1990). Composition and growth of anodic oxide films on iron. Corrosion Science., 31, p. 139.

Grosvenor, A. P., Kobe, B. A., Biesinger, M. C., \& McIntyre, N. S. (2004b). Investigation of multiplet splitting of Fe $2 p$ XPS spectra and bonding in iron compounds. Surface and Interface Analysis, 36(12), p. 1564.

Grosvenor, A. P., Kobe, B. A., McIntyre, N. S., Tougaard, S., \& Lennard, W. N. (2004a). Use of QUASESTM/XPS measurements to determine the oxide composition and thickness on an iron substrate. Surface and Interface Analysis, 36(7), p. 632.

Gu, P., Beaudoin, J. J., Tumidajski, P. J., \& Mailvaganam, N. P. (1997). Electrochemical incompatibility of patches in reinforced concrete. Concrete International, 19, p. 68.

Gui, J., \& Devine, T. M. (1994). The influence of sulfate ions on the surface enhanced raman spectra of passive films formed on iron. Corrosion Science, 36(3), p. 441.

Hansson, C. M., \& Sorensen, B. (1990). The threshold concentration of chloride in concrete for the initiation of reinforcement corrosion. Corrosion Rates of Steel in Concrete, Eds: Berke, N. S., Chaker, V., Whiting, D., p. 3.

Hansson, C. M., Frolund, T., \& Markussen, J. B. (1985). The effect of chloride cation type on the corrosion of steel in concrete by chloride salts. Cement and Concrete Research, 15(1), p. 65. 
Haupt, S., \& Strehblow, H. H. (1987). Corrosion, layer formation, and oxide reduction of passive iron in alkaline solution: A combined electrochemical and surface analytical study. Langmuir, 3(6), p. 873.

Haupt, S., Calinski, C., Collisi, U., Hoppe, H. W., Speckmann, H. D., \& Strehblow, H. H. (1986). XPS and ISS examinations of electrode surfaces and passive layers with a specimen transfer in a closed system. Surface and Interface Analysis, 9(6), p. 357.

Hausmann, D. A. (1967). Steel corrosion in concrete--how does it occur. Materials Protection, 6(11), p. 19.

Hausmann, D.A. (2007). Three Myths About Corrosion of Steel in Concrete, NACE Materials Performance Magazine, August issue, p. 70.

Heusler, K. E. (1995). Fundamental aspects of the passivation of metals and semiconductors. Materials Science Forum, vol. 185-188, p.9.

Heusler, K. E., \& Fischer, L. (1976). Kinetics of pit initiation at passive iron. Materials and Corrosion, 27(8), p. 551.

Hoar, T. P. (1967). The production and breakdown of the passivity of metals. Corrosion Science, $7(6)$, p.341.

Hoar, T. P., Mears, D. C., \& Rothwell, G. P. (1965). The relationships between anodic passivity, brightening and pitting. Corrosion Science, 5, p. 279.

Holliday, J. E., \& Frankenthal, R. P. (1972). Characterization of passivating films on Fe$\mathrm{Cr}$ alloys by soft X-ray spectroscopy. Journal of the Electrochemical Society, 119(9), p. 1190.

Horne, A. T., Richardson, I. G., \& Brydson, R. M. D. (2007). Quantitative analysis of the microstructure of interfaces in steel reinforced concrete. Cement and Concrete Research, 37(12), p.1613. 
Hugot-Le Goff, A., Flis, J., Boucherit, N., Joiret, S., \& Wilinski, J. (1990). Use of Raman spectroscopy and rotating split ring disk electrode for identification of surface layers on iron in 1M NaOH. Journal of the Electrochemical Society, 137, p. 2684.

Hussain, S. E., \& Al-Gahtani, A. S. (1996). Chloride threshold for corrosion of reinforcement in concrete. ACI Materials Journal, 93(6), p. 534.

Hussain, S. E., Rasheeduzzafar, , Al-Musallam, A., \& Al-Gahtani, A. S. (1995). Factors affecting threshold chloride for reinforcement corrosion in concrete. Cement and Concrete Research, 25(7), p. 1543.

Isgor, O. B., \& Razaqpur, A. G. (2006). Advanced modelling of concrete deterioration due to reinforcement corrosion. Canadian Journal of Civil Engineering, 33(6), 707.

Izquierdo, D., Alonso, C., Andrade, C., \& Castellote, M. (2004). Potentiostatic determination of chloride threshold values for rebar depassivation experimental and statistical study. Electrochimica Acta, 49(17-18), p. 2731.

Jäggi S, Elsener B, Böhni H. (2000). Oxygen reduction on mild steel and stainless steel in alkaline solutions. Corrosion of Reinforcement in Concrete-Corrosion Mechanisms and Corrosion Protection, Eds: Mietz J, Polder R, Elsener B., European Federation Publication, EFC 2000; 31, p. 3.

Jain, B. K., Singh, A. K., Chandra, K., \& Saraswat, I. P. (1977). Mössbauer studies of corroded steels. Japanese Journal of Applied Physics, 16, p. 2121.

Janik-Czachor, M. (1981). An assessment of the processes leading to pit nucleation on iron. Journal of the Electrochemical Society, 128(12), p. 513C.

Joiret, S., Keddam, M., Nóvoa, X. R., Pérez, M. C., Rangel, C., \& Takenouti, H. (2002). Use of EIS, ring-disk electrode, EQCM and Raman spectroscopy to study the film of oxides formed on iron in $1 \mathrm{M} \mathrm{NaOH}$. Cement and Concrete Composites, 24(1), p. 75.

Jones, D. A. (1995). Principles and prevention of corrosion ( $2^{\text {nd }}$ ed.). Prentice Hall. 
Kamrunnahar, M., Bao, J. E., \& Macdonald, D. D. (2005). Challenges in the theory of electron transfer at passive interfaces. Corrosion Science, 47(12), p. 3111.

Karadakis, K. (2009). Numerical investigation of the chemistry of the pore solution in the mill scale crevices of carbon steel rebar. M.Sc.Thesis., Carleton University.

Kayyali, O. A., \& Haque, M. N. (1995). The $\mathrm{Cl}^{-} / \mathrm{OH}^{-}$ratio in chloride-contaminated concrete-A most important criterion. Magazine of Concrete Research, 47, p. 235.

Khalil, W., Haupt, S., \& Strehblow, H. H. (1985). The thinning of the passive layer of iron by halides. Materials and Corrosion, 36(1), p. 16.

Kim, K. S., Baitinger, W. E., Amy, J. W. \& Winograd, N. (1974). ESCA studies of metal-oxygen surface using argon and oxygen ion bombardment. Electron Spectroscopy and Related Phenomena, 5, p.351.

Kolotyrkin, J. M. (1961). Effects of anions on the dissolution kinetics of metals. Journal of the Electrochemical Society, 108, p. 209.

Kruger, J. (1976). Passivity and its breakdown on iron and iron base alloys. USA-Japan Seminar, NACE, Houston TX, p. 91.

Kruger, J. (1988). Passivity of metals-A materials perspective. International Materials Reviews, 33, p.113.

Kruger, J. (1989). Nature of the passive film on iron and ferrous alloys. Corrosion Science, $29(2)$, p.149.

Kruger, J. and Rhyne, K. (1981). Nuclear Chemistry Waste Management. 3, p.205.

Kruger, J., \& Calvert, J. P. (1967). Ellipsometric-Potentiostatic studies of iron passivity. Journal of the Electrochemical Society, 114, p. 43.

Kuroda, K., Cahan, B. D., Nazri, G., Yeager, E., \& Mitchell, T. E. (1982). Electron diffraction study of the passive film on iron. Journal of the Electrochemical Society, 129, p. 2163 . 
Lambert, P., Page, C. L., \& Vassie, P. R. W. (1991). Investigations of reinforcement corrosion. 2. electrochemical monitoring of steel in chloride-contaminated concrete. Materials and Structures, 24(5), p. 351.

Landolt, D., Mischler, S., Vogel, A., \& Mathieu, H. J. (1990). Chloride ion effects on passive films on FeCr and FeCrMo studied by AES, XPS AND SIMS. Corrosion Science, 31, p. 431.

Leckie, H. P. (1970). A contribution to the applicability of critical pitting potentials. Journal of the Electrochemical Society, 117, p. 1152.

Leckie, H. P., \& Uhlig, H. H. (1966). Environmental factors affecting the critical potential for pitting in 18-8 stainless steel. Journal of the Electrochemical Society, 113, p. 1262.

Li, L., \& Sagues, A. A. (2001). Chloride corrosion threshold of reinforcing steel in alkaline solutions: open-circuit immersion tests. Corrosion, 57(1), p.19.

Li, L., \& Sagues, A. A. (2002). Chloride corrosion threshold of reinforcing steel in alkaline solutions- cyclic polarization behaviour. Corrosion, 58(4), p. 305.

Li, L., \& Sagues, A. A. (2004). Chloride corrosion threshold of reinforcing steel in alkaline Solutions-Effect of specimen size. Corrosion, 60(2), p. 195.

Lin, L. F., Chao, C. Y., \& Macdonald, D. D. (1981). A point defect model for anodic passive films II. chemical breakdown and pit initiation. Journal of the Electrochemical Society, 128, p. 1194.

Macdonald, D. D. (1992). The point defect model for the passive state. Journal of the Electrochemical Society, 139, p. 3434.

Macdonald, D. D., \& Urquidi-Macdonald, M. (1990). Deterministic models for passivity breakdown. Corrosion Science, 31, p. 425. 
Macias, A., \& Andrade, C. (1983). Corrosion rate of galvanized steel immersed in saturated solutions of $\mathrm{Ca}(\mathrm{OH})_{2}$ in the $\mathrm{pH}$ range 12-13. 8. British Corrossion Journal, $18(2)$, p. 82.

Mahallati, E., \& Saremi, M. (2006). An assessment on the mill scale effects on the electrochemical characteristics of steel bars in concrete under DC-polarization. Cement and Concrete Research, 36(7), p. 1324.

Mammoliti, L. (1995). Evaluation of corrosion inhibitors in synthetic concrete pore solutions. M.Sc. Thesis, Queen's University, Kingston, Ontario, Canada..

Mammoliti, L., \& Hansson, C. M. (2005). Influence of cation on corrosion behavior of reinforcing steel in high-pH sulfate solutions. ACI Materials Journal, 102(4), p. 279.

Mammoliti, L., Brown, L., Hansson, C., \& Hope, B. (1996). The influence of surface finish of reinforcing steel and $\mathrm{pH}$ of the test solution on the chloride threshold concentration for corrosion initiation in synthetic pore solutions. Cement and Concrete Research, 26(4), 545-550.

Mangat, P. S., \& Molloy, B. T. (1992). Factors influencing chloride-induced corrosion of reinforcement in concrete. Materials and Structures, 25(7), p. 404.

Mansfeld, F. (1976). The polarization resistance technique for measuring corrosion currents. Advances in Corrosion Science and Technology, 6, p. 163.

Marcotte, T. D. (2001). Characterization of chloride-induced corrosion products that form in steel-reinforced cementitious materials. Ph.D. Dissertation, University of Waterloo, Canada.

McBee, C. L., \& Kruger, J. (1972). Nature of passive films on iron-chromium alloys. Electrochem.Acta, 17, p. 1337.

McIntyre, N. S., \& Zetaruk, D. G. (1977). X-ray photoelectron spectroscopic studies of iron oxides. Analytical Chemistry, 49(11), p. 1521. 
Mehta, P. K., \& Monteiro, P. J. M. (2005). Concrete: microstructure, properties, and Materials ( $3^{\text {rd }}$ ed.), McGraw-Hill.

Miserque, F., Huet, B., Azou, G., Bendjaballah, D., \& Hostis, V. L. (2006). X-ray photoelectron spectroscopy and electrochemical studies of mild steel FeE500 passivation in concrete simulated water. Journal of Physics, 136, 89-97.

Mohammed, T. U., \& Hamada, H. (2001). A discussion of the paper "Chloride threshold values to depassivate reinforcing bars embedded in a standardized OPC mortar" by C. Alonso, C. Andrade, M. Castellote, and P. Castro. Cement and Concrete Research, 31(5), p. 835.

Mohammed, T. U., \& Hamada, H. (2006). Corrosion of steel bars in concrete with various steel surface conditions. ACI Materials Journal, 103(4), p. 233.

Montemor, M. F., Simoes, A. M. P., \& Ferreira, M. G. S. (1998). Analytical characterization of the passive film formed on steel in solutions simulating the concrete interstitial electrolyte. Corrosion, 54(5), p. 347.

Monticelli, C., Frignani, A., \& Trabanelli, G. (2000). A study on corrosion inhibitors for concrete application. Cement and Concrete Research, 30(4), p. 635.

Moragues, A., Macias, A., \& Andrade, C. (1987). Equilibria of the chemical composition of concrete pore solution: I, comparative study of synthetic and extracted solutions. Cement and Concrete Research, 17(2), p. 173.

Moreno, M., Morris, W., Alvarez, M. G., \& Duffó, G. S. (2004). Corrosion of reinforcing steel in simulated concrete pore solutions: effect of carbonation and chloride content. Corrosion Science, 46(11), p. 2681.

NACE International. (2002) Corrosion costs and preventive strategies in the United States. Report FHWA-RD-01-156. available at: http://events.nace.org/publicaffairs /images_cocorr/ccsupp.pdf(accessed 10 September 2009). 
National Institute of Standards and Technology, Gaithersburg. (2003). NIST X-ray photoelectron spectroscopy database.

Neville, A. M. (1996). Properties of concrete ( $4^{\text {th }}$ ed.), John Wiley \& Sons Inc.

Nmai, C. K. (2004). Multi-functional organic corrosion inhibitor. Cement and Concrete Composites, 26(3), p. 199.

Oh, B. H., Jang, B. S., \& Lee, S. C. (2004). Chloride diffusion and corrosion initiation time of reinforced concrete structures. Proceedings of the International Workshop on Microstructure and Durability to Predict Service Life of Concrete Structures, Japan.

Oh, B. H., Jang, S. Y., \& Shin, Y. S. (2003). Experimental investigation of the threshold chloride concentration for corrosion initiation in reinforced concrete structures. Magazine of Concrete Research, 55(2), p. 117.

Olefjord, I., \& Brox, B. \& Froment, M. (1983). Passivity of metals and semiconductors. Elsevier Science Publishers, Amsterdam.

Ord, J. L., \& De Smet, D. J. (1976). The anodic oxidation of iron: Overpotential analysis for a Two-Phase film. Journal of the Electrochemical Society, 123, 1876.

Page, C. L. (2002). Advances in understanding and techniques for controlling reinforcement corrosion. ICC $15^{\text {Th }}$ International Corrosion Congress, Granada, Spain, p.22.

Page, C. L., \& Vennesland, Ø. (1983). Pore solution composition and chloride binding capacity of silica-fume cement pastes. Materials and Structures, 16(1), p. 19.

Page, C. L., Lambert, P., \& Vassie, P. R. W. (1991). Investigations of reinforcement corrosion. 1. the pore electrolyte phase in chloride-contaminated concrete. Materials and Structures, 24(4), p. 243.

Pettersson, K. (1993). Chloride threshold value and the corrosion rate in reinforced concrete. Proceedings of Concrete 2000, Vol. 1, E\&FN Spon, p. 461. 
Pettersson, K. (1995). Chloride threshold value and the corrosion rate in reinforced concrete. Cement and Concrete Research, 24, p. 461.

Pettersson, K. (1996a). Service life of concrete Structures in saline environment. CBI Report, 3 .

Pettersson, K. (1996b). Factors influencing chloride induced corrosion of reinforcement in concrete. Durability of Building Materials and Components, 1, p. 334.

Pettersson, K., \& Sandberg, P. (1997). Chloride threshold levels and corrosion rates in cracked high performance concrete exposed in a marine environment. The $4^{\text {th }}$ CANMET/ACI International Conference on Durability of Concrete, Sydney, Australia.

Pillai, R. G., \& Trejo, D. (2005). Surface condition effects on critical chloride threshold of steel reinforcement. ACI Materials Journal, 102(2), p. 103.

Poter, B., Parezanovic, I., \& Spiegel, M. (2005). In-situ scanning electron microscopy and electron backscatter diffraction investigation on the oxidation of pure iron. Materials at High Temperatures, 22, 3(4), p. 185.

Pou, T. E., Murphy, O. J., Young, V., Bockris, J. O. M., \& Tongson, L. L. (1984). Passive films on iron: The mechanism of breakdown in chloride containing solutions. Journal of the Electrochemical Society, 131, p. 1243.

Poupard, O., Ait-Mokjtar, A., \& Dumargue, P. (2004). Corrosion by chlorides in reinforced concrete: Determination of chloride concentration threshold by impedance spectroscopy. Cement and Concrete Research, 34, p. 991.

Pourbaix, M. (1973). Lectures on electrochemical corrosion. Plenum Publishing Corporation, New York.

Pourbaix, M. (1974). Atlas of electrochemical equilibria in aqueous solutions, NACE, Houston, TX. 
Pour-Ghaz, M., Isgor, O. B., \& Ghods, P. (2009). Quantitative interpretation of half-cell potential measurements in concrete structures. Journal of Materials in Civil Engineering, 21, p. 467.

Pour-Ghaz, M., Isgor, O. B., \& Ghods, P. (2009). The effect of temperature on the corrosion of steel in concrete. part 1: Simulated polarization resistance tests and model development. Corrosion Science, 51(2), 415-425.

Poursaee, A. (2007). An analysis of the factors influencing electrochemical measurements of the condition of reinforcing steel in concrete structures. $P h D$ Dissertation, University of Waterloo, Canada.

Poursaee, A., \& Hansson, C. M. (2007). Reinforcing steel passivation in mortar and pore solution. Cement and Concrete Research, 37(7), p. 1127.

Rajagopalan, K. S., Venu, K., \& Balakrishnan, K. (1962). Anodic polarization studies in neutral and alkaline solutions containing corrosion inhibitors. I. $\mathrm{NaOH}-\mathrm{NaCl}$ system. Journal of the Electrochemical Society, 109(2), p. 81.

Raktoe, B. L., Hedayat, A., \& Federer, W. T. (1981). Factorial designs. Wiley, New York.

Ramachandran, V. S., \& Beaudoin, J. J. (2001). Handbook of analytical techniques in concrete science and technology, William Andrew Publications

Ramachandran, V. S., Feldman, R. F., \& Beaudoin, J. J. (1981). Concrete science: Treatise on current research, Heyden.

Rasheeduzzafar, H., Hussain, S. E., \& Al-Saadoun, S. S. (1992). Effect of tricalcium aluminate content of cement on chloride binding and corrosion of reinforcing steel in concrete. ACI Materials Journal, 89(1), p. 3.

Rasheeduzzafar, S., Hussain, S. E., \& A1-Saadoun, S. S. (1991). Effect of cement composition on chloride binding and corrosion of reinforcing steel in concrete. Cement and Concrete Research, 21, p.771. 
Refait, P., \& Genin, J. M. R. (1993). The oxidation of ferrous hydroxide in chloridecontaining aqueous media and pourbaix diagrams of green rust one. Corrosion Science, 34(5), p. 797.

Refait, P., Abdelmoula, M., \& Genin, J. M. R. (1998). Mechanisms of formation and structure of green rust one in aqueous corrosion of iron in the presence of chloride ions. Corrosion Science, 40(9), p. 1547.

Rehm, G., Nürnberger, U., \& Fret, R. (1981). Zur korrosion und Spannungsrisßkorrosion von spannstählen bei bauwerken mit nachträglichem verbund. Bauingenieur, 56, p. 275.

Revie, R. W. (2000). Uhlig's corrosion handbook ( $2^{\text {nd }}$ ed.). John Wiley \& Sons Inc.

Revie, R. W., \& Uhlig, H. H. (2008). Corrosion and corrosion control: an Introduction to Corrosion Science and Engineering ( $2^{\text {nd }}$ ed.). John Wiley \& Sons.

Richardson, J. A., \& Wood, G. C. (1970). A study of the pitting corrosion of A1 by scanning electron microscopy. Corrosion Science, 10, p. 313.

Rondelli, G., Lazzari, L., Ormellese, M., Rovoa, R., Perez, E., (2006). Study of carbon steel corrosion inhibition in alkaline solution by means of EIS. Proc. Int. Conf. Passivation of Metals, and Properties of Thin Oxide Layers, Eds. Marcus, P., Maurice, V., Paris, p. 709.

Rubin, J. (1983). Transport of Reacting Solutes in Porous media: Relation between Mathematical Nature of Problem Formulation and Chemical Nature of Reactions. Water Resources Research, 19(5), p. 1231.

Sagues, A. A., Li, L., \& Pickering, H. W. (2000). Crevice effect on corrosion of steel in simulated concrete pore solutions. Corrosion, 56(10), p. 979.

Sakashita, M., \& Sato, N. (1978). Bipolar fixed charge-induced passivity, Passivity of metals, eds. by Frankenthal, R. P. \& Kruger, J., Electrochemical Society, Pennington, NJ, p. 479. 
Samson, E., and Marchand, J. (1999). Numerical Solution of the Extended Nernst-Planck Model. Journal of Colloid and Interface Science, 215, p. 1.

Samson, E., Marchand, J., and Beaudoin, J. J. (1999). Describing ion diffusion mechanisms in cement-based materials using the homogenization technique. Cement and Concrete Research, 29, p. 1341.

Sandberg, P., Pettersson, K., Sorensen, H. E., Arup, H., Nilsson, L. O., \& Ollivier, J. P. (1995). Critical chloride concentrations for the onset of active reinforcement corrosion. RILEM International Workshop on Chloride Penetration into Concrete, Paris, p. 459.

Saremi, M., \& Mahallati, E. (2002). A study on chloride-induced depassivation of mild steel in simulated concrete pore solution. Cement and Concrete Research, 32(12), p.1921.

Sarver, J. M., \& Szklarska-Smialowska, Z. (1984a). A study of the formation and breakdown of passive films on iron in alkaline $\mathrm{Na}_{2} \mathrm{SO}_{4}$ solutions. Proceedings of Electrochemical Society, 84, p. 357.

Sarver, J. M., \& Szklarska-Smialowska, Z. (1984b). A study on the formation and breakdown of passive films on iron in alkaline solutions. Localized Corrosion: Proceedings of an International Symposium Honoring Professor Marcel Pourbaix on His Eightieth Birthday, 84, p. 328.

Sato, N. (1971). Theory for breakdown of anodic oxide films on metals. Electrochim Acta, 16(10), p. 1683.

Sato, N., \& Cohen, M. (1964). The kinetics of anodic oxidation of iron in neutral solution. Journal of the Electrochemical Society, 111, p. 519.

Sato, N., \& Notoya, T. (1967). Measurement of the anodic oxide film growth on iron for hours. Journal of the Electrochemical Society, 114, p. 585. 
Sato, N., \& Okamoto, G. (1981). Comprehensive treatise of electrochemistry. Vol. 4, New York, NY, Plenum Press.

Schiessl. P., \& Raupach M. (1988). Chloridinduzierte Korrosion von Stahl im Beton, Beton-Informationen, , 28, p.33

Schiess1. P., \& Raupach M. (1990). Einfluß der Betonzusammensetzung und der Umgebungsbedingungen auf die chloridinduzierte Korrosion von Stahl im Beton, Beton-Informationen, 30, p. 43.

Schmuki, P., Büchler, M., Virtanen, S., Isaacs, H. S., Ryan, M. P., \& Böhni, H. (1999). Passivity of iron in alkaline solutions studied by in situ XANES and a laser reflection technique. Journal of the Electrochemical Society, 146(6), p. 2097.

Schultze W. \& Stimming U. (1975). Zeitschrift für Physikalische Chemie. NF, 98, p.285.

Schweitzer, P. A. (1989). Corrosion and corrosion protection handbook ( $2^{\text {nd }}$ ed.). CRC Press.

Seah, M. P., \& Dench, W. A. (1979). Quantitative electron spectroscopy of surfaces: A standard data base for electron inelastic mean free paths in solids. Surface and Interface Analysis, 1(1), p. 1.

Siegel, S., \& Castellani Jr, N. J. (1988). Nonparametric statistics, MacGraw-Hill, New York, NY.

Strehblow, H. H., \& Titze, B. (1977). Pitting potential and inhibition potentials of iron and nickel for different aggressive and inhibiting anions. Corrosion Science, 17(6), p. 461 .

Suetaka, W., \& Yates, J. T. (1995). Method of Surface Characterization, Vol.3: Surface infrared and Raman spectroscopy: Methods and applications. Plenum Press, New York.

Tait, W. S., Handrich, K. A., Tait, S. W., \& Martin, J. W. (1993). Analyzing and interpreting electrochemical impedance spectroscopy data from internally coated 
steel aerosol containers. Electrochemical Impedance Analysis and Interpretation: ASTM STP 1188, Eds.: Scully, J. C., Silverman, D. C., Kendig,M. W., p. 428.

Thomas, M. (1996). Chloride thresholds in marine concrete. Cement and Concrete Research, 26(4), p. 513.

Tjong, S. C. (1985). TEM study of the passive film on iron-chromium alloys. Journal of Materials Science Letters, 4(1), p. 6.

Trabanelli, G., Monticelli, C., Grassi, V., \& Frignani, A. (2005). Electrochemical study on inhibitors of rebar corrosion in carbonated concrete. Cement and Concrete Research, 35(9), p. 1804.

Trejo, D., \& Pillai, R. G. (2003). Accelerated chloride threshold testing: Part I - ASTM A 615 and A 706 reinforcement. ACI Materials Journal, 100(6), p. 519.

Tritthart, J. (1989). Chloride binding in cement. I. investigations to determine the composition of porewater in hardened cement. Cement and Concrete Research, 19(4), p. 586.

Tuutti, K. (1982). Corrosion of Steel in Concrete. Swedish Cement and Concrete Institute (CIB), n $4-82$, Stockholm, Sweden.

Venu, K., Balakrishnan, K., \& Rajagopalan, K. S. (1965). A potentiokinetic polarization study of the behaviour of steel in $\mathrm{NaOH}-\mathrm{NaCl}$ system. Corrosion Science, 5, p. 59.

Vetter K. S. (1958). Dicke und Aufbau von passivierenden Oxydschichten auf Eisen. Z. Elektrochem, 62, p. 642.

Williams, D. B., \& Carter, C. B. (2009). Transmission electron microscopy: A textbook for materials science. Vol.4, Springer.

Yonezawa, T., Ashworth, V., \& Procter, R. P. M. (1988). Pore solution composition and chloride effects on the corrosion of steel in concrete, Corrosion Engineering, 44(7), p. 489 . 
Zakroczymski, T., Fan, C. J., \& Szklarska-Smialowska, Z. (1985a). Kinetics and mechanism of passive film formation on iron in $0.05 \mathrm{M} \mathrm{NaOH}$. Journal of the Electrochemical Society, 132, p. 2862.

Zakroczymski, T., Fan, C. J., \& Szklarska-Smialowska, Z. (1985b). Passive film formation on iron and film breakdown in a sodium hydroxide solution containing chloride ions. Journal of the Electrochemical Society, 132, p. 2868.

Zimmermann, L. (2000). Korrosionsinitiierender Chloridgehalt Von Stahl in Beton. Ph.D. Dissertation, ETH Nr. 13870, ETH Zürich.

Zivica, V., Krajci, L., Bagel, L., \& Vargova, M. (1997). Significance of the ambient temperature and the steel material in the process of concrete reinforcement corrosion. Construction and Building Materials, 11(2), p.99. 


\section{Appendix A}

\section{Supplementary TEM Figures}

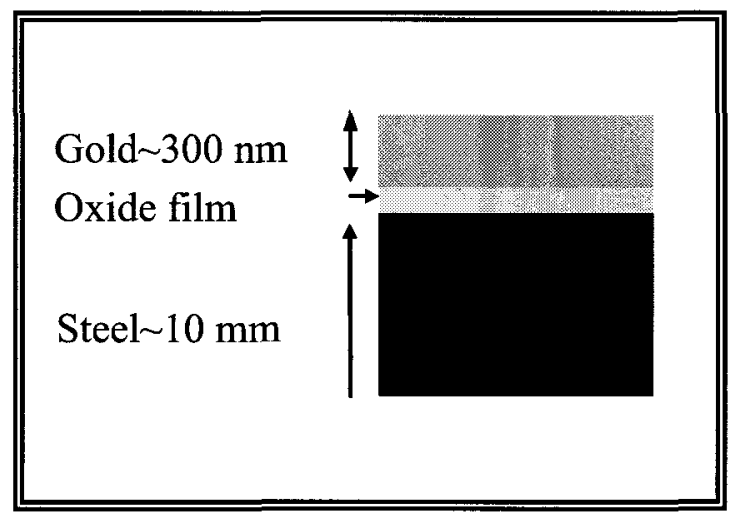

(a)

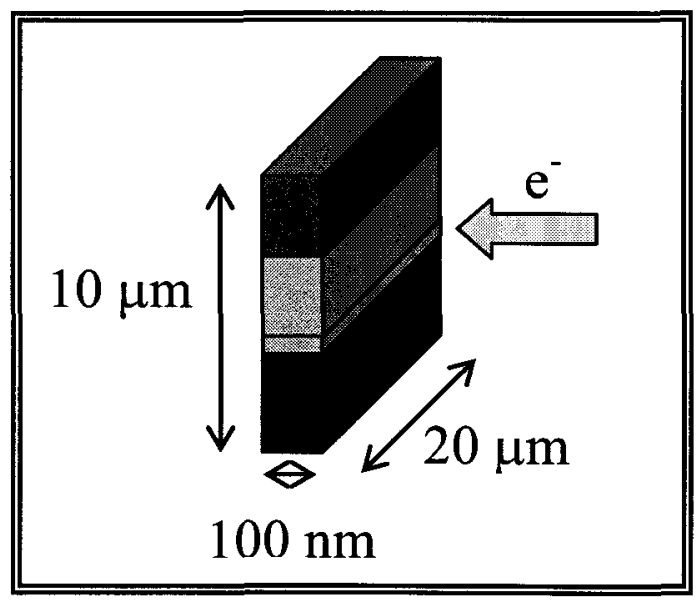

(c)

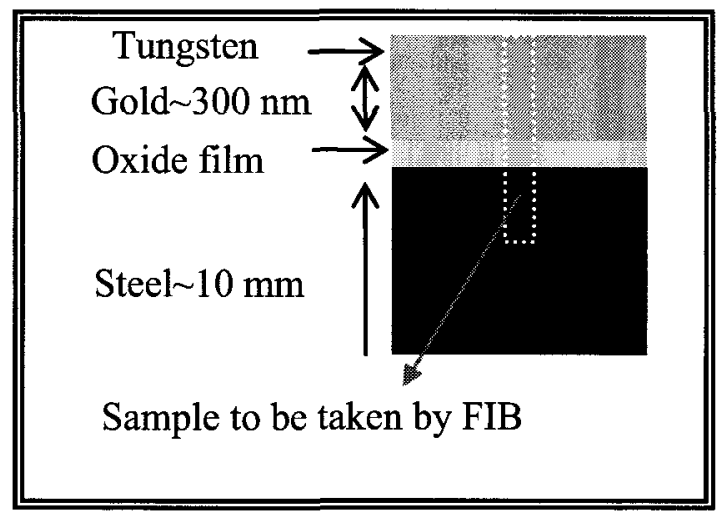

(b)

Figure A.1: Schematic illustration of TEM sample preparation procedure carried out in this study using FIB technique: a) gold coated, cross-sectional view b) Tungsten sputtered, cross-sectional view c)TEM sample taken from the bulk specimen, a 3-D view (Not to scale) 


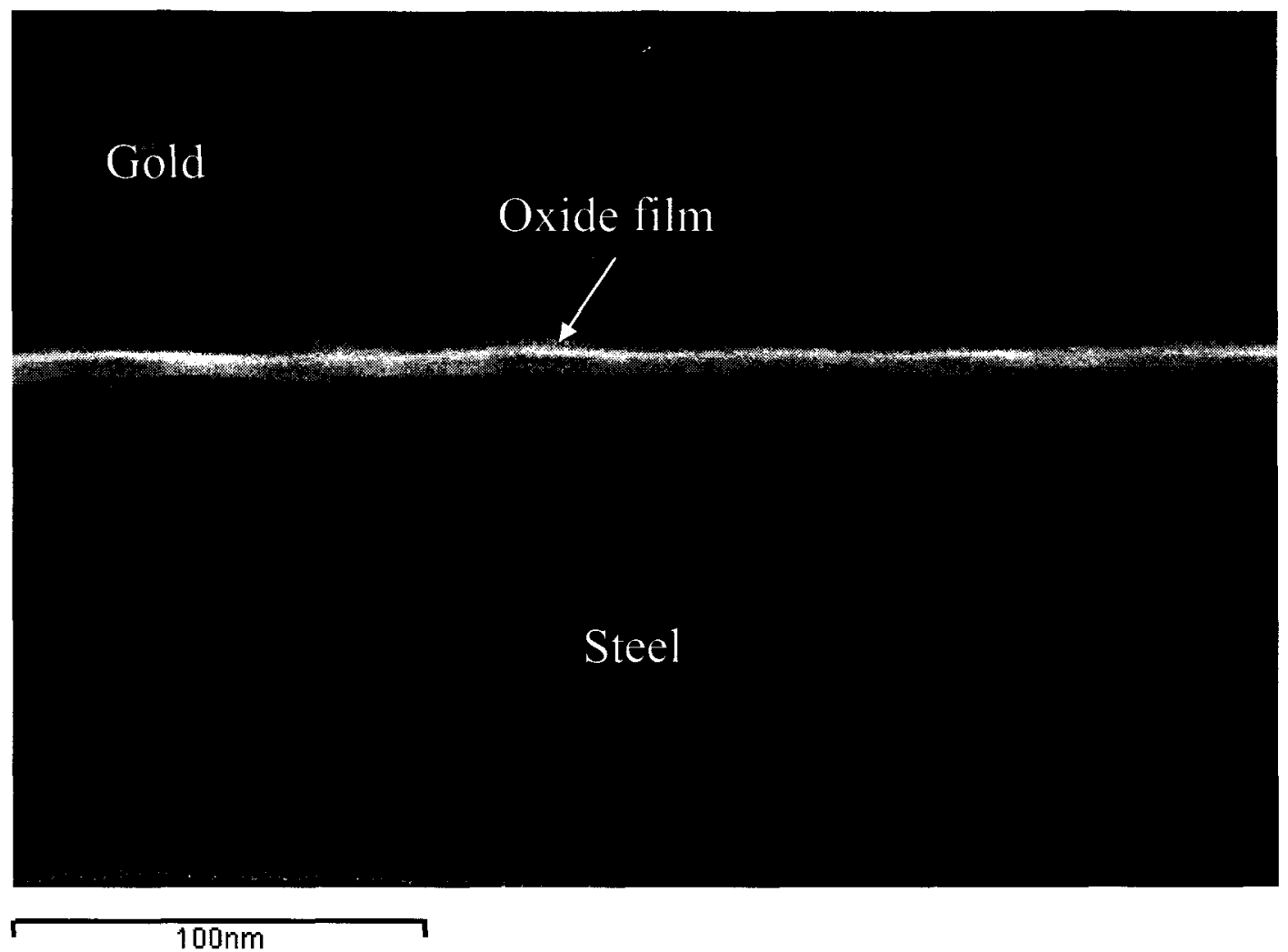

(a)

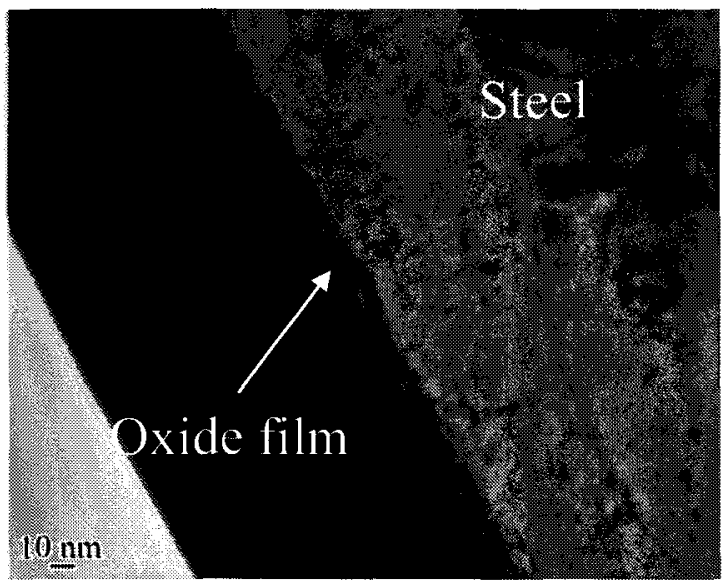

(b)

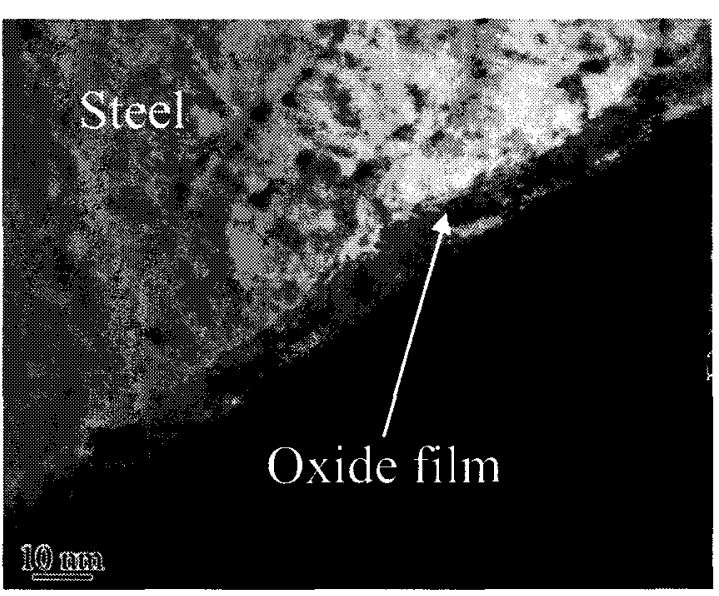

(c)

Figure A.2: TEM images of oxide film in the $\mathrm{CH}$ solution (CH-0): a) a digital TEM micrograph; b) a low magnification TEM micrograph; c) a high magnification TEM micrograph. 


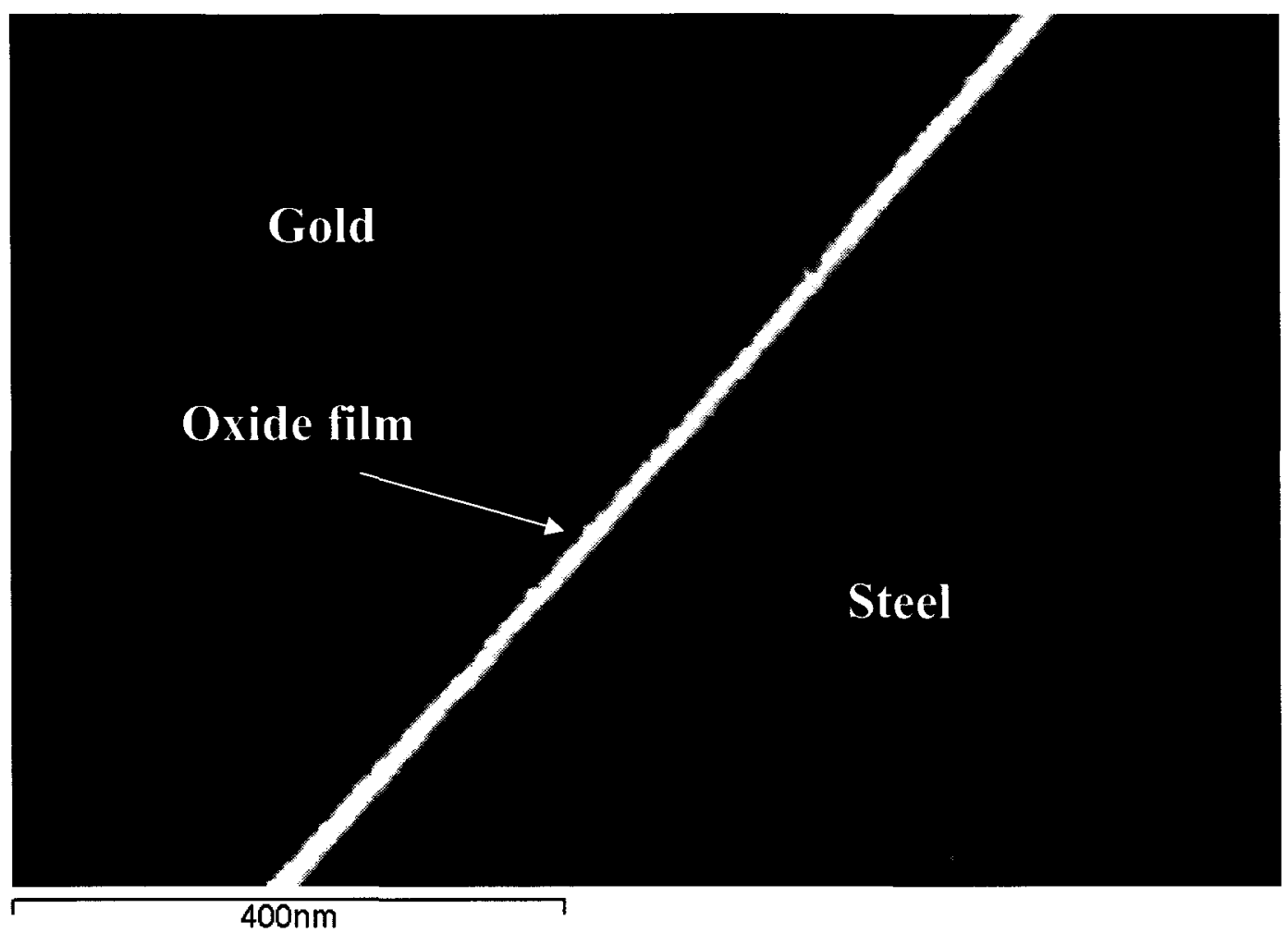

(a)

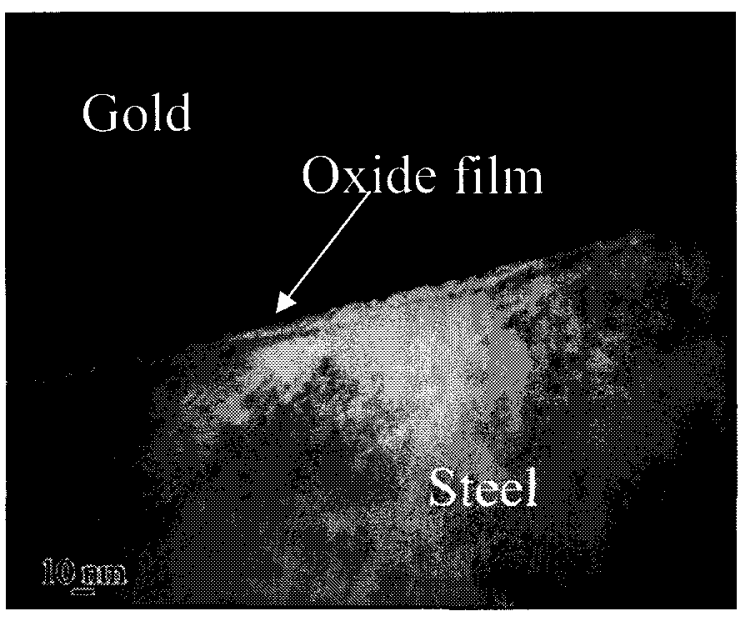

(b)

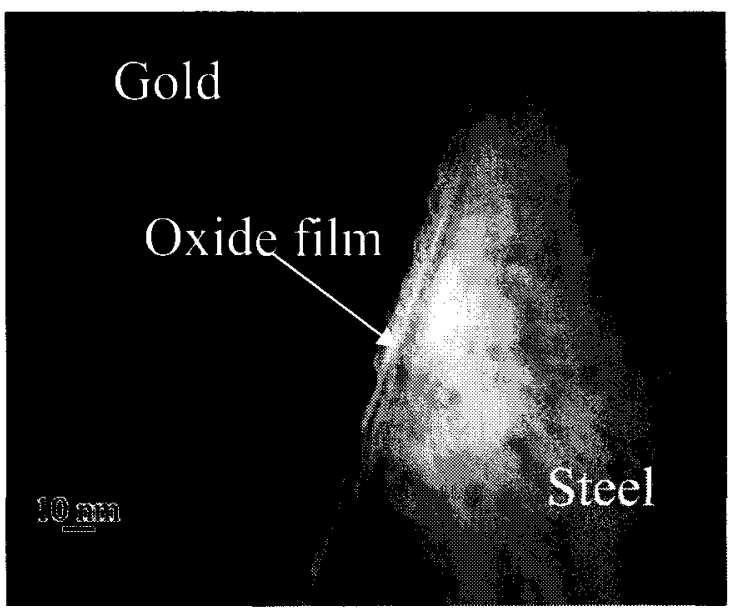

(c)

Figure A.3: TEM images of oxide film in the CP solution (CP-0): a) a digital TEM micrograph; b) a low magnification TEM micrograph c) a high magnification TEM micrograph. 


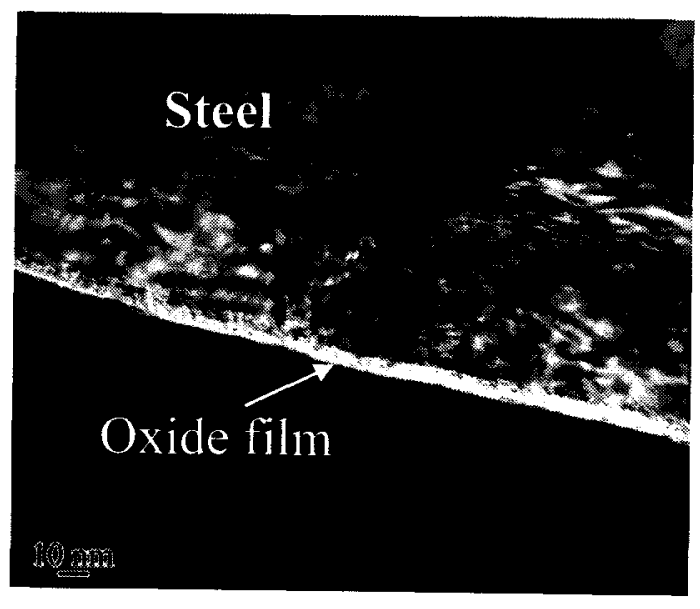

(a)

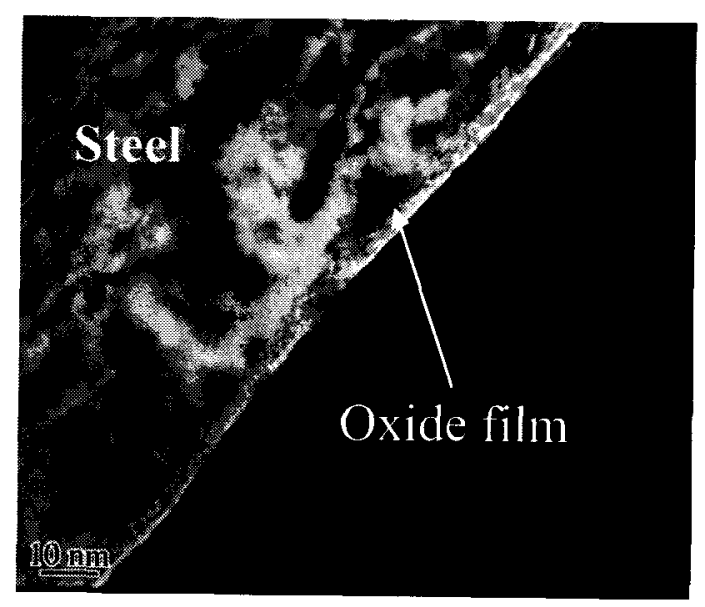

(b)

Figure A.4: TEM images of the CH-1 sample: (a) a low magnification TEM micrograph; (b) a high magnification TEM micrograph. 


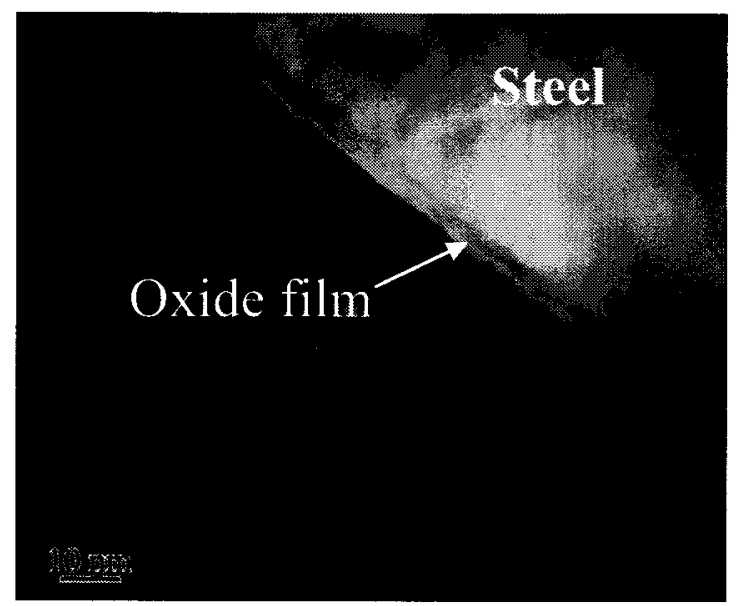

(a)

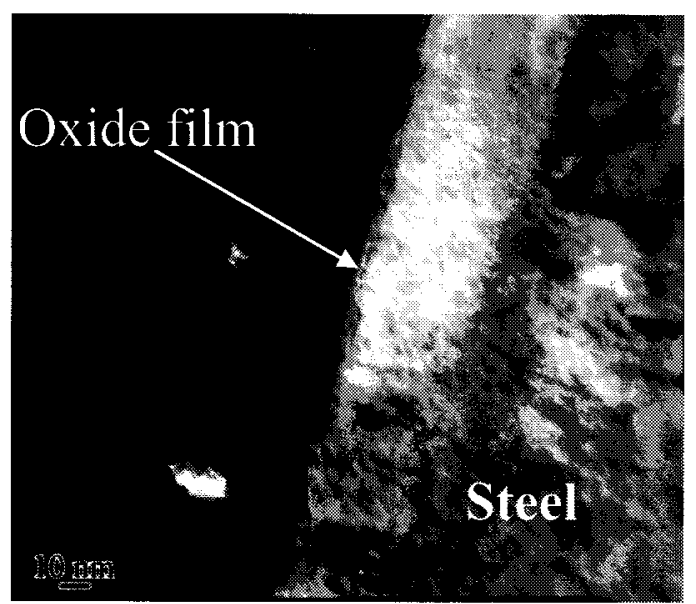

(b)

Figure A.5: TEM images of the CP-1 sample: (a) low magnification TEM micrograph; (b) high magnification TEM micrograph. 


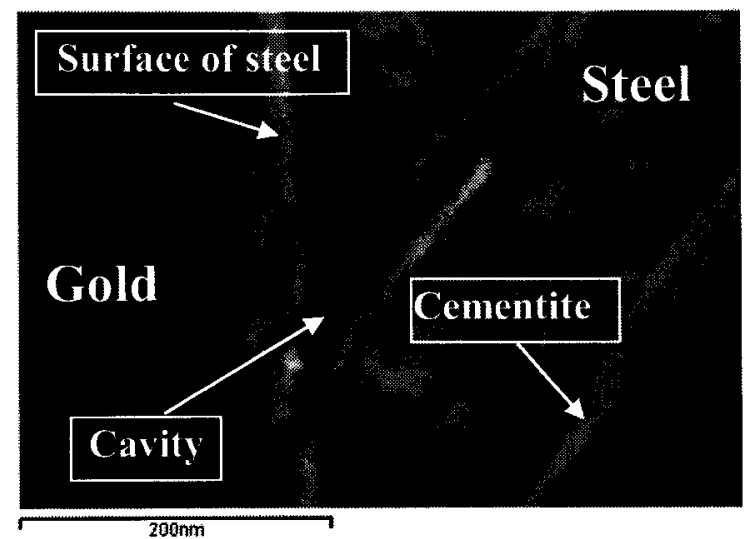

(a)

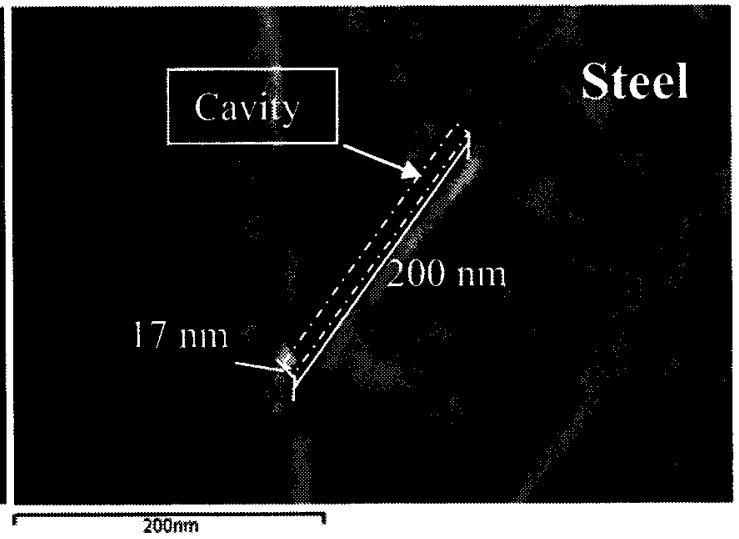

(b)

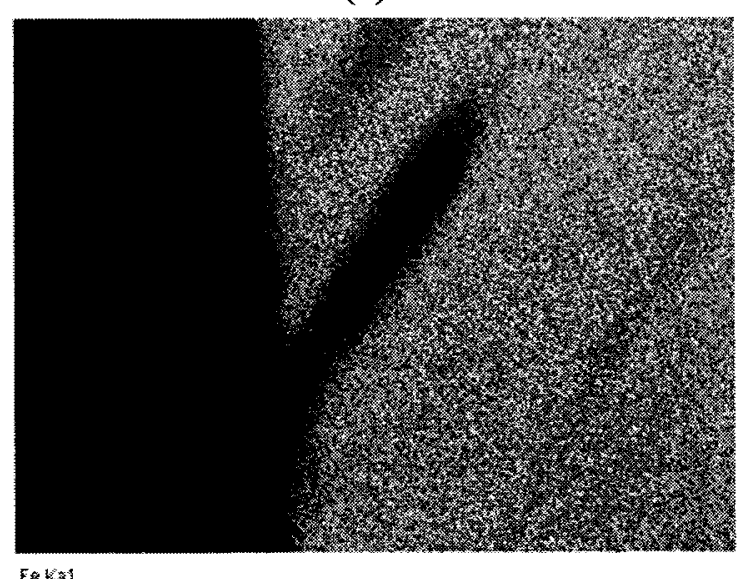

(c)

Figure A.6: TEM images of the steel near the surface for the CP-2 sample: a) a cavity (void) on the surface of steel along the openings of cementite lqmellae; b) dimensions of the cavity (void); c) Fe ka map of Fig. A.6 (a). 


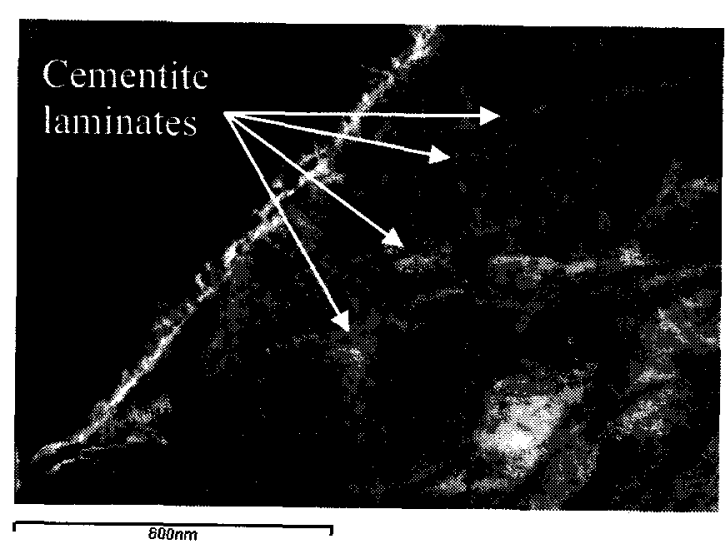

(a)

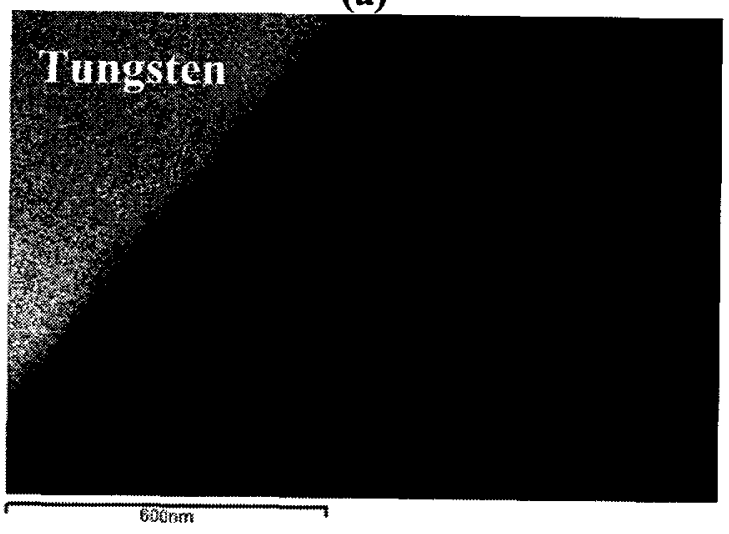

(c)

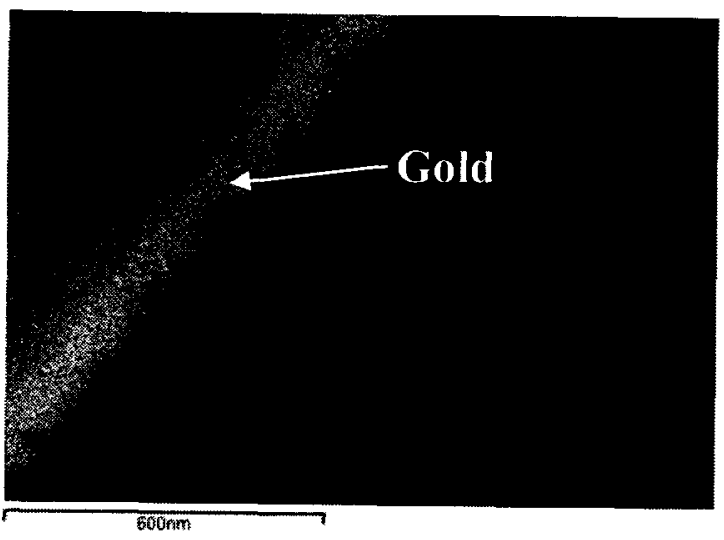

(b)

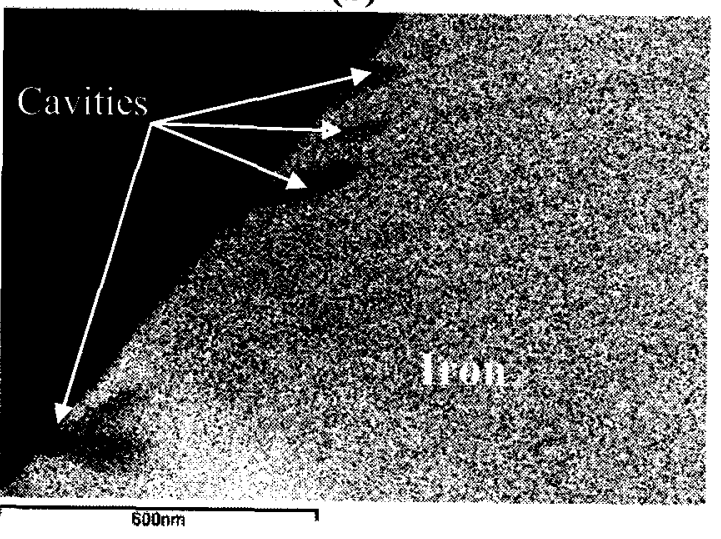

(d)

Figure A.7: Steel near the surface for the CP-2 sample: a) a TEM micrograph showing the cementite lamellae on steel; b) Gold (Au) map of Fig. A.7(a); c) Tungsten (W)map of Fig. A.7(a); d) Iron (Fe) map of Fig. A.7(a). 


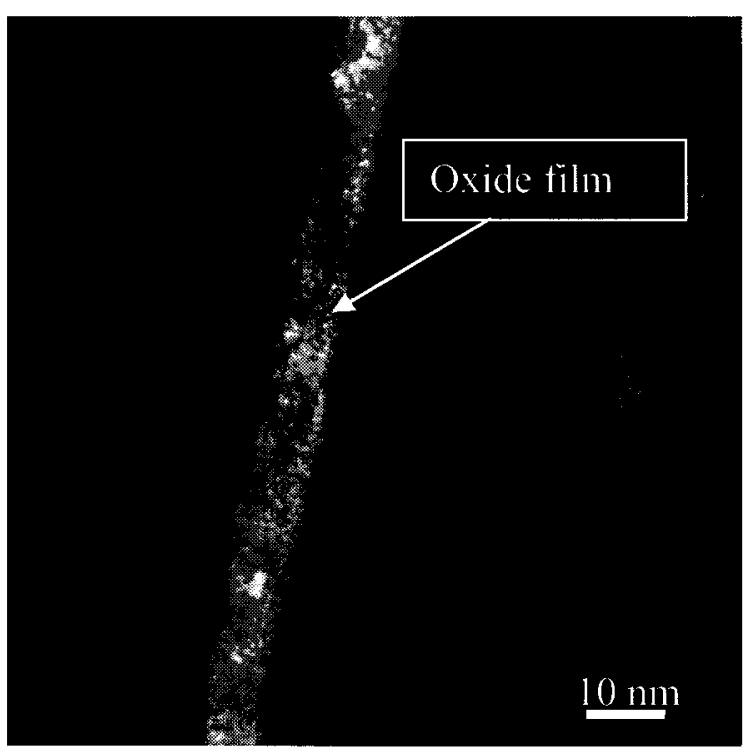

(a)

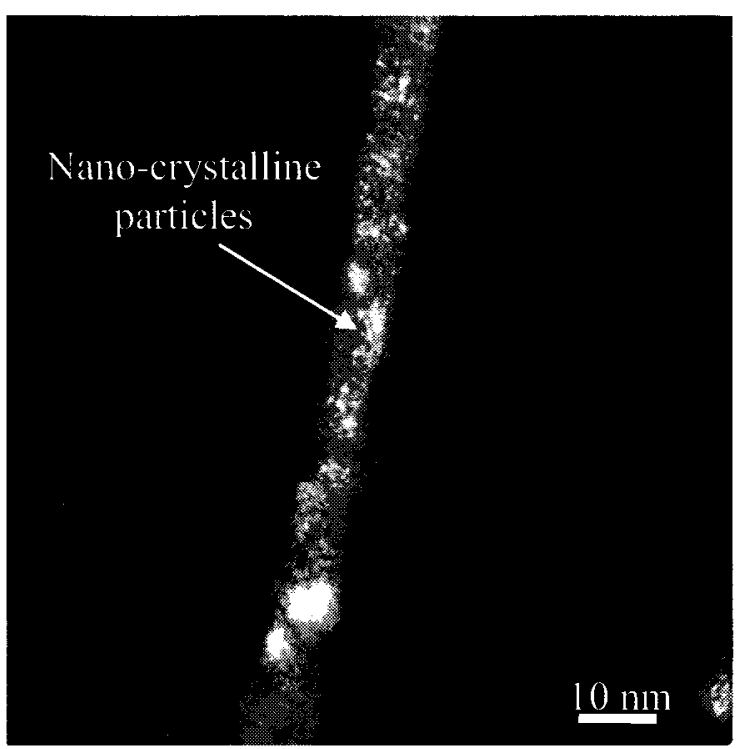

(b)

Figure A.8: TEM dark-field images of the CH-0 sample: a) a single exposure dark-field micrograph; b) a multi-exposure dark-field micrograph. The bright phases indicate the incorporation of nano-crystalline particles in the oxide film. 


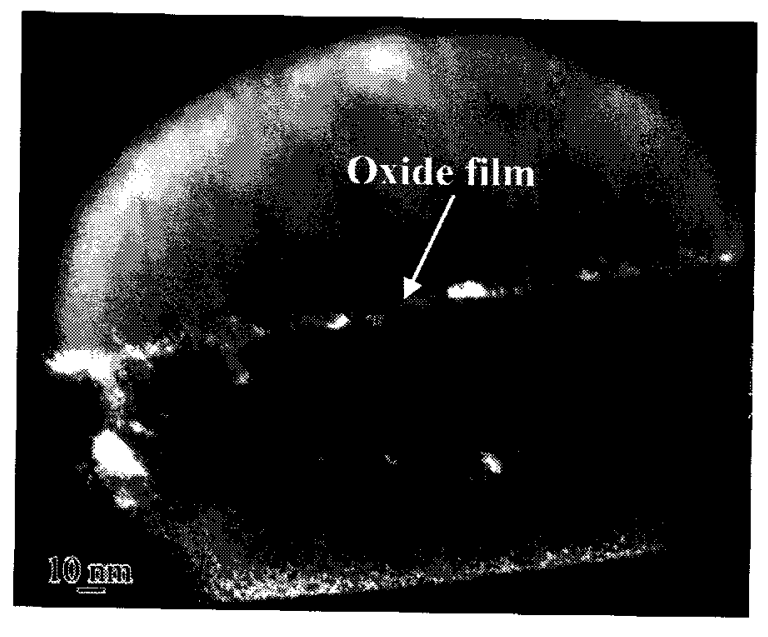

(a)

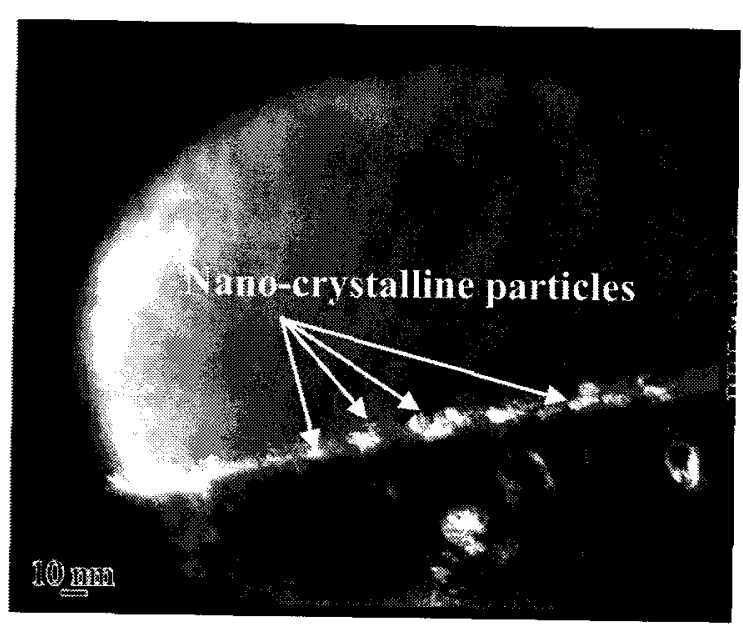

(b)

Figure A.9: TEM dark-field images of the CP-0 sample: a) a single exposure dark-field micrograph; b) a multi-exposure dark-field micrograph. The bright phases indicate the incorporation of nano-crystalline particles in the oxide film. 


\section{Appendix B}

\section{Supplementary XPS Results}

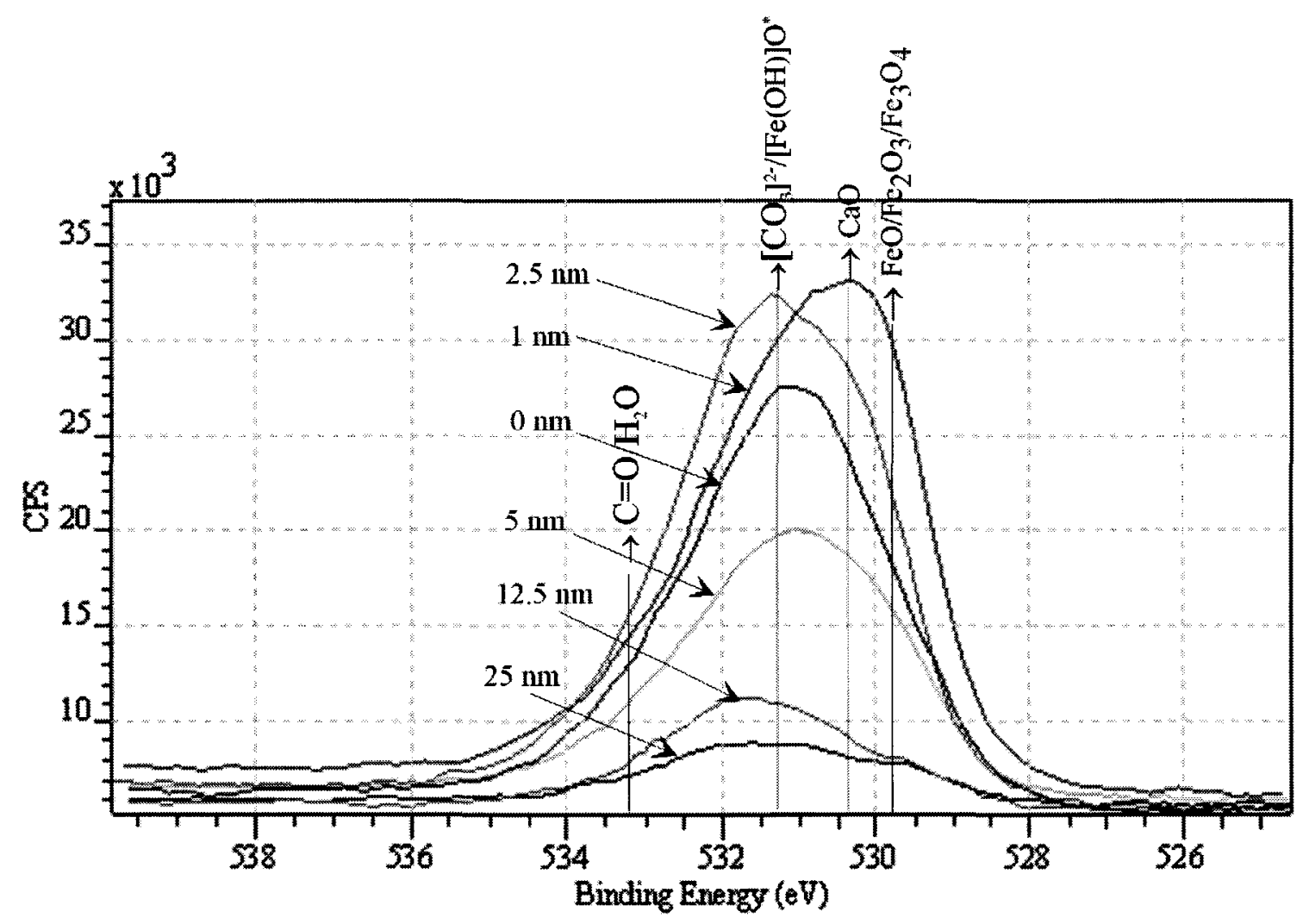

Figure B.1: O 1s high resolution XPS spectra at selected sputtered depths for AE specimen and the binding energy position of various oxygen-incorporated compounds that are present in the spectra 


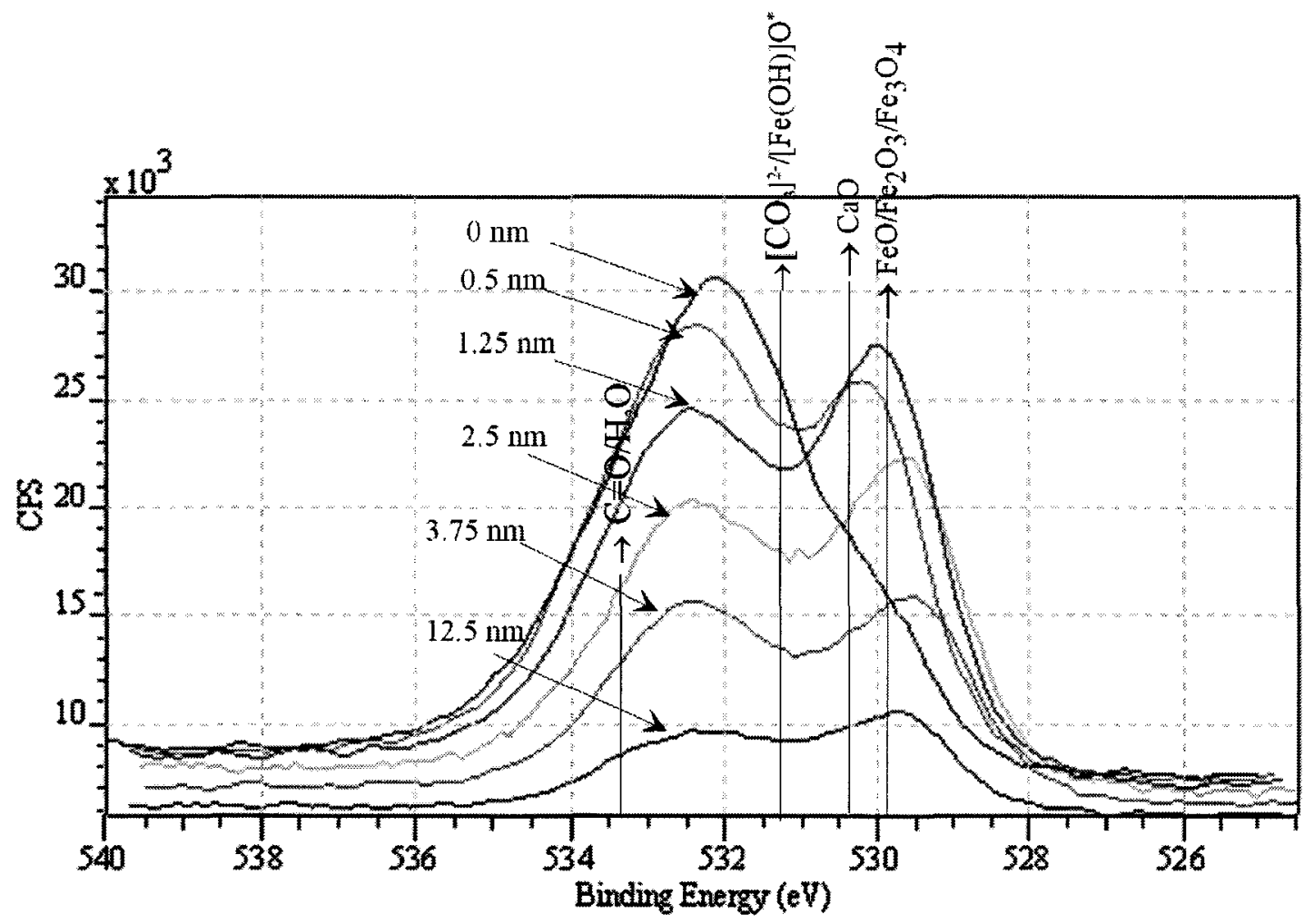

Figure B.2: O 1s high resolution XPS spectra at selected sputtered depths for $\mathrm{CH}-2$ specimen and the binding energy position of various oxygen-incorporated compounds that are present in the spectra. 


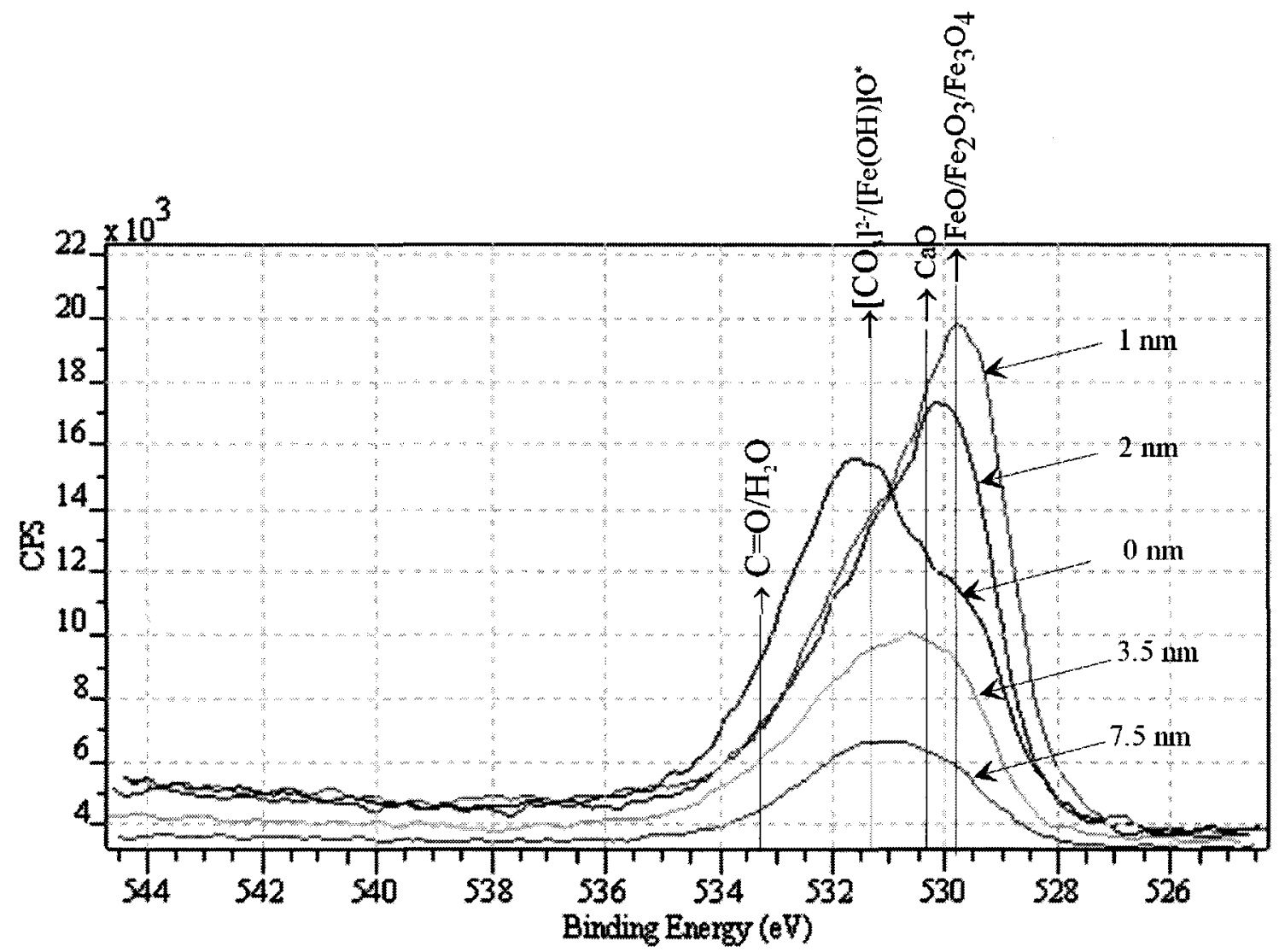

Figure B.3: O 1s high resolution XPS spectra at selected sputtered depths for $\mathrm{CH}-9$ specimen and the binding energy position of various oxygen-incorporated compounds that are present in the spectra. 


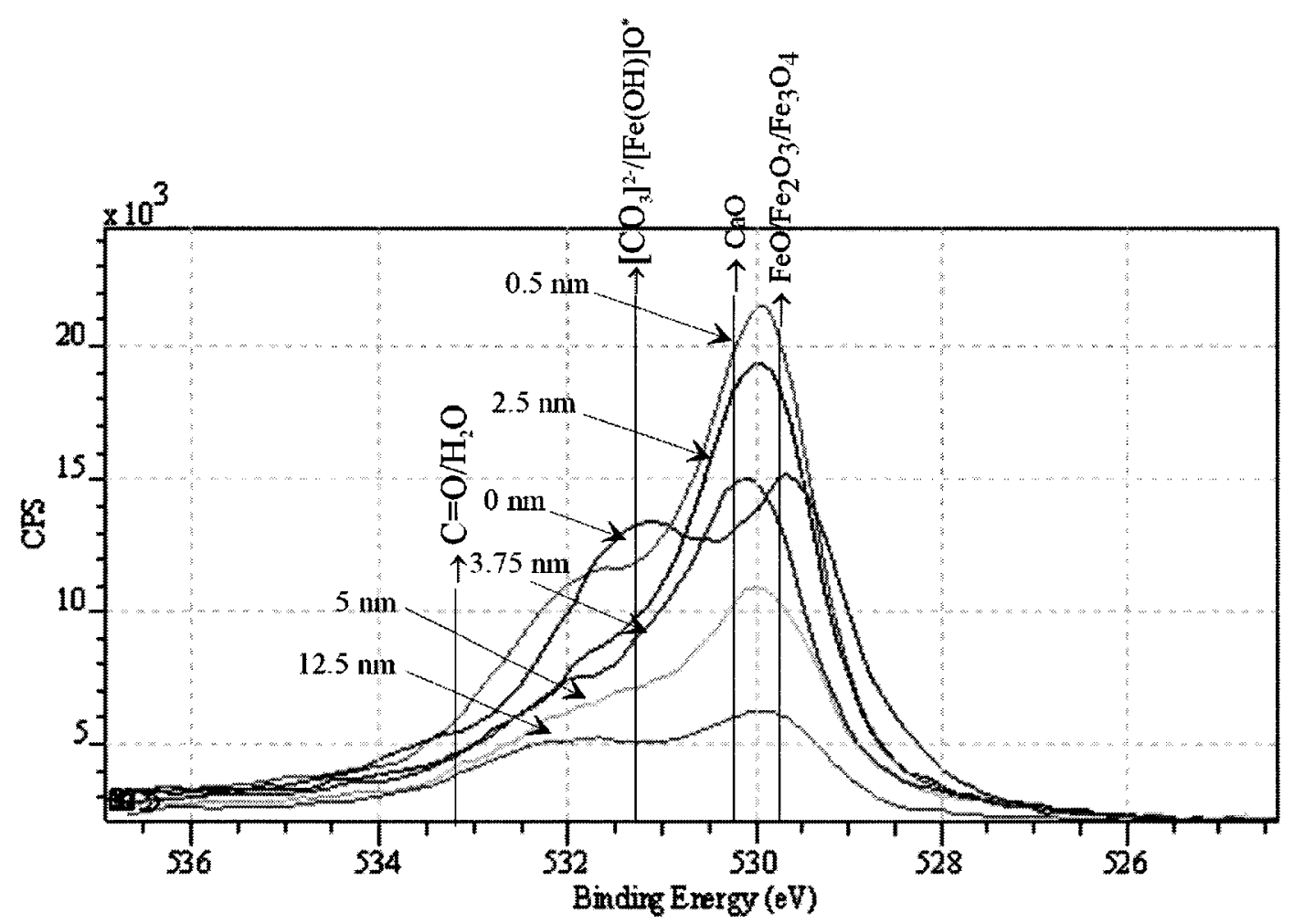

Figure B.4: O 1s high resolution XPS spectra at selected sputtered depths for $\mathrm{CH}-\mathrm{Cl}$ specimen and the binding energy position of various oxygen-incorporated compounds that are present in the spectra. 


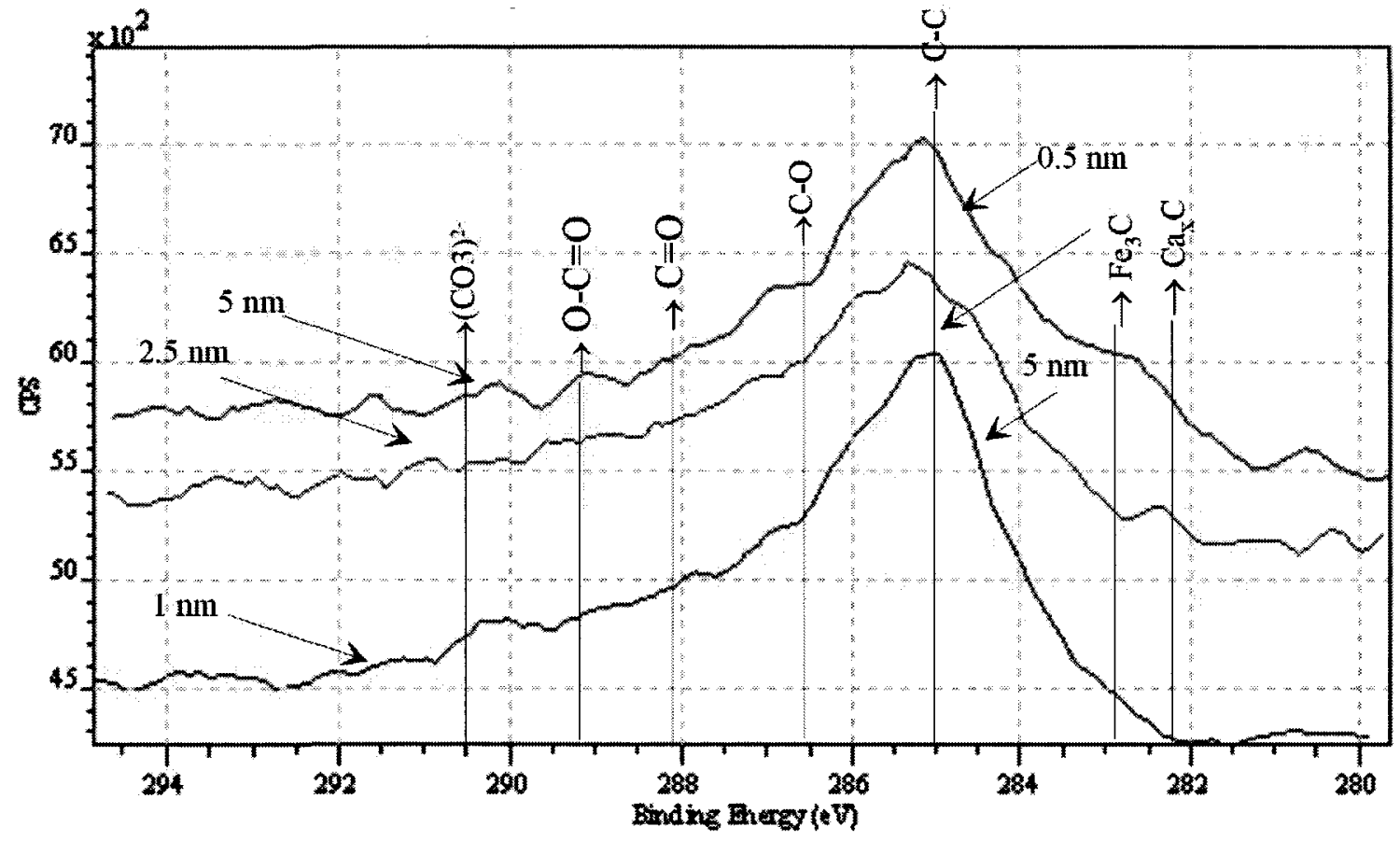

Figure B.5: C 1s high resolution XPS spectra at selected sputtered depths for AE specimen and the binding energy position of various carbon-incorporated compounds that are present in the spectra. 


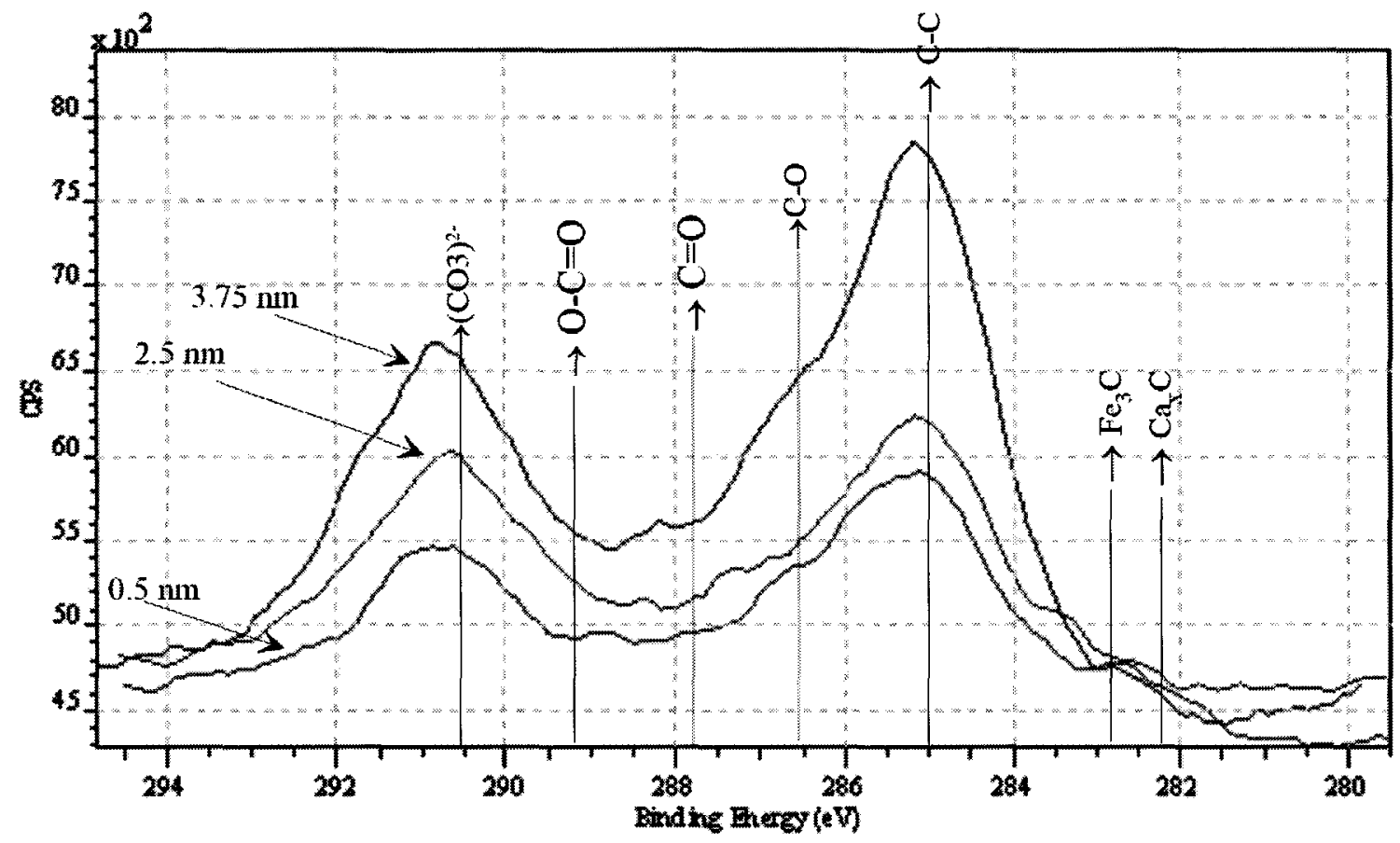

Figure B.6: C 1 s high resolution XPS spectra at selected sputtered depths for $\mathrm{CH}-2$ specimen and the binding energy position of various carbon-incorporated compounds that are present in the spectra. 


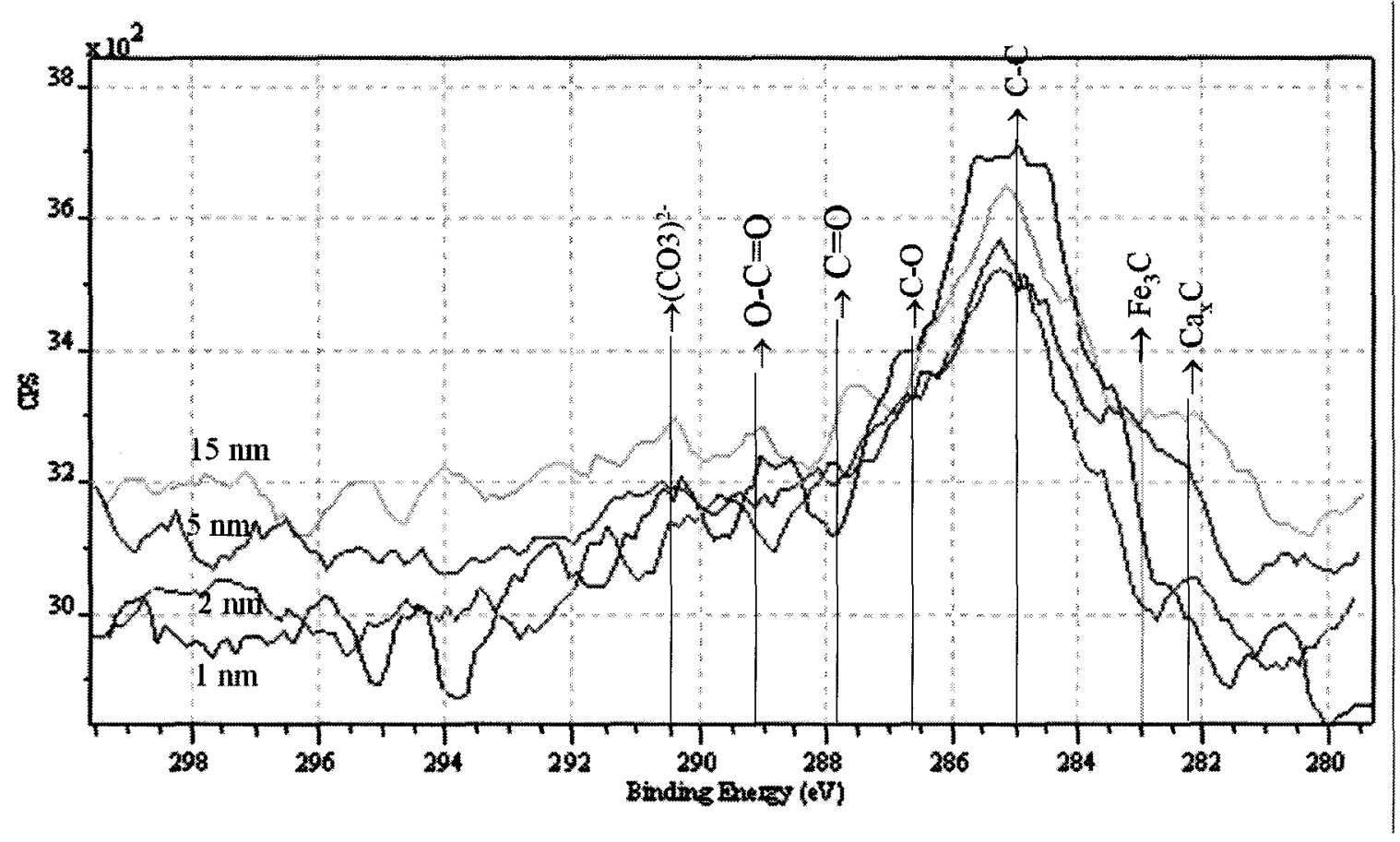

Figure B.7: C 1s high resolution XPS spectra at selected sputtered depths for $\mathrm{CH}-9$ specimen and the binding energy position of various carbon-incorporated compounds that are present in the spectra. 


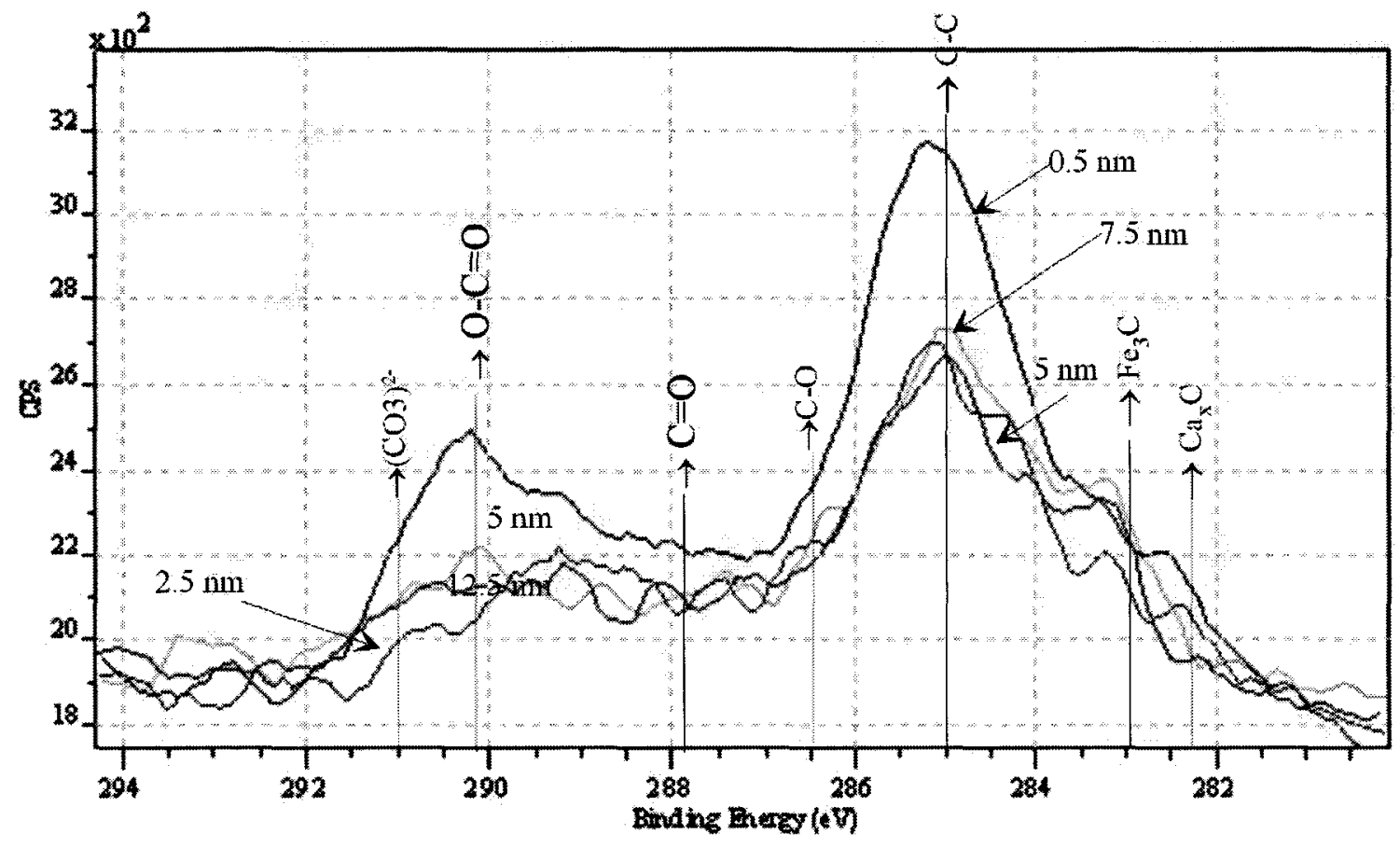

Figure B.8: C 1s high resolution XPS spectra at selected sputtered depths for $\mathrm{CH}-\mathrm{Cl}$ specimen and the binding energy position of various carbon-incorporated compounds that are present in the spectra. 


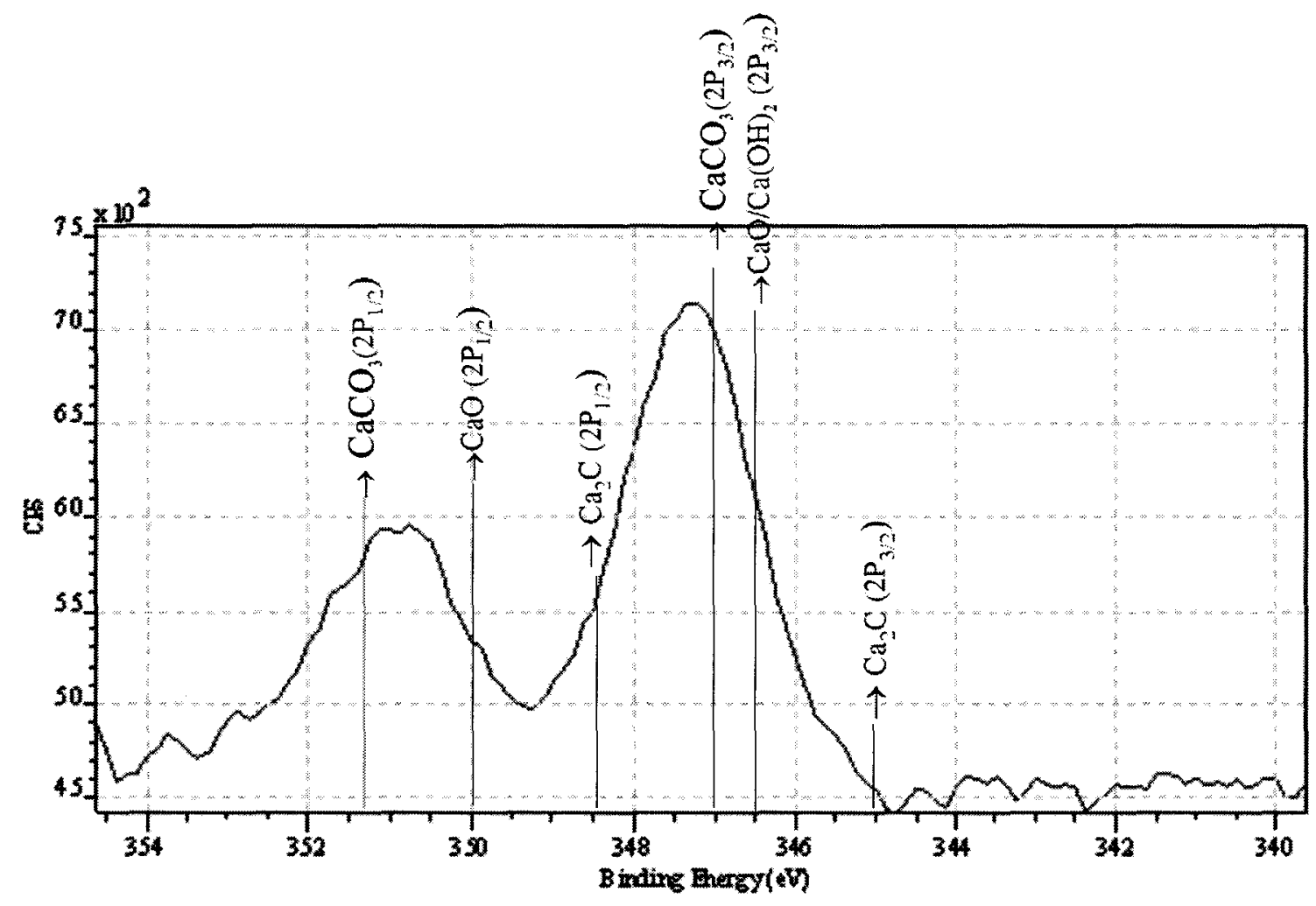

Figure B.9: Ca 2p high resolution XPS spectra at selected sputtered depths for AE specimen and the binding energy position of various calcium-incorporated compounds that are present in the spectra. 


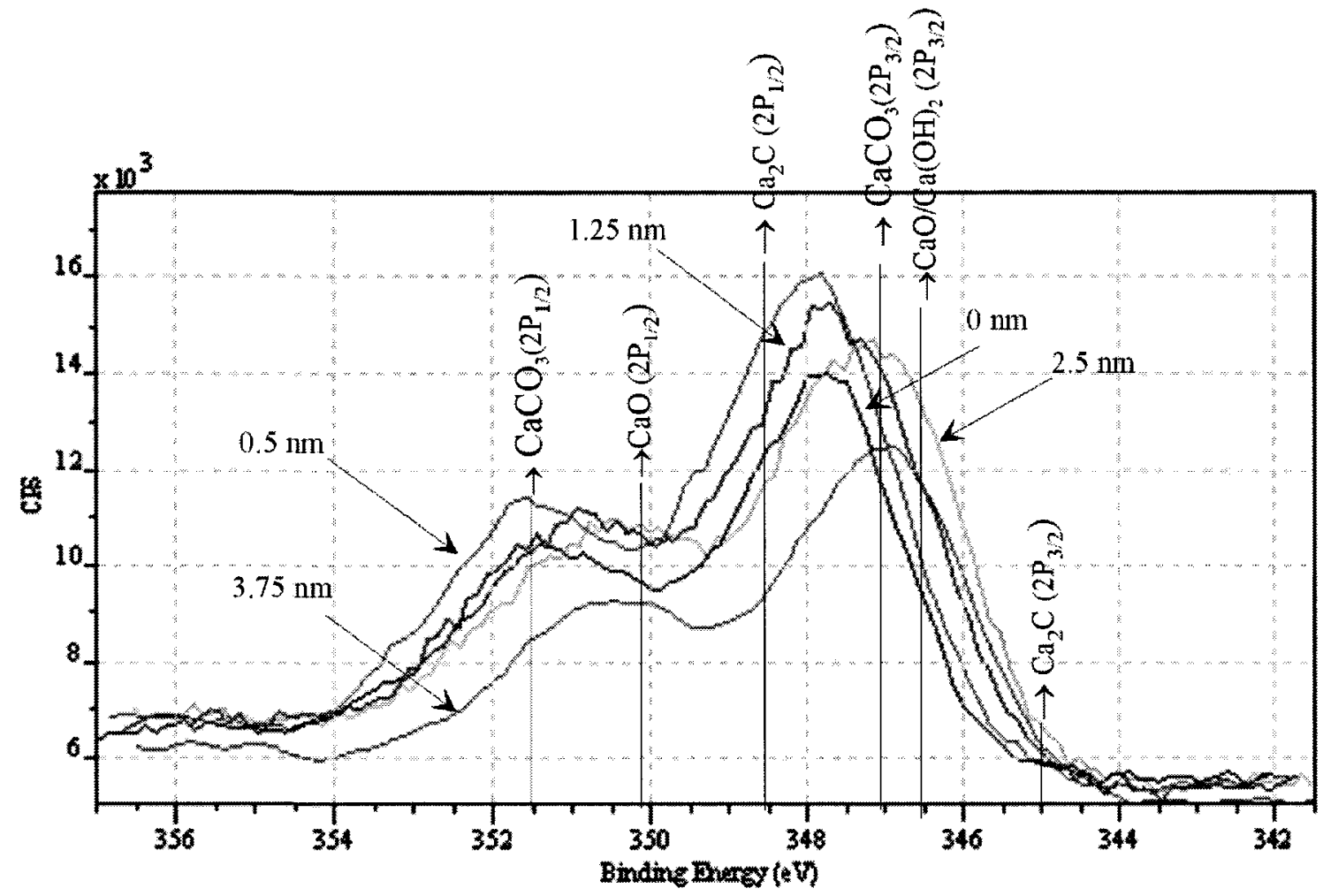

Figure B.10: Ca 2p high resolution XPS spectra at selected sputtered depths for $\mathrm{CH}-2$ specimen and the binding energy position of various calcium-incorporated compounds that are present in the spectra. 


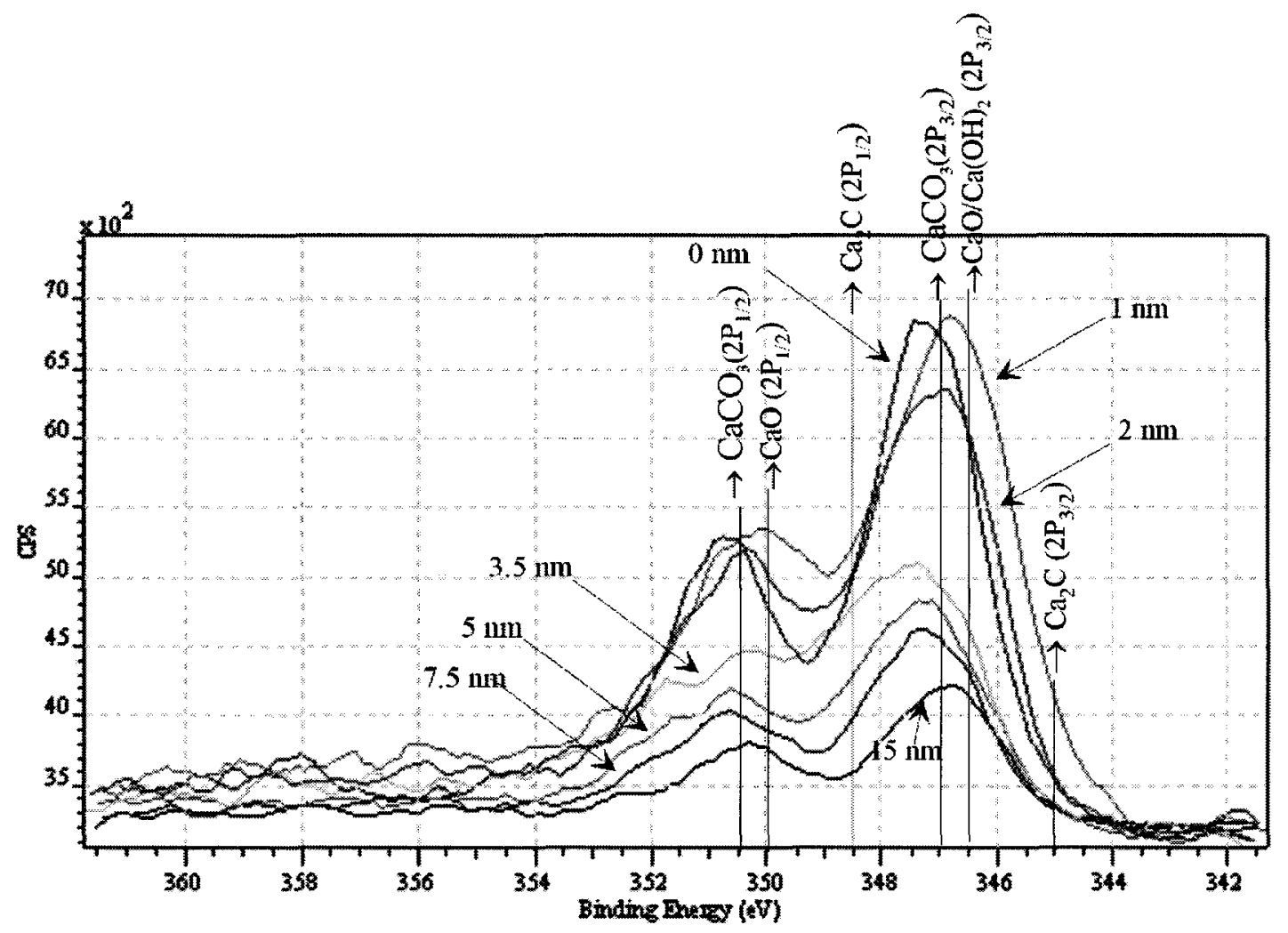

Figure B.11: Ca 2p high resolution XPS spectra at selected sputtered depths for $\mathrm{CH}-9$ specimen and the binding energy position of various calcium-incorporated compounds that are present in the spectra. 


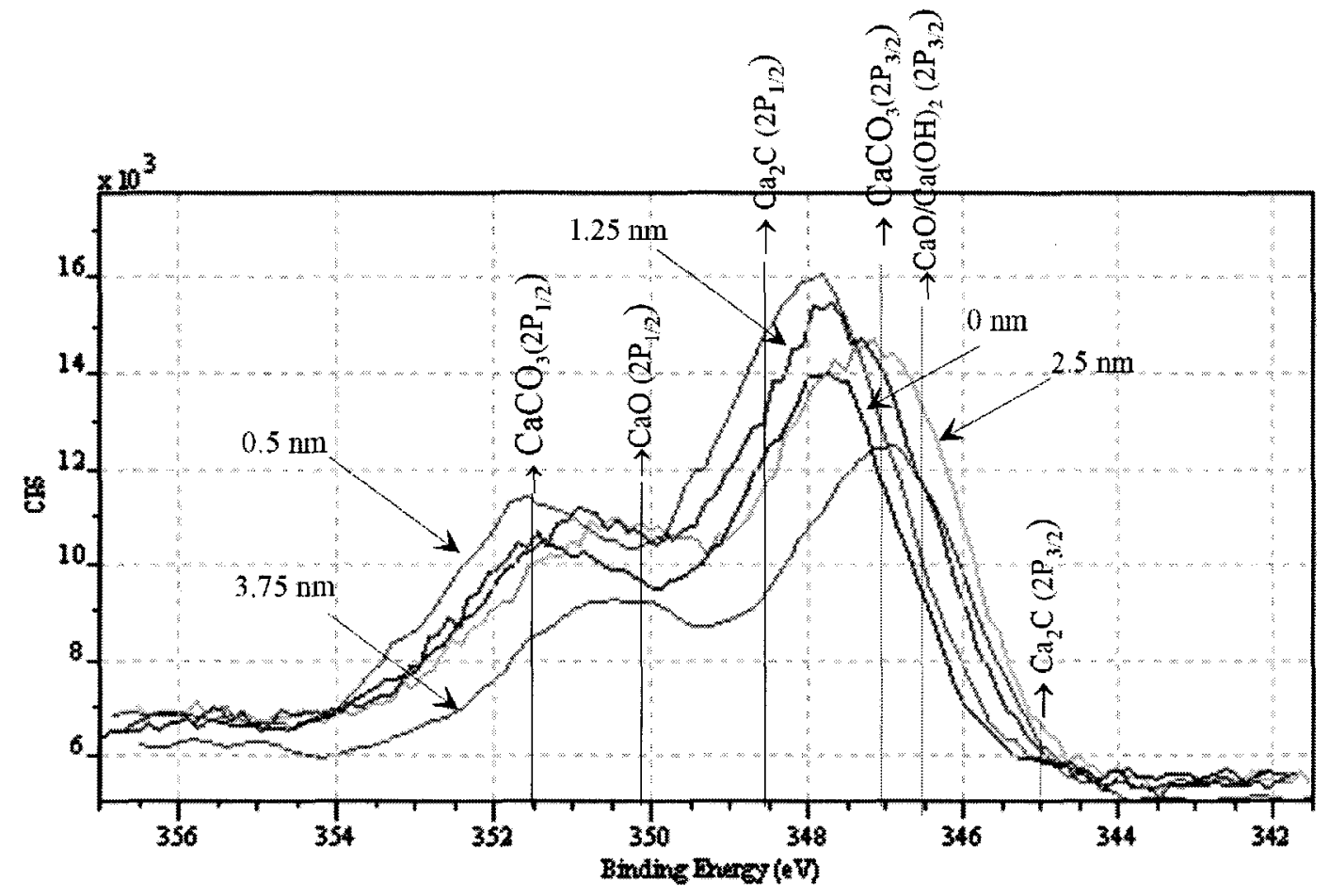

Figure B.12: Ca 2p high resolution XPS spectra at selected sputtered depths for $\mathrm{CH}-\mathrm{Cl}$ specimen and the binding energy position of various calcium-incorporated compounds that are present in the spectra. 


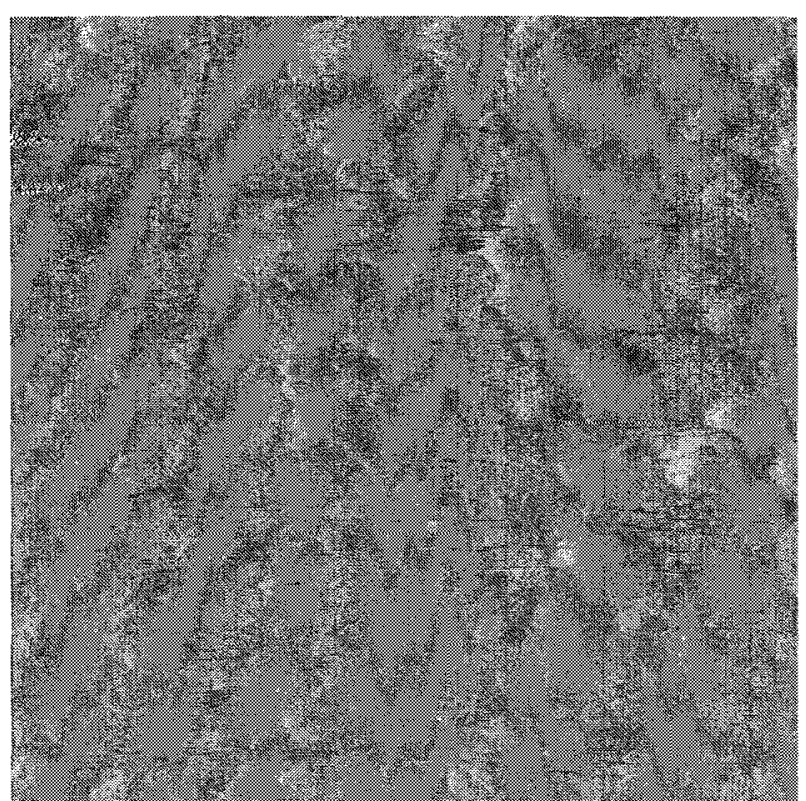

(a)

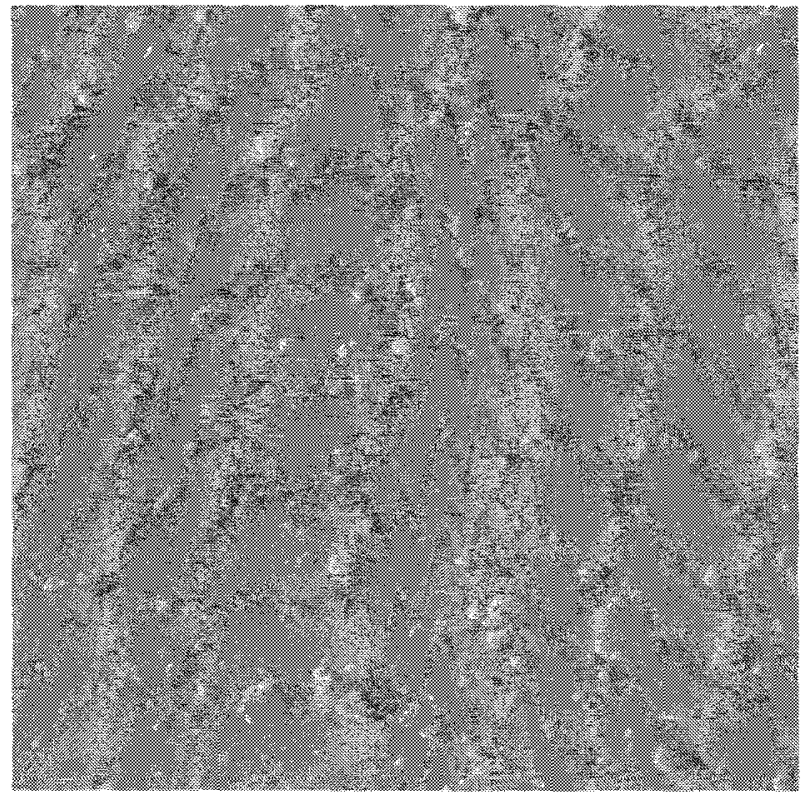

(b)

Figure B.13: AFM topography images of polished rebar cross section: (a) surface area of $1 \mu \mathrm{m} \times 1 \mu \mathrm{m}$; (b) surface area of $10 \mu \mathrm{m} \times 10 \mu \mathrm{m}$, recorded in Contact Mode. 